\title{
L'effectivité du droit à l'eau face au processus de libéralisation du secteur de l'eau en Afrique subsaharienne
}

Citation for published version (APA):

Lawson, N. S. H. A. T. (2012). L'effectivité du droit à l'eau face au processus de libéralisation du secteur de l'eau en Afrique subsaharienne. [Doctoral Thesis, Maastricht University]. Uitgeverij BOXPress. https://doi.org/10.26481/dis.20121115nl

Document status and date:

Published: 01/01/2012

DOI:

$10.26481 /$ dis.20121115nl

Document Version:

Publisher's PDF, also known as Version of record

\section{Please check the document version of this publication:}

- A submitted manuscript is the version of the article upon submission and before peer-review. There can be important differences between the submitted version and the official published version of record.

People interested in the research are advised to contact the author for the final version of the publication, or visit the DOI to the publisher's website.

- The final author version and the galley proof are versions of the publication after peer review.

- The final published version features the final layout of the paper including the volume, issue and page numbers.

Link to publication

\footnotetext{
General rights rights.

- You may freely distribute the URL identifying the publication in the public portal. please follow below link for the End User Agreement:

www.umlib.nl/taverne-license

Take down policy

If you believe that this document breaches copyright please contact us at:

repository@maastrichtuniversity.nl

providing details and we will investigate your claim.
}

Copyright and moral rights for the publications made accessible in the public portal are retained by the authors and/or other copyright owners and it is a condition of accessing publications that users recognise and abide by the legal requirements associated with these

- Users may download and print one copy of any publication from the public portal for the purpose of private study or research.

- You may not further distribute the material or use it for any profit-making activity or commercial gain

If the publication is distributed under the terms of Article $25 \mathrm{fa}$ of the Dutch Copyright Act, indicated by the "Taverne" license above, 


\section{L'EFFECTIVITÉ DU DROIT À L'EAU FACE AU PROCESSUS DE LIBÉRALISATION DU SECTEUR DE L'EAU EN AFRIQUe SUbSAharienNe}

Monsieur N'Sinto H. A. T. LAWSON 
(C) N’Sinto H.A.T. LAWSON, Maastricht 2012

ISBN XXX-XX-XXXX-XXX-X

Printed \& lay-out by: Proefschriftmaken.nl || Printyourthesis.com

Published by: Uitgeverij BOXPress, Oisterwijk 


\title{
L'EFFECTIVITÉ DU DROIT À L'EAU FACE AU PROCESSUS DE LIBÉRALISATION DU SECTEUR DE L'EAU EN AfriQUE SUBSAHARIENNE
}

\author{
DISSERTATION
}

\begin{abstract}
To obtain the degree of Doctor at Maastricht University, on the authority of the Rector Magnificus, Prof. dr. L.L.G. L. Soete in accordance with the decision of the Board of Deans, to be defended in public on Thursday 15 November 2012, at 16.00 hours
\end{abstract}

by

Monsieur N'Sinto H.A.T. LAWSON 


\section{Supervisors}

Prof. Dr. Michael G. FAURE

Prof. Adama KPODAR, Université de Lomé, Togo

\section{Assessment Committee}

Prof. mr. G.E. VAN MAANEN (chairman)

Prof. Xavier THUNIS, Université de Namur

Prof. mr. F.A.M. STROINK 
Les Universités de Lomé et de Maastricht n'entendent donner aucune approbation ni improbation aux opinions émises dans les thèses; ces opinions doivent être considérées comme propres à leurs auteurs. 
A tous ceux qui ont soif et qui sont victimes du manque d'accès à l'eau. 


\section{Remerciements}

Je tiens à remercier tous ceux qui m'ont soutenu moralement et matériellement au cours de ces travaux, plus particulièrement mes directeurs de recherche, Messieurs les Professeurs KPODAR Adama et FAURE Michael dont les conseils et la confiance sans faille ont rendu possible à la fois l'entreprise et l'achèvement de ce travail : qu'ils veuillent bien trouver, dans le présent hommage, l'expression de ma sincère et profonde reconnaissance.

Je tiens également à remercier, pour leurs remarques bienveillantes et utiles, les membres du jury, et au-delà, tous ceux, parents, collègues et amis, qui, chacun à leur manière, ont contribué, par l'aide et les encouragements constants, à l'élaboration de ce document. Une intention particulière à l'endroit des Professeurs SANTOS et KOKOROKO de l'Université de Lomé, de même que les Professeurs DROBENKO à Limoges, HERVOUET à Poitiers et BIOY à Toulouse.

Je ne saurais oublier feu Professeur VIGNON qui est le véritable architecte de ce travail. 
RESUME ET MOTS-CLES - SUMMARY AND KEYWORDS 


\section{Résumé en français}

Considéré comme la nécessité de disposer de suffisamment d'eau potable pour survivre et d'un équipement adéquat pour récupérer les eaux usées, le droit à l'eau peine à trouver ses marques. Et pourtant, rien ne justifie cette nonchalance politique et intellectuelle à l'égard de la matière, car l'eau est incontestablement le premier des besoins essentiels de l'homme. Aussi, importe-t-il de préciser que le manque d'eau constitue la première cause de mortalité dans le monde, devant la malnutrition. L'eau est un enjeu vital pour 2,4 milliards d'individus, qui ne disposent d'aucun assainissement et 1,1 milliard d'hommes n'ont pas accès à l'eau potable. Si le droit à l'eau connaît une affirmation lente et progressive, tel n'est pas le cas du concept de la libéralisation qui est consacré par la plupart des constitutions en vigueur dans les Etats de l'Afrique subsaharienne. L'hésitation de la communauté internationale à consacrer un droit à l'eau non équivoque et effectif, dresse le lit aux partisans de la marchandisation de l'eau. De même, au début des années 1990, les institutions de Bretton Woods, principaux acteurs du développement multilatéral, ont vigoureusement encouragé le recours par les autorités et sociétés publiques à des opérateurs privés pour apporter investissements et efficacité de gestion. Aujourd'hui, à l'heure du bilan l'on découvre un résultat mitigé. Le besoin de rendre effectif ce droit, passe par l'adoption de multiples mesures notamment la protection des ressources en eau et l'urgence de rendre plus lisible les politiques de l'eau.

Mots-clés en français : eau potable, droit à l'eau, libéralisation, service public, Afrique subsaharienne, ressources en eau.

\section{Summary}

Regarded as the need for having sufficient drinking water to survive and adequate equipment to recover used water, the right to water pains to find its marks. And yet, nothing justifies this political and intellectual nonchalance with regard to the matter, because water is incontestably the first of the essential needs for human. As, is essential it to specify that the lack of water constitutes the first cause of mortality in the world, in front of malnutrition. Water is a vital stake for 2,4 billion individuals, who do not have any cleansing and 1,1 billion men does not have access to drinking water. If the right to water knows a slow and progressive assertion, such is not the case of the concept of the liberalization which is devoted by the majority of the constitutions in force in the States of sub-Saharan Africa. The hesitation of the international community to devote an unambiguous and effective right to water, draws up the bed with the partisans of the selling of water. In the same way, at the beginning of the years 1990, the institutions of Bretton Woods, principal actors of the multilateral development, vigorously encouraged the recourse by the authorities and public companies to privates operators to bring investments and efficiency of management. Today, at the moment of the assessment one discovers a mitigated result. The need to make effective this right, passes by the adoption of multiple measurements in particular the protection of the water resources and the urgency to make more readable water policies.

English key words: drinking water, right to water, liberalization, public utility, sub-Saharan Africa, water resources. 
Résumé et mots-clés - Summary and keywords ............................................................... 9

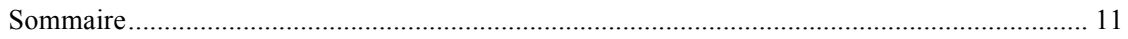

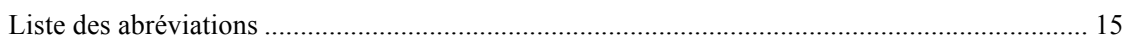

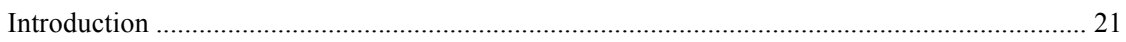

Partie I. La réalisation de la libéralisation du secteur de l'eau ...................................................... 53

Titre I. La pertinence de la désétatisation du secteur de l'eau en Afrique subsaharienne............ 55

Chapitre I. La privatisation du secteur de l'eau juridiquement expliquée................................... 57

Chapitre II. La privatisation du secteur de l'eau économiquement expliquée ............................ 85

Conclusion du titre I de la Ière partie. ............................................................................... 109

Titre II de la Ière partie : Les mécanismes juridiques de la................................................. 111

libéralisation du secteur de l'eau ............................................................................................. 111

Chapitre I. le mode privilégié de la libéralisation du secteur de l'eau : .................................... 113

la délégation du service public de l'eau.................................................................................... 113

Chapitre II. La mise en berne inéluctable des principes du service public .............................. 139

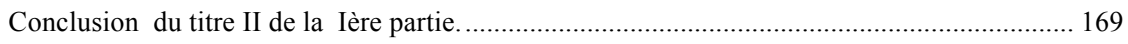

Conclusion de la Ière partie................................................................................................ 171

Partie II. Les aboutissements de la libéralisation du secteur de l'eau ...................................... 173

Titre I. Les appréhensions suscitées par la privatisation du service public de l'eau ................ 175

Chapitre I. Les conséquences néfastes de la libéralisation du secteur de l'eau......................... 179

Chapitre II. La libéralisation du secteur de l'eau,.................................................................... 205

une négation des facteurs de développement ....................................................................... 205

Conclusion du Titre I de la deuxième partie..................................................................... 235

Titre II. La nécessaire amélioration des moyens de l'effectivité du droit à l'eau ................... 239

Chapitre I. Le retour à une étatisation pragmatique du secteur de l'eau .................................. 241

Chapitre II. La nécessité d'une meilleure protection des ressources en eau ............................. 269

Conclusion du Titre II de la deuxième partie. ....................................................................... 295

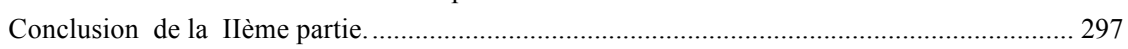

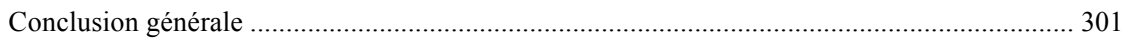

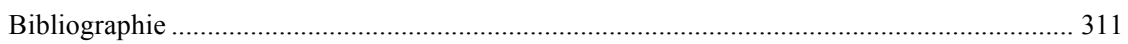

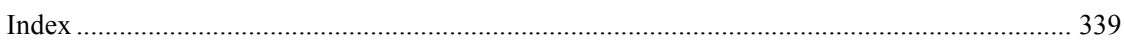

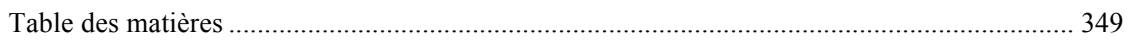


ABN : $\quad$ Autorité du Bassin du Niger

ACDI : $\quad$ Annuaire de la Commission du droit international

ADI : $\quad$ Association de droit international

AFDI : $\quad$ Annuaire français de droit international

Aff.: $\quad$ affaire

AGNU : $\quad$ Assemblée générale des Nations Unies

AJDA : $\quad$ Actualité juridique du droit administratif

AJIL: $\quad$ The American Journal of International Law

al. : $\quad$ Alinéa

AN : $\quad$ Assemblée nationale

ARM : $\quad$ Autorité de Régulation Multisectorielle

Art. : $\quad$ article

Ass. : $\quad$ Assemblée du contentieux du Conseil d'Etat

BAD : $\quad$ Banque Africaine de Développement.

BIT : $\quad$ Bureau International du Travail

BJCL : $\quad$ Bulletin juridique des collectivités locales

$\quad B J C P$ : $\quad$ Bulletin juridique des contrats publics

BO : $\quad$ Bulletin officiel

Bull. civ. : $\quad$ Bulletin des arrêts de la Cour de cassation, chambres civiles

C. civ : $\quad$ Code civil

CAA : $\quad$ Cour administrative d'appel

CAA : $\quad$ Cour administrative d'appel

Cass. civ. : Cour de cassation, chambre civile

Cass. Com. : Cour de cassation, chambre commerciale

Cass. soc. : Cour de cassation, chambre sociale 
Cass. : $\quad$ Cour de cassation

CBLT : $\quad$ Commission du Bassin du Lac Tchad.

CC : $\quad$ Conseil constitutionnel

CC : $\quad$ Conseil constitutionnel

CDI : $\quad$ Commission du droit international

CE : $\quad$ Conseil d'Etat

CE : $\quad$ Conseil d'Etat

CEDE : $\quad$ Conseil Européen du Droit de l'Environnement

CEEAC : $\quad$ Communauté Economique des Etats de l'Afrique

CEMAC : Communauté Economique et Monétaire de l'Afrique Centrale.

CESCR : $\quad$ Comité des droits économiques, sociaux et culturels.

$C f .: \quad$ Confère.

Chron. : $\quad$ Chronique

CIJ : $\quad$ Cour International de la Justice.

CIRDI : $\quad$ Centre international de règlement des différends relatifs à l'investissement

CITES : $\quad$ Convention sur le Commerce International des Espèces de

CJCE : $\quad$ Cour de Justice des Communautés Européennes

CNUCED: Conférence des Nations unies sur le Commerce et le Développement

CODESC : Comité des droits économiques, sociaux et culturels

Coll. : $\quad$ Collection

Concl. : $\quad$ Conclusions

CPJI : $\quad$ Cour Permanente de Justice Internationale

D. : $\quad$ Recueil Dalloz

DIE : $\quad$ Droit International de l'Environnement.

Dir. : $\quad$ sous la direction de. 
Doc. Document.

DSP : $\quad$ Délégation de service public

éd. : $\quad$ éditions

EDCE : $\quad$ Etudes et documents du CE

FAC : $\quad$ Fonds d'Aide et de Coopération et la Culture.

Fasc. : $\quad$ fascicule

FEM : $\quad$ Fonds pour l'Environnement Mondial.

FRS : $\quad$ Fonds pour la recherche stratégique.

GAJA : Grands arrêts de la jurisprudence administrative

GWP: Global Water Partnership

Ibid. : $\quad$ Ibidem

Id. : $\quad$ Idem

IDEF : $\quad$ Institut International de Droit d'Expression et d'Inspiration Françaises.

IDI : $\quad$ Institut de Droit International

IEC : $\quad$ Information Education et Communication.

JOCE : Journal Officiel des Communautés Européennes

LGDJ : $\quad$ Librairie générale de droit et de jurisprudence

LPA : $\quad$ Les Petites Affiches

NEPAD : $\quad$ Nouveau Partenariat pour le Développement de l'Afrique

OCDE : $\quad$ Organisation de Coopération et de Développement Economiques

OMD : $\quad$ Objectifs du Millénaire pour le Développement

OMVS : $\quad$ Organisation de Mise en Valeur du Fleuve Sénégal.

ONU : $\quad$ Organisation des Nations Unies.

Op. cit. : ouvrage précité

p. : $\quad$ page 
Penant : $\quad$ Revue Penant

PIDCP : $\quad$ Pacte international relatif aux droits civils et politiques.

PIDESC : Pacte International relatif aux droits économiques, sociaux et culturels.

Préc. : $\quad$ précité

RCADI : $\quad$ Recueil des cours de l'Académie de Droit International

RGDIP : $\quad$ Revue générale de droit international public

RICR : $\quad$ Revue internationale de la Croix -Rouge

RSA : $\quad$ Recueil des sentences arbitrales

RTDE : $\quad$ Revue trimestielle de droit européen

RUDH : $\quad$ Revue universelle des droits de l'homme

S. : $\quad$ Recueil Sirey

SEEG : $\quad$ Société d'énergie et d'eau du Gabon

SPEN : $\quad$ Société de Patrimoine des Eaux du Niger

t. : $\quad$ tome

TA : $\quad$ Tribunal administratif

TC : $\quad$ Tribunal des conflits

UA : $\quad$ Union Africaine.

UDEAC : Union Douanière et Economique des Etats de l'Afrique

UICN : $\quad$ Union Internationale pour la Conservation de la nature.

UNESCO : Organisation des Nations pour l'Education, la Science

UREF : $\quad$ Université des réseaux d'expression Française

V. : $\quad$ voir

Vol. : $\quad$ Volume 
INTRODUCTION 
«Eau, tu n'as ni goût, ni couleur, ni arôme, on ne peut pas te définir, on te goûte, sans te connaître. Tu n'es pas nécessaire à la vie tu es la vie $»^{1}$. Cette affirmation révèle que l'eau présente une caractéristique très particulière. Elle est incontestablement un bien très essentiel et indispensable non seulement à la vie humaine mais aussi à l'univers tout entier. DROBENKO Bernard écrira à cet effet que «le vivant a besoin d'eau pour exister et préserver ses équilibres, qu'il s'agisse des plantes, des animaux y compris les humains... $»^{2}$ et d'ajouter que «disposer d'eau potable ${ }^{3}$ constitue bien une exigence de vie ». Bien plus, nous pouvons citer le Rapport MALANDAIN selon lequel : "celui qui contrôle le point d'eau dispose du droit à la vie et du pouvoir sur les hommes. Tout au long de notre histoire, l'eau a déterminé l'organisation sociale et politique des peuples $»^{4}$. L'eau est nécessaire à des fins diverses : les usages personnels et domestiques, la production alimentaire et pour l'hygiène du milieu. Elle est essentielle pour obtenir des moyens de subsistance et pour exercer certaines pratiques culturelles et cultuelles. Néanmoins, les ressources en eau doivent être affectées en priorité aux usages personnels et domestiques. Car, l'on a l'impression que l'eau est abondante à surface de la planète Terre. Mais, la réalité, c'est que $98 \%$ de cette eau apparemment abondante est salée. Dans la préface consacrée par LASSERRE Frédéric à l'ouvrage intitulé «Eau douce, la nécessaire refondation $d u$ droit international ${ }^{5}$ » rédigé par PAQUEROT Sylvie, le parrain de l'auteur affirme que «même si l'eau douce constitue seulement $2 \%$ de l'eau totale et qu'un maigre 0,02\% de l'eau totale est mobilisable pour les usages des sociétés humaines, cette quantité d'eau douce disponible représente malgré tout environ $6900 \mathrm{~m} 3$ par personne et par an, un volume très appréciable. Mais cette moyenne statistique renferme de très grandes disparités ». Des disparités parfois criantes voire scandaleuses sur lesquelles nous reviendrons dans les développements ultérieurs.

1 De ST-EXUPERY A., Terre des hommes, Folio, Gallimard, 1991.

2 DROBENKO B., Le droit à l'eau : une urgence humanitaire, Editions Johanet, Paris 2010, p.5

3 Selon les directives de l'OMS, la notion d'eau potable doit être comprise comme une quantité d'eau suffisante, saine, salubre, exempte de pollution microbienne ou chimique, sans risque pour la santé.... $C f$. DROBENKO B. précité.

4 Rapport MALANDAIN, Doc. Ass. nat., n² 2381, nov. 1991.

5 PAQUEROT S., Eau douce, la nécessaire refondation du droit international, Presses de l'Université de Québec, 2005 . 


\section{Qu'est-ce que le droit à l'eau?}

Le droit à l'eau ${ }^{6}$ consiste à assurer l'accès à l'eau potable ${ }^{7}$ et à l'assainissement de la même façon que le droit à la nourriture vise à garantir l'accès à la nourriture et le droit à la santé à assurer l'accès aux soins de santé. L'eau potable comme la nourriture et les soins de santé sont indispensables pour satisfaire le «droit à un niveau de vie suffisant» reconnu par tous les États dans la Déclaration universelle des droits de l'homme en 1948.

Conformément au droit international et au droit national, les pouvoirs publics sont soumis à des obligations importantes concernant l'approvisionnement en eau potable et l'assainissement des eaux usées ${ }^{8}$. Ce droit concerne une quantité limitée d'eau qui permette à l'homme de satisfaire à ses besoins essentiels tels que la boisson, la préparation de la nourriture, l'hygiène et le nettoyage, d'assurer l'abreuvement des animaux de compagnie et d'arroser une petite production vivrière familiale (potager et quelques animaux domestiques) ${ }^{9}$. Le droit à l'eau concerne plus particulièrement les personnes qui n'ont pas accès à l'eau, c'est-à-dire principalement les personnes les plus démunies dans les pays développés et les personnes non desservies en eau dans les pays en développement (zones périurbaines et zones rurales) ${ }^{10}$.

L'affirmation d'un droit suppose de parvenir à cerner précisément son objet, mais pas seulement, puisque l'objet n'est qu'un des éléments constitutifs de ce que doit être un droit. De manière classique, un droit se définit par la réunion de quatre éléments que sont la détermination de son objet, de son titulaire, de celui ou de ceux auxquels il est opposable et enfin, de recours

\footnotetext{
6 Henri SMETS, Le droit à l'eau, Rapport pour le Conseil Européen du droit de l'environnement, 2002, p.37.

$C f$. également la définition proposée par M. J.ZIEGLER dans son rapport sur le droit à l'alimentation présente à l'Assemblée générale des Nations unies (A/56/210) : «Le droit à l'alimentation est le droit d'avoir un accès régulier, permanent et libre, soit directement, soit au moyen d'achats monétaires, à une nourriture quantitativement et qualitativement adéquate et suffisante ». Selon les conclusions du Stockholm Water Symposium (août 2000), « Human right to water basically refers to provision of a minimum amont of safe water required for basic human needs ».
}

7 Selon le Rapport mondial sur le développement humain (2006), plusieurs paramètres déterminent cette qualité, à savoir des paramètres biologique, chimique, organoleptique ou physique,.. Cf. p.51.

8 Art.22 du Pacte international sur les droits économiques, sociaux et culturel « toute personne... est fondée à obtenir la satisfaction des droits économiques, sociaux et culturels indispensables à sa dignité...compte tenu de l'organisation et des ressources de chaque pays. »

9 La Convention des Nations unies sur le droit relatif aux utilisations des cours d'eau internationaux à des fins autres que la navigation (New York, 1997) demande qu'une attention spéciale soit « accordée à la satisfaction des besoins humains essentiels» (art.10) étant entendu qu'il s'agit de la «fourniture d'eau en quantité suffisante pour la vie humaine, qu'il s'agisse de l'eau potable ou de l'eau à réserver aux productions vivrières destinées à empêcher la famine ».

Dans beaucoup de pays, la loi établit une priorité des usages domestiques de l'eau sur les autres usages. En cas de pénurie, il faut réduire les usages non essentiels tels que l'arrosage des jardins ou le nettoyage des voitures ainsi que certains usages agricoles ou hydro-électriques.

10 «.... l'appauvrissement des pays pauvres et la diminution de l'aide au développement rendent le problème de l'accès à l'eau encore plus aigu dans ces pays. »Cf. Henri SMETS. 
pour en assurer la garantie ${ }^{11}$. Les propositions de l'Observation $\mathrm{n}^{\circ} 15^{12}$ ont permis d'esquisser la définition du droit à l'eau et de dégager les critères normatifs.

\section{a. L'esquisse de la définition du droit à l'eau}

L'Observation Générale ${ }^{\circ} 15$ a été longtemps la seule expression du droit à l'eau existant qui faisait autorité, et cette interprétation n'est pas juridiquement contraignante. Le droit à l'eau est « un nouveau droit» de la personne qui sera établi et précisé par les États au fil du temps. L'Observation Générale ${ }^{\circ} 15$ stipule que « le droit à l'eau consiste en un approvisionnement suffisant, physiquement accessible et à un coût abordable, d'une eau salubre et de qualité acceptable pour les usages personnels et domestiques de chacun ».

Selon l'Observation Générale ${ }^{\circ} 15$, le droit à l'eau vise à assurer " une quantité adéquate d'eau salubre [...] pour prévenir la mortalité due à la déshydratation et pour réduire le risque de transmission de maladies d'origine hydrique ainsi que pour la consommation, la cuisine et l'hygiène personnelle et domestique ». L'Observation Générale $n^{\circ} 15$ se rapporte aux ressources en eau requises pour prévenir la famine et les maladies, de même qu'à l'eau requise pour satisfaire aux obligations fondamentales relatives aux droits reconnus par le PIDESC (Pacte International relatif aux droits économiques, sociaux et culturels) $)^{13}$.

Il faut préciser que le concept du droit à l'eau n'avait pas été véritablement défini par la doctrine ni par le législateur. L'on constate que la notion est souvent couplée à celle du droit à l'assainissement, ce qui ne rend pas aisée sa définition. Pour DROBENKO B., ce droit est compris comme la nécessité de disposer de suffisamment d'eau potable pour survivre et d'un équipement pour récupérer les eaux usées ${ }^{14}$. Pour SMETS H., le droit à l'eau couvre un ensemble de droits et d'obligations en matière d'approvisionnement en eau potable et d'assainissement. Il figure dans des dispositions législatives et réglementaires et même dans certaines Constitutions nationales. Il est parfois associé au droit à l'environnement, au droit à la santé ou à la vie et au droit à la protection des ressources naturelles. La mise en œuvre du droit à l'eau signifie que les États clarifient les obligations en matière d'eau et consacrent plus de moyens à l'alimentation en eau saine et à l'assainissement ${ }^{15}$.

Cette définition se fonde notamment sur l'affirmation du CEDE (Conseil européen du droit de l'environnement) que « toute personne a droit à l'eau en quantité et de qualité suffisantes pour sa vie et sa santé » et sur l'affirmation de l'Académie de l'Eau d'un «droit imprescriptible à l'eau pour tous ».

11 PONTHOREAU M.-C., « Constitutions, juges constitutionnels et droit à l'environnement » in L'environnement à quel prix? Sous la direction de Ejan MACKAAY et Hélène TRUDEAU, Les Editions Thémis, Université de Montréal, p.320

12 Le Comité des droits économiques, sociaux et culturels, dans son Observation générale $n^{\circ} 15$ en 2002.

13 Cf. Rapport de l'experte indépendante, de ALBUQUERQUE C., A/HRC/15/31/Add.1

14 DROBENKO B., Le droit à l'eau : une urgence humanitaire, Editions JOHANET, 2010.

15 SMETS H., Le droit à l'eau, précité, p.37 


\section{b. L'énoncé des critères normatifs}

Les critères normatifs, servant à déterminer si une pratique est bonne, sont fondés sur le contenu normatif des droits de l'homme qui concernent l'accès à l'eau et à l'assainissement. Le Comité des droits économiques, sociaux et culturels, dans son Observation générale $n^{\circ} 15$, décrit ce en quoi consiste le droit à l'eau, et l'experte indépendante, dans son rapport sur la question des obligations en rapport avec les droits de l'homme qui concernent l'accès à l'assainissement ${ }^{16}$, décrit le contenu normatif du droit concernant l'accès à l'assainissement. L'experte indépendante a décidé de s'appuyer sur ce cadre pour définir les critères normatifs des bonnes pratiques, à savoir: disponibilité, qualité/sûreté, acceptabilité, accessibilité et coût abordable.

Ces catégories sont étroitement liées entre elles et certaines pratiques répondent à plusieurs critères normatifs ou à un seul. Par exemple, une tarification qui tient compte des difficultés particulières auxquelles sont confrontées les personnes vivant dans la pauvreté peut être évaluée sous l'angle du coût abordable, d'autres critères se révélant ici moins pertinents. Parallèlement, une approche sectorielle relèverait de l'ensemble des critères normatifs. Les pratiques qui contribuent à l'exercice des droits à l'eau et à l'assainissement en faisant jouer au moins un de ces critères seront examinées par l'experte indépendante au moment de dresser son inventaire, pour autant que celles-ci ne faussent ni ne contredisent les autres critères ${ }^{17}$.

En ce qui concerne la qualité de l'eau, l'observation exige que l'eau doive être salubre et acceptable. Enfin, l'accessibilité de l'eau devra répondre aux conditions ci-après: une accessibilité physique (physiquement accessible sans danger), accessibilité économique (offerte à un coût abordable), une non-discrimination (son usage ne doit pas donner lieu à de la discrimination et certains groupes à risque doivent faire l'objet d'une " attention spéciale ») et une accessibilité de l'information (les personnes doivent être en mesure " de rechercher, de recevoir et de répandre des informations concernant les questions relatives à l'eau ». Les États devraient progressivement veiller à ce que chacun ait accès aux services d'eau et que la distribution de l'eau soit équitablement assurée. Lorsque des ressources suffisantes ne sont pas disponibles pour garantir un service de haute qualité, les États devraient d'abord investir dans des services qui donnent la priorité aux besoins des personnes sans accès de base, normalement à travers des services peu coûteux qui peuvent être améliorés plutôt que des services coûteux dont ne bénéficie qu.une faible part de la population. Le Comité confirme que les États parties ont l'obligation fondamentale minimum d'assurer, au moins, la satisfaction de l'essentiel de chacun des droits énoncés dans le Pacte. De l'avis du Comité, il s'agit au minimum d'assurer l'accès à des moyens élémentaires de soins, d'hébergement et de logement ${ }^{18}$.

En ce qui concerne l'assainissement et l'accessibilité en eau salubre et potable, cette obligation minimum consisterait en un approvisionnement suffisant pour prévenir la déshydratation et les maladies ${ }^{19}$. Évidemment, tel que stipulé à l'article 2 (2) du Pacte, cet accès

\section{$16 \mathrm{~A} / \mathrm{HRC} / 12 / 24$}

17 de ALBUQUERQUE C., précité.

18 CESCR, La nature des obligations des États parties, 1990, Observation générale $n^{\circ} 3,14$ décembre, $5^{\text {ème }}$ session E/1991/23, par. 10

19 World Health Organization, op . cit. 
doit être exercé sans discrimination et de façon égalitaire entre les hommes et les femmes ${ }^{20}$. L'État doit donc assurer un minimum de respect des droits fondamentaux et est tenu d'améliorer progressivement le plein exercice des droits relatifs à l'eau potable ${ }^{21}$.

De même, l'article 4 du PIDESC permettant aux États d'établir une certaine limitation aux droits mentionnés, ne peut être invoquée afin de justifier ou limiter l'accès à l'eau potable. En effet, comme le mentionne le Comité du PIDESC : « le Comité tient à souligner que la clause de limitation figurant à l'article $4 \mathrm{du}$ Pacte vise essentiellement à protéger les droits des individus plutôt qu'à permettre aux États de les limiter $»^{22}$.

Chaque État doit s'assurer que les populations ne seront pas privées d'eau du fait d'un changement dans les usages ou de modification des pratiques agricoles ${ }^{23}$. Ce principe découle de celui de la non régression selon lequel on ne peut restreindre les droits acquis sauf en cas de circonstances exceptionnelles. L'obligation de mettre en œuvre comprend trois obligations principales, soit faciliter, promouvoir et assurer l'accès à l'eau. L'obligation de faciliter requiert de l'État qu'il prenne des mesures positives visant à permettre à toutes les populations d'exercer pleinement leur droit à l'eau, notamment par des infrastructures adéquates et par l'adoption de politiques nationales. L'obligation de promouvoir implique que les États diffusent de l'information sur l'utilisation hygiénique de l'eau, sur les dangers reliés à la consommation d'eau insalubre, sur la protection des sources d'eau et sur les méthodes permettant de réduire le gaspillage. Les États doivent finalement assurer la réalisation du droit à l'eau, particulièrement envers les populations vulnérables qui sont le plus susceptibles de voir leur droit brimé24.

À cette fin, les États doivent donc adopter des mesures législatives, se doter d'une stratégie et d'un plan d'action pour l'eau, et, enfin, veiller à ce que l'eau soit accessible à tous à un coût abordable. Ainsi, le prix des services doit être établi sur la base du principe de l'équité afin que ces services, qu'ils soient fournis par des opérateurs publics ou privés soient abordables pour tous, y compris pour les communautés défavorisées ${ }^{25}$. D'ailleurs, la privatisation des services d'eau n'enlève pas les obligations incombant aux États relatives au respect, à la protection et à la mise en œuvre du droit à l'eau potable. En plus des critères normatifs, il faut ajouter les critères intersectoriels notamment la non-discrimination, la participation et la responsabilité sont des éléments fondamentaux des droits de l'homme qui ont conjointement pour effet de donner les moyens d'agir aux personnes désarmées, marginalisées ou exclues.

20 CESCR, op. cit., par. 13.

21 SMETS H., op. cit., p. 35.

22 CESCR, op. cit., par. 28.

23 SMETS H., Ibidem.

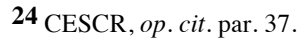

25 Idem, par. 27 : L'équité exige que l'eau ne représente pas une part excessive des dépenses des ménages les plus pauvres par rapport aux ménages plus aisés. 


\section{La délimitation du concept du droit a l'eau}

Le problème de la reconnaissance du droit à l'eau se trouve parfois aggravé par une confusion sémantique. Le droit à l'eau se retrouve noyé des termes proches mais qui n'ont pas forcément la même signification. Et sa propension à se rendre incontournable dans la mise en œuvre d'autres droits de l'homme fait de lui, plus qu'un corollaire, un droit catalyseur. Il s'agira de préciser le concept puis de démontrer que le droit à l'eau n'est pas un droit autonome.

\section{£. La précision de concept}

A s'en tenir à la définition du Comité des droits économiques, sociaux et culturels, le droit à l'eau consiste en un approvisionnement suffisant, physiquement accessible et à un coût abordable, d'une eau salubre et de qualité acceptable pour les usages personnels et domestiques de chacun ${ }^{26}$. De nombreuses conditions sont donc nécessaires pour parler du droit à l'eau et cela facilite l'amalgame qui se fait entre les concepts proches mais différents du droit à l'eau. Il s'agit du droit d'accès à l'eau potable, du droit de l'homme à l'eau, de l'accès à l'eau, de l'approvisionnement en eau, du droit à l'assainissement et bien d'autres encore. Le paradoxe, c'est que dans la majorité des textes ayant abordé la question du droit à l'eau, très peu l'appréhende sous cette expression. D'une part, on peut relever que la tendance est plus du côté du droit d'accès à l'eau ${ }^{27}$.

En réalité, toutes ces notions signifient la même chose : permettre à tous les citoyens d'avoir accès à l'eau potable. La seule explication qui permet de comprendre cette variabilité de terminologie se situe au niveau de la portée du droit à l'eau. En effet, les Etats sont réticents à l'utilisation du droit à l'eau pour éviter d'avoir à répondre des obligations qui y sont inhérentes.

Toutefois, il faut relever que le droit à l'assainissement doit être distingué du droit à l'eau car si les deux sont complémentaires, ils n'ont pas le même objet. L'accès à l'assainissement vise à faire en sorte que chacun ait effectivement accès à des installations d'assainissement acceptables et à un prix abordable qui permettent de satisfaire aux exigences en matière d'hygiène, de dignité humaine, de santé publique et de protection de l'environnement ${ }^{28}$.

La liaison étroite entre droit à l'eau et droit à l'assainissement est inscrite dans certaines Constitutions et dans des lois internes. Le traitement des eaux résiduaires urbaines est considéré comme indispensable dans les pays développés d'autant plus que l'approvisionnement en eau augmente la quantité d'eaux usées.

\footnotetext{
26 Paragraphe 2.

27 Le droit d'accès à l'eau potable et à l'assainissement a été analysé dans plusieurs rapports de la Sous-commission de la promotion et de la protection des droits de l'homme. Il apparaît dans de nombreuses conventions internationales dont le Protocole sur l'eau et la santé (Londres, 1999) qui vient d'entrer en vigueur pour l'Europe.
}

28 SMETS H., idem. 


\section{$\alpha$. La différence entre droit à l'eau et droit de l'eau}

Contrairement à la confusion générale parfois constatée même dans les milieux juridiques, le droit à l'eau est différent du droit de l'eau. Assimiler le droit à l'eau au droit de l'eau ou l'inverse, reviendrait à faire l'amalgame qui est souvent fait entre le droit de l'environnement et le droit à l'environnement ou encore le droit de la santé et le droit à la santé29. Cet amalgame consiste à confondre les droits de l'eau (Water Rights) que possèdent certaines personnes ou collectivités en rapport avec une ressource en eau avec le droit à l'eau potable (Right to Drinking Water) dont devrait bénéficier chaque individu sur une petite partie de l'eau ${ }^{30}$. Comme il a été précédemment défini, le droit à l'eau c'est cette faculté de l'homme à prétendre à un approvisionnement suffisant d'une eau salubre et facilement accessible. Par contre, le droit de l'eau regroupe d'une part l'ensemble des règles régissant la protection des ressources en eau et d'autre part, établit « des processus d'entente et de règlements des différends à propos des eaux partagées entre plusieurs Etats $»^{31}$. Deux objets distincts, mais l'effectivité du premier nécessite que le second soit réalisé. Cela suffit-il pour autant que c'est deux droits soient confondus?

Si le droit à l'eau a des détracteurs, le droit de l'eau est aussi souvent contesté quant à son existence même et en tant qu'ordre juridique autonome. Considéré comme un droit international en construction ${ }^{32}$, le droit de l'eau a été développé à partir des exigences liées à l'usage ou des contraintes résultant de l'appropriation de l'eau ou des milieux ; ces exigences apparaissent complexes et diversifiées. En effet, les questions posées par l'eau, « or bleu » du XXI ${ }^{\mathrm{e} m e}$ siècle, relèvent à la fois de préoccupations quantitatives (risques d'inondations par des excès ou risques de sécheresse par pénurie) et qualitatives (en terme de milieu et en terme de potabilité), sachant que l'eau est quantitativement limitée et non renouvelable. Pour Alan SAOUT ${ }^{33}$, les origines du droit de l'eau remontent à l'Antiquité et il s'est construit par strates successives et non linéaires. En effet, le manque d'unité de la matière a longtemps été illustré par le refus du juriste de recourir à l'expression " droit de l'eau » au profit d'autres appellations, telles que " régime juridique des eaux » ou " droits sur les eaux ». Aujourd'hui cependant, l'expression est utilisée communément, sans toutefois qu'une véritable définition de la matière n'ait été dégagée.

Ce droit de l'eau s'inscrit aujourd'hui à la fois dans un schéma international balbutiant, un cadre régional déterminant et une approche nationale en évolution ${ }^{34}$. Pour corroborer cette affirmation, on peut citer, l'exemple la Convention entre le Conseil fédéral suisse et le

29 On peut multiplier les exemples, le droit du travail et le droit au travail, droit à l'éducation et droit de l'éducation...

30 SMETS H., op.cit.p 27.

31 POYDENOT A., «Le droit international de l'eau, état des lieux », Les Notes d'analyse du CIHEAM, n²9 février 2008, p. 4.

32 DROBENKO B., Droit de l'eau, Gualino éditeur, 2007, p.25.

33 Théorie et pratique du droit de l'eau, Johanet, 2011, 474 p.

34 DROBENKO B., idem, p.331. 
Gouvernement de la République française concernant la protection des eaux du lac Léman contre la pollution ${ }^{35}$. Toutefois, il ne faut pas perdre de vue que le droit à l'eau est un droit composite.

\section{$\beta$. Les imbrications du droit a l'eau (Le droit à l'eau, un droit non autonome)}

L'année 1990 verra l'adoption par la communauté internationale des ONG (organisation non gouvernementale), de la Charte de Montréal, qui s'ouvre sur l'affirmation du droit d'accès à l'eau et de son caractère indissociable des autres droits humains ${ }^{36}$. Il importe de démontrer que le droit à l'eau est au carrefour de nombreux droits dont le droit à la santé et le droit au logement.

\section{$\gamma$. L'importance du droit à l'eau dans le droit à la santé}

Reconnu pour la première fois en $1946^{37}$ au cours de la création de l'Organisation Mondiale de la Santé (OMS), le droit à la santé est proclamé aujourd'hui proclamé par presque tous les Etats membres de l'organisation mondiale. Pour l'OMS, «la possession du meilleur état de santé qu'il est capable d'atteindre constitue l'un des droits fondamentaux de tout être humain, quelles que soient sa race, sa religion, ses opinions politiques, sa condition économique ou sociale $\rangle^{38}$. Ce que confirme la Charte Africaine des droits de l'homme et des peuples, adoptée le 27 juin 1981 à Nairobi au Kenya, lors de la XVIII ${ }^{\text {ème }}$ Conférence de l'Organisation de l'Unité Africaine (OUA) ${ }^{39}$. De même, de nombreux sommets onusiens se référent au droit à la santé. Il faut noter que le droit à la santé figure dans plusieurs paragraphes de la Déclaration et du Programme d'action de Vienne ${ }^{40}$. Le Programme d'action de la Conférence internationale sur la population et le développement ${ }^{41}$ et la Déclaration et le Programme d'action de la quatrième Conférence mondiale sur les femmes contiennent également des définitions concernant, respectivement, la santé génésique et la santé des femmes. D'où il est important, de souligner

35 Conclue à Paris le 16 novembre 1962.

36 PAQUEROT S., précité, p. 234.

37 Confère le rapport de la conférence internationale de la santé qui s'est tenue à New York du 19 juin au 22 juillet 1946. UN doc-E/772 du 11 mars 1947.

38 World Health Organization, The right to water, 2003, p.8,

39 Article 16: «1. Toute personne a le droit de jouir du meilleur état de santé physique et mentale qu'elle soit capable d'atteindre. 2. Les Etats parties à la présente Charte s'engagent à prendre les mesures nécessaires en vue de protéger la santé de leurs populations et de leur assurer l'assistance médicale en cas de maladie ».

$40 \S 11,18,24,31$ et 41 notamment, adoptées par la Conférence mondiale sur les droits de l'homme, tenue à Vienne du 14 au 25 juin 1993.

41 Tenue au Caire du 5 au 13 septembre 1994. 
tout comme Halfdan MAHLER ${ }^{42}$, que «le nombre de robinets par milliers de personnes deviendra un meilleur indicateur de santé que le nombre de lits des hôpitaux $»^{43}$.

Qu'est-ce le droit à la santé ? «A priori, il paraît saugrenu de parler de la santé en tant que droit quand la réalité indique qu'une portion croissante de la population mondiale voit plutôt ses conditions de santé se dégrader et même son existence être menacée... ${ }^{44}$. Aussi, faut-il préciser que le droit à la santé n'est pas synonyme du droit de la santé. En effet, ce dernier «correspond à l'ensemble des règles juridiques qui s'appliquent aux activités de la santé. Ces activités peuvent être curatives ou non médicales comme pharmaceutiques ou autres $»^{45}$. Selon J. MOREAU ${ }^{46}$, le droit à la protection de la santé comprend certaines composantes évidentes et générales, sur lesquelles il ne semble pas utile d'insister : accès des titulaires du droit à un système de soins ${ }^{47}$ et droit de ces derniers à obtenir des prestations sociales ${ }^{48}$.

Le droit à la santé suppose que les pouvoirs publics créent des conditions telles que chacun puisse jouir du meilleur état de santé possible. Ceci implique notamment l'existence de services de santé, des conditions de travail sûres et saines, un logement adéquat et une alimentation nutritive $^{49}$. Il est reconnu par les instruments internationaux des droits de l'Homme et par certains instruments régionaux des droits de l'Homme.

Le Comité des droits économiques, sociaux et culturels, qui surveille l'application de la Convention, a adopté en 2000 une observation générale sur le droit à la santé. Dans son observation générale, il affirme qu'entrent dans le champ du droit à la santé, non seulement la prestation de soins de santé appropriés en temps opportun, mais aussi les facteurs fondamentaux déterminants de la santé, tels que l'accès à l'eau salubre et potable et à l'assainissement, l'accès à une quantité suffisante d'aliments sains, la nutrition et le logement, l'hygiène du travail et du milieu et l'accès à l'éducation et à l'information relatives à la santé, notamment la santé sexuelle et génésique ${ }^{50}$.

Ceci suppose que la réalisation du droit à la santé dépend de différents facteurs qui ne relèvent pas directement des services de santé mais de la réalisation d'autres droits, y compris des droits civils et politiques tels que la participation aux prises de décision et le droit

\footnotetext{
42 Ancien directeur de l'OMS.

43 LARBI BOUGUERRA M., «Eau et santé » in La mise en æeuvre du droit à l'eau, Actes du XXIXème Congrès ordinaire de l'IDEF, Schultess, 2006, p. 125.
}

44 ÖZDEN Melik, « Le droit à la santé, Un droit humain fondamental stipulé par l'ONU et reconnu par des traités régionaux et de nombreuses constitutions nationales », Directeur du Programme Droits Humains du CETIM et Représentant permanent auprès de l'ONU.

45 BERTHIAU D., Droit de la santé, Gualino éditeur, 2007, p. 18.

46 MOREAU J., « Le droit à la santé », AJDA 1998, p. 185.

47 Cons. constit. 18 janvier 1978, déc. $n^{\circ}$ 77-92 DC

48 Cons. constit. 22 janvier 1990 (cons. 26), 29 juillet 1991 (cons. 13, 17 et 19) ; comp. Cons. constit. 16 janvier 1991 (cons. 18).

49 www.who.int/mediacentre/factsheets/fs323/fr/index.html consulté le 27 septembre 2008.

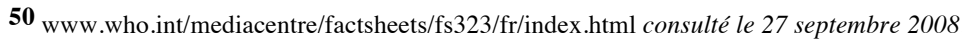


d'association qui sont indispensables, par exemple, à l'élaboration et la mise en place d'un système de santé efficace et non-discriminatoire. Mais, dans le cadre de ces travaux de recherche, nous nous intéresserons uniquement au lien entre droit à l'eau et droit à la santé. Il est une évidence que l'absence d'un accès fiable à l'eau potable limite l'exercice du droit à la santé, un assainissement adéquat est un préalable indispensable à l'accès à l'eau potable car, beaucoup de maladies sont associées à l'assainissement ${ }^{51}$.

\section{ס. La place du droit à l'eau dans le droit au logement}

La définition du droit au logement n'est pas aisée car le droit au logement ne se limite pas au droit à un toit et quatre murs. TRIBILLON J-F., en propose la définition suivante : "le droit d'accéder à un logement décent, convenablement situé, suffisamment desservi par des équipements publics et privés ${ }^{52}$. Le droit au logement implique donc un certain niveau de qualité de l'habitat mais aussi une insertion dans l'espace urbain. On observe alors que plus on élargit la notion de droit au logement plus on l'inscrit dans un droit plus large et qui l'englobe : le droit à la ville qui lui-même recoupe d'autres droits sociaux notamment le droit d'accès aux services publics et le droit à l'eau ${ }^{53}$. Consacré implicitement dans les constitutions de plusieurs Etats, le droit au logement est incontestablement lié au droit à l'eau. Le droit au logement sort le droit à l'eau de l'abstraction et en fait un droit concret.

En 1996, les Chefs d'État et de Gouvernement, réunis lors de la Conférence des NationsUnies relative aux établissements humains (Habitat II) ${ }^{54}$, ont convenu que chacun avait le droit de bénéficier de systèmes d'approvisionnement en eau et d'assainissement. Le droit au logement est reconnu au niveau international dans toute une série de textes qui, s'ils ont été signés par les Etats, peuvent créer des obligations entre eux mais dont les personnes privées ne peuvent se prévaloir, leur portée reste donc très limitée ${ }^{55}$. En effet, le Conseil d'Etat a estimé que le Pacte ne peut être directement invoqué par les particuliers ${ }^{56}$.

La Déclaration universelle des droits de l'Homme $^{57}$ et le Pacte International relatif aux droits économiques, sociaux et culturels ${ }^{58}$ considèrent le droit au logement comme une composante

51 El Hadj GUISSE, « Rapport entre la jouissance des droits économiques, sociaux et culturels et la promotion de la réalisation du droit à l'eau potable et à l'assainissement », E/CN.4/Sub.2/2002/10 du 25 juin 2002, pp. 10-13.

52 GUISELIN Emmanuel-Pie, «Droit au logement: quelle reconnaissance ?», in. SEGAUD M., BRUN J. et DRIANT J.-C., Dictionnaire de l'habitat et du logement, Armand Colin, Paris, 2003.

$\mathbf{5 3}$ http://base.d-p-h.info/fr/fiches/dph/fiche-dph-7301.html consulté le 29 janvier 2010.

54 E/CN. 4/Sub. 2/1996/10

55 UHRY M., «Le droit au logement en Europe », in La ville à l'épreuve du libéralisme, Cahier Voltaire, Aitec, Paris, 2006.

56 CE, 5 mars 1999, Rouquette et autres, AJDA, p. 420 et 462.

57 Article 25, paragraphe 1.

58 Article 11, paragraphe 11. 
essentielle du droit à un niveau suffisant. En effet, un logement convenable doit comprendre certains équipements essentiels à la santé, à la sécurité, au confort et à la nutrition ${ }^{59}$. Aussi, chaque Etat présente-t-il des dispositions législatives et constitutionnelles censées faciliter la mise en œuvre du droit au logement. Mais, cela n'est possible que si le droit à l'eau est effectif. Et c'est dans cette optique que KOTHARI M. déclarait que « la question du droit à un logement convenable a souvent été examinée avec d'autres droits, y compris le droit à la santé, à l'éducation, à l'approvisionnement en eau.... ${ }^{60}$. AUBRY M., ajoutera lors d'un débat parlementaire que «le droit au logement ne se limite pas aux murs, il concerne aussi les moyens que l'on a d'y vivre. Vous avez donc raison d'insister sur les éléments de conforts minimaux que sont le chauffage, l'électricité l'eau et le téléphone... $»^{61}$.

Pour Céline BIGOT, «ces différentes considérations invitent à considérer que le droit à l'eau est avant tout une condition de mise en cuvre des droits de deuxième génération ${ }^{62}$.

\section{De la libéralisation du secteur de l'eau}

Défini comme l'action de libéraliser c'est-à- dire rendre une économie plus libérale, le concept de la libéralisation est parfois assimilé à la notion de privatisation. Notion polysémique, elle est à la base de la doctrine du libéralisme économique. Lorsqu'il est mis en vigueur, l'Etat ne doit pas par son interventionnisme, gêner le libre jeu de la concurrence. En réalité, en Afrique subsaharienne, ces nouvelles politiques économiques, laborieusement mises en œuvre, concernent principalement le secteur moderne, le plus souvent une petite part du pays réel. Les populations restent, en effet, majoritairement rurales et survivent en économie de subsistance, pendant que les périphéries urbaines prolifèrent aux alentours des capitales, offrant un réservoir illimité au développement incontrôlé d'activités informelles, notamment la distribution de l'eau. La libéralisation du secteur de l'eau va consister donc à soumettre l'accès à l'eau au libre jeu de la concurrence par le procédé de la privatisation des entreprises publiques qui géraient la distribution de l'eau.

La probité intellectuelle serait mise à défaut, si l'on ne précise pas qu'en réalité, la privatisation des secteurs étatiques était bien souhaitée, fût ce-t-il sous la pression des financiers internationaux, dans la région. En effet, les esprits étaient préparés à cette révolution des mentalités. Déjà en 1980, le plan de Lagos recommandait aux gouvernements africains un changement des stratégies de développement et préconisait un relatif recul de l'étatisme économique. Plus récemment, la Conférence internationale de Nairobi d'octobre 1986, avait apporté une caution remarquée au secteur privé. Elle demandait aux gouvernements africains «d'adopter des politiques concertées visant à élargir le rôle du secteur privé..., de réduire les

\footnotetext{
59 Observation générale ${ }^{\circ}$ 4, Le droit à un logement suffisant, E/1992/23, par.7

60 Rapport présenté devant la Commission des droits de l'homme, le 03 avril 2001.

61 Ass. Nat. Débats, J.O., p. 4582, 20/05/1999.

$62 C f$. BIGOT C., Le droit à l'eau en droit international et en droit communautaire : contribution à l'étude d'un droit de l'homme et des générations futures en émergence, Thèse, Paris X- Nanterre, 2006.
} 
contrôles administratifs sur le secteur privé, de simplifier les procédures et de promouvoir l'esprit d'entreprise... $»^{63}$.

Il est indéniable que l'eau est une ressource vitale. Aussi, a-t-elle toujours "été associée au territoire et à la souveraineté étatique qui s'y exerce». Par contre sa marchandisation va soulever de multiples interrogations surtout que celle-ci engendre des conséquences dramatiques dans certains milieux comme l'Afrique subsaharienne ${ }^{64}$.

Dès lors, l'on peut se poser la question de savoir si l'eau peut constituer une marchandise ? Une question difficile, dont la réponse varie selon les institutions ou les auteurs qui sont saisis du problème. L'opinion de l'Union Européenne à cet égard nous paraît très remarquable. En effet, selon les autorités de cette institution, "l'eau n'est pas un bien marchand comme les autres mais un patrimoine qu'il faut protéger, défendre et traiter comme tel $»^{65}$. Une position très ambiguë à laquelle semble adhérer le Rapport mondial 2010 de l'OMC sur «le commerce des ressources naturelles ${ }^{66}$. Selon ce rapport, les ressources naturelles comme l'eau ne peuvent faire l'objet d'échanges. Toutefois, le rapport précise que le commerce peut avoir un effet bénéfique sur l'approvisionnement.

A l'opposé de cette attitude ambivalente, la doctrine constate que dans la plupart des Etats, le commerce de l'eau constitue une réalité67. Certes, l'eau potable a un prix et, dans beaucoup de pays, l'eau est fortement subventionnée par l'État, c'est-à-dire que l'acquéreur paye bien moins que le service reçu et ne paye rien pour la matière première elle-même qui est donnée et non vendue $^{68}$. Mais avec la libéralisation du secteur de l'eau, l'on s'interroge sur ce qui adviendra de cette subvention qui amortit le prix de l'eau au robinet. De plus, il n'est pas évident que les opérateurs économiques, souvent des multinationales, qui sont les bénéficiaires de ces contrats de concession, aient vraiment pour objectif, l'extension du réseau de distribution de l'eau. Finalement, l'accès à l'eau devient un problème préoccupant, surtout dans les pays en développement et particulièrement dans les Etats de l'Afrique subsaharienne. Ainsi, va se poser la question de l'existence d'un droit à l'eau qui soit effectif.

63 CHAVANE Bruno, «Bilan et perspectives des privatisations en Afrique francophone: Une étape de la démocratisation? » http://www.ilo.org/public/french/employment/ent/papers/ippred1.html consulté le 20 février 2010 .

64 Le principe de séparation des pouvoirs a été énoncé par le philosophe anglais John LOCKE (1632-1704) dans son «Second traité du Gouvernement Civil» de 1690 et plus tard par MONTESQUIEU (1689-1755) dans "L'esprit des lois » (1748). La séparation des pouvoirs est un principe, une théorie, qui préconise que les trois grandes fonctions de l'Etat (le pouvoir exécutif, le pouvoir législatif et le pouvoir judiciaire) soient chacune exercée par un organe ou une instance différente.

65 Point 1 du préambule de la directive $n^{\circ} 2000 / 60 / C E$ du 23 octobre 2000 établissant un cadre pour une politique communautaire dans le domaine de l'eau. Modifié ; $C f$. JOCE n ${ }^{\circ} 327$ du 22 décembre 2000.

66 OMC, Rapport mondial sur le commerce 2010, «le commerce des ressources naturelles », p. 5 , $46,49,67$.

67 DROBENKO B., précité, p. 138.; Cf. aussi, PAQUROT S., «Et l'eau dans tout ça, une évolution inachevée » dans Le statut des ressources vitales en droit international, Bruylant, 2002, p. 194.

68 SMETS H., L'accès à l'eau potable et le droit international, Académie de l'eau, Janvier 2008, p. 3. 


\section{Le service public de l'eau ${ }^{69}$, une garantie du droit à l'eau}

Dans ses réflexions sur les fondements du service public, J. CHEVALLIER précise que « $d u$ mythe, le service public présente en effet tous les aspects essentiels : l'ampleur et la profondeur des résonances qu'il éveille en chacun; les glissements de signification qu'il autorise; la force agissante attestée par les effets sociaux et politiques qu'il produit ; la tension entre la représentation et la réalité $\gg{ }^{70}$. On retiendra alors que le service public peine à être considérée exclusivement comme un concept strictement juridique car, d'une portée symbolique, la notion véhicule avec elle, bien au-delà, des considérations d'ordre social, économique et politique relevant plus largement du choix d'un modèle de société. En droit français et dans une certaine mesure dans les Etats africains d'expression francophone ${ }^{71}$, le service public est appréhendé «en tant que représentation dramatisée (...) des rapports entre l'Etat et la société civile (...) : idéal social pour les uns, contre modèle pour les autres, il est un lieu privilégié d'investissements affectifs $\gg^{72}$. Ce constat marque la difficulté d'approche d'une notion envers laquelle nul n'est, dès lors, unanime ${ }^{73}$, notamment de par le caractère politique qu'elle sous-tend ${ }^{74}$. Or cette difficulté trouve un écho particulier au regard de la relation que le service public peut entretenir avec la question des droits et libertés, laquelle intéresse de la même manière, dans une perspective différente mais trouvant de nombreux points de rapprochement, les rapports entre l'Etat et l'individu. Au regard des interrogations qu'ils suscitent, du rôle devant être reconnu aux pouvoirs publics et des multiples implications qui s'y jouent, les deux mondes ne semblent ainsi pas si éloignés ${ }^{75}$.

L'une des missions fondamentales que s'est assigné l'Etat, particulièrement les Etats africains sortis du joug colonial, est la prestation de services publics aux citoyens par le biais de leur administration publique. Et la distribution de l'eau devrait occuper un rang privilégié sur cette liste. Par ces prestations, les Etats réalisent leurs objectifs de redistribution sociale et de respect des droits fondamentaux de l'homme tels que précisés dans la plupart des constitutions africaines et dans la déclaration universelle des droits de $1^{1}$ homme ${ }^{76}$. Ces services de base contribuent à

69 Concept désignant « un service d'alimentation en eau potable soumis à des sujetions de service public », $c f$. Art. 2 de la loi $\mathrm{n}^{\circ}$ 2010-006 du 18 juin 2010 portant organisation des services publics de l'eau potable et de l'assainissement collectif des eaux usées domestiques.

70 CHEVALliER J., Le service public, PUF, Collec. « Que sais-je ?», $9^{\text {ème }}$ éd., 2012, p. 3.

71 Les Etats africains, qui ont pour la plupart acquis leur indépendance en 1960, ont organisé leur administration sur le modèle des administrations coloniales.

72 CAILlOSSE J., La constitution imaginaire de l'administration, PUF, 2008, p. 56.

73 CAILlOSSE J., « Service public et concurrence - Le service public entre deux mythologies », Droit ouvrier, avril 2008, p. 199.

74 MOREAU J., Droit administratif, Droit fondamental, PUF, 1989, p. 316.

75 BOYER-CAPELLE C., Le service public et la garantie des droits et libertés, Thèse dactylographiée, Université de Limoges, 2009, pp. 14 et 15.

76 Article 25 de la déclaration universelle des droits de l'homme de 1948 
assurer des conditions de vie honorables, et sont capitaux pour toutes stratégies de lutte contre la misère ${ }^{77}$.

Cependant, de la littérature sur les services publics en Afrique en général et en Afrique subsaharienne en particulier, il ressort que ces services connaissent un mauvais fonctionnement et subissent une forte désagrégation. Ces appréciations négatives sont alimentées par les rapports et écrits sur les pratiques qui gangrènent le secteur public, régi par des logiques clientélistes inégalitaires et souffrant d'un manque croissant de productivité et d'un déficit d'efficacité. En effet, des services de base dans les domaines de l'administration publique, de la santé, de la justice, de l'éducation, de la fourniture en eau et électricité, des télécommunications et de l'information ou encore de la sécurité ne sont pas rendus de manière satisfaisante, et les premières victimes sont les populations les plus pauvres (financièrement et socialement), qui ne peuvent s'offrir l'accès à ces services. La plupart des Etats de l'Afrique subsaharienne semblent être confrontés à ces problèmes identiques ${ }^{78}$.

Alors qu'on pouvait légitimement d'une part, s'accorder avec BOYER-CAPELLE C. ${ }^{79}$ que le service public est un instrument de garantie directement sollicité par les «droits-créances » à l'instar du droit à l'eau et d'autre part, constater que le service public considéré comme garantie des «droits-créances » est de plus en plus consacré dans les lois fondamentales ${ }^{80}$, la vision développée par Pierre DELVOLVE dans son article fondateur ${ }^{81}$ relève en effet, avant tout, d'une défense de la liberté d'entreprendre et de la libre concurrence et d'une contestation de certaines particularités organisationnelles attachées au service public qui tendent à restreindre l'initiative privée.

Ce qui est condamné, plus que toute autre chose, est l'atteinte à la libre conduite des activités privées que sous-tendrait le service public. C'est ainsi par le media de l'inflexion, voire de la négation, de ces libertés économiques que ce dernier est censé constituer une menace envers l'ensemble des libertés publiques. Se manifeste alors la foi en une logique économique libérale qui parait inspirée par les travaux de Friedrich HAYEK. Le choix même du terme de « servitude » pour dénoncer cette menace semble, du reste, faire écho à La route de la servitude ${ }^{82}$, l'un des ouvrages les plus connus de cet auteur réputé pour sa condamnation de l'interventionnisme étatique et de la notion de justice sociale ${ }^{83}$. La justice sociale apparaît en effet pour cet auteur comme une menace importante pour la liberté car susceptible de légitimer le totalitarisme. Cette thèse sera défendue par les institutions de Bretton Woods pour qui il fallait libéraliser les

77 CERAP, «L'Etat et le service public en Afrique de l'Ouest», Colloque sous-régional, Abidjan, 23 et 24 Novembre 2010.

$\mathbf{7 8}$ Ibidem.

79 Le service public et la garantie des droits et libertés, Thèse dactylographiée, Université de Limoges, 2009, précité.

80 Cf. La Constitution de la République du Niger de 2010.

81 DELVOLVE P., « Service public et libertés publiques », RFDA 1985, p. 1.

82 HAYEK F., La route de la servitude, PUF, 1985, pp. 66-67.

$\mathbf{8 3}$ HAYEK F., Droit, législation et liberté, Tome 2 : Le mirage de la justice sociale, Quadrige-PUF, 1995, spéc. p. 75 et $\mathrm{s}$. 
secteurs «économiques » encore aux mains de l'Etat et de ses démembrements. Il s'agit là de faire de l'eau une marchandise même s'il est évident que les populations de l'Afrique subsaharienne n'ont pas les moyens financiers pour se l'offrir. Le service public qui devait constituer une garantie pour l'effectivité des droits émergents comme le droit à l'eau, se retrouve dans le rôle inverse, liberticide à en croire DELVOLVE et HAYEK. En réalité, il s'agit d'un conflit de droits, conflit sur lequel nous reviendrons dans développements ultérieurs.

\section{L'intérêt du sujet : « l'eau, source de vie et objet de droit »}

Alors que plus d'un milliard de personnes sont dépourvues d'eau potable et près de 3 milliards de personnes n'ont pas accès aux services d'assainissement, il est surprenant de constater que peu d'ouvrages ou d'articles ont été consacrés au droit à l'eau.

Mais depuis la Conférence de Mar Del Plata ${ }^{84}$, on assiste à l'émergence d'une littérature engagée sur le thème. Aussi, les associations, les organisations non gouvernementales, les organisations internationales et les étudiants des facultés de droit ou de sciences humaines se sont emparés du sujet. Le constat, c'est qu'il y a un drame de l'eau. Un drame qui n'est pas vécu de la même façon et dont les conséquences sont inestimables.

Habituellement, lorsque le droit intervient en matière d'eau, c'est pour restaurer l'intégrité chimique, physique et biologique des eaux intérieures et des mers. Par ailleurs, son intervention permet de réglementer le déversement de contaminants, les activités affectant les marécages, les déversements accidentels ainsi que l'utilisation et la destruction des déchets. Tel ne sera pas exclusivement le cas dans le cadre des présentes recherches. Celles-ci sont consacrées à l'accès et à la disponibilité de cette matière indispensable et vitale.

\section{Le « drame » de l'eau, un drame inégalement vécu}

L'an 2011, quelque part le long d'une route nationale en Afrique subsaharienne, une file de femmes et d'enfants, chacun muni d'un bidon ou d'une bassine, se précipitent sur l'eau boueuse d'un marigot. Chacun se sert autant qu'il peut et transporte sa corvée sur des kilomètres, pour certains, sur plus d'une dizaine de kilomètres avant de regagner le village. Cette scène, loin d'être isolée, est malheureusement quotidienne. Elle reflète la difficulté qu'éprouvent les populations des pays en voie de développement et particulièrement celles de l'Afrique subsaharienne à s'approvisionner en eau potable. Si pour certaines personnes, la crise de l'eau signifie parcourir chaque jour de longues distances à pied afin de collecter suffisamment d'eau propre ou insalubre - pour survivre, pour d'autres, cela signifie souffrir d'une malnutrition évitable ou de maladies provoquées par les sécheresses, les inondations ou des systèmes d'assainissement inappropriés. Pour d'autres encore, c'est le manque de financements,

84 Première conférence des Nations unies sur l'eau, 14 mars 1977. 
d'institutions ou de connaissances qui empêche de résoudre les problèmes locaux liés à l'utilisation et à la répartition des ressources en eau ${ }^{85}$. Et pourtant l'eau est un besoin vital à la survie des êtres humains et indispensable à la vie en général. Près d'un milliard quatre cent millions ${ }^{86}$ sont "souvent démunis (...) de la denrée la plus précieuse à tout être vivant : l'eau salubre ${ }^{87}$. A ces derniers, la pauvreté interdit toute possibilité de payer l'eau qui leur est nécessaire, ne serait-ce que pour leur consommation journalière de base.

Presque 13 millions d'humains meurent chaque année du manque d'eau potable soit environ 34000 personnes chaque jour ${ }^{88}$. Treize millions d'individus, c'est près de deux fois la population d'un Etat comme le Togo qui périssent chaque année parce que le monde vit d'une part «sous une dictature économique $»^{89}$ et d'autre part dans un individualisme outrancier. Si l'on remonte le temps, cette situation est le résultat de l'absence d'une véritable politique de distribution d'eau aggravée par une quasi inexistence de planification de la distribution d'eau et des difficultés de production et de distribution d'eau potable en milieu rural. Aujourd'hui la situation a évolué et CONAC G. écrira que «les politiques de l'eau touchent au problème actuel le plus crucial en

85 L'eau, une responsabilité partagée, $2^{\text {ème }}$ Rapport mondial des Nations Unies sur la mise en valeur des ressources en eau, 2006; www.unesco.org/water/wwap/index fr.shtml// consulté le 4 février 2007.

86 Selon l'ONU et d'autres spécialistes, la situation va empirer. Les prévisions les plus alarmistes affirment qu'en 2050 près de sept milliards de personnes vivront avec de très maigres ressources en eau. Un des objectifs du Millénaire pour le développement est de réduire de moitié, d'ici 2015, le nombre de personnes qui n'ont pas accès à un approvisionnement suffisant en eau potable et à un système sanitaire de base ; la période $2005-2015$ a été déclarée par l'ONU décennie internationale d'action sur le thème «L'eau, source de vie. »

Sources : Rapport mondial des Nations Unies sur la mise en valeur des ressources en eau : l'eau pour les hommes, l'eau pour la vie ; http://www.unesco.org/water/wwap/index_fr.shtml ; http://www.un.org/french/waterforlifedecade/ )

87 N. TIEN-DUC, L'Humanité mourra-t-elle de soif? Paris, Hydrocom éditions, 1999.

$\mathbf{8 8}$ www.france-libertés

89 «...on constate (...) que les système de gestion des «usages d'aval» (distribution de l'eau potable, assainissement des eaux résiduaires) se privatisent conformément au principe promu par les bailleurs de fonds...» Jacques SIRONNEAU, Revue juridique de l'environnement, $n^{\circ} 3,1998$, p.317

D'autre part, selon le Rapport mondial sur le développement humain 2002 du PNUD, «les multinationales peuvent exercer une énorme influence sur les droits de l'homme, dans leurs habitudes d'emploi, leur impact sur l'environnement, leur soutien aux régimes corrompus ou leur promotion du changement». Aujourd'hui, les 200 premières sociétés contrôleraient le quart des ressources productives mondiales. Les sociétés transnationales ont souvent des revenus maintes fois supérieurs à ceux des gouvernements des pays dans lesquels elles sont implantées. D'après la CNUCED, 29 des 100 entités économiques les plus importantes au monde sont des sociétés transnationales. La concentration a donné naissance à d'énormes sociétés transnationales qui exercent un monopole sur l'ensemble de la chaîne alimentaire, de la production à la distribution au détail en passant par la vente, la transformation et la commercialisation, ce qui a pour effet de restreindre le choix des agriculteurs et des consommateurs. Dix sociétés seulement (dont Aventis, Monsanto, Pioneer et Syngenta) contrôlent un tiers du marché des semences, évalué à 23 milliards de dollars et $80 \%$ du marché des pesticides, évalué à 28 milliards de dollars.

A elle seule, Monsanto contrôle $91 \%$ du marché mondial des semences génétiquement modifiées30. Dix autres sociétés, dont Cargill, concentrent $57 \%$ des ventes des 30 premiers détaillants au monde et comptent pour $37 \%$ des recettes engrangées par les 100 premières sociétés productrices de denrées alimentaires et de boissons. En Afrique du Sud, Monsanto contrôle totalement le marché national des semences génétiquement modifiées, $60 \%$ du marché du maïs hybride et $90 \%$ du marché du blé.

SOURCES : Conseil Economique et social - Commission des droits de l'homme, E/CN.4/2004/10, 9 février 2004 
Afrique, celui de l'alimentation et de la survie de populations entières. Elles conditionnent le développement agricole de la plupart des pays africains $»^{90}$. Toutefois, il faut insister sur l'efficience de ces politiques. Le bilan peut varier dans l'espace et dans le temps. En effet, certains Etats s'évertuent à la promotion d'une politique de l'eau susceptible de soulager les difficultés des populations. Au Bénin, "l'accès des populations les plus défavorisées à l'eau potable et aux services adéquats d'assainissement de base constitue l'une des grandes priorités de la stratégie de croissance pour la réduction de la pauvreté de la République du Bénin. Il $s$ 'agit là d'une déclinaison de l'un des Objectifs du Millénaire pour le Développement (OMD), faisant partie des facteurs de réduction de la pauvreté dans le monde ».

Dans l'hypothèse où "tous les êtres humains sont égaux en dignité et en droit. "1" il est difficile d'admettre que dans la réalité cette conjecture est toujours vérifiée. Si au même instant, l'utilisation "abusive» et le "gaspillage» de l'eau est de "règle» dans les sociétés industrialisées. En Amérique du Nord, chaque habitant consomme en moyenne 350 litres d'eau ${ }^{92}$. Cette eau, il ne va pas la chercher dans une rivière souillée ou dans un marigot pollué. Elle lui est servie à domicile ${ }^{93}$.

Par rapport aux inégalités dans la répartition des ressources en eau d'autres sources indiquent «... que les pays industrialisés disposent de 400 à 600 litres d'eau par jour et par habitant, les [triangles de la soif] du Moyen-Orient au Pakistan ou en Afrique sub-saharienne peinent à atteindre quelques dizaines de litres d'eau par jour et par habitant $»^{94}$. La qualité de l'eau est de moins en moins bonne dans la plupart des régions. Les chiffres montrent que le nombre d'espèces d'eau douce est en recul et que les écosystèmes d'eau douce se détériorent rapidement, souvent plus vite que les écosystèmes terrestres et marins. Le rapport souligne que le cycle hydrologique, nécessaire à la vie, ne peut se dérouler que dans un environnement sain. Pendant ce temps le budget mondial de l'armement frôle les 1000 milliards de dollars chaque année. Les Etats ne peuvent-ils pas consacrer au moins $1 \%$ de ce budget pendant quinze ans pour financer le programme qui amènera l'eau où les habitants n'y ont pas accès, s'interrogea

90 CONAC G., (dir), Les politiques de l'eau en Afrique, Paris, Economica, 1985

91 Article 11 de la constitution de la IV ${ }^{\text {ème }}$ République du Togo

92 A titre d'exemple, les habitants de l'Amérique du Nord consomment 350 litres d'eau par jour. L'industrie utilise 280000 litres d'eau pour produire une tonne d'acier et 700 litres pour produire un kilo de papier, sans parler des déchets et des matières radioactives produits par les activités industrielles qui contaminent les eaux. Il faut ajouter à cela l'agriculture industrielle et intensive qui consomme $80 \%$ des ressources disponibles.

Voir « Le droit d'accès de tous à l'eau potable et aux services d'assainissement ». E/CN.4/Sub.2/1998/7, Cote ONU

Sources : http://www.cetim.ch./fr/interventions_details.php?iid=185/ consulté le 2 janvier 2007.

93 «En ce qui concerne l'eau potable, l'Afrique a une disponibilité moyenne par tête en eau assez faible par rapport au reste du monde, à savoir 16\% d'eau en moins par personne que le reste du monde. De plus, 21 pays d'Afrique subsaharienne ont une moyenne inférieure à la moyenne mondiale. Cette relative rareté de l'eau affecte principalement les pays pauvres et, au sein de ceux-ci, les ménages les plus pauvres. En effet, ceux-ci ont non seulement le plus de difficulté à accéder à l'eau potable mais sont aussi les plus vulnérables en cas de manque d'eau car ils ne disposent pas de réserves de nourriture ni d'épargne afin de faire face à ce déficit. » ...

Ouvrage collectif (PNUD), La lutte contre la pauvreté en Afrique subsaharienne, Economica, 1999, p.229

94

www.notre-planete.info/ consulté le 26 mars 2007. 
Danielle MITTERRAND, ex-présidente de l'association France-Libertés ${ }^{95}$. Il faut aussi indiquer que les politiques économiques internationales telles que les projets de développement ou les politiques commerciales peuvent avoir des effets néfastes sur les droits de l'homme. Ainsi, selon un Rapport d'Amnesty International, la construction de l'oléoduc Tchad-Cameroun financée par la Banque Mondiale a entraîné de nombreuses violations des droits de l'homme (privation de l'accès à l'eau potable, déplacements des fermiers ...). Ces violations de droits de l'homme se traduisent par des confiscations de terres et des expulsions dont les procédures n'ont pas été respectées ${ }^{96}$.

De même, l'Accord Général sur les services de l'OMC (AGCS), de par les privatisations des services publics de base qu'il implique, peut entraîner, dans les pays où il est mis en place des atteintes aux droits de l'homme ${ }^{97}$. En réalité, le droit à l'eau demeure un «parent pauvre » du droit international de l'eau. Cela est d'autant plus vrai pour les accords bilatéraux que pour les accords multilatéraux, puisque il n'y a que peu de traces et, parfois même, des termes peu explicites. Cependant, il y a quelques exceptions notables. Par exemple, le Protocole à la convention d'Helsinki ${ }^{98}$ souligne clairement, le lien entre gestion internationale de l'eau et droit à l'eau. Aussi, faut-il ajouter qu'au niveau des instruments régionaux de droits humains, les références au droit à l'eau sont absentes ou presque. En effet, quelques indications allusives sont rencontrées dans la Charte africaine des droits de l'Homme et dans la Convention interaméricaine des droits ${ }^{99}$.

Les conséquences sont très importantes et s'étendent à tous les domaines. Sur le plan de la santé, il faut souligner que les maladies liées à l'absence d'une eau potable sont très redoutables. Il s'agit en premier lieu des « maladies hydriques » qui sont provoquées par de l'eau contaminée par des déchets humains, animaux ou chimiques. Elles comprennent entre autres le choléra, la typhoïde, la polio, la méningite, l'hépatite A et E, et la diarrhée ${ }^{100}$. De plus, l'eau de la nature est d'autant moins potable aujourd'hui qu'elle est de plus en plus polluée par des substances rejetées par les sociétés humaines. Or cette pollution est parfois telle que même la qualité de l'eau traitée s'en ressent. Les femmes sont les plus touchées par cette situation. Plus de la moitié des 1.4 milliards des personnes qui n'ont pas accès à l'eau sont des femmes et des filles. La IV ${ }^{\text {ème }}$ Conférence mondiale des femmes des Nations Unies en 1995 consacre un chapitre entier dans

95 Interview publiée sur le site web de l'association France-Libertés : http://www.france-libertés.fr// consulté le 15 septembre 2008.

96 Rapport d'Amnesty International, «Contracting out of human rights : the Chad-Cameroon Pipeline Project », disponible sur : web.amnesty.org/library/pdf/POL340122005ENGLISH/ consulté le 7 novembre 2007.

97 MICHAUD E., « Les études d'impact des politiques économiques internationales sur les droits de l'homme. » In A la recherche de l'effectivité des droits de l'homme, Paris, Presses Universitaires de Paris 10, 2008, p. 231.

98 Déclaration d'Helsinki de 1994 : Article 1er - «Le présent Protocole a pour objet de promouvoir à tous les niveaux appropriés, aussi bien à l'échelon national que dans un contexte transfrontière et international, la protection de la santé et du bien-être de l'homme, tant individuels que collectifs, dans le cadre d'un développement durable, en améliorant la gestion de l'eau, y compris la protection des écosystèmes aquatiques, et en s'employant à prévenir, à combattre et à faire reculer les maladies liées à l'eau ».

99 A l'exception, de la Convention de 1979 contre la discrimination à l'encontre des femmes ainsi que dans la Convention sur les droits de l'enfant.

100 Chaque jour, 6000 personnes meurent dans le monde à cause de maladies diarrhéiques. En 2001, on a ainsi dénombré près de 2 millions de morts, dont plus de la moitié sont des enfants. Ces maladies ont ainsi tué plus d'enfants au cours des 10 dernières années que tous les conflits armés depuis la fin de la seconde guerre mondiale. 
son plan d'action sur les femmes et l'environnement dans lequel l'eau est insérée ${ }^{101}$, bien que l'on n'ait pas analysé comment une pénurie d'eau ou sa contamination affecte les femmes disproportionnellement. Un autre type de menace engendrée par la libéralisation du secteur de l'eau est liée à la « réactivation de la doctrine de la divisibilité des droits de l'homme : les droits économiques et sociaux ne seraient pas de véritables droits de l'homme, à l'égard des droits civils et politiques. Or, force est de constater, qu'au-delà des discours lénifiants sur l'indivisibilité des deux catégories de droits, leur divisibilité est bel bien inscrite dans les faits :.., $»^{102}$. De manière plus appréciable encore, la libéralisation du secteur de l'eau ralentit ou paralyse l'éclosion du droit à l'eau.

\section{Les sources du droit à l'eau}

L'eau est une ressource naturelle limitée et un bien public; elle est primordiale à la vie et à la santé. Le droit à l'eau est indispensable pour mener une vie digne. Il est une exigence préalable à l'effectivité des autres droits de l'homme. On distingue d'une part les sources juridiques et d'autre part les sources extra-juridiques. Mais nos développements vont uniquement s'atteler aux sources juridiques. Celles-ci sont de deux ordres, les sources d'origine nationale et les sources d'origines internationales.

\section{a. Le droit à l'eau dans l'arsenal juridique national}

Pour Henri SMETS «le droit interne permet de rendre effectif le droit à l'eau» car celui-ci trouve son fondement dans les politiques de l'eau, de la santé et du logement et est mis en œuvre par les dispositions de droit interne. Il est constitué par un ensemble de dispositions qui visent à garantir l'accès à l'eau et à l'assainissement et qui figurent dans différents codes (code civil, code de l'environnement, santé publique, action sociale, urbanisme, consommation, collectivités locales, etc. $)^{103}$. L'inquiétude de nombreux Etats est que l'existence d'un droit à l'eau ne les contraigne à des objectifs difficilement réalisables. Ce qui explique que lorsqu'ils reconnaissent l'accès à l'eau comme droit fondamental, ils limitent par des clauses restrictives la valeur contraignante de cet engagement. Le récapitulatif de la situation de la reconnaissance du droit à l'eau montre que l'on a deux groupes d'Etats. Le premier proclame l'existence de droit tout en limitant sa portée et le second qui ne le reconnaît que de manière implicite. Au niveau européen, peu d'Etats reconnaissent expressément le droit à l'eau dans leur droit interne. L'alinéa $1^{\text {er }}$ et suivant de l'article 76 de la constitution de la fédération helvétique, dispose : «Dans les limites

\footnotetext{
101 Déclaration de Beijing.

102 FLAUSS J-F., «Le droit international des droits de l'homme face à la globalisation économique », in Commerce mondial et protection des droits de l'homme, Bruylant, 2001, p. 225.
}

103 SMETS H., Le droit à l'eau dans les législations nationales, AESN, Nanterre, octobre 2005, www.academie-eau.org/ consulté le 16 février 2006. 
de ses compétences, la Confédération pourvoit à l'utilisation rationnelle des ressources en eau, à leur protection et à la lutte contre l'action dommageable de l'eau. Elle fixe les principes applicables à la conservation et à la mise en valeur des ressources en eau, à l'utilisation de l'eau pour la production de l'énergie et le refroidissement et à d'autres interventions dans le cycle hydrologique. Elle légifère sur la protection des eaux, sur le maintien de débits résiduels appropriés, sur l'aménagement des cours d'eau, sur la sécurité de barrages et sur les interventions de nature à influencer les précipitations. Les cantons disposent des ressources en eau... $»^{104}$.

La reconnaissance du droit à l'eau par les Etats européens n'est en réalité qu'allusive. Aucun d'entre eux ne proclame avec force et précision l'existence du droit à l'eau. L'Irlande fait quelques références aux ressourcés naturelles notamment l'eau ${ }^{105}$. Toutefois, il faut saluer la volonté du gouvernement irlandais qui considère que l'eau potable est un bien public financé par l'ensemble de la société et qu'il convient de fournir à chaque ménage sans exiger de paiement. En 2000, lors de la discussion de la Directive-cadre sur la politique de l'eau, l'Irlande a refusé de faire payer les services d'eau des ménages et a obtenu l'insertion d'une clause d'exemption ${ }^{106}$.

Cependant, il faut signaler que la Belgique constitue une exception. En effet, la Région de Bruxelles a adopté une ordonnance qui garantit à toute personne le droit à la distribution d'eau potable ${ }^{107}$. De la même façon, la Région wallonne a adopté un décret qui dispose que «toute personne a le droit de disposer d'une eau potable de qualité suffisante pour son alimentation, ses besoins domestiques et sa santé »108.

\section{b. Le droit à l'eau dans les instruments internationaux}

L'analyse de l'arsenal juridique national et international révèle que le droit à l'eau existe bien au niveau des instruments sous-régionaux et régionaux ainsi qu'au niveau des instruments universels.

104 Constitution fédérale de la Confédération suisse du 18 avril 1999 (Etat le 17 mai 2009)

105 Article 10 de l'actuelle constitution

106 Art. 9.4 de la directive européenne établissant un cadre pour une politique communautaire dans le domaine de l'eau. - Non application de la récupération des coûts pour une "activité d'utilisation de l'eau donnée, dans la mesure où cela ne remet pas en question les buts de la présente directive et ne compromet pas la réalisation de ses objectifs". Si cette exception est permise pour l'Irlande, elle devrait pouvoir être utilisée pour d'autres cas similaires car les prélèvements d'eau potable n'ont que rarement un effet négatif sur l'environnement.

107 De la même façon, la Région flamande a adopté un décret qui prévoit que : «Chaque abonné a droit à une fourniture minimale et interrompue d'électricité, de gaz et d'eau à des fin d'utilisation ménagère afin de pouvoir mener une vie d'home suivant le niveau de vie en vigueur.

108 Décret du 15 avril 1999 de la Région wallonne relatif au cycle de l'eau et instituant une entreprise publique de gestion de l'eau (art.1.2, 4.2 et 6.2.5) 


\section{$\alpha$. Le droit à l'eau dans les instruments sous-régionaux et régionaux}

En Afrique, la Charte africaine des droits et du bien-être de l'enfant adoptée à Addis-Abeba en 1990109 prévoit l'obligation pour les États de prendre les mesures nécessaires «pour garantir la fourniture d'une alimentation et d'une eau de boisson saine en quantité suffisante ». Ce principe est réaffirmé dans le Protocole à la Charte africaine des droits de l'homme et des peuples relatifs aux droits des femmes adopté à Maputo en 2003 dont l'article 15 stipule que : «Les Etats prennent les mesures nécessaires pour assurer aux femmes l'accès à l'eau potable $»^{110}$. La Convention africaine pour la conservation de la nature et des ressources naturelles ${ }^{111}$ stipule en son article 7.2 que : " les Parties s'efforcent de garantir aux populations un approvisionnement suffisant et continu en eau appropriée ». La Charte des eaux du fleuve Sénégal signée en mai 2002 par le Mali, la Mauritanie et le Sénégal rappelle que «les principes directeurs de toute répartition des eaux du Fleuve visent à assurer aux populations des États riverains, la pleine jouissance de la ressource, dans le respect de la sécurité des personnes et des ouvrages, ainsi que du droit fondamental de l'homme à une eau salubre, dans la perspective d'un développement durable $»^{112}$. Aussi, importe-t-il de préciser que la Charte africaine des droits de l'homme et des peuples de 1981 reconnaît de manière implicite, le droit d'accès à l'eau. Aux termes de son article 24, l'on peut lire que tous les peuples ont un droit à un environnement satisfaisant et global propice à leur développement. Il n'est pas de doute que le premier élément indispensable à la réalisation d'un environnement satisfaisant et à la promotion du développement est l'eau. On observera que dans le processus du nouveau constitutionnalisme en Afrique, nombreux sont les Etats qui ont inscrit dans leur loi fondamentale, leur attachement aux principes proclamés dans la Charte de $1981^{113}$.

L'approfondissement de l'analyse révèle que la reconnaissance du droit à l'eau n'est pas uniquement l'œuvre des textes africains. Les droits régionaux européen et américain participent également à l'accouchement du droit à l'eau. En effet, le Protocole Additionnel à la Convention américaine relative aux droits de l'homme traitant des droits économiques, sociaux et culturels dite "Protocole de San Salvador » ${ }^{114}$, consacre trois articles qui reconnaissent implicitement le droit à l'eau. En proclamant le « droit à la santé ${ }^{115}$, le droit à un « environnement salubre »" ${ }^{116}$ et

109 En vigueur, ratifiée par 37 États.

11038 signatures et 12 ratifications à ce jour.

111 Adoptée à Maputo en juillet 2003 et signée par 33 États

112 Art. 4.3.

113 On peut citer les cas du Bénin, du Burkina Faso, du Gabon et bien d'autres encore.

114 Adopté à San Salvador, El Salvador le 17 novembre 1988, à la dix-huitième Session ordinaire de l'Assemblée générale.

115 Article 10 : Droit à la santé

1. Toute personne a droit à la santé qui est considérée comme le droit de jouir du meilleur état de santé physique, mentale et sociale.

2. Afin d'assurer le plein exercice du droit à la santé, les États parties s'engagent à reconnaître ce droit comme un bienfait public et notamment à adopter pour garantir l'exercice de ce droit les mesures suivantes: - a. l'octroi des soins primaires de santé, autrement dit, la mise à la disposition de tous les individus et de toutes les familles de la 
le «droit à l'alimentation $»^{117}$, le Protocole reconnaît implicitement le droit à l'eau. Le lien indissociable qui existe entre ces différents objectifs définis dans le protocole d'une part et le droit à l'eau d'autre part, confirme cette reconnaissance.

Par ailleurs, la reconnaissance du droit à l'eau peut être décelée dans certains textes du droit régional européen. Le Protocole sur l'eau et la santé à la convention sur la protection et l'utilisation des cours d'eau transfrontières et des lacs internationaux, signé à Londres le 17 juin 1999, de l'OMS et du Bureau régional de l'Europe, est sans doute le premier texte international de droit positif qui prenne autant position en faveur de l'accès à l'eau salubre pour tous ${ }^{118}$. A l'article 5 de ce texte, il est précisé qu'un accès équitable à l'eau, adéquat de point de vue aussi bien quantitatif, devrait être assuré à tous les habitants, notamment aux personnes défavorisées ou socialement exclues. En dépassant les dispositions de l'article 5 précité, il est n'est plus aisé de rencontrer de textes qui proclament explicitement le droit à l'eau. Ainsi, peut-on déplorer que la Convention de sauvegarde des droits de l'homme et des libertés fondamentales ${ }^{119}$, n'y fasse pas du tout allusion. Finalement, l'on ne peut se réjouir que de quelques unes des déclarations du Comité des ministres du Conseil de l'Europe. Il recommande aux gouvernements: «de reconnaître, au niveau national, un droit individuel, universel et justiciable à la satisfaction des besoins matériels élémentaires des personnes en situation d'extrême précarité » ${ }^{120}$.

Par ailleurs, le Comité des ministres, en révisant la Charte européenne de 1968, a proclamé en octobre 2001 que «toute personne a le droit de disposer d'une quantité d'eau suffisante pour satisfaire à ses besoins essentiels $»^{121}$. Il s'agit là, d'une reconnaissance incontestable de l'existence du droit à l'eau. Toutefois, le droit à l'eau ne figure pas dans la Directive-cadre de $2000^{122}$ qui établit le cadre d'une politique communautaire dans le domaine de l'eau. Citant le Professeur DROBENKO B.123, OLAKA J-M. semble regretter cette situation, lorsqu'il écrit que si les objectifs de la directive doivent contribuer à assurer un approvisionnement

communauté de l'aide médicale essentielle; b. l'extension des services de santé à tous les individus relevant de la juridiction de l'État; c.- l'immunisation complète contre les principales maladies infectieuses; d. La prophylaxie et le traitement des maladies endémiques, professionnelles et autres;

116 Article 11 : Droit à un environnement salubre

1. Toute personne a le droit de vivre dans un environnement salubre et de bénéficier des équipements collectifs essentiels; 2. Les États parties encourageront la protection, la préservation et l'amélioration de l'environnement.

117 Article 12 : Droit à l'alimentation.

1. Toute personne a droit à une alimentation adéquate qui lui assure la possibilité d'atteindre son plein développement physique et son plein épanouissement affectif et intellectuel.

118 OLAKA J-M., Le droit à l'eau, Thèse, Université Jean-Moulin Lyon 3, 2008, p. 154.

119 Texte signé à Rome le 4 novembre 1950.

120 Recommandation R (2000) 3 sur le droit à la satisfaction des besoins matériels élémentaires des personnes en situation d'extrême précarité.

121 Recommandation du Comité des ministres aux Etats membres de la Charte européenne des ressources en eau, Conseil de l'Europe, 17 octobre 2001, Rec. (2001) 14.

122 Directive 2000/60/CE, JOCE L 327, du 22 décembre 2000, p. 1

123 DROBENKO B., « Directive eau : un cadre en trompe-œil ? », Revue européenne de droit de l'environnement, 4/2000, pp. 381-402. 
suffisant en eau de surface et en eau souterraine de bonne qualité pour les besoins d'une utilisation durable, équilibrée et équitable de l'eau, la directive ne distingue pas entre les besoins qu'il s'agit de satisfaire, ce qui aurait pu conduire à la reconnaissance d'un tel droit ${ }^{124}$. Dès lors, on peut conclure que le droit à l'eau n'existe dans le droit communautaire européen que de façon implicite ${ }^{125}$.

\section{$\beta$. Le droit à l'eau dans les instruments universels}

Au plan international, le droit à l'eau est prévu dans deux catégories d'instruments. D'une part, on relève des instruments juridiques contraignants et d'autre part il existe des instruments dont la portée est très limitée. Le tout premier texte qui aborde la question de manière allusive, donc de manière implicite est le Pacte international relatif aux droits économiques, sociaux et culturels adopté par l'Assemblée générale des Nations-Unies en 1966 ${ }^{126}$. La référence à un niveau de vie suffisant par le Pacte permet de penser que l'Assemblée a consacré le droit à l'eau.

Aux termes de l'article 11 du Pacte, un niveau de vie suffisant doit permettre entre autres l'accès à une nourriture, un vêtement et un logement suffisants, ainsi qu'à une amélioration constante de ces conditions d'existence ${ }^{127}$.

Le second élément de référence au Pacte est l'article 12 selon lequel, l'exercice du droit à l'eau devrait assuré par des mesures visant la diminution de la mortalité infantile, ainsi que le développement sain de l'enfant, l'amélioration de tous les aspects de l'hygiène du milieu et de l'hygiène individuelle, la prophylaxie et le traitement des maladies endémiques, professionnelles et autres ${ }^{128}$. Par contre, la lecture de la Convention relative aux droits de l'enfant ${ }^{129}$ et de la Convention sur l'élimination de toutes les formes de discrimination à l'égard des femmes ${ }^{130}$ révèle la reconnaissance du droit à l'eau par la communauté internationale. La première étant ratifiée par 191 Etats alors que la seconde est ratifiée par 170 Etats. Aussi, faut-il saluer la précision du contenu de l'article 14 de la Convention sur l'élimination de toutes les formes de discrimination à l'égard des femmes qui dispose que "les Etats doivent assurer aux femmes le droit de bénéficier de conditions de vie convenables, notamment en ce qui concerne l'approvisionnement en eau ».

124 OLAKA J-M., op. cit. p. 156.

125 Idem.

126 Voir les articles 11 et 12 du Pacte.

127 LAMARCHE L., Perspectives occidentales du droit international des droits économiques de la personne, Bruxelles, Bruylant, 1995, pp. 443-444.

128 OLAKA J-M., op. cit. p. 144.

129 Texte adopté le 20 novembre 1989

130 Convention adoptée le 18 décembre 1979 
De même, les auteurs reconnaissent que certains instruments relatifs au droit à l'eau sont d'une portée aléatoire. Il s'agit par exemple de l'Observation générale $n^{\circ} 15$ sur le droit à l'eau éditée par le Comité des nations unies sur les droits économiques, sociaux et culturels. Le Comité précise que : «le droit humain à l'eau est indispensable pour vivre sa propre existence dans conditions de dignité humaine. Il constitue une pré-condition pour la réalisation des autres droits humains $»^{131}$. Aussi noble qu'elle paraît, l'observation n'a pas de portée de juridique, ce qui explique l'indifférence des Etats à mettre en œuvre ce droit. Enfin, il faut mentionner que de nombreuses déclarations et recommandations internationales ont célébré le droit à l'eau. De la Déclaration de Mar Del Plata en passant par la Déclaration de Dublin sans omettre les Déclarations de Stockholm et de $\mathrm{Rio}^{132}$, toutes ont affiché de manière non équivoque la nécessité de rendre l'eau accessible à tous. Le pas récent le plus décisif a été la reconnaissance solennelle par tous les gouvernements en 2000 de l'objectif de réduire de moitié en 15 ans la proportion des personnes privées d'un tel accès ${ }^{133}$. Mais elles demeurent de simples instruments déclaratoires ${ }^{134}$.

\section{La question de l'existence du droit à l'eau et le débat sur la hiérarchie des droits fondamentaux}

Selon PICARD E., on peut dégager trois conceptions de la catégorie des droits fondamentaux ${ }^{135}$. Reprenant à son compte une conception positiviste qu'il qualifie de pure, les droits fondamentaux seraient " exclusivement les droits consacrés par la constitution $»^{136}$. Cette conception est très répandue dans la doctrine. Il a ainsi pu être relevé que «pour la plupart des auteurs, des droits sont fondamentaux parce qu'ils sont inscrits dans les normes de plus haut degré d'un système juridique $»^{137}$.

La question de la hiérarchie des droits fondamentaux a toujours été, et est encore, fort délicate. Dans cette incertitude, l'affirmation du droit à l'eau risque d'aggraver la polémique. Quel sera le degré de fondamentalité du droit à l'eau ? La réponse à cette question étant déterminant pour l'effectivité de ce droit, nous allons explorer la question en examinant le point de vue de quelques auteurs en la matière. En restant dans la logique de VEDEL G. et RIVERO

131 Voir E/C. 12/2002/11, pp. 1- 4.

132 Rapport de la Conférence des Nations Unies sur l'environnement et le développement durable, Rio de Janeiro, 3-14 juin 1992.

133 « Nous, chefs d'État et de gouvernement, nous décidons....de réduire de moitié, d'ici à 2015, la proportion des personnes qui n'ont pas accès à l'eau potable ou qui n'ont pas les moyens de s'en procurer ». Ce texte qui se réfère aux « moyens » financiers pose la question du caractère abordable du prix de l'eau. Il oblige à s'interroger sur l'accès à l'eau potable pour les pauvres...,

134 On peut citer d'autres instruments à caractère déclaratoire comme le paragraphe 18.47 d'Action 21 , le rapport de la Conférence Internationale sur la population et le développement (le Caire du 5 au 13 septembre 1994)...

135 PICARD E., « Droits fondamentaux », in Dictionnaire de la culture juridique, Paris, PUF, 2003, p. 544.

136 Idem.,

137 CHAMPEIL-DESPLATS V., «La notion de droit fondamental et le droit constitutionnel français », Dalloz, 1995, p. 323. 
J., cette polémique est vite balayée puisque ces auteurs estiment que tous les droits sont identiques ${ }^{138}$. Si tous les droits sont identiques, le droit à l'eau bénéficiera de la même protection notamment la possibilité pour tout citoyen de saisir les tribunaux pour en réclamer le respect. Mais l'obstination d'autres auteurs à distinguer les droits par rapport à leur fondamentalité nous amène à nous interroger sur l'avenir de ce droit fondamental tombé dans l'oubli. En se référant aux réflexions de PICARD E., la hiérarchie des droits fondamentaux se résume à un « échelle de fondamentalité $»^{139}$ dont l'intérêt est de résoudre les conflits entre les droits fondamentaux. Mais pour qu'un tel mode de résolution des conflits soit effectif, il est nécessaire d'isoler les critères de cette hiérarchie, faute de quoi, l'échelle de fondamentalité ne serait qu'une abstraction ${ }^{140}$.

Aussi, faut-il préciser que dans le contexte de la libéralisation du secteur de l'eau, le droit à l'eau ou son effectivité entrera en conflit direct et certain avec la liberté d'entreprendre qui est aussi un "droit fondamental» ou "une liberté fondamentale » et sous-tend la politique de la libéralisation en général. Si la liberté d'entreprendre est constitutionnellement proclamée, le refus de la libéralisation du secteur de l'eau y serait une entrave. Or l'effectivité du droit à l'eau, et donc sa constitutionnalisation, n'est pas forcément compatible avec la liberté d'entreprendre qui sous-tend en droit interne la libéralisation du secteur de l'eau. Du point de vue de la hiérarchie des droits, le droit à l'eau et la liberté d'entreprendre étant des normes placées au même rang, l'effectivité de l'un risquerait de compromettre la réalisation de l'autre. Il faut remarquer que, à première vue, le droit à l'eau et la liberté d'entreprendre semblent a priori incompatibles. Mais à l'analyse, on peut affirmer qu'il est possible de les concilier. La puissance publique prendra à cet effet, les mesures nécessaires, pour que la liberté d'entreprendre ne s'exerce pas au détriment du droit à l'eau, qui au-delà de son caractère individuel, présente également un caractère collectif qui justifierait de faire prévaloir l'intérêt général.

En effet, presque tous les Etats de l'Afrique subsaharienne ont constitutionnalisé la liberté d'entreprendre ou l'une de ses variantes ${ }^{141}$. Revenant sur les critères de la hiérarchie, nous

138 RIVERO J. et VEDEL G., «Les problèmes économiques et sociaux dans la Constitution de 1946 », Droit social, 1947, p. $31 \mathrm{~s}$.

139 PICARD E., «L'émergence des droits fondamentaux en France», AJDA, 1998, nº spécial, pp. 6 s., spécialement pp. $32 \mathrm{~s}$.

140 PLATON S., La coexistence des droits fondamentaux constitutionnels et européens dans l'ordre juridique français, Paris, LGDJ, p. 542.

141 - La Constitution du Mali promulguée par DECRET N 92-0731 P-CTSP en son article 14: «La liberté d'entreprise est garantie dans le cadre des Lois et règlements en vigueur ».

Loi n 2001-03 du 22 janvier 2001 portant Constitution du Sénégal en son article 8 : - La République du Sénégal garantit à tous les citoyens les libertés individuelles fondamentales, les droits économiques et sociaux ainsi que les droits collectifs. Ces libertés et droits sont notamment :

- Les libertés civiles et politiques : liberté d'opinion, liberté d'expression, liberté de la presse, liberté d'association, liberté de réunion, liberté de déplacement, liberté de manifestation.

- les libertés culturelles,

- les libertés religieuses,

- les libertés philosophiques,

- les libertés syndicales,

- la liberté d'entreprendre, 
dirons avec FAVOREU L. ${ }^{142}$ qu'il s'agit de déterminer une variable permettant d'identifier une préséance axiologique ${ }^{143}$ intrinsèque de certains droits sur les autres.

A l'évidence, lorsque l'on considère uniquement la conception du Professeur FAVOREU, on serait tenté d'affirmer que si les politiques africains tiennent à réaliser l'objectif du droit à l'eau effectif, ils doivent faire en sorte que ce droit bénéficie d'un régime de protection renforcée. Et la principale "protection renforcée» dont pourrait bénéficier le droit à l'eau en Afrique subsaharienne, serait la mise en place d'une juridiction constitutionnelle opérationnelle et véritablement indépendante, capable d'invalider toutes lois remettant en cause le libre accès à l'eau ${ }^{144}$.

L'approche du Professeur BREILLAT D. ${ }^{145}$ consiste à examiner le droit en considération de son «emballage». En effet, il préconise "une hiérarchie accordant par exemple davantage d'importance à certains droits fondamentaux selon la nature du texte dans lequel ils sont proclamés ». D'autre part il se réfère à l'ordre dans lequel les droits fondamentaux sont proclamés dans les textes. Quant à GENEVOIS B., il tenta de dégager les paramètres du degré d'importance d'un droit fondamental et distingue « le degré de précision de la norme, l'existence d'exceptions prévues, le degré d'attachement de l'opinion dominante et l'étendue du contrôle du juge sur les actes ou décisions qui le mettent en cause ou en æeuvre ${ }^{146}$. Pour sortir de cette nébuleuse de conceptions en matière d'hiérarchie des normes fondamentales, du moins en ce qui concerne l'affirmation et l'effectivité du droit à l'eau, il nous paraît judicieux d'adhérer à l'approche de VEDEL G. qui considère que tous les droits sont identiques. Nous reviendrons sur la question lorsque nous traiterons des rapports entre l'effectivité du droit à l'eau et l'insoluble débat sur l'indivisibilité des droits de l'homme.

Voir aussi la Constitution du Burkina Faso adoptée par le Référendum du 02 Juin 1991 et révisée en 1997, 2000, 2002) et mise à jour le 20 Septembre 2004, en son article 16 : «La liberté d'entreprise est garantie dans le cadre des lois et règlements en vigueur ».

142 FAVOREU L., «Les libertés protégées par le Conseil constitutionnel » in ROUSSEAU D. et SUDRE F.(dir.), Conseil constitutionnel et Cour européenne des droits de l'Homme (droits et libertés en Europe), Paris, STH, 1990, pp. 33 s., spéc. p. 37.

143 «Qui se rapporte à la théorie des valeurs morales». Dictionnaire Larousse.

144 Ces juridictions sont prévues dans les «nouvelles constitutions ». C'est le cas par exemple du Togo et du Gabon. Cf. la Constitution de la IV ${ }^{\text {ème }}$ République du Togo en son Article 99 : « La Cour Constitutionnelle est la plus haute juridiction de l'État en matière constitutionnelle. Elle est juge de la constitutionnalité de la loi et elle garantit les droits fondamentaux de la personne humaine et les libertés publiques. Elle est l'organe régulateur du fonctionnement des institutions et de l'activité des pouvoirs publics » et la Constitution du Gabon du 26 mars 1991 (modifiée par les lois du 18 mars 1994, du 29 septembre 1995 du 22 avril 1997 et du 11 octobre 2000) en ses articles 83 « La cour constitutionnelle est la plus haute juridiction de l'Etat en matière constitutionnelle. Elle juge de la constitutionnalité des lois et elle garantit les droits fondamentaux de la personne humaine et les libertés publiques. Elle est l'organe régulateur du fonctionnement des institutions et de l'activité des pouvoirs publics. » et 84 « La cour constitutionnelle statue obligatoirement sur : la constitutionnalité des lois organiques et des lois avant leur promulgation, des actes réglementaires sensés porter atteinte aux droits fondamentaux de la personne humaine et aux libertés publiques ».

145 BREILlAT D., «La hiérarchie des droits de l'homme » in Mélanges Philippe ARDANT, Droit et Politique à la croisée des cultures, Paris, LGDJ, 1999, pp. 353 s.

146 BADINTER R. et GENEVOIS B., «Normes de valeur constitutionnelle et degré de protection des droits fondamentaux », rapport présenté par la délégation française à la VIIIème conférence des Cours constitutionnelles européennes (Ankara, 7-10 mai 1990), RFDA, 1990, pp. 317 s., spéc. pp. 331 s. 


\section{La déclaration du 28 juillet 2010 , une étape décisive dans le processus de l'affirmation du droit à l'eau}

Mar Del Plata, Stockholm, Rio, Dublin..., les réunions internationales sur l'eau et sur l'accès à l'eau sont nombreuses, mais aucune d'entre elles n'a proclamé avec précision le droit à l'eau. C'est à l'Assemblée Générale des Nations Unies le 28 juillet 2010 que la communauté internationale s'est prononcé clairement sur le contenu du droit à l'eau.

\section{a. Une proclamation sans équivoque}

A la suite du Rapport ${ }^{147}$ de l'experte du Conseil des Droits de l'Homme, Catarina de ALBUQUERQUE, rapport confirmant l'engagement des Nations Unies à rendre effectif l'accès à l'eau, l'Assemblée générale des Nations Unies adopte, sur proposition de la Bolivie, une résolution intitulée «le droit fondamental à l'eau et à l'assainissement $»^{148}$. Les Etats signataires réaffirment qu'il incombe aux Etats de promouvoir et de protéger tous les droits de l'homme, qui sont universels, indivisibles, interdépendants et intimement liés, et qui doivent être traités globalement, de manière juste et égale, sur un pied d'égalité et avec la même prioritél49. On constate ainsi que la déclaration du 28 juillet 2010 a une portée universelle et les Nations Unies précisent à cet effet que le droit à l'eau est indissociable des autres droits de l'homme. Le contenu du texte de la résolution fait référence à la notion d'accès à l'eau. Le texte précise que l'accès équitable à une eau potable salubre fait partie intégrante de la réalisation des droits de l'homme ${ }^{150}$.

La déclaration du 28 juillet 2010 constitue un tournant décisif dans le processus de formalisation et de reconnaissance du droit à l'eau. Toutefois, la prudence doit être de mise, car plusieurs Etats n'ont pas voté la déclaration au motif que l'accès à l'eau constitue une prérogative étatique relevant de la souveraineté, étant entendu que c'est l'accès à l'eau disponible qui est essentiellement visée dans ce cas ${ }^{151}$. En effet, seulement 122 Etats ont voté pour l'adoption du texte tandis que 41 se sont abstenus. L'acte du 28 juillet 2010 justifie que l'arsenal juridique régional et sous-régional en la matière soit approfondi.

$147 C f$. A/HRC/12/24- $1^{\text {er }}$ juillet 2009.

148 Cf. Résolution A/64/L.63/Rev.1.

149 DROBENKO B., précité, p. 113.

150 Idem.

151 Idem. 


\section{b. Un contenu aux fondements humanitaires}

La rédaction de l'acte du 28 juillet 2010 révèle de nombreuses références à des considérations humanitaires. Elle a ainsi souligné l'importance que revêt l'accès équitable à une eau potable salubre et propre et à des services d'assainissement, cet accès étant primordial à la réalisation de tous les droits de l'homme. De nombreux points sensibles apparaissent dans le texte adopté. En effet, l'Assemblée n'a pas manqué de rappeler la croissance démographique exponentielle actuelle, une croissance qui est à la base du drame de l'eau, mais elle a surtout souligné les impacts environnementaux qui pourront en découler. Sans ignorer les effets du changement climatique, la déclaration du 28 juillet invite les Etats signataires à intégrer le droit à l'eau dans le processus normatif ${ }^{152}$. En réalité, le contenu du droit à l'eau avait été déjà défini à travers les nombreuses conférences qui ont précédé la déclaration du 28 juillet. SMETS H. en a d'ailleurs fait un élément essentiel de son combat pour l'effectivité du droit à l'eau. Il écrira dans cette optique que la reconnaissance du droit à l'eau présente un intérêt tout particulier pour toutes les personnes qui n'ont pas accès à l'eau, en particulier celles en situation de précarité et celles éloignées des sources d'eau potable. Pour SMETH H., lorsque l'eau est difficilement accessible ou que son prix devient inabordable pour certaines personnes vu leur faible niveau de revenu ${ }^{153}$, il est nécessaire que la société intervienne pour leur permettre d'acquérir une quantité limitée d'eau sans y consacrer une part trop importante de leurs revenus ou de leur temps ${ }^{154}$. Enfin, on peut retenir que le droit à l'eau est le droit pour chaque être humain de disposer gratuitement de 50 litres d'eau salubre pour satisfaire ses besoins fondamentaux ainsi que celui de pouvoir bénéficier d'une installation sanitaire, dans tous les domaines de la vie, garantissant l'intimité et la dignité de la personne, tout en permettant le traitement des eaux usées domestiques ${ }^{155}$.

\section{Délimitation du sujet}

L'étendue du thème est très vaste. Il ne nous est pas possible d'explorer toute l'Afrique subsaharienne qui est un ensemble regroupant 48 pays, à la fois homogène en ce sens qu'il cristallise tous les indicateurs de développement « dans le rouge » mais aussi très hétérogène : il y a par exemple la faiblesse du Produit National Brut par habitant, la coïncidence du pouvoir d'achat en Sierra Leone et au Burkina Faso, et la divergence entre celui du Mali et celui du

152 DROBENKO B., précité, p. 115.

153 En France, les personnes les plus exposées à manquer d'eau sont les “sans abri”, les personnes sans domicile fixe (SDF), les immigrés illégaux ("sans papiers"), les gens du voyage, les nomades sédentarisés dans des campements, les occupants sans titre de logements salubres ou insalubres ("squat") et plus généralement beaucoup de personnes dont le revenu individuel est inférieur à $2600 \mathrm{~F} / \mathrm{mois}$ et qui peinent à payer leur eau. En tout, la population ayant des problèmes d'accès d'eau pourrait atteindre 1 à $2 \%$ de la population totale. Dans certains pays en développement particulièrement pauvres, plus de la moitié de la population est mal desservie en eau potable.

154 En Afrique du Sud, on s'efforce de mettre les bornes fontaines à moins de $200 \mathrm{~m}$ des habitations. Selon Wateraid, la distance journalière parcourue par les femmes en zone rurale en Afrique et en Asie pour chercher l'eau serait de $6 \mathrm{~km}$. Lorsqu'il faut parcourir plus d'un km à pied pour accéder à l'eau, la consommation journalière tombe à 10 litres par personne.

155 Cf. Résolution 41/120 de l'AG précitée. 
Gabon. La diversité s'apprécie en termes économiques mais aussi politiques (gouvernance) et culturels, en termes de tailles des marchés à desservir, aussi les 10 millions d'habitants de Lagos n'ont pas grand chose à voir avec les villages du Niger par exemple. Nous n'avons pas la prétention d'étudier au cas par cas le problème de l'accès à l'eau de chacun de ces Etats. L'épine dorsale du sujet étant l'effectivité du droit à l'eau, nous éviterons d'être excessif sur le développement des termes voisins du droit à l'eau comme le droit de l'eau. Par rapport au champ géographique, nous allons prendre, essentiellement, un échantillon d'Etats composés du Togo, du Bénin, du Burkina, du Niger, du Mali, du Sénégal, de la Côte d'Ivoire, du Sénégal, de la Guinée, du Congo, de la RDC, du Cameroun, de la Gambie, de l'Afrique du Sud... Un choix très opportun, car ne pouvant se justifier que par la difficulté d'avoir accès aux informations concernant les autres. A travers une approche d'ensemble, nous évoquerons de manière préférentielle ou contingente les Etats d'expression francophone.

\section{Problématique}

$\mathrm{Au}$ centre de différentes considérations d'ordre économique, environnemental social, politique et géostratégique, l'accès à l'eau sera le principal enjeu des prochains conflits qui pourront toucher notre planète. Les juristes commencent à réviser leur approche par rapport à cette ressource en axant leur centre d'intérêt sur l'accès des plus pauvres à l'eau potable. D'où la reconnaissance du droit à l'eau mais avec des questions très controversées. Sans doute, le droit à l'eau est susceptible de limiter les droits et libertés, notamment la liberté d'entreprendre. Les conséquences juridiques et économiques du droit ne sont pas évidentes. L'intensification des usages de l'eau au $\mathrm{XX}^{\mathrm{ème}}$ siècle, et maintenant au $\mathrm{XXI}^{\mathrm{e} m e}$ siècle, appelle à s'interroger sur les limites de l'approche classique de la nature juridique et économique de l'eau ${ }^{156}$. Jadis, l'eau était une ressource abondante et gratuite ${ }^{157}$. Elle était considérée jusqu'au début du $\mathrm{XX}^{\text {ème }}$ siècle comme une ressource inépuisable partout dans le monde. En dehors des périodes de sécheresses ou de pénuries, les ressources en eau étaient gérées comme un bien public gratuit, disponible pour l'usage des êtres humains. Quel bilan doit-on faire de la politique de distribution de l'eau en Afrique subsaharienne? La mise en œuvre du droit à l'eau doit-elle se faire de manière progressive ou de façon immédiate ${ }^{158}$ ? Par ailleurs, la mise en œuvre du droit à l'eau nécessite-telle une constitutionnalisation de ce droit comme c'est déjà le cas dans certains pays ${ }^{159}$ ? Sur

156 «Les tensions sur la ressource en eau constatées à la suite d'un accroissement de la demande liée à la diversification des usages (irrigation, industrialisation, besoins domestiques...) ont conduit ces dernières années un certain nombre de pays à reconnaître à l'eau le statut du patrimoine commun de la nation et dans certains cas à nationaliser purement la ressource en eau. Plus cette dernière est rare plus la nécessité se fait sentir de procéder à son appropriation collective. ». SIRONNEAU J., op. cit. , p.302

157 MARX K. y a fait allusion en affirmant que : « les forces physiques appropriées à la production telles que l'eau, la vapeur, etc., ne coûtent rien... ». Cf., Le capital, Editions sociales, t.2, p.71

158 «Il a fallu vingt-trois siècles à l'Europe pour faire la Déclaration de 1789 et, environ, un siècle et demi pour consacrer les droits sociaux. L'idée selon laquelle il serait possible de «sauter » les étapes historiques est sans doute fausse. Elles peuvent être vécues en accéléré du fait du progrès des connaissances et de l'essor des techniques de communication. Mais chaque phase de développement doit être franchie avant de parvenir à celle qui verra coexister les droits de l'Homme et le bien être économique et social. »Cf. MADIOT Y., Droits de l'Homme, Paris, Masson, 1990, p.26

159 Certains pays comme l'Afrique du Sud ont inscrit le droit à l'eau dans leur loi fondamentale. 
quelle base juridique s'effectue aujourd'hui la libéralisation du secteur de l'eau? La reconnaissance du droit à l'eau implique-t-elle que les citoyens ont un droit à obtenir sa mise en œuvre, exiger la réalisation des obligations qui en découlent et poursuivre l'Etat qui serait coupable de manquement à ces obligations? Si la doctrine classique du service public «admet de multiples interventions de l'État, en dehors du domaine régalien, pour les activités non rentables, mais aussi là où l'initiative privée est défaillante et dans les situations de monopole $»^{160}$, comment comprendre l'acceptation des Etats de l'Afrique subsaharienne à subir le dictat des institutions financières internationales qui leur imposent la libéralisation des secteurs dits non rentables comme celui de l'eau?

Il se pose la question de savoir comment assurer l'effectivité du droit à l'eau face aux différents enjeux économiques inhérents à sa mise en œuvre.

\section{Méthodologie et annonce du plan}

Le libéralisme économique n'est pas de nature à catalyser le respect de certains droits et libertés contenus dans les instruments internationaux relatifs aux droits de l'homme. C'est dans cette optique que l'Observation $n^{\circ} 15$ recommande que les États qui sont membres d'institutions financières internationales, notamment le Fonds monétaire international, la Banque mondiale et les banques régionales de développement, accordent une plus grande attention à la protection du droit à ces institutions ${ }^{161}$. De même, $1^{\prime}$ Observation $\mathrm{n}^{\circ} 15$ précise que les accords de libéralisation du commerce ne devraient pas entraver ou amoindrir la capacité d'un pays d'assurer le plein exercice du droit à l'eau ${ }^{162}$. Ceci est révélateur de la crise de confiance existant à l'endroit des acteurs de l'eau, particulièrement les multinationales qui, sous l'angle financier, sont les véritables bénéficiaires de la libéralisation. D'autre part nous analyserons les législations sur l'eau de la plupart des Etats africains du sud du Sahara, notamment les codes de l'eau. Il s'agira de relever les dispositions susceptibles de contribuer à la promotion du droit à l'eau et in fine souligner leur côté protecteur des ressources en eau.

Notre démarche analytique se veut aussi comparatiste et descriptive. Elle est basée sur l'exploitation d'une recherche documentaire (bibliographique et internet), qui elle-même est orientée sur l'effectivité d'un droit fondamental qu'est le droit à l'eau. Concrètement, nos recherches sont allées vers les services publics, la théorie des droits fondamentaux, le droit international de l'eau et la politique de l'environnement. Quand bien-même, il s'agit d'une étude sur l'Afrique subsaharienne, nous allons nous référer «abondamment» au droit français notamment, en puisant abondamment dans la jurisprudence et la doctrine française.

160 MILL J. S. cité par Galbraith Pierre LE MASNE (Chercheur au GEDES à l'Université de Poitiers), « Services publics et développement », p. 7.

http://sceco.univ-poitiers.fr/gedes/docs/CollBdxDev PLM.pdf consulté le 25 mars 2011.

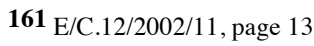

162 Ibidem. 
En résumé, ces travaux constituent un regard sur les utilisations de la ressource en eau et nous amène à établir un état des lieux du droit qui lui est applicable, de l'organisation, de la gestion, et de la gouvernance qui en est faite. D'autre part, il s'agit de proposer des solutions à apporter aux préoccupations nouvelles telles que la quantité d'eau disponible, sa qualité ou encore son prix. Comme DELVOLVE P., l'on s'interrogera, par rapport à l'effectivité du droit à l'eau, sur le lien entre le service public et les libertés publiques ${ }^{163}$, ou se demander sur comment va-t-on appliquer le droit de la consommation au service public de l'eau ${ }^{164}$. Ceci conduira à relever les contradictions de la délégation de service public de l'eau ${ }^{165}$ et inévitablement étudier le rapport entre les lois fondamentales et les contrats publics ${ }^{166}$.

Tout en démontrant que l'effectivité du droit a l'eau est dans l'engrenage de la liberté d'entreprendre, nous expliquerons les fondements juridiques et économiques de la réalisation de la libéralisation du secteur de l'eau (PREMIERE PARTIE). A partir de l'analyse des fondements et des sources du droit à l'eau nous démontrerons que la notion du droit à l'eau demeure encore très controversée. En effet, le droit à l'eau suscite sur le plan doctrinal un débat intense. Le caractère inaliénable et économique de l'eau fait du droit à l'eau un droit particulier, qui non seulement est au centre de la vie mais constitue également la pierre angulaire de nombreux autres droits fondamentaux. Il sera également question de montrer que le droit à l'eau et la libéralisation du secteur de l'eau sont en réalité conciliables tant il est capital aux Etats africains d'œuvrer pour l'effectivité du droit à l'eau à travers une sélection des différentes solutions possibles, en redéfinissant la politique de gestion du secteur de l'eau. Aussi, sera-t-il important de procéder à la mise en œuvre des obligations consécutives à ce droit et la prise en compte des principes du développement durable et de la bonne gouvernance. Il s'agira de montrer que le droit à l'eau est et devra être plus qu'une obligation de moyens pour les pouvoirs publics africains et qu'eu égards aux raisons précédemment évoquées, les Etats de l'Afrique subsaharienne ont l'impérieux devoir de tout mettre en œuvre pour la réalisation effective du droit à l'eau ${ }^{167}$, car la libéralisation du secteur de l'eau a des conséquences néfastes sur l'effectivité du droit à l'eau (DEUXIEME PARTIE).

163 DELVOLVE P., « Service public et libertés publiques », RFDA 1985, p. 2.

164 DELVOLVE P., « La question de l'application du droit de la consommation aux services publics », DA 1993, $\mathrm{n}^{\circ} 10$, p. 3 .

165 DELVOLVE P., « Les contradictions de la délégation de service public », AJDA 1996, p. 681.

166 DELVOLVE P., « Constitution et contrats publics », Mélanges Franck Moderne, Dalloz, 2004, p. 469.

167 Nous démontrerons comme Lionel JOSPIN que «l'eau n'est pas, ..., un produit comme les autres. Elle ne peut entrer dans une pure logique de marché régulé seulement par le jeu de l'offre et de la demande. Un équilibre est à trouver en fonction des capacités contributives de chaque catégorie d'usagers, du coût économique et aussi social de sa participation à l'action commune, et des options politiques qu'il appartient à chaque État de retenir, au titre de ce qu'il considère comme ses intérêts prioritaires ». Source http://www.premier-ministre.gouv.fr, consulté le 12 juin 2001 . 
PARTIE I.

LA REALISATION DE LA LIBERALISATION DU SECTEUR DE L'EAU 
Selon Henri SMETS, dans les pays en développement et en particulier en Afrique subsaharienne, il reste d'importants besoins insatisfaits en eau potable, surtout en dehors des centres urbains. Certains besoins correspondent à une demande solvable qu'il est possible de satisfaire sur une base économique et d'autres à une demande non solvable vu le degré de pauvreté mais qui pourrait devenir solvable grâce au développement économique. Il serait incohérent pour les gouvernements de ces pays de refuser de reconnaître le droit à l'eau sur la base de considérations d'ordre économique après avoir reconnu le droit à l'alimentation comme le droit au logement (inscrits dans le Pacte international relatif aux droits économiques, sociaux et culturels), droits qui ont des incidences financières bien supérieures au droit à l'eau. Par ailleurs, au plan juridique, la reconnaissance explicite du droit à l'eau serait une démarche cohérente pour les très nombreux pays en développement qui ont déjà ratifié l'une des conventions sur la discrimination à l'égard des femmes ou sur les droits de l'enfant qui font chacune référence explicite au droit à l'approvisionnement en eau. Il en serait de même pour les pays qui ont ratifié l'une des conventions régionales sur les droits de l'homme ${ }^{168}$. Alors que l'Etat n'arrive pas à assumer pleinement son rôle en matière de distribution d'eau, les institutions financières internationales « obligent » celui-ci à se désengager totalement du secteur.

Certes, il existe dans chacun des Etats africains du sud Sahara un dispositif juridique propice à cette libéralisation, mais est-il suffisant? Pour expliquer juridiquement la privatisation du secteur de l'eau, il faut rapprocher le processus du principe de la liberté d'entreprendre qu'il faudrait distinguer des droits et libertés voisins ${ }^{169}$. Cette liberté reconnue d'ailleurs par la plupart des constitutions africaines, justifie assurément le début de la désétatisation du secteur de l'eau. S'il est vrai que le secteur était géré essentiellement en régie par l'Etat, sa privatisation va marquer un tournant décisif dans la perspective d'une gestion plus saine. Malheureusement, elle va sonner le glas à l'Etat-providence d'où la dégénérescence du service public de l'eau.

D'autre part, la marchandisation du secteur de l'eau peut s'expliquer sur le plan économique. Longtemps considéré comme un res communis, l'eau ou plutôt l'eau potable a nécessairement un coût financier. De plus, sa distribution requiert un investissement financier énorme. On comprend dès lors que la ressource en eau n'ait pas de valeur économique alors que le service de l'eau a nécessairement un coût. A cet effet, les usagers du service public de l'eau doivent en application du principe de l'utilisateur-payeur, être soumis à une tarification. Celle-ci est diversifiée et parfois adaptée aux revenus des consommateurs. Les lois portant code de l'eau prévoient également la mise en application du principe du pollueur-payeur, principe qui préconise d'une part que l'utilisateur qui pollue l'eau ou les ressources en eau, doit procéder à leur restauration et d'autre part, que toute personne dont l'activité est susceptible de dégrader les ressources, de prendre des dispositions de prévention.

168 SMETS H., Le droit à l'eau, Conseil Européen du Droit de l'Environnement, 2002, p. 32.

169 Par exemple la liberté du commerce et de l'industrie. 
TITRE I.

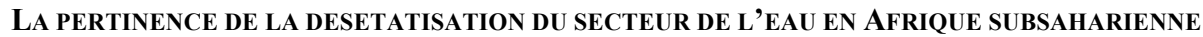


Suite à l'aggravation de la paupérisation économique qui a résulté de la politique de gabegie appliquée dans les années 1970, la plupart des Etats de l'Afrique subsaharienne ont dû s'adresser à partir de 1980-1981 au Fonds Monétaire International et à la Banque Mondiale, dont l'assistance est conditionnée par l'adoption d'un certain nombre de mesures drastiques parmi lesquelles l'on peut mentionner: le contrôle strict du déficit budgétaire par la réduction des dépenses; l'abolition des subventions aux produits de consommation (notamment l'eau), l'accroissement de la pression fiscale et la hausse des tarifs des entreprises publiques... ${ }^{170}$

En fait, toutes ces mesures visent au désengagement économique de l'Etat et, par conséquent, à l'adoption de stratégie de développement libérale conformément aux orientations économiques du FMI et de la Banque Mondiale ${ }^{171}$. Car ainsi que le soulignait le célèbre rapport BERG ${ }^{172}$, «les pouvoirs publics atteindraient plus facilement leurs objectifs de développement économique et social, s'ils réduisaient les attributions administratives trop étendues confiées un peu partout en Afrique au secteur public. Il conviendrait donc d'encourager et d'exploiter l'esprit d'entreprise des particuliers et des sociétés privées qui sont à même de s'adapter à la situation et aux besoins locaux, notamment dans la petite industrie, la commercialisation et les services... $»^{173}$.

Dans l'optique de la mise en œuvre de ces mesures d'assainissement économique, le secteur de l'eau à l'instar de nombreux secteurs publics sera privatisé. Malheureusement, les objectifs escomptés par les institutions de Bretton Woods ne seront pas atteints. Ce qui suscitera de nombreuses polémiques à propos du libéralisme ainsi prôné ${ }^{174}$.

Au-delà de la levée de boucliers des $\mathrm{ONG}$ et d'un certain nombre de personnalités scientifiques $^{175}$ et politiques contre la libéralisation économique et en particulier contre la désétatisation du secteur de l'eau, il faut remarquer que cette dernière possède un fondement juridique (Chapitre I) et ne peut se comprendre que par rapport à l'ampleur de ses enjeux financiers et économiques (Chapitre II).

170 AHADZI K., «Droits humains et développements, théories et réalités », Revue Nigérienne de droit, $\mathrm{n}^{\circ} 3$, Décembre 2000, p. 51.

171 LAIDI Z., Enquête sur la Banque Mondiale, Paris, Fayard ; cité par AHADZI K. dans « Droits humains et développements, théories et réalités », précité, p.52.

172 BERG E., Le développement accéléré en Afrique au sud du Sahara, Programme indicatif d'action, Washington, Banque mondiale, 1981.

173 BANQUE MONDIALE, «Le développement accéléré en Afrique au Sud du Sahara », Programme indicatif d'action, Washington DC, 1982, p. 8 cité par AHADZI K. précité, p.52.

174 LESUEUR J-Y. et PLANE P., Les services publics africains à l'épreuve de l'assainissement : une évaluation économique et sociale, Paris, L'Harmattan, 1994.

$175 C f$., TENIERE-BUCHOT P.F., L'eau, Rapport présenté à l'Académie des sciences morales et politiques à sa séance du 28 janvier 2002. Pour lui, il ne saurait être question de vendre l'eau, bien inaliénable de la personne humaine, droit fondamental, au même titre que les Droits de l'homme. 
CHAPITRE I. LA PRIVATISATION DU SECTEUR DE L'EAU JURIDIQUEMENT EXPLIQUEE 
L'accès à l'eau douce est devenu un des enjeux majeurs pour les sociétés contemporaines caractérisées par une forte croissance des besoins. Face à une population mondiale qui a quadruplé au cours du XX ${ }^{\text {ème }}$ siècle, la demande en eau a été multipliée par sept. Cet enjeu revêt néanmoins une acuité distincte selon les aires géographiques. D'un côté, les pays occidentaux satisfont leurs besoins au prix d'investissements financiers lourds permettant d'accéder, de distribuer et de traiter l'eau douce. De l'autre, les pays du Sud sont confrontés à d'importantes difficultés dont l'intensité dépend de variables physiques, démographiques, économiques et politiques $^{176}$.

À cet égard, l'Afrique subsaharienne se classe parmi les régions les plus désavantagées. Elle est confrontée à un fort « stress hydrique », c'est-à-dire une menace de pénurie d'eau qui pèse sur une partie significative des populations. Au sud du Sahara, près de 300 millions de personnes n'ont pas accès à l'eau potable (plus du tiers de la population du continent ${ }^{177}$ ) et un habitant sur deux souffre de maladies conséquentes de la pénurie ou de la mauvaise qualité de cette matière première essentielle pour la survie humaine. Une aggravation est désormais attendue compte tenu de l'élévation des températures générée par les changements climatiques. Elle préempte le développement des rendements des agricultures africaines demeurées essentiellement pluviales alors que la survie de nombreuses familles repose sur elles. De plus, le secteur va connaître un changement notable à la suite de la vague de privatisation enclenchée sous l'égide des institutions financières internationales.

En réalité, le changement ne touche que la gestion. D'une gestion publique on en est arrivé à une gestion privée. Quant à l'amélioration des conditions d'accès à l'eau, on ne peut en parler de façon péremptoire. La preuve, c'est que la libéralisation du secteur continue de faire l'objet de vives critiques. Toutefois, il faut préciser que la libéralisation a des fondements juridiques incontestables. La politique de désétatisation peut s'expliquer juridiquement à travers l'examen de la notion de liberté d'entreprendre (Section I) et l'étude de son influence sur le rôle de l'Etat en matière de services sociaux (Section II).

\section{Section I. La privatisation du secteur de l'eau à l'aune du principe de liberté d'entreprendre}

L'affirmation de la liberté d'entreprendre remonte à la Révolution française. Mais cette affirmation ne se trouve explicitement nulle part dans la Déclaration des droits de 1789. Pour DELVOLVE P., «ce silence équivaut à une reconnaissance implicite... » ${ }^{178}$. Et MESTRE J-L. de renchérir, affirmant que si la liberté d'entreprendre n'apparaît pas dans la rédaction définitive,

176 MERINO M., «L'eau : quels enjeux pour l'Afrique subsaharienne ? » Note de la FRS, n 20/2008, p. 2.

177 «World Water Forum », Africa Research Bulletin, vol. 43, n³, March 16th-April 15th 2006.

178 DELVOLVE P., Droit Public de l'économie, Dalloz, 1998, p. 106. 
il ne fait aucun doute que les constituants l'ont incluse dans les dispositions des articles 2, 4 et 5 de la Déclaration ${ }^{179}$.

Pour lui, les constituants de 1789 «entendaient bien consacrer la liberté d'entreprendre, la liberté d'user de ses facultés pour parvenir à son épanouissement et à son bien-être matériel, sous la seule réserve de l'obligation de ne pas nuire à autrui... ». Cependant, il faut préciser que ce point de vue ne fait pas l'unanimité. Pour G. QUIOT ${ }^{180}$, «la Déclaration des droits de 1789 ne proclame pas la liberté d'entreprendre ». Si en Afrique, le législateur s'est très peu référé au principe, le constituant des années 1990 l'a formellement consacré. Par exemple, selon la constitution malienne de 1992 promulguée par décret $\mathrm{n}^{\circ}$ 92-0731 P-CTSP en son article 14 : "La liberté d'entreprise est garantie dans le cadre des lois et règlements en vigueur ${ }^{181}$.

La politique de désétatisation peut s'expliquer juridiquement à travers l'examen de la notion de liberté d'entreprendre (§I) et l'étude de sa valeur juridique (§II).

\section{§ 1. Le principe de liberté d'entreprendre}

La libéralisation est incrustée dans l'idéologie libérale portée par le Préambule de la constitution de 1946 et RIVERO J. et VEDEL G. en ont fait écho dans "Les principes économiques et sociaux dans la Constitution de 1946 : le Préambule $»^{182}$. Plus récemment QUIOT G., dans une réflexion « actualisée » a souligné le lien remarqué existant entre le service public et la liberté d'entreprendre ${ }^{183}$. De plus en plus, les libertés économiques et, entre elles, la liberté d'entreprendre acquièrent en importance dans les ordres juridiques actuels. Aux dires de beaucoup, elles sont devenues fondamentales. Pourtant, une telle qualification ne va pas de soi. Elle relève d'un effort de construction, impliquant parfois une torsion de la notion de droit fondamental. Il est d'ailleurs frappant que le glissement de la notion de "droit de l'homme » vers celle de "droit fondamental ", que l'on observe tout particulièrement en France, serve singulièrement une telle construction. Les libertés économiques sont difficiles à appréhender en termes de droits de l'homme, parce qu'elles soutiennent les intérêts des entreprises et elles s'accommodent mieux de la qualification de libertés fondamentales. En effet, tandis que l'homme n'est pas un référent nécessaire pour que la notion de «droits fondamentaux » ait un sens, par

179 MESTRE J-L., «Le conseil constitutionnel, la liberté d'entreprendre et le droit de propriété », Dalloz 1984, Chroniques, p. 1.

180 Professeur de Droit public, CREDECO - Université de Nice - Sophia Antipolis, il publie « Service public national et liberté d'entreprendre ». source non disponible.

181 Voir également l'article 8 de la constitution du Sénégal, l'article 16 de la constitution du Burkina Faso...

182 RIVERO J. et VEDEL G., « Les principes économiques et sociaux dans la Constitution de 1946 : le Préambule », Collection droit social, Vol. XXXI, 1947, p. 13 et Pages de doctrine, L.G.D.J., 1980, p. 135.

183 QUIOT G., « Service public national et liberté d'entreprendre », in G. KOUBI (dir.), Le Préambule de la Constitution de 1946, antinomies juridiques et contradictions politiques, PUF, 1996, p. 187. 
contre, il l'est pour la notion de droit de l'homme ${ }^{184}$. Il s'agira d'examiner le contenu (A) puis la valeur juridique (B) du principe de la liberté d'entreprendre.

\section{A. La précision du contenu}

L'exercice de la liberté d'entreprendre nécessite que l'entrepreneur puisse quotidiennement, dans le cadre de son activité professionnelle, contracter, choisir son cocontractant et fixer librement les conditions du contrat. La création d'une entreprise, son activité et son fonctionnement, sa gestion impliquent la passation d'une multitude de contrats forts variés et si celle-ci n'est pas libre, la liberté d'entreprendre n'existe pas. La liberté contractuelle apparaît donc bien comme une condition de l'effectivité de la liberté d'entreprendre. On ne peut donc pas garantir cette dernière sans garantir la première. Dans le cadre de ces recherches, il s'agira d'expliquer le concept puis de montrer ses limites.

\section{La signification de la notion d'entreprendre}

Au nombre des libertés fondamentales applicables à l'activité économique, et indispensable à une société démocratique ou en voie de démocratisation, figurent la liberté contractuelle, la liberté de la concurrence et la liberté d'entreprendre. La liberté d'entreprendre ou d'entreprise est un principe vivant, mouvant et dont l'application quasi quotidienne illustre sa réalité et son dynamisme. Cette dernière est de loin la clé de voûte d'une liberté économique, qui met l'initiative privée au cœur du développement. L'expansion du secteur privé serait impossible si les citoyens n'avaient pas liberté intemporelle de choisir l'activité de leur choix et le mode d'exercice. C'est l'expression pure de la liberté économique

Souvent confondue à la liberté du commerce et de l'industrie, l'on a longtemps pensé que la liberté d'entreprendre y était incluse ${ }^{185}$. Et à propos du lien présumé entre les deux principes, CAMBY J-P. ${ }^{186}$ s'interrogea s'il fallait " faire un parallèle entre la liberté d'entreprendre et la liberté de l'industrie et du commerce»?

La liberté d'entreprendre a un caractère général et comprend la liberté de commerce et de l'industrie, la liberté professionnelle et la liberté de concurrence. Sommairement, la liberté d'entreprendre présente deux aspects : liberté d'établissement, c'est-à-dire la libre installation

184 CHAMPEIL-DESPLATS V., «La liberté d'entreprendre au pays des droits fondamentaux », Revue de droit du travail 2007, p. 19.

185 Pour DRAGO G., «l'on fait remonter cette liberté au fameux décret d'Allarde, c'est-à-dire à la loi des 2 et 17 mars 1791 » ainsi rédigé : «à partir du $1^{\text {er }}$ avril prochain, il sera libre à toute personne de faire tel négoce ou d'exercer telle profession, art ou métier qu'elle trouvera bon mais elle sera tenue auparavant d'une patente ».

186 CAMBY J-P., Les libertés économiques, sous la direction de DRAGO G. et LOMBARD M., Paris, Editions Panthéon-Assas, LGDJ, 2003, p. 25. 
d'une activité économique et professionnelle; liberté d'exercice d'une activité professionnelle ${ }^{187}$. Se référant à la décision du Conseil constitutionnel du 16 janvier 1982 ${ }^{188}$, par laquelle la liberté d'entreprendre a été consacrée en France, DELVOLVE P. explique que la liberté d'entreprendre "couvre tous les aspects de l'activité économique, et au plus haut niveau de l'ordonnancement juridique, c'est-à-dire le niveau constitutionnel ${ }^{189}$. Il précise ainsi, que la liberté d'entreprendre est à la fois la plus large et la plus haute de toutes les libertés économiques.

Ainsi, présente-t-il la liberté d'entreprendre comme étant composée de la liberté professionnelle (le libre accès à l'exercice de toute activité professionnelle qui est plus vaste que la liberté du commerce et de l'industrie), de la liberté du commerce et de l'industrie qui est une application dans le domaine commercial et industriel de la liberté d'entreprendre et de la liberté de concurrence, prolongement de la liberté du commerce et de l'industrie ${ }^{190}$. Le Conseil d'Etat a confirmé cette présentation en jugeant explicitement que la liberté du commerce et de l'industrie est une composante de la liberté fondamentale d'entreprendre ${ }^{191}$. La liberté d'entreprendre signifie que toute personne physique ou morale de droit privé doit pouvoir fonder l'entreprise de son choix, avoir accès à l'activité professionnelle qu'elle souhaite. Autrement dit, elle signifie la libre création d'entreprise. Une fois l'entreprise fondée, une fois l'accès à l'activité professionnelle garanti, au sens de la seconde branche de la règle, la personne juridique doit pouvoir librement exploiter cette entreprise ou exercer cette activité. C'est pourquoi, les conditions d'accès et d'exercice des différentes professions, notamment l'exigence d'agrément, de compétence, de solvabilité et plus généralement toutes règles de nature réglementaire limitant ou subordonnant l'accès à un professionnel doivent respecter l'équilibre entre la protection de l'intérêt général et l'atteinte à la liberté.

Mais il faut reconnaître que la liberté d'entreprendre ne saurait être absolue, elle doit être limitée et réglementée comme le reconnaissent aussi bien le conseil constitutionnel en France et le constituant africain ${ }^{192}$.

\section{Les limites de liberté d'entreprendre}

SIEYES affirmait que « tout citoyen est pareillement libre d'employer ses bras, son industrie et ses capitaux, ainsi qu'il le juge bon et utile pour lui-même. Nul genre de travail ne lui est

187 BON P., Dictionnaire constitutionnel, PUF, 1992, p.582.

188 C.C., n ${ }^{\circ}$ 81-132 DC, 16 janvier 1982, Loi de nationalisation, Rec. 18

189 DELVOLVE P., Droit public de l'économie, Dalloz, 1998, pp. 108-109.

190 MONPION A., «Le contrôle de l'interventionnisme économique public : l'affaiblissement du principe de liberté du commerce et de l'industrie ? » AJDA 2008 p. 232 et s.

191 Cf. CE 12 nov. 2001, Commune de Montreuil-Bellay, Lebon 551 et CE 22 mai 2003, Commune de Théoule-surMer, Lebon 232.

192 L'exemple du Mali ; Article 14 : «La liberté d'entreprise est garantie dans le cadre des Lois et règlements en vigueur ». Le constituant précise les conditions d'exercice de cette liberté. 
interdit. Il peut fabriquer et produire ce qui lui plaît, et comme il lui plaît ; il peut garder ou transporter à son gré toute espèce de marchandise, et les vendre en gros ou en détail »193. Cet enthousiasme est aujourd'hui dépassé et le Conseil constitutionnel a au fil d'une longue évolution tracé les « asymptotes » de cette faculté.

Dans son entreprise de précision du contenu de la notion d'entreprendre, le Conseil constitutionnel français a évolué dans sa démarche. Dans un premier temps, le Conseil estime à travers la décision du 4 juillet 1989, à propos de la loi modifiant la loi du 6 août 1986 relative aux modalités d'application des privatisations que " considérant que la liberté d'entreprendre n'est ni générale, ni absolue; qu'il est loisible au législateur d'y apporter des limitations exigées par l'intérêt général à la condition que celles-ci n'aient pas pour conséquences d'en dénaturer la portée $»^{194}$. Le Conseil assouplira sa position en ne faisant plus référence à la notion de "d'intérêt général », tout en excluant les atteintes excessives. On peut citer à cet égard la décision du 20 mars 1997 à propos de la loi créant les plans d'épargne retraite: "Considérant que la liberté d'entreprendre, qui n'est ni générale ni absolue, s'exerce dans le cadre des règles instituées par la loi ; que les contraintes établies par le législateur en vue de préserver la sécurité financière des salariés, en ce qui concerne la création, la gestion et le contrôle des fonds d'épargnes retraite ne portent pas à cette liberté des atteintes excessives propres à en dénaturer la portée».

Puis, il revient à une position plus nuancée, notamment dans sa décision du 10 juin 1998 sur la loi d'orientation et d'incitation relative à la réduction du temps de travail en affirmant que: «Considérant, en deuxième lieu, qu'il est loisible au législateur d'apporter à la liberté d'entreprendre qui découle de l'article 4 de la Déclaration de 1789, les limitations justifiées par l'intérêt général ou liées à des exigences constitutionnelles, à la condition que lesdites limitations n'aient pas pour conséquence d'en dénaturer la portée; qu'il revient ailleurs au législateur de fixer les principes fondamentaux du droit de travail, et notamment de poser des règles propres à assurer au mieux, conformément au cinquième alinéa du Préambule de la Constitution de 1946, le droit pour chacun d'obtenir un emploi, tout en ouvrant le bénéfice de ce droit au plus grand nombre d'intéressés ${ }^{195}$.

Enfin le Conseil constitutionnel va achever son entreprise en affirmant que toute limitation doit être justifiée par des exigences constitutionnelles ou par un motif d'intérêt général. Il n'est plus fait mention de ce que la liberté d'entreprendre n'est générale ni absolue. La censure n'est plus limitée au cas où la liberté d'entreprendre serait " dénaturée ». Un certain contrôle de proportionnalité devient possible. La protection de la liberté d'entreprendre en sort sensiblement renforcée. C'est la décision du 27 juillet 2000 qui illustre cette jurisprudence notamment dans son considérant principal au terme duquel : "la liberté d'entreprendre découle de l'article 4 de la Déclaration des droits de l'homme et du citoyen; qu'il est cependant loisible au législateur de lui apporter des limitations justifiées par l'intérêt général ou liées à des exigences constitutionnelles (...) que, par suite, il incombe au législateur, en fixant les règles tendant à la préservation du caractère pluraliste des courants d'expression socioculturels, de veiller à ce que

193 Archives Parlementaires de 1787 à 1860 , p. 260

194 Décision n 89-254 DC du 4 juillet 1989.

195 Décision n 98-401 DC du 10 mars 1998. Le Conseil reprend cette formulation à l'identique concernant la loi relative à la réduction négociée du temps de travail dans sa décision du 13 janvier 2000, Décision n 99-423. 
leur application ne limite pas la liberté d'entreprendre dans des proportions excessives au regard de l'objectif constitutionnel du pluralisme $»^{196}$.

De son côté, le Conseil d'Etat reconnaît la possibilité de limiter la liberté d'entreprendre dans un souci de protection de la santé publique ${ }^{197}$. Le Conseil d'Etat affirme le principe du «libre accès à l'exercice par les citoyens de toute activité professionnelle n'ayant fait l'objet d'aucune limitation légale ${ }^{198}$. En réalité, la loi peut restreindre la liberté d'entreprise, notamment lorsqu'elle soumet une activité à autorisation, elle permet également à l'administration de prendre des mesures restrictives dans le but du maintien de l'ordre public. Le Conseil d'Etat affirme dans un arrêt de principe que le maire détient «le pouvoir de prendre les mesures nécessaires pour remédier aux inconvénients qu'un mode d'exercice de la profession (...) peut présenter pour la circulation et l'ordre public ${ }^{199}$.

Il n'est pas rare de constater que certaines constitutions africaines ont clairement défini les limites. Les limitations d'ordre constitutionnel peuvent concerner les secteurs d'activités privatisables ; la privatisation est alors exclue pour certaines activités jugées stratégiques. C'est ainsi que la Constitution de Guinée-Bissau de 1984 disposait que sont biens d'Etat « le sol, le sous-sol, les eaux, les ressources minérales, les principales sources d'énergie, les forêts, les moyens fondamentaux de production industrielle, les moyens d'information, les banques les assurances, les routes et les moyens de transport essentiels $»^{200}$. Au Mozambique, jusqu'à la révision constitutionnelle de novembre 1990, la Constitution disposait que «les biens de l'Etat jouissent d'une protection spéciale, leur mise en valeur et leur expansion incombent à tous les organes d'Etat, organisations sociales et citoyens ». Toutefois, la complexité des rapports entre la liberté d'entreprendre et d'autres libertés se combine avec celle de leur nature.

\section{B. La nature juridique du concept}

Le choix politico-idéologique adopté par la quasi-totalité des Etats africains en faveur du système d'économie de marché, amène à distinguer les libertés économiques des libertés individuelles classiques.

196 Décision n 2000-433 du 27 juillet 2000 à propos de la loi modifiant la loi nº 86-1067 du 30 septembre 1986 relative à la liberté de communication.

197 V. à propos des dispositions relatives à la lutte contre l'alcoolisme et la tabagie, Cons. const. 8 janv. 1991, décis. n 90-283 DC, JO 10 janv., p. 524

198 CE 22 juin 1963, Synd. du personnel soignant de la Guadeloupe, AJDA 1963.460.

199 CE 22 juin 1963, préc.

200 Article 12 alinéa 2 


\section{Une liberté économique et un droit fondamental}

Comme le souligne Véronique CHAMPEIL-DESPLATS, se demander si la liberté d'entreprendre est un droit fondamental ou un droit de l'homme, peut paraître incongru tant aux lecteurs attentifs des historiens du droit qui se sont attachés à montrer l'importance de cette liberté dans l'esprit des Révolutionnaires en dépit de son absence dans la Déclaration de 1789 que de ceux du droit positif contemporain ${ }^{201}$. Celui-ci en effet, a expressément promu la liberté d'entreprendre au rang des droits et libertés fondamentaux. La question trouve toutefois une nouvelle pertinence si l'on interroge les raisons et les implications d'une telle qualification.

Pour Véronique CHAMPEIL-DESPLATS, "reconnaître le caractère fondamental de la liberté d'entreprendre lui confère notamment une propension au moins équivalente à celle d'autres prétentions légitimes à régir non seulement les rapports de l'individu avec l'Etat (dans une perspective verticale classique en matière de droits de l'homme) mais les rapports interindividuels $»^{202}$. Dans ce cas, une lecture marchande de la liberté d'entreprendre, tout comme d'autres libertés économiques, est susceptible d'entrer en conflit avec d'autres droits fondamentaux de la personne comme le droit à l'eau.

Evoquant la question de la nature juridique de la liberté d'entreprendre, DELVOLVE P. ${ }^{203}$ fait remarquer, en s'appuyant sur la jurisprudence du Conseil d'Etat ${ }^{204}$ que la liberté d'entreprendre peut être classée dans la catégorie des principes généraux du droit ${ }^{205}$.

Il relève d'autre part que la jurisprudence parle de liberté publique ${ }^{206}$ pour catégoriser la liberté d'entreprendre et cite le Conseil d'Etat dans ses décisions rendues le 20 janvier 1964 et le 16 juin $1976^{207}$. Aussi, faut-il préciser que le Professeur DELVOLVE n'a pas manqué de relever l'amalgame que le Conseil d'Etat faisait entre la liberté d'entreprendre et la liberté du commerce et de l'industrie. Cet amalgame fut entretenu par la doctrine, qui «jusqu'en 1998 », soutenait que la liberté de l'industrie et du commerce se subdivisait en deux : liberté d'entreprendre et libre concurrence ${ }^{208}$. En revanche, la question de la nature juridique de la liberté d'entreprendre ne se pose pas dans les mêmes termes en Afrique. En effet, les nouvelles constitutions entrées en

201 CHAMPEIL-DESPLATS V., « La liberté d'entreprendre : un droit fondamental ? »

http://credof.u-paris10.fr/spip.php?article52 consulté le 26 novembre 2009.

202 Ibid., http://credof.u-paris10.fr/spip.php?article52 consulté le 26 novembre 2009.

203 Cf., DELVOLVE P., précité, p.109.

204 CE, 28 octobre 1960, Martial de Laboulaye.

$205 \mathrm{Ce}$ sont des règles réputées non écrites dont le respect s'impose à toutes les autorités administratives. L'expression «principes généraux du droit applicables même en l'absence de texte» est apparue dans la jurisprudence du Conseil d'Etat à propos des droits de la défense avec l'arrêt Aramu (26 octobre 1945)

206 Ensemble des droits fondamentaux individuels ou collectifs reconnus par les textes.

207 CE, 20 janvier 1964, Chambre syndicale des compagnies de change de Lyon et de Marseille, Rec. 20. ; CE, 16 juin 1976, Confédération nationale des auxiliaires médicaux et paramédicaux, Rec. 309.

208 CAMBY J-P., précité. 
vigueur dès le début des années 1990 , consacrent le principe de la liberté d'entreprendre ${ }^{209}$. Ce que semble apprécier le Professeur VIGNON Y., lorsqu'il affirme que «la constitutionnalisation des droits constitue une garantie très sérieuse »210. Il précise que "placés dans un texte constitutionnel, les droits prennent en effet la valeur juridique qui s'y attache, c'est-à-dire la plus élevée dans la hiérarchie des normes ». Seulement, l'exercice de cette liberté constitutionnellement reconnue porte parfois atteinte à la réalisation de l'effectivité du droit à l'eau. Ce conflit ne pourrait être résolu qu'en faisant appel à l'intérêt général.

Si l'on s'est beaucoup interrogé sur la valeur juridique de cette liberté qui permet aux personnes physiques et morales de déployer une activité économique, que peut-on en conclure depuis que le Conseil constitutionnel français l'a déduite de l'article 4 de la Déclaration des droits de l'homme et du citoyen ${ }^{211}$ ?

\section{Une liberté transversale}

La liberté d'entreprendre recoupe plusieurs droits et libertés. Ainsi, l'on constate que les protections de la liberté d'entreprendre nous renvoient à des droits dérivés. Il serait surabondant de revenir sur la notion de la liberté du commerce et de l'industrie que certains avaient substituée à la notion de la liberté d'entreprendre. Par contre, on peut rapprocher la liberté d'entreprendre du principe de la libre concurrence fondée sur l'article 6 de la Déclaration de 1789 qui renvoie au principe de l'égalité devant la commande publique. Le juge constitutionnel l'a illustré à travers la décision $\mathrm{n}^{\circ}$ 2007-556 DC du 16 août 2007 par rapport à la Loi sur le dialogue social et la continuité du service public dans les transports terrestres réguliers de voyageurs ${ }^{212}$. De même, la décision $\mathrm{n}^{\circ}$ 2009-575 DC du 12 février 2009 relative à la Loi pour l'accélération des programmes de construction et d'investissement publics et privés ${ }^{13}$ suscite les mêmes réflexions. Par ailleurs, la liberté d'entreprendre a été précisée à travers la protection spécifique de l'égalité en matière économique notamment par la décision n 81-132 DC du 16 janvier 1982 du Conseil constitutionnel dans ses considérants 29 à 32 .

Par rapport à la protection des contrats légalement conclus, la juridiction constitutionnelle a estimé que "le législateur ne saurait porter à l'économie des conventions et des contrats légalement conclus une atteinte d'une gravité telle qu'elle méconnaisse manifestement la liberté découlant de l'article 4 de la Déclaration des droits de l'homme et du citoyen de $1789 »^{214}$. En effet, le principe de liberté contractuelle possède deux types de fondements, un fondement

${ }^{209}$ L'exemple de l'article 16 de la constitution du 11 juin 1991 du Burkina, précité.

210 VIGNON Y., op. cit., p. 91.

211 CC, $n^{\circ}$ 98-401, Loi d'orientation et d'incitation relative à la réduction du temps de travail, Rec. p. 258

$212 C f$. le considérant 23.

$213 C f$. le considérant 4

214 Décision n 98-401 DC du 10 juin 1998 - Loi d'orientation et d'incitation relative à la réduction du temps de travail (cons.28). 
principal issu de l'article 4 de la Déclaration des droits de l'homme et du citoyen de 1789, et des fondements accessoires issus de l'article 16 de la Déclaration de $1789^{215}$ et de l'Article $8 \mathrm{du}$ Préambule de la Constitution de 1946.

\section{§2. La valeur juridique de la liberté d'entreprendre}

Que ce soit en droit français ou en droit africain, la liberté d'entreprendre a aujourd'hui une valeur constitutionnelle. Mais il faut préciser que cette valeur est longtemps restée controversée (A) avant d'être précisée (B).

\section{A. Une valeur juridique à l'origine controversée}

Si elle peut se définir comme la possibilité pour tout individu de choisir librement une activité professionnelle, qui lui permette d'obtenir ses moyens de subsistance et d'exercer cette activité souverainement. Toute personne doit donc pouvoir créer une entreprise, la liberté d'entreprendre recouvre donc la liberté d'établissement ou d'installation, et diriger et gérer cette entreprise comme il l'entend, la liberté d'entreprendre comprend donc la liberté d'exploitation. Cette liberté est fondamentale pour toute société libérale où la création de richesses doit permettre la prospérité des citoyens et une sorte de «bien-être social ». C'est pourquoi il est surprenant que la Déclaration des Droits de 1 Homme et du Citoyen ne proclame pas expressément la liberté d'entreprendre. Néanmoins, à défaut d'être reconnue explicitement par cette Déclaration, la liberté d'entreprendre l'est implicitement. En effet cette reconnaissance ressort de l'esprit du texte, de la volonté de ses auteurs. Comme le montre le Professeur MESTRE, la liberté d'entreprendre est mentionnée dans les projets présentés par des membres de l'Assemblée Nationale Constituante et il ressort clairement des débats sur le texte définitif que les députés entendaient la prendre en compte. Mais la détermination de sa valeur juridique est malaisée puisqu'il règne autour de ce concept une incertitude sémantique source de conflit.

\section{L'incertitude sémantique}

Si l'on raisonne sur la nature de cette liberté, sur son contenu, on doit observer, qu'elle est historiquement, aussi ancienne que tous les autres principes de 1789. C'est véritablement un

215 Principe de sécurité juridique Décision n² 2002-465 DC du 13 janvier 2003, Loi relative aux salaires, au temps de travail et au développement de l'emploi (cons.8). Cf. également la Décision n 2004-490 DC du 12 février 2004, Loi organique portant statut d'autonomie de la Polynésie française (cons.93). 
acquis de la Révolution. En effet, diverses tentatives avaient déjà été menées mais elles étaient demeurées extrêmement timides. Ce n'est qu'à partir de la Révolution que, véritablement, ce qui s'appellera longtemps la liberté du commerce et de l'industrie (avant que de s'appeler la liberté d'entreprendre), sera proclamé de manière définitive. Cette liberté d'entreprendre, que le Conseil constitutionnel a été amené à ne redécouvrir qu'en 1982, fait donc partie de "notre » héritage juridique à l'instar de toutes les autres libertés proclamées pendant la période révolutionnaire.

D'un point vue conceptuel, on peut considérer que la liberté d'entreprendre est aux personnes morales ce que la liberté est aux personnes physiques. Certes, la liberté d'entreprendre bénéficie aussi aux personnes physiques. Il est toutefois évident qu'elle est plus vitale encore, en tout cas quotidiennement, aux personnes morales, car la liberté des personnes morales, au sens de l'article $4^{216}$, c'est d'abord, et avant tout, la liberté d'entreprendre. Et, sauf à considérer que les personnes physiques et les personnes morales ne sont pas égales en dignité, l'on devrait, en bonne logique, être aussi scrupuleux vis-à-vis des personnes morales à l'égard de leur liberté d'entreprendre, qu'on l'est vis-à-vis des personnes physiques à l'égard de leur liberté au sens de l'article 4.

Enfin, socialement, il faut se rappeler que la liberté d'entreprendre est au cœur de tous nos systèmes ; qu'elle fait partie intégrante de leur définition même ; que l'économie de marché, qui permet et qui traduit la liberté d'entreprendre, est consubstantiellement attachée à la société dans laquelle nous vivons. Il est assez étrange qu'aucune constitution française ne soit allée jusqu'à la proclamer formellement alors que plus personne ne remet véritablement en cause les principes de l'économie de marché. Tout ce qui la menace peut être discuté, aussi bien le collectivisme d'un côté que le capitalisme de l'autre, mais je n'entrerai pas dans ce débat.

\section{Une valeur potentiellement conflictuelle}

C'est le Professeur ESMEIN qui affirmait que " quelque légitimes que soient les droits individuels, ils n'ont pas une portée illimitée. Ils ont, au contraire deux limites nécessaires : le droit égal chez autrui et le maintien de l'ordre public $»^{217}$. Et nous ajouterons une troisième limite qui est l'intérêt général. C'est dire que sans ces restrictions, les droits essentiels proclamés dans les textes fondamentaux seront en conflit insoluble. Et l'enjeu de la reconnaissance du caractère fondamental des libertés économiques comme la liberté d'entreprendre, réside dans les effets et les conséquences auxquels ce qualificatif permet de prétendre, en particulier en cas de conflit avec d'autres droits notamment le droit à l'eau.

216 Art. 4 « La liberté consiste à pouvoir faire tout ce qui ne nuit pas à autrui : ainsi, l'exercice des droits naturels de chaque homme n'a de bornes que celles qui assurent aux autres Membres de la Société la jouissance de ces mêmes droits. Ces bornes ne peuvent être déterminés que par la Loi ».

217 ESMEIN A., Eléments de droit constitutionnel français et comparé, tome 1, Paris, Sirey, 1927, p. 600. 
On a pu affirmer que les droits et libertés fondamentaux sont des droits imprescriptibles et inaliénables ${ }^{218}$ ce qui les rend inconciliables lorsqu'un conflit survient entre eux. Que se passerait-il si le droit à l'eau entrait en conflit ouvert avec la liberté d'entreprendre ?

En admettant la fondamentalité de la liberté d'entreprendre et celle du droit à l'eau, l'on s'inscrit à coup sûr dans une logique conflictuelle. Et paraphrasant CHAMPEIL-DESPLATS V., nous dirons que cette qualité (la fondamentalité) lui (la liberté d'entreprendre) confère en effet une prétention à faire obstacle non plus seulement à l'action de $1^{\prime} E t^{2}{ }^{219}$, mais également à d'autres droits et libertés qui s'inscrivent dans la catégorie traditionnelle des droits de l'homme : dignité, non-discrimination, instruction, santé (donc le droit à l'eau) 220 .

Dans le passé, les libertés économiques et l'économie de marché ont eu besoin du cadre « des droits de l'homme pour assurer leur fonctionnement et leur développement ». De nos jours, une ligne de tension apparaît et remet « en cause l'intimité des rapports entre droits de l'homme et économie de marché $»^{221}$. Les libertés économiques et les droits de l'homme ne s'allient plus dans une perspective commune de limitation de l'action de l'Etat mais ils s'affrontent.

Ce conflit potentiel entre la liberté d'entreprendre et le droit à l'eau prendra des formes diverses. Il peut prendre la forme d'oppositions horizontales directes entre des personnes qui se prévalent des libertés économiques et d'autres qui s'appuient sur le droit de l'homme à l'eau. Elle peut aussi se manifester par une opposition qu'on peut dire « verticale inversée ». Dans ce cas, ce ne sont plus les individus qui opposent leur liberté d'entreprendre aux pouvoirs publics mais ces derniers qui opposent le droit à l'eau à la liberté d'entreprendre.

Par ailleurs, en s'inspirant de l'idée de Kéba M'BAYE222, selon laquelle, il ne saurait y avoir de développement social et économique sans le respect des droits de l'homme, on peut se poser la question de savoir s'il peut y avoir de développement lorsque l'exercice du droit à l'accès à l'eau est restreint. Il faut donc envisager des solutions appropriées qui promeuvent l'accès à l'eau. Traditionnellement, ce type de conflit peut avoir trois issues possibles : la prévalence du droit à l'eau ou "l'issue humaniste ${ }^{223}$ », une conciliation dite "sociale libérale» ou une

218 L'imprescriptibilité signifie qu'un droit ou une liberté fondamentale ne disparaît pas avec son non usage. Pour autant il ne suppose pas que le bénéfice de tels droits est permanent. L'inaliénabilité signifie qu'il ne peut faire l'objet d'une abdication ou d'une aliénation par voie de convention ou d'acte unilatéral. $C f$. DUPRE de BOULOIS X., Droits et libertés fondamentaux, Paris, PUF, 2010, p. 46.

219 Conformément à une conception libérale très classique.

220 CHAMPEIL-DESPLATS V., « La liberté d'entreprendre au pays des droits fondamentaux », Revue de droit du travail 2007, p. 19

221 RACINE J-B, «L'ordre concurrentiel et les droits de l'Homme », Mélanges en l'honneur d'A. PIROVANO, Edition Frison-Roche, 2003, p. 420

222 M'BAYE K., «Les droits de l'homme en Afrique », Revue internationale de droit comparé, Année 1993, Volume 45, Numéro 3, p. 723 - 726.

223 Selon CHAMPEIL-DESPLATS V., la solution humaniste accorde la primauté aux droits de l'homme non marchands sur les libertés économiques soit intégralement, soit partiellement en acceptant des dérogations dûment justifiées au bénéfice des secondes. Cette place subalterne des libertés économiques n'a guère été systématisée que dans des Etats communistes, mais le dirigisme économique n'y a jamais vraiment été justifié au nom de la protection des droits de l'homme. Ailleurs, la subordination des libertés économiques provient essentiellement de justifications à référent collectif exprimant l'intérêt de l'Etat, de la Nation ou de la société : ordre public, intérêt général, solidarité nationale, utilité sociale, santé publique... 
prévalence de la liberté d'entreprendre. Peu d'ordres juridiques recourent exclusivement à l'un de ces modes de résolution. Les ordres juridiques nationaux offrent en général une pluralité de combinaisons en fonction des acteurs qui opèrent les choix, des activités économiques considérées et de l'importance accordée aux droits de l'homme mis à l'épreuve. Ils présentent toutefois des dominantes qui évoluent plutôt depuis une vingtaine d'années au bénéfice des libertés économiques. Ce renouveau des libertés économiques dont celui de la liberté d'entreprendre aura des incidences structurelles sur le plan de l'orientation des économies des Etats de l'Afrique subsaharienne. Le secteur de l'eau en subira de nombreux bouleversements.

\section{B. Une valeur juridique finalement précisée}

La liberté d'entreprendre n'apparait pas dans le texte définitif de la Déclaration des Droits de 1 'Homme et du Citoyen, c'est parce que les rédacteurs ont opté pour un texte concis et synthétique. Elle n'est plus que sous-entendue par ce texte et, notamment, dans la définition donnée à l'article 4 de la liberté mais aussi à l'article 5 . On peut donc voir, conformément à la jurisprudence du Conseil constitutionnel3, dans l'article 4 de la Déclaration des Droits de l'Homme et du Citoyen le fondement de la liberté d'entreprendre. Par ailleurs, sa valeur juridique sera précisée aussi bien au niveau du droit africain qu'au niveau français.

\section{Une valeur constitutionnelle en droit africain et en droit français}

La liberté d'entreprendre est une règle à valeur constitutionnelle, qui trouve sa racine dans la Déclaration des droits de l'homme de 1789. Dans sa décision du 16 janv. 1982, sur les nationalisations, le Conseil constitutionnel estime que " la liberté qui aux termes de l'article 4 de la déclaration consiste à pouvoir faire tout ce qui ne nuit pas à autrui ne saurait elle-même être préservée si des restrictions arbitraires ou abusives étaient apportées à la liberté d'entreprendre ». L'intérêt de la valeur constitutionnelle de la liberté d'entreprendre est de pouvoir l'opposer à la $\operatorname{loi}^{224}$.

Les argumentations du Conseil constitutionnel français ont conduit dans un premier temps à considérer la liberté d'entreprendre comme une liberté de "second rang", même si cette hiérarchisation des droits fondamentaux, " est en elle-même contestable ». La jurisprudence du Conseil constitutionnel sur la liberté d'entreprendre a fluctué au cours des vingt dernières années et a subi des critiques acerbes de la doctrine ${ }^{225}$. Ces flottements ne portent pas sur le fondement

224 KDHIR M., « Le principe de la liberté du commerce et de l'industrie : mythe ou réalité ? », Recueil Dalloz 1994 p. 30 et s.

225 SAVY F., «La constitution des juges », Dalloz. 1983. Chron. 105. Voir aussi J. -L. MESTRE, « Le Conseil constitutionnel, la liberté d'entreprendre et la propriété », Dalloz. 1984. Chron.1. 
de cette liberté226, mais sur son degré de protection, ainsi que sur l'intensité du contrôle de sa limitation par le Conseil.

Pour synthétiser cette évolution, on peut dire qu'à partir d'une formulation initiale protectrice 227 , le Conseil a eu tendance à minorer progressivement la protection de la liberté d'entreprendre. Et ce n'est que depuis $1997^{228}$ que s'opère une évolution inverse, débouchant sur le considérant très ferme figurant dans la décision de janvier $2001^{229}$ relative à l'archéologie préventive. Désormais, la liberté d'entreprendre n'occupe plus de rang subalterne au sein des libertés et le Conseil vérifie que la conciliation opérée par le législateur entre cette liberté et d'autres exigences constitutionnelles ou des motifs d'intérêt général antagonistes n'est pas excessivement ou inutilement déséquilibrée.

Le «parcours » de la liberté d'entreprendre en Afrique n'est pas exactement le même. En effet, le constituant africain n'a pas ou presque utilisé la notion voisine de liberté de commerce et de l'industrie. Sans doute, il a subi l'influence du juge constitutionnel français qui, dès 1982, a consacré l'expression liberté d'entreprendre. Il n'a pas repris exactement l'expression mais il semble lui préférer le « synonyme » de liberté d'entreprise ${ }^{230}$.

Toutefois, son exercice à l'instar du droit français, est soumis au respect des exigences constitutionnelles et la prise en compte des motifs d'intérêt général parmi lesquels on peut ranger l'accès à l'eau. A propos des restrictions à cette liberté, le juge constitutionnel français a précisé qu'elle n'est ni générale, ni absolue et ne peut exister que dans le cadre d'une réglementation instituée par la loi ${ }^{231}$. A l'exemple du constituant malien précité, la plupart des constituants des Etats de l'Afrique subsaharienne ont délimité le cadre d'exercice de cette liberté232. Dès lors, il faut se poser la question de savoir lequel du droit à l'eau et de la liberté d'entreprendre, doit-il primer sur l'autre ? Les deux étant tous droits fondamentaux, le conflit est inévitable mais soluble

226 Art. 4 de la Déclaration de 1789

227 Voir décision du 16 janvier 1982 précitée.

228 Décision n 97-388 DC du 20 mars 1997, Loi créant les plans d'épargne retraite,: « (...) Considérant que la liberté d'entreprendre, qui n'est ni générale ni absolue, s'exerce dans le cadre des règles instituées par la loi ; que les contraintes établies par le législateur en vue de préserver la sécurité financière des salariés, en ce qui concerne la création, la gestion et le contrôle des fonds d'épargne retraite ne portent pas à cette liberté des atteintes excessives propres à en dénaturer la portée ; (...)»

229 Décision n 2000-439 DC du 16 janvier 2001, Loi relative à l'archéologie préventive, « (...) 13. Considérant qu'il est loisible au législateur d'apporter à la liberté d'entreprendre, qui découle de l'article 4 de la Déclaration des droits de l'homme et du citoyen de 1789, des limitations liées à des exigences constitutionnelles ou justifiées par l'intérêt général, à la condition qu'il n'en résulte pas d'atteintes disproportionnées au regard de l'objectif poursuivi ; (...)»

230 A l'exception du constituant sénégalais, l'utilisation de l'expression liberté d'entreprise est généralisée.

231 Décision n 82-141 DC du 27 juillet 1982, Loi sur la communication audiovisuelle, Rec. 48.

232 Sauf l'article 58 de la constitution du Tchad qui ne définit pas les limites: «L'Etat garantit la liberté d'entreprise ». Cela peut être dû à une omission de la part du constituant. 


\section{Une valeur constitutionnelle surestimée}

A partir de la décision de 1982, le Conseil constitutionnel a très souvent été « sommé " par les saisissants, d'utiliser la liberté d'entreprendre. Il n'a prononcé que très peu de censures à ce titre. En vérité, il en a prononcé trois, auxquelles s'en ajoute une quatrième, mais qui portait davantage sur une question d'intelligibilité de la loi ${ }^{233}$ que sur la liberté d'entreprendre. Dans ces trois décisions de censure prononcées en 1988, en 2000 et en 2002, le conseil considère, après avoir « dédouané » la Constitution qui ne peut être ni générale, ni absolue, que ce n'est qu'en cas de dénaturation qu'il peut y avoir une annulation. Il ne s'agit pas de mesurer l'importance d'une liberté au nombre des censures, ce serait complètement absurde. Néanmoins, c'est un indice de ce que cette liberté ne joue pas un rôle équivalent à celui d'autres principes comme, par exemple, le principe d'égalité.

Cette liberté d'entreprendre, de quoi se compose-t-elle ? D'énormément de choses : c'est entreprendre, c'est exploiter, c'est aussi contracter dira Nicolas MOLFESSIS, c'est aussi rechercher. Existe-t-il une différence entre liberté d'entreprendre d'un côté, et liberté du commerce et de l'industrie de l'autre ? À titre personnel, nous sommes tout à fait séduit par ce qu'expose Pierre DEVOLVE lorsqu'il dit, en substance, que la différence entre les deux, c'est la concurrence ; le souci de la concurrence est présent dans la liberté du commerce et de l'industrie, alors qu'il n'est pas, en tant que tel, nécessairement présent, à ce jour du moins, dans la définition de la liberté d'entreprendre. Reste que le sentiment qui se dégage de tout ceci est que, dans la jurisprudence, la liberté d'entreprendre proclamée comme un principe est, en vérité, bien davantage utilisée comme un objectif. En d'autres termes, la liberté d'entreprendre s'apparente moins à un principe constitutionnel qu'à un objectif de valeur constitutionnelle.

Il existe un malaise au point que le Conseil paraît partagé, parfois timoré, sur la façon de concilier harmonieusement les exigences de la liberté d'entreprendre d'un côté, celles de l'intérêt général de la règlementation et de la législation d'autre part. Ceci pose des problèmes d'autant plus épineux que l'on sait très bien que, dans l'avenir, la liberté d'entreprendre va, à un moment ou à un autre entrer éventuellement en conflit, ne serait-ce qu'avec la charte de l'environnement. Celle-ci, à ce jour, n'a pas pleinement donné lieu à un contentieux nourri. Mais il est vrai que, aussi bien à propos de son article 3 sur la prévention des atteintes qu'à propos de son article 4 sur la réparation des dommages, l'on peut imaginer toutes sortes de situations dans lesquelles la liberté d'entreprendre peut, soit être piétinée, soit, au contraire, être invoquée légitimement pour marquer une limite à ce que des exigences environnementales, au demeurant tout à fait défendables, pourraient éventuellement avoir d'excessif.

\section{Section II. La portée de la consécration du principe de la liberté d'entreprendre}

La consécration constitutionnelle de la liberté d'entreprendre va engendrer la rencontre de l'économie de marché et du service public. Arguant de l'idée que la déficience des prestataires publics dans de nombreux pays explique incontestablement le problème de l'approvisionnement en eau, les institutions de Bretton Woods vont les "pousser» à la sortie. Elles vont mettre

233 Décision n 421 DC du 16 décembre 1999. 
l'accent sur la mauvaise gouvernance et la dégradation des infrastructures.

En effet, bon nombre de prestataires publics fonctionnent selon un modèle de service descendant qui n'est ni transparent, ni réceptif aux besoins des usagers. Aussi, n'est-il pas rare de constater qu'une bonne part de l'eau fournie par les services publics n'est pas comptabilisée, soit parce qu'elle fuit des canalisations qui ne sont pas entretenues, soit en raison de systèmes de facturation défectueux ${ }^{234}$. La résurgence de la logique économique dans les politiques de développement des Etats va mettre à mal le secteur public. Les Etats vont se libérer des entreprises publiques qui gèrent l'eau. La désétatisation du secteur de l'eau (§1) sera doublée de la déchéance du service public de l'eau (\$2).

\section{§1. Le début de la désétatisation du secteur de l'eau}

C'est en fait à la diminution de l'interventionnisme étatique en Afrique que l'on va assister. La pression des institutions financières internationales et l'environnement économique régional et sous régional vont contribuer à éclipser l'Etat dans ses fonctions régaliennes et à altérer ses pratiques monopolistiques. La désétatisation du secteur de l'eau sera surtout caractérisée par "l'éclipse de l'Etat providence » (A) et sera le reflet de la crise de l'Etat africain (B).

\section{A. L'éclipse de l' « Etat providence »}

A la faveur des slogans tels que «moins d'Etats, plus de marché » les Etats africains n'ont pas pu résister à la propagation des thèses libérales en Afrique subsaharienne. L'effet d'imitation a joué certainement un rôle non négligeable. Ces conversions au libéralisme sont également le résultat des contraintes financières.

\section{L'origine du phénomène}

La crise de l'Etat-Providence et la mondialisation de l'économie, parallèlement aux travaux sur la réforme de l'Etat, avaient fait éclore le mouvement pour une Charte des services publics... Relisons un article d'humeur de cette époque, signé en 1995 par Georges VEDEL, dans Le Monde « le service public fait chez nous vibrer tous les cœurs ${ }^{235}$. On le défend à Bruxelles [...] A

234 RAPPORT MONDIAL SUR LE DEVELOPPEMENT HUMAIN, Chapitre II : L'eau destinée à la consommation, 2006, p.89.

235 LONG M., «Service public et réalités économiques du XIXe siècle au droit communautaire », RFDA 2001 p. 1161. 
Paris, il paraît être le seul point d'accord entre le gouvernement et les grévistes... On veut l'inscrire dans la Constitution [...] La construction est, en droit, simple [...] : certaines prestations sont indispensables à la Nation. Elles ne peuvent donc être abandonnées aux incertitudes du marché. Seul l'Etat et les personnes publiques décentralisées peuvent les assurer [...] Telle est du moins la théorie [...] Mais en pratique, la loi de continuité est évidemment niée ; la loi de mutabilité ne peut jouer que pour les évolutions acceptées par les agents [...] Est dénié aux autorités politiques le dernier mot des décisions et arbitrages, au moins, sous forme de veto [...] De ce vocable ambigu, unanimement révéré, quelle est la bonne version ? ». En conclusion, le doyen VEDEL s'interroge sur l'opportunité de la Charte : "rédiger un texte qui exalterait les beautés du service public sans en dévoiler la réalité serait un exercice d'utilité douteuse [...] Et comment donner à nos partenaires européens une définition claire de ce que nous proposons?».

Les services locaux, eau, assainissement, déchets, énergie, transports..., relèvent, pour leur part, d'un modèle séculaire renouvelé par les lois de décentralisation. Leur gestion peut être déléguée, par contrat, pour une durée souvent longue, à des entreprises privées, publiques ou d'économie mixte ; si le contrat de gestion est de même type, le statut de l'exploitant suivant la nature juridique et la forme de l'entreprise a, sur la gestion, une incidence qui n'est pas négligeable et qui peut transformer la nature des relations notamment lorsque le concessionnaire est une entreprise publique et monopole national, ainsi que le montrent les rapports entre EDFGDF ou SNCF et les collectivités locales. La Fédération nationale des collectivités concédantes et régies tout en se voulant " l'interlocuteur privilégié de ces entreprises nationales, avec lesquelles elle a des liens étroits, a, pour rénover «l'économie concessionnaire », demandé la renégociation des contrats de concession et l'établissement de nouveaux cahiers des charges ${ }^{236}$.

\section{L'expérience africaine}

La tendance prépondérante en Afrique subsaharienne a été celle d'un accroissement continu de l'emprise étatique sur tous les secteurs ${ }^{237}$ allant même au-delà des secteurs dits régaliens. JACQUEMOT P. parlait ainsi de "l'étatisation outrancière »"238. Certes, le phénomène n'a pas atteint le même degré dans tous les Etats, en raison notamment de la diversité de leurs options politiques. Mais, même dans ceux où la politique défendue par les gouvernants était de créer à terme une économie fondée sur les mécanismes de marché et de faciliter l'éclosion d'un secteur national privé favorable aux capitaux étrangers, les interventions de l'Etat apparaissaient comme le vecteur naturel du développement ${ }^{239}$. Les débats sur le rôle de l'État en Afrique ont

\section{Idem.}

237 Selon FISETTE J. et SALMI M., les États africains sont des États foncièrement centralisateurs; « des raisons idéologiques et historiques expliquent cette tendance. Les actions et mesures prises par les planificateurs africains jusqu'aux années 1980 pour promouvoir le développement des économies sont de façon générale fondées sur une vision du développement qui date des premières années des indépendances ».

http://id.erudit.org/iderudit/022182ar consulté le 3 septembre 2010.

238 JACQUEMOT P., « La désétatisation en Afrique subsaharienne. Enjeux et perspectives », Tiers-Monde, 1988, volume $29, \mathrm{n}^{\circ} 114$, p. 271 et $\mathrm{s}$.

239 Idem. 
considérablement évolué depuis le début des années 1980. En effet, les gouvernements africains ont dû faire face à une crise économique et budgétaire sans précédent et ainsi ont été amenés à questionner et réévaluer l'efficacité des politiques et des stratégies de développement mises de l'avant jusque-là240. Ce qui se traduit dans la pratique par un inégal accès à l'eau caractérisé par des taux de connexion médiocre et des services publics inconstants ${ }^{241}$.

Et à partir du début des années 1990, un consensus de plus en plus large s'est formé autour du constat selon lequel l'excès d'Etat constitue l'un des freins majeurs au développement des économies africaines. Puissamment porté par certaines institutions internationales de financement ${ }^{242}$, le thème du désengagement de l'Etat était au centre de la réflexion politique sur les perspectives d'ajustement structurel des économies du continent. Des critiques sévères se sont élevées d'horizons divers pour fustiger les insuffisances des administrations et des entreprises publiques, y compris celles qui s'occupent de la distribution de l'eau, ou pour dénoncer les dysfonctionnements engendrés par l'omniprésence de l'Etat. A partir de ce moment, les services publics africains entreront dans une phase de privatisation au sens le plus large du terme.

Dès lors, la domination exercée par le postulat du libéralisme, aux termes duquel ce qui est public est intrinsèquement mauvais, va finir par éclipser le «tout Etat». Ainsi, de nombreux experts ont reconnu que le secteur privé et la concurrence seraient les véritables clefs de l'efficacité productive, y compris dans des domaines dits régaliens ${ }^{243}$.

\section{B. Le reflet de la crise de l'Etat africain}

L'écart entre les missions assignées à l'Etat postcolonial dans la phase de construction nationale et la réalité de son action est si profond que nombreux auteurs y voient aujourd'hui «non pas un Etat pathologique mais une donnée structurelle du pouvoir en Afrique» ${ }^{244}$.

240 FISETTE J. et SALMI M., « Le désengagement de l'État en Afrique et les initiatives locales : la naissance de nouvelles formes de partenariat. », Cahiers de géographie du Québec, vol. 35, n 95, 1991, p. 350.

241 JAGLIN S., «L'eau potable dans les villes en développement : les modèles marchands face à la pauvreté », Tiers-Monde, Année 2001, Volume 42, Numéro 166, p. 278.

$\mathbf{2 4 2}$ Le Fonds Monétaire International et la Banque Mondiale.

243 DE BELLESCIZE R., « Grandeur et servitude de la notion de service public constitutionnel », RFDA 2006, p. 1163 et $\mathrm{s}$.

$244 \mathrm{Du}$ BOIS de GAUDUSSON J., Afrique : interrogations sur le rôle économique de l'Etat, Encyclopedia Universalis 1985, p. 132. 


\section{L'Etat surchargé}

La reconnaissance d'un hiatus croissant entre l'instrumentalité de l'Etat et la dynamique sociale est au centre de nombreux travaux de politologie africaniste. Elle est également explicite dans certaines analyses officielles, celle de l'ancien chef de l'Etat sénégalais Abdou DIOUF, par exemple 245 . L'argumentaire défendu par DIOUF s'organise autour d'un constat historique. Le bouleversement des rapports sociaux traditionnels au Sénégal, fondamentalement de type lignager, sous l'effet d'abord de la colonisation puis de l'extension de la traite administrée, a fait reporter sur l'Etat indépendant, en pleine constitution la demande d'assistance liée à la reproduction, individuelle ou collective que la communauté de base ne pouvait plus satisfaire, faute de ressources. Les liens de solidarité communautaires, les systèmes d'entraide familiaux, les mutualités informelles étant devenus incapables de répondre à tous les besoins des membres du groupe, restés présents au terroir ou exilés en ville, un transfert de responsabilité est effectué sans transition sur l'Etat, lui-même prodigue en promesse parce qu'à la recherche de sa légitimité politique.

Ce transfert de charge a progressivement conduit à « une surconsommation d'Etat $»^{246}$. La crise latente du système tient en conséquence è cette impuissance à toutes les doléances de groupe sociaux ayant perdu leur racine sociale et ne trouvant pas dans des organes intermédiaires les structures susceptibles de couvrir leurs besoins. Cette interprétation peut être appuyée par l'exemple des politiques «d'éducation pour tous » ou de «santé pour tous » inscrites dans les premiers plans de développement africains. La même logique d'intégration sociale par l'Etat l'a conduit à absorber, de manière systématique, tous les sortants du système éducatif, de l'enseignement supérieur notamment. Dans certains pays, il était même interdit pour les diplômés du supérieur d'aller chercher un emploi hors du secteur public, l'Etat imposant que le coût de formation qu'il supportait soit valorisé dans son propre secteur. En moyenne, l'absorption des sortants du système éducatif et la création d'emploi public ont contribué pour plus de la moitié à la croissance de l'emploi salarié en Afrique depuis 1960.

La thèse de la surcharge administrative peut être confirmée par les illustrations statistiques mettant en évidence la modestie des ressources publiques au regard des obligations sociales et politiques considérables contractées par l'Etat. Le résultat a été un épuisement progressif de ressources publiques qui s'est traduit notamment par un phénomène de baisse continue des salaires réels des fonctionnaires. Dans l'ex Zaïre, mais il s'agit probablement d'un exemple extrême, l'indice du salaire moyen dans le secteur public n'était que de 26,2 en 1980 et 14,5 en $1983^{247}$. Cette situation a permis la poursuite du recrutement dans la fonction publique, mais elle a rendu inévitable certains phénomènes de fuite : le salaire étant tombé en dessous de la somme nécessaire au seul achat de la quantité de céréales indispensable pour une famille, il n'est plus possible d'attendre du fonctionnaire la moindre activité professionnelle normale.

$245 C f$. son discours devant les élèves de l'Ecole nationale d'Administration et de Magistrature le 7 mai 1985, Le Soleil, Dakar, 8 mai 1985.

246 Cf., OCDE, Coopération pour le développement, Rapport 1986, p. 147.

247 Banque du Zaïre, Kinshasa, Rapports annuels. 


\section{Le déclin de la pratique monopolistique}

Longtemps géré dans un contexte de monopole, le secteur de l'eau à l'instar de nombreux autres secteurs économiques vont quitter l'emprise de l'Etat. Des opérations de restructuration des secteurs publics et parapublics ont été mises en œuvre et la plupart des gouvernements se sont engagés dans une politique de privatisation ${ }^{248}$.

Si le monopole est, selon le Doyen de LAUBADERE, le privilège exclusif que possède une entité de vendre un bien, de fabriquer un produit, et de gérer un service ${ }^{249}$, le fait pour tout autre organisme d'offrir les mêmes biens ou services dans un même espace géographique, annonce la fin de l'emprise exclusive de l'Etat. C'est ce que va connaître d'une certaine manière le secteur de l'eau en Afrique subsaharienne. La nuance vaut la peine, puisque les différentes restructurations opérées n'ont pas abouti absolument à une libéralisation du secteur.

En effet, l'on a assisté non pas à une libéralisation au sens restreint, c'est-à-dire une ouverture du secteur de l'eau au marché, mais une privatisation qui prend le plus souvent la forme allant d'une concession au contrat d'affermage ${ }^{250}$. Cette dernière forme est la plus usuelle en matière de distribution d'eau potable en Afrique subsaharienne ${ }^{251}$. Au Niger, dans le cadre de la réforme institutionnelle du sous-secteur de l'Hydraulique Urbaine, l'État a privatisé l'exSociété Nationale des Eaux (SNE) et a confié à un opérateur privé, par un contrat d'affermage, la production, le transport et la distribution de l'eau dans les centres urbains et semi-urbains.

La SEEN ${ }^{252}$ exploite et gère le service affermé depuis le $1^{\text {er }}$ juin 2001 sous le contrôle de la SPEN (Société de Patrimoine des Eaux du Niger) et de l'ARM (Autorité de Régulation Multisectorielle) selon les règles de l'art définies dans un contrat de performance ${ }^{253}$. Il faut préciser toute de suite, que le cas du Bénin est différent, puisque la restructuration qui y a été opérée ne suit pas la même démarche ${ }^{254}$.

Dans un premier sens la privatisation d'entreprises publiques désigne le transfert à titre définitif d'entreprises du secteur public ou parapublic au secteur privé, sous la forme d'une

248 PLANE P. et LESUEUR J-Y., « Les services publics subsahariens : importance socio-économique et évaluation des d'assainissement », Tiers-Monde, 1994, Volume 35, n40, p. 780.

249 CE, 19 juin 1964, Société des Pétroles Shell Berre, AJDA, 1964, p. $442 .$.

250 Au Cameroun la loi n 98-005 du 14 Avril 1998 portant Code de l'eau a prévu en son article 2 alinéa 3 que « la gestion de l'eau peut, en outre, faire l'objet de concession ou d'affermage, suivant des modalités fixées par un décret d'application de la présente loi ».

251 PLANE P. et LESUEUR J-Y., op. cit., p.792.

252 Société d'Exploitation des Eaux du Niger, filiale de Vivendi Water

$\mathbf{2 5 3}$ http://www.arm-niger.org/secteureau.pdf consulté le 4 octobre 2010.

254 La distribution d'eau potable, elle est assurée en milieu urbain, depuis 1983, par la société Béninoise d'Electricité et d'Eau remplacée en 2004 par la société Béninoise d'Energie Electrique (SBEE) et la société Nationale des Eaux du Bénin (SONEB créée par l'arrêté 2007-18 MMEE/DC/SGM/CTJ/CTREau/DGEau/SA du19 février 2007), dans le cadre du Règlement général du service public de la distribution d'eau potable et industrielle en zone urbaine du 30 octobre 1987. 
cession de titres ou d'actifs, ou d'une cession de contrôle. Elle peut inclure également toute mesure qui opère le transfert temporaire au secteur privé d'activités qui jusque-là avaient été exercées par un organisme public ou parapublic.

Dans une seconde approche, on peut désigner par privatisation d'un secteur, lorsque, à titre principal, l'ouverture dudit secteur à l'initiative privée à travers l'abolition du monopole public et l'octroi de nouvelles licences à d'autres exploitants. Cette ouverture n'est pas toujours accompagnée de l'introduction d'une réelle concurrence entre opérateurs. C'est le cas de la plupart des privatisations du secteur de l'eau en Afrique subsaharienne ${ }^{255}$. L'on passera ainsi d'un monopole public à un monopole privé.

\section{§2. La dégénérescence du service public de l'eau}

A s'en tenir aux idées du Doyen DUGUIT, «le service public est le fondement et la limite du pouvoir gouvernemental " et l'Etat est une "coopération de services publics organisés et contrôlés par des gouvernants $»^{256}$. Pour lui, l'Etat est l'architecte de la solidarité sociale ${ }^{257}$. Les réflexions de Léon DUGUIT conservent leur pertinence aujourd'hui dans les Etats subsahariens. En effet, il est difficile de comprendre que l'Etat, au nom d'une quelconque idéologie libérale et d'orthodoxie financière, renonce à l'une de ses missions traditionnelles. Avant d'examiner la question de la déchéance du service public de l'eau (B), nous allons rappeler la définition du service public (A).

\section{A. La notion de service public}

Il est difficile de s'interroger sur le concept de service public, sans comprendre "l'intérêt général » auquel ils se réfèrent. "Le rôle des services publics est de servir l'intérêt général »: avant les arguments économiques et juridiques, c'est un argument de nature philosophique qui justifie l'existence de services publics ${ }^{258}$.

255 Au Togo, par contrat en date du 13 mai 2003, l'État togolais a concédé à la TDE (Togolaise des Eaux), pour une période de 10 ans renouvelable, l'exclusivité de la production et de la distribution de l'eau potable, de l'assainissement sur l'ensemble du territoire national.

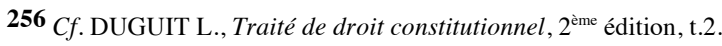

257 PELLETIER M-L., L'entreprise publique de service public : déclin et mutation, L'Harmattan, 2009, p.51.

258 MONTIALOUX Claire, «Service public et intérêt général », La Découverte, Regards croisés sur l'économie, $2007 / 2-N^{\circ} 2$, pages 25 à 26 . 


\section{La définition du concept}

Le concept du service public nait au terme d'une période d'embrouillements en Afrique subsaharienne. L'accession des jeunes Etats à la souveraineté et leur obsession d'asseoir une légitimité incontestable les ont obligés à s'investir dans des infrastructures socio-collectives. En fait, l'émergence de la théorie du service public est complexe. D'abord, l'expression même est ancienne. Elle est utilisée en France dès l'Ancien Régime, invoquée au cours des travaux de l'Assemblée constituante ${ }^{259}$. Vu sous un angle matériel, elle est caractéristique de la plupart des arrêts du Conseil d'Etat et se retrouve sous la plume de différents auteurs spécialistes du droit administratif. Et l'arrêt Blanco ne parle-t-il explicitement du « service public »? Cependant, l'importance de la notion était restée secondaire, au regard des notions d'« intérêt public » ou de « puissance publique ».

Il faut préciser que la mention «service public» ne signifie pas toujours adhésion à la doctrine du service public. En effet, dès 1892, HAURIOU M., avait souligné que le service public constituait la " raison d'être » de l'administration ; mais la référence au service public se trouve intégrée par lui dans un système explicatif plus global, où la notion de puissance publique occupe la place essentielle. Enfin, et surtout, la doctrine du service public elle-même se divise autour de deux tendances, l'une autour de JEZE G., attaché à un point de vue strictement positiviste, l'autre autour de DUGUIT, qui en fera, plus tardivement, la clef de voûte de sa théorie politique : même si les deux courants sont amalgamés par l'« école de Bordeaux » ${ }^{260}$, ils n'en restent pas moins différents, voire opposés sur certains points ${ }^{261}$.

Cette complexité est d'autant plus prononcée que la notion est à la base de la définition de nombreuses notions du droit administratif tels que les contrats administratifs ${ }^{262}$, le domaine public $^{263}$ ou les travaux publics ${ }^{264}$. Et pourtant, «le service public n'est défini dans aucun texte, et une liste des services publics n'existe pas ». La constitution évoque certains services publics qui ne peuvent être supprimés qu'en vertu d'une révision de la norme suprême, comme la justice, la police ou l'armée, mais ceux-ci ne représentent pas l'ensemble des services publics ${ }^{265}$.

Prenant en compte les apports du droit communautaire aux traditions et évolutions du droit français, TRUCHET D. définit le service public comme «une mission créée, définie, organisée et contrôlée par les personnes publiques en vue de délivrer des prestations d'intérêt général à tous ceux qui en ont besoin ${ }^{266}$. La distribution de l'eau ne peut trouver meilleur siège que dans

259 MESTRE J-L., « La notion de service public d'après les débats de l'Assemblée nationale constituante », EDCE, $\mathrm{n}^{\circ} 40,1989$, p. 187 et ss.

260 R. BONNARD, M. REGLADE, L. ROLLAND.

261 CHEVALLIER J., « Le service public : regards sur une évolution », AJDA 1997 p. 8 et ss.

262 Cf. CE, 20 avril 1956, Epoux Bertin, Rec. 167.

263 Cf. CE, 19 octobre 1956, Société Le Béton, Rec.375.

264 Cf. CE, 28 mars 1955, Effimieff, Rec., 617.

265 ROUAULT M-C, BARBE V., et CANEL G., Droit administratif, Gualino Lextenso Editions, 2009, p.128.

266 TRUCHET D., Droit administratif, Thémis droit, PUF, 2008, p.332. 
la définition préconisée par le professeur TRUCHET. A l'évidence, les personnes publiques sont responsables et sont chargées du service public de l'eau et exercent en la matière leur pouvoir réglementaire ${ }^{267}$.

\section{Le « service public en procès »}

En dépit, ou en raison, de son succès, le service public a fait l'objet de critiques de divers ordres, au point que l'on a pu parler de « service public en procès ». Difficile à définir, le concept de service public présente une autre difficulté plus importante. Celle de savoir ce qu'elle recouvre exactement. Car, en la réduisant à la notion d'intérêt général, elle-même complexe, l'on se retrouve dans des situations parfois contradictoires comme l'illustre la jurisprudence administrative en France. Ainsi, il a été jugé que le sport constituait un service public ${ }^{268}$, mais pas les courses de chevaux ${ }^{269}$, tandis que devaient être regardés comme activité de service public les lâchers de taureaux lors des fêtes municipales ${ }^{270}$. Même le doyen HAURIOU, dénonçait une décision du Conseil d'Etat qui avait jugé que le théâtre était une activité de service public ${ }^{271}$. En effet, selon lui, le théâtre a l'inconvénient "exalter l'imagination, d'habituer les esprits à une vie factice et fictive, au grand détriment de la vie sérieuse, et d'exciter les passions de l'amour, lesquelles sont aussi dangereuses que celles du jeu et de l'impétrance $»^{272}$. Difficile ainsi de considérer l'eau, ou précisément le service de distribution de l'eau potable comme étant une activité de service public sans craindre d'être contesté par la jurisprudence ou par la doctrine.

Sur le plan économique d'abord, des contestations s'élèvent : est-il légitime de réserver au service public, en les soustrayant au marché, certaines activités à partir d'un usage abusif de l'idée de monopole naturel, ou de tarifications peu favorables à certaines catégories de consommateurs ? D'autres portent sur le poids excessif des appels des entreprises publiques au marché financier ou des subventions budgétaires qui leur sont consenties, et mettent en cause l'efficacité économique des services publics en doutant qu'ils puissent assumer le meilleur service au moindre coût ${ }^{273}$.

D'une part, et notamment en ce qui concerne les services publics industriels et commerciaux, la mise en œuvre des grands principes de base du service public, principes d'égalité, de continuité, d'adaptation, ne suffit plus à apporter aux usagers de réelles garanties et joue parfois à leur détriment. On peut penser par exemple à la fermeture d'écoles à classe unique ou de bureaux de poste, à la suppression de gares et de voies ferrées en zones rurales et dans les pays de

267 LOPA DUFRENOT M., op. cit., p. 76.

268 CE, 22 novembre 1974, Fédération des industries françaises des articles de sport.

269 CE, 9 février 1979, Société d'encouragement pour l'amélioration des races de chevaux.

270 TC, 22 avril 1985, Laurent.

271 CE, 7 avril 1916, Astruc.

272 SIRINELLI M. et SORIN J., Questions de droit public, Ellipses, 2007, p. 164.

273 LONG Marceau, précité, RFDA 2001 p. 1161. 
montagne. Sans aller jusqu'à leur suppression, on songe également à l'augmentation du coût des services ou à la possibilité de différenciation des tarifs pour des usagers placés dans des situations objectivement différentes. Ainsi, le principe d'égalité ne s'oppose pas à un traitement tarifaire différent pour des usagers d'une même commune selon qu'ils habitent le centre ou des zones plus éloignées ${ }^{274}$.

D'autre part, une certaine confusion existe entre ce qui relève de l'intérêt général, « qui tend, selon les termes du Rapport du Conseil d'Etat de 1999 consacré au concept, à être entendu de façon de plus en plus large par le juge et à recouvrir des activités de plus en plus variées » et, ce qui relève du service public, cette confusion aboutit à une extension indue de la sphère publique comme à un chevauchement qui conduit à parler, à la fois et dans le même temps, de risque de mainmise publique sur l'initiative privée et du risque de contrôle privé sur le service public.

En ce qui concerne le rapport entre citoyens/usagers, si en général, les hommes sont attachés à leurs services publics et reconnaissent la qualité de beaucoup, ils sont souvent réservés sur le fonctionnement d'autres. Ces critiques sont diverses : qualité insuffisante du service, manque de souplesse et d'adaptation aux besoins des usagers, coût jugé excessif. Les mouvements de grève de grande ampleur du type de ceux qu'ont connu les transports publics parisiens à l'hiver 19951996 ont nourri l'acrimonie contre des structures incapables de gérer, sans perturbations majeures pour les usagers, le dialogue social. Les agents de ces services bénéficiant de la garantie de l'emploi font des envieux alors que le pays compte plus de 2 millions de chômeurs, en fait, c'est l'Etat qui est critiqué plus que les présidences et les directions des entreprises de service public. D'où la demande, aussi ancienne que lancinante, d'une législation relative aux procédures de déclenchement des conflits sociaux, comme au service minimum en cas de grève : aucun gouvernement ne s'y est risqué ou n'a persévéré dans de telles tentatives depuis un demi-siècle. Ces critiques n'empêchent pas une vive opposition à la privatisation des services publics : risque d'abandon des segments les moins rentables de l'activité considérée, ou, pour les segments prospères, de recul des " acquis sociaux » : rémunération, stabilité de l'emploi, temps, rythme et conditions de travail ; souvent crainte «d'ajustement» des effectifs (plan de licenciement, de mise à la retraite anticipée.... $)^{275}$.

\section{B. Le service public de l'eau en souffrance}

La problématique de l'eau dans les régions sahéliennes englobe diverses questions, dont celle, prégnante, de l'accès des populations à l'eau potable. Certes, les disponibilités en eau douce sont très disparates selon les pays d'Afrique subsaharienne : le Niger dispose, par exemple, de ressources s'échelonnant entre 0 et $1000 \mathrm{~m} 3$ par personne et par an, alors que son voisin malien jouit de 5000 à $15000 \mathrm{~m} 3$ d'eau ${ }^{276}$. Mais il n'existe pas de corrélation systématique

\footnotetext{
274 Idem.

275 Idem.

276 Cf. Salif DIOP et Philippe REKACEWICZ, Atlas mondial de l'eau, Paris, Autrement, 2003, p.32.
}

Précisions sémantiques : le Programme des Nations unies pour l'Environnement (PNUE) parle de « vulnérabilité hydrique » lorsque les ressources annuelles en eau d'une région sont comprises entre 1700 et $2500 \mathrm{~m} 3$ /pers./an ; 
entre le niveau d'eau douce disponible et l'approvisionnement de la population en eau traitée et purifiée. Ainsi, l'Afrique du Sud dispose de ressources limitées, mais une bonne gestion de la distribution et du traitement de l'eau la positionne au niveau des pays occidentaux, même si subsistent des disparités entre les différentes couches de population de la nation « arc-en-ciel $»^{277}$.

\section{Le paradoxe hydrologique}

Dans un pays comme la République démocratique du Congo, disposant d'un important potentiel hydrique (le fleuve Congo représente à lui seul $33 \%$ du débit fluvial du continent) favorisé par des précipitations élevées, ne bénéficie que d'une faible couverture nationale en eau potable.

Ce qui suppose une priorité, pour les pays en voie de développement : mettre au point un système efficace de distribution d'eau, de traitement des eaux usées et de sensibilisation des consommateurs. Et qui nécessite également des investissements importants pour le maintien ou la rénovation des infrastructures (canalisations) existantes, d'une part, et la mise en place de nouvelles adductions d'eau, d'autre part ${ }^{278}$.

Les capitales de nombreux pays africains sont sujettes à un afflux massif de populations rurales, souvent jeunes, venues dans l'espoir d'un niveau de vie plus confortable et qui participent de l'explosion démographique urbaine. Ainsi, Ouagadougou, la capitale du Burkina Faso, a vu sa population passer de 500000 âmes en 1990 à 1500000 en 2000. Cette urbanisation galopante accroît de façon ostensible le coût des investissements nécessaires dans le domaine de la distribution de l'eau, et pousse à la réflexion les gouvernements africains quant à la solution la plus viable pour résoudre ce problème.

Pour améliorer le fonctionnement des municipalités et des services d'utilité publique, les gouvernements des pays sahéliens cherchent de plus en plus à promouvoir des partenariats entre le secteur public et le secteur privé pour fournir des services d'approvisionnement en eau et d'assainissement.

S'il existe plusieurs modes de gestion des services liés à l'eau, il semble bien que ce soit le modèle français du Partenariat public-privé (PPP) qui emporte les faveurs de la Banque mondiale et de nombreux gouvernements africains (dont ceux du Niger, du Sénégal ou du Tchad). Le PPP s'apparente à un contrat établi entre l'État, à qui revient de définir les règles et les contrôles, tout en assurant les investissements de base nécessaires pour protéger et conserver le « capital» hydrique de la nation, et l'entreprise privée, à qui est confiée la gestion des services d'eau aux

de «stress hydrique » lorsqu'elles sont inférieures à 1 700/pers./an ; et enfin de «pénurie d'eau » lorsqu'elles se situent sous la barre des $1000 \mathrm{~m} 3 /$ pers./an.

277 JACKOWSKA Jonathan, «Des eaux et des idéaux : La question des villes du Sahel », Géopolitique de l'humanitaire, pp. 206-209.

278 Jonathan JACKOWSKA, précité. 
particuliers et aux secteurs économiques.

En 1994, la Banque mondiale accordait un soutien financier et politique à la mise en place du Conseil mondial de l'eau (CME) dont le but était de mettre en place une «politique mondiale de l'eau » dont l'institution financière serait la promotrice. Le CME fut également l'instigateur du Forum mondial de l'eau, manifestation triennale qui se déroule autour du 22 mars et qui regroupe les différents acteurs du domaine de l'eau du monde entier (sociétés privées multinationales, gouvernements, institutions internationales, agences spécialisées, ONG...) ${ }^{279}$.

De facto, un gouvernement comme celui du Niger a adopté le PPP. En effet, en 1996, sous l'effet des plans d'ajustement structurel, le gouvernement nigérien promulgue deux ordonnances qui déterminent la privatisation de treize sociétés ainsi que le cadre juridique de leur vente ${ }^{280}$.

Les défenseurs du PPP et, par conséquent, de l'introduction des entreprises privées dans les services sanitaires des villes africaines usent de divers arguments pour surmonter les réticences émanant notamment de la société civile : échec de la gestion de l'eau par le secteur public, et notamment des subventions; fixation d'un prix de l'eau (le fait d'établir une valeur de l'eau serait le meilleur moyen de prévenir les gaspillages. De plus, un coût de l'eau encouragerait l'adoption d'infra- structures appropriées et stimulerait l'investissement privé nécessaire à l'entretien ou à la rénovation du réseau de canalisations. Le but étant de multiplier le nombre d'abonnés " raccordés » et de les rendre plus indépendants vis-à-vis des porteurs d'eau; lutte contre le volontariat (dont on critique la gestion des services liés à l'eau) ${ }^{281}$.

\section{Des statistiques peu encourageantes}

Avec seulement $56 \%$ de sa population ayant accès à une eau saine, l'Afrique subsaharienne est à la traîne des autres régions en termes d'accès à des sources d'eau de meilleure qualité. Les tendances actuelles semblent indiquer que la région n'atteindra sans doute pas la cible fixée par les Objectifs de développement pour le millénaire (ODM), consistant à donner accès à de meilleurs services d'alimentation en eau à $75 \%$ de la population en $2015^{282}$. Ceci est sans doute le résultat, en partie, d'un service public de l'eau victime des nouvelles orientations économiques.

279 L'année 1996 sera celle de la naissance du Global Water Partnership (GWP) dont le dessein majeur est de mettre en oeuvre, à l'échelle mondiale, la « vision » proposée par le CME, notamment le PPP.

280 Parmi ces sociétés, figurait la Société des eaux du Niger qui sera privatisée en avril 2001 et qui est depuis contrôlée par Veolia Environnement (ex-Vivendi Environnement).

281 Certains principes du PPP cadrent mal avec ceux de plusieurs organisations de la société civile africaine et/ou internationale. Malgré leurs options variables, la plupart des ONG ont été, et demeurent, extrêmement critiques envers ce Partenariat. Les ONG ne forment pas un bloc idéologique monolithique, et leurs réactions à la privatisation progressive des services urbains concernant l'eau, en Afrique sahélienne tendent à le démontrer.

282 BANQUE MONDIALE, «Diagnostics des infrastructures nationales en Afrique : distribution d'eau en Afrique subsaharienne », juin 2008. 
La majorité des citadins qui ne sont pas desservis par une entreprise de services publics ont recours à des puits et forages qui sont la principale source d'alimentation de $24 \%$ de la population urbaine en Afrique. Dans certains pays, comme le Tchad, le Mali, le Nigéria et le Soudan, les puits et les forages constituent la principale source d'alimentation urbaine en eau. Seuls environ $7 \%$ des citadins puisent l'eau destinée à la boisson dans des lacs, étangs, sources ou autres formes d'eau de surface 283 .

L'environnement des services publics a changé avec l'ouverture de notre économie et la mondialisation des échanges. Le retour à des conceptions libérales de l'économie a fait évoluer les moyens d'intervention de l'Etat dans l'économie. On s'efforce de réduire ces interventions, on procède à des dérégulations, on privatise un certain nombre d'entreprises et d'activités. La discipline budgétaire, de plus en plus stricte, conforte cette démarche visant à réduire ou transformer le champ des interventions publiques et leur nature ${ }^{284}$.

Mais, les contrats de distribution d'eau bénéficiés par le secteur privé vont connaître un taux d'échec relativement élevé d'environ $25 \%$, allant jusqu'50\% pour les contrats de location et de concession $^{285}$. En réalité, les concessions ne sont pas la panacée comme en témoignent les échecs aux Comores, en Mauritanie ou au Tchad. Elles ne peuvent réussir que sous condition de prise de risque normal de l'opérateur privé dans un environnement politique et social apaisé et dans un Etat de droit faisant respecter les contrats. Mais il ne faut pas oublier de relativiser cet " échec» puisque certaines privatisations ont été saluées ou du moins ont été épargné des critiques $^{286}$. C'est pourquoi il est difficile de faire des généralisations sur le secteur de l'eau en Afrique subsaharienne. Ce qui est incontestable, c'est l'harmonie qui existe sur la nature économique de l'eau.

283 Idem.

$\mathbf{2 8 4}$ VOISSET M., «Le service public autrement », RFDA, 1995, p. 304

$\mathbf{2 8 5}$ Sources : Base de données des enquêtes AICD DHS/MICS, 2007

286 Le cas de la SAUR (groupe BOUYGUES) en Côte d'ivoire est à examiné sérieusement. Cet opérateur gère depuis 1973 le secteur de l'eau. Il dispose d'un monopole sur le secteur et vient de bénéficier d'un nouveau contrat d'affermage de 15 ans. 
CHAPITRE II. LA PRIVATISATION DU SECTEUR DE L'EAU ECONOMIQUEMENT EXPLIQUEE 
Plusieurs arguments économiques ont milité en faveur de la privatisation de l'eau. Dans un contexte de pénuries et de concurrence des usages marqué par une forte augmentation de la demande en eau, l'eau prend une valeur de plus en plus économique. Comment pouvait -on imaginer le contraire lorsqu'on assiste à un accroissement exponentiel de la démographie assortie d'une urbanisation galopante. Les villes de Lomé, Cotonou, Accra, et bien d'autres ont vu leur population se multiplier en l'espace de quelques années 287 . D'un autre côté, l'agriculture a connu un essor d'une magnitude dépassant les mesures de l'agriculture de subsistance. L'eau est au cœur de « ses progrès ». Et lorsqu'elle ainsi utilisée, elle devient rare et prend de la valeur économique. La diminution de la disponibilité de l'eau concerne surtout l'eau potable et dans une moindre mesure les ressources en eau. Ces dernières sont victimes de la pollution qui se caractérisent entre autres par la salinisation et la contamination de la ressource par le déversement de polluants variés.

Il faut chercher les sources de la dimension économique de l'eau dans les relations internationales. On retiendra que le consensus de Washington, auquel se ralliera l'Organisation mondiale du commerce dès sa création en 1994, constitue, selon ses défenseurs, la seule clef de la réussite économique et donc de la gestion des services publics marchands. Il devient, selon l'expression de Jean ZIEGLER " l'alpha et l'oméga [...] la loi et les prophètes », le seul repère idéologique de la gouvernance économique dans le monde 288 . Le Fonds monétaire international et la Banque mondiale vont soutenir que ce consensus était la seule voie idéale pour les pays du sud de sortir du sous-développement.

C'est pourquoi, la privatisation des services publics «marchands » sera au cœur des programmes d'ajustement structurel qu'ils imposeront à nombre de ces Etats pendant les années 1990. Le consensus de Washington, somme toute, devenait, selon ses concepteurs, une thérapie applicable à tout Etat, à toute économie et à toute époque. Enfin, il faut ajouter que le service de distribution de l'eau est un service à dominante marchande. Elle présente en principe un caractère un caractère industriel et commercial et peut revêtir dans certaines mesures le caractère administratif.

Nous examinerons d'une part le caractère économique indéniable de l'eau (Section I) et d'autre part le service de distribution de l'eau est un service à dominante marchande (Section II).

\section{Section I. L’indéniable dimension économique de l'eau}

Face à l'échec des pouvoirs publics, une des alternatives proposées consiste à généraliser le régime de gestion privée des ressources en eau, afin d'éviter le foisonnement des réseaux informels de distribution d'eau, ou encore la corruption des pouvoirs publics. Cette solution, qui sous-tend la mise en place d'un marché de l'eau, est parfois présentée comme une solution efficace et décentralisée. Sous certaines conditions, elle peut provoquer des incitations à éviter le gaspillage, contribuer à une réallocation efficiente de la ressource et sécuriser des droits d'accès

287 NYASSOGBO G.K., «Comment une capitale devient macrocéphale en Afrique subsaharienne : Le cas de Lomé au Togo (Afrique de 1.Ouest)» in : TALOR J. LENGELLE J.G. and ANDREW C. : Capital Cities. Les capitales. Carleton University Press, Ottawa,1993, pp.325-342.

288 ZIEGLER J., « Schizophrénie des Nations Unies », Le Monde diplomatique, novembre 2001, p.4. 
et d'usage de communautés locales ${ }^{289}$. Cette option consacre la dimension économique de l'eau que les partisans de la gestion publique de l'eau, contestent avec véhémence.

La définition du statut de l'eau est un enjeu dont les implications ne sauraient être sousestimées : les débats théoriques, qui opposent la vision de l'eau comme bien public à l'approche marchande, en passant par la conception éthique de l'eau, ont un prolongement direct dans les modes de gestion effectifs de cette ressource. A l'évidence, l'eau est un bien économique (§1) et le service de distribution d'eau va engendrer des obligations financières à l'endroit des usagers de l'eau (§2).

\section{§1. L'eau, un bien économique}

Rapprocher des logiques économiques une ressource aussi vitale que l'eau suscite de fortes hésitations. Mais les raisons économiques sont incontournables, la qualité de l'eau en dépend. Aussi, pour la première fois, l'ONU va reconnaître la valeur marchande de l'eau lors d'une conférence organisée à Dublin, en mai 1992, sur le thème de l'eau et l'environnement. Cette conférence a donné naissance à la Déclaration dite « de Dublin » sur l'eau et le développement durable. Son principe 4 proclame, en effet, la valeur marchande de l'eau potable en ces termes : "L'eau, utilisée à de multiples fins, a une valeur économique et devrait donc être reconnue comme bien économique ». Mais le dilemme devient profond quand l'on constate que certains Etats reconnaissent le droit à l'eau. Comment reconnaître le droit à l'eau et accepter que l'eau soit vendue? Même la tarification discriminatoire pratiquée ne convainc que partiellement. Après avoir montré le paradoxe des arguments économiques relatifs au statut de l'eau (A) nous traiterons de la tarification de l'eau en Afrique subsaharienne (B).

\section{A. Des caractéristiques économiques spécifiques}

Alors que SMETS H. expliquait que "l'eau est une ressource naturelle et un bien économique mais elle n'est pas une ressource naturelle comme les autres. En effet, elle est sujette à des règles juridiques particulières destinées à en préserver l'usage pour la collectivité où elle se trouve $»^{290}$, Yves THIBAULT DE SILGUY, ancien Commissaire européen, devenu délégué général de Suez en charge des affaires internationales, affirma : "Il est clair que nous ne pouvons pas investir là où il n'existe pas de marché solvable $»^{291}$. Cette pensée traduit clairement le caractère onéreux de l'eau produite par les multinationales qui, à « juste titre », ne peuvent pas fournir de l'eau à des usagers insolvables.

289 BIED-CHARRETON M. et al., «La gouvernance des ressources en eau dans les pays en développement : enjeux nationaux et globaux », De Boeck Université, Mondes en développement, 2006/3 n¹35, p. 49.

290 SMETS H., op. cit., p. 12

291 Interview parue au quotidien Le Monde, 30 mars 2005, p.III. 


\section{Des arguments non négligeables}

Bien que l'eau soit la première substance de la planète, elle n'est formée qu'à hauteur de 2,53 $\%$ d'eau douce, le reste étant de l'eau de mer. Les deux tiers de cette eau douce sont en outre immobilisés dans les glaciers et les neiges éternelles. À l'eau douce accessible dans les lacs, rivières et aquifères s'ajoutent les 8000 kilomètres cubes $(\mathrm{km} 3)$ stockés dans des réservoirs créés par l'homme. Les ressources en eau sont renouvelables (sauf certaines eaux souterraines), avec d'énormes différences de disponibilité selon les régions du monde et des variations considérables, dans de nombreux endroits, en termes de précipitations saisonnières et annuelles. Celles-ci constituent la principale source d'eau pour toutes les utilisations par l'homme et pour les écosystèmes ${ }^{292}$.

La question de l'eau en Afrique subsaharienne ne se pose pas en termes de quantité mais plutôt en termes de disponibilité et d'accès à la ressource. En effet, les experts s'accordent à dire que le potentiel hydraulique dont dispose le continent devrait largement couvrir les besoins de l'intégralité de la population. Cependant, les ressources en eau sont souvent mal réparties, car trop éloignées des grands centres de consommation, et elles sont difficiles à mobiliser pour satisfaire la demande en eau potable des populations urbaines et rurales.

L'accès à l'eau potable constitue dans les quartiers périurbains et dans les établissements humains semi-ruraux et ruraux une difficulté majeure. Ainsi, les services urbains essentiels de base sont quasi inexistants dans certaines zones du fait de la poussée démographique non maîtrisée et d'une déficience d'une prise en charge correcte par les collectivités locales.

L'eau potable présente des caractéristiques économiques spécifiques. Sa production, sa distribution, son assainissement, résultent d'infrastructures très capitalistiques, de longue durée et avec très faible redéploiement, d'ou' un risque élevé pour l'opérateur ; il y a ainsi monopole naturel local. La dimension économique de l'eau potable tient à divers éléments. Pour devenir potable et accessible sous cette forme, elle a d'abord subi une extraction, un traitement et un transport (lié à sa distribution) dégageant un " coût technique» ou « coût d'approvisionnement ». Par ailleurs, cette eau devenue potable, mais qui aurait pu être dirigée vers d'autres usages, représente pour la collectivité une option différente de ce qu'elle aurait été dans un autre cas : on parlera alors de son "coût d'opportunité », tenant compte de la rareté de la ressource afin d'éviter son gaspillage. Enfin, le prélèvement de l'eau, son traitement et son transport même ont éventuellement des conséquences environnementales (par exemple une atteinte des écosystèmes du fait de prélèvements en période de sécheresse), ce qui va ajouter un «coût environnemental» aux précédents ${ }^{293}$.

292 UNESCO, Programme mondial pour l'évaluation des ressources en eau, Rapport mondial sur la mise en valeur des ressources en eau, 2003 , p. 8.

293 VINCENT Is., «Le prix de l'eau pour les pauvres : comment concilier droit d'accès et paiement d'un service ?», Afrique contemporaine - Printemps 2003, p. 122. 


\section{Un prix du service et non de la ressource en eau}

Une telle facturation au coût intégral de l'eau, bien qu'idéale en théorie, n'est toujours qu'un objectif lointain pour les pays développés. Ceci est plus vrai encore dans les pays en développement, où l'on ne vise toujours, au stade actuel, qu'une simple facturation au coût d'approvisionnement dont on reste souvent fort loin. Théoriquement, le prix de l'eau pour les services urbains, par exemple, doit dériver du coût de la ressource, mais ce n'est pas le cas. Ce prix correspond bien aujourd'hui au coût du service de potabilisation et de distribution, mais la ressource demeure gratuite pour tous les usagers : l'eau de surface ou souterraine.

En réalité, le prix de l'eau est la conséquence du prix du service pour traiter, filtrer et distribuer la ressource (service) et pas le prix de la ressource elle-même considérée comme matière première. Ceci constitue une différence essentielle car les autres matières premières ont un prix reflétant leur rareté et l'acheteur paye des royalties au détenteur du gisement.

On pourrait donc en déduire que l'eau n'entre pas sur le marché et que ce n'est pas l'eau qui est vendue mais le service. Elle est en fait attribuée hors marché à des usagers choisis par les pouvoirs publics pour en faire un usage déterminé. Les concessions d'eau sont en principe de durée limitée. Par conséquent, on pourrait considérer que les exportations d'eau ne relèvent pas du droit international du commerce. Une telle conclusion doit être examinée avec grande prudence car l'on assiste à une extension considérable du domaine couvert par le droit du commerce $^{294}$.

\section{B. Des caractéristiques économiques discutables}

L'idée selon laquelle l'eau pour la consommation humaine soit une marchandise dont le prix est fixé par le libre jeu de l'offre et la demande est soutenue par certains économistes mais est loin d'être partagée par tous. En fait, l'idée a du mal à s'imposer malgré le soutien qu'elle a auprès de certaines organisations internationales comme le FMI et la Banque Mondiale. Vues sous un angle social et humanitaire, les caractéristiques économiques se fragilisent.

\section{Les arguments économiques face aux objectifs du millénaire}

L'objectif à atteindre n'est pas mince : en 2004, un sixième de la population mondiale n'avait toujours pas accès à l'eau potable, soit 1,1 milliards de personnes. Compte tenu de la croissance démographique prévue, la réalisation de cet objectif signifie donc de fournir l'accès à l'eau à 2,2 milliards de personnes d'ici l'an 2015, soit 280000 personnes en moyenne par jour. La majorité

294 SMETS H., op. cit. 
des efforts devra être concentrée en Afrique où deux personnes sur cinq n'ont pas accès à l'eau, ce qui correspond au taux d'accès le plus bas au monde ${ }^{295}$.

Dans le monde, des progrès dans l'accès à l'eau ont pourtant été réalisés ces quinze dernières années. Notamment, entre 1990 et 2004, le taux de non accès à l'eau est passé respectivement de $22 \%$ à $17 \%$, soit un peu moins d'un milliard de personnes ayant eu accès à l'eau pour la première fois. Parmi eux, les deux tiers ont eu accès à l'eau courante dans le logement ou la concession, contre un tiers ayant eu accès via des approvisionnements collectifs de type bornesfontaines, puits protégés, forages, pompes, etc.).

C'est l'Afrique sub-saharienne qui enregistre l'un des taux de non accès à l'eau les plus haut au monde avec $44 \%$ de la population de sous-continent, au coude à coude avec l'Océanie (50 \%). Toutefois, dans l'absolu, la plupart des personnes n'ayant pas accès à l'eau se trouvent en Asie du Sud et du Sud-est ${ }^{296}$.

Enfin, si le taux de non accès à l'eau a légèrement diminué dans le milieu rural depuis 1990 , passant de $36 \%$ à $27 \%$, celui-ci semble être stationnaire en zone urbaine du fait de la concentration croissante de la population dans cette zone (avec $5 \%$ de la population n'ayant pas accès en 1990 comme en 2004). En Asie et en Afrique, les projections de population urbaine suggèrent d'ailleurs que les services urbains en termes d'accès à l'eau seront confrontés à un grand défi.

Dans l'ensemble, l'OMD relatif à l'accès à l'eau est en voie d'être réalisé dans le monde, à une année près, soit à quelques cent millions de personnes près. L'OMD relatif à l'accès à l'eau devrait être atteint en 2016 pour l'ensemble du monde. Ce sont l'Inde et la Chine qui ont réalisé des progrès contribuant quantitativement au respect de la date butoir au niveau mondial. Si les tendances se poursuivent, la population n'ayant pas accès à l'eau devrait baisser de 150 millions d'ici à $2015^{297}$.

Toutefois, cette situation générale ne doit pas masquer des différences importantes, entre continent, entre pays, mais également à l'intérieur des pays, via notamment le différentiel entre le milieu urbain et le milieu rural, mais également via des différences entre les populations les plus riches et les populations les plus pauvres dans chaque milieu de résidence.

\section{L'incertitude de l'Afrique subsaharienne face aux enjeux de l'eau}

En Afrique sub-saharienne, la population n'ayant pas accès à l'eau a augmenté de $7 \%$ entre 1990 et 2004, soit une augmentation absolue de 60 millions de personnes. Ainsi, malgré les améliorations, si les tendances actuelles se poursuivent, l'Afrique subsaharienne n'atteindra pas l'OMD concernant l'accès à l'eau en 2015. A ce rythme, l'objectif sera atteint en 2040. Pour que l'Afrique subsaharienne atteigne l'objectif en 2015, il faudrait que le nombre de personnes ayant pour la première fois accès à l'eau soit de 23 millions par an, au lieu des 10 millions par an sur la

\section{WHO/UNICEF, 2000.}

296 DOS SANTOS Stéphanie, « La quête de l'eau en Afrique au Sud du Sahara : pertinence de l'indicateur des OMD pour répondre aux enjeux sanitaires et sociaux? », source non disponible.

297 Idem. 
période 1990-2004. Plus encore, compte tenu de la croissance démographique et si le nombre absolu suit les tendances récentes, aux 314 millions de personnes qui n'ont actuellement pas accès à l'eau, s'ajouteront 47 millions de personnes supplémentaires.

Trois raisons peuvent être invoquées pour expliquer l'insuffisance des progrès en Afrique sub-saharienne. D'une part, au départ des OMD, en 1990, l'Afrique enregistrait déjà l'un des taux d'accès les plus faibles au monde, au coude à coude avec l'Océanie. Ensuite, malgré les progrès réalisés jusqu'à aujourd'hui, ils restent globalement encore insuffisants. Enfin, le continent africain reste encore majoritairement rural. Or, l'accès à l'eau y est, de manière générale, beaucoup plus faible qu'en milieu urbain, et de manière encore plus marquée en Afrique subsaharienne. Si l'on regarde maintenant les faits et tendances par pays ou par milieu, on constate effectivement de très fortes disparités au sein du continent. Dans le rapport de 2006 rédigé conjointement par l'OMS et l'UNICEF, et faisant le point sur les cibles de l'accès à l'eau et de l'assainissement dans le cadre des OMD, un tableau est présenté indiquant la liste des pays les moins développés qui, pour atteindre l'objectif de 2015, auront besoin de multiplier par deux ou plus le taux de premier accès. Sur 24 pays listés, 19 se trouvent sur le continent africain, tous au sud du Sahara. Certains pays, comme la Guinée, le Mozambique, Madagascar, le Togo, le Niger ou la République Démocratique du Congo, devront multiplier ce taux d'augmentation par trois, quatre, cinq ou 14 pour le cas de l' l'Ethiopie. Dans le monde réel, la répartition de l'eau n'est pas faite sur la base d'un marché. Aussi le $\mathrm{CEDE}^{298}$ insiste-t-il sur le fait que «l'accès à l'eau ne peut être soumis au seul jeu des forces du marché». Éviter que l'eau potable ne soit intégralement soumise aux lois du marché apparaît d'autant plus nécessaire que la «main aveugle » de l'économie risque d'oublier les besoins essentiels des plus démunis et de conduire à des situations intolérables puisque l'eau relève d'un monopole naturel ${ }^{299}$.

\section{§2. Les obligations financières des usagers de l'eau}

La relation tarif de l'eau et accessibilité est à la base de toute la problématique du droit à l'eau. En effet, les tarifs déterminent l'accès à l'eau. La plupart des gouvernements réglementent las tarifs afin d'atteindre une série d'objectifs en termes d'équité et d'efficacité. Mais très souvent, le problème réside dans le fait que les structures tarifaires destinées à améliorer l'équité produisent l'effet inverse ${ }^{300}$. Aussi le secteur informel (redistribution d'eau) contribue de façon non négligeable à l'équilibre de la pénurie. Au sein du circuit total, l'achat aux opérateurs informels remplit une fonction régulatrice d'adaptation du service d'eau aux aléas économiques. Cette offre permet à de nombreux ménages de pouvoir disposer chaque jour d'une quantité d'eau potable minimale, en fractionnant leurs dépenses en fonction de leurs revenus quotidiens et irréguliers. Ainsi, le principe de la tarification peut s'expliquer par le principe de l'utilisateur payeur (A) et par la nécessité de prise en charge du coût de restauration et de préservation de la qualité de l'eau : le principe du pollueur-payeur (B).

298 Centre Européen de Droit et de l'environnement.

299 SMETS H., op. cit., p. 12-13.

300 RAPPORT MONDIAL SUR LE DÉ VELOPPEMENT HUMAIN 2006, Chapitre II, p. 84 


\section{A. La tarification de l'eau : le principe de l'utilisateur-payeur ${ }^{301}$}

L'approvisionnement en eau potable a un coût. Avant d'être livrée au domicile des consommateurs, l'eau est captée, traitée, transportée par un organisme disposant de services diversifiés gérés par un personnel polyvalent veillant au bon fonctionnement de la logistique de production et de distribution, de façon permanente. L'eau n'est pas captée de la nappe, ni du cours d'eau pour être livrée directement au domicile des consommateurs. Elle est traitée par des ouvrages qui nécessitent une maintenance et une adaptation permanentes. D'où la prise en compte du financement de ces opérations par le prix de revient de l'eau qui en devient la résultante. Cette considération est omniprésente dans les politiques d'eau potable des Etats de l'Afrique subsaharienne. Cette vision épousée par la plupart des Etats africains est consacrée par l'article 10 de la loi portant régime de l'eau au Cameroun. En effet, il est prévu que «les prélèvements des eaux de surface ou souterraines à des fins industrielles et commerciales sont soumis à l'autorisation préalable et au payement d'une redevance dont le taux, l'assiette et le mode de recouvrement sont fixés par la loi des finances $»^{302}$.

\section{La tarification de l'eau en Afrique subsaharienne}

L'eau potable, en Afrique subsaharienne, est une ressource accessible aux seules classes privilégiées. L'élévation du coût des prestations empêche les catégories défavorisées d'y accéder. Parmi ces prestations, on notera la connexion au réseau, l'abonnement et la fourniture proprement dite.

Le raccordement au réseau représente 10 à $30 \%$ des revenus de ménages africains. Elle coûte en moyenne 100000 francs CFA en Afrique centrale. Ce qui peut être l'équivalent du salaire mensuel d'un professeur de lycée au Congo-Brazzaville, de la rémunération bimensuelle d'un instituteur en République centrafricaine ou au Cameroun et des revenus trimestriels d'un administrateur civil en République démocratique du Congo. La situation s'assombrit encore par l'inaccessibilité aux facilités de payement tel que le payement échelonné, par exemple, pouvant permettre aux familles en cause d'adapter leurs revenus à leurs consommations. Nombreux sont donc les ménages qui, à défaut de revenus suffisants, ne peuvent pas se connecter au réseau public d'approvisionnement en eau potable ${ }^{303}$.

\footnotetext{
301 Principe « utilisateur-payeur », ensemble de règles définies qui permettent de faire une tarification de l'utilisation de l'eau selon les usages, la qualité et la quantité d'eau utilisée. $C f$. article 3 al. 10 du code de l'eau en vigueur au Togo.

302 Loi camerounaise $n^{\circ}$ 98/005 du 14 Avril 1998 portant régime de l'eau.

303 OSSOMBO-YOMBO Rémy, La gestion de l'eau potable dans les grandes agglomérations d'Afrique centrale. Contribution à l'étude d'un service public confronté à la mondialisation, Thèse, Université Jean Moulin - Lyon III, 2007,p. 484.
} 
D'autres usagers, encore, peuvent disposer d'une connexion et d'un abonnement sans avoir les moyens de payer régulièrement leur consommation mensuelle estimée, dans la sous-région, à environ douze mètres cubes par famille pour un coût moyen de 15000 francs CFA $^{304}$.

Les familles vivant en dessous du seuil de pauvreté, tel qu'indiqué par les institutions économiques et financières internationales (disposant de moins de deux dollars par personne et par jour), sont les mêmes familles qui ne peuvent accéder aux soins, ce sont aussi celles qui ne peuvent procurer une bonne éducation à leurs enfants en raison de l'élévation du coût des études ; ce sont les mêmes catégories sociales qui ne peuvent accéder à la culture en raison de leur déconnexion du monde culturel ; ce sont les mêmes classes qui ne peuvent accéder au service de l'électricité en raison du coût important que représentent le raccordement et l'abonnement à ce service. L'absence d'une politique solidaire en faveur de ces franges démographiques fait d'elles de grands exclus de la modernité 305 .

\section{Une tarification diversifiée}

On distingue plusieurs types de tarification dont la tarification progressive, la tarification volumétrique et la tarification forfaitaire. En Afrique, celle qui prédomine est la tarification progressive qui a le mérite d'instaurer une tranche dite sociale. Elle consiste à prévoir le bénéfice d'une première tranche à tarif réduit et de surtaxer les consommations importantes. A titre d'exemple, on peut citer le code de l'eau en vigueur au Mali en son article 54 où il est prévu que : «... Les tarifs des volumes d'eau consommés par les consommateurs domestiques doivent obligatoirement comprendre une tranche sociale à tarif préférentiel dont le niveau en $\mathrm{m} 3$ ne peut être supérieur à un seuil fixé par directive de la Commission de régulation. Les consommations au-delà de cette tranche sociale peuvent être facturées selon des tarifs progressifs par tranches, compatibles avec la capacité de paiement des usagers, la structure des consommations et la viabilité financière de l'exploitation... $»^{306}$.

La tarification par échelons progressifs a pour but de matérialiser différents objectifs de politique publique. Appliquer un prix faible ou la gratuité dans la première tranche tarifaire permet d'améliorer l'accessibilité. Par exemple, la ville de Durban en Afrique du Sud fournit gratuitement 25 litres d'eau par jour ${ }^{307}$. Si elle semble avoir l'adhésion de nombreux Etats ${ }^{308}$, elle ne résout pas pour autant le problème du nombre de connexions encore très limité.

304 OSSOMBO-YOMBO Rémy, précité.

305 OSSOMBO-YOMBO Rémy, op. cit., p. 485.

$306 C f$. loi nº2- 006/ du 31 janvier 2002 portant code de l'eau au Mali.

$307 C f$. également la loi nationale de l'eau de 1998 prévoit en son article 56 la différenciation des tarifs de l'eau selon la situation économique des utilisateurs. La tarification est généralement progressive avec une première tranche gratuite de $200 \mathrm{l}$ /jour (6 kl/mois par ménage) ou 25 l/jour par personne. Le taux d'impayés est élevé dans la population pauvre.

308 Algérie, Bénin, Botswana, Côte d'Ivoire, Ghana, Guinée, Kenya, Madagascar, Maroc, Cf. SMETS H., La mise en æuvre d'un tarif progressif équitable pour l'eau potable, Académie de l'eau, 2011, p. 7. 
$\mathrm{Au}$ Burkina-Faso, seuls $60 \%$ des habitants sont desservis en eau. Les ménages urbains consacrent une part très importante de leurs revenus à l'eau ${ }^{309}$. Un tiers des ménages urbains consacrent plus de $3 \%$ à l'eau. La première tranche d'eau à bas prix a été réduite de $10 \mathrm{~m} 3$ à 6 $\mathrm{m} 3$ par mois. Le prix de la première tranche est 5,5 fois plus faible que celui de la tranche au delà de $30 \mathrm{~m} 3$. L'eau aux bornes-fontaines est vendue $45 \%$ au dessus du prix de la première tranche ${ }^{310}$.

Dans un jugement du 30 avril 2008, le Tribunal de Johannesburg a précisé que le volume de 25 1/jour par habitant gratuit était trop faible pour la bonne santé des usagers pauvres et devrait être augmenté pour atteindre 50 1/jour (ville de Phiri, Soweto). Le juge considère que le volume supplémentaire d'eau gratuite pour les ménages pauvres pourrait être obtenu en ne donnant pas aux ménages aisés de l'eau à titre gratuit (création de catégories d'usagers et subventions croisées entre eux $)^{311}$.

A Soweto, le nouveau système de tarification ${ }^{312}$ (consommation moyenne : $11 \mathrm{kl} / \mathrm{mois}$ par ménage) engendre deux situations : une tranche de la population correspondant à $45 \%$ ne paye pas l'eau et $55 \%$ paye $5 € /$ mois. Il se base sur des compteurs à prépaiement (rabais de $20 \%$ ) en plus des $6 \mathrm{kl} / \mathrm{mois}$ gratuits pour tous $(10 \mathrm{kl} /$ mois pour les ménages enregistrés comme indigents) ${ }^{313}$. La subvention pour un ménage consommant $15 \mathrm{kl} /$ mois dépasse $66 \%$. Lorsque les plus démunis payent leur eau, ils y consacrent moins de 3\% de leurs revenus.

\section{B. La prise en charge du coût de restauration et de préservation de la qualité de l'eau : le principe du pollueur-payeur ${ }^{314}$}

Les ressources en eau sont limitées. Dans les pays en développement, la rareté de l'eau est principalement imputable à la sécheresse du climat. Mais elle peut provenir également d'une mauvaise gestion des ressources en eau, à leur détérioration sous l'effet de la pollution et aux pressions auxquelles elles sont soumises du fait de la rapidité de l'urbanisation et de la croissance de la population. Il est donc important que lorsque du fait de l'homme, les ressources en eau sont dégradées, celui-ci contribue à la remise en l'état du milieu. Le principe de la remise en l'état est issu du principe de la responsabilité civile de la réparation des dommages. Il est

309 KOUANDA I. et MOUDASSIR M., «Social policies and private sector participation in water supply - the case of Burkina Faso », UNRISD, 2007.

310 SMETS H., De l'eau potable à un prix abordable, Académie de l'eau, 2008, p. 129-130.

311 SMETS H., idem.

312 MAS J.P., Communication à l'ENGEES, janvier 2008.

313 A Johannesburg, le nombre d'indigents «enregistrés» est très inférieur au nombre de pauvres selon le recensement (400 000). La High Court a décidé en avril 2008 que le placement «obligatoire » de compteurs à prépaiement dans une banlieue pauvre était discriminatoire. Un supplément d'eau de $4 \mathrm{kl} / \mathrm{mois}$ a été donné aux indigents (48 249 personnes sur 90 400). Il existerait un phénomène de stigmatisation sociale à se faire enregistrer comme « indigent ».

314 Principe «pollueur-payeur », selon lequel le pollueur devrait se voir imputer les dépenses relatives non seulement à la lutte contre la pollution des eaux mais aussi aux mesures préventives engagées par les pouvoirs publics. $C f$. article 3 al. 9 du code de l'eau en vigueur au Togo. 
inspiré des dispositions de l'article 1382 du code civil selon lequel tout fait quelconque qui cause dommage à autrui oblige son auteur à le réparer. Il est d'une très grande importance en droit de l'environnement puisqu'il permet de pouvoir restaurer les écosystèmes dégradés.

\section{Les fondements de la théorie du principe du pollueur-payeur}

Provenant de la théorie économique des externalités qui est devenue un sujet traditionnel de la science économique depuis les travaux de PIGOU, le principe du pollueur-payeur n'est pas à l'origine un concept juridique ${ }^{315}$. C'est par le droit international qu'un véritable principe juridique s'est forgé ${ }^{316}$. On ne verra sa première consécration textuelle qu'avec l'article $191 \S 2$ du traité sur le fonctionnement de l'Union Européenne. Aussi peut-on lire à cet article que «la politique de l'Union dans le domaine de l'environnement [...] est fondée sur les principes de précaution et d'action préventive, sur le principe de la correction, par priorité à la source, des atteintes à l'environnement et sur le principe du pollueur-payeur $»^{317}$. Essentiellement rattachée à la théorie des externalités, celle-ci ne le consacre pas en tant que tel et n'implique pas l'imputation automatique des coûts d'une nuisance à celui qui la cause. Et comme le soulignent MACKAAY E. et ROUSSSEAU S., si l'on fait abstraction des coûts de transaction, il n'est pas nécessairement plus efficace de donner à la victime de la pollution un droit à réparation que de reconnaître au pollueur le droit de polluer ${ }^{318}$. En réalité, le principe vise à imputer au pollueur le coût des mesures de prévention et de lutte contre la pollution pour éviter les distorsions de concurrence qui pourraient résulter, dans le commerce et les investissements internationaux, de l'octroi d'aides étatiques aux entreprises polluantes. Il est donc incontestable que, sans être absente, la préoccupation environnementale n'a pas été un souci primordial dans gestation du principe $^{319}$. L'illustration vient des travaux de l'OCDE, qui jouissent d'une grande autorité sur le plan international. Ils ont inspiré les autorités européennes qui ont explicité le principe dans plusieurs recommandations. En effet, le Conseil des Ministres a ainsi adopté le 3 mars 1975 une recommandation relative à l'imputation des coûts et à l'intervention des pouvoirs publics en matière d'environnement ${ }^{320}$.

315 PIGOU A.C., The Economics of Welfare, London, Mac Millan, 1958.

316 HERTZOG R., «Les trois âges de la fiscalité de l'environnement », Les cahiers du CNFP, n³8, avril 1993, p. 136.

317 THUNIS X., «Le principe du pollueur-payeur : de l'imputation des coûts à la détermination des responsabilités », in Stratégies de développement durable. Développement, environnement ou justice sociale?, 2009 , pp. 169 et suiv.

318 Analyse économique du droit, Dalloz, Thémis, 2008, p. 182 et suiv.

319 THUNIS X., Le principe du pollueur-payeur : de l'imputation des coûts à la détermination des responsabilités, op. cit.

320 75/436/Euratom/C.E.C.A./C.E.E., J.O.C.E., n L 194 du 25 juillet 1975. Voir également PÂQUES M., Fondements et principes du droit de l'environnement de l'Union européenne, Université de Liège, fac de droit, octobre 2003, p. 48 et suiv. 
Le principe va se diffuser en droit européen et en droit international puis être reconnu dans les droits nationaux. En Europe, il va être repris dans plusieurs programmes d'action qui orientent la politique de la Communauté à moyen terme dans le domaine de l'environnement et, plus récemment, du développement durable, il subira des variations dans sa signification mais va conquérir sa force obligatoire aussi bien dans le droit communautaire originaire ${ }^{321}$ que dans le droit dérivé 322 .

En droit international, un nombre croissant de textes se réfèrent au principe, soit en le mentionnant soit en le définissant. Les définitions se ressemblent, les rédacteurs ayant tendance à se copier les uns sur les autres. Qu'il suffise de mentionner le principe 16 de la Déclaration de Rio de 1992 selon lequel "les autorités nationales devraient s'efforcer de promouvoir l'internalisation des coûts de protection de l'environnement et l'utilisation d'instruments économiques, en vertu du principe selon lequel c'est le pollueur qui doit, en principe, assumer le coût de la pollution, dans le souci de l'intérêt public et sans fausser le jeu du commerce international et de l'investissement $»$. Cette disposition, qui n'a pas un caractère impératif, met l'accent sur la fonction d'internalisation et d'intégration du principe. Elle en adopte, semble-t-il, une version large. Le coût, dont il est question sans autre précision, comprend le coût des mesures de prévention et de lutte contre la pollution ainsi que le coût des dommages causés par celle-ci.

Le principe du pollueur-payeur est adopté par le droit national. La plupart des législations nationales dans l'espace européen ont intégré le principe du pollueur-payeur dans leur code de l'environnement ou dans le code de l'eau. En Afrique subsaharienne, les législations sur le secteur de l'environnement et en particulier la législation sur l'eau, ont insisté sur la mise en œuvre de ce principe. Ainsi, si aux termes de l'article $1^{\text {er }}$ du code de l'environnement au Togo, la conservation de l'environnement, le maintien ou la restauration des ressources que la nature offre à la vie humaine, sont d'intérêt général, il en est de même pour la prévention ou la limitation des activités susceptibles de le dégrader et d'entraîner des atteintes à la santé des personnes ou à leurs biens ${ }^{323}$. C'est dans cette optique que le législateur a précisé que toute personne qui produit ou détient des déchets dans des conditions de nature à entraîner des effets nocifs sur le sol, la flore et la faune, à dégrader des sites ou les paysages, à polluer l'air ou les eaux, et d'une façon générale à porter atteinte à l'environnement, est tenue d'en assurer ou d'en faire assurer l'élimination conformément aux dispositions de la présente loi et des règlements pris pour son application ${ }^{324}$. Par ailleurs, on peut citer le législateur burkinabé qui a prévu que ceux qui, par leur activité, rendent nécessaires ou utiles des interventions publiques ou privées en vue de préserver ou de restaurer la qualité de l'eau, de répondre aux besoins correspondant aux utilisations qui en sont faites ou d'assurer la conservation des écosystèmes aquatiques, supportent la charge de ses interventions ou contribuent à leur financement ${ }^{325}$. Enfin, pour le

321 Depuis 1992, il figure dans le traité C.E. Depuis l'entrée en vigueur du Traité de Lisbonne le $1^{\text {er }}$ décembre 2009, il est repris à l'article $191 \S 2$ du traité sur le fonctionnement de l'Union européenne (TFUE), sous le titre XX consacré à l'environnement. Voir les analyses de THUNIS X. dans «Le principe du pollueur-payeur : de l'imputation des coûts à la détermination des responsabilités», in Stratégies de développement durable. Développement, environnement ou justice sociale ?, 2009, pp. 169 et suiv.

322 Des références au principe du pollueur-payeur apparaissent aussi dans le droit communautaire dérivé c'est-àdire dans les actes et les mesures que les institutions communautaires prennent sur la base des traités.

323 Cf. Loi n ${ }^{\circ} 88-14$ du 3 novembre 1988 instituant Code de l'environnement en vigueur au Togo.

$324 C f$. Article 35 de la loi n ${ }^{\circ} 88-14$ du 3 novembre 1988 instituant Code de l'environnement en vigueur au Togo.

$325 C f$. Article 47 de la loi no 002-2001 du 8 février 2001 portant loi d'orientation relative è la gestion de l'eau. 
législateur togolais, les usines, les entreprises industrielles commerciales et les établissements sanitaires doivent se doter de mécanismes d'épuration des eaux usées, et traiter ces eaux conformément aux normes ou standards de rejet fixées par arrêté conjoint des ministres chargés de la santé, de l'environnement et de l'eau ${ }^{326}$.

\section{L'eau et la mise en œuvre du principe du pollueur-payeur}

Comme le souligne SMETS H., il existe au moins deux définitions du PPP. Au terme de la première qualifiée de stricte, le pollueur ne supporte que les frais engagés pour éviter une pollution, pour limiter les émissions, pour combattre une pollution afin de réduire les dommages. Il prend également à sa charge le coût des mesures administratives directement liées à la prévention des pollutions, telles que les mesures de surveillance, inspection, autorisation, etc. Par contre, sont exclus les coûts de remise en état d'un site ou d'un milieu pollué, ainsi que l'indemnisation des dommages causés par la pollution. L'interprétation stricte du principe est celle préconisée par les pollueurs car elle justifie une délimitation rigoureuse des coûts leur incombant. Ils allèguent en effet que dans la mesure où ils mettent en œuvre et financent les dispositifs antipollution exigés d'eux en vertu de règlements ou d'engagements volontaires, ils n'ont pas d'autres dépenses à supporter ${ }^{327}$.

La définition large du PPP, plus favorable aux victimes, permet d'inclure l'ensemble des externalités et d'aboutir ainsi à une internalisation totale. Les coûts imposés au pollueur dans cette acception comprennent les mesures de prévention et de lutte contre la pollution mais aussi les coûts des dommages ${ }^{328}$. L'attitude des pouvoirs publics et de la communauté internationale peut osciller entre ces deux pôles du PPP, car l'adhésion à l'une ou l'autre définition implique le recours à des instruments différents, et correspond en définitive à un choix politique ${ }^{329}$.

La prise en charge de ces coûts concerne plutôt les personnes morales auxquelles l'Etat a délégué le service public de l'eau. C'est pourquoi le législateur a défini les mesures à prendre par le concessionnaire afin d'assurer la protection des ouvrages et installations de même que les mesures à prendre par le concessionnaire pour éviter la dégradation de la qualité des ressources en $e^{330}{ }^{330}$. Il peut s'agir aussi d'entreprises autres que celles qui exploitent les réseaux de distribution d'eau potable. Ainsi, toute personne physique ou morale, publique ou privée exerçant une activité, source de pollution ou pouvant présenter des dangers pour la ressource en eau et l'hygiène du milieu doit envisager toute mesure propre à enrayer ou prévenir le danger constaté ou présumé. Il doit en supporter les coûts de ses activités polluantes ${ }^{331}$.

326 Cf. Article 59 du Code de l'eau en vigueur au Togo.

327 VAN LANG A., Droit de l'environnement, $3^{\text {ème }}$ édition, Thémis Droit PUF, 2011, p.119.

328 Idem.

329 SMETS H., « Examen critique du PPP », in Les hommes et l'environnement, Mélanges KISS A., Frison-Roche, 1998, p. 79.

330 Cf. article du code de l'eau en vigueur au Togo.

$331 C f$. Article 16 de la LOI Nº2- 006/ DU 31 JAN.2002 PORTANT CODE DE L'EAU au Mali 
Ces mesures correspondent bien à l'esprit des recommandations de l'Observation générale $\mathrm{n}^{\circ} 15$ sur le droit à l'eau. En effet, le Comité précise que l'obligation de protéger signifie que les États parties sont tenus de «prendre les mesures législatives et autres nécessaires pour empêcher, par exemple, des tiers de refuser l'accès en toute égalité à un approvisionnement en eau adéquat, et de polluer ou de capter de manière injuste les ressources en eau, y compris les sources naturelles, les puits et les systèmes de distribution d'eau» ${ }^{332}$. Lorsque les services sont privatisés, les États doivent mettre en place un système réglementaire efficace prévoyant un contrôle indépendant, une participation véritable de la population et des sanctions en cas d'infraction ${ }^{333}$.

Ces mesures peuvent se comprendre également si l'on se met dans une perspective de la gestion intégrée de l'eau. Vu sous cet angle, il paraît nécessaire de souligner d'une part les coûts reportés sur l'usager de l'eau du fait de l'inapplication du principe pollueur-payeur et, d'autre part, les coûts différés sur les générations futures du fait de l'inaction, voire l'incurie des pouvoirs publics à maitriser les sources de pollutions des milieux aquatiques, y compris en l'absence d'assainissement. Malgré que de nombreux Etats disposent d'un code de l'environnement et d'un code de l'eau, il est constaté à la lumière des rapports des organisations onusiennes, que ces textes ne sont pas du tout respectés ${ }^{334}$.

\section{Section II. Le service de distribution de l'eau, un service à dominante marchande}

La question de savoir quelle est la nature exacte d'un service de distribution d'eau est loin d'apparaître, sur le plan contentieux, dénuée de tout intérêt. En effet, on sait que conformément à une jurisprudence ancienne et bien établie, tout litige concernant les rapports d'un service public avec l'un de ses usagers doit normalement relever, selon qu'on reconnait audit service un caractère administratif ou au contraire un caractère industriel et commercial, d'un ordre de juridiction différent : les tribunaux de l'ordre administratif étant en principe compétents dans le premier cas, ceux de l'ordre judiciaire l'étant quant à eux, sauf hypothèses particulières, dans le second. Il s'agira d'expliquer que le service de l'eau revêt en principe un caractère industriel et commercial $(\S 1)$ puis de montrer que ce service peut aussi être dépourvu de ce caractère $(\S 2)$.

\section{§1. Un service revêtant en principe un caractère industriel et commercial}

Le commissaire du gouvernement MATTER ${ }^{335}$ établissait, au sein des services pris en charge par des personnes publiques, une distinction décisive. Les uns, les services publics administratifs, comme la police, la justice, l'enseignement, le fisc ... sont de l'essence même de l'Etat ou de l'administration publique. Les autres, ceux de type industriel et commercial, eau,

\footnotetext{
332 Cf. Paragraphe 23.

333 Cf. Paragraphe 24.

334 DROBENKO B., op. cit., p. 129.

335 Cf. Conclusions sous TC, 22 janv. 1921, Société commerciale de l'Ouest africain, R., 91.
} 
gaz, électricité, transport... sont de nature privée et, s'ils sont entrepris par l'Etat et les autres personnes publiques, ce n'est qu'occasionnellement, accidentellement, parce que nul particulier ne s'en est chargé, et il importe de les assurer dans un intérêt général ${ }^{336}$. L'intérêt initial de la distinction opérée par le commissaire du gouvernement MATTER est la détermination de la juridiction compétente en cas de litige. Aussi, faut-il préciser que contrairement au commissaire du gouvernement MATTER qui distingue aisément les services publics administratifs des services publics industriels et commerciaux, l'identification des derniers n'est pas toujours aisée.

En effet, l'opposition entre ce qui est administratif et ce qui est industriel et commercial est fort jugée artificielle ${ }^{337}$. Ce que l'analyse du droit positif met en évidence lorsqu'il est conclu au flou, à l'ambiguïté de la distinction des services publics industriels et commerciaux, c'est l'absence d'une différenciation objective. Pour le Professeur J-F LACHAUME, il n'existe pas ou pratiquement, objectivement des SPIC ou des SPA ${ }^{338}$. La distinction ne concerne pas «les activités en soi »: il n’y a pas d'activités par nature administratives ou industrielles ou commerciales $^{339}$. Ce serait prendre le contre-pied des conclusions du commissaire du gouvernement MATTER dans l'affaire dite Bac d'Eloka ${ }^{340}$. Il faut d'une part procéder à l'identification du SPIC (A) et examiner comment ce caractère peut être reconnu au service de distribution de l'eau (B).

\section{A. L'identification du service public industriel et commercial}

S'il n'a jamais été contesté que l'administration puisse recourir à la gestion privée pour certains aspects des activités des services publics, l'idée que les services entiers puissent fonctionner sous le régime de la gestion privée a été perçue par une large partie de la doctrine de la première moitié du XX $\mathrm{XX}^{\text {ème }}$. Les critères d'identification du service public industriel et commercial ne sont pas faciles à manier. Esquissés par l'arrêt SCOA du 22 janvier 1921, ils ont été révélés par la jurisprudence Union syndicale des industries aéronautiques. Celle-ci oblige à recourir à un faisceau d'indices pour distinguer le service public industriel et commercial du service public administratif.

336 LACHAUME J.-F. (dir.), Droit administratif, Les grandes décisions de la jurisprudence, Thémis PUF, $15^{\text {ème }}$ édition, 2010, p. 373.

337 DUPUIS G., GUEDON M.J., et CHRETIEN P., Droit administratif, Armand Colin, $7^{\text {ème } e ́ d i t i o n, ~ 2000, ~ p . ~} 427$.

338 LACHAUME J-F, BOITEAU C., PAUliat H., Grands Services publics, Armand Colin, $2^{\mathrm{ème}}$ édition 2000, p. 60 .

339 GUGLIEMI G., KOUBI G., Droit du service public, Montchrestien, Coll. « Domat/Droit public », 2000, p. 76.

340 GARCERIES S., L'élaboration d'une notion juridique de service public industriel, Thèse dactylographiée, Université de Cergy-Pontoise, 2010, p. 14. 


\section{Les critères d'identification du service public industriel et commercial}

Ces indices peuvent être tirés de l'objet du service, de l'origine de ses ressources et des modalités de son organisation et de son fonctionnement ${ }^{341}$. Selon la présentation doctrinale la plus fidèle des données jurisprudentielles d'identification du service public industriel et commercial, constitue un tel service, en l'absence de qualification législative dépourvue d'ambiguïtés lui conférant un tel caractère, celui qui « aux trois points de vue -objet, origine des ressources, modalités de fonctionnement - ressemble à une entreprise privée $»^{342}$.

Si le service est essentiellement financé par les usagers et a un objet et des modalités de fonctionnement comparables à l'activité que pourrait exercer une entreprise privée. L'objet du service doit comprendre les opérations de production, de distribution ou d'échange effectuées, dans les mêmes conditions qu'un particulier ${ }^{343}$. En captant, puis traiter, et distribuer l'eau moyennant rémunération, les sociétés délégataires exploitent indubitablement un service public industriel et commercial. Ainsi, ne pourra être industriel et commercial qu'un service public dont les ressources proviennent pour l'essentiel d'un prix perçu sur les usagers qui se rapproche du coût réel du service.

L'importance du critère du mode de financement dans la détermination du caractère administratif ou industriel et commercial d'un service public semble se renforcer, peut-être du fait de sa simplicité d'utilisation, de son objectivité plus grande que les deux autres critères tirés de l'objet du service et des modalités d'organisation et de fonctionnement de celui-ci ${ }^{344}$.

\section{L'intérêt de la distinction}

Le fonctionnement du service public industriel e commercial est régi en principe par le droit privé. Les relations que son exploitant entretient avec les usagers résultent normalement des contrats de droit privé et forment un bloc de compétence judiciaire : ainsi, le voyageur victime d'un dommage causé par la SNCF à laquelle il est lié par un contrat de transport, doit poursuivre la responsabilité de cette dernière devant le juge judiciaire qui appliquera les règles du code $\operatorname{civil}^{345}$.

La situation individuelle des agents est également de droit privé, même lorsqu'elle est régie par des statuts spéciaux établis par décret et dérogatoires au code du travail dans quelques grandes entreprises publiques comme la RATP ou la SNCF. Les relations du service avec les tiers et ses fournisseurs sont en principe de droit privé ${ }^{46}$.

341 LOMBARD M. et DUMONT G., Droit administratif, Dalloz, 2005, p. 282.

342 R. CHAPUS, Droit administratif général I, $15^{\text {ème }}$ éd., 2001, $\mathrm{n}^{\circ} 767$

343 DEBBASCH C.et COLIN F., op. cit., p. 338.

344 DEBBASCH C.et COLIN F., op. cit., p. 339

345 TRUCHET D., Droit administratif, Thémis PUF, 2009, p. 348.

346 Idem. 
Dans cet océan de droit privé émergent pourtant quelques ilots de droit administratif et de compétence du juge administratif. C'est le cas pour la situation du chef de service et du comptable s'il a le statut de comptable public. D'autre part les dommages causés aux tiers par les ouvrages publics utilisés par le service et par les travaux publics menés pour lui. L'illustration la plus spectaculaire est concerne les mesures générales d'organisation du service adoptées par ses organes de direction : ces mesures sont des actes réglementaires en vertu de la jurisprudence Jamart. Aussi, l'on va oser dire avec certains auteurs que cette théorie de distinction SPA/SPIC est ambigüe ${ }^{347}$.

Ainsi, Bertrand SEILLER va-t-il affirmer que les champions de l'Ecole du service public ne pouvaient évidemment admettre que le droit privé puisse s'introduire dans la gestion des services publics, ce qui revenait à abattre l'édifice minutieusement bâti sur la notion de service public ${ }^{348}$. « De deux choses l'une, ou une activité est un service public ou elle ne l'est pas. Si elle est un service public, elle a toujours les mêmes caractères; toutes les conséquences qui se rattachent à ce qu'elle est un service public doivent s'appliquer et toute distinction entre de prétendus services publics d'autorité et de gestion doit être définitivement écartée sous peine de tomber dans la confusion ou l'erreur $»^{349}$.

\section{B. Le service de distribution d'eau, un service public à caractère industriel et commercial}

Comme le démontre une jurisprudence aussi ancienne que constante, le fait de reconnaître à un service de distribution d'eau un caractère industriel et commercial, et ce, quel que soit son mode de gestion, ne saurait véritablement surprendre ; il s'agit là d'une solution classique qui, affirmée déjà il y a plusieurs décennies par le CE le 6 mai $1931^{350}$, n'a jamais cessé, depuis lors, d'être consacrée à de multiples reprises par le juge ${ }^{351}$ mais reprécisée très récemment par la jurisprudence Mme Alberti-Scott ${ }^{352}$. D'autre part il faut préciser que le législateur en Afrique n'a pas passé sous silence la question.

347 Bertrand SEILLER, « L'érosion de la distinction SPA-SPIC », AJDA 2005 p. 417.

$348_{\text {Idem. }}$.

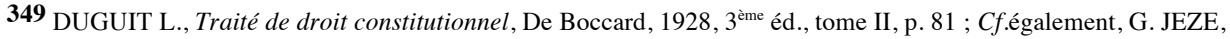
note sous T. confl. 11 juillet 1933, Dame Mélinette, RD publ. 1933, p. 426.

350 Cf. CE 6 mai 1931, Tondut, Lebon, p. 477, S., 1931.III.81..

351 Voir, CE Sect., 13 janv. 1961, Département du Bas-Rhin, Lebon, p. 38 ; AJDA, 1961.235; CE 21 avr. 1961, Dame Agnési, Lebon, p. 253.

352 Tribunal des conflits, 21 mars 2005, Alberti-Scott 


\section{Le service de distribution d'eau, un service public industriel et commercial : la qualification du juge français}

En désignant expressément le service de distribution d'eau comme un service public industriel et commercial, le Conseil d'Etat en France, a donc réglé une question d'importance tout en consacrant, en l'espèce, une solution classique, à défaut d'être systématique. Mais une solution qui, malgré les règles rappelées précédemment, ne l'a cependant nullement empêché de se reconnaître compétent et de juger l'affaire au fond : l'acte litigieux entrant dans l'une des rares exceptions jurisprudentielles à la politique de privatisation globale des relations entre l'usager et le service public industriel et commercial.

La décision du Tribunal des conflits intervenue dans l'affaire Alberti-Scott c/ Commune de Tournefort est intéressante notamment en ce qui concerne le critère d'identification du service public industriel et commercial, qui semble s'écarter de celui utilisé jusqu'ici, encore que la portée du changement, si changement il y a, reste à préciser ${ }^{353}$.

Par la décision Alberti-Scott, le Tribunal des conflits, s'agissant d'un service public communal de distribution de l'eau tout au moins, reconnait à ce service un caractère industriel et commercial, ce qui ne rompt pas avec la jurisprudence la mieux établie, mais par un raisonnement qui semble s'écarter de celui élaboré à partir des conclusions de P. LAURENT. Le service public de distribution de l'eau en cause dans la décision commentée ici était susceptible de soulever une difficulté quant à la détermination de son caractère dès lors qu'on lui appliquait le critère traditionnel de distinction service public administratif/service public industriel et commercial. Si l'objet du service public en cause ne fait pas obstacle au renversement de la présomption d'administrativité, il n'en va pas de même de l'origine des ressources et surtout des modalités de fonctionnement.

Il semblait donc a priori difficile de renverser dans l'affaire Alberti-Scott la présomption d'administrativité du service public en cause, encore que, s'agissant du service de l'eau, le Tribunal des conflits n'hésitait pas, dans certaines décisions, à prendre des libertés avec le critère traditionnel de distinction service public administratif/service public industriel et commercial ; la jurisprudence Thomas ${ }^{354}$ le montre bien à propos justement du service public de l'eau.

\section{La position du législateur africain}

Les textes se rapportant à la distribution de l'eau en Afrique subsaharienne ne précisent nullement la nature du service de distribution de l'eau. Mais l'examen des codes de l'eau qui ont traité de la délégation du service public de l'eau révèle qu'il s'agit bien d'un service public industriel et commercial. En effet, aux termes de l'article 53 du code de l'eau en vigueur au Mali ${ }^{355}$, l'accès au service public de l'eau, que ce soit aux bornes fontaines ou aux branchements

\footnotetext{
353 Jean-François LACHAUME, «L'identification d'un service public industriel et commercial : la neutralisation du critère fondé sur les modalités de gestion du service », RFDA 2006 p. 119.

354 T. confl., 19 févr. 1990, Thomas, Lebon, p. 618.

355 Cf. Loi portant code de l'eau au Mali, précité.
} 
individuels, doit toujours être payant. La délégation étant accordée à des personnes morales de droit privé qui se rémunèrent sur les usagers par le biais des redevances, on ne peut pas objectivement les exclure de la catégorie des services publics industriels et commerciaux.

La société d'exploitation des eaux du Niger (SEEN) est quant à elle une société de droit privé, liée à l'État par un contrat d'affermage pour l'exploitation du service public de production, de transport et de distribution des eaux. A s'en tenir à l'article 13 de la loi 2000-12 qui assigne à cette société des missions relatives notamment à l'exploitation du service du public de production, de transport et de distribution des eaux ; l'exploitation et l'entretien de l'infrastructure et du matériel d'exploitation; le renouvellement du matériel d'exploitation, des branchements et des compteurs ; la maîtrise d'œuvre pour la réhabilitation, le renouvellement et l'extension des réseaux financés sur fonds propres, on peut également le classer dans la catégorie des services publics industriels et commerciaux ${ }^{356}$.

Une « autopsie » de la réglementation en vigueur en la matière pourrait constituer a priori un sérieux indice au profit du caractère industriel et commercial dudit service : puisque aussi bien ce dernier est-il clairement allégué, à côté d'autres services s'étant vu reconnaître par le juge un tel caractère à l'instar, par exemple, des services d'assainissement, des services de production et de distribution du gaz et de l'électricité ou des abattoirs publics, par le titre VII du livre III du code des communes relatif aux dispositions particulières à certains services industriels et commerciaux. Situation qui permettrait dès lors d'agréer, dans le service de distribution d'eau, une sorte de service public industriel et commercial «par détermination de la loi357».

Mais à s'en tenir uniquement à la constance de la jurisprudence administrative française, on pourrait être tenté de voir, dans le service de distribution d'eau, l'exemple type de ces services publics ayant pour particularité de n'avoir qu'un seul caractère, toujours identique : que ce soit un caractère administratif à l'instar, par exemple, des caisses de crédit municipal ${ }^{358}$ ou à l'instar, encore, du service extérieur des pompes funèbres ${ }^{359}$.

\section{§2. Un service parfois dépourvu de caractère commercial}

Il peut arriver que le service public de l'eau soit dépourvu du caractère industriel et commercial, ou dans certaines hypothèses, revêtir et le caractère de SPIC et celui de SPA. J.-F. LACHAUME avait envisagé l'existence du «service public à double visage $»^{360}$. Aussi, la décision du Conseil d'Etat en date du 7 mai 1982 (Arrêt Verdier) ${ }^{361}$, va rendre beaucoup plus

356 ALTERNATIVES ESPACE CITOYENS, Le droit à l'eau potable au Niger, précité, p.16.

357 J.-C. DOUENCE, op. cit. : J.-F. LACHAUME en ce sens, $c f$. Grandes décisions de la jurisprudence administrative, PUF, 6e éd., 1991, p. 223.

358 T. confl., 15 janv. 1979, Caisse de crédit municipal de Toulon, Lebon, p. 658 ; AJDA 1979.33, note J.-M. PONTIER ; JCP 1980.II.19328, note KERNINON.

359 T. confl. 20 janv. 1986, Préfet, commissaire de la République de la région d'Ile-de-France c/ SA Rabiot et M. Bouissous, AJDA 1986.267, note L. RICHER.

360 « Brèves remarques sur les services publics à double visage », RFDA 2003, p. 662.

361 CE 7 mai 1982, Verdier, Lebon, tables, p. 554. 
difficile, sinon carrément impossible, une reconnaissance simple des situations aboutissant à l'identification du service public industriel et commercial. Cet arrêt ayant en effet jugé qu'«eu égard aux conditions de son fonctionnement ", une régie municipale de distribution d'eau pouvait présenter «le caractère non d'un service public industriel et commercial mais d'un service public administratifi». Pour une première fois, une confusion va régner en la matière (A), ce qui explique donc la précarité de l'identification du caractère du service de distribution de l'eau (B).

\section{A. La confusion semée par la jurisprudence «Verdier»}

La décision du Conseil d'Etat en date du 7 mai 1982 a laissé penser que le service public de l'eau pouvait revêtir le caractère administratif. Mais à ce jour le juge n'a pas osé confirmer cette décision qui en la matière demeure une décision isolée. Non pas qu'il n'en est pas été tenté, car on a été surpris par la démarche qu'il a adoptée dans l'affaire Alberti-Scott précitée, en neutralisant le critère de l'objet du service public mais en a conclu au caractère industriel et commercial. L'examen attentif de la jurisprudence relative auxdits services s'avère-t-il, pour sa part, beaucoup plus éclairant ? Même s'il convient de se garder, en la circonstance, de toute conclusion hâtive et définitive, il semble néanmoins possible de répondre positivement. En effet, cet examen permet de tirer plusieurs enseignements essentiels ${ }^{362}$.

\section{Une jurisprudence isolée}

Le premier enseignement est clair : il réside dans le caractère tout à fait exceptionnel et, à notre connaissance en tout cas, tout à fait isolé, revêtu par l'arrêt Verdier : le juge n'ayant rendu, aucun autre arrêt ayant conclu à la nature administrative d'un service public local de distribution d'eau. Constat dont il est à peine besoin de souligner toute l'importance : non pas qu'il justifie, bien entendu, d'ignorer ou de méconnaître cet arrêt ; mais bien plutôt parce qu'il permet de relativiser sensiblement la démarche développée par certains auteurs consistant à présenter ce type de service comme pouvant constituer indifféremment, selon les cas et à l'image par exemple de services tels que les piscines ou campings municipaux, tantôt un service public administratif, tantôt au contraire un service public industriel et commercial.

Démarche dont le principal inconvénient, est de se révéler sinon totalement inexacte d'un point de vue strictement juridique, du moins, dans la mesure où elle aboutit finalement à considérer qu'un arrêt puisse faire une jurisprudence, beaucoup trop peu nuancée pour ne pas susciter de sérieuses réserves : que ne dirait-on pas, en effet, de celui qui, s'appuyant sur un arrêt d'espèce ayant admis qu'un service public industriel et commercial puisse être gratuit ${ }^{363}$, soutiendrait que la gratuité n'est pas considérée par le juge comme étant par principe exclusive de toute commercialité ?

362 BORGETTO M., «Sur la nature juridique du service de distribution d'eau et le traitement jurisprudentiel du principe d'égalité », RFDA 1993 p. 673

363 En ce sens, cf. T. confl. 6 nov. 1967, Sébie c/ Entraide sociale des Basses-Pyrénées et Compagnie d'Assurance «La Foncière », Lebon, tables, p. 813. 
C'est dire qu'on peut déjà proposer, à ce stade de l'analyse, une interprétation quelque peu différente, sur la forme en tout cas, de la précédente : interprétation qui consisterait à dire que la règle, en ce qui concerne les services locaux de distribution de l'eau, est qu'ils constituent des services publics à caractère industriel et commercial, le fait qu'ils puissent se voir reconnaître, « eu égard à leurs conditions de fonctionnement ", un caractère administratif constituant indéniablement une exception en l'occurrence extrêmement rare puisque demeurée jusqu'ici unique ; interprétation, encore, qui aurait pour elle d'avoir été explicitement reprise et adoptée, dans ses conclusions rendues dans l'affaire Bachelet ${ }^{364}$, par le commissaire du gouvernement STIRN : ce dernier ayant en effet directement affirmé, sans qu'il ait jugé utile, ce faisant, d'évoquer l'arrêt Verdier, que «le service de distribution d'eau (revêtait) en principe un caractère industriel et commercial $»^{365}$.

\section{Une jurisprudence en marge de la législation en vigueur}

L'examen des textes applicables pourrait constituer a priori un sérieux indice en faveur du caractère industriel et commercial dudit service : puisque aussi bien ce dernier est-il expressément évoqué, à côté d'autres services s'étant vu reconnaître par le juge un tel caractère à l'instar, par exemple, des services d'assainissement, des services de production et de distribution du gaz et de l'électricité ou des abattoirs publics, par le titre VII du livre III du code des communes relatif aux Dispositions particulières à certains services industriels et commerciaux. Situation qui permettrait dès lors de saluer, dans le service de distribution d'eau, une sorte de service public industriel et commercial «par détermination de la loi $»^{366}$.

D'autre part, l'étude des législations se rapportant au service de distribution d'eau montre que celles-ci ne font pas explicitement mention de la nature exacte du service public de l'eau. Toutefois, les indices ne manquent pas pour étayer une certaine idée de SPIC. Si l'on s'en tient aux critères classiques de la jurisprudence, le service public de l'eau pourrait revêtir le caractère industriel et commercial. En effet, le service public de l'eau comprend bien la production et la distribution de l'eau, le critère dit de l'objet ne fait donc pas de doute. Quant au second critère relatif aux origines des ressources, il ne peut faire a priori l'objet de contestation, le service de l'eau étant toujours payant. Evoquant le critère se rapportant aux modalités de fonctionnement du service, il semble là également ne point souffrir de contestation possible. Le service public de l'eau étant en grande partie délégué à des concessionnaires de droit privé, on voit mal comment leur gestion échappera aux techniques de gestion des entreprises privées.

On peut imaginer que le service public de l'eau peut être pour partie un SPA et pour l'autre partie un SPIC. Les ports autonomes en sont une illustration parfaite. Enfin, on constate que l'incertitude de la notion de SPIC et le caractère mixte de son régime juridique ont introduit une grande complexité.

364 Conclusions qui ont été publiées aux CJEG, 1991, p. 164-165.

365 BORGETTO M., op. cit., RFDA 1993 p. 673

366 Pour reprendre ici l'expression utilisée par J.-C. DOUENCE, op. cit. : thèse qui semble désormais avoir la faveur de J.-F. LACHAUME, en ce sens, $c f$. Grandes décisions de la jurisprudence administrative, PUF, $6^{\mathrm{èm}}$ éd., 1991, p. 223. 


\section{B. La précarité de l'identification du caractère du service de distribution de l'eau.}

Dans la pratique, les choses se présentent beaucoup plus embrouillées. Pour s'en convaincre, on pourrait faire état, tout d'abord, de la multitude des opinions doctrinales émises en la matière. En effet, tous les auteurs sont loin d'avoir, sur la question, un point de vue identique ; s'il en est certains qui n'opèrent pas véritablement de distinction entre le service de distribution d'eau et d'autres services voyant leur caractère fluctuer selon les cas, il en est aussi beaucoup pour qui le caractère industriel et commercial de ce service ne saurait faire l'objet, par principe, d'aucune discussion : soit qu'ils tiennent l'arrêt Verdier pour tout à fait exceptionnel et isolé, soit surtout, et c'est l'attitude la plus fréquente, qu'ils l'ignorent purement et simplement dans leurs commentaires $^{367}$.. Mais là, bien évidemment, n'est pas l'essentiel : l'essentiel est bien plutôt dans l'examen attentif sinon des différents textes applicables, du moins de l'ensemble de la jurisprudence rendue à propos de ce type particulier de service public local ${ }^{368}$.

\section{L’incohérence du juge en la matière}

Réagissant par rapport à l'affaire précitée du Tribunal des conflits, Thomas du 19 janvier 1990, décision dans laquelle le Tribunal juge que le service de l'eau est un service public industriel et commercial même si la somme forfaitaire demandée à l'usager " est inférieure au coût réel du service assuré », le Professeur CHAPUS avait constaté que « si le Tribunal des conflits a voulu étonner, il a certainement réussi... $»^{369}$. C'est reconnaître toute l'incohérence du juge dans les affaires relatives au service de distribution d'eau.

Lorsque les critères d'identification du service public industriel et commercial (SPIC) ne sont pas réunis, le juge n'hésite pas à écarter la qualification de SPIC si l'on n'est pas dans le domaine de l'eau, mais opère des gymnastiques pour retenir la qualification de SPIC lorsqu'il s'agit de l'eau. En effet, entre les décisions Thomas et Alberti-Scott, est venue s'intercaler la décision Préfet de Mayotte ${ }^{370}$ qui semblait revenir à l'utilisation orthodoxe du critère de distinction service public administratif/service public industriel et commercial en refusant de reconnaître un caractère industriel et commercial à des distributions de pétrole lampant à la population de Mayotte par les autorités de la République dès lors qu'elles « sont faites à un prix de vente sensiblement inférieur à leur coût ».

367 C'est la raison pour laquelle, d'ailleurs, tous les commentaires relatifs au service de distribution d'eau antérieurs à 1982 voient dans celui-ci un service revêtant toujours un caractère industriel et commercial. En ce sens, $c f$. par ex. G. Le BONHOMME, La distribution publique d'eau potable en France, mémoire DES de Paris I, 1974, p. 39-40 ; P. DEGUIGNET, « Le service public de la distribution d'eau potable », Administration, $n^{\circ} 96,1977$, p. 32 et s.; «La gestion en régie des services publics de l'eau et de l'assainissement », Rev. fin. communales, 1979, p. 82 et s.; Le Maire face au problème de l'eau, La VI ${ }^{\text {ème }}$ communale et départementale, $\mathrm{n}^{\circ} 618,1980$, p. 125 et s. ; J.-C. DOUENCE, « La gestion du service public local », Rép. coll. loc. Dalloz, 6122-4, etc.

368 BORGETTO M., précité, RFDA 1993 p. 673.

369 CHAPUS R., op. cit.

370 T. confl., 24 oct. 1994, Lebon, p. 607. 
Le juge des conflits, tout au moins s'agissant du service public de distribution de l'eau, n'a donc pas abandonné sa jurisprudence Thomas en admettant, à nouveau, dans la décision AlbertiScott qu'il n'existe pas d'incompatibilité entre le caractère industriel et commercial du service et une sous-tarification de la fourniture à l'usager. Alors qu'une telle situation est de nature à différencier fortement le service public en cause des modes de financement des entreprises privées, même si le Tribunal exclut expressément, ce que ne faisait pas la décision Thomas, qu'un service public de distribution d'eau puisse être industriel et commercial «lorsque son cô̂t ne fait l'objet d'aucune facturation périodique à l'usager », c'est-à-dire que sont maintenus d'une part le principe de l'incompatibilité entre gratuité totale et caractère industriel et commercial d'un service public et, d'autre part, l'exigence que le prix payé par l'usager, si modeste soit-il, prenne en compte, d'une façon ou d'une autre, le volume de la consommation de celui-ci ${ }^{371}$.

Le raisonnement suivi par le Tribunal des conflits dans la décision Alberti-Scott s'écarte de celui traditionnellement suivi par les juges pour, dans le silence des lois, distinguer les deux catégories de services publics, tout en débouchant sur une solution confirmant une jurisprudence qui, pour l'essentiel, a toujours considéré le service public de distribution de l'eau comme industriel et commercial, ce qui correspond d'ailleurs à la façon dont le code général des collectivités territoriales traite ce service, sans compter que le législateur finira bien par lui reconnaître un jour cette qualité. En tout cas, la décision stabilise, si besoin était, cette qualité en évitant de la rendre également tributaire d'autres conditions, sous réserve de l'exigence d'une facturation périodique.

\section{Les divergences de l'opinion doctrinale}

Sur la question de la nature du service de l'eau, les auteurs n'ont pas un point de vue similaire. Ainsi on constate que certains d'entre eux n'opèrent pas indubitablement de distinction entre le service de distribution d'eau et d'autres services voyant leur caractère fluctuer selon les cas. Il en est aussi pour qui le caractère industriel et commercial de ce service ne saurait faire l'objet, par principe, d'aucune discussion. Aussi, vont-ils considérer la jurisprudence Verdier comme une décision littéralement exceptionnelle et isolée. C'est d'ailleurs l'opinion dominante, osons-nous affirmer, et à cet effet, celle-ci va « mépriser » la jurisprudence Verdier.

Finalement, la théorie de bloc de compétence judiciaire appliquée aux relations d'un service public à caractère industriel et commercial avec l'un de ses usagers, demeure consolidée car la doctrine ne semble pas accentuer les rares fissures qu'elle connaît. Cette privatisation ainsi que l'incompétence des tribunaux administratifs qui en résulte revêt, on le sait, un caractère extensif : s'appliquant à tous les services quel que soit leur mode de gestion, concernant tous les litiges relatifs à l'exécution ou l'inexécution des prestations du service, insuffisance, interruption de ce dernier ${ }^{372}$, jouant à l'égard de tous les contrats passés avec l'usager effectif, que ceux-ci se caractérisent ou non par la présence de clauses exorbitantes ${ }^{373}$, affectant aussi bien les usagers en

371 LACHAUME J.F., op. cit.

372 T. confl. 27 janv. 1964, Pradal, préc.; T. confl. 28 juin 1976, Burlat c/ Cne de Somme-Suippe, AJDA 1976.570, note F. MODERNE

373 CE, Sect., 13 oct. 1961, Sté des Ets Companon-Rey, Lebon, p. 567 
situation régulière que ceux en situation irrégulière ${ }^{374}$, elle se révèle suffisamment forte et prégnante non seulement pour faire échec à des notions aussi importantes que les notions de travaux et d'ouvrages publics, mais encore pour interdire au candidat-usager la possibilité d'intenter un recours pour excès de pouvoir contre une décision individuelle prise par l'autorité assurant l'exploitation du service ${ }^{375}$. Solution qui n'est d'ailleurs pas forcément, on doit le rappeler des plus opportunes dans la mesure où elle aboutit à ce qu'un candidat-usager se voyant refuser l'accès à ce dernier ne puisse se porter, contrairement à une ancienne jurisprudence beaucoup plus satisfaisante, que devant un juge dont rien ne prouve qu'il « soit aussi bien armé que son homologue administratif pour apprécier la légalité d'un acte unilatéral individuel émanant d'une autorité administrative $\gg . .$.

Au-delà de la solidité de la règle d'identification des SPIC et SPA, la question préoccupe toujours la doctrine et elle s'interroge parfois sur l'opportunité de cette distinction. S. BOUSSARD écrira, "L'éclatement des catégories de service public et la résurgence du service public par nature ${ }^{376}$. Le Professeur B. SEILLER, publie «L'érosion de la distinction SPASPIC $»^{377}$. On peut citer également P. SANDEVOIR, qui avait publié « Les vicissitudes de la notion de service public industriel et commercial» dans les Mélanges offerts au Professeur STASSINOPOULOS ${ }^{378}$.

374 T. confl. 5 déc. 1983, Niddam c/ SNCF, Lebon, p. 541.

375 CE 21 avr. 1961, Dame Agnesi, Lebon, p. 254

376 RFDA 2008 p. 43.

377 Précité.

378 LGDJ 1974. p.317 
CONCLUSION DU TITRE I DE LA IERE PARTIE. 
L'intervention du secteur privé, qui dispose d'un savoir-faire immédiatement mobilisable, a souvent permis de créer, réhabiliter ou/et pérenniser le bon fonctionnement de services d'eau que les autorités publiques n'avaient pas les moyens, humains, techniques, financiers..., d'assurer dans de bonnes conditions.

Cependant, l'irruption du secteur privé dans la gestion de ces services a modifié les rôles et responsabilités respectifs des autorités publiques et du secteur privé. Les observateurs s'accordent à dire que le passage à une gestion privée ou semi-privée doit être soigneusement préparé, d'autant qu'une fois effectué il est souvent difficile de revenir en arrière ${ }^{379}$.

Or, beaucoup d'États négligent l'importance ou échouent à mettre en place un cadre juridique, institutionnel et économique suffisant, c'est-à-dire une réglementation qui permette de savoir quelles sont les responsabilités de chacun à tous les stades de la privatisation, l'adaptation ou la création d'instances de régulation indépendantes spécifiques, et un financement du service d'eau qui combine de façon optimale les tarifications, taxes et transferts ${ }^{380}$.

Autorités publiques et entreprises peuvent également échouer à identifier les groupes d'usagers (et pour quel service ils sont prêts à payer), à prendre en compte le rôle joué par les fournisseurs préexistants (petits opérateurs privés, associations d'usagers, souvent informels) et à créer le climat de transparence et de participation des usagers qui aurait permis un passage de relais compris et accepté, en particulier en ce qui concerne les modifications de la tarification et leur pertinence dans le cadre local ${ }^{381}$.

379 CNUCED, World Investment Report 2008, Transnational Corporations and the Infrastructure Challenge, Genève/New York, Nations Unies, 2008. p. 150.

380 Idem., p. 150-151 ; Voir aussi OCDE, De l'eau pour tous..., op. cit., p. 14-17

381 ÉTIENNE J., HENRY A., « Eau et assainissement en Afrique : croyances, modes et modèles », in IDDRI, Eau : encadrer les partenariats public-privé, Paris, IDDRI, 2003, p. 23-24. 
TITRE II DE LA IERE PARTIE : LES MECANISMES JURIDIQUES DE LA LIBERALISATION DU SECTEUR DE L'EAU 
Contraints par les institutions financières à libéraliser le secteur de l'eau, les Etats africains du sud Sahara vont hésiter face aux modèles de libéralisation à choisir. Finalement, le modèle privilégié de la libéralisation du secteur de l'eau sera la délégation du service public de l'eau. Aussi, de nombreux Etats ont-ils opté pour ce modèle et ont choisi soit la concession, soit l'affermage ou la régie intéressée.

Authentiques contrats administratifs, les conventions issues de la délégation de service public doivent respecter un certain nombre d'engagements consignés dans un cahier des charges. Véritable bouclier contre les dérives, le cahier des charges définit les obligations incombant aux délégataires et prévoit dans certains cas les sanctions applicables lorsque les clauses y insérées ne sont pas respectées.

En délégant le service public de l'eau, les délégataires risquent de malmener les principes du service public. Ces principes désignés sous l'expression lois de Rolland vont être mis en berne. En effet, les entreprises privées bénéficiaires de la délégation ne visent en réalité que la réalisation du profit maximum. Ceci les écarte de l'objectif de la réalisation des missions d'intérêt général qui incombe à l'Etat.

Dès lors, ce n'est plus une surprise le constat selon lequel les délégataires ne prennent pas en compte le principe d'égalité si important dans un Etat qui inspire à la promotion des droits sociaux. De même, le principe de continuité qui est le véritable instrument de cohésion étatique est vite mis est mis en veilleuse. Dans ce contexte, les nouvelles lois dites lois émergentes ne pourront recevoir application. Dans l'ensemble on note une désillusion totale par rapport à ces principes, qu'ils soient classiques ou qu'ils soient nouveaux.

Après avoir démontré que le modèle privilégié de la libéralisation du secteur de l'eau est la délégation du service public de l'eau (Chapitre I), nous montrerons que cette situation engendrerait nécessairement la mise en berne des principes du service public (Chapitre II). 
ChAPITRE I. LE MODE PRIVILEgIE DE LA LIBERALISATION DU SECTEUR DE L'EAU : LA DELEGATION DU SERVICE PUBLIC DE L'EAU 
A l'instar du service public, la délégation du service public est un concept qui ne cesse d'alimenter les chroniques depuis quelques années. Tous deux sont en profonde mutation. Le premier, mythe fondateur, profondément lié au statut de l'Etat, est soumis aux changements de notre temps qui sont ceux de la libre concurrence, du libéralisme et de la mondialisation. Le second, a été créé de toutes pièces par la doctrine et avalisé par la suite par le législateur comme pour attirer dans l'escarcelle de la transparence les contrats qui ont pour objet de confier à un tiers l'exécution d'un service public. Cette catégorie est ancienne et s'est historiquement distingué des marchés publics.

«Le concept de délégation ne serait-il pas alors un ensemble vide, une mode sémantique échafaudée sur l'autel de la transparence? "» s'interrogèrent AUBY J.F. et RAYMUNDIE O. ${ }^{382}$. Bref, il aurait été créé par inadvertance et ne serait qu'une modernisation conjoncturelle du droit des contrats de gestion des services publics, et plus particulièrement, de celui des concessions. Ce contrat est, en principe, un contrat de droit public ou contrat administratif puisqu'il a pour objet de confier l'exécution d'un service public. La catégorie de contrats qui tire son unité de l'objet consistant à opérer la dévolution d'un service public a été isolée depuis longtemps, même si ses contours exacts ne sont pas toujours bien déterminés en dehors de la concession et de l'affermage. Un auteur avait proposé de dénommer ces contrats « contrats de service public » ${ }^{383}$.

Hubert-Gérald HUBRECHT distingue trois catégories de contrats de service public : les contrats de dévolution du service, les contrats de mandat, les contrats d'association. C'est à la première catégorie que se rattachent les délégations de service public. En réalité, les dénominations utilisées pour désigner ces contrats varient d'un contexte à un autre et dépendent des personnes qui les utilisent. On peut dire les expressions ont évolué. Du contrat de service public, on est passé par le contrat de gestion déléguée avant d'aboutir à l'expression de délégation de service public. En droit français, l'expression « contrats de service public » figurait à l'article 30-III de la loi no 92-125 du 6 février 1992 relative à l'administration territoriale de la République, devenu l'article L. 2121-12 du CGCT, selon lequel dans les communes de 3500 habitants et plus « si une délibération concerne un contrat de service public, le projet de contrat ou de marché accompagné de l'ensemble des pièces peut, à sa demande, être consulté à la mairie par tout conseiller municipal dans les conditions fixées par le règlement intérieur ». Dans la doctrine administrative a prévalu l'expression «contrat de gestion déléguée », qui a parfois été utilisée par le législateur ${ }^{384}$; l'article L. 5212-12 du CGCT utilise l'expression " délégation de gestion d'un service public ».

En fin de compte, les textes législatifs emploient la qualification « convention de délégation de service public » (loi du 6 février 1992 ; loi du 29 janvier 1993 ; CGCT, art. L. 1411-1 et s. ; NCP, art. L. 121-12). Les deux expressions, gestion déléguée et délégation de service public, doivent être réputées synonymes.

Le concept va ainsi pénétrer le langage juridico-politique aux lendemains des pressions des institutions financières internationales sur les Etats de l'Afrique subsaharienne appelés à moderniser leur système économique. La plupart des gouvernements africains ont consacré ces cinquante dernières années des efforts importants pour construire des infrastructures et

382 Cf. AUBY J.F. et RAYMUNDIE O., Le service public, le Moniteur, Paris 2003, p. 405.

383 HUBRECHT H.-G., Les contrats de service public, Thèse Bordeaux, 1980.

384 Loi n$^{\circ}$ 93-23 du 8 janvier 1993 relative à la législation dans le domaine funéraire, art. ${ }^{\text {er }}$ CGCT, art. L. 2223-19 
notamment des infrastructures hydrauliques malheureusement mal gérées ${ }^{385}$. Le processus de réorganisation des économies nationales et de l'économie mondiale, engagé à la fin des années 1970 en réponse à la crise de rentabilité va ainsi toucher le secteur de l'eau. La recherche des justifications juridiques aux nouvelles politiques économiques, oriente à examiner les désengagements effectués dans le secteur de l'eau sur le plan de la délégation du service public de l'eau (Section I) et du respect des cahiers des charges des délégataires (Section II).

\section{Section I. La délégation du service public de l'eau}

«La notion de service public remplace le concept de souveraineté comme fondement du droit public. Assurément cette notion n'est pas nouvelle. Du jour même où sous l'action de causes très diverses (...) s'est formée la distinction entre gouvernants et gouvernés, la notion de service public est née dans l'esprit des hommes. En effet, dès ce moment on a compris que certaines obligations s'imposaient aux gouvernants envers les gouvernés et que l'accomplissement de ces devoirs était à la fois la conséquence et la justification de leur grande force $»^{386}$. C'est en ces termes que le Professeur DUGUIT révélait la notion de service public en 1913. Cette notion vient "préfigurer un Etat transcendé par les liens de solidarité ou un interdépendance sociale ${ }^{387}$, un $"$ Etat de service public » selon les termes de PISIER KOUCHNER E. ${ }^{388}$. DUGUIT affirme qu'à l'intérieur de la nation, dans la limite du territoire occupé par cette nation, les gouvernants, différenciés des gouvernés et monopolisant la force, doivent employer cette force pour organiser et contrôler le fonctionnement des services publics ${ }^{389}$. Dans cette optique, l'Etat peut souverainement décider de la méthode qui convient à la gestion d'un service public. Le cas du service public de l'eau en est une illustration. En effet, au gré de ses « intérêts », l'Etat gère directement le service public de l'eau ou le confie à d'autres personnes morales à travers les contrats de délégation.

Aborder la question de la délégation du service public de l'eau revient à s'attarder sur la notion de délégation de service public. Si la plupart des Etats de l'Afrique subsaharienne ont opté pour la délégation du service public de l'eau, il faut préciser que l'opération est basée sur des textes juridiques. Ces derniers distinguent plusieurs cas de délégation, et donnent souvent des détails sur l'objet et les autorités signataires de la délégation. Ainsi, aux termes de la loi : "une délégation de service public est un contrat par lequel une personne morale de droit public confie la gestion d'un service public dont elle a la responsabilité à un délégataire public ou privé, dont la rémunération est substantiellement liée aux résultats de l'exploitation du service. Le délégataire peut être chargé de construire des ouvrages ou d'acquérir des biens nécessaires au

\footnotetext{
385 Idem.

386 DUGUIT L., Les transformations du droit public, Paris, Colin, 1913, p. 33
}

387 GARCERIES S., L'élaboration d'une notion juridique de service public industriel et commercial, Thèse, Université de Cergy-Pontoise, 2010, p.427.

388 PISIER KOUCHNER E., Le service public dans la théorie de l'Etat de Léon Duguit, Paris, LGDJ, BPD, volume XV, 1972, p. 23.

389 DUGUIT L., Traité de droit constitutionnel, Paris, Ed. de Boccard, tome II, $3^{\text {ème }}$ éd. 1928. 
service ». En France, un avis du Conseil d'Etat, confirmé par la loi MURCEF ${ }^{390}$, affirme que le caractère administratif d'un service public n'interdit pas à la collectivité d'en confier l'exécution à des personnes privées sous réserve que ces services ne soient pas par leur nature ou la volonté du législateur au nombre de ceux qui ne peuvent être assurés que par la collectivité publique. Nous allons examiner respectivement les dimensions de la délégation $(\S 1)$ puis les exigences juridiques de la délégation du secteur de l'eau (\$2).

\section{$\S 1$. Les contours de la délégation de service public}

La convention de délégation de service public a pour objet la délégation d'un service public. Cette condition limite le champ des conventions de délégation de service public à la fois positivement et négativement. En premier lieu, l'activité déléguée à l'opérateur économique doit répondre aux critères du service public. La distribution de l'eau répond d'une certaine manière à ces critères. Il faut préciser que selon la jurisprudence française le service peut se définir comme étant une activité d'intérêt général exercée par une personne publique ou sous son contrôle ${ }^{391}$. Toutefois, il n'est pas nécessaire que la personne privée cocontractante de la personne publique ait été investie de prérogatives de puissance publique pour admettre qu'elle s'est vue déléguer une mission de service public. Cela apparaît dans la décision du Conseil d'Etat du 22 février 2007. Cette décision dénommée "arrêt $A P R E P^{392}$ » par la doctrine précise que «indépendamment des cas dans lesquels le législateur a lui-même entendu reconnaître ou, à l'inverse, exclure l'existence d'un service public, une personne privée qui assure une mission d'intérêt général sous le contrôle de l'administration et qui est dotée à cette fin de prérogatives de puissance publique est chargée de l'exécution d'un service public».

Par ailleurs, «même en l'absence de telles prérogatives, une personne privée doit également être regardée, dans le silence de la loi, comme assurant une mission de service de public lorsque, eu égard à l'intérêt général de son activité, aux conditions de sa création, de son organisation ou de son fonctionnement, aux obligations qui lui sont imposées ainsi qu'aux mesures prises pour vérifier que les objectifs qui lui sont assignés sont atteints, il apparaît que l'administration a entendu lui confier une telle mission ». D'autre part, la convention ne peut porter sur la délégation d'une activité de service public considérée comme susceptible de faire l'objet d'une délégation à une personne privée. Il en va ainsi des prérogatives de police. Ainsi, est nulle une convention de stationnement payant, car elle s'analyse en une délégation des prérogatives de police du stationnement du maire de la commune au profit d'une société privée ${ }^{393}$.

Le lien unissant le délégant au délégataire doit être nécessairement contractuel. La loi définit la délégation de service public par l'instrument juridique réalisant la délégation. La notion de

\footnotetext{
390 Cf. article 3 de la loi MURCEF n 2001-1168 du 11 décembre 2001 (L. 1411-1 du CGCT)

391 CE, 20 juillet 1990, Ville de Melun.

392 Association du personnel des établissements pour inadaptés.

$393 \mathrm{CE}, 1^{\mathrm{er}}$ avril 1994, Commune de menton.
} 
contrat se trouve mise en exergue ce qui, ipso facto, fait sortir du champ de la définition toutes les délégations « statutaires », c'est-à-dire prévues par détermination de la loi ou du règlement. Le délégataire doit être chargé de la gestion et de l'exploitation du service public. A défaut, le contrat pourrait n'être qu'une fourniture de moyens pouvant relever du code des marchés publics. Par ailleurs, cette définition réaffirme le principe de la distinction entre la maitrise du service public et sa gestion. Seule une collectivité publique peut détenir la maîtrise d'un service public, c'est-à-dire qu'elle peut seule détenir les compétences pour le créer, l'organiser, lui donner les moyens de sa mission et en assumer le contrôle. La gestion proprement dite concerne uniquement l'exécution du service, conformément au cadre juridique préalablement établi. Le principe général du droit administratif selon lequel la collectivité confie, sous son autorité et son contrôle, la gestion du service public dont elle a la responsabilité, se trouve ainsi réaffirmée.

Selon le juge administratif, la délégation de service public doit opérer « la dévolution du service, c'est-à-dire qu'il doit bien avoir pour objet d'organiser le service $»^{394}$. Pour P. TERNEYRE et C. MAUGÜE, "il faut que le délégataire ait été véritablement chargé de sa gestion et qu'il ne se borne pas simplement à fournir l'un de ses éléments ou à lui apporter une collaboration $»^{395}$. Le délégataire doit avoir un rôle propre et disposer d'un degré suffisant d'autonomie et des responsabilités suffisamment étendues. "Le simple fait d'être autorisé ou habilité à exercer une activité de service public ne signifie pas qu'il y ait délégation de service public $»^{396}$. Toutefois, la délégation n'est pas totale, en ce sens que la collectivité demeure toujours, in fine, responsable du service public. Cette originalité a orienté la doctrine vers la recherche d'une distinction entre gestion directe, confiée au délégataire, et gestion indirecte, dont reste titulaire la collectivité ${ }^{397}$.

Enfin, la rémunération est substantiellement assurée par les résultats de l'exploitation d'un service public ${ }^{398}$. La délégation du service public est une catégorie générique qui recouvre plusieurs types de contrats ${ }^{399}$. Pour mieux maîtriser le concept, nous allons étudier la concession (A) puis les autres cas de délégation(B).

\section{A. Le cas de la concession}

$\mathrm{Au}$ sens strict du terme, la concession est caractérisée par le fait qu'au début de l'exploitation, le concessionnaire fasse l'avance des frais de premier établissement du service (construction du réseau et de tous les ouvrages nécessaires au fonctionnement du service) et du fonds de roulement nécessaire à l'exploitation. D'autre part, pendant toute la durée de la concession, le

394 CAA Marseille, 4 juill. 2005, BJDCP 2006, $\mathrm{n}^{\circ}$ 45, p. 113,

395 C. MAUGÜE et P. TERNEYRE, « Les délégations de service public en question », CJEG avr. 1997., p. 132

396 C. MAUGÜE et P. TERNEYRE, «Les délégations de service public en question », précité, p. 134.

397 G. GLENARD, « La notion de délégation dans le droit de la délégation de service public », CJEG avr. 1997, p. $131 \mathrm{~s}$.

398 Principe de la rémunération substantielle, c'est-à-dire, celui de l'aléa financier.

399 TRUCHET D. , Droit administratif, Thémis Droit PUF, 2009, p. 342. 
concessionnaire exploite le service à ses risques et périls, qu'il en assume la direction, qu'il choisisse, rémunère et surveille lui-même le personnel du service, qu'il entretienne et renouvelle lui-même, à ses frais, les installations et qu'il achète tout l'outillage et le matériel nécessaires à l'exploitation. Enfin, en échange de ces services, le concessionnaire est rémunéré par la perception directe de redevances sur les usagers, lesquelles doivent normalement lui permettre de couvrir les intérêts et l'amortissement du capital qu'il a engagé et de dégager un bénéfice net dont il garde tout le profit pour lui-même. Au-delà de ces considérations, il faut en cerner la notion et tenter de comprendre pourquoi les Etats africains ont une préférence pour ce modèle.

\section{La notion de concession}

La concession désigne une variété de délégation de service public. C'est un acte par lequel une personne, privée ou publique appelée concessionnaire, s'engage à gérer pour une personne publique appelée le concédant, un service public, ou à réaliser un travail moyennant une rémunération qui peut prendre diverses formes ${ }^{400}$. Elle avait été la première fois définie par le commissaire du gouvernement CHARDENET dans l'affaire du «Gaz de Bordeaux»" ${ }^{401}$ en ces termes : "C'est le contrat qui charge un particulier ou une société d'exécuter un ouvrage public ou d'assurer un service public, à ses frais, avec ou sans subvention, avec ou sans garantie d'intérêts, et que l'on rémunère en lui confiant l'exploitation de l'ouvrage public ou l'exécution du service public avec le droit de percevoir des redevances sur les usagers de l'ouvrage ou sur ceux qui bénéficient du service public ».

Dans les années 1990, la concession était la principale formule d'investissement privé dans le secteur de l'eau. Les firmes privées nationales et étrangères assumant la responsabilité du financement et de l'exploitation des systèmes. Le concessionnaire est souvent rémunéré sur les factures des usagers, et non directement par la puissance publique. Certains concessionnaires ont pu réduire les fuites d'eau, accroître l'approvisionnement, multiplier les compteurs ainsi que les recettes, tout en étendant la couverture. Mais certaines multinationales comme Suez, Veolia Environnement, Thames Water/RWE,...se sont retirées de ce type d'engagement parfois sous la pression des instances gouvernementales et des réglementations.

C'est une formule assez souple qui consiste à confier la direction de l'entreprise à un opérateur privé dans le cadre d'objectifs et de moyens définis de façon contractuelle. Plusieurs formules juridiques sont possibles. La convention de concession de service public, la Côte d'Ivoire l'adoptée dès le début de l'indépendance, avant même que ne commencent les pressions des institutions financières internationales, pour la distribution d'eau en zones urbaines, assurée par une société d'économie mixte, la SODECI, où l'actionnaire majoritaire est le groupe Bouygues ${ }^{402}$.

400 DEBBASCH C. et COLIN F., op. cit., p. 460.

401 CE 30 mars 1916.

402 Bernard CONTAMIN, «Entreprises publiques et désengagement de 1'Etat en Côte-d'Ivoire, A la recherche des privatisations », références non disponibles. 


\section{La préférence des Etats au modèle de la concession}

La majorité des Etats de l'Afrique subsaharienne ont adopté la technique de la concession et l'ont consacrée dans leur loi portant code de l'eau. On peut citer quelques exemples notamment, le cas de la République mauritanienne où aux termes de l'article 46 de l'ordonnance portant code de l'eau ${ }^{403}$, «des concessions de service public fondées sur l'utilisation de l'eau sont accordées, pour une durée déterminée, aux personnes morales publiques. Elles peuvent, dans certains cas, être accordées à des personnes morales privées ou à des personnes physiques exerçants une activité présentant un caractère d'intérêt général ». D'autre part, l'on peut évoquer le Togo et le Sénégal où le législateur a admis la technique de la concession comme moyen de gestion du service public de l'eau. Pour le législateur togolais, "le prélèvement, l'accumulation et l'utilisation des eaux de surface et souterraines effectués au moyen d'ouvrages, installations et travaux permanents, et destinés à la production et distribution d'eau potable, ou à la production d'énergie électrique, dans le cadre d'un service public ${ }^{404} \gg$. Le législateur sénégalais à l'instar de son homologue mauritanien évoque la concession pratiquement dans les mêmes termes. Selon lui, des concessions de service public fondées sur l'utilisation des eaux sont accordées, pour une durée déterminée, aux personnes morales publiques ou privées ou aux personnes physiques exerçant une activité présentant un caractère d'intérêt général ${ }^{405}$.

Au Mali, c'est un contrat de concession de 20 ans qui a été signé au profit de l'entreprise nationale EDM, qui dessert (Mali) 16 centres, avec vente simultanée de $60 \%$ du capital d'EDM à l'opérateur stratégique, Saur et IPS WA (membre du groupe Aga Khan). Le contrat signé en janvier 2001 a rencontré des difficultés dès le départ. En août 2005, après une tentative de renégociation avortée, Saur a revendu ses parts à son partenaire, IPS WA, qui les a lui-même revendues à l'Etat, ce qui revient de facto à une renationalisation ${ }^{406}$.

La préférence des Etats à ce type de contrat peut s'expliquer par le fait qu'il présente certaines caractéristiques offrant les moyens de s'assurer de la continuité du service de l'eau. En effet, le contrat de concession est un acte mixte car il comporte deux types de clauses. Les unes sont dites clauses réglementaires parce que le concédant peut les modifier à tout moment, s'il estime que l'intérêt général commande ces modifications, on peut citer l'exemple du tarif de l'eau. Aussi, faut-il ajouter qu'il y a plus d'un siècle, la concession a offert au Conseil d'Etat en France l'occasion de construire sa jurisprudence relative aux contrats administratifs ${ }^{407}$. A côté des clauses réglementaires, on trouve les clauses contractuelles qui ne peuvent être modifiées unilatéralement ${ }^{408}$. Qu'en est-il des autres contrats voisins ?

$403 C f$. Ordonnance ${ }^{\circ}$ 85-144 du 4 juillet 1986 portant code de l'eau en Mauritanie.

$404 C f$. Article 19 al. 2 du code de l'eau en vigueur au Togo, précité.

$405 C f$. Article 27 du code de l'eau en vigueur au Sénégal.

406 TREMOLET S., « Un point sur les privatisations de l'eau en Afrique », Annales des mines avril 2006, p. 61.

407 Cf. CE, 10 janv. 1902, Cie nouvelle du gaz de Déville-Lès-Rouen.

408 DEBBASCH C. et COLIN F., op. cit., p. 461. 


\section{B. Les autres cas de délégation}

C'est l'alinéa 2 de l'article 45 du code de l'eau en vigueur au Mali qui englobe bien la situation des autres cas de délégation du service public de l'eau. En effet, la délégation de gestion peut couvrir différents modes de délégation de gestion, à savoir la concession d'ouvrage, l'affermage ou la gérance, ainsi que toute variante ou combinaison de ces trois conventions ${ }^{409}$. Les variantes des conventions de délégation vont au-delà de l'affermage et de la gérance précitée, on peut ajouter la régie intéressée et le bail emphytéotique.

Quant au contrat de gérance, il est défini comme un contrat par lequel un maître d'ouvrage confie à un tiers, contre rémunération, le mandat de réaliser les activités techniques et commerciales nécessaires au bon fonctionnement des installations d'eau, mais conserve tous les risques techniques et commerciaux inhérents à ces activités, y compris la responsabilité et le financement des investissements de renouvellement et extension du réseau ${ }^{410}$.

\section{Le contrat d'affermage}

Dans la convention d'affermage, le délégataire (le fermier) reçoit les ouvrages et installations de la personne publique et les utilise pour faire fonctionner le service public en se rémunérant sur l'usager ${ }^{411}$. Le critère du financement des investissements permet de distinguer l'affermage de la concession. L'affermage n'est en définitive qu'un type de concession dans laquelle les frais de premier établissement ont été exposés par la collectivité publique. Le fermier reçoit donc les ouvrages déjà réalisés et n'a qu'à assurer la gestion du service et faire l'avance du seul fonds de roulement. Le fermier ne peut, en principe, exécuter pour le compte du bailleur des travaux autres que ceux d'entretien courant, à la charge de tout locataire.

Il convient, en effet, de ne pas écarter l'application de la réglementation sur les marchés publics et notamment la mise en concurrence des entrepreneurs, sous le prétexte de l'existence d'une convention d'affermage. Bien entendu, comme dans la concession stricto sensu, le fermier assure l'exploitation à ses risques et périls et est rémunéré au moyen de redevances prélevées directement sur les usagers. Enfin, pour payer les intérêts et rembourser les annuités des emprunts qu'elle a dû contracter pour construire les ouvrages, la collectivité concédante doit demander à son fermier d'encaisser pour son compte une " part collectivité » sur les usagers du service.

Pour illustrer ce type de délégation, on peut citer le contrat d'affermage de 10 ans avec obligations d'investissements limitées que le gouvernement du Sénégal a signé avec l'entreprise nationale SDE, qui dessert 56 centres urbains, avec prise de participation par Saur et des privés locaux. Le contrat, signé en avril 1996, a apporté des améliorations notables tant du point de vue technique que financier ${ }^{412}$. D'autre part, lorsque le gouvernement de Guinée Conakry avait

\footnotetext{
$409 C f$. la loi n ${ }^{\circ} 02-006 /$ du 31 jan.2002 portant code de l'eau.

$410 \mathrm{Cf}$. Chapitre I de la loi nº2- 006/ du 31 jan.2002 portant code de l'eau au Mali.

411 TRUCHET D., Droit administratif, Thémis PUF, $2^{\text {ème } e ́ d i t i o n, ~ 2009, ~ p . ~} 343$

412 Sophie TREMOLET, op. cit.
} 
décidé en 1989 de privatiser la gestion de l'eau potable, il avait opté pour le contrat d'affermage. La période d'affermage a été caractérisée par un faible niveau de rendement technique des installations ${ }^{413}$.

\section{La régie intéressée}

En ce qui concerne la régie intéressée, il s'agit d'un mode de gestion dans lequel le régisseur intéressé assure l'exploitation du service public, mais sur le plan financier se contente d'effectuer les opérations de dépenses et de recettes pour le compte du délégant. Il perçoit en contrepartie une rémunération du délégant dont le montant est en général fonction du chiffre d'affaires réalisé et des résultats liés à la qualité de la gestion pratiquée ${ }^{414}$.

Considérée souvent comme à mi-chemin entre la régie et la concession, la régie intéressée se caractérise principalement par deux critères dont l'un financier et l'autre organique. En effet, le régisseur est rémunéré suivant un procédé qui doit l'inciter à améliorer et sa gestion. D'autre part, le régisseur agit pour le compte de la collectivité publique qui demeure chargée de la direction et de l'exploitation du service. Contrat très ancien mais autonome par rapport à la concession et l'affermage, la régie intéressée doit reposer sur une rémunération qui fasse que le régisseur soit substantiellement rémunéré par les résultats de l'exploitation. A défaut, la jurisprudence requalifie le contrat de régie intéressée en marché public.

La rémunération du régisseur intéressée n'est pas assurée directement par le solde du compte d'exploitation, mais indirectement par la collectivité publique qui verse une rémunération forfaitaire à l'exploitant, à laquelle s'ajoute un intéressement au résultat. Il est également possible que le régisseur encaisse pour le compte de collectivité les sommes auprès des usagers et les lui reverse après avoir perçu sa propre rémunération ${ }^{415}$.

De la gestion déléguée, la régie intéressée se rapproche par le fait que le régisseur apparaît comme un entrepreneur dont la rémunération n'est pas fixe comme celle du gérant, mais variable, calculée en fonction du pourcentage du chiffre d'affaires, généralement complétée d'une prime de productivité. Elle s'en éloigne cependant par le caractère de « régie » qui fait que le régisseur agit pour le compte de la collectivité, et ce, à la différence de la concession ${ }^{416}$. Il en résulte, du moins, au niveau des règles comptables, que les sommes perçues sur les usagers et encaissées par le régisseur sont la propriété de la personne publique et qu'elles sont soumises au contrôle comptable et financier applicable aux régies d'avances.

413 GARANE A., « La mise en œuvre du droit à l'eau en Afrique de l'Ouest », in GRISEL G., La mise en æuvre du droit à l'eau, Actes du XXIX ${ }^{\text {ème }}$ Congrès ordinaire de l'IDEF, Schuthess, 2006, p. 219.

414 DEBBASCH C. et COLIN F., précité.

415 CE, 24 avril 1989, Sté Monégasque des entreprises J-B Pastor et fils, Lebon, p. 663.

416 CE, $1^{\text {er }}$ février 1989, Socea-Balency, RDP 1989, p. 1816. 


\section{§ 2. Les exigences juridiques de la délégation du secteur de l'eau}

Si en général, le délégataire dispose par son contrat d'un monopole dans la gestion et l'exploitation du service, il n'en reste pas moins que la participation du secteur privé dans la gestion de l'eau est l'objet principal des délégations du service public de l'eau. Ceci est vrai a fortiori en Afrique, où les bailleurs de fonds internationaux répandent le bien fondé des privatisations depuis des années maintenant, et ont souvent fait de l'introduction d'un opérateur privé dans la gestion des services d'eau une condition sine qua non de leur soutien financier ${ }^{417}$. Nous examinerons d'une part les droits des délégataires (A) puis les autorités délégantes signataires (B).

\section{A. Les droits des délégataires}

Pour mieux comprendre les droits des délégataires qui sont multiples, il est important d'examiner la nature juridique du lien qui existe entre les délégataires et les autorités délégantes.

\section{La nature du lien de délégation}

L'intérêt porté à la nature de l'habilitation est d'ordre théorique : en principe, l'habilitation contractuelle ne peut être modifiée qu'après accord des volontés des parties, tandis que l'habilitation unilatérale n'est modifiable que par la seule volonté de l'autorité qui l'accorde. De plus, il faut parfois prendre garde à la terminologie utilisée qui est peut être trompeuse: l'habilitation peut revêtir une forme unilatérale et connaître un régime juridique de fond proche du contrat. Inversement, l'habilitation peut être de forme contractuelle et rendre impersonnelle et uniforme l'apparence contractuelle, par exemple, dans les marchés de fourniture et de service, les droits et obligations des parties sont très largement déterminés par les cahiers des charges établis par la seule administration. Pourtant, il n'y en a pas moins de contrat.

La délégation de service public se traduit par l'existence d'un lien contractuel, expression de la volonté des parties. Ce lien présente certaines spécificités. Il s'agit d'abord d'un contrat administratif, qui porte sur l'organisation d'un service public. En effet, le contrat a pour objet de confier au cocontractant "l'exécution même d'un service public». Dès lors, il est incontestable que les « les règles générales applicables à l'ensemble des contrats administratifs » trouveront à s'appliquer. Aussi, l'autorité peut modifier unilatéralement le contrat, le résilier et exercer un étroit contrôle sur le gestionnaire. La jurisprudence fait découler de la notion d'organisation du service public un certain nombre de conséquences. Elle considère que les clauses des charges ayant pour objet d'organiser le service public ont un caractère réglementaire. Présentent

417 Sophie TREMOLET, précité, p. 59. 
notamment un caractère réglementaire les clauses relatives aux horaires ${ }^{418}$, celles relatives à la sous-traitance ${ }^{419}$, aux pouvoirs de contrôle ${ }^{420}$.

Ces clauses réglementaires produisent des effets sur les tiers et les usagers ${ }^{421}$ qui peuvent attaquer par la voie du recours pour excès de pouvoir la clause litigieuse, notamment les tarifs, la surtaxe $^{422}$, mais pas leur redevance à la charge du concessionnaire ${ }^{423}$, ni les droits d'entrée. Le droit de la concurrence, tel qu'appliqué par la juridiction judiciaire, ne saurait autoriser le juge judiciaire à remettre en cause le pouvoir d'organisation du service public ${ }^{424}$. Le juge judiciaire ne saurait apprécier la légalité des dispositions réglementaires d'un cahier des charges ou d'un règlement de service ${ }^{425}$.

Le contenu du contrat de délégation est libre sous réserve de dispositions qui seraient contraires aux règles d'ordre public. En ce sens, il n'existe plus de contrat «type » imposé aux collectivités. En France, la technique de l'habilitation unilatérale à gérer le service public a été longtemps considérée comme ne débouchant pas sur une véritable dévolution du service, celle-ci permettant d'abord d'obtenir un régime juridique privilégié. C'est pourquoi, jusqu'au début du siècle, la doctrine a longtemps hésité à présenter ce procédé comme un mode de transfert du service public. Elle a, par la suite corrigé son point de vue ${ }^{426}$ et admis que le procédé s'accommodait finalement assez bien à la fois des exigences du service public et de la nature de la personne privée investie de la mission de service public, dès lors que cette habilitation empruntait, par nombre de ces aspects, au domaine contractuel et qu'elle était plus négociée qu'imposée.

\section{Les prérogatives des délégataires}

Les droits les plus importants du délégataire, ceux en général en vue desquels il a contracté, sont des droits financiers. Ils revêtent plusieurs significations. D'une part, le délégataire est rémunéré à raison du service qu'il gère ou des travaux qu'il réalise. Cette rémunération prend la forme de redevances perçues sur les usagers du service. Le Conseil d'Etat a qualifié de redevances les sommes versées par les usagers des autoroutes à péage ${ }^{427}$. A contrario, si la

418 CE, 21 déc. 1906, Synd. des propriétaires et contribuables du quartier Croix- de Serguey-tivoli.

419 CE, 24 janv. 1990, Martinettti, Lebon p. 587.

420 TA Marseille, 12 avril 1994, Porelli, req. 924729.

421 CE ass. 10 avril 1996, Cayzeele, req. ํ3라. Lebon p.274.

422 CAA Marseille, 13 déc. 2001, Union fédérale des consommateurs, req. № 99-2298

423 TA Dijon, 9 mai 1995, Mme Bessis, req. ํ 94202, AJDA 1995, p. 941.

424 TC, 6 juin 1989, Préfet de Paris de la région Ile-de-France.

425 TC, 2 mai 1977, Paraïso/Sté Lyonnaise des eaux, Lebon p. 739.

426 SABIANI F., «L'habilitation des personnes privées à gérer un service », AJDA 1977, p.6.

427 CE, 13 mai 1977, Cie industrielle et financière des autoroutes, D. 1978, 130. 
rémunération n'est pas substantiellement assurée par les résultats de l'exploitation, il ne peut s'agir d'une délégation du service public ${ }^{428}$.

Comme toute personne appelée à gérer un service public, le délégataire dispose de prérogatives exorbitantes du droit commun attachées à ce dernier. Le concessionnaire peut, pour les besoins du service, procéder à l'expropriation et les travaux qu'il réalise peuvent être des travaux publics ${ }^{429}$. Le contrat de concession peut conférer au bénéficiaire le droit : d'établir, après approbation des projets par l'autorité concédante, tous ouvrages utiles, d'occuper les parties du domaine public nécessaires à ses installations ${ }^{430}$.

Le délégataire bénéficie d'un droit à être aidé par l'administration en cas de survenance d'évènements présentant un caractère anormal et imprévisible, entraînant un bouleversement du contrat, et dans lesquels le délégataire n'a aucune responsabilité : c'est la théorie de l'imprévision ${ }^{431}$. Bien que le délégataire exploite à ses risques et périls, l'intervention de l'administration est justifiée par la nécessité d'assurer la continuité du service public, que la ruine du délégataire mettrait en cause ${ }^{432}$.

\section{B. Les autorités signataires}

Leurs prérogatives varient en fonction du type de régime constitutionnel en vigueur dans l'Etat concerné.

\section{La distinction des autorités délégantes}

La convention de délégation de service public s'appuyant sur la notion de service public, les autorités compétentes pour organiser un service public sont des personnes de droit public : l'Etat, les collectivités territoriales et les établissements publics. On pourrait conceptuellement envisager qu'une personne morale de droit privé soit investie d'une mission de service public et qu'elle puisse conclure avec des tiers des contrats qui auraient le caractère de délégation de service public. Un tel schéma ne correspond pas, à notre connaissance au droit positif qui réserve la conclusion de contrat de délégation de service public aux personnes morales de droit public. Il n'est cependant pas certain que la jurisprudence n'évolue pas sous l'influence des textes communautaires. Ceci, en effet, assujettit les marchés des entités publiques et privées à des règles de mise en concurrence lorsque celles-ci sont dotées de droits spéciaux ou exclusifs ou subventionnées par la puissance publique.

428 CE, 15 avril 1996, Préfet des Bouches-du-Rhône c/ Commune de Lambesc, Rec., 274.

429 DEBBASCH C. et COLIN F., précité, p. 463.

430 Cf. Article 76 de la loi n $98-755$ du 23 décembre 1998 portant Code de l'Eau en Cote d'Ivoire.

431 CE, 30 mars 1916, Cie d'éclairage de Bordeaux, GAJA.

432 DEBBASCH C. et COLIN F., précité. 
En Afrique subsaharienne, on distingue essentiellement deux autorités délégantes, à savoir l'Etat et les collectivités territoriales. Mais il faudrait faire attention car, certains Etats comme le Togo, semblent ne pas reconnaître aux collectivités territoriales le pouvoir de signer les concessions. C'est ce qui ressort des dispositions du code de l'eau en vigueur depuis l'année 2010. En effet, on peut lire que «la signature de la concession est autorisée par décret en conseil des ministres. Les aménagements, ouvrages et travaux soumis au régime de la concession font l'objet d'une étude d'impact environnemental préalable dans les cas prévus par la législation sur l'environnement. Le contenu, la méthodologie et les procédures des études d'impact environnemental sont réglementés par le ministre chargé de l'environnement, en accord avec le ministre chargé de l'eau $\gg^{433}$. Alors que les nouveaux textes sur la décentralisation reconnaissent aux collectivités territoriales des compétences en matière de gestion et de distribution de l'eau, on se demande s'il s'agit d'une lacune de la part du législateur, ou si cette omission est volontaire afin de limiter les prérogatives transférées aux collectivités décentralisées.

Sans reconnaître explicitement le pouvoir des autorités décentralisées à octroyer des concessions, le législateur malien semble en envisager la possibilité. En effet, il prévoit que «dans les villages, centres ruraux et semi-urbains, une délégation de gestion peut être attribuée à une association d'usagers pour autant que celle-ci soit régulièrement constituée conformément à la réglementation en vigueur et soit dotée de la personnalité morale ${ }^{434}$. De même, il reconnaît que dans les mêmes collectivités, le service public de l'eau ne peut pas être exploité en régie directe par les communes maîtres d'ouvrage. Mais en cas de déchéance de l'exploitant ou de l'association d'usagers et dans l'impossibilité de trouver un autre exploitant, la commune maitre d'ouvrage peut avec l'accord du ministre chargé de l'Eau potable mettre en place une régie autonome. La gestion en régie directe du service public de l'eau est interdite dans les centres urbains ${ }^{435}$.

\section{Les pouvoirs des autorités délégantes}

Les autorités exécutives sont responsables de la politique de distribution d'eau. Pour ce faire, elles sont les seules à décider de l'opportunité d'accorder la délégation du service public de l'eau à une personne morale de droit privé. Selon le code de l'eau en vigueur au Togo, la signature de la concession est autorisée par décret en conseil des Ministres ${ }^{436}$. Si les aménagements, ouvrages et travaux soumis au régime de la concession font l'objet d'une étude d'impact environnemental préalable dans les cas prévus par la législation sur l'environnement, le contenu, la méthodologie et les procédures des études d'impact environnemental sont réglementés par le ministre chargé de l'environnement, en accord avec le ministre chargé de l'eau.

En République du Congo, afin de procéder au choix du délégataire, l'Etat publie un appel d'offres précisant la nature et les principales conditions du contrat de délégation qu'il envisage

433 Cf. Article 19 du code de l'eau en vigueur au Togo, précité.

$434 C f$. article 45 du code l'eau en vigueur au Mali, précité.

435 Idem.

$436 C f$. Art. 19 du code de l'eau précité. 
de conclure et spécifiant précisément les critères sur lesquels il se fonde pour départager les candidats. L'Etat examine les propositions qu'il reçoit en réponse à l'appel d'offres et choisit le délégataire sur la base des critères spécifiques dans l'appel d'offres. Le contrat de délégation revêt une forme écrite. Il est signé par le ministre chargé de l'eau et ratifié par décret en Conseil de ministres ${ }^{437}$.

La même logique est adoptée par le législateur ivoirien selon qui l'autorité chargée de l'eau et les Ministères compétents peuvent confier, à toute personne physique ou morale, le service public d'exploitation des eaux, des ouvrages et aménagements hydrauliques. Ces modes d'exploitation sont approuvés selon les cas par décret pris en Conseil des Ministres ${ }^{438}$. Ceci cadre bien avec la politique nationale de gestion des eaux, des aménagements et ouvrages hydrauliques qui est également définie par décret pris en Conseil des Ministres ${ }^{439}$.

\section{Section II. Le respect des cahiers des charges des délégataires}

Le cahier des charges est un «document administratif détaillant généralement avec minutie, les obligations et éventuellement les droits de certains contrats administratifs comme les concessions... $\gg^{440}$. La question de leur contenu est abordée dans les différents codes de l'eau en vigueur en Afrique subsaharienne. Par exemple, aux termes de l'article 28 du code l'eau du Sénégal ${ }^{441}$, « les concessions de service publique sont approuvés par décret pris sur rapport des Ministres chargés de l'Hydraulique et de l'Assainissement et du Ministre de tutelle de l'organisme concessionnaire, après avis du Ministre chargé des Domaines. Ce décret fixe dans chaque cas les clauses et conditions de la concession ». Le cahier des charges renferme un certain nombre d'obligations $(\S 1)$ auxquelles le délégataire est tenu de respecter sous peine de sanctions (§2).

\section{§ 1. Le cahier des charges, un bouclier contre les dérives}

En théorie, le cahier des charges est un bouclier à toutes épreuves contre les dérives du marché, afin de préserver le caractère de droit à la vie que représente l'accès à l'eau pour chaque humain. Après y avoir défini la quantité d'eau nécessaire de façon quotidienne à la vie de chaque individu, l'Etat doit exiger dans le contrat, que le mètre cube d'eau paie non seulement le service de l'eau, mais qu'il soit fixé en fonction du pouvoir d'achat d'unité monétaire des couches

437 Article 62 de la Loi n¹3-2003 du 10 avril 2003 portant Code de l'eau en République du Congo

438 Article 75 du code de l'eau en vigueur en Côte d'Ivoire.

$439 C f$. Article 55 du code précité.

440 GUILLIEN R. et VINCENT J. (sous la direction), Lexique des termes juridiques, Dalloz, 2001, p.80.

441 Loi n 81-13 du 4 mars 1981 portant Code de l’Eau. 
sociales les plus démunies ${ }^{42}$ (A) puis les exigences juridiques de la délégation du secteur de l'eau (B).

\section{A. Le contenu des cahiers}

Les contrats de délégation sont des contrats d'adhésion, car ils comportent toujours certaines clauses qui ne sont pas susceptibles de discussion, ces clauses étant insérées dans les contrats dans le but d'une protection de l'intérêt général ${ }^{443}$.

\section{Les obligations incombant aux délégataires}

L'analyse des différents codes de l'eau révèle que les obligations incombant aux concessionnaires du service public de l'eau sont en réalité nombreuses. Les obligations du délégataire sont de deux catégories. La première renvoie à la qualité de la prestation et la seconde précise les obligations comptables et financières du délégataire.

En se référant aux termes des articles 12, 13 et 14 du cahier des charges pour la délégation de gestion des adductions d'eau potable dans les centres semi-urbains et ruraux en vigueur en République du Mali, on note d'une part que la conception et la réalisation des travaux, l'exploitation et l'entretien des installations doivent respecter les dispositions administratives et techniques en vigueur, notamment en matière de santé publique. D'autre part, il est précisé que les ouvrages de production et d'adduction doivent être exploités conformément aux règles de l'art, dans le souci de garantir la conservation du patrimoine productif et de la ressource en eau elle-même. Aux termes de l'article 20 du code de l'eau en vigueur au Togo, toute concession relative au domaine public de l'eau donne lieu à l'établissement d'un cahier des charges, qui précise notamment, l'objet de la concession, le débit concédé, le mode d'utilisation des eaux, les obligations du concessionnaire, la redevance à verser par le bénéficiaire de la concession, la durée de la concession ${ }^{444}$, les conditions de renouvellement des équipements, la nature des ouvrages et le délai d'exécution des diverses tranches des installations et aménagements prévus, les mesures à prendre par le concessionnaire afin d'assurer la protection des ouvrages et installations, les mesures à prendre par le concessionnaire pour éviter la dégradation de la qualité des ressources en eau. Il précise entre autre que la concession ne confère à son titulaire aucun droit de propriété sur le domaine public de l'eau. Enfin, le délégataire s'engage à fournir toute l'eau nécessaire aux besoins publics et privés à l'intérieur du périmètre de délégation. Si les installations deviennent insuffisantes pour satisfaire à ces besoins, le délégataire devra informer dans les meilleurs délais la Collectivité Territoriale qui prendra toute disposition pour rétablir la situation.

442 ANOUGOU J. P. T., «L'eau, bien public, bien privé : l'Etat, les communautés locales et les multinationales », p. 161.

443 DEBBASCH C. et COLIN F., précité, p. 475

$\mathbf{4 4 4}$ Trente (30) ans au maximum. 
L'eau distribuée devra répondre constamment aux exigences imposées par la réglementation en vigueur. Le délégataire devra vérifier la qualité de l'eau distribuée aussi souvent qu'il sera nécessaire, se conformer à cet égard aux prescriptions du Ministère chargé de la Santé et donner toute facilité pour l'exercice de contrôles sanitaires, visites, prélèvements et analyses. Il sera toujours responsable des dommages qui pourraient être causés par la mauvaise qualité des eaux. L'ensemble des analyses et frais afférents (autocontrôle et programme réglementaire) est à la charge du délégataire y compris les prélèvements ${ }^{445}$.

Toutefois, un contrat de concession mal écrit et dont le cahier des charges est inadapté et contenant des dispositions erronées et lacunaires (surtout celles relatives aux conditions tarifaires) peut compromettre la réalité du droit à l'eau. Il y a lieu de s'interroger sur la fermeté du contrôle prévu dans les cahiers de charge, d'autant plus qu'en concédant le service de l'eau, certains Etats se privent des moyens nécessaires pour le suivi des activités de l'opérateur privé. Par exemple, à l'article 20 du code de l'eau en vigueur au Togo, il est mentionné que «le niveau de participation de l'Etat au capital social ne peut excéder 10\%». Dans ces conditions, l'Etat aura très peu d'influence sur les décisions qui seront prises ${ }^{446}$.

Concernant les obligations comptables et financières, il est généralement prévu que le délégataire exploite les installations à ses risques et périls. En échange de la perception du prix de vente de l'eau, il prend en charge les dépenses suivantes, qui comprennent des provisions, redevances et taxe. D'abord le délégataire assure à ses frais l'exploitation et l'entretien des installations (station de pompage, réservoirs, conduite de refoulement et réseau de distribution). En particulier, il paye les fournitures nécessaires (gasoil, lubrifiants, pièces détachées, matériaux pour les branchements particuliers, éléments de conduites pour les réparations de fuites) et rémunère tout le personnel nécessaire. Des provisions pour renouvellement, réparations et extension des réseaux liés à cette DSP sont généralement prévues. Le délégataire est entièrement responsable de ces sommes tant qu'elles n'ont pas été déposées sur le compte bancaire prévu à cet effet. La société bénéficiaire de la délégation a souvent des charges fiscales à payer. La nature de ces charges varie en fonction des Etats. Ces charges fiscales vont de simples redevances à l'impôt minimum forfaitaire.

Le délégataire est tenu d'élaborer et de mettre à jour les documents relatifs aux usagers et les points de distribution. Concernant les usagers, le délégataire doit élaborer le contrat type d'abonnement pour les branchements particuliers. En ce qui concerne les points de distribution, le délégataire devra fournir le détail des ventes d'eau journalières par borne-fontaine, des sommes collectées par borne-fontaine et tenir un registre des abonnés.

\section{Le contrat de délégation de service public de l'eau, un contrat intuitu personae}

Le contrat de délégation est conclu en considération de la personne du délégataire. Le délégataire ne peut céder le contrat à un tiers que sur autorisation expresse donnée par décret en

445 Voir aussi, le Cahier des charges pour la gestion déléguée du service public de l'eau potable / Programme LEHDADA/Lot 1 de la République islamique de Mauritanie, février 2009.

446 Cf. loi n ${ }^{\circ} 2010-004$ portant code de l'eau du 14 juin 2010. 
Conseil des ministres. Le délégataire peut sous-traiter une partie des obligations qui sont mises à sa charge. Il demeure alors pleinement responsable de la bonne exécution du service délégué visà-vis de l'Etat. La sous-traitance n'est en outre autorisée que si son objet n'a pas une étendue telle que le délégataire perde en fait la maîtrise opérationnelle du service délégué ${ }^{447}$. Le contrat de sous-traitance est conclu "intuitu personae » et il ne peut y avoir de cessions de droits sous peine de résolution immédiate aux torts et griefs du cocontractant ${ }^{448}$. Le concessionnaire demeure toujours responsable envers l'Etat et les tiers des obligations imposées par le cahier des charges de la concession. L'intuitu personae est une référence soit à la personne elle-même, soit à celle du cocontractant. Quelle que soit la personne visée, c'est de son identité qu'il est question. Si l'intuitu personae évoque d'abord les éléments qui individualisent officiellement une personne, une seconde définition énonce que l'identité est aussi l'ensemble des traits physiques et moraux qui caractérisent un être précis, et font qu'il est une personne unique. On ne considère donc pas nécessairement l'identité juridique. C'est d'ailleurs le plus souvent l'identité qualifiée de morale qui entre en ligne de compte, c'est-à-dire que seront prises en compte essentiellement les qualités de la personne: sa compétence, son imagination, son savoir-faire, son habileté, sa réputation, son appartenance à une profession, voire sa moralité, comme l'a évoqué dans son arrêt du 3 novembre 1994, la cour d'appel de Paris.

En passant en revue les textes sur l'eau dans les autres Etats, on constate que la mention du caractère intuitu personae du contrat de délégation n'est pas expresse. Toutefois, d'autres dispositions sur par exemple la question de la sous-traitance révèlent que les différents contrats conclus sur la gestion de l'eau prennent en compte la considération de la personnalité du délégataire. En effet, au Togo, on note qu'en cas de changement de propriétaire, ayant droit, titulaire de la concession, les bénéfices et les charges de la concession sont transférés de plein droit au nouveau propriétaire ou ayant droit qui doit déclarer le transfert au ministre chargé de l'eau dans un délai de trois mois à compter de la date de la mutation ${ }^{449}$. Certaines dispositions du code de l'eau sénégalais font également penser à la prise en compte de ce caractère: «L'autorisation est personnelle sauf en ce qui concerne les personnes morales de droit public pouvant en bénéficier. L'autorisation personnelle ne peut être transmise ou cédée à des tiers autres que les héritiers du bénéficiaire, sauf en vertu d'une autorisation donnée en la même forme que l'autorisation primitive $»^{450}$.

En droit français, la règle de l'intuitu personae prévoit que la procédure soit conforme au principe d'égal accès des candidats à l'octroi à la délégation. Pour ce faire, elle fait l'objet d'une publicité préalable sous la forme d'un avis d'appel public à la concurrence publié au Bulletin d'Annonces des Marchés publics voire au Bulletin Officiel Européen des Marchés publics et dans une revue spécialisée ou un quotidien d'annonces légales. Après réception des candidatures, il appartient à la commission des délégations de service public d'établir la liste des candidats admis à présenter une offre. Un document de consultation, présentant les caractéristiques du service délégable, est envoyé aux candidats retenus. Sur cette base, ils élaborent leurs offres dans un délai qui doit être adapté à la complexité du service à gérer ${ }^{451}$.

$447 C f$. Article 65 de la loi portant code de l'eau en vigueur au Congo précitée.

448 CE 15 Mai 1957 ALLARD Jin, AJDA 1957 p.254.

449 Article 22 code de l'eau du Togo précité.

450 Art. 14.

451 FOILLARD Ph., Droit administratif, Paradigme, 2011, p. 214. 


\section{B. Le contrôle de la délégation de service public}

Le contrôle porte essentiellement sur les résultats obtenus par les délégataires et sur le respect des normes techniques relatives aux installations d'approvisionnement en eau potable ${ }^{452}$. Il serait tout à fait paradoxal que la délégation des services publics de l'eau ne soit pas contrôlée, car le manque de contrôle ou même son insuffisance rendra incertaine l'effectivité du droit à l'eau. Dans cette optique, il faut analyser les mécanismes de contrôle, afin de s'assurer que le principe de la séparation des fonctions de contrôle et de régulation est garanti. Selon la Directive $\mathrm{n}^{\circ} 05 / 2005 / \mathrm{CM} / \mathrm{UEMOA}$ portant contrôle et régulation des marches publics et des délégations de service public dans l'Union Economique et Monétaire Ouest Africaine, les Etats devront s'engager à mettre en œuvre des procédures et mécanismes garantissant la séparation et l'indépendance des fonctions de contrôle et de régulation des marchés publics et des délégations de service public. En réalité on distingue deux contrôles majeurs.

\section{Le contrôle précédant la signature de la délégation}

En droit français, l'inobservation des différentes règles encadrant la passation des délégations de service public ${ }^{453}$ peut être sanctionnée d'un référé précontractuel formé avant que le contrat ne soit signé devant le président du tribunal administratif territorialement compétent. Ce dernier peut adopter des mesures provisoires, par exemple, injonction à l'auteur du manquement de se conformer à ses obligations, suspension de la passation du contrat ou de l'exécution des décisions s'y rapportant, mais il peut adopter aussi des mesures définitives comme l'annulation des décisions litigieuses ou la suspension de clauses devant figurer dans le contrat. Le recours est ouvert à toute personne ayant un intérêt à conclure la délégation de service public et susceptible d'être lésée par le manquement invoqué. Le juge doit se prononcer dans les vingt jours de sa saisine. Son ordonnance n'est susceptible que d'un recours en cassation dans les quinze jours, devant le Conseil d'Etat.

Aux termes de la Directive de l'UEMOA ${ }^{454}$ précitée, il importe également que les Etats de l'Afrique subsaharienne s'engagent à mettre en place des entités administratives centrales, déconcentrées, ainsi que décentralisées de contrôle des délégations de service public. Ces entités auront pour fonction notamment de contrôler l'application de la législation et de la réglementation sur les délégations du service public, sans préjudice de l'exercice des pouvoirs généraux de contrôle des autres organes de l'Etat. Elles devront émettre les avis et accorder les autorisations et dérogations nécessaires à la demande des autorités contractantes lorsqu'elles sont prévues par la réglementation en vigueur. Par ailleurs, ces entités devront, en plus d'assurer, en relation avec l'organe de régulation, la formation, l'information et le conseil de l'ensemble des acteurs de la commande publique sur la réglementation et les procédures applicables, elles contribueront, en relation avec l'organe de régulation, à la collecte d'informations et de

$452 C f$. Art. 18 de la loi n 2010-006 du 18 juin 2010 portant organisation des services publics de l'eau potable et de l'assainissement collectif des eaux usées domestiques, précitée.

453 CE, 24 octobre 2001, Collectivité territoriale de Corse.

454 Cf. Article 4. 
documents en vue de la constitution d'une banque de données. Manifestement, il s'agit des organes de contrôle concurrents, puisqu'il existe déjà des juridictions administratives susceptibles d'apprécier la légalité $a$ priori et a posteriori de la délégation de service public de l'eau.

Par ailleurs, il faut saluer les dispositions à caractère éthique contenues dans cette Directive. Sans doute, elles contribueront à dissuader de tout tripatouillage de la réglementation en vigueur en matière de délégation de service public. En effet, aux termes de l'article 6 de la Directive, il est prévu que les Etats africains subsahariens s'engagent à adopter les mesures en vertu desquelles, les agents de l'administration, et plus généralement, l'ensemble des personnes morales de droit public et de droit privé455, ainsi que toute personne intervenant, à quelque titre que ce soit, dans la chaîne de conclusion des délégations de service public, soit pour le compte d'une autorité contractante, soit pour le compte d'une autorité d'approbation, de contrôle ou de régulation soient soumis aux dispositions des réglementations communautaires et nationales interdisant les pratiques frauduleuses et les actes de corruption. Chaque Etat doit s'engager en outre à prendre des mesures visant à adopter et à faire respecter les codes d'éthique prohibant tout conflit d'intérêts dans la passation des marchés.

\section{Le contrôle de légalité du contrat de délégation de service public}

Il comporte un certain nombre de particularités. En droit français, le Préfet dispose du droit de déférer au tribunal administratif, alors même que cette procédure constitue un contentieux de l'excès de pouvoir qui normalement n'est ouvert qu'à l'encontre des actes unilatéraux. Lorsqu'il assortit sa demande d'une demande de suspension, dans les dix jours de la transmission obligatoire du contrat, ce dernier est automatiquement privé d'effet, jusqu'à ce que le juge ait statué sur la suspension sollicitée ou à défaut, pour un mois maximum.

Le délégataire a, dans le cadre de son contrat, une obligation de résultats. Il rend compte à l'autorité organisatrice en lui livrant son rapport annuel de gestion. La collectivité doit apporter un regard critique sur le rapport du délégataire tant sur les aspects techniques que sur les aspects comptables ainsi que sur le respect des clauses contractuelles qui peuvent avoir une incidence non négligeable sur le tarif du délégataire. Bon nombre de contrats récents assortissent d'un intéressement ou d'une pénalisation le respect de clauses techniques, par exemple, l'amélioration du rendement du réseau d'eau potable, ou le nombre de jours de respect de l'arrêté d'autorisation de rejet de la station d'épuration.

La collectivité peut, pour l'assister, faire appel à des conseils extérieurs spécialisés dans cette discipline à l'interface entre approche technique, approche environnementale, approche économique et sociale, au cœur du développement durable. Les moyens de contrôle dont dispose la collectivité sont multiples. Le contrôle d'origine sur le contrat est celui de la commission d'appel d'offres qui doit analyser les propositions élaborées par chacun des candidats avant d'en proposer la signature à la personne responsable, puis suivra, celui du préfet au titre de son contrôle de légalité. Les exemples de l'intervention du juge administratif dans ce contrôle de légalité des contrats de DSP sont nombreux. Ainsi, le titulaire d'un contrat d'affermage, condamné par la juridiction judiciaire à indemniser les usagers pour interruption de service

$455 C f$. les articles 4 et 5 de la Directive $\mathrm{N}^{\circ} 5 / 2005 / \mathrm{CM} / \mathrm{UEMOA}$ portant procédures de passation, d'exécution et de règlement des marchés publics et des délégations de services publics. 
public, peut demander réparation auprès du juge administratif lorsque la personne publique est tenue responsable de ce préjudice. L'étendue des réparations dues par celle-ci ne peut être déterminée en fonction de l'évaluation du juge judiciaire, dans la mesure où cette personne publique n'était pas partie dans ce litige, mais par le juge administratif456. De même, dans le cadre d'une délégation de service public pour la réalisation de travaux et l'exploitation d'un réseau urbain, en cas de litige, si le tribunal administratif ne dispose pas d'informations suffisantes relatives aux compétences de la communauté d'agglomération délégante, il ordonne une expertise ${ }^{457}$. Le conseil d'État annule les décisions du préfet (Le préfet du Nord a refusé à la commune de Mons-en-Barœul, l'attribution du fonds de compensation pour la TVA qu'elle sollicitait, au titre de dépenses d'investissement correspondant à des travaux réalisés sur son réseau communal de télédistribution par câble) en relevant que les travaux menés par la commune avaient pour objet d'élargir les services télévisés d'information et de divertissement proposés à l'ensemble des habitants de la commune et, que si le réseau ainsi aménagé avait été utilisé par une société privée chargée par convention avec la commune de commercialiser certains des services proposés, cet usage était resté très accessoire pour les années en cause ${ }^{458}$.

L'importance des contrôles auxquels sont soumises les conventions de gestion déléguée témoigne du souci de protéger les intérêts des usagers à travers ceux de la collectivité publique concédante. De plus, il est normal que le concessionnaire soit confronté à des contrepouvoirs parce qu'il est généralement en situation d'exclusivité et parce qu'il est délégataire de prérogatives de puissance publique. L'un des objectifs de la loi Sapin qui l'a amendée a été d'accroître la transparence vis-à-vis de la collectivité concédante et des usagers du service délégué. Le contrôle revêt un double aspect : interne à la convention, d'une part, et externe, d'autre part. Le contrôle interne est celui du concédant. Selon un adage classique, «le concessionnaire gère, l'administration contrôle ». L'autorité concédante est comptable vis-à-vis des usagers du bon fonctionnement du service. La collectivité concédante a le droit de contrôler les comptes du concessionnaire. En France, les plus petites collectivités éprouvent quelque difficulté à faire le poids face à de puissants cocontractants. Elles peuvent trouver de l'assistance auprès d'organismes indépendants mais elles estiment que des progrès restent à faire pour mieux équilibrer les rapports de force contractuels, notamment au stade du contrôle.

Les tiers, s'ils peuvent classiquement demander l'annulation d'actes détachables de la convention de délégation de service public peuvent faire directement de même à l'égard des clauses réglementaire y figurant ${ }^{459}$. Le juge administratif français, confronte, lors de son contrôle, l'acte contractuel aux dispositions du droit français comme communautaire de la concurrence ${ }^{460}$. Dans le but d'éviter la déqualification de nombreux contrats de délégation de service public, le juge se réfère souvent à la notion de risque d'exploitation pour vérifier si le critère financier est rempli. Il s'agit de déterminer si l'entreprise assume un risque réel dans l'exploitation du service public. Ce risque qui pourra consister soit dans le fait de réaliser des pertes soit, à tout le moins, dans celui de ne pas réaliser de bénéfices. Peu importe, dès lors, que la rémunération soit perçue directement auprès des usagers ou qu'elle découle d'un payement versé directement par la personne publique. L'essentiel est que le montant total de cette

456 Conseil d'Etat, 5 juin 2009, n²95837, Commune de Richardmenil.

457 TA Chalons-en-Champagne, 30 novembre 2006, n0601311, Société Kéolis.

458 Conseil d'Etat, 24 mai 2006, n²74810, Ministère de l'Intérieur c/ Commune de Mons-en-Baræul.

459 Cf. CE, 10 juillet 1996, Cayzeele, précité

460 CE, sect., 3 novembre 1997, Sté Million et Marais, RD publ. 1998, page 260. 
rémunération soit fonction du comportement des usagers, et ce dans les proportions suffisamment importantes pour que l'on puisse considérer qu'un risque pèse véritablement sur l'exploitant. Le Conseil d'Etat en a décidé ainsi lorsqu'une partie variable de la rémunération versée par la commune pour l'exploitation d'un service de traitement de l'eau est fonction de la quantité d'eau consommée par les usagers ${ }^{461}$. De même, commet une erreur de droit le juge du fond qui ne prend en compte pour vérifier si le critère financier de la délégation est satisfait, que le paiement des usagers et non ceux effectués par le département alors que cette rémunération varie avec le nombre d'usagers ${ }^{462}$. En revanche, le critère financier n'est pas rempli, alors même que le cocontractant de la personne publique facture directement le prix des repas d'un centre aéré sur les usagers, dans la mesure où la commune prend en charge la différence entre le prix payé par les usagers et le prix réel et que le nombre d'usagers du service est insusceptible de varier substantiellement d'une année sur l'autre. Dans cette hypothèse, il n'est guère pas possible de considérer qu'un réel risque d'exploitation pèse sur le cocontractant de l'administration ${ }^{463}$.

\section{§ 2. Les sanctions de l'inobservation du contenu des cahiers}

Les sanctions de l'inobservation du contenu des cahiers des charges sont multiples. Elles vont de la déchéance qui peut s'analyser soit en résiliation juridictionnelle soit en résiliation unilatérale de l'administration fondée sur des textes législatifs, et enfin aux sanctions pénales et pécuniaires. Nous allons nous intéresser essentiellement à la déchéance (A) avant d'examiner les autres cas de sanction (B).

\section{A. La déchéance}

Elle constitue l'une des sanctions les plus infligées aux cocontractants de la collectivité publique en cas d'inobservation des clauses de la convention de délégation de service public. Elle a été recommandée par le législateur dans le cadre des sanctions correspondant au non respect des dispositions qui fondent les délégations du service public de l'eau.

\section{La signification de la notion}

C'est le retrait d'un droit, soit à titre de sanction soit en raison du non respect de ses conditions d'exercice. C'est dans cette logique que le législateur togolais affirme que la déchéance de la concession peut être prononcée pour utilisation des eaux à des fins autres de

\footnotetext{
461 CE, 28 juin 2006, Requête $n^{\circ} 288459$, Syndicat intercommunal d'alimentation en eau de la moyenne vallée du Giers.

462 CE, 7 novembre 2008, Requête n² 291794, Département de la Vendée.

463 CE, 5 juin 2009, Requête n²98641, Société Avenance.
} 
celles autorisées ou hors de la zone d'utilisation fixée, pour non-paiement des redevances aux termes fixés, pour non utilisation des eaux concédées dans les délais fixés dans le cahier des charges, non respect des obligations à caractère sanitaire et en général, non respect des conditions précisées par la concession ${ }^{464}$. En cas de déchéance de la concession, le ministre chargé de l'eau peut ordonner la remise des lieux en l'état initial et, le cas échéant, faire effectuer d'office cette remise en l'état aux frais du concessionnaire déchu.

Dans son arrêt Syndicat intercommunal Guzet-Neige ${ }^{465}$, le Conseil d'Etat apporte une précision intéressante concernant le régime de la déchéance, qui constitue la sanction la plus sévère à l'encontre du délégataire du service public. Cette mesure, également appelée résiliation aux torts du contractants, ne doit en effet intervenir que lorsque les sanctions pécuniaires et coercitives se révèlent véritablement insuffisantes pour assurer l'exécution du contrat. Elle ne peut donc être qu'une sanction exceptionnelle justifiée par une faute d'une particulière gravité. Or, si cette sanction existe même dans le silence du contrat, les clauses de ce dernier peuvent revêtir une importance cruciale. En effet, alors qu'en principe seul le juge détient le pouvoir de prononcer une déchéance, les documents contractuels peuvent stipuler valablement que l'administration pourra elle-même appliquer cette mesure résolutoire ${ }^{466}$.

Par contre, la révocation ou modification de la concession dans le cas où les eaux concédées doivent faire l'objet d'une autre utilisation dans l'intérêt public, conformément aux dispositions d'un schéma directeur d'aménagement et de gestion des eaux, donne lieu à indemnité correspondant à la valeur du préjudice subi.

\section{L'illustration jurisprudentielle}

Les parties au contrat peuvent subordonner la possibilité de prononcer la déchéance du concessionnaire à la condition du respect par le concédant de ses engagements. Si le juge administratif est saisi d'une telle demande de déchéance, il lui appartient de vérifier si la condition retenue par les parties était ou non remplie.

Dans l'affaire SIGN ${ }^{467}$ du 19 octobre 2001, le Conseil d'Etat a refusé de prononcer la déchéance du concessionnaire car cette condition n'était pas remplie. Selon le Conseil d'Etat, «contrairement à ce que soutient le syndicat requérant, les parties au contrat de concession n'ont méconnu aucune règle d'ordre public en subordonnant ainsi la possibilité de prononcer la déchéance du concessionnaire à la condition du respect par le concédant des engagements qu'il avait par ailleurs souscrits ; qu'il appartenait au juge du contrat de concession, saisi d'une demande tendant à ce qu'il prononçât la déchéance, de vérifier si cette condition était remplie ».

Il faut souligner qu'il existe une différence entre la déchéance sanction forte qui prive le concessionnaire d'indemnité et le pouvoir de résiliation unilatérale pour motif d'intérêt général

\footnotetext{
464 Article 25 du code de l'eau précité.

465 Cf. CE, 19 octobre 2001, Requête n²212677

466 Cf. CAA Versailles, 7 mars 2006, contentieux nº4VE01381 publié au Recueil Lebon, Commune Draveil c/ Sté Via Net Works.

467 Conseil d'Etat, 19 octobre 2001, n² 212677, SYNDICAT INTERCOMMUNAL DE GUZET-NEIGE.
} 
impliquant l'indemnisation du cocontractant. S'agissant du pouvoir de résiliation unilatérale pour un motif d'intérêt général, toute clause niant ou limitant ce pouvoir est réputée nulle ${ }^{468}$.

Il est de jurisprudence constante que le contrat peut prévoir les conditions de forme de la déchéance (respect de droits de la défense, mise en demeure préalable, pour une hypothèse de mise en demeure caduque, ${ }^{469}$. Mais l'arrêt SIGN est intéressant dans la mesure où il juge également légales les stipulations contractuelles fixant des conditions de fond au prononcé de la déchéance.

En l'espèce, un protocole d'accord annexé à un avenant de 1990 à un contrat de concession de remontées mécaniques conclu en 1969 subordonnait la déchéance au fait que le concédant n'ait pas respecté et exécuté les obligations imposées au protocole (en l'occurrence des obligations financières). Les parties contractantes n'ont donc méconnu aucune règle d'ordre public en limitant contractuellement le pouvoir du concédant de prononcer la déchéance du concessionnaire.

Dans un premier temps, le tribunal administratif de Toulouse a prononcé la déchéance du concessionnaire à la demande du syndicat intercommunal. Dans un second temps, le Conseil d'Etat a confirmé l'arrêt de la Cour administrative d'appel de Bordeaux annulant le jugement du tribunal administratif, sur la base du raisonnement précédemment exposé.

\section{B. Les autres sanctions}

Elles sont nombreuses, mais on peut distinguer d'une part les sanctions pécuniaires et d'autre part les sanctions non pécuniaires.

\section{Les sanctions pécuniaires}

Les inobservations du contenu des cahiers des charges indexés aux contrats de délégations ont amené le législateur à envisager des sanctions à caractère civil et pénal. La préoccupation est de dissuader les délégataires contre toute tentation de réduire la qualité de l'eau distribuée ou dégrader les ressources en eau. Certaines dispositions ont vocation à réparer les dommages causés par le délégataire, et d'autres ont une vocation exemplaire.

Au Sénégal, le législateur a prévu que toute personne qui aura volontairement ou indûment utilisé des eaux destinées à l'irrigation par nature ou par des dispositions réglementaires sera punie d'un emprisonnement de deux mois et d'une amende de 20.000 francs à 2.000.000 de francs ou de l'une de ces deux peines seulement ${ }^{470}$. D'autre part, toute personne qui aura

\footnotetext{
468 CE, 6 mai 1985, Association Eurolat, Rec. p. 141

469 Cf. CE, 8 février 1999, Ville de Montélimar, Actualité juridique n5, pp. 52 et 54

470 Cf. Article 100 du code de l'eau.
} 
introduit des matières susceptibles de nuire à la salubrité de toute eau, ou abandonné des objets, des corps putréfiables dans les anfractuosités naturelles ou artificielles sera punie d'un emprisonnement de deux mois à deux ans et d'une amende de 20.000 francs à 2.000 .000 de francs ou de l'une de ces peines seulement ${ }^{471}$.

Au Congo, le fait de capter à titre habituel des eaux du domaine public hydraulique sans être bénéficiaire sur ce site des droits de captage ou d'en capter des volumes excédant ceux sur lesquels portent les droits de captage est puni d'une peine d'amende de 250000 francs CFA et d'un emprisonnement de 2 à 6 mois, ou de l'une de ces deux peines seulement ${ }^{472}$. Le fait de déverser et de déposer sur le sol ou d'enfouir dans l'eau, ou dans le sous-sol, des substances polluantes au sens de l'article 20 est puni d'une peine d'amende de 50000 à 5000000 francs CFA et d'un emprisonnement de 2 mois à 1 an, ou de l'une de ces deux peines seulement. S'il entre en voie de condamnation, le tribunal ordonnera en outre la confiscation des substances polluantes ${ }^{473}$.

De la même façon, le législateur togolais a prévu que les infractions au code de l'eau et aux dispositions prises pour son application sont constatées par des procès-verbaux établis en quatre exemplaires par les agents assermentés transmis au procureur de la République, à la hiérarchie de l'instrumentaire, au ministre chargé de l'eau et notifiés au délinquant ${ }^{474}$. Selon l'article $158 \mathrm{du}$ même code, le procès verbal de constatation doit comporter notamment l'identité des personnes impliquées, les circonstances de l'infraction, les explications des auteurs présumés et des témoins éventuels ainsi que les éléments faisant ressortir la matérialité des faits et leur imputabilité aux auteurs présumés.

\section{Les sanctions non pécuniaires}

Elles sont de plusieurs ordres. La mise en régie consiste à exécuter tout ou partie du marché avec le matériel et le personnel du titulaire, à ses frais et risques ${ }^{475}$. Elle ne met pas un terme aux relations contractuelles, le contrat n'est même pas suspendu. L'entreprise reste titulaire du marché.

La résiliation du marché consiste à mettre fin au marché pour l'avenir. La résiliation peut être simple : les conséquences de la résiliation sont supportées par l'administration. En effet, aux frais et risques du titulaire : l'administration résilie le marché pour confier les mêmes prestations à une autre entreprise. Dans ce cas, le titulaire écarté supporte les conséquences financières de la passation de ce marché de remplacement. L'administration doit préciser dans sa décision de résiliation qu'il s'agit d'une résiliation aux frais et risques du titulaire, à défaut elle sera considérée comme pure et simple.

$471 C f$. Article 101 du code de l'eau.

472 Cf. Article 89 du code de l'eau.

$473 C f$. Article 91 du code de l'eau.

474 Cf. Article 157

475 CCAG-Travaux, art 49-1 
L'administration a toujours le droit de résilier un contrat pour faute, après mise en demeure, même si le contrat ne le prévoit pas ${ }^{476}$. Toutefois, les parties doivent être liées par un contrat. De même, les sanctions ne pourront donc plus être prononcées après la réception des travaux ${ }^{477}$.

Le contrat peut subordonner l'application des sanctions à certaines conditions. En effet, il a été jugé récemment que le contrat pouvait prévoir que les sanctions ne seraient pas applicables lorsque l'administration n'a pas exécuté ses propres obligations ${ }^{478}$.

Il faut ajouter que la mise en œuvre des sanctions est conditionnée par 1'exigence d'une faute, caractérisée par la violation d'une clause du contrat ou d'un manquement à une obligation de résultat. Aussi, la faute doit être suffisamment grave. Le juge contrôle l'existence de la faute et sa gravité. Enfin, la faute doit être imputable au cocontractant. Comme exemples de faute, on peut citer les fausses déclarations, la sous-traitance occulte, l'abandon du chantier, retards «considérables» dans l'exécution des prestations ${ }^{479}$, les malfaçons importantes ou si l'entrepreneur tarde à y remédier ${ }^{480}$.

476 CE 30 septembre 1983, requête n 26611, SARL COMEXP., Rec. CE p. 393.

477 CE 31 mars 1954, requête $n^{\circ}$ 9880, SA Entreprise industrielle de travaux publics.

478 CE 19 octobre 2001, Syndicat intercommunal de Guzet-Neige, RFDA 2001, p. 1314

479 CE, 16 octobre 1978, Gotteland, RDP 1980 p. 1484.

480 CE 5 mai 1958, Association syndicale autorisée pour l'assainissement des marais de La Fosse, Lebon p. 260 
Chapitre II.

LA MISE EN BERNE INELUCTABLE DES PRINCIPES DU SERVICE PUBLIC 
Le souvenir du célèbre arrêt $\mathrm{SCOA}^{481}$ consacrant la gestion privée du service public nous serait d'une grande utilité dans notre démarche. Car comme l'affirme Le BERRE C., "la rencontre de l'économie de marché et du service public est marquée du sceau de la contrainte et de l'épreuve » ${ }^{482}$. Une affirmation suffisante pour que d'aucuns s'exclament : "L'idée de service public est-elle encore soutenable? $»^{483}$. En effet, l'Administration confie souvent la gestion d'un service public à une personne privée, morale ou physique. Elle recourt fréquemment à cette stratégie pour remettre contractuellement la gestion d'un service public ou faire exécuter un travail particulier avec lequel une convention de concession a été conclue. Malheureusement, le procédé contribue couramment à des déviations favorisées par l'absence de transparence et l'insuffisance des contrôles ${ }^{484}$.

Nous aurions pu intituler ce chapitre non pas la mise en berne des principes du service public, mais plutôt le maintien ou le prolongement de la mise en berne des lois du service public. Car, en réalité, la privatisation opérée à partir des années 1980 n'est pas à l'origine des « galères » des services publics en Afrique subsaharienne, on y notait bien avant le début de la vague de libéralisation, un dysfonctionnement chronique. Donc, la libéralisation n'a donc fait que contribuer à aggraver une situation déjà en déliquescence, la posture des lois de Rolland était déjà peu enviable. Devenues gestionnaires des services publics de l'eau à la suite de la libéralisation, les multinationales devraient en principes respecter les lois de Rolland en participant à la « continuité essentielle à la vie nationale $»^{485}$.

Nous montrerons que la gestion privée du service de l'eau est peu compatible avec les lois du service public (Section I) et ne favorise pas l'émergence des nouvelles lois du service public (Section II).

\section{Section I. La gestion privée du service public de l'eau, une gestion peu conciliable avec les lois de Rolland}

Il n'est pas abusif de constater avec le Doyen CHAPUS que "les activités de plus grand profit ne sont pas des services publics $»^{486}$. A contrario, l'on peut s'interroger sur la situation des activités de moindre profit. Sont-elles de la catégorie des services publics ? Au-delà de la controverse, l'on peut déjà affirmer que le service de distribution de l'eau n'est pas une affaire de moindre profit, pour preuve, ce ne sont pas de «petites épiceries» qui s'agitent autour du marché de l'eau. Il s'agit des multinationales dont les gains sont "principalement au moins, destinés à être utilisés par les entreprises elles-mêmes en vue d'étendre ou consolider les bases

481 Cf., T. confl. 22 janv. 1921, Lebon 91.

482 Le BERRE C., « La logique économique dans la définition du service public », RFDA 2008, p.50.

483 CHEVALIER J.-M. (dir.), L’idée de service public est-elle encore soutenable ?, Paris, PUF, 1999.

484 LINOTTE D. et ROMI R., Services publics et droit économique, $5^{\text {ème }}$ édition, Paris, Litec, 2003, p. 315.

485 Cf., CE 7 août 1909, Arrêt Winkell, Rec., p. 826.

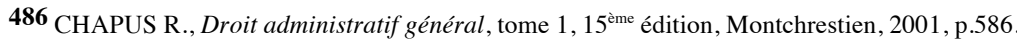


de leur prospérité $»^{487}$. Redoutant les excès pouvant résulter de la privatisation, CHEVALLIER J. souligne que « dès l'instant où des capitaux privés entrent dans une entreprise, une contrainte nouvelle apparaîtrait, celle de la rentabilité financière $»^{488 .}$

L'on a beau prévoir des cahiers de charges pour obliger les opérateurs auxquels l'Etat concède le service public de l'eau ${ }^{489}$ à respecter les obligations essentielles inhérentes au service public, la réalité est que l'Etat n'a pas toujours les moyens de contrôle du respect des obligations contenues dans ces cahiers. Aussi, doit-on s'interroger sur la teneur même de ces cahiers de charges. Les obligations qui y sont souvent consignées répondent-elles au respect des principes qui sous tendent le fonctionnement des services publics? L'eau constitue un " bien essentiel », et de plus, à la différence de l'électricité, c'est un « don du ciel », ce qui rendrait inacceptable sa « marchandisation » et donc l'intervention des firmes privées. Ces critiques oublient que le développement des infrastructures en eau potable depuis le XIX ${ }^{\text {ème }}$ siècle n'a, en grande partie, été rendu possible que par la concession et que la gestion déléguée du service public n'implique pas l'abandon du service public ${ }^{490}$. Malheureusement, le gestionnaire privé est enclin à négligé les lois du service public $(\S 1)$ et le contexte économique international entretient cette fâcheuse tendance $(\S 2)$.

\section{§ 1. Le gestionnaire privé peu préoccupé par les lois du service public}

Les services publics quelle que soit leur nature (SPA ou SPIC) sont soumis, de manière générale, aux mêmes règles communes ; ce sont les lois du service public. Ces lois du service public trouvent l'essentiel de leur fondement dans la jurisprudence du Conseil d'Etat. A partir des décisions jurisprudentielles, une systématisation doctrinale a été opérée par le Professeur Louis ROLLAND au cours des années $1930^{491}$.

Aussi, en s'appropriant les explications que le président LATOURNERIE donnait du service public, dans ses conclusions sur l'arrêt Etablissements Vezia : "le service public est celui dont l'exécution est regardée par le législateur comme assez important à l'intérêt public pour être assuré par l'ensemble des procédures du droit public, du moins celles qui sont nécessaires pour atteindre le but de ce service ", l'on admettrait pas la moindre dérogation au respect des lois du

487 Idem.

488 CHEVALLIER J., «La nouvelle réforme des télécommunications : ruptures et continuité », RFDA, 1996, p. 941

${ }^{489}$ Art. 44 alinéa 2 du code de l'eau de la République du Mali : « ... service public est délégué à des exploitants dans le cadre de Délégation de gestion de service public délivré dans les conditions prévues par la présente loi». Article 45 : «L'exercice du service public de l'eau, ainsi que le développement et / ou la gestion et la maintenance des installations d'eau sont déléguées à des exploitants avec obligations de service public définies par la Convention de Délégation de Gestion à laquelle est annexé un cahier de charges ».

490 RICHER L., « Les services de l'eau potable et de l'assainissement dans la loi sur l'eau. La loi à la traîne de la jurisprudence », AJDA 2007 p. 1168.

491 Cf. COMBEAU P., Cours de droit public économique, Site de l’Université Numérique Juridique Francophone 
service public ${ }^{492}$. Dans ses réflexions, Pierre SANDEVOIR, s'inquiétait « que le service public industriel et commercial est à lui seul une authentique contradiction. Par l'utilisation de cette notion, il est demandé à un organisme quelconque [...] de protéger l'intérêt général tout en agissant selon les méthodes du secteur privé. A cet organisme, il faut donc réaliser ouvre altruiste avec des moyens égoïstes, il faut se révéler puissance publique sous une allure de simple citoyen ${ }^{493}$. De même, Jean RIVERO notait que la poursuite simultanée de la satisfaction d'un besoin d'intérêt général et de la rentabilité, voire du profit, est le problème essentiel posé par la notion de service public industriel et commercial ${ }^{494}$.

D'autre part, selon l'Acte uniforme relatif au droit des Sociétés Commerciales et du Groupement d'Intérêt Economique 495 en vigueur dans la plupart des Etats de l'Afrique subsaharienne, la société commerciale est créé par deux ou plusieurs personnes qui conviennent, par un contrat, d'affecter à une activité des biens en numéraire ou en nature, dans le but de partager le bénéfice ou de profiter de l'économie qui pourra en résulter. Les associés s'engagent à contribuer aux pertes dans les conditions prévues par l'Acte uniforme. "La société commerciale doit être créée dans l'intérêt commun des associés ». Ceci renforce l'opinion selon laquelle, la société libérale et la croissance qu'elle entraîne par leur nature même, créent des tensions économiques et sociales. Elle suscite l'inégalité qu'il ne s'agit pas de nier. Toutefois, pour ceux qui en ont réellement besoin, " le devoir de solidarité implique qu'à titre individuel ou collectif, soit apportée une réponse tout en laissant la société fonctionner naturellement ${ }^{496}$.

Sans aucun doute, la commercialité a des répercussions importantes sur le régime du service (public de l'eau). Les décisions sur le prix restent dans l'ensemble contenues dans des actes administratifs, mais ils s'inspirent de calculs économiques et constituent le pivot du système de gestion. On trouve là les transports urbains, les services d'eau et d'assainissement, le chauffage urbain... ${ }^{497}$.

Un commerçant privé cherche à satisfaire une multitude d'intérêts particuliers, et se trouve pris dans une relation individuelle avec chacun de ses clients, qu'il voit tous différemment. Le service public, lui, cherche à satisfaire un intérêt général unique, et se trouve pris dans une relation globale avec la collectivité abstraite des usagers, qu'il voit tous identiquement. Pour le commerçant, tous les clients sont concrets et différents ${ }^{498}$ et pour le service public, tous les usagers sont abstraits et identiques ${ }^{499}$. Il est évident que la recherche de l'intérêt commun des associés n'est point favorable à la réalisation de l'intérêt général dont les «principes » dits de

492 VEROT C., «L'utilité sociale sans le service public : à propos de la mission assurée par le gestionnaire privé d'un centre d'aide par le travail », Revue de droit sanitaire et social 2007, p. 499.

493 SANDEVOIR P., «Les vicissitudes de la notion de service public industriel et commercial», Mélanges STASSINOPOULOS, p. 317

494 RIVERO J., « Les deux finalités du service public industriel et commercial », CJEG, 1994

495 Acte adopté le 17 avril 1997 et paru au JO OHADA nº 2 du $1^{\text {er }}$ octobre 1997

496 SORMAN G., La solution libérale, Fayard, 1984, p.270

497 ENCOUA D., FLOCHEL L., « La tarification : du monopole à la concurrence régulée », AJDA 1997, p. 254.

498 Il y a autant de relations commerciales différentes qu'il y a de clients.

499 QUIRINY B., « Les droits de l'usager face au droit du marché », RFDA 2008 p. 20. 
Rolland garantissent l'effectivité. Nous montrerons que le secteur privé est peu soucieux du principe d'égalité (A) puis du principe de continuité (B).

\section{A. Le secteur privé peu soucieux de l'effectivité du principe d'égalité}

La notion d'égalité est une notion qui oscille, son sens varie en fonction du contexte dans lequel elle est utilisée. Nous la réduirons à l'égalité d'accès au service public et à l'égalité dans le fonctionnement du service public. La logique égalitariste qui doit fonder les politiques de l'eau est basée sur une conception socialiste de l'Etat. Cette conception conduit à justifier les interventions de l'autorité publique dans des domaines variés pour des raisons qui tiennent à la préservation ou à la garantie de l'intérêt général. L'autorité publique doit mettre en œuvre tout ce qui est possible pour limiter les iniquités, mais également pour identifier et repérer la nature des besoins de la population ${ }^{500}$.

L'Etat est donc l'autorité compétente pour définir le contenu des obligations de service public imposées aux entreprises privatisées et par ce biais, il détermine également quelles sont les activités effectuées dans un but d'intérêt général. Pour respecter les principes propres au service public dégagés par ROLLAND et plus précisément le principe d'égalité d'accès au service public, la détermination au niveau national des obligations de service public indispensable pour éviter la création de disparités entre les usagers ${ }^{501}$.

La vitalité de la question de l'accès à l'eau devait normalement pousser les concessionnaires du service public de l'eau à tenir compte de la situation financière de chaque citoyen dans le cadre de leur politique de tarification. En règle générale les usagers du service public bénéficient de tarification différenciée lorsque l'intérêt général le recommande ${ }^{502}$. A fortiori, les concessionnaires devraient s'y atteler surtout si l'on se réfère aux cahiers de charges inhérents à leur contrat. Aussi, les "nécessités d'intérêt général» doivent-elles être, selon la formule jurisprudentielle, " en rapport avec les conditions d'exploitation du service » ou avec "l'objet du service $»^{503}$.

500 TIZIO S., «Entre Etat et marché. Une nouvelle régulation sanitaire pour les pays en développement ? TiersMonde, Année 2004, Volume 45, Numéro 179, p. 643 - 663.

501 BIRRAUX D., Service public et entreprises privatisées, Université Pierre Mendès France, Mémoire Master II, 2006, p. 41.

$\mathbf{5 0 2}$ En France, la discrimination fondée sur des critères de ressources des familles a suscité une jurisprudence controversée, lorsqu'il a été jugé, à propos d'une école de musique : «Les différences de revenus entre les familles des élèves n'étaient pas constitutives, en ce qui concerne l'accès au service public, de différences de situation justifiant des exceptions au principe d'égalité qui régit cet accès ; [...] compte tenu de l'objet du service et de son mode de financement, il n'existait aucune nécessité d'intérêt général justifiant pour la fixation des droits d'inscription, une discrimination fondée sur les seules différences de ressources entre ces usagers.. » CE Sect., 26 avril 1985, Ville de Tarbes;

503 Cf., CE Sect., 19 décembre 1979, Meyet; CE Sect., 10 mai 1974, Denoyez et Chorques, préc. 


\section{Le principe d'égalité difficile à appliquer}

Déjà dans $l^{\prime} E$ thique à Nicomaque ${ }^{504}$, ARISTOTE envisage les deux types d'égalité possibles. L'une, absolue ou arithmétique, suppose un rapport de réciprocité et tend vers une égalité complète de la prestation et de la contrepartie. Déterminante pour la justice commutative ${ }^{505}$, cette égalité simple traite tous les individus comme schématiquement égaux. L'autre, proportionnelle ou géométrique, correspond à la justice distributive. Elle définit un traitement différent selon les personnes dans la répartition des biens et des charges. Chaque individu dans une communauté est récompensé ou grevé de charges en fonction de ses mérites.

L'égalité et la justice résident dans la juste proportion. Relative, l'égalité suppose une certaine dose d'incertitude juridique. Si le principe d'égalité suppose « une vocation à participer à la répartition des droits ${ }^{506} »$, il ne l'assure pas. C'est un principe dynamique qui offre de multiples possibilités d'interprétation divergentes ${ }^{507}$. Des lois sont donc nécessaires pour traduire cette égalité de tous dans le contenu même des droits, pour préciser les modalités de cette répartition. En réalité, il s'agit d'un principe contesté. Face à une société de plus en plus complexe, à la pluralité des définitions « du juste » qu'elle propose et aux inégalités des chances qu'elle sécrète, le principe d'égalité est remis en cause par l'émergence de l'équité 508 .

Incontestablement, l'équité est le soubassement commun des systèmes juridiques et, dans une démocratie, on ne saurait choisir des lois qui ne seraient pas équitables ${ }^{509}$, tout comme l'on ne saurait instituer un service public inéquitable. Tout le droit n'est qu'une déclinaison de l'équité, notion abstraite qui le domine et l'imprègne tout entier. Concept téléologique et non écrit, l'équité s'opposerait à l'égalité qui ne relèverait pas de l'ordre des fins poursuivies par la société mais de celui des moyens. Cette différenciation n'est pas aussi évidente, à la fois parce que l'égalité emporte une vision substantielle de la société, mais aussi parce que l'équité tend à se concrétiser dans des applications immédiates. Ces rapports entre égalité et équité ont, de tout temps, fait l'objet de réflexions juridiques ou philosophiques ${ }^{510}$.

Somme toute, il est existe une différence entre les activités privées et les services publics. Les premières observent souvent mieux que les seconds la continuité et l'adaptation continue, et même l'égalité. Mais elles ne le font qu'envers une clientèle solvable qu'elles peuvent sélectionner, ce qui est aux antipodes de l'égalité devant le service public. Les services publics, en effet, doivent respecter, et même promouvoir l'égalité ; les entreprises privées peuvent la

504 Voir, ARISTOTE, Ethique à Nicomaque, livre V, chap. 5-7

$\mathbf{5 0 5}$ Concept philosophique signifiant l'échange de droits et devoirs fond\& sur l'égalité des personnes par opposition à la justice distributive.

506 PELISSIER G., Le principe d'égalité en droit public, coll. Systèmes, LGDJ, 1996.

$\mathbf{5 0 7}$ BELLOUBET-FRIER N., « Le principe d'égalité », AJDA 1998, p. 152.

$\mathbf{5 0 8}$ Rapport du Conseil d'Etat, EDCE 1996, p. 13 et ss.

509 BRAIBANT G., « Nouvelles réflexions sur les rapports du droit et de l'équité », Rev. fr. adm. publ. 1992, p. 687.

510 Idem. 
pratiquer, mais c'est pour elles un choix de gestion, pas une obligation ni un objectif. En pratique cependant la différence de comportement est parfois si ténue que la distinction devient négligeable ${ }^{511}$.

Or, le «développement humain $»^{512}$, notamment celui dans les Etats de l'Afrique subsaharienne, repose sur la notion d'équité et d'égalité, de répartition équitable des bénéfices de la croissance, de l'égalité des chances et des opportunités pour tous, sans distinction de sexe, d'origine ou de religion. Reprenant le président BOUFFANDEAU, cité par Maxime LETOURNEUR ${ }^{513}$, nous dirons que: "les principes généraux du droit sont une cuvre constructive de la jurisprudence, réalisée pour des motifs supérieurs d'équité, afin d'assurer la sauvegarde des droits individuels des citoyens ». Ce que PORTALIS avait déjà exprimé en affirmant que: "l'équité est le retour à la loi naturelle dans le silence, l'opposition ou l'obscurité des lois positives ».

Concrètement, l'eau courante atteint plus d'habitants des zones urbaines africaines que n'importe quel autre type d'alimentation en eau - même si sa quote-part est moindre qu'au début des années 1990. Les plus récentes données disponibles sur 32 pays dans la base de données AICD ${ }^{514}$ suggèrent qu'en Afrique subsaharienne $39 \%$ de la population urbaine est raccordée à un réseau de distribution d'eau courante, par rapport à $50 \%$ au début des années 1990. Les bornes-fontaines publiques, également alimentées par les services publics, viennent en seconde position, desservant $24 \%$ de la population.

L'accès à l'eau potable dans les centres urbains et ruraux du Burkina Faso est globalement de $60,2 \%$... Conscient de cette situation, le gouvernement burkinabè, s'est doté d'un Plan National Eau Potable et Assainissement qui prévoit de relever les défis prescrits dans les Objectifs du Millénaire pour le Développement dont il est signataire. Ce plan prévoit d'augmenter d'ici 2015 le taux d'accès à l'eau à un seuil de $80,1 \%$ et le taux de couverture des populations par un système d'assainissement adéquat à $50,4 \%$ à la même échéance ${ }^{515}$.

L'analyse montre que la majorité de ceux qui n'ont pas accès aux services publics des eaux vivent trop loin du réseau de distribution, même si certains ne sont pas raccordés tout en en étant proche. Aujourd'hui dans les agglomérations urbaines africaines, les ménages s'approvisionnent surtout auprès d'un ensemble de sources souterraines privées. Cette catégorie comprend les familles utilisant une source souterraine privée située dans les limites de leur terrain. Même si aucune loi n'est a priori contrevenue, ce mode d'approvisionnement est informel puisqu'aucun contrôle systématique n'existe quant à la disponibilité, la pérennité et la qualité de la ressource.

511 TRUCHET D., « Unité et diversité des grands principes du service public », AJDA 1997 p. 38

512 Concept d'origine onusienne, Il sous-entend un équilibre entre la croissance économique et la croissance démographique et une répartition ciblée des ressources visant à améliorer en priorité les conditions de vie des couches de population les plus démunies et les plus vulnérables.

513 LETOURNEUR M., « Les principes généraux du droit », EDCE, 1951.

514 Africa Infrastructure Country Diagnostic- Cette base de données, qui inclut des enquêtes menées de 1990 à 2006, incorpore 32 pays dont 24 ont plus de deux points temporels permettant une analyse des tendances. On remarque un large chevauchement entre ces 32 pays et les 24 pays ciblés par les Diagnostics des infrastructures nationales en Afrique.

515 WETHE J., Rapport : Systèmes énergétiques : Vulnérabilité - Adaptation - Résilience, HELIO International, 2009, p. 12. 
En l'absence de règles explicites concernant l'accès à ces points d'eau, les vendeurs et les usagers s'accordent sur des règles qui leur sont propres, des règles qui créent des exclus et qui méprisent les principes classiques du service public.

Les inégalités s'intensifient dans les zones rurales, puisque les entreprises privées ne s'y installent guère, préférant la clientèle à faible risque constituée par les riches habitants des villes et laissant au secteur public altéré la tâche de s'occuper du reste de la population ${ }^{516}$.

Finalement, la plupart des citadins qui ne sont pas desservis par une entreprise de services publics ont recours à des puits et forages qui sont la principale source d'alimentation de $24 \%$ de la population urbaine en Afrique ${ }^{517}$.

L'on pourrait affirmer qu'en misant sur les grandes firmes internationales, on a admis que les services d'eau des villes grandes et moyennes pouvaient être déconnectés de ceux du reste du pays. Or, dans des pays faiblement urbanisés, cela revient à accepter de concentrer les moyens, la réflexion, l'expertise sur une minorité d'habitants. C'est aussi entretenir des illusions coûteuses comme au Mali, où la privatisation d'EDM (Mali) puis la résolution des conflits ont mobilisé de manière disproportionnée les dirigeants maliens et la communauté des bailleurs de fonds pour une entreprise qui fournit de l'eau à moins de $10 \%$ de la population malienne ${ }^{518}$. En l'an 2000 , $72.7 \%$ des Sénégalais avaient accès à l'eau potable. Ce taux est de $83.7 \%$ en milieu urbain et de $67.9 \%$ en milieu rural. Les objectifs fixés à l'horizon 2015 sont de porter ces taux à $88 \%$ en milieu et $82 \%$ en milieu rural ${ }^{519}$. A l'évidence, le respect du contenu des clauses du cahier des charges dans ces différents cas est très approximatif.

\section{La distribution d'eau potable et la rupture de l'égalité devant et dans le service}

La proclamation de l'égalité des hommes devant la loi ou leur égalité en droits et obligations dans leur vie quotidienne par des lois fondamentales des Etats de l'Afrique subsaharienne, équivaut en principe, à une reconnaissance implicite du principe d'égalité devant et dans le service. Mais il n'est pas difficile, dans la pratique, d'observer la rupture permanente du principe. L'omission de transposition du principe dans les textes législatifs et réglementaires régissant l'organisation et le fonctionnement du service de l'eau potable, l'absence d'une autorité administrative indépendante chargée de lutter contre toutes les formes de discrimination et

516 L'OMC ET L'ACCORD GENERAL SUR LE COMMERCE DES SERVICES: les enjeux pour la santé publique, Internationale des services publics, http://www.world-psi.org/ consulté le 23/11/09.

517 Sudeshna BANERJEE, Heather SKILLING, Vivien FOSTER, Cecilia BRICEÑO-GARMENDIA, Elvira MORELLA, et Tarik CHFADI, Distribution d'eau en milieu urbain en Afrique subsaharienne, juin 2008.

518 BOtTON S., «Les multinationales de l'eau et les marchés du Sud », Débats et Controverses, juin 2007, n $^{\circ}$, p. 14 et 15 .

519 THIAM A., «Les problèmes de l'eau et d'accès a l'eau potable au Sénégal », World Citizens Assembly on Water (WCAW), Kuala Lumpur (Malaysia), 27-30 October 2005. 
l'absence d'une protection juridictionnelle de l'égalité sont autant de vecteurs de la rupture du principe $^{520}$.

Cette violation commande, tout de même, la distinction entre la rupture d'égalité entre les usagers candidats au service et l'inégalité de traitement entre usagers effectifs fautifs dans la fourniture des prestations ${ }^{521}$.

Le droit au raccordement au réseau de l'eau potable de toutes les personnes situées dans une zone couverte par la distribution, sous réserve de la réunion de certaines conditions, n'est toujours garanti comme par exemple en République Centrafricaine, au Congo, en RDC et au Cameroun. Pour les mêmes conditions, en effet, certaines personnes peuvent attendre deux, trois, six mois voire une année si ce n'est plus tandis que d'autres peuvent y accéder facilement, sans observer le délai d'attente statutaire, selon qu'elles sont une autorité ou selon qu'elles ont des affinités personnelles avec des collaborateurs du service. Aussi conclut-on à l'existence des conditions formelles et des conditions réelles d'accès au raccordement. L'impartialité du service reste de plus en plus remise en cause.

$\mathrm{Si}$, en France, certains cahiers de charges-types ${ }^{522}$ protègent le droit des riverains au raccordement, du moins dans l'hypothèse d'une gestion déléguée, en Afrique subsharienne, par contre, l'égalité des candidats à l'abonnement reste encore inobservée. Si le refus de connexion aux personnes situées en dehors du périmètre couvert par la distribution peut être admissible pour des raisons dont l'objectivité ne suscite aucun doute, le non-raccordement, dans une aire desservie, des usagers candidats au service restent ressentie par les intéressés comme un acte discriminatoire. Même si les refus exprès sont rares, mais le dépassement des longs délais d'attente et le silence injustifié des opérateurs face à ce dernier, restent assimilés par les intéressés à des refus ${ }^{523}$. Dans les grandes métropoles de la sous-région, la différence entre usagers reste perceptible dans l'exécution des opérations matérielles courantes du service. La plus grande partie de ces inégalités, dénoncée à Bangui, à Brazzaville, à Kinshasa comme à Yaoundé, survient quand l'organisme gestionnaire inflige et met en œuvre des sanctions à l'égard des usagers débiteurs.

Si, en effet, les usagers ordinaires peuvent immédiatement se voir suspendre ou interrompre la fourniture faute du paiement de leur facture sans parfois se voir adressés un courrier de rappel, ni se voir proposer le moindre échéancier, ce n'est pas toujours le cas pour d'autres usagers " privilégiés ", notamment des autorités, qui se servent de leur situation sociale pour échapper aux sanctions sus-indiquées, mettant ainsi en difficulté les services d'eau dans le recouvrement de leurs créances. L'expérience prouve que ces usagers " intouchables » ne payent pas toujours

520 OSSOMBO-YOMBO R., La gestion de l'eau potable dans les grandes agglomérations d'Afrique centrale. Contribution à l'étude d'un service public confronté à la mondialisation, thèse, Université Jean Moulin - Lyon III, 2007, p. 646.

$\mathbf{5 2 1}$ Idem.

522 L'article 16 du cahier des charges-type de 1947 pour la concession prévu par le décret n ${ }^{\circ}$ 7- 1554 du 13 août 1947 dispose : «Sur tout le parcours des canalisations, le concessionnaire sera tenu de fournir de l'eau dans les conditions prévues au présent cahier des charges à tout propriétaire qui demandera à contracter un abonnement d'une année au moins ». De même, aux termes du cahier des charges type pour l'affermage prévu par le décret du 17 mars 1980, «dans les conditions prévues au présent cahier des charges et sur tout le parcours des canalisations, le fermier est tenu de fournir l'eau à tout propriétaire qui demandera à contracter un abonnement de six mois au moins » (art.13)

$\mathbf{5 2 3}$ OSSOMBO-YOMBO Rémy, précité. 
leurs consommations d'eau, même après la cessation de leurs fonctions. Ces impayés, qui viennent s'ajouter à la dette de l'Etat vis à vis du service, participent à l'augmentation exponentielle du déficit budgétaire des sociétés de gestion d'eau de la sous-région. En réponse à la division de fait des usagers du service en usagers " sacrés » et usagers "vulnérables », certaines familles procèdent à des branchements frauduleux, qui constituent, au demeurant, un phénomène insoluble, représentant d'énormes pertes pour des opérateurs d'eau, dans toutes les agglomérations des pays du sud 524 .

\section{B. Le secteur privé indifférent au principe de continuité}

Malgré les efforts conjoints du gouvernement, des bailleurs de fond et de nombreuses ONG, la situation en milieu rural en Afrique subsaharienne est l'une des plus préoccupantes au monde. En République du Madagascar, seul $12 \%$ de la population a accès à l'eau potable ${ }^{525}$. La continuité, qui évoque les idées d'enchaînement et de prolongement dans l'espace et dans le temps, n'est pas inconnue du droit « africain ». Considérée par CHEVALLIER J. comme un « socle commun à tous les services publics $»^{526}$ la continuité du service public, principe à valeur constitutionnelle, est un des fondements de la théorie des services publics et la source d'une abondante jurisprudence des juridictions administratives. La continuité territoriale n'est pas, non plus, inconnue.

\section{La signification du principe de continuité}

En droit français, l'entreprise qui gère le service public est d'abord soumise à des obligations de fonctionnement du service au nom du principe de continuité. A défaut de respecter ces obligations, l'usager peut déférer au juge administratif le refus de l'autorité gérante de faire fonctionner le service conformément au principe de continuité. Le principe de continuité suppose que tous les services publics " doivent fonctionner sans heurts, sans à-coups, sans arrêts $»^{527}$.

La continuité implique la régularité du service, et dans certaines hypothèses, la permanence : tel est notamment le cas lorsque le service permet d'assurer l'ordre public comme la police, ou lorsqu'il vise à satisfaire des besoins vitaux, comme les services de secours. Quant aux services publics ne répondant pas à ces impératifs, le principe de continuité a vocation à s'appliquer dans

524 Idem.

$\mathbf{5 2 5}$ Mission économique, Fiche de synthèse, Eau potable et eau usées à Madagascar, 2005.

526 CHEVALLIER J., « Regards sur une évolution », AJDA 1997, nº spécial, p. 8.

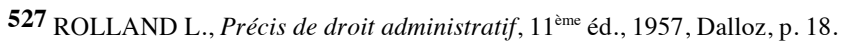


le cadre des horaires d'ouverture. Mais dans tous les cas, les usagers ont droit au fonctionnement normal du service.

Pour le commissaire de gouvernement TARDIEU, la continuité « est de l'essence du service public " ${ }^{528}$. Chacun sait qu'elle fonde un nombre élevé de règles importantes du droit administratif. On a tout dit des inconvénients d'un «Etat à éclipses ». Un service public qui interrompt ses prestations en dehors du règlement qui le régit cesse de répondre à l'intérêt général qui le fonde et altère par là même la justification de son existence. On observe d'ailleurs un déplacement de l'objet de la continuité : naguère principalement envisagée dans sa dimension temporelle, elle l'est désormais aussi dans sa dimension spatiale, et comme le fait remarquer TRUCHET D., le principe implique donc le fonctionnement ponctuel et régulier des services publics mais se rapporte également aujourd'hui de plus en plus souvent à une logique de continuité spatiale préconisant le maintien d'une certaine cohérence dans la localisation de ces services $^{529}$. Aussi, si la continuité évoque les idées d'enchaînement et de prolongement dans l'espace et dans le temps, la continuité du service public, principe à valeur constitutionnelle, est, quant à elle, un des fondements de la théorie des services publics et la source d'une abondante jurisprudence des juridictions administratives. La «continuité territoriale » n'y est pas, non plus, inconnue $^{530}$. Normalement, l'Etat impose au titre des missions de service public la fourniture, car "la cohésion territoriale interdit de laisser des zones entières dépourvues des services sans lesquels il n'est pas de vie économique et sociale moderne, et donc en retrait par rapport à l'activité et au niveau de vie du reste du pays $»^{531}$.

De même, Charles DEBBASCH le souligne en des termes presque similaires. En effet, les usagers peuvent exiger que le service fonctionne de manière régulière conformément aux règles en vigueur. Le service doit être accessible ${ }^{532}$. Pour obtenir satisfaction, les usagers peuvent s'adresser à la personne gestionnaire du service mais lorsqu'il s'agit d'un concessionnaire, l'administration est toujours responsable du bon fonctionnement ${ }^{533}$.

\section{L'ineffectivité du principe de continuité dans les services publics de l'eau en Afrique subsaharienne}

La question de l'eau en Afrique ne se pose pas en termes de quantité mais plutôt en termes de disponibilité et d'accès à la ressource. En effet, les spécialistes ne s'accordent-ils pas à dire que le potentiel en ressources en eau dont disposent les Etats africains pourrait largement couvrir les

528 Cf., CE, 7 août 1909, Winkell, op. cit.

529 TRUCHET D., « Unité et diversité des “"grands principes”' du service public », AJDA,

1997, nº spécial, p. 38.

$\mathbf{5 3 0}$ MADIOT Y., « Service public et aménagement du territoire », AJDA 1997, p. 83.

$\mathbf{5 3 1}$ THIRY B., « Les conceptions de l'intérêt général dans l’Union Européenne », EDCE, n50, 1999, p. 403.

$\mathbf{5 3 2}$ Cf., CE, 25 juin 1969, Vincent, Rec., 334

$\mathbf{5 3 3}$ Cf., CE, 27 novembre 1935, Baudoin, Rec., 1105 
besoins de l'intégralité de la population. Malheureusement, ces ressources sont souvent mal réparties, car trop éloignées des grands centres de consommation, sont difficilement mobilisables pour satisfaire la demande en eau potable des populations urbaines et rurales. La répartition régionale fait apparaître que, dans certaines zones, l'eau potable est accessible à plus de la moitié de la population.

L'accès à l'eau potable constitue dans les quartiers périurbains et dans les établissements humains semi-ruraux et ruraux une difficulté majeure. Ainsi, les services urbains essentiels de base sont quasi inexistants dans certaines zones du fait de la poussée démographique non maîtrisée et d'une déficience d'une prise en charge correcte par les collectivités locales. Par exemple, les populations résidant dans les grandes villes camerounaises Douala et Yaoundé, ne sont pas toujours sûres d'avoir un approvisionnement en eau potable ${ }^{534}$.

Derrière le débat sur les services publics en milieu rural, s'en profilait un autre, beaucoup plus important, portant non pas directement sur le concept de service public mais sur les principes régissant le fonctionnement des services publics, principes qui en « constituent l'ossature en droit français contemporain ${ }^{53}$ ». Les problèmes qui se posaient dans les zones en voie de dépeuplement se posaient également, plus récemment et sous une autre forme, dans des zones urbaines très denses, connaissant des difficultés spécifiques. La réflexion sur le service public et l'aménagement du territoire peut alors s'élaborer sur des bases nouvelles ${ }^{536}$. Une première orientation réside dans l'affirmation, qui n'est guère contestée, affirmation selon laquelle le service public «est une notion juridique forte parce qu'elle constitue l'un des moteurs de la cohésion sociale ${ }^{537} »$.

\section{§ 2. La question récurrente de la gratuité de l'eau}

En janvier 2009, le gouverneur de Californie, M. SCHWARZENEGGER, a décrété subitement l'état d'urgence pour l'eau ${ }^{538}$. Il s'agit d'une décision sans précédent dans un pays développé mais qui témoigne du caractère vital de l'eau quelque soit l'endroit. Ce caractère vital explique que certaines personnes défendent l'idée de la gratuité de l'eau. En effet, la jurisprudence allemande considère que la coupure d'eau est contraire à l'article $1^{\text {er }}$ de la Constitution et la jurisprudence espagnole qu'elle constitue un "moyen de pression excessif » sur le débiteur ${ }^{539}$. L'enjeu de l'eau aujourd'hui réside spécialement dans la capacité de chaque Etat à mettre en place des systèmes qui arrivent à concilier un objectif d'égal accès de tous, en

$\mathbf{5 3 4}$ Conseil Européen du droit de l'environnement, Le droit à l'eau en Afrique et en Europe, Académie de l'eau, France, 2005, p. 85 .

$\mathbf{5 3 5}$ MOREAU J., Droit administratif, PUF, 1989, p. 333.

$\mathbf{5 3 6}$ MADIOT Y., « Service public et aménagement du territoire », AJDA 1997 p. 83.

$\mathbf{5 3 7}$ STIRN B., «La conception française du service public », CJEG 1993, p. 299.

$538 \mathrm{~N}^{\circ} 4070$ - Rapport d'information de M. Jean GLAVANY, http://www.assemblee-nationale.fr/13/rapinfo/i4070.asp consulté le 15 janvier 2012.

539 Conseil d'Etat, L'eau et son droit, Rapport, La documentation, 2010, p.83. 
tenant compte des différences de potentialités financières des individus, comme l'expérimente à ce jour la ville du Cap en Afrique du Sud ${ }^{540}$. En général, le service public est institué dans l'intérêt général. Aussi, l'importance de la question d'accès à l'eau amène à s'interroger sur l'opportunité ou non de faire de la distribution de l'eau un service public gratuit. La gratuité noue ainsi depuis toujours avec l'égalité "un lien étroit et incontestable » ${ }^{541}$ car apparaît comme " l'expression d'un principe de solidarité dont la définition juridique est l'égalité devant les droits de l'homme ${ }^{542}$, révélatrice de la supériorité de l'intérêt général face à une logique purement marchande. Si le principe en lui-même paraît être insaisissable (A), il convient de procéder dans certaines circonstances à un aménagement $(\mathrm{B})$.

\section{A. L'insaisissable principe de gratuité des services publics}

«La gratuité de l'eau est un mythe préjudiciable aux populations. La gratuité n'existe pas: il y a toujours quelqu'un qui paye! Quand ce n'est pas le consommateur, c'est le contribuable... et quand ce n'est pas le contribuable d'aujourd'hui, c'est celui de demain via le déficit et la dette $»^{543}$. Aucun texte juridique ne présente de définition de la gratuité. Rares sont les textes qui y font référence. La notion de gratuité ne détient pas de validité juridique dans un système de droit libéral. Elle revêt un caractère fragile et aléatoire dans le droit de la santé. Elle ne correspond pas à un principe juridique moteur de l'action des pouvoirs publics en ce domaine, ni à une règle de droit imposable à l'ensemble des personnes engagées dans la mise en œuvre de ce droit. Cette perplexité entre la gratuité et le coût réel a préoccupé VIRCOULON Thierry, qui, dans "L'eau gratuite pour tous ? L'exemple de la nouvelle politique de l'eau en Afrique du Sud» démontre que, depuis 1994, le gouvernement sud-africain a oscillé entre deux politiques opposées, l'expansion des infrastructures et la facturation au coût réel, pour finalement inventer en 2000 un moyen terme original et équilibré qui associe gratuité et recouvrement des coûts ${ }^{544}$.

540 JAGLIN S., «Services d'eau et construction métropolitaine au Cap (Afrique du Sud) : les difficultés de l'intégration urbaine », Revue française d'administration publique, $\mathrm{n}^{\circ} 107,2003$.

$\mathbf{5 4 1}$ KOUBI G, «La décomposition de la notion de gratuité en droit administratif français », JCP A, 2003, p. 770.

542 HERTZOG R., Recherche sur la gratuité et la non gratuité des services publics, Thèse Strasbourg, 1972,p. 169.

543 GLAVANY Jean, Rapport d'information sur "La géopolitique de l'eau », http://www.assembleenationale.fr/13/rap-info/i4070.asp consulté le 15 janvier 2012.

$\mathbf{5 4 4}$ VIRCOULON T., «L'eau gratuite pour tous ? L'exemple de la nouvelle politique de l'eau en Afrique du Sud » Afrique contemporaine, $\mathrm{n}^{\circ} 205$, De Boeck Université, 2003/1 242 pages.

http://www.cairn.info/resume.php?ID ARTICLE=AFCO 2050135 consulté le 27 juillet 2006. 


\section{Le principe de la non-gratuité des services publics}

Le concept même de gratuité, ou de non-gratuité, mérite d'être éclairé, car, au sens économique, un service public n'est jamais gratuit. Il relève en effet toujours d'un coût et n'est considéré, de manière quelque peu artificielle, comme gratuit que lorsqu' « aucune participation financière n'est demandée à l'usager du service pris en tant que tel en contrepartie directe de la prestation qui lui est accordée » ${ }^{54}$. Le service public « gratuit» est ainsi celui qui est financé par l'impôt ou par d'autres ressources, et non par un prix perçu directement sur l'usager. En cela, il offre la possibilité de bénéficier de la prestation sans avoir à en débourser directement le prix, concrétisant une égalité d'accès aux implications assurément favorables envers les droits et libertés.

En droit français, il n'existe pas de principe général de gratuité des services publics. Le principe a été consacré par la jurisprudence du Conseil d'Etat dans l'affaire Société Direct Mail Promotion $^{546}$. Aussi bien le juge n'a-t-il jamais fait allusion à un principe général de gratuité des services publics, qui n'aurait eu aucun sens depuis l'apparition des services industriels ou commerciaux, et a-t-il formellement décidé « que le moyen tiré de la violation d'un principe de gratuité du service public administratif ne peut en tout état de cause être que rejeté» ${ }^{547}$. De même, le Conseil constitutionnel n'a pas reconnu à la gratuité des services publics le statut de principe à valeur constitutionnelle ${ }^{548}$. Quant à la doctrine, elle s'est montrée distante à l'égard de cet «introuvable principe de gratuité » ${ }^{549}$. Le législateur malien n'est pas resté en marge de cette logique et précise avec fermeté que "l'accès au service public de l'eau, que ce soit aux bornes fontaines ou aux branchements individuels, doit toujours être payant $»^{550}$. Ce sur quoi le législateur burkinabè semble être d'accord puisqu'il affirme que l'utilisation de l'eau exige de chacun qu'il participe à l'effort de la Nation pour en assurer la gestion ${ }^{551}$.

Le Contrat Mondial de l'Eau ${ }^{552}$ met l'accent sur le droit individuel à l'eau et plaide pour la fourniture gratuite à tout le monde de l'eau nécessaire à la satisfaction des besoins essentiels (c'est-à-dire que la première tranche est payée par la collectivité) tandis que la consommation d'eau au-delà de cette première tranche serait intégralement payée par les utilisateurs. Selon R. PETRELLA, "ce n'est pas parce qu'un service a un coût que cela doit se traduire par un prix sur le marché. L'ensemble des coûts encourus pour la distribution et le traitement de l'eau doit être pris en charge par la collectivité selon des modalités de financement collectif (taxation,

545 LACHAUME J-F., BOITEAU C. et PAUliat H., Droit des services publics, Armand Colin, $3^{\text {ème }}$ éd., 2004, p. 446.

${ }^{\mathbf{5 4 6}}$ Cf., CE, Ass., 10 juillet 1996, Sté Direct Mail Promotion, Lebon, p. 297.

547 Idem.

$\mathbf{5 4 8} C f$., CC, 12 juillet 1979, Ponts à péage, AJDA, 1979.46

549 HERTZOG R., « Le prix du service public », AJDA, 1997 p. 55.

$550 \mathrm{Cf}$. article 54 de la loi n ${ }^{\circ} 02-006 /$ du 31 janvier 2002 portant code de l'eau au Mali.

$\mathbf{5 5 1} C f$. Article 47 alinéa 1 de la loi d'orientation du 8 février 2001.

552 Cf. R. PETRELLA : Le manifeste de l'eau, Éditions Labor, Bruxelles, 1998 , p.2. 
emprunts publics). Assurer l'accès à l'eau pour tous est une responsabilité collective, qui revient aux pouvoirs publics au plan local comme national $»$. Cette approche ne recueille toujours pas le soutien des pouvoirs publics qui cherchent plus à réduire les subventions pour l'eau qu'à augmenter la prise en charge des coûts de l'eau potable.

En effet, les fondateurs de la doctrine du service public n'ont pas fait de la gratuité un de ses principes constitutifs. HAURIOU et DUGUIT expriment des thèses qui tiennent davantage de la doctrine politique que de l'analyse juridique. ROLLAND, écrivant pourtant après l'apparition des SPIC (services publics à caractère industriel et commercial), évoque pendant un certain temps, d'une manière du reste peu convaincante, un principe de gratuité, dont il ne précise pas la portée $u_{\text {utile }}{ }^{53}$. Ceci explique d'ailleurs le fait que l'emploi du terme de gratuité n'est pas ordinaire dans les discours juridiques. Il est d'usage peu fréquent dans les dispositifs des lois et règlements et est d'utilisation circonspecte dans les actes contractuels ou unilatéraux et d'utilité indistincte dans la communication associative et sociale. KOUBI G. dira que ce «mot de gratuité dissimule plus qu'il ne dévoile » ${ }^{554}$.

Dans l'élaboration des textes et lors de la rédaction des codes, cette unité linguistique problématique ne rendrait compte que d'un prétendu désintéressement dans l'activité des sujets de droit. Or, " d'emblée, la gratuité paraît suspecte : tantôt, elle apparaît comme feinte et, traduisant les détours de l'intérêt, elle sera sanctionnée au titre de manœuvre frauduleuse; tantôt, dans des cas exceptionnels, elle passera pour sincère, mais révélera du fait même un dangereux [penchant] dans le chef de son auteur. Sensiblerie, inclination quasi coupable qui pourrait bien entretenir un soupçon de dérangement mental... ${ }^{555}$ ». Si dans la réflexion philosophique, la gratuité est objet de connaissance en tant qu'elle serait un acte de penser, en droit, elle est objet d'étude en ce qu'elle se présenterait sous la forme d'un acte de dire ou de faire.

\section{Les exceptions}

Paradoxalement, des services publics habituellement payants sont quelque fois assurés gratuitement par leur gestionnaire suite à une intervention du législateur. En effet, pour préserver un intérêt social, les usagers les plus défavorisés peuvent bénéficier des activités de distribution d'eau et de fourniture d'énergie sans contrepartie financière ${ }^{556}$, au moins dans certaines périodes de l'année et dans certaines circonstances restrictivement définies par les textes ${ }^{557}$.

553 Recherches sur la gratuité et la non-gratuité des services publics, Thèse dactyl., Strasbourg, 1972,; Cf. également Alain-Serge MESCHERIAKOFF, Droit des services publics, PUF 1991, p. 195.

$\mathbf{5 5 4}$ KOUBI G., « Réflexions sur la gratuité dans le droit de la santé », Revue de droit sanitaire et social 1999 p. 1.

$\mathbf{5 5 5}$ OST F., Entre droit et non-droit : l'intérêt - essais sur les fonctions qu'exerce la notion d'intérêt en droit privé, vol. 2, Droit et intérêt, éd. Fac. Univ. de Saint Louis, Bruxelles, 1990, p. 47.

$556 C f$. Article 43-5 al 1 de la loi 88-1088 du 1er décembre 1988: «toute personne éprouvant des difficultés particulières du fait d'une situation précaire a droit à une fourniture d'eau, d'énergie et de services téléphoniques ».

557 ROYON S., La tarification des services publics à l'épreuve du droit du marché, Mémoire, Master II Recherche Droit public, Grenoble, 2005, p. 10. 
Selon la doctrine, la règle de gratuité lève en effet toute limitation d'accès au service tenant aux capacités financières des usagers et permet ainsi à tous de bénéficier des prestations offertes en les plaçant abstraitement dans la même situation de départ à cet égard. Jean DU BOIS de GAUDUSSON rappelle en ce sens que la gratuité des services publics est "la seule formule capable d'assurer réellement et parfaitement l'égalité entre tous les usagers ${ }^{558}$, approche confirmée par Catherine TEITGEN-COLLY, pour laquelle "la gratuité du service public apparaît sans conteste le moyen le meilleur pour assurer l'accès au service de tous les citoyens puisqu'il évite la sélection par l'argent ${ }^{559}$. En 1954, SALOMON J., ajoutait déjà dans ses travaux de thèse que "l'inégalité économique étant un mal quasi-incurable, seule la gratuité des services permet de faire de l'égalité autre chose qu'une froide fiction juridique. (...) Aussi, la gratuité est la seule formule qui permette d'assurer pratiquement l'égalité entre tous les usagers du service public $»^{560}$.

Il serait vain de dresser une liste exhaustive des textes juridiques dans lesquels serait retranscrite l'idée de gratuité sous sa forme nominale ou selon une proposition adjectivale (gratuit/e) ou adverbiale (gratuitement). La signification du mot et de ses dérivés varie selon les contextes, selon les domaines, selon les objets ; elle se diversifie selon les sujets et selon les actions ou opérations ; elle se modifie selon les protagonistes d'un acte ou d'une activité toujours fondé/e sur un ou plusieurs intérêts - alors que d'une part, l'intérêt peut être dit, quel que soit l'espace de sa perception, « général » donc à redéfinir à chaque fois, et d'autre part, les différents intérêts en présence quels qu'ils soient, peuvent être divergents ou convergents. Ces mobilités dans l'appréciation des intérêts ternissent la valeur intrinsèque de la notion de gratuité, souvent comprise dans la réalisation d'une « activité désintéressée » ${ }^{561}$.

Certains services publics sont donc gratuits en vertu d'une règle constitutionnelle ou législative ${ }^{562}$. Ainsi "l'organisation de l'enseignement public et laïque à tous les degrés » est proclamée comme étant un devoir de l'Etat par le préambule de la Constitution du 27 octobre 1946, maintenu en vigueur par celui de la Constitution du 4 octobre 1958. Le juge a fait une application nuancée de cette disposition. S'il condamne les paiements dans l'école élémentaire ${ }^{563}$, il n'a cependant pas condamné toute forme de participation financière des étudiants au budget des établissements d'enseignement supérieur ${ }^{564}$.

Ainsi, les interventions du service départemental d'incendie et de secours qui se rattachent directement à leurs missions de service public définies à l'article L. 1424-2 CGCT (code général des collectivités territoriales) sont gratuites, mais une participation au frais être demandée aux bénéficiaires pour les autres interventions ${ }^{565}$. Relativement à la gratuité des services ou

558 DU BOIS DE GAUDUSSON J., L'usager du service public administratif, L.G.D.J, 1974, p. 170.

559 TEITGEN-COLLY C., La légalité de l'intérêt financier dans l'action administrative, Economica, 1981, p. 394.

560 SALOMON J., L'égalité des individus devant le service public, Thèse, Grenoble, 1954, p. 291

561 RICHER L., dir. L'activité désintéressée, réalité ou fiction juridique?, Economica, 1981.

562 TRUCHET D., précité, p. 353.

563 CE 10 janvier 1986, Commune de Quingey, Leb.p. 3

564 CE Ass. 28 janvier 1972, Conseil transitoire de la faculté des lettres et sciences humaines de Paris, AJDA 1972 p. 109 et chron. p. 90. Les droits universitaires ont leur fondement dans une loi de 1951 dont le juge ne veut pas apprécier la constitutionnalité.

565 Cf. Art. L.1424-2 CGCT 
équipements publics régie par la loi, on peut citer aussi, les vaccinations obligatoires ou l'usage des autoroutes « en principe $»^{566}$, ou encore des ponts ${ }^{567}$ (loi du 30 juillet 1880 ).

\section{B. L’admissible gratuité de la distribution de l'eau}

Comme les transports publics et la gestion des déchets, et à l'opposé de l'électricité ou des télécommunications, les services d'eau devraient être essentiellement des services de proximité. Certes, il ne faut pas oublier le caractère très capitalistique des équipements hydrauliques, qui s'amortissent sur plusieurs années, et ne dégagent que des faibles marges. La gratuité des services publics a une valeur juridique tout a fait incertaine. Elle ne peut être opposée dans le cas de services publics industriels ou commerciaux, ni lorsque le service public en cause, quelle que soit sa nature, est concédé, le concessionnaire se rémunérant directement les usagers. Son caractère de principe est actuellement largement contesté par la doctrine ${ }^{568}$. Il ne s'agit pas d'une des lois du service public dégagées par ROLLAND, et le Conseil d'Etat lui a récemment dénié toute valeur de principe ${ }^{569}$. Mais le Conseil constitutionnel ${ }^{570}$ s'était prononcé bien avant pour refuser une valeur constitutionnelle à la gratuité des services publics. Cependant, et bien que dans des secteurs d'activité de plus en plus réduits, celle-ci reste une notion applicable à certains services publics.

\section{Les raisons d'instituer la gratuité de l'eau : la pauvreté des Etats de l'Afrique subsaharienne}

"Le caractère central de l'eau pour la survie humaine et l'absence de substitut en font un enjeu social et politique majeur. A cela, il faut ajouter une dimension idéologique et même religieuse. L'eau sera considérée comme un don de la nature ou de Dieu qui devrait être accessible à tous sans prix et sans limites $»^{571}$.

Le constat qui constitue le point de départ de toute étude relative à la pauvreté en science juridique, aussi difficile à admettre serait-il, est que la pauvreté n'est pas définissable en droit. Certes, les textes juridiques de différents États peuvent déterminer un « seuil de pauvreté » à

$\mathbf{5 6 6}$ Code de la voirie routière, art. L. 122-4

567 CE Sect. 16 février 1979, Comité d'action et de défense des intérêts de l'île d'Oléron et Bource franc, AJDA septembre 1979 , p. 54

$\mathbf{5 6 8}$ KOUBI G., précité.

569 CE, Ass., 10 juillet 1996, Sté Direct Mail Promotion, précité.

$\mathbf{5 7 0} C f$., CC, 12 juillet 1979, Ponts à péage, précité.

$\mathbf{5 7 1}$ MENARD C., «Enjeux d'eau : la dimension institutionnelle », Tiers-Monde, Année 2001, Volume 42, Numéro 166 , p. $259-274$ 
partir duquel seraient développées des mesures spécifiques pour contribuer à la lutte contre les exclusions ou pour porter remède aux situations d'extrême pauvreté, mais la plupart du temps, les critères d'évaluation des cas à traiter reposent sur une définition économique, financière et monétaire, plus que sociologique ou psychosociologique, de la pauvreté. La définition proposée par les économistes retient donc souvent l'attention des pouvoirs publics : « Est pauvre celui qui a un revenu insuffisant pour atteindre un niveau de vie minimum. " L'indétermination même des notions, qu'il s'agisse d'un « revenu insuffisant» ou d'un « niveau de vie minimum », influence l'ensemble des politiques publiques qui seraient mises en œuvre dans le but de lutter contre la pauvreté. La définition de la pauvreté doit alors, à chaque fois, dans chaque État, être considérée comme contingente, incluse dans un flou permanent, concernant pourtant des positions individuelles, familiales, collectives, comprise dans un cheminement allant de la difficulté d'assurer sa survie, particulièrement liée à un " droit à la vie » toujours à repenser, jusqu'à la difficulté de vivre des privations temporaires ou d'assumer des frustrations ${ }^{572}$

L'eau est au cœur de toutes les activités qui permettront d'atteindre la plupart des Objectifs du Millénaire pour le Développement. Aussi, en ce qui concerne l'objectif 7, les dirigeants des pays du monde ont convenu de réduire de moitié, au plus tard en 2015, la proportion d'individus qui n'ont pas accès à l'eau potable et aux services d'assainissement de base ou qui n'ont pas les moyens de s'en procurer. Les Objectifs du Millénaire pour le Développement adoptés par le Sommet du Millénium et le Sommet Mondial, sont une interpellation de la communauté internationale et des nations à disposer d'un cadre de mise en cohérence, de synergie et d'articulation des actions afin d'atteindre une échelle significative dans la lutte contre la pauvreté dans les pays en développement et singulièrement en Afrique Subsaharienne ${ }^{573}$.

\section{L'expérience de l'Afrique du sud}

La gratuité générale a été mise en œuvre en Irlande et dans une certaine mesure en Afrique du Sud et en Région flamande. La seconde approche, moins ambitieuse, est d'autant moins coûteuse qu'il y a moins de personnes pour qui l'eau est d'un prix inabordable. L'écart de coûts entre ces deux premières solutions permettrait de financer l'équipement en eau potable et assainissement des régions mal desservies.

Aussi, quoique anecdotique que cela puisse paraître, en République sud africaine, au temps fort de la ségrégation raciale, l'eau était fournie gratuitement aux populations urbanisées et, même aux pires moments de l'insurrection des townships dans les années 1980, Pieter W. BOTHA ne s'est jamais résolu à utiliser l'arme de l'eau, c'est-à-dire à cesser de subventionner ce service public, tant cela aurait été impopulaire à l'intérieur comme à l'extérieur du pays. Ainsi, à cette époque, le service public de l'eau était, pour les pauvres, soit inexistant soit gratuit, et, en ville, ceux qui en bénéficiaient vivaient aux temps «édéniques » de l'ignorance du

572 KOUBI G., « La pauvreté, comme violation des droits humains », Revue internationale des sciences sociales, 2004/2 - N 180 , p. 361.

573 Processus d'Elaboration du PAGIRE du Cameroun : Document d'Orientation, p.10 
principe «consommateur-payeur $»^{574}$. Du point de vue de l'accès à l'eau potable, le sousdéveloppement sud-africain était paradoxal car non général : l'eau était fournie gratuitement à une fraction de la population noire en dépit du racisme institutionnel qui prévalait à l'époque.

Le fait que la matière première, la farine, soit un bien privé et que l'eau soit un bien commun distribué par des canalisations occupant le domaine public et bénéficiant souvent de subventions semble être totalement ignoré, seule restant en place la logique du marché et la volonté de ne pas faire de pertes sur la vente de chaque litre d'eau.

L'eau des plus démunis ne doit pas être financée par les distributeurs d'eau car ceux-ci n'ont aucune responsabilité en matière d'action sociale et ne sont pas chargés de s'occuper plus particulièrement des besoins fondamentaux de leurs clients pauvres. Selon cette approche, cette préoccupation sociale relève des pouvoirs publics (contribuables) et des œuvres caritatives qui peuvent organiser des distributions gratuites ou donner des aides financières comme elles le font pour le pain. Dans certains cas, les distributeurs d'eau ne sont pas autorisés par la loi ou les municipalités à introduire des différenciations de prix dans l'eau des ménages et sont amenés de ce fait à exclure les usagers les plus pauvres de l'accès à l'eau.

\section{Section II. La gestion privée de l'eau et l'émergence des nouvelles lois du service public}

A la suite de la circulaire du 26 juillet $1995^{575}$ en France, DONIER Virginie affirme qu'il faut donner corps à de nouveaux principes au nombre desquels figurent la qualité, l'accessibilité, la simplicité, la rapidité, la transparence, la médiation, la participation et la responsabilité. Plus récemment, la circulaire du 2 mars 2004 a permis l'élaboration d'une charte de l'accueil des usagers afin d'assurer le respect des principes de transparence et d'accessibilité, et afin de promouvoir un accueil de qualité. Cet afflux de nouveaux principes tend à " complexifier » le droit commun des services publics puisque de nouvelles règles s'imposent désormais au côté des principes fondamentaux.

Après avoir expliqué la controverse sur les nouvelles lois $(\S 1)$, nous montrerons qu'il est illusoire de croire que la gestion privée prenne en compte les lois émergeant du service public $(\S 2)$.

$\mathbf{5 7 4}$ VIRCOULON T., « L'eau gratuite pour tous ? L'exemple de la nouvelle politique de l'eau en Afrique du Sud », op. cit., p. 136.

$\mathbf{5 7 5}$ Circulaire relative à la préparation et à la mise en œuvre de la réforme de l'Etat et des services publics, JO 28 juill. 1995, p. 11217 


\section{$\S 1$. La controverse sur les nouvelles lois du service public}

Parmi les principes nouveaux, certains sont en vérité appliqués depuis longtemps. D'autres, la qualité, la simplicité, l'accessibilité,..., traduisent des exigences de bonne gestion (B) qui dépendent bien davantage de mesures administratives et financières que de la proclamation de principes qui leur apporterait peu d'effectivité supplémentaire. Avant même de s'y attarder nous montrerons que ces principes ou lois sont contestés (A).

\section{A. Des « lois » initialement réfutées dans leur existence}

L'on notera que c'est principalement la doctrine qui s'est méfié des nouveaux principes. Préoccupés par l'unité mythique des lois de ROLLAND, les auteurs hésitent à conférer aux nouveaux principes une existence juridique autonome.

\section{La méfiance doctrinale}

Peu d'auteurs considèrent que ces nouvelles règles constituent toutes de véritables lois du service public ; la classification adoptée dans les différents ouvrages distingue généralement les principes fondamentaux et les nouvelles règles de fonctionnement. Stéphane BRACONNIER établit ainsi une distinction entre les «lois réelles» du service public et les "principes virtuels» qu'il qualifie « d'exigences a-juridiques $»^{576}$.

Pour Pierre-Laurent FRIER les nouveaux principes ne peuvent être élevés au rang de loi du service public, il s'agit simplement de " règles de bonne gestion $»^{577}$. A cet égard, il faut d'ailleurs rappeler que la charte des services publics du 18 mars 1992 n'associait pas expressément ces nouvelles règles aux principes traditionnels puisqu'elle établissait une distinction entre les «principes fondamentaux » et les «principes d'action ». De manière générale, la doctrine apparaît rétive face à l'émergence et à la consécration de nouvelles lois du service public, même si certains auteurs n'hésitent cependant pas à admettre l'apparition de nouveaux principes fondamentaux ${ }^{578}$.

Et comme la doctrine "marque le pas », la question de l'apparition de nouvelles lois du service public mérite d'être posée. Et pour y répondre, il faut, au préalable, définir la notion de

576 BRACONNIER S., Droit des services publics, PUF, Thémis droit public, 2003, p. 269 et s.

577 FRIER P.-L., Précis de droit administratif, Montchrestien, 2003, p. 227.

578 lachaume J.-F., Boiteau C. et PaUliat H., Droit des services publics, p. 433 et s: ces auteurs considèrent que le principe de neutralité constitue la quatrième loi du service public. 
loi du service public, afin d'établir un cadre de référence permettant d'analyser ces nouveaux principes. En d'autres termes, quels sont les critères communs aux principes fondamentaux, critères auxquels doivent satisfaire les nouvelles règles de fonctionnement pour appartenir aux lois du service public?

Ces lois se caractérisent de prime abord par leur généralité dans la mesure où elles ont vocation à s'appliquer à l'ensemble des services publics, ce qui ne signifie pas, pour autant, qu'elles s'appliquent avec la même intensité à toutes les activités. En outre, il s'agit de principes généraux du droit, ce qui leur confère une valeur et donc une force juridiques. Le principe de continuité a été élevé au rang de principe «fondamental » dans l'arrêt du Conseil d'Etat du 13 juin 1980, Mme Bonjean, et il a valeur constitutionnelle ${ }^{579}$.

Le principe d'égalité revêt lui aussi une double qualification juridique au regard de la jurisprudence administrative ${ }^{580}$ et constitutionnelle ${ }^{581}$. Quant à la valeur juridique du principe d'adaptation, certains auteurs lui dénient toute qualification de principe général du droit ${ }^{582}$, mais en raison des conséquences attachées à ce principe à l'égard des agents du service, des usagers et des cocontractants de l'administration, il semble constituer lui aussi un principe général du droit $^{583}$. En revanche, à la différence des principes d'égalité et de continuité, il n'a pas fait l'objet d'une consécration constitutionnelle.

\section{Les principes exclus des lois du service public ${ }^{584}$}

L'enracinement du service public aboutit à parer la gestion publique d'une supériorité de principe par rapport à la gestion privée. Le service public devrait être accessible et fonctionner au meilleur coût possible. Les services publics sont censés être mieux à même de préserver et de garantir les intérêts du public, tout en oeuvrant pour la réduction des inégalités ; aussi, leur extension paraît-elle devoir s'imposer ainsi que le prévoyait DUGUIT ${ }^{585}$.

579 V. en ce sens la décision du Conseil constitutionnel du 25 juillet 1979, Droit de grève à la radio et à la télévision, Rec. p. 33.

580 CE, Sect., 9 mars 1951, Société des concerts du conservatoire, Lebon p. 151.

581 Cons. const., décis. $n^{\circ}$ 79-107 12 juill. 1979, Loi relative à certains ouvrages reliant les voies nationales ou départementales, Rec. p. 31

582 A.-S. MESCHERIAKOFF, Droit des services publics, précité, p. 175 ; G. J. GUGLIELMI et G. KOUBI, Droit du service public, Montchrestien, Domat de droit public, 2000, p. 428 : selon ces auteurs, le principe d'adaptation « ne détient pas la valeur d'un principe général du droit, mais il est bien un principe directeur des services publics ».

$\mathbf{5 8 3}$ J.-P. VALETTE, Le service public à la française, Ellipses, 2000, p. 91-92 ; D. TRUCHET, Unité et diversité des « grands principes » du service public, AJDA 1997, numéro spécial, p. 38; J.-F. LACHAUME, C. BOITEAU et H. PAULIAT, Droit des services publics, op. cit. p. 367. L'absence de droit acquis au maintien d'un service public facultatif constitue sans nul doute un principe général du droit : V. en ce sens L. CLUZEL, Le service public et l'exigence de qualité, thèse dactylographiée, Paris II, 2004, p. 533, publiée aux éditions Dalloz 2006.

$\mathbf{5 8 4}$ DONIER V., « Les lois du service public : entre tradition et modernité », RFDA 2006 p. 1219

$\mathbf{5 8 5}$ CHEVALLIER J., «Le service public : regards sur une evolution », AJDA, 1997, p. 8. 
Aux principes traditionnels tendent à s'ajouter d'autres principes dont la portée reste cependant controversée. Si les principes de neutralité et de transparence régissent le fonctionnement de tous les services publics, d'autres font en revanche l'objet d'une application diversifiée. Tel est notamment le cas du principe de gratuité, qui se révèle être en réalité un principe inexistant car aucune disposition n'impose, de manière générale, la gratuité des services publics. En effet, la gratuité ne figure pas plus que par le passé au nombre des principes traditionnels. La neutralité se distingue désormais de l'égalité avec laquelle elle était confondue $^{586}$. De même les principes de simplicité, d'accessibilité, de sécurité, de participation, ou encore de qualité doivent, eux aussi, être exclus de la catégorie des lois du service public. En raison de leur imprécision et de leur inconsistance, ces principes restent de simples règles de bonne gestion, ils n'ont pas vocation à devenir de véritables lois du service public. On a pu les considérer comme des lois incomplètes ou des principes en devenir. Même si les juges des services publics ont toujours refusé de consacrer ces principes, il faut reconnaître que certains, comme celui de la qualité, étaient déjà pris en compte par l'administration.

\section{B. Des « lois » toutefois indispensables au fonctionnement du service public}

Les nouvelles lignes directrices qui guident le fonctionnement des services publics ont permis de préciser les lois traditionnelles et ont contribué à les enrichir. Elles remettent en cause l'unité qui caractérise les principes fondamentaux et posent la question de leur «adoption » par les lois dites de ROLLAND.

\section{Une ligne de conduite incontournable}

Parmi les nouvelles règles d'action affirmées par la Charte des services publics du 18 mars 1992 et par la circulaire du 26 juillet 1995 relative à la préparation et à la mise en œuvre de la réforme de l'Etat et des services publics, figure le principe de simplicité. Cet objectif s'est notamment traduit par l'adoption de trois lois de simplification du droit, par le recours à la codification et par la mise en place de l'administration électronique en vue de faciliter les échanges entre l'administration et les usagers ${ }^{587}$. Toutefois, si l'effort de simplification est de plus en plus prégnant, ce principe ne bénéficie pas d'une force juridique suffisante pour être intégré au sein de la catégorie des lois du service public : en effet, si le Conseil d'Etat s'assure désormais que le pouvoir réglementaire a édicté des normes compréhensibles ${ }^{588}$, rien ne semble

586 CE,16 octobre 2000, requête $n^{\circ} 217801$, Association Promouvoir.

$\mathbf{5 8 7}$ CANTERO A. et S. BOYTCHEV S., Quelles avancées juridiques en matière " d'administration électronique » ?, JCP A 7 nov. 2005, p. 1639 ; V. également l'ordonnance $\mathrm{n}^{\circ} 2005-1516$ du 8 décembre 2005 relative aux échanges électroniques entre les usagers et les autorités administratives et entre les autorités administratives, JO 9 déc., p. 18986 (un projet de loi de ratification a été présenté en Conseil des ministres le 22 février 2006).

$\mathbf{5 8 8}$ CE, 24 mars 2006, Société KPMG et autres, $\mathrm{n}^{\circ} 288460$ 
obliger l'administration à simplifier les procédures existantes, édictées par des normes antérieures à l'arrêt du 24 mars 2006.

Le principe de la simplification entretient les liens étroits avec le principe d'accessibilité dans la mesure où l'effort de simplification permet de rendre le droit plus accessible à l'égard des usagers. Mais ce principe d'accessibilité dispose, lui aussi, d'une force juridique limitée à l'égard de l'administration, il demeure une simple ligne de conduite même si certains progrès ont été accomplis, notamment sous l'influence du droit communautaire. En effet, l'accessibilité ne revêt pas seulement une acception intellectuelle, elle peut également recevoir une signification économique ou géographique, comme en témoigne la notion de service universel qui impose un tarif abordable et une prestation accessible sur l'ensemble du territoire.

Les lois de ROLLAND ont vu leur signification évoluer face à l'apparition de nouvelles exigences. Aussi, le principe d'égalité connaît-il désormais de nouvelles implications liées essentiellement à la notion d'accessibilité tarifaire. De même, les conséquences découlant du principe de continuité se sont multipliées : à la continuité temporelle, il faut peut-être ajouter la continuité spatiale des services publics. L'apparition de nouvelles lignes directrices a donc suscité certaines adaptations au sein des lois du service public ${ }^{589}$.

\section{A la quête d'une adoption par Rolland}

D'une manière générale, les principes traditionnels du régime des services publics ne forment plus un ensemble homogène voué à satisfaire l'intérêt général. La recherche de nouveaux principes attachés au régime des services publics devient alors déterminante afin de relégitimer l'action publique ${ }^{590}$.

Dans cette perspective d'évolution du concept de service public, les usagers doivent demeurer au centre des préoccupations. De toute évidence, les revendications citoyennes, l'insatisfaction des usagers ${ }^{591}$ imposent une réforme ou du moins une clarification, du régime juridique des services publics afin de répondre à la satisfaction de l'intérêt général, finalité du service public.

Pour l'heure, ces nouveaux principes ne constituent pas tous de nouvelles lois du service public ; seule la neutralité semble pouvoir revêtir une telle qualification. Certains principes sont néanmoins amenés à jouer un rôle particulier et peuvent être assimilés à des lois du service public en devenir, telles, la qualité et l'accessibilité. Cela tend par conséquent à démontrer que

$\mathbf{5 8 9}$ DONIER V., « Les lois du service public : entre tradition et modernité », précité.

$\mathbf{5 9 0}$ THOMAS I., «Le principe de participation des usagers au fonctionnement des services publics », RFDA 2004 p. 330 .

591 ROUBAN L., «Le service public face à l'opinion: la fin des clivages idéologiques ? », Rev. adm. 1998, n 305, p. 645-652. 
l'évolution du droit commun des services publics n'est pas achevée ; cette évolution connaîtra sans doute de nouveaux développements à l'avenir.

L'apparition de nouvelles lois du service public et l'évolution intrinsèque des principes traditionnels sous l'influence des règles de bonne gestion ne remettent pas fondamentalement en cause l'unité théorique qui caractérise le droit commun des services publics. Au-delà de leur adaptation, ces principes subsistent et constituent encore aujourd'hui un socle commun à tous les services publics. Pourtant, si ces principes ont effectivement vocation à régir l'ensemble des services, ils ne s'appliquent pas nécessairement de la même manière. L'expérience de la privatisation du secteur de l'eau en Afrique en est une illustration.

\section{§ 2. Les désillusions de la privatisation par rapport aux lois émergeant du service public}

Les principes émergents restent, de manière générale, des principes d'action, ils pâtissent d'ailleurs d'une portée juridique limitée puisqu'ils ont généralement été énoncés par de simples circulaires. La simplicité, l'accessibilité, la sécurité, la responsabilité, la participation, la qualité et l'efficacité ne sont pas vouées à devenir des lois du service public ; il s'agit simplement d'objectifs ou encore de vœux destinés à orienter les politiques de modernisation du service public. Ces principes « relèvent davantage de la profession de foi, voire de l'incantation que de la conceptualisation opératoire $»^{592}$. Si les lois du service public constituent des principes intangibles, dont la pérennité est avérée, la longévité des principes émergents semble beaucoup plus incertaine ; ils ne peuvent donc être associés aux lois du service public reconnues ou en devenir. La catégorie des principes fondamentaux ne doit pas être excessivement étendue, sous peine de perdre de sa cohérence et de son intérêt. Par rapport à la spécificité de la question de l'eau, nous allons nous intéresser uniquement aux principes d'accessibilité (A) et de qualité (B).

\section{A. Le principe d'accessibilité en pointillés}

La notion d'accessibilité géographique n'est pas nécessairement opposable à l'administration, qui conserve généralement la possibilité de supprimer un service public au nom du principe d'adaptabilité.

592 MODERNE F., «Les mutations des services publics en droit français», in Service public et Communauté européenne : entre l'intérêt général et le marché, sous la direction de R. KOVAR et D. SIMON, La Documentation française, Tome 1, 1998, p. 3 . 


\section{L'idée d'accessibilité en matière de service public}

L'accessibilité économique («affordability» ou caractère abordable) correspond à la notion qu'un bien semble avoir un prix «normal» ou n'est pas jugé comme étant d'un prix « inabordable» par l'usager. Il s'agit d'une notion subjective assez mal définie car l'eau est généralement un poste relativement peu important dans le budget de la plupart des ménages et qui varie d'un pays à l'autre en fonction de situations locales telles que l'extension des réseaux et de l'histoire ${ }^{593}$. Analysant les problèmes récents de l'eau dans le monde, l'expert français NGUYEN Tien- Duc explique : "Au sein d'un pays, la logique du marché (de l'eau potable) frappe de manière disproportionnée les plus démunis, en particulier ceux qui n'ont pas les moyens de s'acquitter de leurs factures. Pour ces derniers, l'eau est et restera un bien inaccessible, à moins qu'une politique interventionniste et des mesures appropriées ne soient arrêtées en leur faveur par les pouvoirs publics : rendre obligatoire la fourniture d'eau aux couches pauvres des villes ; définir un tarif socialement acceptable et l'appliquer ${ }^{594}$.

L'accessibilité du service, qu'elle soit intellectuelle ou matérielle, demeure totalement une ligne de conduite ; si elle a permis de mettre en évidence une nouvelle figure de l'usager, la portée normative des règles afférentes à la qualité de citoyen reste incertaine. Néanmoins, ces règles ne sont pas intégralement dépourvues d'effet car elles exercent une certaine influence sur la théorie du service public en favorisant sa mise à jour. L'accessibilité trouve son fondement dans la notion de cohésion sociale et territoriale. Celle-ci semble liée à une exigence d'accessibilité de tous à des services ou prestations, quels que soient les conditions sociales ou le lieu de résidence. Mais, toujours en raison de sa généralité, la notion de cohésion sociale et territoriale ouvre sur des réalités et des degrés d'exigence qui différent selon les Etats.

Le plus souvent, la notion de cohésion sociale et territoriale n'apparaît pas explicitement dans les textes constitutionnels, législatifs ou décrétaux. En revanche, elle est mobilisée dans les travaux préparatoires, les exposés de motifs ou les écrits doctrinaux se rapportant aux différentes formes d'intervention publique. Par exemple, en France, le rapport de Renaud DENOIX de SAINT MARC sur le service public ${ }^{595}$ ouvre le chapitre consacré à la doctrine du service public en affirmant que, " fondamentalement, les services publics sont faits pour satisfaire et assurer en même temps la cohésion sociale ». D'un autre côté, le caractère général de la notion de cohésion sociale et territoriale permet de justifier des actions ou des mesures publiques assez diverses orientées vers l'accessibilité de tous aux services. Paraphrasant Véronique CHAMPEILDESPLATS dans ses travaux sur les «services publics et droits fondamentaux dans la construction européenne ", il convient de s'interroger sur la pertinence d'une référence à la notion de cohésion sociale et territoriale ou aux droits fondamentaux pour justifier que des interventions publiques sous forme de services d'intérêt économique général soient exemptées de l'application des règles communautaire de la concurrence.

593 SMETS H., Le droit à l'eau, CONSEIL EUROPÉEN DU DROIT DE L'ENVIRONNEMENT, 2002, p. 41.

594 Tien-Duc NGUYEN, L'humanité mourra-t-elle de soif?, op. cit.

595 Le Service public, Rapport au Premier ministre, collection des rapports officiels, La Documentation française, 1996, p. 49. 
Sans doute, la notion de cohésion sociale et territoriale et les droits fondamentaux sont bien porteurs d'un pouvoir de justification de l'intervention publique. Mais, comment peut-on concilier les règles de gestion privée et la sauvegarde d'un droit fondamental comme le droit à l'eau?

\section{La cohésion sociale et territoriale et les droits fondamentaux ${ }^{596}$}

On a vu précédemment que la notion de cohésion sociale et territoriale est communément associée à un ensemble de dispositifs concrets (normes spéciales de coupure des prestations, péréquation tarifaire...) permettant d'atteindre l'objectif dont elle est réputée porteuse. Le principe d'un appel aux droits fondamentaux pour justifier l'intervention publique est en revanche moins fréquent, notamment en France, car la reconnaissance de droits et libertés fondamentaux est traditionnellement conçue comme un moyen de protéger les personnes contre l'Etat. Il reste que le droit comparé montre que la justification de l'intervention publique par l'invocation de droits fondamentaux, et plus particulièrement par la nécessité de garantir leur exercice, n'a rien d'exceptionnel. On observe, de façon plus ou moins explicite, de façon plus ou moins systématique selon les ordres juridiques, une justification commune de l'intervention publique par la volonté de garantir l'exercice des droits fondamentaux des individus ${ }^{597}$.

En France, le lien entre les services publics et les droits de l'homme a été magistralement énoncé par René Capitant le 8 mars 1946 au cours des débats de l'Assemblée nationale constituante : "La liberté a besoin, pour être effective, que l'Etat organise ces grands services publics, ces grandes institutions sociales qui sont le moyen pour l'Etat de remplir les obligations nouvelles qu'il contracte envers l'individu et de distribuer à chacun les soins, l'éducation, les secours à défaut desquels il n'y a pas de sécurité sociale et, par conséquent, pas de liberté véritable» ${ }^{598}$.

Il reste que dans bon nombre de systèmes nationaux, les droits fondamentaux permettent d'exiger des autorités publiques certaines prestations positives et, parfois même, de condamner les Etats pour omission ${ }^{599}$. Dès lors, le désengagement de l'Etat dans le secteur de l'eau ne peut susciter que des inquiétudes notamment sur la question de l'accessibilité. Après plus de décennies de privatisation, on peut affirmer, "en ce qui concerne l'efficacité du service, la couverture et l'extension du réseau ont profité aux zones densément peuplées, l'incitation pour élargir l'accès aux zones éloignées et non couvertes n'étant pas suffisante ${ }^{600}$. En conséquence,

596 CHAMPEIL-DESPLATS V., «Services d'intérêt économique général, valeurs communes, cohésion sociale et territoriale », AJDA 1999 p. 959.

597 Voir Antoine LYON-CAEN, Véronique CHAMPEIL-DESPLATS, Services publics et droits fondamentaux dans la construction européenne, Institut international de Paris-La Défense, Dalloz-Sirey, 2001. (non commercialisé).

$\mathbf{5 9 8}$ JO 9 mars 1946, p. 645

599 Trib. constit. 127/1994 du 5 mai 1994, BOE 31 mai 1994

600 Rapport du groupe de travail animé par Monsieur le professeur Jean-Claude BERTHELEMY, professeur à l’Université Paris 1 - Panthéon Sorbonne, Libreville, les 12 et 13 avril 2006. 
dans de nombreux pays, les objectifs de desserte n'ont pas été atteints, même si la qualité du service a été globalement améliorée (création de nouveaux services, baisses des ruptures d'approvisionnement et des pertes).

\section{B. L'importance du principe de qualité en matière de service public de l'eau}

Le catalogue des nouveaux principes tournant autour des services publics ne serait pas complet si l'on ne mentionnait pas le principe de qualité. La qualité répond à une appréciation subjective reposant sur l'aptitude du service à satisfaire les besoins des usagers, et plus globalement, l'intérêt général ${ }^{601}$. La qualité n'a pas vocation à être érigée au rang de loi du service public disait Virginie DONIER ; il ne s'agit pas d'une loi en devenir, mais davantage d'une philosophie guidant les politiques de modernisation des services publics et imposant la satisfaction des besoins des usagers dans des conditions économiques optimales. La qualité inonde en effet l'ensemble du droit commun des services publics, c'est une ligne de conduite qui tend à réunir l'intérêt général et les intérêts des usagers. Elle ne nous semble pas devoir être intégrée parmi les lois en devenir, il s'agit simplement d'un principe directeur qui oriente le service public vers la satisfaction des intérêts des usagers et non vers la seule satisfaction d'un intérêt général désincarné602.

\section{Le concept de la qualité du service public}

L'exigence de la qualité des services publics se révèle de plus en plus importante à mettre en œuvre. La notion de « qualité » demeure, en effet, une notion fondamentalement subjective qui reçoit, de surcroît, de multiples acceptions. Souvent synonyme d'efficacité ou de performance, elle implique également la simplicité, la rapidité ou encore l'accessibilité, autant de notions qui dépassent la summa divisio ${ }^{603}$ des services publics ${ }^{604}$. Deux grandes visions de la qualité des services publics se dégagent néanmoins, selon que l'on analyse les services publics de puissance publique ou les services publics économiques ${ }^{605}$.

601 Rapport remis au Premier ministre par Y. Cannac, La qualité du service public, 18 juin 2004, consultable sur le site www.dusa.gouv.fr./ consulté le 5 janvier 2005.

$\mathbf{6 0 2}$ DONIER V., « Les lois du service public : entre tradition et modernité », op. cit., p. 1219.

603 Locution latine qui littéralement signifie: « la division la plus élevée ». La summa divisio est donc la division la plus élevée avant d'arriver au genre qui est la classe qui englobe l'ensemble des espèces (autres classes). L'expression summa divisio est fréquemment employée dans le domaine juridique pour signaler les divisions principales comme: droit public / droit civil; droit des personnes / droit des biens; responsabilité contractuelle / responsabilité délictuelle...

604 BRACONNIER S., Droit des services publics, PUF, p. 329.

605 Idem. 
L'accès au service public est sans doute la dimension de la qualité qui transcende le plus la distinction entre services publics de puissance publique et services publics économiques. Si elle se révèle, primitivement, orientée vers des critères non économiques ${ }^{606}$. Le refus de raccordement au réseau d'eau potable de terrains non constructibles n'est légal que s'il est justifié par un motif de la bonne gestion et de la préservation de la qualité du service de la distribution de l'eau ${ }^{607}$.

La qualité est en réalité une notion pluridimensionnelle qui suppose une définition au cas par cas, en fonction de l'objet du service et de la prestation fournie ; il s'agit d'un concept nébuleux qui ne peut recevoir de définition univoque. Par ailleurs, elle ne constitue pas, pour l'heure, un principe juridique contraignant : si la qualité semble devenir une véritable exigence censée guider la gestion des services publics, ce principe reste dépourvu de sanction, il demeure un simple objectif visant à orienter les politiques de modernisation des services publics ${ }^{608}$.

En effet, les engagements pris en faveur de la qualité ne se traduisent pas nécessairement par l'émergence d'un droit à la qualité au profit des usagers puisque ces engagements ne sont pas toujours assortis de sanctions : si certaines entreprises publiques ont effectivement prévu une compensation en cas de non-respect de leurs engagements, les démarches de qualité engagées par les services publics administratifs ne présentent pas toujours un caractère contraignant, l'usager ne peut donc pas invoquer un droit à la qualité609. En réalité, l'exigence de la qualité ne doit pas faire l'objet de discussion lorsqu'il s'agit de la qualité de l'eau, tout comme dans le service public hospitalier, qui tend à être de plus en plus soumis à une obligation de sécurité et, implicitement, de qualité610. Cependant, certains auteurs voient dans la qualité une loi du service public en devenir : selon Lucie CLUZEL, le principe de qualité « semble pouvoir combler les lacunes des principes traditionnels, impuissants à rendre compte des nouvelles exigences qui s'imposent désormais aux services publics ». Dès lors, " il peut être vu comme un " grand principe en puissance » distinct des autres principes parce que répondant à une problématique nouvelle ${ }^{611_{\rangle}}$.

S'il apparaît que l'objectif de qualité bénéficie désormais d'une application généralisée puisque tous les services publics semblent être concernés par une démarche de qualité, la qualité ne dispose pas, à l'heure actuelle, d'une force juridique suffisante pour pouvoir être intégrée au sein des lois du service public en devenir. Certes, elle fait parfois naître une véritable obligation assortie de sanctions, mais l'inconstance de cette contrainte invite à exclure la qualité de la catégorie des lois du service public « en puissance».

606 CE, 27 juin 1994, Charpentier, Rec., p. 334.

607 BRACONNIER S. op. cit., p. 333.

608 VOISSET M., «Qualité, adaptabilité, mutabilité », in Service public et Communauté européenne : entre l'intérêt général et le marché, tome 2, op. cit. p. 397

609 DONIER V., « Les lois du service public : entre tradition et modernité », précité.

610 Cette obligation s'applique notamment lorsque le service fait usage de produits de santé : sur ce point, on peut se reporter à CE, 9 juill. 2003, Assistance publique-Hôpitaux de Paris, Lebon p. 338 ; AJDA 2003, p. 1946, note M. DEGUERGUE.

611 CLUZEL L., Le service public et l'exigence de qualité, thèse dactylographiée, Paris II, 2004, Dalloz 2006. p. 569 
A la différence du principe de transparence qui a été imposé à l'administration, et qui fait partie des lois en devenir, la qualité est le plus souvent soumise à la définition et à la bonne volonté de celle-ci ; il ne s'agit pas toujours d'un principe contraignant. Le droit d'accès aux documents administratifs peut être juridiquement sanctionné, tandis que le droit à la qualité n'est pas nécessairement invocable car il n'est pas toujours associé à une obligation de résultat. La transparence fait peser de véritables obligations sur l'administration, ce qui n'est pas forcément le cas de la qualité.

\section{La réalité de la qualité de l'eau distribuée par les concessionnaires}

Privatisation et démonopolisation s'accélérant ces dernières années dans les Etats de l'Afrique subsaharienne, les références du droit à la notion de qualité se sont multipliées, liant de plus en plus cette exigence de qualité à une meilleure reconnaissance des droits des citoyens.

Aux termes de l'article 51 Loi n ${ }^{\circ} 81-13$ du 4 mars 1981 portant code de l'Eau de la République du Sénégal : «les eaux d'alimentation doivent satisfaire les normes de potabilité en vigueur, notamment en ce qui concerne leurs caractéristiques physiques, chimiques, biologique et bactériologiques $»^{612}$.

Les articles 78, 79 et 80 du code de l'eau ${ }^{613}$ en vigueur en République de Côte d'Ivoire prévoient des dispositions presque identiques. En effet, peut-on y lire que «l'eau destinée à la consommation humaine doit être conforme aux normes de potabilité fixées par arrêté conjoint de l'Autorité chargée de l'eau et du Ministre chargé de la Santé ». D'autre part " quiconque offre au public de l'eau en vue de l'alimentation humaine, à titre onéreux ou à titre gratuit et sous quelque forme que ce soit y compris la glace alimentaire, est tenu de s'assurer que cette eau est potable et conforme aux normes en vigueur ». Enfin, "l'utilisation d'eau pour la préparation et la consommation de toute denrée et marchandise destinées à l'alimentation tant humaine qu'animale doit répondre aux normes d'hygiène et de santé publique ».

La qualité de l'eau pose la question de l'élaboration du cadre normatif, du contrôle, des outils de celui-ci, des infrastructures, de la formation du personnel et des effets de leur intervention sur le terrain. Le système de contrôle présente des faiblesses. Au Burkina, jusqu'à récemment, en l'absence de normes nationales et de tout texte sur le contrôle de la qualité, les autorités appliquaient les normes de l'OMS ${ }^{614}$.

Enfin, sur la question de la qualité de l'eau, la Banque mondiale souhaitait accroître la qualité des ressources distribuées par l'entrée d'opérateurs privés dans la gestion de l'eau. Cependant,

612 Voir également ; Loi n ${ }^{81-13 ~ d u ~} 4$ mars 1981 portant Code de l'Eau Art. 52. : Des décrets pris sur rapport des Ministres chargées de l'Hydraulique et de l'Assainissement, classent les cours d'eau, canaux, lacs, étangs ou eaux souterraines en fonction des usages auxquels leurs eaux sont destinées : - Consommation humaine ; - Production d'aliments pour l'alimentation humaine ; - Elevage, agriculture, pisciculture, sylviculture ; - Utilisation minière hydroélectrique ; - Navigation ; - Activités récréatives.

613 LOI n98-755 du 23 décembre 1998 Portant Code de l'Eau

614 LOPA DUFRENOT M., op. cit., p. 101. 
l'absence de transparence sur les méthodes de contrôles employées, notamment quant à l'indépendance de la procédure, et le manque de communication publique des résultats de ces analyses à la société civile et aux autres parties intéressées ne plaide pas en leur faveur et ne peut être que condamnée ${ }^{615}$. La reconnaissance d'un droit à la qualité pour les citoyens dans les services publics régaliens ou sociaux touche ainsi aux ressorts les plus profonds de l'Etat et est susceptible de remettre en question le système juridique ${ }^{616}$.

615 FIDH, Rapport : Mission internationale d'enquête, Droit potable au Niger, p. 39.

616 VOISSET M., «La reconnaissance, en France, d'un droit des citoyens à la qualité dans les services publics », RFDA 1999 p. 743. 
En 1913, le Doyen DUGUIT expliquait que la conscience moderne " n'admet pas par exemple que l'Etat n'intervienne pas dans le service d'enseignement. ...elle n'admet pas par exemple que l'Etat n'organise pas des services d'assistance ${ }^{617} »$. Et le doyen BONNARD abondera dans le même sens, en soulignant, même s'il adoptait un point de vue organiciste, que les services publics sont « destinés à donner satisfaction à certains besoins et intérêts individuels et collectifs de la nation $»^{618}$.

Tous les Etats africains ont opté pour des politiques allant dans la mise en place d'infrastructures sociocollectives gérées par des régies. Le service public était donc incontournable et fonctionnait avec ses tares. Au début des années 1980, il a été opéré un changement dans les politiques économiques et sociales de ces Etats. Si, bien avant cette date, la tendance était une implication de ces Etats dans l'économie et dans les secteurs sociaux, afin de pallier le manque d'initiative privée, une sorte de révolution va s'opérer avec le Consensus de Washington. Un processus de désétatisation va se répandre et va toucher presque l'ensemble des Etats d'Afrique subsaharienne. Dans ce nouvel élan de libéralisation, ces Etats vont opter pour la délégation de service public et spécifiquement dans le cas de la distribution de l'eau. Les spécialistes vont, en marge des conséquences sociales de ce libéralisme, s'interroger sur son influence sur les principes juridiques qui gouvernent le fonctionnement du service public. En droit français, la situation est bien consignée par Christophe Le BERRE dans son article « $L a$ rencontre de l'économie de marché et du service public ${ }^{619}$.

Pour cet auteur, la pénétration de la logique économique dans la définition du service public n'échappe pas à la problématique de la " crise » du service public qu'aurait notamment ressuscitée la nouvelle donne communautaire à l'orée des années quatre-vingt dix. Le débat a cependant évolué et la bannière du " service public à la française » fait long feu. Il est aujourd'hui admis que l'économie de marché et les règles de droit qui en assurent le bon fonctionnement amarrent le service public à un contexte concurrentiel mais n'en signifient pas la disparition ${ }^{620}$. D'ailleurs, si l'on doit déceler une influence des premières sur le second, c'est davantage le régime des services publics qui est touché, en particulier leur statut de monopole ou l'octroi de privilèges d'exclusivité, non la définition du service public. La problématique de la libéralisation des services collectifs a même apporté un éclaircissement en permettant de distinguer les missions de service public de leurs modalités d'organisation ${ }^{621}$.

617 Les transformations du droit public, Armand Colin, 1913, p. 48, réédition La Mémoire du droit, 1999

618 Précis élémentaire de droit administratif, Sirey, 1926, p. 15

619 Le BERRE Ch., « La logique économique dans la définition du service public », RFDA 2008 p. 50.

620 LACHAUME J.-F, « Réflexions naïves sur l'avenir du service public », in Mélanges L. Labetoulle, Dalloz, 2007 , p. 519.

621 du MARAIS B., Droit public de la régulation économique, Presses de Sciences Po et Dalloz, 2004, p. 137. 
Souvent présenté comme un principe récent, l'interventionnisme étatique est apparu en réalité depuis des siècles. On se souvient de COLBERT et KEYNES. L'invitation de l'Etat à intervenir dans l'économie ne doit pas avoir pour conséquence la négation des libertés économiques. Le privilège conféré à l'initiative privée par rapport à l'activité économique des personnes publiques a été contredit par l'ensemble des politiques d'intervention publique et par la jurisprudence du Conseil d'Etat avalisant par exemple l'existence des SPIC. Il peut y avoir des restrictions et elles doivent être justifiées par l'intérêt général. Encore faut-il préciser la nuance entre les expressions «intérêt général», "utilité publique» et «nécessité publique». Pour DELVOLVE P., le vocabulaire n'a pas été toujours d'une égale rigueur. La «nécessité publique » paraît limiter les interventions de l'Etat à des cas exceptionnels ; "l'utilité publique » est déjà une expression plus souple; celle d' "intérêt général» est encore plus approximative et laisse place à une appréciation largement subjective. Elle est finalement le dénominateur commun vers lequel ont convergé toutes les formules et en fonction duquel les restrictions aux libertés économiques et à la propriété se trouvent admises ${ }^{622}$.

Et, en revisitant les origines du droit à l'eau, il est difficile de ne pas considérer la gestion et la distribution de l'eau comme une nécessité publique. Dans bien d'autres situations, situations apparemment manquant de raisons suffisantes, l'intérêt général, pour justifier la qualification de service public, le juge administratif a cependant considéré qu'il y avait du service public ${ }^{623}$. Ainsi, le Conseil d'Etat reconnaît la création d'un service public par les autorités locales alors même que l'activité était susceptible de concurrencer l'initiative privée et ce dès lors, en application de la jurisprudence dite du « socialisme municipal » ${ }^{624}$. Ce ne serait pas une situation nouvelle car le service public de l'eau a toujours existé, et c'est le nouveau régime auquel il sera soumis qui suscite la polémique. A s'en tenir aux législations en vigueur dans ces Etats, le service public de l'eau ne va pas disparaître. Cependant, il risque d'être compromis comme nous l'avons souligné dans nos développements précédents. Les principes ou les « lois » garantissant le bon fonctionnement d'un tel service risquent tout simplement de ne pas être respectés.

De toute façon, les relations entre l'Etat, personne morale de droit public détentrice de la souveraineté sur un territoire donné à l'égard d'une population donnée, et le marché, lieu de rencontre de l'offre et de la demande, font l'objet de théories contradictoires et se sont montrées particulièrement versatiles au fil de l'histoire. $\mathrm{Si}$, pour les libéraux, le marché est capable de s'autoréguler, les interventionnistes considèrent au contraire que les interventions publiques sont nécessaires pour satisfaire l'intérêt général. En France, COLBERT a préconisé de façon précoce l'intervention de l'Etat dans l'économie. Les partisans de KEYNES considèrent pour leur part, que l'Etat doit corriger les défaillances du marché et soutenir la croissance et l'investissement. Et surtout, veiller sur le social. La question de l'accès à l'eau est si importante, si vitale que lorsque sa réalisation se trouve en conflit avec la plénitude d'autres droits et libertés, l'on doit trouver un moyen de les concilier. La libéralisation du secteur de l'eau va engendrer des conséquences dont l'ampleur sera difficile à contenir.

622 Cf. TRUCHET D., Les fonctions de la notion d'intérêt général dans la jurisprudence du Conseil d'Etat, LGDJ, 1977. Voir aussi, DESWARTE MP., « L’intérêt général », RDP, 1988.1289.

623 CE, 24 novembre 1933, Zenard, à propos de la création d'une boucherie municipale ; CE, 15 février 1956, Siméon, à propos de la création d'un service communal de fabrication de glace alimentaire.

624 CE, Sect., 30 mai 1930, Chambre syndicale du commerce en détail de Nevers, Rec. P. 583. 
PARTIE II.

LES ABOUTISSEMENTS DE LA LIBERALISATION DU SECTEUR DE L'EAU 
Alors que le droit à l'eau peine à s'affirmer, la libéralisation du secteur semble ralentir le processus de son émergence. Il est difficile de concilier les intérêts des multinationales avec l'intérêt général. La crise de l'Etat-Providence est certaine en Afrique, à cause du manque de moyens budgétaires. Mais cette crise implique une recomposition dans l'offre de protection sociale. De multiples organisations volontaires se sont substituées à l'Etat: mutuelles, associations, clubs, etc... Par nature, ces organisations sont décentralisées, plus proches des besoins et de la demande des citoyens. Ainsi à travers la crise de l'Etat, doit-on voir une forme particulière d'organisation qui se recompose ? Le marché du développement? Les réalités socioéconomiques de l'Afrique ont conduit à la réalisation de systèmes différents d'approvisionnement en eau potable (AEP) des populations. La plupart des pays d'Afrique ont établi à l'occasion de divers ateliers nationaux et internationaux sur le secteur de 1'AEP, la liste des localités à équiper en système d'AEP urbain et celles qu'il faut équiper en système d'hydraulique villageoise. C'est ainsi que des systèmes d'AEP de type urbain sont réalisés dans plusieurs petits centres urbains alors que la population et les structures économiques sont insuffisantes dans ces petits centres urbains pour assurer une économie d'échelle dans les coûts d'investissement et le prix de revient de l'eau distribuée. Il se pose alors des problèmes d'accessibilité des ménages.

Depuis 50 ans, le PNUD a ainsi recensé 37 cas de violences entre Etats portant sur la question de l'eau, dont 30 ont eu lieu au Moyen-Orient. Cependant, la sagesse des hommes a, à ce jour, permis de mettre un terme aux querelles entre "rivalis », pour reprendre l'expression latine signifiant littéralement, « qui tire son eau du même cours d'eau ». Plus de 3600 traités ont ainsi pu être signés, rendant effectif le concept naissant d' « hydrodiplomatie ${ }^{625}$.

Lorsque des entreprises privées interviennent, ce qui doit être une décision politique, le problème central est de trouver les voies et moyens de préserver un service public équitable et géré efficacement. Sur le continent africain, il n'est plus question de nouveaux grands contrats et il s'agit surtout d'essayer de pérenniser ceux qui existent. Certains se portent plutôt bien et évoluent (comme le très ancien SODECI ${ }^{626}$ en Côte d'Ivoire, le plus récent contrat SEEG au Gabon, ou encore le contrat SDE au Sénégal). En définitive, beaucoup de ces contrats suscitent de nombreuses réserves et les résultats d'ensemble sont, de l'avis même d'experts de la Banque mondiale et de l'OCDE, mitigés ${ }^{627}$.

625 GALLAND Franck, «L'eau : une priorité stratégique », sources non disponibles.

626 Société de distribution d'eau de la Côte d'Ivoire

${ }^{627}$ BOTTON S, « Les multinationales de l'eau et les marchés du Sud : pourquoi Suez a-t-elle quitté Buenos Aires et La Paz ? » Collection débats et controverses $-\mathrm{n}^{\circ} 1-$ page 11 
TITRE I.

LES APPREHENSIONS SUSCITEES PAR LA PRIVATISATION DU SERVICE PUBLIC DE L'EAU 
La libéralisation des services publics industriels et commerciaux finit par toucher tous les secteurs, y compris ceux de l'eau et de l'assainissement. Si, en Europe, ce phénomène est largement lié au développement du marché unique, en Afrique, il est dû surtout à la gestion peu ou très peu décente de la question de l'accès à l'eau. Au sein des Etats européens, parmi les services publics en réseau, ceux de l'eau semblent avoir été exclus des restructurations observées dans les autres secteurs jusqu'à très récemment, si ce n'est que le recours à la gestion déléguée et à différentes formes de privatisation s'est fortement développé au cours des vingt dernières années $^{628}$. En constatant les désillusions de la privatisation des entreprises publiques en Afrique, le Professeur VIGNON avait affirmé que « de nombreux gouvernements désireux de contenir le déficit de leurs finances publiques, sans alourdir le poids de la fiscalité, ou sans accroître le volume de leur endettement, ont opté pour le transfert au secteur privé d'un grand nombre de leurs entreprises ${ }^{629} »$. La quintessence de nos travaux est de savoir si les services de l'eau sont susceptibles de faire l'objet des mêmes restructurations que les autres services à caractère industriel et commercial, en se demandant si les services de l'eau peuvent être considérés comme des services d'intérêt général au même titre que les autres. Cette problématique découle fondamentalement de la nature même du bien qu'est l'eau. Cette position rejoint le consensus dégagé lors du Forum Mondial de l'eau en 2000 définissant l'eau comme un bien économique.

Toutefois, la privatisation du secteur de l'eau ne peut réussir que " $s$ 'il existe des Etats démocratiques forts, capable d'empêcher au marché d'embrigader les populations dans une logique économique suicidaire. Or, la capacité de régulation des Etats s'effrite au jour le jour, depuis Thomas HOBBES ${ }^{630}$, et devient même nébuleuse aujourd'hui avec la mondialisation actuelle de l'économie ». A ce jour, un des enjeux majeurs pour sécuriser les besoins en eau est la gestion de la ressource en partant de ses valeurs économiques, sociales et culturelles dans tous ses usages, afin de se diriger vers une tarification des services de l'eau qui reflète le cout d'acheminement et de potabilisation de l'eau, tout en maintenant une place centrale a la nécessité d'accès universel a l'eau, en tant que bien commun. Par ailleurs, la Banque mondiale et le Fonds Monétaire International avaient entrepris une promotion agressive en faveur de la privatisation de l'eau dans les pays en développement. La porte est ainsi grand ouverte pour que les grandes compagnies transnationales des eaux tirent des profits des systèmes de distribution des eaux et du traitement des eaux usées ${ }^{631}$.

628 MOREL B., Pour une gestion raisonnée de l'eau et de ses services en Europe , Memoire de $4^{\text {ème }}$ annee d'I.E.P., Strasbourg, Juin 2007, p. 7.

629 VIGNON Y., «Réflexions sur la privatisation des entreprises publiques en Afrique », Annales de l'Université du Bénin, Série Droit Economie, Tome XVI, 1996, p. 214.

630 Pour HOBBES, « l'Etat est superstructure qui viendra réguler le secteur public, principale zone de conflit entre les hommes, à cause du politique dont la légitimité se trouve dans la démocratie, tout en laissant la régulation privée aux particuliers après en avoir défini les modalités ». $C f$. ANOUGOU J. P. Thiéry, «L'eau, bien public, bien privé : l'Etat, les communautés locales et les multinationales » in L'eau, patrimoine commun de l'humanité, Alternatives Sud, Vol. VIII, $\mathrm{N}^{\circ} 4,2001$, p.163.

631 BARLOW M., «Privatisation de l'eau et des autres ressources environnementales », Source : wwww.urfig.org./ consulté le 26 juin 2007. 
En vertu de cette nouvelle disposition de l'OMC ${ }^{632}$, une règle nationale qui protège l'eau en tant que service public et droit fondamental pourrait être considérée comme un « obstacle non tarifaire » au commerce. Elle pourrait donc être éliminée tout comme d'autres règles visant à limiter la privatisation. Aussi, cet organisme a-t-il lancé en 1994 L'Accord général sur le commerce des services ${ }^{633}$.

Aussitôt, de nombreuses voix se sont élevées pour reprocher à l'AGCS d'avoir un objectif, dans le cas particulier des services, qui se concentrerait uniquement sur la marchandisation et la valorisation commerciale des services publics essentiels, prenant ainsi le pas sur le bien-être réel des populations concernées. Concilier service public et concurrence, ..., est chose difficile. Avec l'interprétation de règles d'esprit parfois opposé, vivons-nous une simple évolution de la notion de service public, dont l'histoire a montré la plasticité et les capacités d'adaptation ? Un renouveau ? ou une révolution, une rupture pouvant se traduire par l'abandon des mots mêmes de " service public » ..., au profit de notions telles que l'utilité publique, par exemple, au moins pour les services publics industriels et commerciaux ${ }^{634}$ ?

632 L'eau est clairement un «bien» dans l'Accord général sur les tarifs douaniers et le commerce (GATT 94). L'article 11 exclut déjà toute restriction quantitative sur l'exportation d'un bien tout en permettant des mesures tarifaires telles que taxes et régimes du double prix. Pourtant, le nouveau texte propose d'éliminer de tels contrôles sur les exportations, rendant ainsi illégale toute exportation d'eau en gros pour des raisons commerciales.

633 AGCS ou en anglais GATS pour General Agreement on Trade in Services.

634 VOISSET M., « Le service public autrement », RFDA 1995 p. 304. 
CHAPITRE I. LES CONSEQUENCES NEFASTES DE LA LIBERALISATION DU SECTEUR DE L'EAU 
La libéralisation du secteur de l'eau confirme la dimension économique de l'eau potable qui repose sur plusieurs éléments. Proposée par les institutions de Bretton Woods comme la solution ultime au problème d'accès à l'eau, la libéralisation s'est révélée plutôt controversée. Pour devenir potable et accessible sous cette forme, elle a d'abord subi une extraction, un traitement et un transport lié à sa distribution dégageant un «coût technique » ou "coût d'approvisionnement ». Par ailleurs, cette eau devenue potable, mais qui aurait pu être dirigée vers d'autres usages, représente pour la collectivité une option différente de ce qu'elle aurait été dans un autre cas : on parlera alors de son " coût d'opportunité », tenant compte de la rareté de la ressource afin d'éviter son gaspillage. Enfin, le prélèvement de l'eau, son traitement et son transport engendrent des conséquences diverses en l'occurrence des conséquences sociales, environnementales, économiques... Aussi, la libéralisation du secteur de l'eau va transformer va remettre en cause l'Etat dans son approche régalienne. D'un côté, l'on va assister au recul de l'établissement public et d'autre part, le service de distribution de l'eau va se dégrader davantage.

Nous allons examiner d'une part les déboires engendrés par la libéralisation du secteur de l'eau (Section I) avant de montrer comment elle révèle par moment le déni de l'Etat (Section II).

\section{Section I. Les déconvenues engendrées par la libéralisation du secteur de l'eau}

BOURDIEU écrivait : "Le fatalisme des lois économiques masque en réalité une politique, mais tout à fait paradoxale, puisqu'il s'agit d'une politique de dépolitisation; une politique qui vise à conférer une emprise fatale aux forces économiques en les libérant de tout contrôle et de toute contrainte en même temps qu'à obtenir la soumission des gouvernements et de citoyens aux forces économiques et sociales ainsi libérées ${ }^{635}$. Et c'est dans cette optique que s'inscrivait le Consensus de Washington selon lequel il faut prôner favorable à l'émergence des investissements privés. Malheureusement, ces investissements ne profitent pas forcément aux populations des Etats africains subsahariens. Cette libéralisation, loin de favoriser l'amélioration des services de distribution de l'eau (§1), va plutôt causer sa décadence d'où la désillusion des usagers $(\S 2)$.

\section{§1. L'amélioration du service de distribution de l'eau, un espoir non tenu}

Contrairement aux prévisions des grands théoriciens de l'économie libérale qui pensent que la libéralisation est le remède miracle à toute gestion défaillante, la libéralisation du secteur de l'eau a montré les insuffisances de cette orientation. Ainsi, faut-il examiner l'essor de l'approvisionnement alternatif non institutionnel (B) mais avant, nous allons examiner l'essor mitigé de la marchandisation (A).

635 BOURDIEU P., cité par ZIEGLER J., Les nouveaux maîtres du monde et ceux qui leur résistent, Fayard, Paris, p. 66 . 


\section{A. L'essor mitigé de la marchandisation de l'eau}

Selon PETRELLA R. ${ }^{636}$, le rapport sur l'eau qui est présenté au Forum mondial de l'eau, à Istanbul, a réaffirmé le lien entre pauvreté et problème d'accès à l'eau. Cette avancée date de 2006, avec l'élaboration du programme des Nations unies pour le développement. Pour le reste, PETRELLA déplore le fait que «la position des Nations unies reste sous l'influence du Conseil mondial de l'eau (CME) [qui est lui-même sous l'influence] ... des multinationales du secteur ». Cet organisme de droit privé a réussi à s'imposer comme un acteur incontournable auprès de nombreux gouvernements et institutions internationales.

\section{Un essor justifié}

Il est déplorable de constater qu'en Afrique, on est arrivé à une situation d'échec quasi général des régies publiques. Ainsi à Kinshasa, on constate un pillage de l'eau potable, une extension de la corruption, des trafics d'eau, et le fait notable que l'Etat et les entreprises publiques, utilisateurs importants, sont les premiers à ne pas payer. La régie publique, par exemple la REGIDESO ${ }^{637}$ en RDC, ne recouvre quant à elle que $15 \%$ de ses recettes normales du fait de ces dysfonctionnements. Pour la majorité des populations n'ayant pas accès à l'eau des régies publiques, les prix sont très élevés. Au cul des camions le mètre cube revient à 7 dollars contre quelques centaines de francs congolais au départ ${ }^{638}$. Les contre performances de la gestion publique constatées ont catalysé la prise en main par les trois grands leaders mondiaux de l'eau: Veolia-Vivendi (Algérie, Burkina Faso, Gabon, Maroc, Niger, Tchad) ; Saur (Afrique du Sud, Côte d'Ivoire, Centre Afrique, Mali, Mozambique, Sénégal) ; Suez (Afrique du Sud, Cameroun, Guinée-Bissau, Maroc).

La multiplication des entreprises de distribution d'eau a connu une expansion vers la fin des années 1980 et tout le long des années 1990. Les trois groupes historiques de la gestion de l'eau en France, vont s'étendre sur les cinq continents. La lyonnaise des eaux, la Compagnie générale des Eaux et la Saur, forte de leur expérience française et de l'assise financière qu'elle leur procure, conduisent une expansion internationale qui paraît irrésistible ${ }^{639}$.

La conséquence est la vente systématique de l'eau quelque soit le revenu des citoyens. Alors que bien avant l'émergence et la prolifération de la technique de la délégation, la vente de l'eau n'était pas généralisée dans ces Etats. Les bornes-fontaines offraient de l'eau gratuitement aux populations démunies. L'essor des investissements dans le secteur de l'eau et ses conséquences

636 Article paru le 16 mars 2009 dans L'Humanité.

637 La REGIDESO (pour Régie de distribution d'eau) est une entreprise publique de la République démocratique du Congo chargée de la distribution d'eau potable sur l'ensemble du territoire national, autant en zone urbaine qu'en zone rurale. Cette entreprise publique autonome est sous la tutelle des départements de Mines et Énergie et du Portefeuille. La compagnie fut créée en 1933

638 CAMDESSUS M., Eau, le scandale de la soif dans le monde, Paris, R. Laffont, 2004

639 TAITHE A., op. cit., p. 42. 
néfastes en ce qui concerne l'accès à l'eau, ne devraient constituer une surprise car l'entreprise privée a pour vocation la recherche du profit. On comprend donc pourquoi il y a eu cette ruée vers l'or bleu en Afrique subsaharienne. Toutefois, il faut le souligner, la délégation de service n'a pas été littéralement généralisée.

\section{Un essor mâtiné}

Mais les conflits surgissent dès la moitié des années 1990, quand les opérateurs privés entendent faire payer des usagers fraîchement raccordés, qui n'ont ni la culture du paiement d'un bien jusqu'alors largement subsidié par la puissance publique, ni, le plus souvent, les moyens de payer. De nombreuses luttes se succèdent sur les cinq continents. La tenue des premiers grands forums altermondialistes publicise le thème du refus de la «marchandisation » de l'eau ${ }^{640}$.

Les entreprises d'Afrique de l'Ouest ont en général des tarifs plus élevés que celles d'Afrique de l'Est. Cette comparaison, peut cacher des différences liées à la valorisation de la monnaie locale par rapport à l'euro, le franc CFA restant une monnaie forte grâce à la protection accordée par la Banque de France. Ces tarifs plus élevés permettent aux entreprises d'Afrique de l'Ouest de recouvrer une part plus importante de leurs coûts opérationnels que leurs homologues d'Afrique de l'Est, à l'exception notable de l'Ouganda qui recouvre une part importante de ses coûts opérationnels $(120 \%)$ avec des tarifs acceptables. Par contre, les entreprises publiques en Zambie comme en Tanzanie appliquent des tarifs extrêmement bas, ce qui rend leur santé financière fragile et se reflète dans des performances techniques et commerciales médiocres ${ }^{641}$

\section{B. L'essor de l'approvisionnement alternatif non institutionnel}

En réalité l'expression «approvisionnement alternatif» n'a de sens que si l'on compare ce mode d'approvisionnement à celui des réseaux d'adduction d'eau. Avant même l'apparition des Etats sur le continent, il n'existait pratiquement pas de structure collective d'approvisionnement en eau. Les populations s'approvisionnaient en grande majorité dans les marigots ou dans les cours d'eau. Ceci explique la tolérance acceptée à l'endroit de l'approvisionnement alternatif quand bien même, cette pratique fait l'objet de diverses controverses.

640 LAIME M., « La marchandisation de l'eau s'accélère », Le Monde Diplomatique du 19 mars 2008, http://www.monde-diplomatique.fr/carnet/2008-03-19-La-marchandisation-de-1-eau consulté le 31/07/08.

641 Idem. 


\section{Une pratique socialement acceptable}

L'incapacité des multinationales bénéficiaires des délégations de service public de distribution de l'eau oblige certains des usagers de recourir à des solutions alternatives ou parallèles. Cette réaction est une réponse à la défaillance, à la rupture voire à l'inexistence du service public de l'eau. Des solutions de substitution sont toujours possibles lorsqu'un service public s'avère défaillant. Les exemples sont multiples : en effet, quand surviennent les dysfonctionnements dans la fourniture publique de l'électricité, les usagers peuvent recourir aux régénérateurs électriques, à l'énergie issue des panneaux solaires, bref d'autres formes d'énergie en vue de satisfaire des besoins domestiques, industriels privés et même des besoins d'intérêt général. En cas d'inexistence du service d'assainissement à l'échelle communale, des particuliers ou des industriels peuvent recourir à la fosse septique dans le but d'évacuer des eaux usées.

De la même manière, lorsqu'apparaissent des difficultés d'approvisionnement en eau potable en réseau public, les populations s'approvisionnent en eau courante autrement, soit en captant directement les eaux brutes superficielles ou souterraines, soit en achetant l'eau minérale en bouteille ou encore en l'achetant au revendeur ou au voisin. Même si le recours à des prestations de substitution peut parfois être interprété comme un luxe, il est d'abord une nécessité642. En Afrique subsaharienne, le recours à l'approvisionnement en eau hors réseau est une réponse aux dysfonctionnements techniques de la distribution publique.

Cette technique n'est plus qu'une réalité sociologique pouvant s'analyser par ses origines lointaines et sa consécration par le droit moderne. Le captage de la ressource des cours d'eau et des nappes, est une pratique ancienne. Cette technique a eu également cours dans des pays du nord jusqu'à une époque récente. En France, par exemple, des réseaux de distribution de l'eau potable, jusqu'au début des années 1940, n'existaient que dans les grands centres urbains ${ }^{643}$. Les populations des communes rurales s'alimentaient en eau en la prélevant directement des puits ou des cours d'eau. Près d' $1 \%$ des communes ne sont pas encore équipées d'un réseau de distribution d'eau potable. Si le captage direct de l'eau des sources précitées devient marginal dans les pays du nord, il reste encore très répandu en Afrique. Ainsi des populations captent gratuitement l'eau des rivières, des étangs, des lacs, des fleuves et des nappes pour subvenir aux besoins domestiques ${ }^{644}$.

Mais c'est surtout une seconde approche qui consacre explicitement le droit des populations au captage des eaux douces, des nappes ou des cours d'eau, dans les limites d'usage domestique. Il en est ainsi de l'article 11 du Code de l'eau du Congo-Brazzaville qui le prévoit, en ces termes: «Toute personne détenant un titre régulier d'occupation d'un fonds peut capter et traiter, dans la limite de ce qui est nécessaire pour son usage personnel, les eaux d'une source qui y prend

\footnotetext{
642 OSSOMBO R., précité, p. 573.

643 CATY G., « Histoire d'eau, Frotin «Curator aquarum », RA, 1966, p.339 ; SAYOUR A., « Aspects économiques et financiers du problème des distributions d'eau », $R A, 1953, \mathrm{p} .367$.
}

644 OSSOMBO R., précité. 
naissance, d'un cours d'eau qui le borde ou le traverse ou d'une nappe constituée dans son sous-sol $[\ldots] »^{645}$.

L'approvisionnement alternatif est pratiqué sous plusieurs formes : revente par camionsciternes, livraison à domicile par des marchands colporteurs, revente au voisinage par des abonnés au service, etc. Ces services alternatifs participent considérablement de la satisfaction des besoins d'eau des familles non abonnées à la distribution publique ou victimes des dysfonctionnements périodiques de la même distribution. A Bangui, à Brazzaville, à Kinshasa et à Yaoundé, la distribution de l'eau hors réseau représente plus de la moitié de l'approvisionnement global. La reconnaissance sociale du phénomène ne le décharge pas de critiques. Appréhendé sous tous ses angles, l'approvisionnement alternatif informel en eau peut poser de sérieux problèmes juridiques aussi à l'égard des distributeurs publics (ou délégués) qu'à l'égard des pouvoirs publics et des populations.

\section{Une pratique préjudiciable pour les usagers et les concessionnaires}

Le développement de la fourniture concurrente de l'eau peut constituer un handicap à l'essor du réseau, jadis public mais aujourd'hui concédé, dès l'instant où des usagers potentiels, enclins à des prestations moins contraignantes en termes d'accès, rechignent à s'abonner au service public. De plus, l'eau des revendeurs coûte plus chère que celle vendue par les gestionnaires de réseau. Son prix est soumis aux conjonctures techniques de la distribution publique. Les revendeurs spéculent quand intervient l'intermittence des prestations fournies par les opérateurs historiques. En Afrique centrale, elle peut coûter deux à trois fois plus chère et peut représenter près du tiers du chiffre d'affaires de la vente globale de l'eau ${ }^{646}$. Par ailleurs, le système est souvent pratiqué en dehors de tout cadre juridique.

Dans toutes les grandes métropoles de la sous-région, il est fréquent de constater des cas de revente clandestine de l'eau par des abonnés du service au voisinage, en toute illégalité. Dans certains cas, ils peuvent alimenter jusqu'à une dizaine de familles et même tout un quartier. Il est vrai qu'en termes de vente au mètre cube, le gestionnaire du réseau public n'est directement pas perdant, mais en termes des revenus d'abonnement, la rémunération de dix familles, par exemple, associées à leurs consommations, devraient lui rapporter plus. Les pertes subies par les organismes en charge de la distribution publique de l'eau sont donc considérables. De même, les vendeurs à la criée, les transporteurs d'eau par camions-citernes ou par charrette ne sont pas souvent titulaires d'un titre qui leur permet d'exercer cette activité. Ils ne sont pas non plus imposables lorsqu'on sait que leurs revenus mensuels dépassent souvent ceux des agents d'exécution de l'organisme gestionnaire du réseau public, même si les conditions de travail ne

645 Cf. également l'article 10 de l'avant-projet de Code de l'eau de la République démocratique du Congo: « Des prélèvements et utilisations de l'eau du domaine public hydraulique à des fins domestiques peuvent être librement pratiqués. Sont considérés comme étant pratiqués à des fins domestiques, tous prélèvements et utilisations de l'eau destinée exclusivement à la satisfaction des personnes physiques dans les limites des quantités d'eau nécessaires à la consommation humaine, aux soins d'hygiène, au lavage et aux productions végétales ou animales réservées à l'alimentation de la famille $\mathrm{d}$ ces personnes $»$.

646 BAUER A., « Accès à l'eau : la décennie perdue », Les Echos, 24 novembre 2006. 
sont pas les mêmes. Ainsi, l'approvisionnement informel rivalise fortement avec l'approvisionnement public.

Cette concurrence est tout à fait déloyale, puisqu'il n'existe aucun contrôle sérieux de la prestation. Cette absence de contrôle augmente les risques sanitaires humains liés à l'usage de cette eau. Alors que l'eau fournie par les opérateurs publics reste d'une qualité douteuse, du moins dans la plupart des cas, des risques sanitaires des usagers sont donc décuplés en consommant l'eau issue de cet approvisionnement parallèle. Même si dans une confusion générale, il est fait allusion à des maladies liées à la consommation d'une eau de mauvaise qualité, dans la sous-région, il n'a jamais été précisé qu'une grande partie des pathologies en cause provient, sans doute, de ce réseau parallèle d'approvisionnement en eau. Ces risques sanitaires ne concernent pas que des populations urbaines des pays d'Afrique subsaharienne, mais aussi celles de tous les pays en développement non raccordées au réseau. Chaque année, dans les pays en développement, surviennent des cas pathologiques issus de cet approvisionnement de substitution. Certains peuvent d'ailleurs prendre des allures épidémiques telles que celles survenues en Afrique du sud et au Nigeria, au début des années $2000^{647}$. Vue ainsi, la revente de l'eau reste donc l'un des facteurs de l'aggravation de l'expansion des maladies hydriques dans les villes d'Afrique centrale et dans bien d'autres agglomérations de l'Hémisphère sud. Si les risques sanitaires existent dans la distribution publique de la ressource, ils sont, par contre, quintuplés ou décuplés dans l'hypothèse d'une distribution informelle ${ }^{648}$.

\section{§2. La désillusion des usagers du service de distribution de l'eau}

L'espoir, suscité au lendemain de l'accession des Etats africains à la souveraineté internationale par rapport à la possibilité offerte aux jeunes Etats de s'offrir les infrastructures socio collectives devant contribuer à l'épanouissement des citoyens, a été vite déçu. D'une part la mauvaise gestion n'a pas permis la réalisation de ces ouvrages, d'autre part le remède proposé par les institutions financières internationales a montré ses limites. L'universalisation de l'eau potable a échoué (A) et cela a engendré un impact sanitaire notable (B).

\section{A. L'échec de l'universalisation de l'eau potable}

L'universalité ou l'universalisation de l'eau implique ${ }^{649}$ ainsi une couverture nationale, une prise en charge publique et parfois privée des consommations des populations les plus démunies et la réalisation d'investissements nécessaires aux adaptations et aux évolutions du service par

647 OSSOMBO R., précité, p. 580.

648 Idem.

649 SMETS H., Pour un droit à l'eau potable, Ed. Académie de l'eau, Nanterre, 2004, p.65 ; « Les implications juridiques et économiques du droit à l'eau potable », Communication présentée aux Vème Journées scientifiques du Réseau droit de l'environnement de l'Agence universitaire francophone tenues du 6 au 8 novembre 2004 à Bucarest. 
les pouvoirs publics. Ce qui rapproche le service de l'eau du " service universel » tel qu'il est expliqué par la plupart des auteurs. Le service universel consiste à l'accès un service de base sur l'ensemble du territoire d'ne certaine qualité à un prix abordable justifiant une exception à l'application de la concurrence entre personnes en principe privées. Cette optique est différente de la notion française du service public qui jusqu'à maintenant supposait l'idée d'une intervention de la puissance publique contrôlant l'accomplissement des missions d'intérêt général. Si le service universel n'est pas obligatoirement public il a tout de même une vocation commune avec le service public à la française. C'est un instrument de cohésion sociale écrira DEBBASCH C ${ }^{650}$., et c'est justement dans cette direction que nous orientons notre réflexion. Nous constatons malheureusement les tentatives de l'universalisation de l'eau potable ont échoué.

\section{Sur le plan financier}

L'échec de l'universalisation de l'eau potable dans les Etats de l'Afrique subsaharienne, tout au moins dans son volet financier, découle d'un défaut de coordination des acteurs associés au financement du service : Etats concernés, institutions financières multilatérales, pays riches et des multinationales. Pendant les années 1960 pour certains et 1970 pour d'autres, ces Etats ont créé les sociétés nationales de gestion d'eau. Capitalisées avec des recettes fiscales de jeunes Etats, ces sociétés avaient la mission de distribuer de l'eau sur tout le territoire national. Elles ont, jusqu'au début des années 1970, constitué des supports considérables à la politique de l'aménagement du territoire. On ne doit pas perdre de vue qu'à cette époque, le taux de la croissance urbaine était inférieur à celui qui frôle les $5 \%$, à ce jour ${ }^{651}$. Il était donc possible pour les autorités locales et nationales de contenir cette croissance et, de l'assortir de la construction des infrastructures adéquates connues. Mais le manque de ressources financières adéquates a vite hypothéqué cette ambition. L'essentiel des ressources des collectivités publiques provenant des impôts, celles-ci ne sont pas arrivées à rendre effectif le rêve de l'eau pour tous. Sans le soutien de l'Etat, les communes ne peuvent construire les ouvrages hydrauliques. Les impôts locaux seuls ne pouvaient suffire pour financer les opérations d'extension, d'agrandissement du réseau d'eau potable. La situation va s'aggraver avec l'explosion démographique des grandes villes au lendemain des années 1960. Cette hausse de la population provient, en partie, de l'exode rural. Dans ces pays où le taux de chômage franchit facilement les $50 \%$, la ville est perçue comme le seul cadre d'intégration sociale et professionnelle. C'est pourquoi, les flux migratoires interviennent vers les plus grands centres urbains. Cette augmentation de la population a eu (et aura toujours) les incidences sur la distribution de l'eau potable, de l'électricité et l'assainissement. Lorsque la demande est, en effet, supérieure à l'offre, les capacités de production des réseaux finissent par s'avérer insuffisantes. Ainsi se brise le rêve d'une eau accessible à tous à un vil prix.

Dans un rapport conjoint, l'Organisation mondiale de la santé (OMS) et le Fonds d'urgence des Nations Unies pour l'enfance (UNICEF) ont souligné qu'en Afrique, les financements

650 DEBBASCH C. et COLIN F., Droit administratif, précité, p. 364.

$651 C f$. Atlas de l'Afrique, Editions du Jaguar, $2^{\text {ème }}$ éd. Paris, 2000, p.52. 
extérieurs représentent plus du tiers des ressources affectées au service public de l'eau potable ${ }^{652}$. Quelques années plus tard, l'Organisation des Nations Unies a noté que « rares sont les pays en développement à considérer l'eau comme un élément clé de leurs projets et budgets nationaux $»^{653}$. Face à l'insuffisance de leurs ressources dans le financement du service, le recours, par ces Etats, à des financements alternatifs, externes notamment, devient une nécessité. C'est pourquoi, ils ont contracté plusieurs prêts auprès de banques privées du nord en vue du financement de grands travaux, sauf que ces fonds ont été mal utilisés.

\section{Sur le plan politique}

Les Etats africains n'ont pas toujours élaboré une politique de l'eau très lisible. Il importe cependant de souligner l'influence de la Conférence des Nations Unies sur l'eau de Mar Del Plata en 1977 à laquelle ces Etats ont participé. En effet, ayant reconnu implicitement, à l'issue de cette conférence, l'eau potable comme un bien fondamental dont l'accès à tous doit être un droit, certains ont commencé par mettre sur pied une politique appréciable de l'eau. Les principes découlant de cette rencontre internationale n'ayant aucun caractère contraignant, ces Etats n'avaient pourtant aucune obligation de les transposer dans leur droit interne. On suppose aussi que ces pays sont censés respecter un droit à l'eau potable devant découler du droit à un environnement sain que proclame la Charte africaine des droits de l'homme et des peuples ${ }^{654}$ et leurs constitutions respectives ${ }^{655}$. En appliquant les critères du service universel (ou d'autres critères standard) au service de l'eau potable des Etats d'Afrique subsaharienne, on découvre aisément que cette activité, à l'échelle urbaine, n'obéit à aucune logique de « répartition ». Le service, en effet, n'obéit ni au critère de l'accès généralisé des populations au réseau, ni à celui de la couverture territoriale, fût-elle urbaine, ni à celui du caractère abordable du prix des prestations, ni à celui de la prise en charge des consommations des usagers les plus démunis par les pouvoirs publics encore moins à celui de la tarification sociale (péréquation).

L'égal accès au service n'est pas garanti à tous par l'organisme public chargé d'en assurer la tutelle. Déjà, la connexion au réseau n'est réservée qu'à des classes moyennes et aux catégories privilégiées. Le caractère élevé du coût de celui-ci disqualifie souvent les classes défavorisées du bénéfice de la prestation. Même lorsqu'elles possèdent des moyens financiers nécessaires à ladite connexion, ces classes n'ont aucune assurance dans l'accomplissement de la démarche. Peuvent également intervenir plusieurs délais d'attente confirmant l'existence d'autres paramètres entre autres subjectifs et parfois non conventionnels.

Les critères d'accès au réseau ne sont donc pas toujours objectifs, et de nombreux observateurs s'accordent à soutenir que les pouvoirs ne fournissent aucun effort pour mettre fin à

$\mathbf{6 5 2} \mathrm{OMS} / \mathrm{UNICEF}$, Rapport sur l'évaluation de la situation mondiale de l'approvisionnement en eau potable et de l'assainissement en 2000, Genève, 2000, p.16

653 ONU, «L'eau : une responsabilité partagée », Deuxième rapport mondial sur la mise en valeur des ressources en eau, Paris, New York, 2006, p.44.

$\mathbf{6 5 4} C f$. Article 24 de la Charte africaine des droits de l'homme et des peuples de 1981 : «Tous les peuples ont droit à un environnement satisfaisant et global, propice à leur développement ».

$655 C f$. art.53 de la Constitution de la République démocratique du Congo du 18 février 2006 ; Cf. également art.27 de la Constitution béninoise du 11 décembre 1990 ; 
ce défaut de transparence en rendant, par exemple, opposable le droit au raccordement des foyers situés dans les zones desservies par le réseau public de distribution d'eau. Il ne fait guère de doute que la couverture urbaine en approvisionnement en eau potable en Afrique subsaharienne reste encore très faible. Contrairement à certaines métropoles du continent qui ont franchi le taux de desserte de $80 \%{ }^{656}$, rares sont les communes qui atteignent les $50 \%$. L'eau n'est, en effet, desservie que dans certains quartiers, en particulier les zones résidentielles et le centre-ville. Il se trouve qu'une grande partie des populations habite des quartiers périphériques. Donc, elles sont ipso facto déconnectées au réseau. Par ailleurs, les Etats de l'Afrique subsaharienne n'ont pas mis en place des politiques publiques tendant à la prise en charge des consommations d'eau des démunis par les pouvoirs publics ni une tarification inspirée de la péréquation entre consommateurs. L'universalisation de l'eau potable en Afrique est donc aussi un échec qui se manifeste également sur le plan sanitaire ${ }^{657}$.

\section{B. L'impact sanitaire de la libéralisation du secteur de l'eau}

La pénurie d'eau potable est la première cause de mortalité au monde avec 3 millions de victimes annuelles ${ }^{658}$, soit la population d'un Etat comme le Congo-Brazzaville, les Emirats Arables Unis, le Libéria, la Lituanie, le Panama et l'Uruguay. Le phénomène devance ainsi le paludisme, le sida et les accidents. Ces victimes sont, à $90 \%$, constituées d'enfants de moins de cinq ans.

\section{Une mise en péril de la sécurité alimentaire}

L'eau potable est une composante non substituable de la sécurité alimentaire. Contrairement à une conception très répandue, la sécurité alimentaire ne se réduit pas à la consommation de la nourriture, mais elle est aussi extensible à l'usage de l'eau potable. Selon l'Organisation Mondiale de la Santé, un individu doit boire deux litres d'eau par jour pour se maintenir en bonne santé. Le corps humain est essentiellement composé d'eau puisque celui d'un adulte sain de $70 \mathrm{~kg}$ en contient environ 45 litres. La teneur moyenne en eau varie au cours de la vie : de 97 $\%$ chez le foetus de deux mois, elle tombe à $75 \%$ chez le nouveau né, à $65 \%$ chez l'adulte et à $55 \%$ chez le vieillard. Cette quantité peut aussi varier d'un individu à un autre. Elle peut être de $58 \%$ chez une personne maigre et de $67 \%$ chez une personne ronde. L'eau, souvent stockée dans les cellules, représente environ $40 \%$ du poids du corps de l'adulte de $70 \mathrm{~kg}$ et joue un rôle capital dans la constitution du sang et du plasma. La répartition de l'eau dans l'organisme humain varie selon l'organe et les tissus ${ }^{659}$.

656 BRIAND A., LEMAITRE A., « La privatisation de la distribution d'eau potable en Afrique : une aubaine? », Actes de la journée d'étude « Les territoires d'eau », Université d'Artois, Arras, 26 mars 2004, p.98.

657 OSSOMBO R., précité, p. 428.

$\mathbf{6 5 8}$ Selon le rapport PNUE-OMM-GIEC, « Le changement climatique et l'eau » de 2008, le nombre de catastrophes naturelles entre 1950/1980 et 1996/2005 a doublé au plan mondial. Cf. aussi DAVID C., « Les batailles de l'or bleu », L'Expansion, juillet-août 2006, p.64.

659 OSSOMBO R., op. cit. 
L'eau représente dans les substances cérébrales $80 \%$; elle correspond à environ $30 \%$ dans le cour, les poumons et le sang. Le squelette humain en contient environ $20 \%$. Les muscles, quant à eux, contiennent environ la moitié de l'eau de l'organisme humain. Les substances blanche et grise du système nerveux en contiennent respectivement 70 et $80 \%$. Les échanges d'eau entre le corps et l'extérieur sont importants et s'évaluent, chez chaque individu, à deux litres par jour. Les pertes se font selon quatre modes différents : la perspiration, la respiration, l'excrétion et la transpiration. Ces pertes ne peuvent être compensées qu'en buvant de l'eau dans des quantités précitées. D'où la recommandation médicale d'en boire environ deux litres par jour.

Chez l'enfant et surtout chez un nourrisson, les besoins en eau sont trois à six fois plus importants que chez l'adulte. Malgré ces échanges permanents, la concentration en eau reste remarquablement constante. Si la vie exige la consommation individuelle de telles proportions d'eau, on conçoit que l'absence de la ressource peut provoquer de graves risques sanitaires, parfois mortels. Un individu peut avoir des graves complications sanitaires en perdant les dix pour cent d'eau que contient son corps et en mourir avec $20 \%$. On meurt donc plus vite de soif que de faim. C'est à cette situation que sont confrontés des milliers d'individus insuffisamment approvisionnés en eau potable, en Afrique centrale, en milieu urbain notamment. Le manque d'eau, en effet, cause la mort à de milliers d'enfants dans la sous-région, victimes de dysenterie amibiase, de déshydratation et de choléra ${ }^{660}$. Aussi, il faut préciser que dans l'hémisphère sud 600 enfants de moins de cinq ans décèdent chaque jour des conséquences du manque d'eau potable $^{661}$, ce que confirme également le Rapport 2006 conjoint de l'Organisation mondiale de la Santé et du Fonds des Nations Unies pour l'enfance.

\section{L'aggravation des risques de maladie}

C'est au regard des conséquences humaines de la privation d'eau potable et de l'absence d'équipement d'hygiène qu'il est nécessaire d'être attentif. A cet égard, les chiffres précités sont révélateurs ${ }^{662}$. Selon l'Organisation Mondiale de la Santé, chaque individu doit disposer d'une quantité suffisante lui permettant de satisfaire des besoins autres qu'étancher sa soif. Si les deux litres sont indispensables dans la perspective du maintien en vie d'un être humain et de la compensation des pertes encourues, il lui en faut, en revanche, une autre quantité d'eau pour préparer des aliments, se laver ...bref la satisfaction des " besoins élargis ", pour vivre en conformité avec les règles d'hygiène.

Le volume d'eau nécessaire à la préparation des repas est fonction du nombre de personnes qui composent le foyer. Il y faut au moins une vingtaine de litres d'eau par jour. La quantité d'eau nécessaire au respect d'une hygiène minimale varie selon les saisons, les Etats et l'environnement dans lequel évoluent les individus. Quoiqu'il en soit, on aura toujours besoin d'eau pour se laver, laver des vêtements, laver la voiture, etc. La non-disposition d'une quantité

660 Déclaration de Lamine CISSE, représentant de l'Organisation Mondiale de la Santé au Congo-

Brazzaville, lors de la célébration annuelle de la journée mondiale de la santé fixée au 7 avril, Brazzaville, 7 avril 2003.

$661 C f$. N. TEC-DUC, L'humanité mourra-t-elle de soif? , op. cit..

662 DROBENKO B., Le droit à l'eau : une urgence humanitaire, précité, p. 20. 
d'eau suffisante en Afrique centrale, en milieu urbain, crée toute une « sociologie familiale » dite de « l'eau potable ». Certaines familles urbaines n'ont pas accès à une quantité d'eau minimale nécessaire à la satisfaction de leurs « besoins élargis ».

Ces difficultés d'accès modifient donc leurs comportements. Elles obligent certains foyers à affecter prioritairement l'eau à la préparation des repas et à la boisson au détriment des soins corporels de ses membres et aux dépens du nettoyage du linge sale, par exemple. Des populations sont contraintes, par pénurie d'eau potable, au non-respect des règles élémentaires d'hygiène. Cette contrainte aggravant les risques de maladies hydriques et d'autres pathologies dues au défaut d'hygiène, réduit, par conséquent, l'espérance de vie des individus, estimée à 40 ans dans les pays de la sous-région.

\section{Section II. La libéralisation du secteur de l'eau, un déni de l'Etat}

Comparée à celle des Etats occidentaux, l'histoire de l'approvisionnement en eau est différente dans les pays en développement. Pendant l'ère coloniale, alors que les pays colonisateurs développaient leurs réseaux publics dans les villes d'Europe, dans les colonies, la distribution d'eau était réservée à l'élite coloniale. Les restrictions étaient de nature à la fois économique et politique. Dans les cas où les réseaux ont été étendus, les usagers devaient acquitter une redevance correspondant au coût réel, sans possibilité d'interfinancement, ce qui veut dire que le service était inabordable pour les masses populaires ${ }^{663}$. Les relations économiques échappent de plus en plus aux frontières nationales. La mondialisation et la création de blocs économiques régionaux ont modifié le rôle de l'État. L'abaissement des barrières commerciales et la circulation internationale des flux de capitaux ont largement transformé les conditions du développement de l'infrastructure et des services d'utilité publique. La génération précédente n'a pas connu les entreprises de télécommunication transnationales qui, aujourd'hui, peuvent mobiliser des volumes de capitaux considérables et s'ériger en chefs de file du progrès technique: ainsi, une poignée de compagnies dominent actuellement le secteur des télécommunications ${ }^{664}$.

Plus la privatisation s'étend, plus le phénomène des économies d'échelle est transféré de l'État à des sociétés transnationales dont la taille, la puissance et la portée augmentent. Ces sociétés, qui dominent les services d'utilité publique et le secteur public à l'échelle internationale, sont capables d'investir les bénéfices réalisés (souvent en régime de monopole) sur certains marchés établis pour financer la conquête de nouveaux marchés. D'une part, l'Etat perd ses moyens d'action $(\S 1)$, et d'autre part cette substitution de l'Etat par les multinationales est synonyme d'une violation du principe de non ingérence (§II).

663 NILSSON D., « Public Service for a chosen few? Water and sanitation service provision in Kampala, Uganda, under colonial rule», RKTF Suède, Communication à la conférence de l'IWHA, décembre 2005, Paris.

664 Les recherches sur lesquelles reposent ces Débats actuels et analyses ont été effectuées par l'auteur du texte initial, M. BRENDAN Martin : Public World, 83 Alkham Road, London N16 6XD, Angleterre.] 


\section{§ 1. La réduction de l'Etat à un « corps sans organes »}

L'analyse de la structure institutionnelle publique fait fréquemment usage de comparaisons organicistes. Très tôt, les établissements publics ou les services publics sont qualifiés de " cellules composantes de ce corps qu'est l'Etat ${ }^{665}$ », d' " organes » de l'Etat ${ }^{666}$ ou de " démembrements ${ }^{667}$ » qui sont "incorporés ${ }^{668}$ à son administration. Progressivement, les organes de l'Etat que sont les établissements publics se réduisent, écartés par un libéralisme qui entend limiter la place et le rôle de l'Etat dans la société civile et qui considère les établissements publics inutiles car sclérosants. C'est une même quête de liberté qui est à l'œuvre chez ARTAUD $^{69}$ et dans le retrait des établissements publics. L'Etat-providence a succédé à Dieu dans la maitrise et le contrôle des personnes (physiques ou morales) et ce n'est plus à l'emprise de Dieu que les libéraux entendent s'échapper, mais à celle de l'Etat. D'où l'idée de réduction de l'Etat à un corps sans organes, sans établissements publics. La réduction de l'intervention de ces derniers permettrait un gain de liberté pour l'initiative privée et le recours à d'autres formes sociales pour la gestion des services publics ${ }^{670}$. Il faut préciser que ce recul de "l'icône » de la personne publique n'est pas définitif (A), certains Etats ont vite fait de retourner à l'ancien procédé de gestion publique de l'eau(B).

\section{A. Le recul éphémère du procédé de l'établissement public industriel et commercial}

Pour familière qu'elle nous soit devenue, la notion d'établissement public ne repose pas sur des fondements juridiques clairement établis ${ }^{671}$ en droit français. C'est la jurisprudence et la

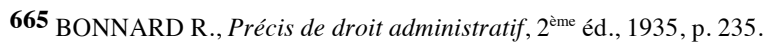

666 AUCOC L., Conférences sur l'administration et le droit administratif faites à l'Ecole des ponts et chaussées, Paris, Dunod, t. 1, p. 307 s. ; L. BLUM, Concl. sur CE 21 juin 1912, Demoiselle Pichot c/ Asile national de la Providence ; D. 1915. 3.9.

667 Note anonyme sous Cass., 28 oct. 1885, Cazentre c/ Enregistrement, préc.; L. Blum, concl. sur CE, 21 juin 1912, Demoiselle Pichot c/ Asile national de la Providence, D. 1915. 3. 9.

668 HAURIOU M., note sous T. confl., 9 déc. 1899, Association syndicale du canal de Gignac, S. 1900. 3. 49. L'idée de «vitalisme social » développée par Hauriou fait également penser aux comparaisons organicistes, in La théorie de l'institution et de la fondation (essai de vitalisme social), Les cahiers de la Nouvelle journée, 1925, $\mathrm{n}^{\circ} 23$, p. 89.

669 ARTAUD A., Pour en finir avec le jugement de Dieu, Euvres Complètes, Gallimard, tome XIII, p. 104. ; Voir aussi SAUVAGNARGUES A., « Corps sans organes », in M. MARZANO, Dictionnaire du corps, PUF, 2007.

670 BEROUJON F., «Le recul de l'établissement public comme procédé de gestion des services publics », RFDA 2008 p. 26

671 Le terme, consacré par ses premières mentions dans le code civil en 1804 et dans la loi de finances pour 1813, est progressivement apparu dans les décennies suivant la Révolution française. Il était alors essentiellement employé pour définir les institutions ou les entités qui se substituèrent aux « gens de mainmorte » - communautés, congrégations, collèges ou hôpitaux - qui bénéficiaient d'une pérennité d'existence leur permettant d'échapper aux 
doctrine qui ont progressivement dessiné les contours de la notion ${ }^{672}$. Suite, à cet effort doctrinal qui avait permis de la doter d'une densité dont elle manquait jusqu'alors, la formule de l'établissement public connut un vif succès puis s'éclipsa ${ }^{673}$. Sur le continent africain également, le législateur ne s'est pas montré très dynamique sur le concept. Il est souvent utilisé sans être défini. Toutefois, l'on rencontre de plus en plus textes qui définissent clairement la notion. C'est le cas du législateur congolais qui revient sur une précédente définition du fait de la confusion qu'elle engendrait ${ }^{674}$. Aux termes de l'article 2 de la nouvelle loi relative à l'établissement public, «l'établissement public est toute personne morale de droit public créée par l'Etat en vue de remplir une mission de service public $»^{675}$.

Revenant sur la question du déclin de l'établissement, on peut citer l'exemple du Sénégal où en 1996, la SONEES a été privatisée par création d'une société de patrimoine SONES, et d'une société d'affermage, la Société des Eaux (SDE). Tandis que la SONES est restée entièrement publique et sert de maître d'œuvre pour les Plans eau financés par les bailleurs de fonds à des conditions concessionnelles, la SDE est contrôlée à 50,5\% par la SAUR (groupe BOUYGUES), dont le contrat de dix ans est en cours de renouvellement. D'autre part, dès 1998 les autorités maliennes se sont engagées à ouvrir le capital d'EDM à un partenaire stratégique après une période transitoire de deux ans. Puis, en 2000, le groupement formé de SAUR et IPS W.A. (filiale sénégalaise du fonds de l'Aga Khan) a remporté l'appel d'offre international pour le rachat de $60 \%$ des parts de la société, et a signé deux contrats de concession pour vingt ans pour l'eau et l'électricité,

Le recul de l'établissement public est parfois amplifié par un postulat erroné, selon lequel l'établissement public aurait été conçu pour la gestion des services publics. Il ressort en effet de la genèse de l'établissement public que celui-ci n'a pas été créé pour prendre en charge les missions de service public, assumées par les collectivités, ce qui explique la raison pour laquelle le service public n'est pas un élément constitutif de la définition de l'établissement public. Cela fournit une explication supplémentaire du recul de l'établissement public : n'ayant pas spécialement été conçu pour assurer la gestion des services publics, il n'est pas surprenant qu'à un moment donné, il cesse de remplir une fonction qui ne lui a été assignée que par opportunité676. Il importe donc d'analyser d'abord la notion d'établissement public.

droits de succession sous l'Ancien Régime et dont la création était soumise à une autorisation de l'Etat, selon un principe posé depuis le XVIIème siècle et réaffirmé par une ordonnance royale de 1749 .

672 D'un point de vue pratique d'une part, le contentieux des établissements publics a très tôt permis de préciser les règles régissant leur fonctionnement, que ce soit en ce qui concerne leurs droits d'agir en justice (Cass., 22 mai 1822, Lafarge), de transiger (CE, 28 février 1825, Gelly), ou de posséder des biens et d'être légataires (Cass.,18 novembre 1834, Feuchères). Par un arrêt Caisse d'épargne de Caen du 5 mars 1856 (Cass., D. 1856 1. 121), la jurisprudence a en outre nettement distingué les établissements publics, personnes publiques faisant partie intégrante de l'administration, des établissements d'utilité publique.

673 Les Etudes du Conseil d'Etat, Les établissements publics, 2009, pp. 10 et 11.

$674 C f$. Loi n 78-002 du 06 janvier 1978 portant dispositions générales applicables aux entreprises publiques en RDC.

$675 C f$. Loi n 08/009 du 07 juillet 2008 portant dispositions générales applicables aux établissements publics en RDC.

676 BEROUJON F., précité. 


\section{Les vices d'une notion empiriquement construite}

Il s'agit d'une catégorie juridique du droit administratif présentant des traits vigoureux d'originalité. L'établissement était toute entité de droit public dotée de la personnalité juridique et chargée de la gestion d'une activité de service public dans le cadre limité de sa spécialité677.

La faiblesse conceptuelle de la notion d'établissement public est immédiatement perçue par la doctrine. A côté de l'unique personne morale de droit public jusqu'alors connue qu'est l'Etat et dont la représentation idéelle est forte, émerge une personne morale aux contours mal définis et à la signification vague. Et HAURIOU a vite fait de les stigmatiser en précisant que ce sont des « institutions ambiguës ». Toutefois, en constatant que le Conseil d'Etat fait émerger la catégorie des établissements publics sans l'asseoir sur des fondements solides, il tente, comme l'ensemble de ses contemporains, de lui trouver une assise dans le service public et les définit comme des services publics spéciaux personnifiés ${ }^{678}$.

En réalité, la notion de service public, ou plutôt l'idée d'utilité publique pour éviter les anachronismes, n'apparaît ainsi pas première dans la jurisprudence pour caractériser l'établissement public. Par exemple, en France, la Cour de cassation pour caractériser l'établissement public en 1856, affirme qu' «il faut surtout une ingérence publique dans la gestion de l'institution ». C'est l'organisation administrative, la tutelle et l'origine publiques, qui conduisent les juridictions à distinguer entre les établissements publics et les établissements d'utilité publique ${ }^{679}$.

DUGUIT tente d'asseoir théoriquement l'établissement public sur la notion de service public. La démarche mérite toutefois une attention spéciale parce qu'il ajoute un élément qui montre qu'il a pleinement conscience des origines exactes de l'établissement public : il indique en effet qu'il s'agit d'un « service public patrimonialisé ${ }^{680} 》$. Il caractérise ainsi l'établissement public par son activité, mais également par l'aspect patrimonial qui a présidé à sa création. Toutefois, il insiste longuement sur le caractère de service public de l'établissement public et en cela, il ne diffère pas fondamentalement d'AUCOC et HAURIOU, dont les développements trouvent ensuite un prolongement dans la thèse de R. DRAGO ${ }^{681}$.

Le recul de l'établissement public dans la gestion des services publics est porteur de significations diverses. Empiriquement lié à la puissance publique, conceptuellement relié au service public, l'établissement public voit son évolution rejaillir sur ces deux référents. Le déclin

677 Aujourd'hui, cette originalité s'est estompée sous l'influence de diverses causes.

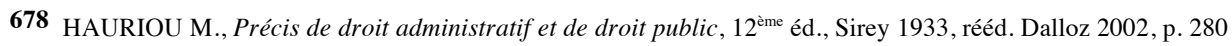
et, pour la difficulté à appréhender la notion, note (3) p. 281.

679 CE 22 mai 1903, Caisse des Ecoles du VIe arrondissement de Paris, D. 1904, 3, 1, concl. J. ROMIEU, S. 1905, 3, 33, note M. HAURIOU ; cf. également Rép. Dalloz, suppl., t. VII, 1891, «Etablissement public »; Rép. Dalloz 1913, «Etablissement public ».

680 DUGUIT L., Traité de droit constitutionnel, vol. II, Paris, 1928, $3^{\text {ème }}$ éd., p. 20, spéc. p. 343 s.

681 DRAGO R., Les crises de la notion d'établissement public, Pedone, 1950. 
de l'établissement public rend compte d'un retrait de la puissance publique dans la gestion du service public.

Plus largement, le mouvement qui affecte l'établissement public reflète l'évolution actuelle du droit administratif. Une scission s'opère au sein même de ce droit qui ne s'applique plus de manière uniforme. Là où la puissance publique demeure, le droit administratif continue de s'appliquer pleinement; là où elle se retire et laisse le service public dans les mains de la gestion privée, l'application du droit administratif n'est plus totale, mais combinée avec des règles qui proviennent d'ensembles normatifs différents. Le phénomène est ancien mais son renouvellement renforce la désunion du droit administratif. Parallèlement, le recul de l'établissement public modifie l'organisation institutionnelle publique. A un Etat dont les établissements publics constituaient les organes, succède un Etat « désorganisé », qui évoque la métaphore du " corps sans organes ».

\section{La primauté accordée aux délégations contractuelles}

L'un des effets de la pression libérale sur la délégation de la gestion des services publics est d'imposer le contrat comme le principal mode de gestion des services publics ${ }^{682}$. Le recul de l'établissement public traduit la montée en puissance du contrat en droit administratif, à plusieurs niveaux. En cela, cette évolution épouse parfaitement celle qui touche actuellement le droit administratif général et le contentieux administratif.

Tout d'abord, le recul du procédé de l'établissement public dans la gestion des services publics met en avant de nouveaux modes de gestion, tant publics que privés. Or, qu'ils soient publics ou privés, ces nouveaux modes laissent une large place au contrat. C'est ainsi que l'arrêt Commune d'Aix en Provence consacre le principe du contrat de délégation de service public pour confier la gestion d'un service public à un tiers ${ }^{683}$. Ensuite, le droit administratif institutionnel est lui-même pénétré par le contrat puisque les groupements d'intérêt public, nouveaux gestionnaires publics, sont constitués par convention passée entre plusieurs personnes morales, de nature publique ou privée.

Le recul de l'établissement public au profit de la gestion privée peut également s'expliquer par la volonté de transformer les « usagers de l'action sociale » ${ }^{684}$ en consommateurs de prestations contractuellement définies. C'est quasi-unanimement que la doctrine a considéré que l'individualisation et la contractualisation des prestations de services publics fournies aux usagers était le gage d'une amélioration de la situation de ces derniers ${ }^{685}$. Quel que soit le regard porté sur

682 CE Sect., 6 avr. 2007, Commune d'Aix-en-Provence, AJDA 2007, 1020, chron. F. LENICA et J. BOUCHER ; JCP A 2007, et p. 2128 note J.-M. PONTIER ; p. 2111, note M. KARPENSCHIF ; C. et marchés publics 2007, comm. 191, G. Eckert ; RFDA 2007. 812, concl. F. SENERS , et p. 821, note J.-Cl. DOUENCE .

683 CE, Sect., 6 avr. 2007, Commune d'Aix-en-Provence, préc.

684 HUMBERT Ch. (dir.), Les usagers de l'action sociale, Sujets, clients, bénéficiaires ?, L'Harmattan, 2000.

685 BEROUJON F., L'application du droit de la consommation aux gestionnaires de services publics - Eléments de réflexion sur l'évolution du droit des services publics, Thèse dactyl. Grenoble II, 2005, p. 340 s. 
le phénomène, on observe une nouvelle immixtion du contrat en droit administratif, par l'intermédiaire du droit des contrats. Depuis l'arrêt Société des eaux du Nord ${ }^{686}$, les actes administratifs unilatéraux sont soumis à des règles dont le champ d'application est expressément cantonné aux contrats. Il existe ainsi une volonté d'intégrer au sein du régime juridique des actes administratifs unilatéraux, des règles applicables aux contrats ${ }^{687}$.

Le mouvement de recul de l'établissement public s'insère parfaitement dans l'évolution particulière du droit administratif, qui se désunit en deux éléments, gouvernés l'un par la puissance publique, l'autre par le contrat. Là où la puissance publique s'exerce, le droit administratif est d'application totale. Là où la puissance publique se retire, le contrat s'impose et seuls l'encadrent quelques vestiges du droit administratif mélangés à des règles d'ensembles normatifs issus du droit privé. En admettant l'amalgame entre l'entreprise publique et établissement public ${ }^{688}$, nous affirmerons qu'en France, « du fait des privatisations, le poids des entreprises publiques dans l'économie française s'est considérablement amoindri ${ }^{689} »$. Alors que les entreprises publiques représentaient $19,3 \%$ des effectifs et $25 \%$ de la valeur ajoutée de l'économie nationale en 1985, elles n'emploient plus que 7,8\% de la population active et ne réalisent plus que $11,5 \%$ de la valeur ajoutée en 2000 . Le secteur public représente encore $29 \%$ des actifs physiques des sociétés non financières en 2000 (cette importance tient aux investissements des entreprises de réseau), mais le chiffre était de $47 \%$ en 1985. En 1985, les entreprises publiques employaient $60 \%$ des salariés des banques et des assurances, alors qu'aujourd'hui leur importance dans ce secteur est résiduelle. Le secteur public est constitué désormais de quelques grands réseaux (EDF et GDF...) ; la privatisation de certains de ces réseaux est prévue ou en cours.

\section{B. Le retour de l'établissement public dans la gestion du service public de l'eau}

Récemment, on a assisté dans de nombreux pays à un retour des services publics de distribution d'eau qui est souvent le résultat de programmes politiques ou la conséquence de mouvements sociaux d'opposition à la privatisation ${ }^{690}$.

686 CE, sect., 11 juil. 2001, Lebon 348; BJCP 2001. 519, concl. C. BERGEAL.

$\mathbf{6 8 7}$ BEROUJON F, «L'analyse des effets des actes administratifs : une nouvelle source de flexibilité du droit », RRJ 2008.

688 Différentes techniques juridiques ont été utilisées pour constituer les entreprises publiques. Certaines empruntent la forme de l'Etablissement public à caractère industriel et commercial. D'autres utilisent une structure de droit privé, celle de société. Voir, DEBBASCH C. et COLIN F., Droit administratif, $8^{\text {ème }}$ édition, Economica, 2007, p.303.

689 Voir Rapport INSEE, 2002

690 De nombreux exemples d'exploitations publiques sont repris dans un ouvrage publié par le TNI « Reclaiming Public Water », disponible à l'adresse : http://www.tni.org/books/publicwater.htm/ 30/08/07. 


\section{L'échec annoncé de la privatisation du secteur de l'eau en Afrique}

L'échec de l'expérience de la privatisation confirme que le secteur public doit être le premier vecteur de l'expansion future, comme c'était déjà le cas dans le passé. L'étude de la Banque mondiale sur les politiques d'infrastructures publiée en juillet 2003 notait que les capitaux privés représentaient moins de $10 \%$ de l'investissement total réalisé pendant la décennie précédente dans le secteur de l'eau dans les pays en développement et concluait que «la Banque va devoir promouvoir plus activement l'investissement et l'offre de services par le secteur public ${ }^{691}$. Les contrats privés n'ont pas permis d'obtenir les investissements attendus dans les infrastructures attendus. Au bout de quinze années, les investissements consentis par les opérateurs privés n'ont permis de raccorder que 600.000 foyers en Afrique subsaharienne et en Asie du Sud et de l'Est soit moins d'un pour cent des personnes qui auraient dû être raccordées dans ces régions pour répondre aux OMD. On peut comparer ce chiffre avec les résultats obtenus par la « décennie de l'eau » des années 1980, pendant laquelle le financement était assuré par le secteur public, et qui fut généralement considérée comme un échec alors qu'elle a permis de ramener la proportion de personnes privées d'un approvisionnement en eau salubre de 56\% en 1980 à $31 \%$ en $1990^{692}$, un résultat bien supérieur à celui obtenu par la privatisation.

En prenant l'exemple du Mali, «il a été notamment révélé le très faible niveau de réalisation des investissements contractuels, la non maîtrise des pertes dans les réseaux et le non respect de nombreuses dispositions majeures des cahiers de charge » (insuffisance d'entretien du système électrique,...). La gestion des contrats a été, en outre, rendue particulièrement difficile en raison d'imprécision et de contradictions de certaines dispositions ainsi que de l'incohérence et de l'inadéquation avérées des formules d'indexation tarifaire. La réaction de l'Etat malien a été de renégocier avec le partenaire stratégique un nouveau cadre contractuel, en vue d'assurer de façon durable et mutuellement avantageuse la gestion déléguée des services publics de l'électricité et de l'eau potable ${ }^{693}$. Finalement SAUR International s'est retiré et la part de l'Etat dans le capital d'EDM SA est ainsi passée de 40 à $66 \%{ }^{694}$.

Les grands groupes internationaux du secteur de l'eau ont essuyé de sérieux revers, n'ayant pas engrangé des bénéfices suffisants dans les pays en développement. Depuis 2003, ils ont donné un coup de frein à leurs politiques d'expansion. Le groupe Suez, le plus implanté dans les pays en développement, a annoncé en janvier 2003 son intention d'y réduire sa présence d'un tiers et de ne plus procéder à l'avenir qu'à des investissements financés par ses activités propres,

$691 C f$. «Public solutions for private problems? - responding to the shortfall in water infrastructure investment 2003». www.psiru.org/reports/2003-09-W-strats.doc consulté le 16 septembre 2007.

692 PNUD (1990), Rapport mondial sur le développement humain. Concept et mesure du développement humain, cité par Brian MATHEW dans Ensuring Sustained Beneficial Outcomes for Water and Sanitation Programmes in the Developing World, IRC Occasional Paper Series 40; p.12

http://www.irc.nl/page/27612. consulté 24 juillet 2007.

693 IPS WA s'est proposé, avant la fin du processus de négociation, de racheter les actions détenues par SAUR International, et d'en rétrocéder une partie à l'Etat malien

694 BERTHELEMY J.-C., « Rapport : les privatisations en zone franc », Libreville, les 12 et 13 avril 2006, p.17. 
échappant ainsi aux risques de change, et qui auraient un taux de rentabilité défini695. Depuis 2002, toutes les multinationales essayent de se défaire de leurs activités dans le secteur de l'eau, mais elles ont souvent eu du mal à trouver des acquéreurs. Bechtel a mis en vente ses participations dans le secteur de l'eau pendant plus d'un an; elles ont finalement été rachetées par une banque publique de développement.

La filiale des eaux du groupe Bouygues, SAUR, numéro quatre mondial, était en vente depuis deux ans avant d'être rachetée par un investisseur financier qui n'a accepté de reprendre que ses activités en Europe. Thames Water, numéro trois mondial de la distribution d'eau, a été officiellement proposé à la vente en novembre 2005 pour être finalement vendu à un investisseur financier en octobre 2006 après s'être défait de la plupart de ses activités dans les pays en développement. En 2006, le groupe Suez lui-même a fait l'objet de deux offres publiques d'achat de sociétés intéressées pas ses activités dans les secteurs du gaz et de l'électricité, aucun candidat ne voulant de son département international de l'eau.

\section{Les causes de l'échec de la privatisation du secteur de l'eau}

Dans certains cas, au niveau des obligations contractuelles, les résultats sont mitigés notamment en ce qui concerne l'accroissement de la desserte et l'amélioration de la qualité du service (Mali). Les raisons sont diverses : l'on constate un flou dans la définition des obligations contractuelles lors de l'opération de cession ou leur non-respect par le repreneur a pu conduire à une gestion moins efficace de l'entreprise. Par ailleurs, la desserte est de moindre qualité et les taux d'investissement sont faibles, voire inexistants. Enfin, l'on note une défaillance des repreneurs ou de mauvais gestionnaires, lorsque le monopole public est remplacé par un monopole privé sans régulation, ou lorsque les repreneurs privés n'ont pas mesuré l'ampleur de l'enjeu (faible intérêt pour l'entreprise).

Par ailleurs, les sociétés privées ne se sont pas montrées plus efficaces que les opérateurs publics $^{696}$. Les enseignements tirés d'études réalisées sur tous les continents indiquent que le régime de propriété ne semble pas avoir d'impact prononcé sur l'efficacité. On ne peut plus postuler que le secteur public est moins efficace que le secteur privé, comme l'explique un rapport de la Banque mondiale : «... en général, on ne note pas de différence statistiquement significative entre le rendement des exploitants publics et privés dans ce secteur... S'agissant des entreprises de service public, il semble généralement que le régime de propriété ne compte pas autant qu'on le prétend parfois. La plupart des rapports comparatifs entre pays ne relèvent pas d'écarts statistiquement notables au niveau des résultats obtenus par les opérateurs publics

\footnotetext{
695 SUEZ met en place un plan d'action 2003-2004 : recentrage, désendettement, rentabilité accrue. Paris, 9 janvier 2003. www.suez.com.

696 Pour plus de détail sur la question, voir «The relative efficiency of public and private sector water» $C f$. traduction «L'efficacité relative du secteur public et privé de l'eau ».www.psiru.org/reports/2005-10- -effic.doc consulté 11 novembre 2005.
} 
et privés ${ }^{697} \gg$. Certaines privatisations sont cependant parfois trop récentes pour permettre d'apprécier la rentabilité des investissements ou l'évolution des indicateurs microéconomiques.

\section{§ 2. La violation du principe de non ingérence}

Le principe de non ingérence trouve son fondement dans la Charte des Nations Unies ${ }^{698}$ qui rappelle avec force qu' " aucun Etat ni groupe d'Etats n'a le droit d'intervenir, directement ou indirectement, pour quelque raison que ce soit, dans les affaires intérieures ou extérieures d'un autre Etat. En conséquence, non seulement l'intervention armée, mais aussi toute autre forme d'ingérence et ou toute menace, dirigées contre la personnalité d'un Etat ou contre ses éléments politiques, économiques et culturels, sont contraires au droit international... Aucun Etat ne peut appliquer ni encourager l'usage de mesures économiques, politiques ou toute autre nature pour contraindre un autre Etat à subordonner l'exercice de ses droits souverains ou pour obtenir de lui des avantages de quelque ordre que ce soit ». Aux yeux des Etats subsahariens en particulier et des pays sous développés en général, le principe de non ingérence revêt une grande importance au point qu'ils y attachent divers corollaires notamment la souveraineté sur les ressources naturelles ${ }^{699}$. L'eau, ressource naturelle qui plus est vitale, «a toujours été associée au territoire et donc à la souveraineté étatique qui s'y exerce »700. Inéluctablement, la privatisation du secteur de l'eau, lorsqu'elle imposée de l'extérieur, met ces Etats dans l'impossibilité de choir librement un système économique (A) et ceux-ci voient leurs ressources naturelles aliénées (B).

\section{A. L’impossibilité de choisir librement un système économique}

S'appuyant sur son dernier rapport présenté à l'Assemblée générale (A/58/330), ZIEGLER J. prend pour point de départ le fait que, dans de nombreuses régions du monde, les sociétés transnationales ont acquis un pouvoir sans précédent sur l'alimentation et l'eau et qu'il n'existe aucun système cohérent qui les empêche d'abuser de ce pouvoir.

\footnotetext{
697 Infrastructure performance and reform in developing and transition economies: evidence from a survey of productivity measures. A. ESTACHE, S. PERELMAN, L. Trujillo World Bank Policy Research Working Paper 3514, février 2005.

http://wdsbeta.worldbank.org/external/default/WDSContentServer/IW3P/IB/2005/03/06/000090341 200503061014 29/Rendered/PDF/wps3514.pdf consulté le 30 mars 2005.

698 Résolution 2625 (XXV) du 4 octobre 1970

699 FEUER G. et CASSAN H., Droit international du développement, Précis Dalloz, 1991, p. 30.

700 PAQUEROT S., Le statut des ressources vitales en droit international, Bruylant, 2002, p. 194.
} 


\section{Une mainmise des multinationales sur l'eau}

Les sociétés multinationales exercent un contrôle de plus en plus important sur l'approvisionnement en eau, qui fait l'objet d'une libéralisation croissante dans toutes les régions du monde. La privatisation des services relatifs à l'eau a déjà été menée à bien dans de nombreux pays, dont la Côte d'Ivoire, Madagascar, le Maroc, le Nigéria, le Sénégal, la Tunisie... Dans de nombreux cas, ces opérations se sont faites en grande partie parce que le FMI et la Banque mondiale avaient posé la participation du secteur privé aux services relatifs à l'eau comme condition préalable à l'octroi de prêts et de subventions. Deux sociétés, Veolia Environnement, anciennement Vivendi Environnement, et Suez Lyonnaise des Eaux, contrôlent une grande majorité des concessions privées dans le monde entier ${ }^{701}$.

Or, le principe de non ingérence ne vise pas seulement les Etats. Il concerne aussi les sociétés multinationales. Et les pays en développement leur reprochent de s'ingérer dans leurs affaires intérieures. On trouve dans quelques dispositions qui ont trait au devoir de ces sociétés de respecter la souveraineté des Etats d'accueil. Par exemple, la résolution $3201(\mathrm{~S}-\mathrm{VI}) \mathrm{du} 1^{\mathrm{er}}$ mai 1974 pose comme principe l'instauration du nouvel ordre la « réglementation et supervision des activités des sociétés multinationales par l'adoption de mesures propres à servir l'intérêt de l'économie nationale des pays où ces sociétés multinationales exercent leurs activités sur la base de la souveraineté entière de ces pays ${ }^{702}$.

Ainsi, tout pays, dont les ressources et richesses naturelles se trouvent entre des mains étrangères, doit-il pouvoir recouvrer l'intégralité des droits normalement attachés à la souveraineté. Par extension naturelle de cette idée, on ajoute qu'un Etat souverain ne peut être contraint de céder contre son gré à des étrangers les droits qu'il détient normalement sur les richesses situées sur son territoire ${ }^{703}$.

Pour l'heure, le droit international ne semble pas, apte à résoudre cette équation dans la mesure où la logique de marché s'y trouve de plus en plus largement intégrée, logique qui n'a jamais su, à ce jour, résoudre les enjeux de redistribution : "Si l'eau est un bien économique, c'est avant tout un bien social. C'est l'égalité d'accès à un bien commun qui doit être construite et défendue déclarera HESSEL S., lors de la Conférence internationale sur l'eau et le développement durable $»^{704}$.

701 ZIEGLER J., Rapport: Droit à l'alimentation, COMMISSION DES DROITS DE L'HOMME, $\mathrm{E} / \mathrm{CN} .4 / 2004 / 10$.

702 FEUER G. et CASSAN H., précité, p. 30.

$\mathbf{7 0 3}$ Idem.

$\mathbf{7 0 4}$ Déclaration de Paris, 21 mars 1998. 


\section{Le principe d'autodétermination économique compromis}

Les sociétés transnationales ont souvent des revenus maintes fois supérieurs à ceux des gouvernements des pays dans lesquels elles sont implantées. D'après la CNUCED, 29 des 100 entités économiques les plus importantes au monde sont des sociétés transnationales ${ }^{705}$. La concentration a donné naissance à d'énormes sociétés transnationales qui exercent un monopole sur l'ensemble de la chaîne alimentaire, de la production à la distribution au détail en passant par la vente, la transformation et la commercialisation, ce qui a pour effet de restreindre le choix des agriculteurs et des consommateurs.

Et c'est avec suspicion que les Etats subsahariens entrevoient les sociétés multinationales opérant sur leur territoire. Une inquiétude qu'ils ont exprimée dans plusieurs textes internationaux. Le plus significatif est la Déclaration économique des Non-alignés d'Alger de 1973 dont la section 7 se lit ainsi : "Les chefs d'Etat et de gouvernement dénoncent devant l'opinion publique mondiale les pratiques inadmissibles des sociétés transnationales qui portent atteinte à la souveraineté des pays en voie de développement et qui violent les principes de non ingérence et du droit des peuples à l'autodétermination, conditions fondamentales pour le progrès politique, économique et social de ces pays».

Pour mieux comprendre cette suspicion, il faut se référer à l'influence que les sociétés-mères exercent sur les filiales installées dans ces Etats. C'est une emprise difficile à saisir, dans la mesure où elle peut s'exercer de manière très variée, en particulier par la maîtrise de la politique des prix, du choix de la technologie, des nominations aux postes-clés, de la détermination des marchés, etc. Dans ces conditions, les multinationales peuvent exercer une énorme influence sur les droits de l'homme, en l'occurrence le droit à l'eau, et sur l'environnement ${ }^{706}$.

\section{B. L'aliénation du droit de souveraineté permanente sur les ressources naturelles}

Le principe de souveraineté sur les ressources naturelles a été introduit dans les débats des Nations Unies à la suite de la demande des pays colonisés et des pays en développement de pouvoir bénéficier de l'exploitation de leurs ressources naturelles. Un long processus tenu au sein de l'ONU a fini par consacrer le principe de la souveraineté permanente sur les ressources naturelles. C'est ainsi que l'Assemblée générale a solennellement proclamé le droit de tout État de disposer librement de ses richesses et de ses ressources naturelles. Aujourd'hui, chaque État dispose, en droit, d'une souveraineté pleine et entière sur ses richesses et ressources naturelles ${ }^{707}$.

705 CNUCED, communiqué de presse TAD/INF/PR/47, 12 août 2002.

706 Voir Rapport mondial sur le développement humain 2002 du PNUD.

707 Ainsi l'article 47 du Pacte international relatif aux droits civils et politiques et l'article 25 du Pacte international relatif aux droits économiques, sociaux et culturels spécifient: "aucune disposition du présent pacte ne sera interprétée comme portant atteinte aux droits inhérents de tous les peuples à profiter et à user pleinement et librement de leurs richesses et ressources naturelles ». 
Ce principe s'adresse à tous les États ainsi que l'a rappelé l'Assemblée générale des Nations Unies dans bon nombre de ses résolutions ${ }^{708}$. De plus, la Cour internationale de justice a affirmé, dans son arrêt sur le Timor oriental, que le droit des peuples à disposer d'eux-mêmes constitue une coutume générale du droit international contemporain et "tel qu'il s'est développé à partir de la Charte et de la pratique de l'Organisation des Nations Unies, est un droit opposable erga omnes $\left.{ }^{709} \gg\right\rangle^{710}$. La souveraineté sur les ressources naturelles étant l'un des principaux corollaires du droit des peuples à disposer d'eux-mêmes, on peut légitimement penser que cette souveraineté est également un droit opposable erga omnes. D'autre part, l'article premier du PIDESC énonce que " tous les peuples peuvent disposer librement de leurs richesses et de leurs ressources naturelles, (...) en aucun cas, un peuple ne pourra être privé de ses propres moyens de subsistance ». Si cet article énonce le droit collectif qu'ont les peuples d'accéder et de jouir de leurs richesses, c'est qu'il reconnait ainsi également le droit à l'eau, puisque l'accès à l'eau est un moyen de subsistance à la fois pour les individus et pour les peuples ${ }^{711}$. Ce droit implique donc que le déni d'accès à l'eau pour un peuple serait incompatible avec les obligations des Etats.

Bien que ce principe - le droit des peuples à disposer librement de leurs richesses et de leurs ressources naturelles- soit reconnu par les Etats et par la jurisprudence internationale comme une norme incontournable du droit international, il n'est pas respecté par tous. Le cas des multinationales illustre de façon insidieuse cette violation.

\section{La théorie du droit de souveraineté permanente sur les ressources naturelles}

Le principe de souveraineté permanente sur les ressources naturelles s'applique normalement à tous les peuples. Il faut pourtant relever à cet égard l'imprécision du langage employé par les Nations Unies concernant la souveraineté sur les ressources naturelles. Parfois l'Assemblée générale emploie le terme «État» pour évoquer le titulaire de la souveraineté sur les ressources naturelles et parfois elle emploie le terme de "peuples» ou "nations». Ces expressions se rencontrent parfois successivement dans le même texte ${ }^{712}$.

\footnotetext{
708 Voir notamment résolutions : Souveraineté permanente sur les ressources naturelles, Rés. AG 2386 (XXIII), Doc.Off. A.G.N.U., 23e sess., 1968, 26; Déclaration sur le progrès et le développement dans le domaine social, Rés. AG 2542 (XXIV), Doc.Off. A.G.N.U., 24e sess., 1969, 51; Souveraineté permanente des pays en voie de développement sur leurs ressources naturelles et expansion des sources intérieures d'accumulation aux fins du développement économique, Rés. AG 2692 (XXV), Doc.Off. A.G.N.U., 25e sess., 1970, 69 ; Souveraineté permanente sur les ressources naturelles, Rés. AG 3171 (XXVIII), Doc.Off. A.G.N.U., 28e sess., supp. n³0, Doc. NU A/9400, (1973), 55.

$\mathbf{7 0 9}$ Obligation opposable à tous, pas seulement aux parties prenantes.

710 Timor oriental (Portugal c. Australie), arrêt, [1995],C.I.J, Rec. 102, par.29.

711 BOVEN Van T., « Human Right and Rights of peoples » 1995 E.J.I.L. 3, 6

712 Droit d'exploiter librement les richesses et les ressources naturelles, Rés. AG 626 (VII), Doc. Off AG NU, 7ème sess., supp. $\mathrm{n}^{\circ} 20,(1952), 18$.
} 
Le débat sur le principe de souveraineté sur les ressources naturelles s'est poursuivi par la création en 1958, à travers la résolution 1314 (XIII) du 12 décembre 1958713, de la Commission pour la souveraineté permanente sur les ressources naturelles. Cette Commission était « chargée de procéder à une enquête approfondie sur la situation de cet élément fondamental [qu'est le «droit de souveraineté permanent sur leurs richesses et leurs ressources naturelles»] du droit des peuples et des nations à disposer d'eux-mêmes ». On peut relever dans le corps de cette résolution une innovation importante. C'est celle qui déclare que le «droit des peuples et des nations à disposer d'eux-mêmes comprend un droit de souveraineté permanent sur leurs richesses et leurs ressources naturelles ». De liberté d'exploitation, qui paraissait dans la résolution 626 de 1952, nous sommes passés au droit de souveraineté permanent.

Les peuples et les nations ne sont pas seulement libres d'exploiter leurs richesses et ressources naturelles, ils sont surtout et avant tout souverains permanents sur ces richesses. Cette idée sera reprise quelques années plus tard dans la célèbre résolution 1803 du 14 décembre 1962. Même si cette formule semble être passée inaperçue à l'époque, la création de cette Commission a eu au moins le mérite de marquer une progression importante dans le processus normatif qui entourait le principe de souveraineté sur les ressources naturelles ${ }^{714}$.

En effet, dire que la souveraineté sur les ressources naturelles est permanente, signifie qu'elle est inaliénable. L'inaliénabilité se trouve proclamée par la résolution 1803 (XVII) et par la plupart des textes ultérieurs ${ }^{715}$. Déclarer cette souveraineté permanente et inaliénable signifie d'une part qu'aucune aliénation en concession n'est valable sans le consentement de l'État territorial, d'autre part que cet État a, à tout instant, le droit de prendre ou de reprendre le contrôle des richesses aliénées ${ }^{716}$.

Selon SOHNLE J., "le principe de la souveraineté permanente sur les ressources naturelles, pertinent en matière des ressources en eau, date de la grande époque des pays en développement et constitue l'un des aspects majeurs du droit au développement. Ainsi, toute mesure prise en vue de la gestion des richesses et des ressources naturelles, doit se fonder sur la reconnaissance $d u$ droit inaliénable de tout Etat de disposer librement de celles-ci, conformément aux intérêts nationaux et dans le respect de l'indépendance économique des Etats $»^{717}$.

$\mathbf{7 1 3}$ Recommandations concernant le respect, sur le plan international, du droit des peuples et des nations à disposer d'eux-mêmes, Rés. AG 1314 (XIII), Doc. Off. A.G.N.U., 13ème sess., supp. n¹8, (1958), 27.

714 ROSENBERG D., Le principe de souveraineté des États sur leurs ressources naturelles, Paris, L.G.D.J., 1983, p. 134.

715 Résolution 2158 (XXI) (Souveraineté permanente sur les ressources naturelles, Rés. AG 2158 (XXI), Doc. Off A.G.N.U, 21e sess., supp. $n^{\circ} 16$, Doc. NU A/6518, (1966), 29) et 3171 (XXVIII) (Souveraineté permanente sur les ressources naturelles, Rés. AG 3171 (XXVIII), Doc.Off. A.G.N.U, 28e sess., supp. n³0, Doc. NU A/9400, (1973), 55) et les résolutions du Conseil économique et social: Souveraineté permanente des pays en voie de développement sur leurs ressources naturelles, Rés. CES 1737 (LIV), Doc. Off. CES NU, $54^{\text {ème }}$ session, supp. $n^{\circ} 1$, (1973), 2 ; Souveraineté permanente sur les ressources naturelles, Rés. CES 1956 (LIX), Doc. Off. CES NU, $59^{\text {ème }}$ session, supp. $\mathrm{n}^{\circ} 1$, Doc. NU E/5731, (1975), 10.

716 FEUER G., La théorie de la souveraineté sur les ressources naturelles dans les résolutions des Nations Unies, Paris, Université René Descartes, 1983, p.16.

717 SOHNLE J., Le droit international des ressources en eau douce: solidarité contre souveraineté, La documentation française, Paris, 2002, p. 252. 
Qu'en est-il de la privatisation du secteur de l'eau en Afrique subsaharienne ? Les conditions de la privatisation de l'eau en Afrique correspondent parfaitement aux schémas «colonialistes »; on trouve presque exclusivement des opérateurs britanniques, portugais et allemands dans les pays qui faisaient autrefois partie de l'empire britannique ou portugais, ou de l'empire allemand avant la première guerre mondiale, tandis que les opérateurs français contrôlent les anciennes colonies françaises. Il n'est donc pas surprenant que ce processus de privatisation soit ressenti comme une résurgence des liens coloniaux ${ }^{718}$. Il faut préciser que l'on ne doit pas perdre de vue que le droit à l'eau impose aux Etats parties trois types d'obligations: celle de respecter ce droit, celle de le protéger et celle de l'appliquer. L'obligation de protéger exige des Etats parties qu'ils empêchent des tierces parties d'interférer de quelque manière que ce soit dans la jouissance du droit à l'eau ${ }^{719}$.

\section{Une théorie peu respectée par les multinationales}

Partant de l'idée que «le droit de souveraineté permanente des peuples et des nations sur leurs richesses et leurs ressources naturelles doit s'exercer dans l'intérêt du développement national et du bien-être de la population de l'Etat intéressé ${ }^{720}{ }_{\|}$, l'on constate que la privatisation du secteur de l'eau participe à l'aliénation des ressources naturelles dénoncée par diverses associations et ONG. Aujourd'hui, de plus en plus de voix sonnent l'alarme, les environnementalistes, les associations de droits de l'homme, des organismes de recherche, les agences internationales et nombre de communautés à travers le monde. Les ressources en eau potable sont limitées. Or la consommation d'eau par habitants double tous les vingt ans, soit plus de deux fois le taux de croissance démographique. L'agriculture intensive, l'irrigation massive, la construction de grands barrages, les déchets toxiques, la déforestation et les pollutions urbaines et industrielles ont tellement endommagé les eaux de surface que nous devons désormais puiser dans les réserves souterraines à un point tel que la nature n'est pas capable de recycler ces « prélèvements $»^{721}$.

Du coup, tirant profit de l'ouverture du marché de l'eau, une poignée d'entreprises transnationales s'approprie les services publics de gestion des eaux et parfois des ressources en eau dans le monde entier, avec pour conséquences une augmentation des tarifs pour les populations locales. Pour ces entreprises, l'eau doit être considérée comme n'importe quel autre

718 HALL D. et LOBINA E., Rapport réalisé à la demande de l'Internationale des Services Publics, novembre 2006, www.world-psi.org. Consulté le 22 novembre 2006.

719 Par tierces parties, on entend notamment des individus, des groupes, des entreprises ou d'autres entités ainsi que des agents agissant sous leur autorité.

720 Résolution 1803 (XVII) de l'Assemblée générale en date du 14 décembre 1962.

721 BARLAW M., « A qui appartient l'eau ? », Projet Planète Bleue et Tony Clarke, Polaris Institute.

http://www.courrierdelaplanete.org/70/article2.html consulté le 6 janvier 2011. 
bien marchand, dont l'usage est régi par le principe du profit. Les ressources se trouvant ainsi aliénées ${ }^{722}$.

722 Selon le magazine Fortune, les profits annuels de l'industrie de l'eau, proches de 1000 milliards de dollars, atteignent désormais $40 \%$ de ceux réalisés dans l'industrie pétrolière et sont supérieurs aux profits du secteur de la pharmacie. 
Chapitre II. La Liberalisation DU SECTEUR DE L'EAU, UNE NEGATION DES FACTEURS DE DEVELOPPEMENT 
Indubitablement, la privatisation, synonyme de marchandisation de l'eau, risque de contrarier la poursuite de l'un des objectifs du millénaire relatif à l'accès à l'eau potable si les politiques ne prennent pas les dispositions nécessaires pour contenir les déviances possibles. Et, par ricochet, la réalisation des autres objectifs se trouve également gravement compromise dans la mesure où l'on ne peut améliorer l'état sanitaire de la population sans accès à l'eau potable. Pas plus que l'on ne peut, dans ces conditions, significativement mener la lutte contre la pauvreté ${ }^{723}$. La rareté 724 de l'eau, la contamination de l'eau ${ }^{725}$ et du sol et le manque d'accès à l'eau pour les pauvres comptent parmi les principaux obstacles à la jouissance complète du droit à l'eau. Le Rapporteur spécial de la Sous Commission pour la Prévention de la Discrimination et la Protection des Minorités ${ }^{726}$ a identifié les causes du drame à l'eau. Il cite l'horrible gestion de l'eau douce, le manque de planification, l'inégalité de la distribution de l'eau potable et d'assainissement, l'augmentation régulière du coût de l'approvisionnement en eau potable et la privatisation des Entreprises d'Etat liées aux services d'approvisionnement en eau de boisson.

Les Etats se sont désengagés de l'exploitation des points d'eau sans que les communes ne soient en mesure de prendre en charge cet aspect en raison de l'absence de transfert de ressources financières du budget national au niveau communal tel que prévu par le cadre légal de la décentralisation. De même, on dénote actuellement l'inexistence de tout mécanisme de solidarité entre usagers d'une part et entre communes d'autre part. Ce désengagement « contribue» de façon la plus significative à la négation du droit à l'eau. Car, "l'idée radicale d'équilibrer le prix par une offre constante et une demande sans cesse croissante » de l'eau soutenue par les institutions spécialisées de la Banque Mondiale ${ }^{727}$ ne permet pas aux populations des pays de l'Afrique subsaharienne de s'approvisionner en eau potable. Heureusement, aujourd'hui "même le FMI, grand promoteur de la privatisation de services de réseau tels l'eau et l'énergie dans les pays en voie de développement, est de plus en plus sceptique $»^{728}$ sur la question.

723 SANI ADAMOU M., «Problématique de la Privatisation de la Distribution de l'Eau en Afrique », http://www.alternativeniger.org/spip.php?article145\&debut articles rubrique=90 27/03/2006.

724 Comme l'indique NGUYEN-TEN-DUC, auteur d'un ouvrage de synthèse au titre choc (L'humanité mourra-telle de soif? , éd. Hydrocom, 1999) si le volume total de l'eau sur terre est évalué à 1400 millions de kilomètres cubes, $98 \%$ de ce volume sont trop salés pour être utilisables par l'homme, et sur les $2 \%$ restant, seuls $0,15 \%$, soit 40 000 kilomètres cubes, participent au cycle de l'eau (évaporation, pluie, ruissellement, infiltration...) et sont donc utilisables pour les besoins humains.

725 Une eau de mauvaise qualité joue un rôle clé dans les mauvaises conditions de vie et les grands problèmes de santé du monde. Les maladies diarrhéiques et le paludisme ont tué quelque 3,1 millions de personnes dans le monde en 2002. Quatre-vingt-dix pour cent de ces victimes étaient des enfants de moins de cinq ans. On estime que quelque 1,6 millions de vies pourraient être sauvées chaque année si l'on améliorait les conditions d'accès à l'eau potable, les services d'assainissement et l'hygiène.

726 «The right of access of everyone drinking water supply and sanitation services » Document de travail preparé par El Hadji GUISSE, Rapporteur Spécial, Sous-commission pour la prévention de la discrimination et la protection des minorités, Cinquième Session, Conseil économique et social, E/CN.4/ Sub.2/1998/7, 10 juin 1998, paragraphe 29.

www.worldwaterday.org/wwday/2001/lgfr/thematic/hmnrights.html consulté le 17/05/2009.

727 N.TIEN-DUC, idem .

728 Voir, « L'or bleu, un bien commun convoité » dans CIPRA INFO, n 79, 2006. 
Il est regrettable qu'en matière de politique d'eau, tous les Etats africains ont marginalisé les milieux ruraux au profit des centres urbains ${ }^{729}$. En effet il est difficile voire impossible de trouver l'eau potable en zone rurale ${ }^{730}$. Cela témoigne du peu de considération que les gouvernants africains accordent à une matière sans laquelle aucune vie et de surcroît humaine n'est possible.

La question est d'autant plus préoccupante que depuis le début du 3ème millénaire, la Banque Mondiale s'implique fortement dans la privatisation et de la marchandisation de l'eau. En effet, en l'an 2000 le FMI «a formellement lié son aide à la privatisation des services de d'eau, à divers degrés, dans le cas de douze pays les plus pauvres: l'Angola, le Bénin, la Guinée-Bissau, le Honduras, le Nicaragua, le Niger, Panama, le Rwanda, Sao Tomé, le Sénégal, la Tanzanie et le Yémen... ».

Les privatisations des services publics en réseau se traduisent le plus souvent dans un premier temps par une progression des tarifs, ce qui pénalise en premier lieu les consommateurs les plus défavorisés, s'il n'a pas été convenu de clauses tarifaires explicites dans le contrat de cession. Le principal facteur expliquant cette hausse des tarifs provient de la structure des comptes de l'entreprise publique lors de la cession. Une société publique très soutenue avant la privatisation ou en difficulté structurelle sera contrainte de procéder à une hausse de ses tarifs, dans un premier temps. Obstacle au développement social (Section I), la privatisation est aussi une menace sur la protection des ressources en eau (Section II).

\section{Section I. La privatisation du secteur de l'eau, un frein au développement social}

$\mathrm{Au}$ sud du Sahara, les femmes passent encore des heures à transporter de l'eau pour leur famille et à faire de longues queues aux points d'eau. Dans ces pays, le problème de l'eau exacerbe les inégalités entre hommes et femmes et porte préjudice à l'éducation des enfants. En effet, l'une des plus importantes conséquences positives d'un meilleur accès à l'eau est le gain de temps pour les femmes et les jeunes filles. Le ravitaillement en eau fait partie d'une division du travail fondée sur le sexe. Les normes sociales et culturelles influencent la répartition du travail au sein des familles en Afrique subsaharienne. Le ravitaillement en eau étant une tâche particulièrement réservée aux femmes ${ }^{731}$.

De plus, il faut le souligner, ces programmes de libéralisation sont motivés par des considérations d'ordre budgétaire. Confrontés à une crise financière sans précédent, les gouvernements cherchent à obtenir des ressources financières substantielles des cessions d'actifs pour combler leurs déficits budgétaires, tout en supprimant les subventions accordées aux

729 Voir, ALACHI E. K., Orientations de la politique nigérienne d'exploitation et de gestion des ressources en eau, Mémoire DEA Droit et politique de l'environnement, Université de Lomé, Faculté de Droit, 2002-2003.

730 котоКо M., L'Intervention de l'Etat dans la gestion de l'eau potable au Togo, Mémoire DEA Droit et politique de l'environnement, Université de Lomé, Faculté de Droit, 1999-2000.

731 Les enquêtes menées à ce sujet au Bénin, à Madagascar, à Maurice et en Afrique du Sud mettent en évidence des différences allant de 5 à 7 heures par semaine. Sources : Rapport mondial sur le développement humain 2006, p. 87. 
services publics, notamment celui de l'eau, grevant lourdement les ressources de l'Etat ${ }^{732}$. Ce que Pierre LE MASNE illustre bien dans un article publié sur le lien entre le service public et le développement. En effet, Pierre LE MASNE fait remarquer que «les politiques économiques d'inspiration néolibérale menées depuis plus de 20 ans ont conduit un peu partout dans le monde à des privatisations et à un affaiblissement des services publics $\gg{ }^{733}$. Aussi, simultanément le développement des pays en voie de développement se retrouve compromis ${ }^{734}$. Dans un contexte marqué par les politiques d'ajustements structurels, l'exigence des institutions de Bretton Woods a de sérieuses conséquences sur la population entière mais plus singulièrement pour les femmes. Aujourd'hui, partout dans le monde, nombreux sont ceux qui postulent que l'eau en tant que droit fondamental, soit exclue des négociations de $1^{\prime} \mathrm{OMC}^{735}$. Car lorsque l'eau est déclarée légalement comme une marchandise sous les régimes des accords internationaux, régionaux ou bilatéraux ${ }^{736}$, cela viole l'accord sur les droits sociaux et culturels ainsi que d'autres lois internationales. Nous montrerons ainsi que la libération engendre une aggravation de fracture sociale $(\S 1)$ et qu'elle est susceptible de freiner la dynamique de la protection des droits sociaux $(\S 2)$.

\section{$\S 1$. Un facteur d'aggravation de fracture sociale}

Avec la privatisation de la distribution de l'eau, le droit à l'eau potable devient plus abstrait pour les classes populaires. En effet la difficulté d'accès liée, entre autres, au coût très élevé amène plus souvent ces dernières à recourir à des stratégies qui compromettent leur santé (fonçage de puits dans les domiciles en milieu urbain, consommation des eaux souillées, utilisation des eaux usées pour le maraîchage...). Aussi, faut-il préciser que si en général, les pauvres sont les principales victimes (A) de la nouvelle situation, celle-ci touche particulièrement les femmes (B).

732 SAKHO P. O., «La politique de privatisation du gouvernement : enjeux et perspectives », Revue du Conseil économique et social $n^{\circ} 2$, févr.-avr. 1997, pp. 20-22.

733 LE MASNE P., Chercheur au GEDES à l’Université de Poitiers.

$\mathbf{7 3 4}$ LE MASNE P., « Services publics et le développement », sources non disponibles.

735 L'Accord général sur le commerce des services (AGCS) a été établi en 1995 dans le cadre de l’Organisation mondiale du commerce. Son objectif est de promouvoir une plus grande libéralisation des services. Le traité considère la gestion et la distribution de l'eau comme des services environnementaux. L'Union européenne, en tant que membre de l'OMC et région d'origine des plus grandes entreprises de distribution d'eau, exige que le marché de l'eau soit libéralisé dans plus de 60 pays en développement et, ce faisant, incite fortement à la commercialisation de l'eau partout dans le monde. Le statut de l'eau dans le cadre de l'AGCS est toujours en cours de négociation. Au sein du Contrat mondial de l'eau, le Réseau œcuménique de l'eau exige que cette ressource soit retirée de ces négociations commerciales et de toutes les autres.

736 Déclaration de Dublin. 


\section{A. Les pauvres, principales victimes de la privatisation de l'eau}

La privatisation est souvent considérée comme préjudiciable aux pauvres, puisqu'elle entraîne la suppression des subventions et donc l'augmentation du prix des produits et des services dont ils ont besoin, l'eau, l'électricité et les transports publics. Toutefois, il faut cerner la notion de pauvre. Selon le Professeur KOUBI, la notion de pauvreté n'est pas définissable en droit ${ }^{737}$. Certes, les textes juridiques de différents États peuvent déterminer un "seuil de pauvreté » à partir duquel seraient développées des mesures spécifiques pour contribuer à la lutte contre les exclusions ou pour porter remède aux situations d'extrême pauvreté, mais la plupart du temps, les critères d'évaluation des cas à traiter reposent sur une définition économique, financière et monétaire, plus que sociologique ou psychosociologique, de la pauvreté. La définition proposée par les économistes retient donc souvent l'attention des pouvoirs publics : " Est pauvre celui qui a un revenu insuffisant pour atteindre un niveau de vie minimum ${ }^{738}$.

\section{Le revenu est un facteur décisif pour l'accès à l'eau}

En partant de l'expérience des habitants de Conakry (capitale de la Guinée), on constate que le montant du revenu des ménages étant difficile à obtenir en raison du contexte (réticence culturelle, analphabétisme, revenu erratique des populations non salariées), nous avons développé un indicateur de richesse à partir de ce que possède le ménage (électroménager, voiture). Ceci a permis d'indexer tout ménage interrogé selon une des trois catégories suivantes : le revenu faible correspondant à un ménage dont les équipements ont une valeur estimée inférieure à 1,3 million de francs guinéens ; le revenu élevé correspond au ménage dont les équipements ont une valeur supérieure à 2,6 millions de francs guinéens; le revenu moyen étant compris entre les deux ${ }^{739}$.

Chez les ménages à revenu élevé, $87 \%$ des répondants ont utilisé le branchement individuel pour l'eau de boisson alors que chez les ménages à faible revenu, c'est seulement $28 \%$ des ménages interrogés qui se tournent vers cette modalité pour le même usage. Une différence très forte se dessine entre les deux classes de revenu quant à l'utilisation de cette modalité qui fournit l'eau du réseau à domicile, une eau considérée de meilleure qualité pour la boisson par rapport aux trois autres modalités restantes. Quant aux ménages à revenu moyen, $51 \%$ ont recours au branchement individuel. Ainsi, plus le revenu du ménage est élevé, plus ce dernier utilise ce mode d'approvisionnement pour la boisson; par contre, plus son revenu est faible, plus il a recours à cette modalité de façon sélective. Il se dégage une relation positive entre mode d'approvisionnement pour boisson et classe de revenu. Quant à l'utilisation de la borne-fontaine,

737 KOUBI G., «La pauvreté, comme violation des droits humains », Revue internationale des sciences sociales, $2004 / 2$ - N 180 , p. 361 .

$738_{\text {Idem. }}$.

739 BAH A., DIALlO A. et MORIN R., «Approvisionnement en eau des ménages de Conakry », Afrique contemporaine 2007/ 1, $\mathrm{N}^{\circ} 221$, p. 227-228. 
$19 \%$ des ménages à revenu moyen s'en servent pour l'eau de boisson, contre seulement $6 \%$ des répondants à revenu élevé, mais $13 \%$ chez les répondants à revenu faible ${ }^{740}$.

La faible utilisation de la borne-fontaine pour se procurer l'eau de boisson par les trois catégories de revenus des répondants pourrait être due aux difficultés d'accès qui démotiveraient certains ménages. Les principales plaintes des répondants qui y ont recours pour leur eau de boisson sont les suivantes : les irrégularités dans la distribution et les temps d'attente prolongés en période d'affluence (c'est là où ressort l'important rôle joué par la femme) ; le nombre insuffisant et l'inégale répartition des bornes-fontaines entre les cinq communes, la commune de Kaloum avec 10748 ménages comptant $39 \%$ des pompes de la ville alors que les deux plus grandes communes (Ratoma et Matoto) avec un total de 104444 ménages ne regroupent que 11 $\%$ des pompes ; la malpropreté des lieux immédiats où la pompe est installée, la boue que forment autour d'elles les éclaboussures et l'eau renversée, utilisée par certains pour laver les légumes ou leurs jeunes enfants. Il arrive parfois, selon certains fontainiers rencontrés, qu'hommes, femmes et enfants transforment l'alentour de la pompe en un lieu de lavage pendant les coupures d'électricité. Mais ce sont aussi toutes les activités domestiques qui se font sur place : la lessive, la vaisselle, etc., sans parler des taximen qui viennent pour y laver leur véhicule ${ }^{741 .}$

\section{Les pauvres au centre des inégalités observées dans la distribution de l'eau}

Les inégalités d'accès à l'eau se traduisent par une grande variabilité des consommations spécifiques. Les statistiques officielles font état de consommations moyennes de 200 litres $/ \mathrm{hab} / \mathrm{j}$ pour les habitants raccordés et de 45 litres /hab/j pour ceux qui dépendent de bornes-fontaines. La réalité est souvent faite de disparités plus grandes encore entre ménages aisés, gros consommateurs742, et les plus pauvres dépendant de solutions collectives ou de revendeurs. Certes, le raccordement au réseau ne constitue pas une garantie suffisante contre le rationnement, et des disparités de consommation, subies, existent aussi entre les abonnés en raison de l'irrégularité des débits et des coupures. Lorsqu'il n'est pas organisé en fonction de prescriptions politiques, pour préserver certains quartiers et leurs clientèles, le vannage peut en outre être soumis à des pressions et marchandages individuels ${ }^{743}$.

Quelles qu'en soient les causes, ces disparités entre consommations spécifiques témoignent de l'inégal accès à l'eau. L' "apartheid hydrique » de Windhoek en Namibie, qui a encouragé les consommations somptuaires des riches ménages blancs, tandis que les ménages noirs raccordés consommaient moins de 70 litres $/ \mathrm{hab} / \mathrm{j}$ et les squatters guère plus de 201 litres $/ \mathrm{hab} / \mathrm{j}$, n'est que l'exacerbation d'une inégale répartition de la ressource, très fréquente dans les villes en développement.

740 Idem.

741 BAH A., DIALLO A. et MORIN R., op. cit.

742 Parfois plus de 500l/hab/j

743 JAGLIN S., «L'eau potable dans les villes en développement : les modèles marchands face à la pauvreté », Tiers-Monde Année, Volume 42, Numéro 166, pp. 280-281 
La persistance de ces inégalités et les enjeux sanitaires liés à la mauvaise desserte des pauvres ne sont évidemment pas la seule ni même la plus déterminante des causes qui ont conduit aux réformes institutionnelles entreprises dans les années 1980. Dans la mesure où celles-ci sont accompagnées d'une redéfinition des objectifs à atteindre en terme d'universalisation du service, "l'eau du robinet pour tous » ne serait plus à l'ordre du jour et l'on préfère désormais évoquer la question en termes d'accès à l'eau potable, il est néanmoins utile de rappeler la façon dont ces réformes abordent la question de la pauvreté et du financement des transferts sociaux.

L'on avait pu proposer pour résoudre le problème de l'accès difficile à l'eau, le recours aux subventions, mais celles-ci n'ont pas du tout résolu le problème. Elles sont la plupart du temps, mal utilisées ou dans certains cas détournées. L'exemple des subventions au kérosène en Ethiopie, illustre bien la situation. Dans les communes urbaines d'Éthiopie en 1996, près de 86 pour cent des subventions sur le kérosène tombaient dans l'escarcelle des non-pauvres, puisque la consommation de ce produit augmente avec le revenu. De plus, même lorsque le marché officiel est abondamment subventionné, de nombreux pauvres sont contraints, faute d'accès officiel, d'acheter sur le marché secondaire, les riches profitent là encore de la faiblesse des prix officiels ${ }^{744}$. Les subventions reçues par les entreprises publiques ne contribuent donc pas toutes à réduire la pauvreté, parce que ce sont souvent les groupes les plus riches qui en bénéficient. Les entreprises publiques ont conduit à verser des rentes à une clientèle relativement limitée. Les données font apparaître un double impact des privatisations sur les prix. Dans le secteur des télécommunications, l'intensification de la concurrence provoquée par l'octroi de nouvelles licences de téléphonie fixe ou mobile a généralement conduit à une baisse des tarifs. Mais l'on observe un phénomène inverse dans les secteurs de l'énergie et de l'eau. Les subventions des prix de l'eau et de l'électricité ayant été une pratique courante, nombre des titulaires d'un bail ou d'une concession ont dû réajuster leurs prix pour rentrer dans leurs frais d'acquisition ${ }^{745}$.

Dans de nombreux cas (comme en Ouganda, en Zambie et au Zimbabwe), les tarifs ont été augmentés avant la privatisation pour limiter le besoin de financement des entreprises et séduire des acheteurs stratégiques. Il y a, bien entendu, eu des exceptions. La concession accordée en 1997 pour la Société d'énergie et d'eau du Gabon (SEEG) a conduit à une diminution des tarifs de l'eau et de l'électricité. Cela tient à la fois à la conception du contrat de concession, qui énonçait clairement les objectif de qualité et de couverture, et au long processus préparatoire de restructuration, qui a duré près de dix ans ${ }^{746}$.

744 Repères, $\mathrm{n}^{\circ}$ 14, octobre 2005, www.oecd.org/dev/reperes consulté le 12 avril 2006.

$\mathbf{7 4 5}$ Idem.

$\mathbf{7 4 6}_{\text {Idem }}$. 


\section{B. Les femmes, grandes perdantes de la privatisation de l'eau}

Les femmes jouent un rôle important dans la gestion des ressources hydriques. C'est presque toujours à elles qu'incombe la collecte, l'utilisation et la gestion de l'eau servant à la famille ainsi que la responsabilité des cultures irriguées et pluviales. En raison de ces rôles, les femmes ont des connaissances étendues en matière de ressources en eau, y compris leur qualité et leur fiabilité, leurs restrictions et les méthodes correctes de stockage, et c'est d'elle finalement que dépend le succès des politiques et programmes d'irrigation et de mise en valeur des eaux ${ }^{747}$. Les femmes sont les plus touchées par cette situation. Plus de la moitié des 1.4 milliards des personnes qui n'ont pas accès à l'eau sont des femmes et des filles. La 4ème Conférence mondiale des femmes des Nations Unies en 1995 consacre un chapitre entier dans son plan d'action sur les femmes et l'environnement dans lequel l'eau est insérée ${ }^{748}$, bien que l'on n'ait pas analysé comment une pénurie d'eau ou sa contamination affecte les femmes disproportionnellement

\section{La situation précaire de la de la femme en milieu rural et urbain}

Le droit des femmes à l'eau est, dans le meilleur des cas, précaire. Du moment qu'elles dépendent de la petite irrigation ou de l'irrigation manuelle, elles ont du mal à combattre la sécheresse. Souvent, les techniques disponibles sont inadaptées, telles les pompes munies de manches qu'elles ne peuvent toujours atteindre ou actionner ou encore qu'elles n'ont pas appris à réparer ${ }^{749}$. Les pratiques culturales des femmes doivent normalement s'adapter au degré d'humidité du sol qui, à son tour, dépend des aléas climatiques et de l'état des sols. Lorsque les stratégies de survie des femmes entraînent l'érosion, leurs pratiques agricoles peuvent représenter de graves sources d'instabilité pour les bassins versants ${ }^{750}$.

En zone rurale, les femmes et les enfants fournissent la quasi-totalité de l'eau servant à la famille. Cette eau est utilisée comme boisson, dans la transformation et la préparation des aliments, pour se baigner et faire la lessive, pour irriguer les jardins familiaux et pour abreuver le bétail. Les femmes connaissent l'emplacement, la fiabilité et la qualité des ressources locales en eau. Elles la collectent, la stockent et en contrôlent l'utilisation et la salubrité. Elles recyclent l'eau, utilisant les eaux grises pour la lessive et l'irrigation et le trop-plein pour le bétail ${ }^{751}$.

747 http://www.fao.org/FOCUS/F/Women/Water-f.htm consulté le 19 mars 2009.

748 Déclaration de Beijing.

749 http://www.fao.org/FOCUS/F/Women/Water-f.htm consulté le 19 mars 2009.

750 Idem.

751 Idem. 
Les femmes font de multiples usages des sources d'eau qu'elles exploitent au maximum, et veillent à ce qu'elles ne soient pas polluées. Etant donné la multiplicité de leurs besoins souvent concurrentiels (eau pour le bétail et pour la consommation humaine) sans compter le temps et les ressources nécessaires, elles ne peuvent toujours éviter la contamination de l'eau disponible. A mesure que l'homme, les animaux et le ruissellement provenant de l'agriculture contaminent les sources d'eau, que s'accroît la sécheresse ou que se dégradent les sources du fait d'une mauvaise gestion des bassins versants, les femmes et les enfants doivent parcourir de plus longues distances pour se procurer de l'eau. Quelque 30 pour cent des femmes en Egypte marchent tous les jours pendant plus d'une heure pour trouver l'eau nécessaire au ménage. Dans certaines régions d'Afrique, les femmes et les enfants consacrent huit heures par jour à la collecte de l'eau.

Les difficultés d'accès à l'eau et sa faible qualité nuisent non seulement à l'élevage et aux activités agricoles des femmes, mais aussi à la quantité de travail qu'elles doivent destiner à la collecte au stockage, à la protection et à la distribution de l'eau. Ces facteurs nuisent également à leur santé et à celle de leurs familles. Toutes les maladies liées à l'eau, notamment celles transmises par l'eau et certains vecteurs, frappent des millions de pauvres chaque année. Les femmes doivent soigner les personnes affligées de paludisme, d'onchocercose, de bilharziose et de diarrhée et fournir leur propre main-d'œuvre en substitution de celle des malades ${ }^{752}$. Mais en ville, les difficultés rencontrées par les femmes sont particulières. L'eau, contrairement à celle recueillie en milieu rural, a un coût et celui-ci risque d'être répercuté sur le prix.

Selon Nici NELSON ${ }^{753}$ dans certaines villes du Kenya, comme Mathare, les femmes estiment que se procurer de l'eau leur donnait beaucoup de mal qu'à la campagne. Beaucoup de ces femmes venaient de régions bien irriguées du Kenya et n'avaient pas l'habitude des régions semi-arides où le point d'eau le plus proche est à plusieurs kilomètres. Elles appelaient Mathare "la cité de la boue et de la poussière », où il fallait continuellement laver les corps et les vêtements. Même dans une agglomération aussi pauvre et marginale que Mathare, la ville impose un degré de propreté et d'élégance plus grand que la campagne ${ }^{754}$.

D'un côté, la ville de Mathare par certain côté est sûrement plus sale que la campagne. La densité élevée des habitations, les tas d'ordures, le manque de latrines et d'installations sanitaires, auxquels s'ajoute le manque de soins généralisé des visiteurs qui, constamment, arpentent la vallée en quête de bière, de compagnons ou de femmes, font que très souvent les terrains vagues et les voies de passage sont couverts de déchets, de détritus et d'excréments. Les mères en sont conscientes et ne cessent de lutter, bien que sans succès, contre les ivrognes du quartier; elles ressentent le besoin de laver leurs enfants beaucoup plus souvent qu'à la campagne, car « on ne sait où il a été joué et avec quoi $»^{755}$.

752 http://www.fao.org/FOCUS/F/Women/Water-f.htm consulté le 11 novembre 2009.

753 NELSON N., «L'importance de l'eau pour les femmes africaines vivant dans un bidonville : le cas de Mathare Valley, faubourg de Nairobi (Kenya)», Tiers-Monde, Année 1980, Volume 21, Numéro 84, p. 866.

$\mathbf{7 5 4}$ Idem.

755 Idem. 


\section{Le risque de l'aggravation de la situation des femmes}

Dans plusieurs pays du monde, il en est de même pour l'eau. Les femmes et les filles, responsables de l'approvisionnement en eau et des tâches domestiques de leur foyer, doivent passer plusieurs heures par jour à marcher jusqu'à la source d'eau (parfois potable, parfois non potable) la plus proche. Une Sud-Africaine témoigne : «Dans les communautés rurales, nos principaux problèmes sont les suivants : couvrir de longues distances à pied, 2 à 3 kilomètres par jour, pour avoir accès à un réseau public d'approvisionnement en eau; porter sur nos têtes de lourds jerricanes de 20 à 25 litres d'eau à chaque voyage; faire la queue pendant des heures au point d'approvisionnement; et si l'eau est contaminée à cet endroit, c'est tout le village qui est menacé» ${ }^{756}$. Cette femme n'est pas la seule : le Programme des Nations unies pour l'environnement (PNUE) estime que, pour le transport de l'eau en Afrique du Sud, l'ensemble des femmes parcourent quotidiennement une distance qui équivaut à 16 allers-retours TerreLune $^{757}$.

L'impact du manque d'eau sur la vie des filles et des femmes est renversant. Il est d'autant plus surprenant pour les Occidentaux que nous sommes peu habitués à voir l'eau comme une préoccupation quotidienne, comme le projet d'une journée. En effet, selon le Programme des Nations unies pour le développement (PNUD), « la corvée consistant à aller chercher l'eau et à la transporter sur de longues distances fait que des millions de filles ne vont pas à l'école, ce qui les voue à un avenir caractérisé par l'analphabétisme et des choix limités ${ }^{758}$. Non seulement la corvée d'eau est-elle une entrave à la scolarisation des filles, mais le manque de latrines dans les écoles est problématique pour les jeunes filles qui ont leurs règles mensuellement. Le PNUD estime que la moitié des filles d'Afrique abandonne l'école primaire en raison du manque d'installations sanitaires appropriées ${ }^{759}$.

En plus du fait que plusieurs femmes ne pourront recevoir l'éducation de base qui leur permettrait d'élargir leurs possibilités, mais elles doivent de plus prendre soin des membres de leur famille aux prises avec des maladies liées au manque d'eau potable. Le manque d'eau a donc des conséquences désastreuses sur l'éducation des filles et sur leur capacité à avoir une vie économiquement et politiquement productive, qui garantit le développement de leur plein potentiel.

On assiste alors à la mise en place d'un cercle vicieux : les femmes sont sous-représentées au sein des instances décisionnelles (politiques) et intellectuelles (universités) de plusieurs sociétés. Aussi, il faut préciser que la privatisation de l'eau peut constituer des obstacles aux progrès de l'éducation en faveur des enfants défavorisés, en particulier des filles. Car, " en dépit d'une scolarisation primaire quasi universelle dans de nombreuses parties du monde, des failles

756 World Water Council. 2005. <http://watercouncil.org/index.php ?id=23\&L=1>. Le 19/03/2006.

757 PNUE (Programme des Nations unies pour l'environnement).

<http://hqweb.unep.org/OurPlanet/imgversn/152/french/glance.html>. le 19/03/2006.

758 PNUD (Programme des Nations unies pour le développement). 2006.

<http://hdr.undp.org/en/media/HDR_2006_Presskit_FR.pdf>. Consulté le 19/03/2006.

759 Idem. 
demeurent. Dans les pays à IDH faible, près de 3 enfants sur 10 en âge d'aller à l'école primaire n'y sont même pas inscrits. Cette situation s'explique par de multiples contraintes, dont certaines sont environnementales. L'absence d'électricité, par exemple, a des effets à la fois directs et indirects. L'accès à l'électricité permet un meilleur éclairage qui allonge le temps d'étude, ainsi que l'emploi de cuisinières modernes qui réduisent le temps passé à collecter le combustible et l'eau $\gg^{760}$.

\section{§2. Une obstruction à la dynamique des droits sociaux}

La privatisation du secteur de l'eau peut perturber la promotion et l'éclosion des autres droits sociaux. La question de la primauté des droits humains sur les accords de commerce. Interprétant de façon limitative les principes du droit international, voire les sélectionnant à son gré, l'OMC, sous prétexte de vouloir « dépolitiser » le commerce, tente de fait de se dégager des obligations nées de la préséance du droit relatif aux droits de l'homme sur tout autre traité international, notamment commercial. Il est clair que l'OMC doit être intégrée au système onusien, afin de devenir pleinement responsable au regard de la Charte des Nations-Unies ${ }^{761}$. Les pays notamment les pays européens ne sauraient ignorer ni pour les autres ni pour eux mêmes les problèmes de l'exclusion, ceux de la santé, de la faim, du logement, du niveau de vie décent et de l'accès à l'eau. Il faut donc cerner la notion même des droits sociaux (A) et expliquer qu'à l'instar des autres droits sociaux, le droit à l'eau n'est point considéré (B).

\section{A. L'ambiguïté du concept des droits sociaux}

Historiquement contestés, les droits sociaux ont été reconnus sous l'influence des thèses marxistes, thèses selon lesquelles ces droits apparaissent comme des libertés réelles opposées aux libertés classiques qualifiées de bourgeoises. Ces droits qui « sèment» le doute et le flou dans nos esprits, ces droits désignés sous plusieurs vocables notamment celui de droits-créances, jouent cependant un rôle important dans l'épanouissement de l'être humain et à l'affirmation de la dignité de l'être humain ${ }^{762}$. SAINT-JAMES V. en a consacré quelques articles dont les plus remarquables portent sur le « droit à la santé ${ }^{763}$ » et le « droit au logement $»^{764}$. Un droit-créance d'accès à l'eau pourrait être indirectement rattaché à deux normes constitutionnelles : le droit au

760 PNUD, Rapport sur le développement humain 2011, p. 7.

$\mathbf{7 6 1}$ http://www .fidh.org/DROITS-DE-L-HOMME-ET-COMMERCE-INTERNATIONAL-LE

762 SAINT-JAMES V., «Réflexions sur la dignité de l'être humain en tant que concept juridique du droit français », D. Chron., 1997, p. 61.

763 SAINT-JAMES V., « Le droit à la santé dans la jurisprudence du Conseil constitutionnel », RDP 1997, p. 457.

764 SAINT-JAMES V., « Faut-il désespérer du droit au logement ? », JCP A 2008, n 1-2, p. 31. 
logement et à la protection de la santé publique ${ }^{765}$. L'effectivité de certains de ces droits est nulle ou incertaine comme c'en est le cas du droit à l'eau aujourd'hui ${ }^{766}$.

\section{La notion de droits sociaux}

L'appellation «droits sociaux» recoupe tous les droits par rapport à un terrain social. Qu'estce que le «social »? Est-il le phénomène fondateur des droits dits « économiques et sociaux ?». $\mathrm{Au}$ sens étroit, le social suppose les seules relations du travail (rapports employeurs et employés), incluant la protection sociale des loueurs de services, et son extension aux agents publics, professionnels libéraux et aux anciens travailleurs. Le social, c'est tout ce qui a été conquis dans un rapport de force avec le libéralisme sauvage et qui met en cause l'Etat mais aussi et surtout, à l'intérieur même de chaque Etat, interpelle les interlocuteurs sociaux, acteurs économiques ${ }^{767}$.

$\mathrm{Au}$ sens large, le social c'est ce qui touche l'être humain en tant que tel, sans distinction inégalitaire, dans sa qualité et son niveau de vie matériel et culturel. Il est l'expression de la lutte contre les exclusions de toutes natures. Il s'élargit à tout ce qui est désormais le combat pour le développement humain ${ }^{768}$.

En France, la proclamation des droits sociaux remonte à la Constitution de 1848. En effet, l'article VIII du préambule recommande à la République le «soin: par une assistance fraternelle, d'assurer des citoyens nécessiteux, soit en leur procurant du travail dans les limites de ses ressources, soit en donnant, à défaut de la famille, des secours à ceux qui sont hors d'état de travailler $\gg^{769}$. Le Comite des Droits Economiques, Sociaux et Culturels s'est inquiété à propos de la non-prise en compte du droit à l'eau. Les droits sociaux sont très variés et leur mise en œuvre ainsi que leur respect semblent parfois compliqués. La qualification même de ces droits est souvent controversée. Pour certains, le droit au travail, le droit au logement ou le droit à l'eau par exemple sont davantage des principes que des droits. L'Etat ne serait pas forcé de les mettre en œuvre. C'est un objectif à atteindre. Le rapport de ces droits, à la loi et au juge est donc ambigu. Les juges sont tenus de faire respecter les droits, tous les droits ; mais s'ils sont trop actifs ils risquent d'être accusés de mettre fin à la séparation des pouvoirs entre le législateur et le judiciaire ${ }^{770}$. C'est dans cette optique que Albert JACQUART affirma que : « le respect de la

765 Le droit à l'eau potable est inséparable du droit au logement décent, progressivement affirmé par le législateur à partir des années 1980 et reconnu comme un objectif à valeur constitutionnelle par le Conseil constitutionnel en 1995

766 FIAILAIRE J. et MONDIELLI E., Droits et libertés publiques, Ellipses, 2005, p. 25.

767 http://perso.orange.fr/credh.benar/wazak.htm

768 Voir les critères très détaillés exposés dans les rapports annuels du PNUD, qui étudient également les liens du développement humain avec la croissance économique.

769 FIAILAIRE J. et MONDIELLI E., op. cit., Ellipses, p. 61.

770 PUTELAT E., La question de la justiciabilité des droits sociaux à l'épreuve du droit européen, Séminaire de droit européen, Université de Strasbourg, 2004-2005. 
loi est toujours préférable, mais il est parfois nécessaire d'aider le législateur à la transformer ou à l'interpréter dans un sens plus favorable aux personnes défavorisées $»^{771}$.

\section{Des droits indivisibles et interdépendants}

Les droits économiques, sociaux et culturels sont aussi largement reconnus dans les systèmes de droit interne, mais pas dans la même mesure que les droits civils et politiques. Des dizaines de constitutions nationales, comme celles de l'Afrique du Sud, de la Finlande et du Portugal par exemple, reconnaissent expressément les droits économiques, sociaux et culturels comme des droits pleinement justiciables. La protection et la promotion de ces droits sont citées parmi les obligations générales de l'Etat dans le domaine juridique et politique dans de nombreuses autres constitutions nationales, y compris celles de l'Inde, des Pays-Bas et du Mexique. Tous les Etats, ou presque, ont des lois internes incorporant des éléments des droits économiques, sociaux et culturels. Le statut juridique de ces droits n'est donc pas contesté. Seul un petit nombre de systèmes internes incorporent tous les éléments de tous les droits économiques, sociaux et culturels, mais la grande majorité des Etats a ratifié les instruments internationaux qui reconnaissent ces droits et adopté des lois nationales et locales visant à leur donner effets.

Il n'en a pas toujours été ainsi. Et même, les militants des droits de l'homme ont déployé des efforts immenses pour obtenir dans les textes et en pratique la reconnaissance de l'interdépendance des droits. Les principes de l'indivisibilité et de l'interdépendance sont au cœur des droits de l'homme, de même que la dignité inhérente à la personne humaine, la participation et l'égalité des sexes.

Le caractère indivisible et interdépendant de tous les droits de l'homme signifie que les droits économiques, sociaux et culturels s'appliquent à tous les individus sur un pied d'égalité et sans discrimination, qu'ils donnent lieu à des obligations concrètes de l'Etat, qu'ils sont justiciables et que les hommes et les femmes peuvent et doivent les faire valoir ${ }^{772}$. Tous les droits doivent être traités comme égaux par les institutions nationales des droits de l'homme dans leurs efforts pour protéger et promouvoir les droits de l'homme. Tous les traités relatifs aux droits de l'homme contiennent des dispositions qui ont un rapport direct avec les droits économiques, sociaux et culturels. Même le Pacte international relatif aux droits civils et politiques et d'autres conventions qui, en apparence, traitent exclusivement des droits civils et politiques, en reconnaissant le droit à la vie, à l'égale protection de la loi et à la liberté de réunion, reconnaissent en même temps indirectement des éléments constitutifs des droits économiques, sociaux et culturels. Dans de nombreux pays, les tribunaux l'ont d'ailleurs reconnu. Par exemple, de nombreux tribunaux ont jugé que le droit à la vie suppose nécessairement d'autres droits essentiels à une qualité élémentaire de vie, comme le droit à l'éducation et aux soins de santé Le maintien des deux catégories traditionnelles de droits est de plus en plus dépourvu de sens et d'intérêt, et résulte de la méconnaissance et d'une mauvaise interprétation du droit relatif aux

771 JACQUART A., L'équation du nénuphar, Calmann-Lévy, 1998, p.190.

772 Haut Commissariat des Nations Unies aux droits de L'Homme, Droits économiques, sociaux et culturels, Manuel destiné aux institutions nationales des droits de l'homme, Série sur la formation professionnelle $\mathrm{N}^{\circ} 12$, NATIONS UNIES New York et Genève, 2004, p.4 
droits de l'homme et des violations de ce droit. Beaucoup des droits de la personne sont perméables par essence. Les droits civils et politiques et les droits économiques, sociaux et culturels devraient être fusionnés et non pas clivés ${ }^{773}$. Ainsi, toutes les normes relatives aux droits de l'homme pourront être utilisées pour appuyer les revendications de droits économiques, sociaux et culturels. Une telle approche unifiée des droits de l'homme englobe les principes de l'égalité et de la non-discrimination, qui sont le socle du droit relatif aux droits de l'homme.

Ces principes supposent l'égalité de traitement, l'égale protection de la loi, l'égalité des chances et l'égalité effective. Bien qu'associés en général aux droits civils et politiques, ces principes s'appliquent de la même façon aux droits économiques, sociaux et culturels. Leur importance continuera de grandir, en particulier à mesure que de nouvelles formes d'égalité effective seront reconnues et qu'il sera admis que les droits à l'égalité entraînent pour les Etats une obligation positive d'agir, et non une simple obligation d'abstention ${ }^{774}$.

\section{B. L'accès à l'eau, un droit sacrifié comme les autres droits sociaux}

Il n'est pas surprenant que les droits sociaux, le droit à l'eau en particulier, soit à la traîne. Car il est une vieille conception qui relègue le ces droits sociaux au second plan. La faiblesse du cadre normatif dans les pays du Sud et l'inexistence d'un cadre législatif international sont deux problèmes majeurs ne font qu'aggraver la situation.

\section{Les droits sociaux en souffrance en Afrique subsaharienne}

En Afrique sub-saharienne, cette inquiétude est reprise en écho par les syndicats et les organisations non gouvernementales qui cherchent à avoir une meilleure visibilité sur un avenir qui risque d'être hypothéqué si rien n'est fait pour sauvegarder l'accès des plus pauvres aux services les plus élémentaires comme l'eau, l'éducation, la santé, le transport, l'électricité et les télécommunications. Un document inédit du syndicat de la Société des Télécommunications du Mali illustre cette inquiétude en évoquant l'illusion donnée au consommateur de lui laisser le choix du produit (bien ou service), accentuée par tout un tapage médiatique qui frise le viol de son intégrité mentale ${ }^{775}$.

773 SCOTT C., «The interdependence and permeability of human rights norms: towards a partial fusion of the International Covenants on Human Rights», Osgoode Hall Law Journal, vol. 27, no 4, 1989; voir en particulier les pages 851 à 878 .

774 Schacter c. Canada (1990), Cour fédérale d'appel du Canada, 2 F.C. 129. Voir aussi HUNT Paul, Reclaiming Social Rights: International and Comparative Perspectives (Aldershot, Dartmouth Publishing Company, 1996), p. 95 à 106.

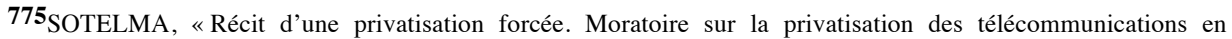
Afrique », Document inédit publié le 13 juillet 2005. 
On retrouve, cependant dans la littérature des opinions moins négatives sur le rapport entre privatisations et les droits économiques, sociaux et culturels. C'est ainsi qu'un auteur, se basant sur les commentaires généraux du Comite des Nations Unies pour les Droits Economiques, Sociaux et Culturels sur la nature des obligations des Etats-parties au Protocole International sur les droits, économiques, sociaux et culturels, affirme que le Droit international est neutre sur la privatisation et ne saurait être interprété en faveur ou a l'encontre de cette politique. Selon lui, le Comite note que les termes « entreprendre les mesures nécessaires....par les moyens appropries » incluent toute forme de gouvernance économique à la seule condition que les principes démocratiques et les droits de l'homme soient respectes ${ }^{776}$.

A partir d'exemples subsahariens, l'on peut souligner deux caractéristiques importantes. D'abord, l'inégale diffusion de la privatisation et de la libéralisation des services d'eau est productrice de nouvelles disparités. Ensuite, dans les aires des monopoles juridiques délégués, l'intensité variable des efforts de libéralisation est créatrice de fortes disparités intra-urbaines et rurales $^{777}$. Aussi, le problème crucial auquel la privatisation est censée remédier est celui de l'accès limité aux services publics des consommateurs en milieu urbain et rural. L'intervention d'un cadre réglementaire approprié permettra sans doute de réguler à la fois l'accès et les tarifs. Par exemple, en Côte d'Ivoire, des mesures ont été prises pour contribuer à améliorer la couverture des réseaux d'eau et d'électricité dans les zones rurales. La ville d'Abidjan comptait, en 2001, 48 pour cent des branchements, 65 pour cent du chiffre d'affaires et seulement 52 pour cent des coûts de production, en raison de la proximité de la source d'eau. Aucune des agglomérations secondaires en Côte d'Ivoire ne serait intrinsèquement rentable et ces zones ne pourraient être approvisionnées en eau sans les excédents dégagés à Abidjan ${ }^{778}$.

\section{L'évolution du droit positif en la matière}

Le droit positif se défait lui aussi progressivement de la conception rigide de la séparation des droits civils et politiques/droits économiques, sociaux et culturels. Il suffit de rappeler, à titre purement exemplatif, l'insistance du IV ${ }^{\text {ème }}$ paragraphe du préambule de la Charte africaine des droits de l'homme et des peuples sur le caractère indivisible et interdépendant des droits de l'homme. Dans le même sens, les résolutions des Nations-unies qui affirment l'indivisibilité des droits de l'homme sont très nombreuses. La plus connue est incontestablement la Résolution $\mathrm{n}^{\circ}$ 60/251 adoptée par l'AGNU le 15 mars 2006 qui stipule que : «tous les droits de l'homme sont

776 Commentaires Généraux n. 3, La nature des obligations des Etats-parties (art.2, para 1 PIDESC), 1990, NU Doc. E/1!/23, Annexe III.

777 JAGLIN S., « Vingt ans de réformes dans les services d'eau urbains d'Afrique subsaharienne : une géographie de la diversité », Séminaire de recherche du GDR Rés-Eau-Ville (CNRS 2524) «L'eau à la rencontre des territoires », Montpellier, France, 27-28 et 29 mai 2004.

778 TREMOLET, S., « Multi-utilities and Access », Public Policy for the Private Sector, Note ${ }^{\circ}$ 248, Groupe de la Banque mondiale, Washington, D.C., juin 2002. 
universels, indivisibles, indissociables et interdépendants et se renforcent mutuellement, comme d'égale importance, et qu'il faut se garder de les hiérarchiser ou d'en privilégier certains ${ }^{779}$.

Le Comité des droits économiques, sociaux et culturels a, lui aussi, déclaré que l'adoption d'une classification rigide des droits économiques, sociaux et culturels qui les placerait, par définition, en dehors de la juridiction des tribunaux serait arbitraire et incompatible avec le principe de l'indivisibilité et de l'interdépendance des deux types de droits de l'homme. Elle aurait en outre pour effet de réduire considérablement la capacité des tribunaux de protéger les droits des groupes les plus vulnérables et les plus défavorisés de la société ${ }^{780}$.

En droit international, il a été pendant longtemps prétendu que les auteurs du PIDESC n'avaient pas voulu que les droits consacrés soient justiciables, et que c'est pour cette raison qu'ils n'auraient pas inséré dans ce pacte un pendant de l'article 2 du PIDCP consacrant le droit au recours en droit interne en cas de violation. Le même courant renchérit en prétendant que ceci explique également que le comité n'eût été créé que longtemps après l'entrée en vigueur du pacte. Sur le droit au recours prétendument non envisagé par le PIDESC, le comité des droits économiques, sociaux et culturels, interprétant l'article 2 de ce pacte dans une de ses observations générales, affirme que l'Etat qui chercherait à se justifier du fait qu'il n'organise pas de recours contre les violations des droits économiques, sociaux et culturels devrait établir que ce recours ne constitue pas un moyen approprié au sens de l'article 2 du PIDESC ou que ce recours est, eu égard aux autres moyens organisés, surplus ${ }^{781}$. Le comité en conclut que cela est très difficile à montrer et que les autres moyens risquent d'être inopérants s'ils ne sont pas renforcés ou complétés par des recours juridictionnels ${ }^{782}$.

Quant à l'absence initiale du comité, à supposer même qu'elle ait un lien avec la volonté d'empêcher la justiciabilité des DESC, sa création, en 1985, par le conseil économique et social devrait être interprété comme un changement de cap, changement que confirme le protocole facultatif au PIDESC entré en vigueur en 2008 et accroissant la compétence du CDESC jusqu'à l'habiliter à recevoir les communications individuelles et à conduire des enquêtes.

\section{Section II. La privatisation du secteur de l'eau, une menace sur la protection des ressources en eau}

L'Etat est comptable vis-à-vis de la population de l'accès à l'eau, de l'organisation de la disponibilité de la ressource et de la surveillance de sa potabilité. Il est également chargé de négocier le droit international de l'eau, de définir le droit national, de réglementer l'usage de la

779 DUMONT H., Systèmes internationaux de protection des droits de l'Homme, Syllabus de Cours, Université du Burundi, Chaire UNESCO en Education a la paix et Résolution Pacifique des conflits, 2008- 2009, inédit, p. 44.

780 Amnesty International, Projet de fiche sur l'application du PIDESC en droit interne et la justiciabilité des DESC, p.2, inédit.

781 L'article 2 du PIDESC oblige en effet tous les Etats parties à user de tous les « moyens appropriés » en vue d'assurer le plein exercice des droits que le pacte consacre.

782 DUMONT H., op.cit., p 51. 
ressource et d'organiser la conciliation entre ses différents usages. Aussi, en sa qualité de législateur et de régulateur du secteur de l'eau, il détermine les modes de gestion les plus efficaces des différents services ainsi que leurs règles de financement et incite à leur mise en place effective. Somme toute, l'Etat joue aussi un rôle essentiel dans la normalisation en matière de santé publique ou d'environnement ${ }^{783}$.

La question de la protection des ressources en eau provient du fait que l'eau est devenue un enjeu symbolique de la rhétorique internationale. D'un côté, les mouvements altermondialistes la défendent comme un droit et un bien commun non marchand et veulent appuyer les déshérités des bidonvilles qui en sont privés. Ils dénoncent l'oligarchie mondiale de l'eau et le contrôle des agences de l'eau par le politique, le lobby agricole ou certaines multinationales. De l'autre, les multinationales de l'eau ainsi que les agences d'aide, notamment la Banque mondiale, mettent en avant l'efficacité et la rentabilité de la gestion d'activités très capitalistiques ${ }^{784}$. La question est en fait très complexe. L'eau est un bien fluide au cœur des écosystèmes. Elle est une ressource vitale non substituable et un symbole de la fragilité de la vie. Cette ressource planétaire tend à se raréfier et est très inégalement distribuée à l'échelle de la planète. Elle a aussi une dimension locale et régionale du fait de son coût de transport conduisant à des écarts entre ressources disponibles et capacités d'accessibilité selon les territoires. La concession de ces services au profit des multinationales met en péril la ressource $(\$ 2)$ et participe à la négligence des eaux souterraines $(\S 2)$.

\section{§1. Des ressources en péril et à la merci du commerce international}

La perte de la souveraineté nationale sur les ressources en eau est difficile à qualifier d'autant plus que l'OMC insiste sur le fait que les accords qui sont le fruit des négociations sont acceptés de plein gré par les gouvernements souverains et que, par conséquent, elle ne les impose pas aux États. Mais elle ne dit pas tout, car les accords de libéralisation peuvent avoir des conséquences imprévues. L'AGCS ayant pour seul objectif de promouvoir le commerce international, il laisse de côté les questions telles que l'équité en matière de services de santé et de l'eau. Pour mieux cerner la question, il importe de définir la notion de ressources en eau. Après avoir défini le concept des ressources en eau (A) nous montrerons comment le Comité du commerce et de l'environnement de l'OMC a progressé dans son approche (B).

783 Etudes et documents du Conseil d'Etat, L'eau et son droit, 2010, p. 24.

784 HUGON P., «Vers une nouvelle forme de gouvernance de l'eau en Afrique et en Amérique latine », Dalloz/I.R.I.S., Revue internationale et stratégique, 2007/2 - №66, p. 65. 


\section{A. La définition des ressources en eau}

En droit interne, notamment dans la plupart des Etats de l'Afrique subsaharienne, l'expression est utilisée dans de nombreux textes sans être définie ${ }^{785}$. Néanmoins, il faut saluer l'initiative du législateur ivoirien qui, dans l'article $1^{\mathrm{er}} \mathrm{du}$ Code de l'Eau ${ }^{786}$, assimile les termes « eau» et " ressources en eau», et affirme que "les termes eaux et ressources en eau sont utilisés de façon interchangeable ». En revanche, en droit international, sa définition manque de précision.

\section{L'approche des ressources en eau en droit interne}

L'eau constitue sans aucun doute un bien très spécifique ${ }^{787}$, le système juridique qui lui est applicable étant autonome, mélangeant dans une large mesure règles de droit privé et règles de droit public ${ }^{788}$. Les lois se sont succédé depuis la fin du 19ème siècle, l'une des plus remarquables et des plus récentes étant la loi nº 92-3 du 3 janvier 1992 sur l'eau $^{789}$. L'eau se décline ainsi en eau dormante, eau souterraine, eau pluviale, eau de mer, source et eau courante, chacune faisant l'objet d'une réglementation propre (même si l'on peut noter une tendance récente de la réglementation à réduire les catégories, en distinguant les « eaux superficielles » des « eaux souterraines »). Si l'on s'arrête sur l'hypothèse des cours d'eau, telle la rivière dans l'affaire ici commentée, il convient d'en distinguer plusieurs types ${ }^{790}$ : le cours d'eau domanial, le cours d'eau non domanial et le cours d'eau mixte (qui répond à un régime juridique intermédiaire). Le cours d'eau domanial relève du droit public et est insusceptible d'appropriation privée, au contraire du

785 Voir l'art.4 de la Loi nº/94/ 005/CTRN du 15 février 1994 portant code de l'eau en Guinée : « Les ressources en eau de la République de Guinée font partie intégrante du Domaine public naturel de l'Etat. En tant que telles, et sous réserve des dispositions du présent Code, elles ne sont pas susceptibles d'appropriation. Cependant elles peuvent faire l'objet d'un droit d'utilisation de nature précaire et limitée soumis au régime de l'autorisation préalable ».

Voir aussi l'article 149 de la Loi 016/PR du 18 août 1999 portant Code de l'eau: «L'allocation des ressources en eau doit à tout moment tenir compte des besoins sociaux et économiques des populations. L'alimentation en eau potable des populations demeure, dans les cas l'élément prioritaire dans l'allocation des ressources en eau ».

786 LOI n $98-755$ du 23 décembre 1998

787 Sur la délicate classification de l'eau parmi les meubles ou les immeubles, Voir. R. SAVATIER, préc., $\mathrm{n}^{\circ} 17$; égal., GAONAC'H A., La nature juridique de l'eau, éd. Johanet, 1999.

788 MALLET-BRICOUT B., « Droit des biens », Recueil Dalloz 2007 p. 2490.

789 JO 4 janv. p. 187 ; D. 1992. Lég. 104.

790 Depuis une loi $n^{\circ}$ 64-1245, 16 déc. 1964 relative au régime et à la répartition des eaux et à la lutte contre leur pollution ; V. en particulier M.-L. MATHIEU-IZORCHE, Droit civil. Les Biens, Sirey, 2006, n 246 s. ; GODFRIN P. et DEGOFFE M., Droit administratif des biens, $7^{\text {ème }}$ éd., Colin, 2005, p. 35 s. 
cours d'eau non domanial, qui relève en principe du droit civil, encadré par le droit de l'environnement ${ }^{791}$.

Il faut ajouter que le code civil organise de façon sommaire le régime juridique des eaux courantes. Un droit d'usage tempéré par le droit de propriété est prévu. L'eau qui s'écoule ou l'eau courante, régie par les articles 640, 644 et 645 du code civil, peut se présenter sous deux grandes formes : les eaux de ruissellement et l'eau des cours d'eau. Le régime des eaux de ruissellement est défini par l'article 640 en vertu duquel : "Les fonds inférieurs sont assujettis envers ceux qui sont plus élevés à recevoir les eaux qui en découlent naturellement sans que la main de l'homme y ait contribué. Le propriétaire inférieur ne peut point élever de digue qui empêche cet écoulement. Le propriétaire supérieur ne peut rien faire qui aggrave la servitude du fonds inférieur. " De portée très générale, cet article enserre les propriétaires voisins dans des obligations réciproques au regard des eaux qui s'écoulent d'un fonds à l'autre. L'eau des cours d'eau, très convoitée dans la France métropolitaine du XIXème siècle, fait l'objet de l'article 644 : "Celui dont la propriété borde une eau courante, autre que celle qui est déclarée dépendance du domaine public (par l'article 538 au titre « De la distinction des biens ») ${ }^{792}$, peut s'en servir à son passage pour l'irrigation de ses propriétés. Celui dont cette eau traverse l'héritage peut même en user dans l'intervalle qu'elle y parcourt, mais à la charge de la rendre, à la sortie de ses fonds, à son cours ordinaire. » L'article 645 s'efforce de prévenir les conflits d'usage que la mention de l'irrigation pourrait susciter à l'intérieur du monde agricole ou avec lui : " $S$ 'il s'élève une contestation entre les propriétaires auxquels ces eaux peuvent être utiles, les tribunaux, en prononçant, doivent concilier l'intérêt de l'agriculture avec le respect dû̀ à la propriété... » La rédaction de ces trois articles est toute d'équilibre : si un droit sur l'eau semble reconnu, une obligation en découle immédiatement pour le voisin, pour le propriétaire du fonds supérieur, pour celui du fonds inférieur ou à l'égard de celui à qui l'eau peut être utile ${ }^{793}$.

\section{L'approche des ressources en eau en droit international}

De même, étudier le statut de l'eau en droit international pose un défi particulier. De quoi parle -t- on ? L'eau existe-t-elle en droit international ? De la molécule au cycle hydrologique, en passant par les écosystèmes aquatiques, la définition de l'objet mérite d'être examinée d'autant plus que "ce n'est qu'au XX'ime siècle que concept de ressource en eau a fait son apparition $^{794} \gg$. En réalité, les concepts actuellement admis par le droit international ne correspondent véritablement ni aux caractéristiques spatiales ni aux caractéristiques

791 Pour une démonstration des imbrications du droit civil et du droit de l'environnement dans cette matière, Voir. MARC P., Les cours d'eau et le droit, préf. R. ROMI, éd. Johanet, 2006 ; égal., MOISAN M., Essai sur le droit et l'administration des cours d'eau en France, th. Paris 1, 1996.

792 L'article 538 du code, qui figure au titre « De la distinction des biens » et définit la consistance de ce domaine, a été abrogé par l'ordonnance no 2006-460 du 21 avril 2006 relative à la partie législative du code général de la propriété des personnes publiques. Le toilettage du code reste à parfaire.

793 RAPPORT CE 2010, Le droit et son eau, p.52.

794 MARGAT J., «Vers une nouvelle culture de l'eau», dans BINDE J., Les clés du XX ème siècle, Paris, UNESCO/Seuil, 2000, p. 124. 
fonctionnelles de l'eau. En remontant à la formalisation des règles concernant les cours d'eau internationaux au congrès de Vienne en 1815, nombreux sont les auteurs qui utilisent l'expression d'internationalisation indiquant clairement par là qu'à cette époque, le caractère international d'un cours d'eau n'était pas fondé sur sa nature mais bien sur la volonté des Etats et sur une fonction, en l'occurrence, la navigabilité ${ }^{795}$. La navigation ayant été l'usage principal considéré en droit international jusqu'au début du $\mathrm{XX}^{\mathrm{ème}}$ siècle, il était normal que le concept utilisé alors corresponde à cette considération restreinte. Mais avec la multiplication des usages de l'eau, cette définition apparaît désuète puisque c'est l'eau en tant que telle et non la voie de circulation, qui doit devenir objet de règles.

Aussi, le caractère vital de l'eau appelle-t-elle une rupture par rapport à la considération traditionnelle de cette ressource, fut-elle territorialement partagée. Dès lors qu'elle est vitale, une ressource ne concerne plus seulement les intérêts des Etats, sujets traditionnels du droit international mais bien l'être humain, entré dans le droit international par la porte des droits de l'homme, car c'est du droit à la vie dont il s'agit ${ }^{796}$.

C'est le projet du Traité de Bellagio qui le premier, va prendre en compte l'intégration des eaux souterraines transfrontalières. En effet, Le projet de Traité dit de «Bellagio » sur les eaux souterraines transfrontalières ${ }^{797}$ applique de façon spécifique aux eaux souterraines les principes précédemment mis en exergue notamment par les «Règles d'Helsinki», à savoir "l'unité de gestion» (respect des eaux souterraines et reconnaissance des interrelations entre ressource en eau superficielle et eau souterraine) et la « communauté d'intérêts », "l'utilisation optimale et la conservation sur une base raisonnable et équitable incluant la protection de l'environnement souterrain $\gg^{798}$ le recours possible à une commission internationale autorisée à déclarer des zones de protection, d'alerte à la sécheresse, à élaborer des plans d'urgence, l'échange réciproque d'informations résultant des inventaires quantitatif et qualitatif et des mesures de contrôle prescrites par chacun des Etats ${ }^{799}$. Le projet de traité prévoit également la mise en œuvre de plans de gestion de la ressource très complets dans les zones de protection (incluant notamment des mesures préventives et des mesures de suppression de la dégradation des eaux, des schémas de surveillance) ainsi que des plans "sécheresse » à l'initiative de la commission (condition de la déclaration d'alerte ou d'urgence, désignation des aquifères à utiliser en cas de sécheresse, diminution graduelle des prélèvements autorisables etc...). Enfin, un mécanisme très sophistiqué de résolution des différends comportant plusieurs degrés et des délais intermédiaires, accorde d'importants pouvoirs à la commission. En effet d'en un premier temps, celle-ci s'efforce de résoudre le différend. Si elle n'y parvient pas, le(s) gouvernement(s) demande(nt) un rapport à une commission d'enquête. La commission poursuit ses efforts sur la base du rapport et si un

795 CAPONERA D. A., «Shared waters and international law » dans BLAKE G. H., HILDESLEY W. J., PRATT M. A., SCHOLFIEL C.H. (dir.), The peaceful management of transboundary Resources, Boston, GRANHAM et Trotman/Martinus Nijhoff, p. 121.

796 PAQUEROT S., Eau douce, la nécessaire refondation du droit international, Presses de l'Université du Québec, 2005, p. 6.

797 Transboundary Groundwaters : the Bellagio Draft Treaty", C.I.R.T., éd. augmentée et revue par MM. D. DAYTON et A.E. UTTON, été 1989 Washington D.C.

798 Cf. article 2

$799 C f$. articles 3 à 5 . 
accord s'avère impossible, les gouvernements s'engagent à avoir recours à la médiation, la conciliation, l'arbitrage, la Cour internationale de Justice ou à tout autre moyen adéquat ${ }^{800}$.

On voit là tout l'intérêt que de telles dispositions pourraient avoir au Proche-Orient, notamment concernant l'aquifère montagneux de Judée-Samarie, Israéliens et Palestiniens devant pouvoir négocier pour parvenir à un règlement fondé sur les principes de «communauté d'intérêt» et de "part raisonnable et équitable » ${ }^{801}$. Ces efforts en vue d'une codification apparaissent certes prometteurs, d'autant que les eaux souterraines respectent encore moins les frontières que les eaux superficielles et que de nombreux aquifères se trouvent partagés par deux ou plusieurs pays dans des zones arides, risquant ainsi d'engendrer des conflits plus difficiles à résoudre qu'en matière d'eaux superficielles. Toutefois à supposer que le projet de Traité soit ratifié par un nombre suffisant d'Etats, une volonté politique demeurera indispensable pour entamer la négociation en cas de contentieux. Sans avoir la naïveté de prétendre que l'existence de règles de droit pourraient systématiquement empêcher les Etats de recourir à la guerre pour régler leurs différends, une accélération du processus s'impose toutefois.

\section{B. Le baroud d'honneur du Comité du commerce et de l'environnement de l'OMC}

Le domaine classique de l'OMC est très vaste. Il s'agit du secteur des marchandises, du secteur des droits de propriété intellectuelle et du secteur des investissements. Cette réflexion prendra en compte l'impact de l'idéologie libérale sur la politique de l'eau en Afrique subsaharienne. Aussi nous irons au-delà de la simple présentation du domaine classique de l'OMC pour démontrer qu'au sein de l'institution, il existe une obédience en faveur d'une vision non mercantiliste de l'eau.

\section{L'étendue du domaine}

Le secteur des marchandises est très vaste. Le commerce international des marchandises regroupe les secteurs économiques réintégrés tels que l'agriculture, les textiles et les vêtements et les secteurs nouvellement intégrés tels que la propriété intellectuelle et les investissements liés au commerce et l'ensemble des transactions physiques. Le secteur des marchandises est en grande partie composé de flux physiques de produits qui traversent les frontières nationales. Ces produits peuvent être des produits de base ou « matières premières $»^{802}$ ou des produits manufacturés. La notion du produit de base est, au sens de la définition proposée par la Charte de

800 Cf. articles 15 et 16 .

801 SHUVAL Hillel. I. « Le problème du partage de l'eau entre Israel et les Palestiniens - A la recherche d'une solution équitable », Revue Monde arabe-Maghreb - Machrek $n^{\circ} 138$ oct. - déc. 1992, pp. 34 et s.

802 LUSSON-LEROUSSEAU, «Les accords de produits de base, instruments de régulation des marchés », R.G.D.I.P. 1981, p.5-37. 
la Havane ${ }^{803}$ de 1948 dont la ratification devait aboutir à la création de l'Organisation Internationale du Commerce (OIC), " tout produit de l'agriculture, des forêts ou de la pêche, et tout minéral, que ce produit soit sous sa forme naturelle ou qu'il ait subi la transformation qu'exige communément la vente en quantité importante sur le marché international ».

Cette définition, qui a cours malgré l'échec de la création de l'OIC, saisit le produit de base comme une ressource naturelle. Or, c'est l'utilisation abusive et la commercialisation à grande échelle des ressources naturelles qui entraînent des pressions sur l'environnement. Certains Etats en développement comme la Côte d'Ivoire et le Nigeria ont bâti l'essentiel de leurs stratégies de développement économique sur l'utilisation abondante, voire abusive des ressources naturelles, tels que les bois tropicaux, le pétrole, les produits agricoles de rente. Les produits manufacturés sont les produits qui ont subi entièrement ou partiellement le processus de transformation industrielle 804 .

L'expansion du commerce international a commencé avec la révolution industrielle au XIX $^{\text {ème }}$ siècle. Celle-ci s'est intensifiée après la seconde guerre mondiale qui ouvre la voie à la reconstruction et à la réalisation des infrastructures économiques dans les Etats dévastés par la guerre. L'industrie, en tant qu'ensemble des activités économiques ayant pour objet l'exploitation des ressources naturelles et leur transformation en produits finis ou semi-finis, ne vise pas prioritairement la protection de l'environnement. De nombreux produits et techniques industriels utilisés de manière intensive sont générateurs de pollution ${ }^{805}$.

Au cours de ces dernières décennies, les effets nocifs du Les investissements internationaux sont à inscrire au rang des secteurs qui, dans leur réalisation, ont plus ou moins directement des impacts sur l'état de l'environnement. Les sociétés transnationales investissent dans les Etats qui leur accordent des facilités tant au niveau juridique qu'au niveau politique.

Les considérations environnementales jouent un rôle déterminant dans l'attrait, le rejet ou la délocalisation des entreprises. Les investissements ont été insérés à l'OMC pour autant qu'ils soient liés au commerce international. Cette extension n'est pas globale mais elle est plutôt connexe dans la mesure où il existe un régime juridique spécifique aux investissements internationaux.

\section{Le sursaut du Comité du commerce et de l'environnement de l'OMC}

Le Comité du commerce et de l'environnement de l'OMC (CCE) est chargé d'identifier les catégories de biens et services environnementaux qui devraient faire l'objet de la réduction des obstacles tarifaires. A ce sujet, les négociations entre les Etats afin de définir ces biens se révèlent particulièrement difficiles. A ce propos, il est intéressant de mentionner que le CCE a

\footnotetext{
803 Cf. article 56 .

804 GADJI A. Y., Libéralisation du commerce international et protection de l'environnement, Thèse, Université de Limoges, 2007, p. 33.

$\mathbf{8 0 5}$ Idem.
} 
suggéré aux États de fournir des exemples pratiques quant aux biens et services qu'ils désireraient inclure dans cette catégorie.

Dans le cadre de ces négociations, il a été mis en relief qu'il faudra tenir compte des intérêts des pays en développement dans l'identification de cette catégorie de biens et services, de façon à améliorer le transfert de technologies vers ceux-ci en la matière ${ }^{806}$.

Quelques Etats membres de l'OMC ont préconisé d'inclure certaines activités reliées à l'eau, telles que le captage, la distribution et la gestion des eaux usées dans la définition des biens et services environnementaux ${ }^{807}$. Tout en reconnaissant que la libéralisation pouvait inciter à un développement durable, les membres ont toutefois rappelé le droit de réglementer l'accès à leurs ressources naturelles et de sauvegarder leurs objectifs de politique générale publique.

Les Etats qui se sont exprimés en faveur de la prise en compte de l'eau dans la définition des biens et services environnementaux ont relevé le caractère particulier de l'eau du point de vue humain en insistant sur le besoin d'assurer l'accès à l'eau et, du point de vue environnemental, en mettant en exergue la protection environnementale nécessaire de cette ressource.

Dans le préambule de l'Accord de Marrakech, les membres de l'OMC ont affirmé que « leurs rapports dans le domaine commercial et économique devraient être orientés vers le relèvement des niveaux de vie [...] tout en permettant l'utilisation optimale des ressources mondiales conformément à l'objectif de développement durable, en vue à la fois de protéger et préserver l'environnement et de renforcer les moyens d'y parvenir d'une manière qui soit compatible avec leurs besoins et soucis respectifs à différents niveaux de développement économique »808. En outre, adoptée lors de la quatrième conférence ministérielle, la Déclaration de Doha édicte : " Nous réaffirmons avec force notre engagement en faveur de l'objectif du développement durable, tel qu'il est énoncé dans le Préambule de l'Accord de Marrakech. Nous sommes convaincus que les objectifs consistant à maintenir et à préserver un système commercial multilatéral ouvert et non discriminatoire, et à cuvrer en faveur de la protection de l'environnement et de la promotion du développement durable peuvent et doivent se renforcer mutuellement ${ }^{809}$.

Du fait que les négociations n'ont pas encore abouti eu égard à l'identification de ces biens et services, il est difficile de tirer des conclusions à ce sujet. Il faut toutefois mentionner que l'inclusion de certaines catégories relatives à l'eau dans la classification des biens et services environnementaux ainsi que l'affirmation du caractère particulier de cette ressource naturelle par les États membres de l'OMC pourront contribuer à déterminer si l'eau a une place dans le régime

\footnotetext{
806 Comité du commerce et de l'environnement (CCE), Communication du Secrétariat de l'OCDE sur les travaux de l'OCDE relatifs aux biens et services environnementaux à la réunion de la session extraordinaire du Comité du commerce et de l'environnement des 12 et 13 octobre 2004, OMC Doc. TN/TE/INF/8 (2004)
}

$\mathbf{8 0 7}$ Voir OMC, CCE, Accès aux marchés pour les biens environnementaux - communication des Communautés européennes, OMC Doc. TN/TE/W/47 (2005) et OMC, CCE, Accès aux marchés pour les biens environnementaux - addendum des Communautés européennes, OMC Doc. TN/TE/W/47/Add.1 (2005); OMC, CCE, Biens environnementaux - communication de la Nouvelle-Zélande, OMC Doc. TN/TE/W/46 (2005). Voir supra pour les implications de cette inclusion à l'égard de l'AGCS.

$\mathbf{8 0 8} C f$. Accord de Marrakech, préambule.

809 Déclaration de Doha 
commercial international. D'autre part, la thèse mercantile a pris également le pas sur la protection des eaux souterraines.

Il est à noter que l'OMC elle-même précise dans son dernier rapport que des ressources naturelles comme l'eau ne peuvent faire l'objet d'échanges, tout en soulignant que le commerce peut avoir un effet bénéfique sur l'approvisionnement mais aussi qu'il peut contribuer à l'épuisement des ressources et peut accélérer l'appauvrissement des réserves ${ }^{810}$.

\section{§2. L'inconsistance du cadre juridique des eaux souterraines}

Au niveau international, très peu de conventions traitent des eaux souterraines bien que l'on puisse aussi considérer, en principe, que les accords qui utilisent la notion de bassin devraient s'appliquer de fait aux eaux souterraines. Toutefois, les législations internes consacrent de plus d'espaces à la notion des eaux souterraines. De même, les avancées constatées ne résolvent pas toujours le problème de l'emprise des multinationales sur les ressources en eau. Tout en reconnaissant qu'il existe un dispositif juridique perfectible (A) il faut saluer les avancées significatives dans certains accords (B).

\section{A. Un dispositif juridique perfectible}

En reprenant CHEMILLIER-GENDREAU ${ }^{811}$, nous affirmerons que les années récentes ont vu se multiplier les textes visant à préserver les eaux dans ce continent. Comme dans le cas des accords africains, on pourrait s'interroger sur l'efficacité de ces textes. A l'évidence, ces différentes mesures ne suffisent pas pour mettre à l'abri les ressources en eau des multinationales.

\section{L'absence de législations spécifiques à l'eau}

En Afrique très peu d'Etats sont dotés d'une législation spécifique sur l'eau. En Afrique francophone subsaharienne, le Sénégal, comme précédemment indiqué, est un pionnier dans ce domaine, avec l'adoption d'un premier Code de l'eau en 1981812. Il a été suivi par le Cameroun

$\mathbf{8 1 0}$ DROBENKO B., Le droit à l'eau : une urgence humanitaire, précité, p.138.

$\mathbf{8 1 1}$ «Le crépuscule du Rhin », Le Monde diplomatique, février 1988.

812 Loi nº $81-14$ du 4 mars 1981 portant Code de l'eau (gou.sn/textes.code_eau.pdf). 
en $1984^{813}$, le Tchad en $1999^{814}$, le Burkina Faso en $2001^{815}$, le Mali en $2002^{816}$, le CongoBrazzaville en $2003^{817}$, le Togo en $2010^{818}$. En l'absence des données concernant les Etats anglophones du continent, nous nous sommes imposé une prudence quant à tous développements relatifs à leur implication dans la préservation des ressources en eau.

$\mathrm{Au}$ niveau interne, nombreux sont les Etats qui commencent par mettre sur pied une législation en la matière. En se référant à la loi n L/94/ 005/CTRN du 15 février 1994 portant code de l'eau en Guinée notamment dans ces articles 23 et suivants, l'on note l'existence de plusieurs mesures notamment, "l'établissement de périmètre de protection autour des sources et des points d'eau captée pour la consommation humaine pouvant comporter les prescriptions relatives à l'utilisation des sols, du sous-sol et des eaux superficielles "; "la délimitation des zones de sauvegarde des ressources en eaux souterraines dans lesquelles les modalités de protection et les conditions d'exploitation de la ou des nappes d'eau souterraines peuvent comporter les prescriptions relatives à l'utilisation des sols, du sous-sol et des eaux superficielles ", et diverses mesures assurant la protection des eaux souterraines ${ }^{819}$. De même la loi n $98-755$ du 23 décembre 1998 portant Code de l'Eau en Côte d'ivoire prévoit en son article 25 que "nul ne doit empêcher le libre écoulement des eaux de surface et des eaux souterraines ». En examinant l'ordonnance n ${ }^{\circ} 85-144$ du 4 juillet 1986 portant code de l'eau en vigueur en Mauritanie, l'on constate que les eaux souterraines sont réglementées ${ }^{820}$ et classées $^{821}$.

$\mathbf{8 1 3}$ La loi du 5 décembre 1984 portant régime de l'eau a été abrogée par une autre loi du 14 avril portant régime de l'eau.

$\mathbf{8 1 4}$ Loi 016/PR du 18 août 1999 portant Code de l'eau.

$\mathbf{8 1 5}$ Loi n $^{\circ} 002-2001 / \mathrm{AN}$ du 8 février 2001 portant loi d'orientation relative à la gestion de l'eau.

$\mathbf{8 1 6}$ Loi du nº2-006 du 31 janvier 2002 portant Code de l'eau.

${ }^{817}$ Loi n $13-2003$ du 10 avril 2003 portant Code de l'eau

$\mathbf{8 1 8}$ Code de l'eau précité.

$819 C f$. Art.24.- «Les modalités d'établissement des périmètres de protection, de délimitation des zones de sauvegarde des ressources en eau et de délivrance des autorisations de forage sont déterminées par Arrêté du Ministre chargé de l'hydraulique».

820 Cf. Art.49.- " Le captage des eaux souterraines au moyen d'un puits d'une capacité inférieure ou égale à deux mètres cube par jour et dont le débit ne dépasse pas cinq cent litres par heure, pour la satisfaction des besoins domestiques individuelles, est autorisé à seule charge pour le propriétaire de déclarer l'ouvrage suivant les formes prévues par l'arrêté pris sous l'autorité de l'Article 33 ».

$821 C f$. Art.50- Les eaux souterraines sont classées en zone I, II et III.

Sont classées en zone I:

1. les bassins ou l'utilisation des nappes souterraines approche des limites de leurs sources;

2. les bassins qui alimentent les localités desservies par un service public de distribution;

3. les bassins où existe un danger potentiel d'intrusion saline ou d'une quelconque substance organique pouvant altérer la potabilité de l'eau ou sa salubrité.

Sont classées en zone II :

1. les nappes situées dans les roches métamorphiques et des mauritanides, 
Cette insuffisance juridique est génératrice de situations, souvent défavorables à l'eau et à l'environnement, d'une manière générale. Cette absence de législation particulière sur l'eau peut constituer, pour certains Etats, un obstacle à l'adaptation des différentes utilisations aux ressources hydrauliques disponibles, à l'échelle du territoire national. Le problème se pose, cependant, avec moins d'acuité dans d'autres Etats de la sous-région moins confrontés à des consommations massives d'eau découlant notamment des activités agricoles et industrielles et, générateurs de conflits entre utilisations ${ }^{822}$. Il est regrettable qu'une vingtaine d'Etats seulement, sur la cinquantaine que compte le continent, soient dotés d'un cadre juridique régissant les différentes utilisations des ressources hydrauliques. En Afrique centrale, on doit le rappeler, seuls le Cameroun, la République du Congo et le Tchad en font partie. Dans son deuxième rapport mondial sur l'eau, l'Organisation des Nations Unies a dénoncé l'inertie des Etats du sud qui n'ont pas adopté, avant 2005, un cadre juridique prévoyant une gestion intégrée des ressources en eau (GIRE), conformément aux engagements pris lors du Deuxième sommet mondial sur le développement durable de Johannesburg de 2002823. L'on peut citer l'accord sur le Plan d'action pour la gestion écologiquement rationnelle ${ }^{2}$ du bassin commun du Zambèze ${ }^{824}$ et bien d'autres encore ${ }^{825}$. Certains traités de délimitations de frontières prévoient l'usage commun des eaux souterraines, mais la répartition des eaux des nappes aquifères demeure aujourd'hui l'exception, alors même qu'elles comptent pour une part de plus en plus importante de l'approvisionnement en eau douce.

\section{Les conséquences des insuffisances législatives}

L'une des incommodités pouvant résulter de l'inexistence d'une législation conséquente est l'impuissance de l'Etat face à la généralisation de certains comportements collectifs « anormaux ». Malgré son faible taux d'industrialisation et un recours restreint aux techniques agricoles modernes, l'Afrique, à l'instar d'autres continents du sud, présente les eaux les plus polluées de la planète par des effluents industriels et urbains. De rares industries, en effet, n'y traitent pas leurs déchets. Ceux-ci finissent par, soit être enfouis dans le sous-sol, soit par être déversés dans les cours d'eau le plus proche au grand dam de la flore et de la faune fluviales et des populations riveraines. De même les effluents urbains, les déchets ménagers et les eaux usées de toute origine, industrielle ou domestique, sont déversés dans des lacs, des rivières et des lacs en toute

2. les schistes précambriens et les granito - gneiss de l'Aftout- Guidimagha,

3. les grès quartzites ordoviciens de l'Assaba-Taguant,

4. les grès infracambriens de l'affolé, les schistes et petites des hodhs.

Sont classées en zone III tous les autres bassins du territoire national.

822 OSSOMBO R., précité, p.101.

$\mathbf{8 2 3}$ ONU, «L'eau : une responsabilité partagée », Deuxième rapport mondial sur la mise en valeur des ressources en eau, Paris, New York, mars 2006.

$\mathbf{8 2 4}$ Harare, 28 mai, ILM, vol. 1988, p. 1109

825 L'Accord de 1963 relatif à la navigation et à la coopération économique entre les Etats du bassin du Niger, de la Convention sur la mise en valeur du bassin du Tchad... 
impunité. Ces pratiques sont courantes à Bangui, Brazzaville et Kinshasa situées au bord l'Oubangui pour la première et au bord du Congo pour les secondes. On voit bien que les cours d'eau sont des égouts à ciel ouvert; ce qui constitue une source de dégradation chronique de la qualité des eaux superficielles ${ }^{826}$.

Il est vrai que le traitement des eaux usées nécessite beaucoup de moyens financiers et que, même dans les pays développés, la dépollution des eaux susmentionnées ne s'est pas généralisée. En France, par exemple, certaines grandes villes (cas de l'agglomération de Marseille notamment) ne se sont dotées de stations d'épuration des eaux qu'au cours de la décennie $1990^{827}$ et le taux de dépollution des eaux usées n'est que de $45 \%$ contrairement à d'autres pays du nord comme les Pays-Bas où il atteint $100 \%$. Cela inquiète d'ailleurs une grande partie de l'opinion publique et les pouvoirs publics quant à l'atteinte des objectifs fixés par la directive du Conseil et du Parlement européen de 2000 établissant un cadre pour une politique communautaire dans le domaine de l'eau.

Donc, si ces Etats disposaient d'une législation sur l'eau, les cours d'eau, les nappes et même les eaux territoriales marines ne constitueraient pas de dépotoirs de déchets et effluents de toute nature. On s'aperçoit aisément qu'ils contribuent, par leur passivité ou leur non créativité, à la dégradation de la qualité des mêmes eaux qui sont prélevées à des fins de production d'eau potable. Le dernier inconvénient de l'absence d'une législation particulière sur l'eau est celui du risque permanent de l'arbitraire administratif et judiciaire. En effet, lorsque l'administration chargée de la gestion des eaux n'exerce pas ses compétences, le risque de l'arbitraire administratif est élevé. Comme on l'a souligné dans les précédents développements, l'Administration peut être tentée de faire abusivement usage de ses prérogatives de puissance publique pour maintenir l'ordre public à travers des sanctions administratives. Les pouvoirs de l'Administration seront alors illimités.

Certes, au nom de la protection de l'eau, les collectivités publiques peuvent faire usage des sanctions administratives classiques pour mettre fin aux différentes atteintes aux ressources, mais le risque de la rupture de l'égalité entre les destinataires des sanctions en cause peut aussi être grand. Pourront ainsi survenir le clientélisme et le favoritisme qui constituent deux entraves majeures à la neutralité de l'action administrative dès l'instant où le juge administratif ne disposera pas de moyens pour en contrôler l'étendue, le cadre et les limites des sanctions précitées. Or s'il y a un cadre juridique précis, l'Administration sait à quoi s'en tenir et à quoi elle est tenue. Il n'est pas exclu qu'il y ait des dérives, même sous l'empire d'un cadre juridique, car l'existence de celui-ci n'est pas nécessairement un gage à la neutralité administrative. On retrouve dans d'autres textes dans d'autres régions du monde, cette difficulté à atteindre un équilibre entre le commerce international et la préservation des ressources en eau.

Ainsi, l'inexistence d'un cadre juridique prévoyant la répression par voie administrative des différentes atteintes aux ressources peut donner des pouvoirs illimités à la puissance publique. On voit bien qu'on est loin des Etats du nord (tel le cas de la France) où des sanctions administratives sont susceptibles de contrôle par le juge administratif dans leur mise en œuvre. On n'est loin de l'Europe dont la directive cadre sur l'eau de 2000 prévoit la prononciation et l'application des sanctions administratives à l'encontre des auteurs de la dégradation qualitative

\footnotetext{
826 Idem.

827 Idem.
} 
et quantitative de l'eau sous certaines conditions ${ }^{828}$. On est toujours loin de l'Europe où la Cour européenne des Droits de l'Homme dispose d'un droit de regard permanent sur les Etats quant à la régularité des sanctions pouvant être prononcées d'office par l'Administration à l'encontre des particuliers ou des personnes morales de droit privé829.

\section{B. Des avancées significatives dans certains accords}

Il faut saluer les efforts quoique insuffisants de la communauté internationale par rapport à la protection des ressources en eau et les acquis en matière de précision du statut juridique de l'eau.

\section{Les accords signés par les Etats africains en la matière}

L'examen des accords signés entre les pays africains depuis la décolonisation, révèle que ceux-ci s'intègrent en général dans l'évolution des principes du droit international. Ceux-ci peuvent fournir un cadre normatif apte à garantir les contrôles, les transferts et la distribution des ressources en eau.

Dans la plupart des traités, l'on remarque que, pris de manière globale, ils préconisent que les Etats parties fassent les choses en commun; les accords sont multifonctionnels; les pays africains semblent accepter le principe selon lequel le bassin de drainage doit être l'unité de base géographique de la coopération y compris dans les secteurs de développement non reliés à l'eau.

L'idée que les cours d'eau internationaux sont une ressource commune ou partagée est prédominante dans le Protocole du Southern African Development Community (SADC) de $1995^{830}$ et l'accord entre la Namibie et l'Afrique du Sud mentionne à son premier article «le développement et l'utilisation des ressources en eau d'intérêt commun ${ }^{831}$. Les pays en voie de développement se caractérisent, en dépit de progrès dans certains domaines, par un déficit en infrastructures et services de base, que la communauté internationale s'est engagée à combler

828 LEBRIER S., «Les sanctions administratives de la police des eaux et des milieux aquatiques. Conditions de mise en œuvre de l'article L.216-1 du Code de l'environnement », RD.rur. 303, mai 2002, p.276.

829 BONICHOT J-C., « Les sanctions administratives en droit français et la Cour européenne des droits de l'homme », AJDA, 2001, nº spéc. p.73.

830 Protocol on Shared Waterhouse Systems in the Southern African Development Community Region, 28 août 1995, entré en vigueur le 29 septembre 1998.

831 Agreement between Namibia and South Africa on the Establishment of a Permanent Water Commission, 14 septembre 1998, 32 ILM 1147, 1993. 
partiellement d'ici 2015832: chaque année, 11 millions d'enfants meurent du sous-développement (malnutrition, hygiène, mortalité maternelle) et l'accès à l'eau potable est loin d'être généralisé.

\section{La contribution de Nations-Unies}

En effet, aux termes des travaux réalisés à ce jour cette qualification est attribuée à des biens qui contribuent à la réalisation des droits fondamentaux de l'être humain, au nombre desquels le logement, l'éducation, l'eau potable et l'assainissement. Pour ce faire, ce bien doit répondre à certains critères. En effet, le bien doit pouvoir bénéficier à l'ensemble de la société, les règles du marché étant en incapacité de satisfaire seules les besoins. La consommation de ce bien par un acteur ne peut limiter les besoins de ce bien pour un autre acteur. D'autre part, il est difficile voire impossible d'exclure un acteur de la consommation d'un bien. Enfin, un critère d'universalité, c'est-à-dire qu'il doit concerner plus d'un groupe de pays, ne pas exclure certaines catégories socio-économiques de ces bénéfices qui doivent être durables.

Or s'il n'existe pas à ce jour de réelles politiques internationales de l'eau, il existe bien une exigence pour une action concertée de l'ensemble des acteurs internationaux pour satisfaire au moins aux besoins fondamentaux pour chaque être humain. Les caractéristiques de ces biens sont déterminées en particulier par leur fonction dans la réalisation de ces droits fondamentaux. Ils deviennent ainsi l'un des éléments clés de la réalisation des objectifs du millénaire. En considérant ces divers critères, l'eau répond bien aux divers aspects de la définition, elle doit donc bénéficier de ce statut de bien public mondial. Mais il s'agirait alors d'en définir les modalités de gestion.

832 Objectifs de développement du Millénaire. 
CONCLUSION DU TITRE I DE LA DEUXIEME PARTIE. 
L'annonce de la libéralisation du secteur de l'eau a suscité une grande inquiétude dans les milieux politiques et a amené bon nombre d'intellectuels à réfléchir sur les conséquences de cette décision. En effet, de nombreux auteurs se sont évertués à démontrer la crise de l'eau, certains la prévoyant inéluctable, immédiate, voire violente avec l'identification de zones qualifiées "hydro-conflictuelles $»^{833}$. Aussi, aborder la crise de l'eau, conduit à préciser comment les sociétés déterminent les politiques publiques et génèrent un droit qui en détermine les statuts et les protections.

En réalité, les conséquences de la libéralisation du secteur de l'eau sont de divers ordres. La libéralisation du secteur de l'eau va remettre en cause les principes fondamentaux du service public tel le principe de continuité et celui d'égalité. L'opérateur privé auquel est confié la mission de distribuer l'eau et d'œuvrer pour l'effectivité de l'accès à l'eau pour tous, ne montre pas toujours la volonté d'accomplir fidèlement sa mission. Et pourtant, les Etats africains ont tous ou presque, adopté une législation, parfois un code de l'eau afin de contrôler les usages, organiser la police de l'eau et instaurer le cas échéant des sanctions en cas d'atteinte à l'eau et au milieu aquatique ${ }^{834}$. Comme nous l'avons démontré dans nos développements, la libéralisation du secteur de l'eau aura des impacts inévitables sur les ressources en eau, d'où l'urgence de tout mettre en œuvre pour que la qualité de ces ressources soit préservée. Ceci suppose une action à l'échelle nationale et internationale. Le plus préoccupant, ce sont les conséquences sanitaires et parfois socio-cultuelles que pourrait amplifier la libéralisation du secteur de l'eau en Afrique subsaharienne. De toute façon, les statistiques, nous en avons évoquées, sont plutôt décourageantes. Il s'agit donc d'une problématique à multiple facettes, mais du point de vue pratique, l'effectivité du droit à l'eau constitue un atout pour résoudre ce problème.

Comme le dit si bien JAGLIN S., il s'agit selon cet auteur, d'arriver à se poser les vraies questions, celles qui peuvent véritablement contribuer à améliorer la situation de l'accès à l'eau. Elles sont nombreuses: "une régie publique à moderniser, un partenariat avec le privé à consolider, une association d'usagers à professionnaliser; ou des échelles ? Une législation nationale à toiletter, un réseau municipal à étendre, un système de mini-réseaux régional à organiser ; ou de la chronologie ? Un service public ancien à adapter, un service public à inventer ou refonder... ». La question d'une participation du secteur privé aux services de l'eau ne devrait se poser qu'une fois l'approche de gestion déterminée. En effet, le recours à des solutions économiques et techniques ne constitue pas nécessairement la solution à la recherche d'un intérêt commun au partage de la ressource. Ainsi, la contestation dont fait l'objet le secteur

\footnotetext{
$\mathbf{8 3 3}$ Voir les opinions de SHIVA V., «La guerre de l'eau », Paragon, 2003 ; Voir aussi NGUYEN T-D., « La guerre de l'eau aura-t-elle lieu ?», Editions Johanet, 1999.; Voir également DROBENKO B., Le droit à l'eau : une urgence humanitaire, Editions Johanet, 2010.
}

Toutefois, il faut préciser qu'il existe des opinions plus nuancées. En effet, Selon Aaron WOLF et son équipe (Université de l'Oregon), une seule guerre ouverte aurait été directement provoquée par la répartition des usages de l'eau. Elle aurait opposé deux cités de la Mésopotamie, Umma et Lagash, pour l'appropriation de palmeraies il y a 4 500 ans. Cf. BEACH Heather L., HAMMER Jesse, HEWITT J. Joseph, KAUFMAN Edy, KURKI Anja, OPPENHEIMER Joe A., WOLF Aaron T., Transboundary freshwater dispute resolution. Theory, practice and annotated references, New York, United Nations University Press, coll. Water ressources management and policy, 2000,324 p.

834 Voir la loi n²010-004 du 14 juin 2010 portant code de l'eau en République du Togo ; Voir aussi loi n 98 -755 du 23 décembre 1998 portant code de l'eau en République de Côte d'Ivoire. Voir également loi n02-006 du 31 janvier 2002 portant code de l'eau en République du Mali. 
privé s'avère être le symptôme d'un bouleversement plus profond, qui dépasse même ce qui a trait à la seule libéralisation du secteur ${ }^{835}$.

835 JAGLIN S., «Quelques pistes de réflexions sur les PPP à partir des expériences africaines », in BOTTON Sarah (dir.), Les multinationales de l'eau et les marchés du sud : pourquoi Suez a-t-elle quitté Buenos Aires et La Paz?, Gret, coll. Débats et controverses, 2007, 84 p. 
TITRE II.

LA NECESSAIRE AMELIORATION DES MOYENS DE L'EFFECTIVITE DU DROIT A L'EAU 
Dans le «Dictionnaire du droit international public», SALMON J. définit l'effectivité comme le caractère de ce qui existe en fait. Selon cet auteur, l'effectivité est la qualité d'une situation juridique qui correspond à la réalité, d'une compétence qui s'exerce réellement. Elle produit des effets en droit, dans les conditions prévues par l'ordre juridique international luimême et joue, en conséquence, un rôle dans de nombreuses institutions de droit international ${ }^{836}$. Pour le Professeur KPODAR A., l'effectivité « c'est le caractère d'une règle de droit qui produit l'effet voulu, qui est appliqué réellement $»^{837}$. La signature par les Etats des différentes conventions relatives à l'affirmation du droit à l'eau doit s'accompagner d'un cadre législatif conséquent au niveau interne. Evoquant la mise en œuvre du droit a l'eau potable au Cameroun, ATONTSA M. J838 affirme que l'effectivité du droit à l'eau est davantage l'œuvre du droit international, lato sensu, que l'œuvre du droit national camerounais lui-même. En effet, selon elle, pour que la mise en œuvre du droit à l'eau «soit effective, il faudrait que les différents acteurs y compris les individus soient intégrés dans le processus et que les instruments conventionnels ratifies au plan international soient effectivement mis en cuvre dans la pratique ».

Comme le disait aussi F. MAYOR ${ }^{839}$, la véritable question ne se limite pas à la proclamation du droit à l'eau mais plutôt à la question de savoir comment faire pour que ce droit soit effectif : "Si l'accès à l'eau est aujourd'hui considéré comme un droit fondamental, il nous incombe à tous de réfléchir aux responsabilités qu'implique l'exercice de ce droit », dans les pays en voie de développement. Ces textes renvoient à la protection de droits de l'homme ayant de lien direct avec l'affirmation du droit à l'eau, alors que les autres s'inscrivent dans l'optique de protection des ressources en eau, une protection sans laquelle l'effectivité du droit à l'eau serait illusoire. Pierre SANE nous invite à repenser la pauvreté comme une violation des droits humains. Cela implique d'analyser les concepts et les enjeux relatifs à la pauvreté considérée comme une violation des droits humains, et plus précisément d'aboutir à la formulation d'une assise conceptuelle de la problématique qui appréhende la pauvreté sous cet angle. Il est donc nécessaire de se demander s'il existe, au sein de ce vaste corpus législatif et jurisprudentielle sur les droits humains, quelque chose comme un droit incontestable à l'eau. Le droit régional et sous régional «manque de fermeté» par rapport à l'effectivité du droit à l'eau ${ }^{840}$. Il faut donc effectuer un retour intelligent et pragmatique à une étatisation du secteur de l'eau (Chapitre) et il est nécessaire d'œuvrer pour une meilleure protection des ressources en eau (Chapitre II).

836 SALMON J., Dictionnaire de droit international public, Bruxelles, Bruylant, 2001, pp 411-412. On peut comparer cette définition à celle donnée le lexique juridique : Principe invoqué pour justifier la reconnaissance ou l'opposabilité d'une situation ou d'un fait réellement établis. (Raymond GUILLIEN et Jean VINCENT, Lexique des termes juridiques, Dalloz, 2001).

837 KPODAR A., «Les droits catégoriels : philosophie et spécificité », Revue béninoise des sciences juridiques et administratives, $\mathrm{n}^{\circ} 18,2007$, p. 46.

838 ATONTSA M. J. C., Droit international de l'environnement et mise en ceuvre du droit a l'eau potable en Afrique centrale : le cas du Cameroun, Thèse, Université de Maastricht, 2011, p. 94.

839 Ancien Directeur de l'Unesco.

840 Cf. «Pauvreté et droits humains : la question de la discrimination économique systémique avec quelques propositions pratiques de réforme» par Christian ARNSPERGER, Revue internationale des sciences sociales, 2004/2 - N 180, pages 323 à 333 . 
CHAPITRE I. LE RETOUR A UNE ETATISATION PRAGMATIQUE DU SECTEUR DE L'EAU 
«Si le rapport entre l'opérateur privé, la collectivité locale et l'État sont uniquement des rapports de force, alors dans ce cas-là, vous n'aurez jamais de service public, puisqu'il n'y a pas de confiance $»^{841}$.

La déception issue de la libéralisation du secteur de l'eau amène à s'interroger sur le bienfondé de ce processus. Si l'on ne peut pas y renoncer totalement, il importe tout de même d'y apporter quelques correctifs. Il s'agira de mélanger à la gestion privée des techniques qui relèvent essentiellement des procédés de l'Etat interventionniste. A partir du XIX ${ }^{\text {ème }}$ siècle, S. MILL, constate que l'évolution de la production est loin d'avoir toutes les suites souhaitables supposées, qu'il laisse de côté une immense partie de la population et ne résout pas le problème social. Toutes sortes de réformes sont mises en avant, d'inspiration socialiste ou non ; elles insistent souvent sur la nécessité d'une action forte de l'État et de ses services publics ${ }^{842}$. Ce qui signifie qu'il serait, même au XXI ${ }^{\text {ème }}$ siècle, irréaliste d'occulter les capacités de l'Etat à œuvrer pour l'effectivité du droit à l'eau.

Focaliser le débat sur la seule "privatisation » est contre-productif, "que la délégation soit adaptée à certaines configurations ne fait pas de doute, qu'elle soit une solution universelle est peu probable ${ }^{843}$. Car la gestion de l'eau et particulièrement celle du service public de l'eau revêt un caractère d'intérêt général. Mais les rapports entre les concepts de la libéralisation du secteur de l'eau et celle de l'intérêt général sont, a priori, opposés. A l'instar de nombreux concepts juridiques, l'intérêt général est une notion qui embarrasse le juriste.

Il faut comprendre que les espoirs suscités par la libéralisation n'ont pas été réalisés. Le drame de l'eau est toujours d'actualité et parfois l'on a l'impression que la situation s'est aggravée. Les ressources en eau sont mal réparties car elles sont trop éloignées des grands centres de consommation. Ainsi, elles sont difficilement mobilisables pour satisfaire la demande en eau potable des populations urbaines et rurales. En prenant l'exemple de la ville de Dakar, on constate que le réseau de distribution d'eau demeure toujours médiocre, avec des pertes qui étaient d'environ 30\% lors de la reprise de la SDE par la SAUR en 1996. La doctrine néoclassique qui s'impose au début du XX ${ }^{\text {ème }}$ siècle ne raisonne plus dans le cadre de la théorie des droits ou de la théorie de l'utilité publique. Néanmoins, pour un auteur comme WALRAS L., l'État a de nombreuses tâches à accomplir. Il doit produire des services d'intérêt public comme les tribunaux, les routes, les écoles. Il doit intervenir dans les situations de monopoles naturels, soit pour exercer lui-même le monopole, soit pour l'organiser dans l'intérêt social. Dans le cas des réseaux municipaux d'eau ou de gaz, WALRAS L. estime la concurrence impossible, et le monopole inévitable 844 .

Après avoir démontré que le service public de l'eau est un service public objectivement

841 Bernard BARRAQUE in Sarah BOTTON, Les multinationales de l'eau et les marchés du Sud: Pourquoi Suez a-t-elle quitté Buenos Aires et La Paz? Gret - Collection débats et controverses - $\mathrm{n}^{\circ} 1$ - page 34

842 LE MASNE P., op. cit.p. 2.

843 Sylvy JAGLIN, «Vingt ans de réformes dans les services d'eau urbains d'Afrique subsaharienne : une géographie de la diversité », Séminaire de recherche du GDR Rés-Eau-Ville (CNRS 2524) L'eau à la rencontre des territoires, Montpellier, France, 27-28 et 29 mai 2004, Cybergeo, p. 2.

$\mathbf{8 4 4}$ LE MASNE P., « Services publics et développement », op. cit., p. 7. 
d'intérêt général, nous expliquerons pourquoi il est nécessaire que la distribution de l'eau fasse l'objet d'un transfert vers les collectivités décentralisées. Nous montrerons que le service public de l'eau est un service public objectivement d'intérêt général (Section I) et démontrer qu'il peut revêtir aussi le caractère d'un service public d'intérêt local (Section II).

\section{Section I. Le service public de l'eau, un service public objectivement d'intérêt général}

L'intérêt général fait partie des nombreux concepts juridico-politiques qui sont, comme LOCKE l'a bien montré, indéfinissables. Ces concepts, qui expriment en apparence une idée simple, prennent des sens variables selon le contexte et les individus qui les emploient. Ce sont des concepts, et l'intérêt général en est un exemple frappant, sans « essence» fixe. Même en nous limitant à son emploi juridique, nous devons reconnaître que ce concept n'a jamais eu de définition claire et stable ${ }^{845}$. Tout à la fois «notion-mère $»^{846}$, "épine dorsale $»^{847}$, «âme $»^{848}$, "pierre angulaire de l'action publique» ${ }^{849}$, l'intérêt général est au cœur du droit public et constitue "l'alpha et l'oméga du droit administratif»" ${ }^{850}$. Selon le Doyen VEDEL, l'intérêt général est « indéfinissable $»^{851}$ et il est rangé dans la catégorie des notions à contenu variable ${ }^{852}$.

Et comme l'exprimait Didier TRUCHET ${ }^{853}$, on " considère volontiers la définition et la poursuite de l'intérêt général comme un monopole de l'Etat $»$. En effet, il est admis que la notion d'intérêt général ne peut être définie que par les autorités politiques légitimement élues et politiquement responsables, dans le respect de la constitution et sous le contrôle du juge. Elles seules, peuvent décider du choix à opérer entre deux objectifs, l'effectivité du droit à l'eau et le respect la liberté d'entreprendre, qui sont tous deux apparemment importants. Mais, il faut

845 Pour D. LOSCHAK, il s'agirait d'une «notion fonctionnelle» c'est-à-dire n'ayant aucune homogénéité conceptuelle et se caractérisant uniquement par la fonction qu'elle remplit qui seule lui confère une véritable unité. En droit public, les «notions fonctionnelles» s'opposeraient aux «notions conceptuelles », telles les notions de fonctionnaire, de commune, de département..., dont le contenu est déterminé à l'avance et qui lient le juge administratif. Cf. Le rôle politique du juge administratiffrançais, L.G.D.J., 1972, pp. 138-149.

846 WALINE M., « Préface » in Juris-Classeur Administratif, 1952 p. 9.

$\mathbf{8 4 7}$ Conseil d'Etat (Section du rapport et des études), L'intérêt général, Rapport public 2002, EDCE n 53, Paris, La documentation française, 2002, p.227.

848 RIVERO J., « Droit public et droit privé : conquête ou statu quo ? », D. 1947, Chroniques XXV, p. 69.

849 Conseil d'Etat (Section du rapport et des études), L'intérêt général, op. cit. p. 245.

850 Ibid., p. 271

851 VEDEL G., «Préface» in RANGEON F., L'idéologie de l'intérêt général, Paris, Economica, «Politique comparée », 1986, p. 3.

852 CLAMOUR G., Intérêt général et concurrence : essai sur la pérennité du droit public en économie de marché, Thèse, Université Montpellier I, 2004, p. 16.

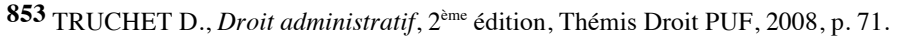


préciser que dans nos développements, nous allons aussi emprunter des termes voisins à la notion d'intérêt général comme la nécessité publique ${ }^{854}$, l'utilité publique, l'intérêt public...

«Source de vie et de développement social, frontière naturelle, voie de communication, l'eau et ses usages intéressent le droit depuis ses origines. Ainsi, le plus vieux traité répertorié porte sur le partage, en 3100 av. J.-C., des eaux de l'Euphrate ${ }^{855}$ ». Le caractère vital de l'eau est aussi bien historique qu'actuel, surtout si l'on fait une approche géostratégique de sa disponibilité. En effet, "la disponibilité en eau douce a toujours conditionné l'emplacement des zones de peuplement $»^{856}$. Il apparaît donc que l'eau ou plutôt le service public de l'eau revêt un caractère d'intérêt général évident (§1), d'où la nécessité de l'ériger en service public constitutionnel (\$2) si l'on veut atteindre l'objectif de l'effectivité du droit à l'eau.

\section{§1.L'évidence du caractère d'intérêt général du service public de l'eau en Afrique subsaharienne}

En consacrant la gestion de l'eau comme un mission d'intérêt général, comme une créance sur la société internationale au profit de tout être humain, c'est faire un choix idéologique et politique. Lorsque, dans une société donnée, les gouvernants décident de ce que doit y être la place de l'Etat, et incidemment celle de l'administration, bras séculier de l'Etat, et déterminent, et bornent par là même, le rôle de l'Etat. Cette gestion ainsi qualifiée bénéficie alors d'un régime juridique particulier, donc dérogatoire au droit de la concurrence. Le droit de chacun d'accéder à une eau potable pour satisfaire ses besoins vitaux est un droit de l'homme fondamental, depuis l'origine de la naissance de l'homme, qu'il convient de consacrer pour sa gestion la notion de « service public », « service universel », de « public utility » ${ }^{857}$ ou de « public services » selon la conception que l'on a de la place de l'intérêt général dans la société, et incidemment selon la

$\mathbf{8 5 4}$ Cette expression remonte à la Déclaration de 1789, notamment en son article 4 qui précise que seule la nécessité publique peut justifier la privation de la propriété.

$\mathbf{8 5 5}$ http://www.fd.ulaval.ca/site//fichier1109.pdf consulté le 23 février 2009.

856 TAITHE Alexandre, L'eau. Un bien? Un droit? Tensions et opportunités, UNICOMM, 2008

857 Le Public utility se définit alors le plus souvent comme une situation de monopole naturel justifiant ainsi non seulement une intervention publique tant sur les prix que sur les modalités de fourniture du service, et par suite, la soumission de ce service à un régime juridique dérogatoire au droit commun, le droit de la concurrence. En effet, l'utilité de ce service fait qu'il doit être distribué de manière équitable et non discriminatoire, ce qui justifie des «entorses juridiques» aux lois du marché (Rapp (L.), L'expérience américaine, in Le service public, AJDA, $\mathrm{n}^{\circ}$ spécial, juin 1997, p. 159-164).

Par suite, pour les américains, l'intervention publique n'est que résiduelle et limitée, elle ne permet pas, en principe, aux personnes publiques de prendre totalement et directement en charge l'activité d'intérêt public. Ce sont les personnes privées qui normalement gèrent les Public utilities, quitte à ce que cette gestion bénéficie d'un régime juridique dérogatoire au droit de la concurrence afin de permettre à la société de bénéficier du service ainsi géré et considéré comme utile voire indispensable au regards des besoins sociaux. Mais, ces entorses sont strictement entendues et contrôlées par des agences fédérales. Cf. MARAIS (du) (B.), Droit public de la régulation économique, Presses de sciences po et Dalloz, 2004, p. 94-96. 
vision que l'on a de ce que doit être et rester la place de l'homme dans la société moderne ${ }^{858}$. Les conséquences dramatiques du manque d'eau sur les plans sanitaire, social et culturel démontrent bien que le service de distribution devrait être érigé en mission d'intérêt général. D'autre part, l'exploitation mal encadrée et non contrôlée des ressources en eau par les multinationales, peut dégrader les réserves en eau et compromettre ainsi l'accès à l'eau d'où l'impérieuse nécessité de faire de la protection des ressources en eau une mission d'intérêt général. Si la distribution de l'eau constitue une urgence humanitaire (A) sa promotion relève d'un enjeu d'intérêt général (B).

\section{A. La distribution de l'eau, une urgence humanitaire}

Inégalement répartie, l'eau a toujours été la priorité des priorités car elle est d'une importance vitale pour l'humanité entière. C'est ce qui explique que certains auteurs comme DROBENKO B. ont parlé d'une urgence humanitaire pour souligner l'importance du droit à l'eau.

\section{Une urgence humanitaire justifiée par l'inégale répartition}

En effet, «...les ressources par habitant varient de plus de un à mille entre l'Alaska (180 $000 \mathrm{~m} 3$ par an) et la péninsule arabique (moins de 200) »859. Dans le Monde, alors que la Terre contient seulement 3\% d'eau douce ${ }^{860}$ seuls neuf pays ${ }^{861}$ concentrent $60 \%$ des ressources en eau douce. De plus, là où elle est abondante, elle devient souvent impropre à la consommation sous l'effet des pollutions qu'elle subit depuis plus d'un siècle du fait principalement des pratiques industrielles et agricoles intensives. Ce phénomène vient s'ajouter à des besoins domestiques (arrosage des jardins et pelouses, lavage des véhicules automobiles, par exemple) voire à l'entretien et à la préservation des espaces publics. Tout cela répond certes à une demande de confort, mais cette dernière est très gaspilleuse en eau de qualité et génère aussi un surcoût économique sensible tant pour le consommateur que pour la collectivité. Par suite, le niveau des nappes d'eau souterraine connaît une baisse inexorable et quasi-généralisée à travers le Monde ${ }^{862}$.

$\mathbf{8 5 8}$ Stéphane GUERARD, op. cit.

859 THEYS J., «L'environnement au XXIe siècle. Entre continuité et ruptures », Futuribles, 1999, n²39-240, p. 12.

860 Les trois quart de cette eau se situent d'ailleurs aux pôles ou en haute montagne sous forme de glace ou de neige.

$\mathbf{8 6 1}$ Ce sont le Brésil, le Canada, la Chine, la Colombie, les Etats-Unis d'Amérique, l'Inde, l'Indonésie, le Pérou et la Russie.

862 Stéphane GUERARD, «La gestion démocratique de l'eau : Un enjeu mondial d'intérêt général », source non disponible. 
De surcroit, les mégalopoles en plein développement aujourd'hui, telles que Lagos, Le Caire, ... ont tendance à consommer plus d'eau que les endroits peu habités à cause, en particulier, d'un grand nombre d'usages collectifs de l'eau, tels que les piscines, les hôpitaux, les crèches, ou encore, les commerces. La sur-urbanisation concentrée de certains Etats, même en voie de développement, génèrent une concentration des problèmes tant économiques qu'écologiques ${ }^{863}$.

"Cette surconsommation domestique, associée au développement des surfaces agricoles irriguées ${ }^{864}$ et à l'activité industrielle, a des conséquences désastreuses sur l'environnement. Les eaux usées sont toujours plus nombreuses, les engrais et les pesticides polluent chaque jour un peu plus les ressources d'eau douce et réduisent ainsi le volume d'eau potable disponible dans le monde $»^{865}$.

"Compte tenu de (...) la distribution attendue de la population sur le globe on estime que d'ici 30 ans plus d'un tiers de la population mondiale souffrira d'une grande pénurie d'eau ${ }^{866}$, et plus de 50\% en Méditerranée, ce qui laisse penser que les conflits autour de l'appropriation des ressources en eau risquent effectivement de s'intensifier ${ }^{867}$.

Il est donc plus que temps d'utiliser les ressources en eau de façon plus optimisée ${ }^{868}$, parce que l'eau, même si elle recouvre les trois quarts de la planète, n'est pas finalement inépuisable ${ }^{869}$. Et ce, d'autant plus que la sécheresse, liée au réchauffement climatique planétaire, se développe ${ }^{870}$. Il reste que l'accès à l'eau demeure un objectif à concrétiser. En effet, mal répartie elle est également mal distribuée.

863 Idem

864 «Pour nourrir les 8 milliards d'individus qui peupleront la Terre en 2025, il faudra cultiver toujours plus. Depuis le début du siècle, la surface des terres irriguées a déjà été multipliée par cinq. Aujourd'hui, l'agriculture engloutit $70 \%$ des prélèvements d'eau dans le monde. Dans certains pays situés en Afrique ou en Asie, elle consomme jusqu'à $90 \%$ de l'eau puisée » $C f$. Le MARCHAND V., L'eau. Source de vie, source de conflits, éditions Milan, Collection «Les essentiels Milan», 2003, p. 55.

$\mathbf{8 6 5}$ Le MARCHAND V., op. cit., p. 53.

866 Et, en 2050, ce seront les deux tiers de la planète qui pourraient être touchés par de graves pénuries d'eau.

867 THEYS J., op. cit., p. 12.

868 Voir, sur cette question : TROUILLY P., «Le principe de la gestion équilibrée de la ressource en eau est-il devenu inutile ?», Environnement, 7/2004, p. 7-9.

869 PAPON P., « Mieux gérer l'océan mondial », Futuribles, février-mars 1999, n²39-240, p. 23-35.

870 «La France a d'ailleurs connu dans un passé récent quelques étés particulièrement chauds (1976, 1989, 1990 et 1991 (voire 2003), par exemple) qui ont provoqué une situation de sécheresse assez exceptionnelle. Les français ont alors pris conscience qu'ils n'étaient pas à l'abri d'un manque d'eau, plaie que l'on attribue en général au tiersmonde tropical. Ces sécheresses hexagonales ont été telles que des mesures ponctuelles de rationnement de la consommation d'eau ont dû être prises : il a fallu gérer la pénurie, il serait temps désormais de la prévenir » (GUERARD S., Prendre en compte le cadre juridique de l'eau, in Guide pratique de la gestion de l'eau, éditions Weka, octobre 2003, p. 1 du Chapitre 2). 


\section{Une urgence humanitaire expliquée par des conséquences sociales alarmantes}

L'eau est la première cause de mortalité et de morbidité au monde, de façon directe ou indirecte. Trois millions d'enfants meurent chaque année avant l'âge de cinq ans du fait du manque d'accès à une eau potable. Les épidémies de paludisme et de dengue qui affectent plusieurs centaines de millions de personnes sont les plus grandes épidémies mondiales ${ }^{871}$.

De plus, "l'eau est un élément essentiel pour la production de cultures vivrières. On estime que $40 \%$ de l'alimentation mondiale est produite par des systèmes d'agriculture irriguée. A l'avenir, la croissance de la population et l'évolution des modes d'alimentation nécessiteront une augmentation de la production agricole qui ne pourra provenir que d'une meilleure utilisation de l'eau en agriculture $»^{872}$.

De surcroît, il importe de souligner avec force qu'à la différence du pétrole, l'eau n'est pas remplaçable, elle n'a pas de substitut. Ainsi, plus de 7000 sites de baignade ont été supprimés, sans explication, des listes officielles des Etats-membres de l'Union européenne, leur évitant ainsi l'application des nouvelles règles communautaires sur la qualité des eaux de baignade. La Commission européenne soupçonne que ces Etats ont préféré fermer ces sites au lieu de les nettoyer, et ce, d'autant plus que la nouvelle directive concernant la gestion de la qualité des eaux de baignade a été publiée au Journal officiel de l'Union européenne ${ }^{873}$ le 4 mars 2006. Pourtant, les Etats ont jusqu'au 24 mars 2008 pour la transposer dans leur droit national et jusqu'au $1^{\text {er }}$ janvier 2015 pour la mettre en application ${ }^{874}$.

In fine, il faut préciser que "la moitié des grands fleuves et lacs mondiaux sont pollués. La moitié des zones humides a disparu depuis le début du XX'ìme siècle (...) L'eau est une ressource naturelle limitée. Cependant la demande augmente et la ressource est de plus en plus dégradée ${ }^{875}$. Il est donc urgent de s'assurer que la ressource elle-même est suffisamment protégée, ce qui n'est pas forcément le cas lorsque le service de distribution de l'eau est confié à des entreprises privées.

871 GUENE C., Rapport sur la proposition de loi sur la coopération internationale des collectivités territoriales et des agences de l'eau dans les domaines de l'alimentation en eau et de l'assainissement, Sénat, n³47, 16 juin 2004, p. 6.

872 GUENE C., op. cit., p. 7.

873 (JOUE)

874 AJDA, 17 avril 2006, n 15 , p. 795.

$\mathbf{8 7 5}$ GUENE C., op. cit., p. 8. 


\section{B. La promotion de l'accès à l'eau, un enjeu d'intérêt général.}

Si l'on s'en tient aux différentes constitutions en vigueur dans les Etats de l'Afrique subsaharienne, les préoccupations relatives à l'eau constituent bien des questions d'intérêt général. En effet, la gestion de l'eau ne peut être correctement effectuée qu'à partir d'un réseau développé et bien entretenu. Ce n'est qu'à ce prix, parfois très élevé au demeurant, que l'accès à l'eau et le droit y afférent, et ce, au bénéfice de tout être humain, peuvent être assurés. Aussi, doit-on comprendre la notion de développement durable et démontrer en quoi le concept de développement durable est d'intérêt général.

\section{Le développement durable, une mission d'intérêt général}

Considéré comme un «développement qui répond aux besoins du présent sans compromettre la capacité des générations futures à répondre aux leurs $»^{876}$, le concept de développement durable est une notion à géométrie variable ${ }^{877}$.

L'apparition du concept de développement durable remonte à une quarantaine d'années. Il est le fruit d'une prise de conscience des problèmes environnementaux liés aux activités humaines. Cette notion est aujourd'hui ancrée dans les langages courant, politique, économique, scientifique, écologique et bien évidemment juridique. C'est le fruit d'une longue maturation qui débute nettement en 1972 lors de la Conférence des Nations unies sur l'environnement humain tenue à Stockholm (Suède). Elle débouchera sur la Déclaration de Stockholm ${ }^{878}$. C'est le début de gestation du concept de développement durable même s'il ne porte pas à cette époque cette dénomination ${ }^{879}$. Le rapport Brundtland a été, en 1987, le « véritable catalyseur $\gg$ du ce concept. C'est lors de la Conférence des Nations unies sur l'environnement et le développement, tenue à Rio de Janeiro (Brésil) en 1992, qu'il connait une consécration du fait de sa proclamation dans la fameuse Déclaration de Rio ${ }^{880}$. Dès lors, le concept a fait l'objet d'une affirmation renouvelée dans différents instruments juridiques

876 Définition retenue dans le Rapport de la Commission mondiale sur l'environnement et le développement encore appelée Commission Brundtland, du nom de Mme Gro Harlem Brundtland à l'époque Premier Ministre norvégien. Ce rapport, publié en anglais sous le titre Our Common Future, Oxford University Press, Oxford/New York, 1987, a été traduit en français sous le titre Notre avenir à tous, Le Fleuve, Montréal, 1988.

877 Fortuné B. AHOULOUMA, op. cit., p. 14.

$\mathbf{8 7 8}$ Nations unies, «Rapport de la Conférence des Nations unies sur l'environnement », Stockholm, 5-16 juin 1972, Doc. NU A/ CONF. 48/Rev. 1, 1972.

879 BARTENSTEIN (K.), « Les origines du concept de développement durable », R.J.E. 3/2005, p. 290 et 291

$\mathbf{8 8 0}$ Nations unies, Conférence des Nations unies sur l'environnement et le développement, « Déclaration de Rio sur l'environnement et le développement» du 13 juin 1992, Rapport de la Conférence des Nations unies sur l'environnement et le développement, Doc. NU A/CONF. 151/26 (vol. I), annexe I (1992) 
internationaux $^{881}$. La Conférence de Johannesburg (Afrique du Sud), tenue en 2002, a conforté la reconnaissance universelle du concept. Le développement durable, qui concilie deux objectifs a priori inconciliables (protection de l'environnement et développement), a, dès lors, fait l'objet de plusieurs développements ${ }^{882}$. Ils révèlent la complexité du concept.

Dès lors que les lois fondamentales des différents pays africains subsahariens proclament le droit du citoyen à un environnement sain, on peut y déceler la volonté de la puissance publique de faire de la question de l'environnement une préoccupation d'intérêt général. La consécration constitutionnelle même si elle n'est pas suffisante, elle demeure décisive dans la prise en compte de l'environnement comme une préoccupation d'intérêt général.

\section{La mise en ouvre de la préoccupation d'intérêt général}

Certains des Etats africains du Sud Sahara ont consacré le droit à l'eau au niveau constitutionnel. L'État du Niger a proclamé explicitement le droit à l'eau potable à travers l'article 12 de sa nouvelle Constitution adoptée par référendum en octobre 2010. Cet article dispose clairement que «chacun a droit à la vie, à la santé, à l'intégrité physique et morale, à une alimentation saine et suffisante, à l'eau potable, à l'éducation et à l'instruction dans les conditions définies par la loi» ${ }^{883}$. L'article 216 al. 4 de la Constitution de Gambie dispose que "l'Etat s'efforce de faciliter un égal accès à l'eau propre et saine ». Pour le constituant éthiopien, les politiques doivent viser à fournir à tous les éthiopiens un accès à une eau propre «dans la mesure où les ressources du pays le permettent... $»^{884}$. La Constitution du Nigeria ${ }^{885}$ demande à l'État de veiller à ce que chaque citoyen bénéficie de «suitable and adequate shelter, food, water supply, ... $»^{886}$. La Constitution de la Zambie ${ }^{887}$ en son article 112 déclare que "L'État s'efforcera de fournir une eau propre et saine ». Dans son article 14, la Constitution de l'Ouganda reconnaît avec précision le droit de l'homme à l'eau ${ }^{888}$. A la faveur de la nouvelle majorité au pouvoir au lendemain de la chute du régime ségrégationniste, le droit à

881 Nations unies, Actions 21 du 13 juin 1992, Doc. NU A/CONF. 151/26/Rev. 1 ; Nations unies, Comité intergouvernemental sous l'égide du P.N.U.E., « Convention sur la diversité biologique », du 5 juin 1992, Doc. NU UNEP/Bio. Div/N7-INCC.5/4.

$\mathbf{8 8 2}$ BARTENSTEIN K., op. cit., p. 289-297

$\mathbf{8 8 3}$ ALTERNATIVE ESPACES CITOYENS, Le droit à l'eau potable au Niger, Rapport d'analyse des politiques publiques et du financement du secteur de l'eau de 2001 à 2010, p.12.

$\mathbf{8 8 4}$ Article 90 de la constitution éthiopienne

$\mathbf{8 8 5}$ Constitution de 1989

886 Art.17.2.d.

$\mathbf{8 8 7}$ Constitution de 1996

888 «L'Etat s'efforce de réaliser les droits fondamentaux de tous les ougandais à la justice sociale et au développement économique et garantit notamment que : tous les ougandais jouissent des droits d'opportunité ainsi qu'un accès à l'éducation, aux services de santé, à une eau propre et saine, à un abri décent, à des éléments adéquats, à la nourriture, la sécurité et aux prestations de pension et de retraite ». 
l'eau est devenu un droit protégé par la Constitution en Afrique du Sud qui proclame que «chaque personne a le droit à un accès à de l'eau en quantité suffisante ». Il faut signaler que la nouvelle constitution du Congo (République démocratique) prévoit que : «le droit à un logement décent, le droit d'accès à l'eau potable et à l'énergie électrique sont garantis »889.

\section{§2.La nécessité d'ériger la distribution de l'eau en service public constitutionnel}

On s'attendait à ce que les sociétés multinationales soient attirées par des perspectives de marchés très rémunérateurs et à ce que les populations réagissent positivement, devant la corruption et l'inefficacité du secteur public dénoncées par la Banque mondiale. Les politiques des bailleurs de fonds lui accordaient une place telle qu'un représentant de la Banque mondiale déclara lors d'une conférence internationale en 2000 qu'il n'y avait "pas d'alternative » à la privatisation ${ }^{890}$. Avec les échecs constatés çà et là, on ne peut plus postuler que le secteur public est moins efficace que le secteur privé, comme l'explique un rapport de la Banque mondiale : « en général, on ne note pas de différence statistiquement significative entre le rendement des exploitants publics et privés dans ce secteur. S'agissant des entreprises de service public, il semble généralement que le régime de propriété ne compte pas autant qu'on le prétend parfois. La plupart des rapports comparatifs entre pays ne relèvent pas d'écarts statistiquement notables au niveau des résultats obtenus par les opérateurs publics et privés ${ }^{891}$.

On constate maintenant que cette expérience fut, à certains égards, un échec. Les contrats privés n'ont pas permis d'obtenir les investissements attendus dans les infrastructures attendues. Au bout de quinze années, les investissements consentis par les opérateurs privés n'ont permis de raccorder Afrique subsaharienne que moins d'un pour cent des personnes qui auraient dû être raccordées dans ces régions pour répondre aux $\mathrm{OMD}^{892}$. A côté de la tentation d'appeler à une nationalisation des services de distribution de l'eau privatisés, il faut envisager leur constitutionnalisation, celle-ci constituera une meilleure garantie pour leur efficacité. Après avoir examiné l'intérêt de la constitutionnalisation du service public (A), nous en étudierons la modalité (B).

889 Cf. Article 48 : « La loi fixe les modalités d'exercice de ces droits ».

890 John BRISCO, dans un exposé prononcé au nom de la Banque mondiale au $2^{\text {ème }}$ Forum mondial de l'eau, la Haye (Pays-Bas), mars 2000.

891 Infrastructure performance and reform in developing and transition economies: evidence from a survey of productivity measures. A. ESTACHE, S. PERELMAN, L. TRUJILLO World Bank Policy Research Working Paper 3514 , février 2005.

http://wdsbeta.worldbank.org/external/default/WDSContentServer/IW3P/IB/2005/03/06/000090341 200503061014 29/Rendered/PDF/wps3514.pdf consulté le 6 mars 2005.

892 Voir PSIRU Pipe Dreams, March 2006 www.psiru.org 


\section{A. L'intérêt de la constitutionnalisation du service public}

L'introduction dans un texte constitutionnel de nouvelles dispositions, autres que celles qui visent à organiser les pouvoirs, traduit la prise en compte d'un intérêt jugé fondamental par le constituant et pour la Nation que régit cette Constitution ${ }^{893}$. L'intérêt de la constitutionnalisation des services publics est qu'ils doivent être gérés par des organismes publics et cela, à la différence des services publics nationaux simplement législatifs qui peuvent être gérés par des organismes de droit privé. Il est vrai qu'il y a des exceptions à ce principe, comme le confirme le cas où le Conseil constitutionnel qui après avoir affirmé que le recouvrement d'un impôt ne peut être effectué que par des services ou des organismes placés sous l'autorité de l'Etat ou son contrôle, admet cependant que des personnes privées soient habilitées à y procéder des l'instant qu'elles " exercent une mission de service public et sont placées sous la tutelle de l'Etat ou son contrôle $»^{894}$. De plus, il faut remarquer également que l'exception qui est souvent apportée à la gestion de certains services publics constitutionnels ne correspondant pas à des fonctions de souveraineté. Il s'agit par exemple des services publics de l'enseignement ou de la protection de la santé895.

\section{L'apparition de la constitutionnalisation du service public de l'eau}

L'idée n'est pas inédite, puisque le 31 octobre 2004 en Uruguay, un amendement constitutionnel sur la question de l'eau soumis à la population par voie de référendum a été approuvé par $62,75 \%$ des votes exprimés. Cet amendement comportait plusieurs éléments, stipulant notamment que l'approvisionnement en eau par canalisation et l'assainissement sont des droits de l'homme fondamentaux et que les considérations sociales priment sur les arguments économiques s'agissant des politiques de l'eau, et aussi que «le service public des égouts et le service public de distribution d'eau pour la consommation humaine seront assurés exclusivement et directement par des entités publiques ${ }^{896}$.

«Le droit administratif est mort, vive le droit constitutionnel jurisprudentiel». C'est en substance ce que pense le Doyen FAVOREU dans son article sur «l'apport du Conseil Constitutionnel au droit public», paru à la revue Pouvoirs en 1991897, lorsqu'il annonce

893 MATHIEU B., X ${ }^{\text {èmes }}$ Journées juridiques franco-chinoises Paris 11-19 octobre 2006

894 Cons. Const. N0 90-258 DC, 28 déc. 1990, loi de finances pour 1991, RFDC N 5, p. 145.

895 AUBY J-F. et RAYMUNDIE O., op. cit., p. 180.

896 Uruguay, Article 47 de la Constitution.

http://www.aguayvida.org/home/legislacion.htm\#Reforma Constitucional.

"El servicio público de sane amiento y el servicio público de abastecimiento de agua para el consumo humano serán prestados exclusiva y directamente por personas jurídicas estatales .”

897 Louis FAVOREU, « L'apport du Conseil Constitutionnel au droit public », Pouvoirs, 1991, n 13, p 17 
par une déclaration prospective, mais quelque peu péremptoire que «les grands problèmes de droit public (...) ne verront plus leur solution dépendre du droit administratif et de la juridiction administrative, lesquels auront surtout à trancher les problèmes "ordinaires" de l'administration ». Si le Professeur FAVOREU ne prédit rien de moins que le phénomène de constitutionnalisation de l'ensemble des branches du droit, il rend indirectement mais incontestablement service à la promotion de l'effectivité du droit à l'eau.

\section{Le service public constitutionnel}

La notion de service public constitutionnel est née tardivement. Elle apparaît pour la première fois dans la décision "Privatisations» des 25 et 26 juin 1986, dans laquelle le conseil constitutionnel fait état de services publics dont la nécessité découle de principes ou de règles de valeur constitutionnelle. Ce que le Professeur FAVOREU va désigner par commodité, service public constitutionnel ${ }^{898}$.

Relativement au contenu même du concept, le Professeur FAVOREU allait-il nous mettre en garde en affirmant qu'à "vrai dire, malgré l'intervention de nouvelles décisions en 1996, la notion reste toujours à définir : on sait, en effet, quels sont les services publics qui ne sont pas constitutionnels ; mais il est toujours difficile de dire ce qu'ils sont, et de dresser leur liste exacte $»^{899}$.

Célébrée par une partie de la doctrine, la constitutionnalisation du service public ne fait pas toujours l'unanimité. Certains auteurs ont pu s'interroger sur leur pertinence. C'est le cas notamment de P. ESPLUGAS qui se demandait si la constitutionnalisation du service public n'est pas « un tigre de papier $»^{900}$. Le Conseil constitutionnel a eu l'opportunité, à quelques occasions, d'indiquer ce que l'on ne peut pas considérer comme services publics constitutionnels mais sans expliciter véritablement les raisons de son refus de voir ces services publics figurer dans la liste de ceux exigés par la Constitution. Il a ainsi décidé « qu'aucune des entreprises qui figurent sur la liste mentionnée à l'article 4 de la loi [liste de 65 entreprises, essentiellement des secteurs bancaire et des assurances] ne peut être regardée comme exploitant un service public dont l'existence et le fonctionnement seraient exigés par la Constitution $»^{901}$. Et va dans le même sens l'affirmation selon laquelle le « service public du crédit » ne répond pas non plus aux

898 Cf. Louis FAVOREU, RDP, 1989, p. 461 ; Louis FAVOREU et Loïc PHILIP, Les grandes décisions du Conseil constitutionnel, Sirey, $5^{\text {ème }}$ éd., $n^{\circ} 41$, pp. 680-682

899 FAVOREU L., « Service public et Constitution », AJDA, 1997 p. 16

900 ESPLUGAS P., « La constitutionnalisation du service public : un tigre de papier ? », RA 1996, n 290, p. 162. $C f$. également ESPLUGAS P., «A propos de la décision du Conseil constitutionnel de 23 juillet 1996 - Les télécommunications : un nouveau service public constitutionnel », RA 1996, p. 510.

901 C. C. ${ }^{\circ}$ 86-207 DC des 25-26 juin 1986 (cons. 54) 
conditions exigées. De même, le Conseil constitutionnel a estimé, en 1988, que « la distribution de prêts bonifiés n'a pas le caractère d'un service public exigé par la Constitution $»^{902}$.

Dans sa décision du 23 juillet $1996^{903}$, le juge constitutionnel ne qualifie pas le service des télécommunications de service public constitutionnel : on notera d'ailleurs que, dans leur saisine, les députés requérants reconnaissaient eux-mêmes que ledit service n'était pas de ceux dont « la nécessité [...] découle de principes ou de règles de valeur constitutionnelle $»^{904}$ et que cela recueillait l'approbation du secrétaire général du gouvernement dans ses observations. Le Conseil constitutionnel, quant à lui, se borne à constater à propos dudit service, que "le législateur a confirmé sa qualité de service public national » sans utiliser de formules semblables à celles citées plus haut. Et s'il subsistait quelque doute, celui-ci serait levé par le commentaire autorisé du secrétaire général du Conseil constitutionnel, Olivier SCHRAMECK, selon lequel le Conseil constitutionnel " n'a pas jugé nécessaire d'apprécier si l'existence du service public en cause était exigée par un autre principe ou disposition à valeur constitutionnelle $»^{905}$.

En effet, les services publics constitutionnels s'imposent aux autorités publiques ${ }^{906}$. Mais le Conseil constitutionnel n'a pas défini ni la nature ni la liste des services public constitutionnels. En analysant les textes constitutionnels notamment le préambule de la constitution de 1946 en France, on pourrait considérer comme tels les services qui sont directement liés à la défense nationale, la justice, l'enseignement public à tous les degrés et le service public de la santée07.

D'autre part, la notion de service public constitutionnel et de service public national ne se recouvrent pas. Tous les services publics constitutionnels sont des services publics nationaux, mais tous les services publics nationaux ne sont pas des services publics constitutionnels ${ }^{908}$. Cette précision peut être rapprochée aux débats de doctrine opposant la thèse selon laquelle, il est des services publics "par nature ", dont l'existence est indispensable à la réalisation de l'interdépendance sociale et s'impose donc au gouvernement, et la conception dite objective du service public de Gaston JEZE, selon laquelle sont uniquement services publics les besoins d'intérêt général que les gouvernants d'un pays donné, à une époque donnée, ont décidé de satisfaire par le procédé du service public. Si l'on insère la question de l'accès à l'eau dans ce débat, elle prendra bien la couleur de l'une ou l'autre des thèses.

902 Décision $n^{\circ}$ 87-232 DC du 7 janvier 1988, Mutualisation de la Caisse nationale de crédit agricole, cons. 30, Rec. jur. const. I-320).

$903 \mathrm{~N}^{\circ}$ 96-380 DC, Entreprise nationale Télécom, JO 27 juillet 1996, p. 11408

904 JO 27 juillet 1996, p. 11410

905 FAVOREU L., « Service public et Constitution », AJDA, 1997 p. 16

906 AUBY J-F. et RAYMUNDIE O., Le service public, Le Moniteur, Paris 2005, p. 179.

907 LOMBARD M. et DUMONT G., Droit administratif, $6^{\text {ème }}$ édition, Dalloz, Paris, p. 281.

908 AUBY J-F. et RAYMUNDIE O., précité. 


\section{B. La conséquence de la constitutionnalisation : la nationalisation du secteur de l'eau}

La conséquence immédiate de la constitutionnalisation du service public de l'eau est de procéder à la nationalisation du secteur de l'eau. Concept ayant connu son essor sous l'influence du mouvement des pays du Tiers Monde, la nationalisation a permis dans une certaine mesure à ces Etats de valoir leur droit de souveraineté sur leurs ressources économiques. Quid de la signification de la nationalisation?

\section{La signification du concept de nationalisation}

L'expression nationalisation dissimule une idée de contrôle collectif et c'est pourquoi, au premier abord, il peut attirer la sympathie. Deux raisons ont présidé, historiquement, aux nationalisations, un peu partout dans le monde. Dans certains cas, il s'agissait de permettre le développement de secteurs jugés économiquement trop stratégiques, ou trop onéreux en termes d'investissements, pour en laisser la propriété et la responsabilité aux intérêts privés : on pense ici aux télécommunications, aux chemins de fer, etc. Par ailleurs, de nombreuses nationalisations de ressources ont eu pour motif une réappropriation par des collectivités de leurs ressources et des bénéfices de leur exploitation : on pense à l'hydroélectricité ou à l'amiante ici mais aussi aux mines, à la forêt, au pétrole, etc. dans d'autres pays.

Dans les deux cas toutefois, et c'est là l'élément important, c'est à des ressources, des biens ou des services de nature économique que s'est généralement appliquée la nationalisation. Il s'agit fondamentalement d'un acte juridique visant à transférer la propriété. Or, assurer la propriété collective, particulièrement dans le contexte actuel de la mondialisation de l'économie et de ses règles dominantes, celle du capitalisme de marché, ne peut en aucun cas constituer un instrument efficace pour protéger une ressource vitale telle que l'eau de l'application de ces règles de marché, de sa marchandisation.

On peut alors retenir que la nationalisation est l'opération par laquelle la propriété d'une entreprise privée est autoritairement transférée au secteur public. Elle se distingue de l'expropriation en ce qu'elle porte sur une entreprise avec l'ensemble de son patrimoine, alors que l'expropriation ne porte que sur des biens immeubles ${ }^{909}$.

Il faut ajouter que la décision de nationaliser relève de la compétence du législateur mais demeure contrôlée par le juge constitutionnel. Ce qui suppose qu'il faut identifier les textes à caractère constitutionnel qui président à la procédure de nationalisation. Ces références ne sont pas explicites, il faut les déceler de certaines dispositions parfois peu précises. Le préambule de la Constitution de 1946 en France, prévoit que tout bien, toute entreprise dont l'exploitation a ou acquiert les caractères d'un service public national ou d'un monopole de fait doit devenir la propriété de la collectivité ${ }^{10}$. Selon le constituant burkinabé, les richesses et les ressources

909 DELVOLVE P., Droit Public de l'économie, Dalloz, 1998, p. 169.

910 HUBRECHT H-G, Droit public économique, Dalloz, 1997, p. 101. 
naturelles appartiennent au peuple. Elles sont utilisées pour l'amélioration de ses conditions de $v^{e^{911}}$. La Constitution en vigueur au Sénégal prévoit en son article 15 que le droit de propriété est garanti par la Constitution. Il ne peut y être porté atteinte que dans le cas de nécessité publique légalement constatée, sous réserve d'une juste et préalable indemnité912. Il faut ajouter le cas du Mali où le constituant a prévu à l'article 13 que «le droit de propriété est garanti. Nul ne peut être exproprié que pour cause d'utilité publique et contre une juste et préalable indemnisation $»^{913}$.

Partant de ces indications que l'on retrouve également dans la constitution française, la juridiction constitutionnelle française conclut que le législateur est le seul compétent et qu'il exerçait dans ce domaine une compétence de nature discrétionnaire. Ce qui signifie qu'il n'est jamais obligé de nationaliser et qu'il ne connaît guère de limites quant aux nationalisations auxquelles il décide de procéder ${ }^{914}$.

Dès lors, on retrouve à propos des nationalisations la même marge d'appréciation du législateur que pour les autres atteintes au droit de propriété et la liberté d'entreprendre. Spécialement en 1982, il ressort des travaux préparatoires de la loi que le législateur a entendu fonder les nationalisations sur son utilité pour donner aux pouvoirs publics les moyens de faire face à la crise économique, de promouvoir la croissance et de combattre le chômage et relèveraient ainsi de l'intérêt général ${ }^{915}$.

\section{La portée de la nationalisation}

Dans deux pays au moins, la privatisation de la distribution d'eau a été décrétée illégale. En 2004, le parlement néerlandais a voté à l'unanimité, moins un petit parti de droite, une loi interdisant à une entreprise privée d'exploiter un réseau public de distribution d'eau. Le texte stipule que les services de distribution d'eau potable ne peuvent être assurés que par des entités publiques ou contrôlées à $100 \%$ par les pouvoirs publics ${ }^{916}$.

911 Constitution du Burkina Faso; Adoptée par le Référendum du 2 Juin 1991 et révisée par les lois constitutionnelles du 27 janvier 1997, du 11 avril 2000 et du 22 janvier 2002. Article 14.

912 Loi n $^{\circ} 2001-03$ du 22 janvier 2001 portant Constitution au Sénégal

913 Décret n 92-0731 P-CTSP portant promulgation de la Constitution du Mali

914 HUBRECHT H-G., op. cit., p.102.

915 DELVOLVE P., op. cit.

916 En septembre 2000, le ministre néerlandais de l'Environnement, Jan PRONK, présente un projet de loi visant à empêcher les sociétés privées de fournir des services d'eau. La production et la distribution de l'eau potable demeureraient le droit exclusif des entreprises publiques d'eau dans leurs régions de distribution. Une loi est rédigée en 2001 à cet effet, mais après que le gouvernement néerlandais eut démissionné (après la publication du rapport sur la non-intervention des soldats néerlandais chargés du maintien de la paix, lors du massacre de Srebrenica en Bosnie in 1995), le gouvernement qui lui succède laisse le projet de loi sur les tablettes. La loi peut être considérée comme le suivi donné à un document gouvernemental publié en 1997 et prévoyant clairement que seules les sociétés d'État pourraient obtenir les concessions pour l'approvisionnement en eau. 
Un Etat n'est pas souverain s'il ne peut disposer pleinement et librement de ses ressources notamment de ses ressources en eau. Si celles-ci sont entre les mains étrangères, sa souveraineté risque d'être purement aléatoire. La nationalisation est une condition du développement, et peut revêtir un caractère urgent, voire humanitaire. La nationalisation permet seule à l'Etat de maîtriser les richesses économiques du pays et de promouvoir une véritable politique nationale de développement ${ }^{917}$. Elle servira à tout Etat qui y recourt de forger et de reconquérir l'identité nationale. En effet, une distribution discriminatoire et parcellaire de l'eau sur un territoire donné du fait des considérations mercantilistes, ne favorise pas l'édification d'une nation déjà victime d'une régionalisation exacerbée. Pour les pays du Tiers Monde, la nationalisation emporte plusieurs conséquences notamment l'application du droit interne et non du droit international. L'Etat s'estime en effet seul juge des motifs pour lesquels il nationalise les entreprises privées. En ce qui concerne le cas des multinationales de l'eau, les raisons sont évidentes. Celles-ci ne sont arrivées à honorer leurs engagements, à savoir l'extension des réseaux et la permanence de la fourniture de l'eau aux citoyens.

On comprend alors que tout droit d'exploitation de ressources naturelles, d'exploitation d'entreprises industrielles et commerciales accordé par les Etats a par essence un caractère précaire et révocable. D'autre part, la thèse de la nationalisation se retrouve fragilisée par diverses contestations de la part des sociétés victimes de l'opération. Ainsi, les nationalisations ont fait naitre des actions sur la non-reconnaissance de l'effet extraterritorial des nationalisations contraires à l'ordre public des pays occidentaux ${ }^{918}$.

L'incertitude du droit positif en la matière est évidente. L'écart qui sépare les positions les plus radicales des pays développés et des pays en développement laisse planer un doute profond sur l'existence en matière de nationalisation, d'un droit positif accepté par tous. Les divergences qui se manifestent dans les solutions adoptées par les sentences arbitrales récentes confirment cette impression ${ }^{919}$.

Aujourd'hui, la plupart des partenaires en reviennent, après de nombreux détours aux principes formulés par la résolution 1803 - XVII qui déclare dans son paragraphe 4 que : "la nationalisation, l'expropriation ou la réquisition devront se fonder sur des raisons ou des motifs d'utilité publique, de sécurité ou d'intérêt national... ». Enfin, il faut préciser que la suspicion avec laquelle les pays en développement considéraient les sociétés multinationales opérant sur leur territoire n'était point un secret. De nombreux textes internationaux se sont l'écho de cette méfiance et le plus significatif est la Déclaration économique des Non alignés d'Alger de 1973 dont la section 7 se lit ainsi : Les Chefs d'Etat et de gouvernement dénoncent devant l'opinion publique mondiale les pratiques inadmissibles des sociétés transnationales qui portent atteinte à

La loi a finalement été adoptée par la chambre principale du parlement néerlandais (Tweede Kamer) le 9 décembre 2003, avec l'appui de tous les grands partis, sauf un, et par la chambre secondaire (Eerste Kamer) le 7 septembre 2004. Elle entrera en vigueur dès sa publication dans la gazette officielle, qui fait suite à l'assentiment royal.

La loi vise uniquement la provision d'eau potable dans les foyers, non les égouts ni le traitement des eaux usées.

http://meteopolitique.com/Plan/Fiches/environnement/eau/privatisation/Thematique/privatisation/pays/PaysBas/a01.htm consulté le 12 mars 2012.

917 FEUER G et CASSAN H. , Droit international du développement, Dalloz, 1991, p. 211.

918 Cf. TGI Paris, 28 novembre 1972. A propos des nationalisations du cuivre chilien.

919 FEUER G et CASSAN H., op. cit., p. 213. 
la souveraineté des pays en voie de développement et qui violent les principes de non ingérence et $d u$ droit des peuples à l'autodétermination, conditions fondamentales pour le progrès politique, économique et social de ces pays».

\section{Section II. Le service public de l'eau, un service public d'intérêt local}

A s'en tenir uniquement aux trois premiers alinéas de l'article 46 du code de l'eau en vigueur au Mali, les acteurs du service public de l'eau potable sont l'État, les maîtres d'ouvrage, les exploitants et la commission de régulation. L'état assure la définition de la politique nationale d'alimentation en eau potable et le développement du service public de l'eau à l'échelle du pays. Les maîtres d'ouvrage sont soit l'État, soit les collectivités territoriales, selon le niveau d'intérêt de l'activité concernée et dans le respect des lois de décentralisation.

Le cadrage des fonctions de l'État par rapport à l'implication du secteur privé avec les résultats décevants enregistrés, amène à orienter les réflexions sur les capacités des collectivités locales à prendre en compte la distribution de l'eau. Outre ces aspects financiers, l'État se désengage de plus en plus des fonctions de maitrise d'œuvre : réalisation de chantiers, maintenance des équipements et des infrastructures, gestion et exploitation du service de l'eau, etc. Si l'on doit exclure le secteur privé, le désengagement de l'Etat devra s'analyser en un transfert des compétences hydrauliques vers les collectivités locales.

La décentralisation en cours ou établie depuis quelques années en Afrique subsaharienne fait ou a fait émerger un nouvel acteur que sont les collectivités locales : communes, communautés rurales ou régions. Ces nouveaux acteurs sont dans certains cas de plus en plus impliqués dans le secteur de l'eau allant parfois jusqu'à une maîtrise d'ouvrage délégué des infrastructures hydrauliques. Ce constat est largement ressorti du séminaire organisé en 1994 à Paris par le pSEau sur la gestion du service de l'eau dans les centres secondaires du Bassin du fleuve Sénégal (Mali, Mauritanie, Sénégal) ${ }^{920}$. Ce fut l'occasion d'échanger sur les expériences conduites et de constater que les enjeux et problématiques auxquels les acteurs du secteur devaient faire face étaient communs. Il est donc fondamental de faire le point sur la situation des pays de l'Afrique subsaharienne. La décentralisation de l'eau potable dans les collectivités locales, devrait associer les autorités municipales à la gestion du service car celles-ci restent les grands « exclus » de la vie des services publics marchands exploités sur le territoire de leur commune. Elle devrait favoriser l'éclosion d'une nouvelle approche du service avec une appréciation inédite de l'intérêt général : la capacité des élus à identifier certains besoins de leurs administrés et d'y trouver des solutions appropriées à la différence des autorités nationales souvent déconnectées des réalités locales. Afin de mieux comprendre la problématique de la décentralisation de l'eau, nous étudierons d'une part les fondements légaux du transfert de compétence $(§ 1)$ puis la mise en œuvre de la gestion décentralisée du service de l'eau (§2).

$920 C f$. « La gestion du service de l'eau dans les centres secondaires du bassin du fleuve Sénégal », Bernard COLLIGNON, Didier ALLELY, pS-Eau, Éditions du Gret, août 1995. 


\section{§1. La redéfinition des compétences dans le cadre de la décentralisation}

Dans tout ou partie du territoire d'une collectivité territoriale, l'Etat peut déléguer à celle-ci, aux conditions qu'il définit conformément à la loi, certaines de ses compétences relatives à l'utilisation de l'eau. Cette délégation concerne la gestion du service public de distribution d'eau potable ou l'utilisation de l'eau à des fins agricoles, aquacoles, pastorales, industrielles, touristiques ou de production d'énergie ${ }^{921}$. Il sera important de préciser les compétences des autorités décentralisées (A) et celles des autres acteurs (B).

\section{A. Les compétences des autorités décentralisées}

Le désengagement de l'Etat a été accéléré sous l'impact du libéralisme engendré par le Consensus de Washington au terme duquel un ensemble d'accords informels ont été signés par les institutions de Bretton Wood, les grandes multinationales, la Bourse américaine, le Trésor américain, les gouvernements américain et britannique. Ce désengagement a favorisé à certains points de vue l'émergence des collectivités décentralisées.

\section{Une décentralisation consacrant le droit des collectivités à l'autogestion de l'eau}

En réalité le processus de décentralisation n'est pas récent. Les premières expériences en la matière datent de la période coloniale avec les collectivités rurales de plein ou moyen exercice, et ont été suivies, au lendemain de l'indépendance, de la recherche d'une organisation territoriale optimale en vue d'une déconcentration et d'une décentralisation efficaces. Les constitutions des Etats de l'Afrique subsaharienne ont presque toutes consacré le découpage du territoire en collectivité territoriale. Une collectivité territoriale forme une subdivision du territoire, dotée de la personnalité juridique définie comme l'aptitude à être titulaire de droits et obligations et de l'autonomie financière922.

La décentralisation consacre le droit des collectivités territoriales à s'administrer librement et à gérer des affaires propres en vue de promouvoir le développement à la base et de renforcer la gouvernance locale. L'Etat transfère aux régions et aux communes, diverses compétences limitativement fixé par la loi sur la décentralisation. Ce transfert s'accompagne de ressources nécessaires, des service correspondants et, de plein droit, de la mise à la disposition des biens

$921 C f$. article 107 du code de l'eau en vigueur au Togo, précité.

922 LOPA DUFRENOT M., op. cit., p. 89. 
meubles et immeubles. La loi sur la décentralisation énumère souvent les domaines se rapportant à la gestion de l'eau, dans lesquels les collectivités doivent intervenir ${ }^{923}$.

La décentralisation de l'eau potable dans les centres urbains de l'Afrique subsaharienne et, même dans toutes les villes des pays du sud, devrait associer les autorités municipales à la gestion du service car celles-ci restent les grands " exclus » de la vie des services publics marchands exploités sur le territoire de leur commune. Elle devrait favoriser l'éclosion d'une nouvelle approche du service avec une appréciation inédite de l'intérêt général : la capacité des élus à identifier certains besoins de leurs administrés et d'y trouver des solutions appropriées à la différence des autorités nationales souvent déconnectées des réalités locales ${ }^{924}$.

\section{L'adoption d'une législation facilitant la délégation de la gestion aux collectivités décentralisées}

L'intérêt du système a conduit certains pays à adopter des législations sur l'eau permettant à l'Etat de déléguer la gestion du service aux collectivités locales sans pourtant vouloir généraliser l'opération. Ainsi la loi camerounaise n98-005 du 14 avril 1998 portant régime de l'eau dans son article 2 dispose que: "l'eau est un patrimoine commun de la Nation dont l'Etat assure la protection et la gestion et facilite l'accès à tous. Toutefois, l'Etat peut transférer tout ou partie de ses prérogatives aux collectivités territoriales décentralisées [...]».

Ainsi que l'illustre la loi sur décentralisation en République du Bénin en son article 93, la commune a la charge de la fourniture et de la distribution d'eau potable ${ }^{925}$. Ce transfert peut donc concerner plusieurs prérogatives en matière de politiques d'eau dont celles concernant le service de l'eau potable ${ }^{926}$. De même l'article 40 de la loi relative à la décentralisation et aux libertés locales au Togo ${ }^{927}$, précise que «l'Etat transfère aux collectivités territoriales, dans leur ressort territorial respectif, des compétences dans les matières suivantes: ... énergie et hydraulique...; ;

Les grandes métropoles font ainsi partie des collectivités locales sur le territoire desquelles la décentralisation de l'eau potable s'impose. L'eau pourrait ainsi y devenir un enjeu politique local ${ }^{928}$. Car à la différence des pays du nord où ce domaine occupe une place importante dans la vie communale, depuis quelques décennies voire des siècles, il est encore loin d'être au cœur des préoccupations des élus locaux de la sous-région.

923 Idem.

924 Rémy OSSOMBO-YOMBO, op. cit., p. 674.

925 Loi N ${ }^{\circ}$ 97-028 du 15 janvier 1999 portant organisation de l'administration territoriale de la République du Bénin.

926 Idem

927 Loi n 2007-011 du 13 mars 2007 relative à la décentralisation et aux libertés locales

928 MENARD C., «Enjeux d'eau : une dimension institutionnelle », Revue Tiers Monde, n¹66, avril-juin 2001, p.259. 
Par ailleurs, les grandes agglomérations disposent souvent d'une fiscalité propre pouvant leur permettre de financer partiellement le service. Mais cette expérience ne pourrait réussir si les Etats cessent de verser des subventions ponctuelles ou permanentes à des organes gestionnaires. Car si l'Etat n'accompagne pas financièrement ce transfert de gestion, on assistera à un " déchargement » de l'Etat sur les collectivités locales et confirmera nombre de thèses empreintes de réticence défendues en défaveur de la décentralisation ${ }^{929}$. Il faudrait qu'un cadre juridique soit établi, qui garantisse le financement des compétences ainsi transférées. Etant donné l'actuelle incapacité des Etats à fournir l'eau potable aussi bien dans les métropoles que dans les communes rurales, il ne faudrait pas que ce transfert s'érige en transmission "d'inefficacité » de l'État à ces communes. C'est pourquoi, une prudence doit être observée lorsqu'il s'agit de recommander la décentralisation généralisée de l'eau potable à ces États. Le défaut de clarté des modalités de financement du transfert des compétences dans le cadre de la décentralisation de certains services n'est pas un dysfonctionnement propre aux pays du sud.

\section{B. La compétence des acteurs autres que les communes}

En dehors de l'Etat et de ses démembrements, d'autres structures interviennent dans la distribution de l'eau potable. Certaines ont même été consacrées par le code de l'eau dans certains pays. Mais nous allons nous intéresser uniquement à la contribution des organisations non gouvernementales et aux interventions des structures villageoises.

\section{Le concours des organisations non gouvernementales}

Il faut saluer la compétence des acteurs autres que ceux qui sont désignés comme collectivités décentralisées. Les ONG ont un rôle vital pour ce qui est de modeler et d'appliquer la démocratie participative. Leur crédibilité réside dans le rôle judicieux et constructif qu'elles jouent dans la société. Les organisations officieuses ou officielles de même que les mouvements au niveau des communautés devraient être reconnus comme partenaires dans l'exécution d'Action $21^{930}$. La nature du rôle indépendant joué par les ONG dans la société exige une participation réelle; c'est pourquoi l'indépendance est une qualité majeure de ces organisations et l'une des conditions d'une participation effective ${ }^{931}$.

929 ROSENBAUM A., « Gouvernance et décentralisation : leçons de l'expérience », R.F.A.P, nº88, octobrenovembre 1988, p.507 ; D. OLOWU, « Evaluation des politiques de décentralisation en Afrique dans les années quatre-vingt-dix », R.F.A.P, $\mathrm{n}^{\circ} 88$, octobre-novembre 1998, p.516.

930 L'Action 21 (ou Agenda 21) est, comme l'indique son nom, un plan d'action pour le XXI ${ }^{\text {ème }}$ siècle adopté par 173 chefs d'État lors du sommet de la Terre, à Rio de Janeiro, en 1992.

931 RAPPORT DE LA CONFERENCE DES NATIONS UNIES SUR L'ENVIRONNEMENT ET LE DEVELOPPEMENT (Rio de Janeiro, 3-14juin1992), Distr. GENERALE A/CONF.151/26 (Vol. III) 14 août 1992, Chapitre 27. 
Le besoin de promouvoir un idéal commun à tous les secteurs de la société constitue l'un des principaux défis que la communauté internationale doit relever dans ses efforts visant à remplacer des modes de développement non viables par un processus de développement écologiquement rationnel et durable. L'édification de cet idéal commun reposera sur la volonté de tous les acteurs d'instaurer une véritable collaboration et un dialogue au sein de la société tout en reconnaissant les rôles, les responsabilités et les capacités respectives de chacun.

Les organisations non gouvernementales, y compris les organisations sans but lucratif représentant les groupes visés dans l'Action 21, possèdent une expérience, une compétence et des capacités solides et diverses dans des domaines qui présentent un grand intérêt pour l'application et le suivi de programmes de développement durable écologiquement rationnels et socialement responsables, tels que ceux qui sont proposés dans le programme Action 21. L'ensemble des ONG constitue donc un réseau mondial qu'il faudra mettre à contribution et renforcer pour appuyer les efforts visant à atteindre ces objectifs communs ${ }^{932}$.

Pour que ces organisations puissent pleinement jouer leur rôle et notamment dans le secteur de l'eau, il faudra favoriser l'établissement de liens de communication et de coopération aussi étroits que possible entre les organisations internationales, les gouvernements et les administrations locales et les $\mathrm{ONG}^{933}$, dans le cadre des institutions chargées de mener à bien Action 21 et des programmes conçus à cet effet. Ces organisations doivent aussi renforcer les relations de coopération et des communications entre elles afin de participer de manière plus efficace à la mise en œuvre d'un développement durable.

\section{Les structures villageoises de gestion de l'eau}

Comme le soulignait LOPA DUFRENOT, dans les années soixante-dix, l'Etat se chargeait de la maintenance et de l'entretien des ouvrages hydrauliques. Or, le système administratif et financier centralisé existant a montré rapidement des limites caractérisées principalement par son manque d'efficacité. Au début des années quatre-vingt, l'Etat a confié la gestion des ouvrages ruraux à des structures issues des communautés villageoises de base. Celles-ci, sous des appellations différentes assurent, jusqu'à présent, le service permanent de la distribution d'eau, la vente et la prise en charge de l'entretien et du renouvellement des ouvrages, tout en faisant respecter les règles d'hygiène et d'assainissement autour des points d'eau ${ }^{934}$.

Aujourd'hui, les codes de l'eau ont institué « les conseils régionaux et locaux de l'eau » qui devront émettre un avis sur toutes questions relatives à l'eau soumises par l'Administration chargée de l'eau. A cet effet, ils pourront formuler des propositions relatives à la gestion des ressources en eau du bassin ou sous-bassin hydrographique ou des systèmes aquifères. Ils devront ensuite formuler des propositions de solutions à tous conflits d'usage de l'eau et proposer

932 Idem.

933 Idem.

934 LOPA DUFRENOT M., op. cit., p. 90. 
la révision du Schéma directeur d'aménagement et de gestion des eaux, d'en assurer le suivi et l'évaluation au niveau régional et local ${ }^{935}$.

Sans doute, le principe d'une gestion décentralisée des équipements villageois s'avère favorable en termes de responsabilités, de proximité et de paiement des cotisations. Or, la constitution et l'organisation des structures communautaires cristallisent de sérieuses difficultés auxquelles la réforme du système de gestion des infrastructures hydrauliques lancées en l'an 2000 tente de s'attaquer. La pierre d'achoppement la plus remarquable est l'absence de personnalité morale des comités de gestion. De cette lacune, découle une succession d'insuffisances dans la constitution et le fonctionnement de ces structures, ainsi que la gestion qu'elle assure. La réforme récente du système de gestion entend substituer aux organismes de gestion une nouvelle structure territoriale.

\section{§2. Une gestion décentralisée de l'eau assortie des principes de gouvernance}

La réorganisation des dispositions de gouvernance en matière d'eau est étroitement liée à l'évolution du processus national de décentralisation. Alors que la décentralisation politique a été réalisée du moins en ce qui concerne l'adoption des textes de base, il reste à les traduire dans les faits et à les appliquer. Aussi en matière de gestion du service public de l'eau, certains principes sont indispensables à mettre en œuvre. Irina BOKOVA (Directrice générale de l'UNESCO) affirmait à l'occasion de la journée mondiale de l'eau tenue le 22 mars 2010 sous le thème « De l'eau propre pour un monde sain », que "pour que les populations humaines et les écosystèmes se développent, l'eau doit être propre, elle doit rester propre et surtout elle doit être accessible à tous ». Pour y arriver, il faut pour cela, en dehors d'autres mesures sus-évoquées, l'information et la participation du public au processus décisionnel (dans le domaine du droit a l'eau potable). L'information et la participation étant des conditions indispensables pour l'amélioration de la qualité des décisions ainsi que leur application, elles doivent être complétées par la sensibilisation et la concertation dans la prise de décision des projets y relatifs ${ }^{936}$. Nous insisterons sur le principe d'information (A) et le principe de participation (B).

\section{A. Le principe d'information}

Progressivement affirmé, le droit à l'eau a été le résultat de l'admission unanime par la doctrine que l'accès à l'eau fait partie de la dignité humaine. Tout être humain, tout individu, est donc à la fois citoyen, à la fois usager du service public de distribution de l'eau. Dans ce sens, Sylvie PAQUEROT, dans «L'urgence, reconnaître le droit d'accès à l'eau " ${ }^{937}$, reconnaît le caractère

$935 C f$. Article 69 du code l'eau en vigueur au Mali, précité.

936 ATONTSA M., op. cit.p. 149.

937 in L'eau, Res publica ou marchandise?, sous la direction de Riccardo PETRELLA. 
indispensable et essentiel de l'eau. De même, reconnaissant l'existence implicite d'un droit d'accès à l'eau, Henri SMETS explique que le droit au logement implique l'eau puisqu'un logement sans eau est un logement insalubre. Ces explications ont pour objectif de reconnaître au moins à l'usager particulier des droits à l'information originellement consacrés pour le citoyen.

\section{Le droit à l'information, un droit reconnu à l'usager du service public de l'eau}

L'usager du service de l'eau peut, en effet, se prévaloir des procédures d'information qui ne lui sont pas réservées en tant qu'usager, mais qui sont ouvertes à tout citoyen. L'accès aux documents administratifs ou l'étude d'impact en sont des exemples. Quand l'usager / citoyen adhère à une de ses procédures, il agira en tant qu'usager, motivé par sa condition personnelle de destinataire du service. C'est bien un citoyen qui s'informe sur le service de distribution d'eau, la qualité du «produit» desservi ou l'exécution d'un ouvrage lié à ce service. Mais il cherche à s'informer surtout, par principe, parce que c'est à lui que l'eau est desservie.

Pour les possibilités d'information originellement ouvertes à tous les citoyens (comme l'accès aux documents administratifs), les usagers y ont accès à travers une démarche active. Cette possibilité est prévue par la loi, mais c'est à l'usager de chercher à s'informer. A l'inverse, pour certains droits d'information prévus spécifiquement par la loi pour les abonnés du service de distribution d'eau, comme le contenu de la facture, ou la note annuelle informative sur la qualité de l'eau (jointe à la facture), c'est à l'administration ou à la personne privée délégataire du service qu'il revient d'informer de droit les usagers.

La problématique de l'information, s'agissant d'un service public de distribution d'eau, elle porte sur divers aspects (prix, qualité de l'eau, exécution du service, incidence des projets sur l'environnement, etc.) ayant ainsi comme source le droit administratif, le droit de l'environnement, et même, le droit de la consommation. Il y a des obligations d'information prévues par la loi, et d'autres (plus rares) qui ne sont prévues que par les contrats passés par les distributeurs. Ces derniers peuvent également développer des pratiques spontanées d'information de l'usager, surtout pour ceux qui sont abonnés.

\section{L'efficacité l'information portée à l'endroit des usagers}

En France, l'information des usagers abonnés est plus efficace que celle des usagers nonabonnés. Tous, bien évidemment, ont accès aux informations affichées dans les mairies et aux documents administratifs au sens de la loi de 1878. Ce sont des droits accordés à tout citoyen. Toutefois, les distributeurs d'eau, n'ont pas d'obligations actives et directes d'information vis-àvis des usagers non-abonnés. Ils y procèdent néanmoins, indirectement, puisque la loi et les cahiers des charges leur imposent des devoirs d'information envers l'administration (compte rendu technique et financier annuel, par exemple), qui fait le " pont» pour informer les citoyens. De plus, la loi prescrit que les personnes publiques ou privées chargées d'une mission de service 
public sont tenues de communiquer les documents administratifs qu'elles détiennent à toute personne qui en fait la demande. En revanche, les distributeurs ont des obligations positives et directes d'information des abonnés, comme le contenu et la clarté des factures envoyées.

Cette inégalité d'information entre les usagers abonnés et non-abonnés ne pose pour autant pas de problèmes pour un même foyer. Elle peut en poser davantage dans le cas d'immeubles collectifs d'habitation, où il n'y a qu'un seul abonné, le syndic ou le propriétaire de l'immeuble, pour plusieurs ménages d'usagers non-abonnés. Cette problématique sera pourtant en grande partie résolue avec la reconnaissance du droit de tout propriétaire d'immeubles collectifs d'habitation à l'individualisation des contrats de distribution d'eau. Cette modification qui va peut-être avoir une conséquence négative pour l'ensemble des usagers, à savoir, l'augmentation du prix de l'eau, puisqu'il y aura une augmentation des coûts du service, va, au moins, avoir une conséquence positive : l'augmentation des possibilités d'information pour les habitants des immeubles collectifs.

La distinction entre les usagers particuliers, qui font usage de l'eau pour satisfaire leurs besoins personnels, et les usagers professionnels, industriels et agriculteurs implique également une différence de traitement quant au devoir d'information et de conseil imposé par le droit de la consommation. Le juge français refuse d'appliquer le droit de la consommation pour les usagers industriels, en raison de la finalité de l'usage d'eau consommée (finalité professionnelle) et de l'absence de fragilité (caractère soupçonné pour les consommateurs en général) devant le distributeur. Les industriels, "usagers personnes morales», ne peuvent pas non plus bénéficier directement des outils d'information prévus pour les citoyens, comme les études d'impact ou l'accès aux documents administratifs. Or, s'ils ne peuvent pas y accéder directement en tant que personnes morales, ils le peuvent à travers leurs « représentants », les dirigeants.

\section{B. Le principe de participation}

Chacun a le droit de participer au processus de prise de décisions qui affectent leur droit à l'eau et à l'assainissement. Des efforts particuliers doivent être faits pour assurer une représentation équitable dans la prise de décisions de groupes vulnérables ou traditionnellement marginalisés, en particulier les femmes. Les communautés ont le droit de déterminer la nature de leurs services de l'eau et d'assainissement, le type de gestion de ces services et, lorsque cela est possible, de choisir de gérer leurs services eux-mêmes avec l'aide de l'Etat ${ }^{938}$.

938 E/CN.4/Sub.2/2005/25 page 9. 


\section{La signification de la notion}

La plupart des dictionnaires français définissent le terme «participer » comme «prendre part à ${ }^{939}$ (quelque chose). Les auteurs du Lexique des termes juridiques définissent la participation comme un "principe d'aménagement du fonctionnement des institutions politiques et administratives ainsi que de la gestion des entreprises privées, et qui consiste à associer au processus de prise de décision les intéressés (citoyens, administrés, salariés) ou leurs représentants ${ }^{940}$.

Elle constitue ainsi un mécanisme juridique qui consiste à associer les personnes concernées au processus décisionnel. Comme l'information, la participation peut parfois s'avérer indispensable dans l'équilibre des rapports entre différents sujets de droit. Dans ce cas, elle sera l'un des piliers de la cohésion sociale et de la sécurité juridique. Le Conseil d'Etat encourage l'émergence de nouveaux principes liés au fonctionnement des services publics, au premier rang desquels le principe de participation. Par ailleurs, la participation est revendiquée constamment par les usagers dans la mesure où elle est régulièrement présentée comme "un facteur essentiel $d u$ rééquilibrage des services publics ${ }^{941}$ à leur profit. A cet effet, différentes modalités de mise en œuvre de la participation ont été instituées et appliquées au fonctionnement de certains services publics. Néanmoins, le développement des instruments de mise en œuvre de la participation ne repose sur aucun principe de portée générale, ils comblent alors un vide juridique en la matière ${ }^{942}$. D'une manière générale, la participation relève d'une légitimité démocratique différente de celle résultant du suffrage universel, consacrée par notre régime constitutionnel. Dès lors, existe t-il, et, peut-il exister, un principe de participation des usagers au fonctionnement des services publics? Il est important d'expliquer qu'un principe de participation des usagers au fonctionnement des services publics est effectivement en voie de constitution. Ce qui, nous amènera logiquement à s'interroger sur ses modes de mise en œuvre, révélateurs de l'existence ou du moins de la présence du principe dans le fonctionnement des services publics.

\section{Un principe juridique encore en gestation}

Le principe de participation «ne correspond à aucun principe juridique positif des services publics $»^{943}$. En effet, tous les services publics ne sont pas soumis à une règle commune

939 Le Larousse de Poche, Ed.2003, p.585.

940 R. GUILLIEN, J. VINCENT, S. GUINCHARD, G. MONTAGNIER (dir.), Lexique des termes juridiques, Dalloz, $8^{\text {ème }}$ édition. Paris, 1990 , p.361.

941 LECERF M., Droit des consommateurs et obligations des services publics, éd. d'organisation, Paris, 1999, p. 148.

942 THOMAS Isabelle, « Le principe de participation des usagers au fonctionnement des services publics », RFDA 2004 p. 330

943 MAILLARD DESGRÉES du LOÛ D., Droit des relations de l'administration avec ses usagers, PUF, 2000, p. 
imposant la participation des usagers au fonctionnement du service. Cependant, le régime juridique des services publics ne méconnaît pas l'existence du principe. Sans être inscrit dans la liste des principes généraux, les références à son existence sont nombreuses et anciennes. Ainsi, des éléments de nature juridique diverse suscitent la participation des usagers au fonctionnement de certains services publics. Par ailleurs, la participation correspond à une volonté politique, celle d'instaurer une plus grande démocratie participative. A ce titre, l'apport du droit communautaire en la matière se révèle substantiel.

La recherche de la valeur d'un principe est symptomatique de toute étude juridique. De cette valeur dépendra la normativité du principe. Reconnaître une valeur juridique de droit positif à la participation, consisterait à l'appliquer à l'ensemble des services publics et le rendre opposable devant toute juridiction administrative. Une telle conjoncture ne s'applique pas en l'espèce. D'une part, différentes dispositions juridiques poussent à croire à l'existence du principe au sein du régime des services publics ; d'autre part, la confrontation du principe à la hiérarchie des normes conduit à réfuter son existence ${ }^{944}$.

L'intérêt porté à la participation n'est pas un phénomène récent et n'a pas concerné prioritairement les services publics. La théorie de la nationalisation industrialisée, mise en œuvre après la Seconde Guerre mondiale, a conduit à une nouvelle gestion de l'entreprise publique reposant sur la participation des travailleurs. Puis la démarche s'est naturellement prolongée en faveur des usagers des services publics. Imposé ou conseillé, soit par des textes de nature législative ou réglementaire, soit par l'institution de contrats proposés par certains grands services publics à leurs usagers, le développement de la participation s'est effectué de manière très progressive dans différents services publics et à des degrés très variables. Ces avancées dans la mise en œuvre d'un principe de participation au fonctionnement des services publics ont néanmoins été consacrées par des textes de portée générale lui offrant une place toute particulière dans le régime juridique des services publics. Cependant, la valeur juridique de ces textes demeure relative ${ }^{945}$.

Dès 1945, le législateur français a organisé la participation de l'usager à la gestion des entreprises publiques. La présence de l'usager dans les conseils d'administrations des entreprises publiques en constitue une des premières expressions. Ainsi, les lois de nationalisation, du 8 avril $1946^{946}$, disposent que les conseils d'administration doivent être composés de cinq représentants de l'Etat, cinq représentants du personnel et cinq représentants des usagers. De même, la loi du 27 juin $1964^{947}$, portant statut de l'Office de radiodiffusion télévision française, précise dans son article 3 que « le conseil d'administration se compose de quatorze à vingt-huit membres dont une moitié représente l'Etat et l'autre moitié est constituée par des représentants des auditeurs et téléspectateurs, de la presse écrite et du personnel de l'Office ainsi que par des personnalités

40 .

944 THOMAS Isabelle, op. cit.

945 Idem.

946 Loi n 46-628 du 8 avril 1946, sur la nationalisation de l'électricité et du gaz, JO 9 avr. ; Rect JO 17 avr. et 3 mai 1946.

947 Loi n 64-621 du 27 juin 1964, portant statut de l'Office de radiodiffusion-télévision française 
hautement qualifiées... ». Ces différentes lois institutionnalisent la gestion tripartite des entreprises publiques, y compris pour celles gérant un service public ${ }^{948}$.

948 THOMAS Isabelle, op. cit. 
Chapitre II.

LA NECESSITE D'UNE MEILLEURE PROTECTION DES RESSOURCES EN EAU 
Il s'agira de dépasser le cadre d'une législation à valeur symbolique, car ceci constitue un problème sérieux à résoudre au niveau de l'application des normes dans les pays en développement. En effet, dans de nombreux Etats en développement, on peut constater une profusion de lois sur la protection environnementale qui, sur le papier, garantissent une excellente protection et annoncent de sévères sanctions. Mais, dans la pratique, ces lois sont impraticables $^{949}$. De ce point de vue, il apparaît nécessaire de bien situer les enjeux et déterminer le cadre des évolutions. Comme le démontre le Rapport du Fonds de nations Unies pour la population, la situation planétaire de l'eau révèle de nombreuses inquiétudes regroupées autour de deux problèmes ${ }^{950}$. D'une part, l'eau ressource naturelle non renouvelable n'est pas répartie de manière égale sur la planète. Aussi, la croissance démographique et le développement des activités humaines entraînent-ils des besoins croissants, ce, de manière exponentielle. Cela signifie donc qu'il faudrait développer une politique de l'eau fondée non sur une offre susceptible de connaître une croissance exponentielle, mais sur une maîtrise de la demande ${ }^{951}$.

D'autre part, la dégradation de l'eau et des systèmes aquatiques se développe. Outre le fait que des êtres humains meurent par la consommation d'eaux polluées, la dégradation de ces eaux pose la question des usages présents et à venir, de la satisfaction qualitative des divers besoins en eau ${ }^{952}$. Appréhendant ces difficultés sus-mentionnées, DONZIER J.-F. affirme que «la disponibilité des ressources en eau continentale, en quantité et en qualité suffisantes, risque de devenir, d'ici une génération, un véritable enjeu de développement économique et social dans la plupart des pays de notre planète... $\rangle^{953}$. De plus, la gestion de l'eau est compromise par le statut juridique aléatoire de l'eau (Section I). Toutefois, il faut saluer l'apport du droit international de l'eau, droit qui participe également à l'effectivité du droit à l'eau (Section II).

\section{Section I. Une protection de l'eau compromise par le statut juridique ambigü de l'eau}

Les périls liés à un manque tant quantitatif que qualitatif d'accès à l'eau exhortent les responsables politiques à initier des stratégies pour mieux gérer de façon intégrée des ressources en eau devenues de plus en plus limitées et vulnérables. Ces stratégies contribueront à l'amélioration de la qualité des services de production/distribution de l'eau. Ceci est indispensable si l'on évalue l'implication de l'eau en matière de développement humain et économique en Afrique subsaharienne.

949 FAURE M., L'analyse économique du droit de l'environnement, METRO, Bruylant, 2007, p. 300.

950 L'état de la population, www.unfpa.org/swp

951 Agenda 21 adopté à Rio en juin 1992, Chapitre 18, point.2.

952 DROBENKO B. (sous la direction), «Vers une stratégie de gestion durable des fleuves », Les cahiers du CRIDEAU No8, PULIM, 2003, p. 15.

953 DONZIER J.F., « La réforme des institutions clé de l'amélioration de la gestion de l'eau en Amérique Latine », in Vers une stratégie de gestion durable des fleuves, Les cahiers du CRIDEAU n8, PULIM, 2003, p. 45. 
Habituellement, les stratégies de l'eau sont élaborées à partir de l'analyse des contraintes et des potentialités que présente le secteur de l'eau en Afrique subsaharienne; elles se réfèrent également aux engagements pris par les autorités étatiques en faveur de la réduction de la pauvreté et de l'amélioration du cadre et des conditions de vie des populations. Mais en plus de ces contraintes, la politique de l'eau est intrinsèquement liée au statut juridique de l'eau. "Ce statut constitue un vecteur pouvant contribuer à satisfaire le droit à l'eau » écrira DROBENKO B. ${ }^{954}$

Il faut remonter au droit romain pour constater que l'eau fut classée parmi les « res communis» c'est-à-dire chose appartenant à tout le monde et non comme un bien ayant une valeur pécuniaire et susceptible d'appropriation privée ${ }^{955}$. Une classification qui sera reprise par le code civil français encore en vigueur dans bien d'Etats de l'Afrique subsaharienne. Aussi, peut-on y lire qu' "il est des choses qui n'appartiennent à personne et dont l'usage est commun à tous $»^{956}$. L'analyse comparative des différents codes de l'eau en vigueur dans certains de ces Etats montre que l'eau rélève, sous différentes formulations, d'un statut se référant à des prérogatives de puissance publique ${ }^{957}$. L'on note des expressions comme domaine public, domaine hydraulique, domaine de l'Etat, patrimoine commun de la Nation... La détermination du statut juridique de l'eau (§1.) constitue donc un élément décisif pour la compréhension de la portée de l'admission de l'eau comme un élément du domaine public (\$2.).

\section{§1. La détermination du statut juridique de l'eau}

L'eau douce indispensable à la vie, au développement et à l'environnement est une ressource limitée et vulnérable. Elle ne constitue que 2,5\% du volume de toute l'eau de la planète. Aujourd'hui, un humain sur trois n'a pas accès à l'eau potable, ou alors, vit dans une situation de "stress hydrique » en ne disposant pas de suffisamment d'eau par rapport à ses besoins réels. L'actuel essor démographique urbain (5 milliards de personnes vivront en zone urbaine en 2030) implique une augmentation des besoins en eau douce. L'eau constitue une ressource provenant de la nature qui ne peut être gérée comme un bien ordinaire selon les critères et les impératifs de rentabilité économique. Sa gestion doit être exercée dans l'intérêt de tous d'autant que cette ressource est limitée. La détermination du statut de l'eau doit permettre de répondre à la fois à la satisfaction des besoins fondamentaux, à la préservation quantitative et qualitative de l'eau et des milieux aquatiques ainsi qu'au contrôle des usages. Nous montrerons d'une part, que l'eau est un élément du domaine public (A) et d'autre part, qu'elle est considérée comme un patrimoine commun (B).

954 DROBENKO B., Le droit à l'eau : une ingérence humanitaire, précité p. 137.

955 LOPA DUFRENOT M., précité, p.63.

956 Article 714 du code civil.

957 DROBENKO B., op. cit. 


\section{A. L'eau, élément du domaine public}

L'intérêt d'une distinction entre domaine public et domaine privé vient du fait que les personnes publiques exercent un droit de propriété sur leur domaine privé. Ainsi, propriétaires de leur domaine privé, le sont-elles de leur domaine public ? Pendant longtemps on a répondu par la négative quand bien même, ces personnes exerçaient un droit de surveillance sur le domaine public. Au XIX ${ }^{\text {ème }}$ siècle, les auteurs disaient que les personnes publiques n'étaient pas propriétaires de leur domaine public ${ }^{958}$. C'est inexact. En observant les solutions jurisprudentielles - c'est la jurisprudence qui fait le droit positif- l'on remarque que celles-ci considèrent que les personnes publiques sont propriétaires de leur domaine public. En effet, le droit de propriété est le pouvoir d'utiliser le bien dont on est propriétaire (possibilité d'aliénation du bien). Cependant les biens du domaine public sont inaliénables donc les personnes publiques ne sont pas propriétaires. Sans ajouter à la confusion, nous allons faire confiance aux différents codes de l'eau qui proclament l'appartenance de l'eau au domaine public. Ce statut de domanialité publique légalement affirmé n'est pas sans portée juridique. Si les ressources en eau font partie intégrante du domaine public, il faut retenir que ceci entraîne une conséquence inéluctable. Celle de l'inaliénabilité de ces ressources si importantes à la vie de l'Homme.

\section{L'affirmation du principe}

Il n'est pas très compliqué de constater les multiples énonciations de l'eau au domaine public de l'Etat. Tous les codes de l'eau où les lois y faisant office énoncent sans détour l'existence d'un domaine public de l'eau. S'inspirant de la loi ancienne, la loi du 8 février $2001^{959}$ adoptée par le législateur burkinabè, définit le domaine public de l'eau comme comprenant l'eau dans ses divers états physiques et situations géomorphologiques et les ouvrages publics affectés ou nécessaires à sa gestion. Ce qui rejoint parfaitement l'état de la doctrine française sur la définition du domaine public. En effet, selon le droit français, le domaine public est considéré comme une partie du patrimoine des personnes publiques soumise à un régime de droit public. Ce régime très protecteur comprend le domaine public naturel (en l'occurrence l'eau) et le domaine public artificiel ${ }^{960}$.

Parmi les Etats pionniers ayant adopté un code de l'eau, on peut citer la Mauritanie. Cet Etat sahélien dont la plus grande partie de sa superficie est désertique, a pris la mesure de l'importance de l'eau très tôt et a édicté son code de l'eau dès 1984. Aussi, l'eau a été considérée comme faisant partie du domaine public. Selon l'article 3 du code «les ressources en eaux superficielles, souterraines ou atmosphériques, où qu'elles soient, situées dans les limites du

\footnotetext{
958 Selon BERTHELEMY.

959 Article 5 de Loi d'orientation relative à la gestion de l'eau au Burkina précitée.

960 GUILLIEN R. et VINCENT J. (dir.), Lexique des termes juridiques, Dalloz, 2001, p. 211.
} 
territoire national, sont un bien collectif et, à ce titre, font partie intégrante du domaine public de l'Etat qui est inaliénable et imprescriptible $»^{961}$.

Sur la question, le législateur togolais adopte la même démarche et affirme que l'eau fait partie du domaine public puis précise que le domaine public de l'eau comprend les cours d'eau, les lacs naturels ou artificiels, les eaux souterraines, les atmosphériques... ${ }^{962}$. De son côté, le législateur malien énonce que l'eau est «un bien relevant du domaine public » et que "son usage appartient à tous pourvu qu'il ne soit pas contraire à l'intérêt public $»^{963}$. Selon l'article 25 de la loi portant loi-cadre relative a la gestion de l'environnement au Cameroun, les eaux continentales constituent un bien du domaine public dont l'utilisation, la gestion et la protection sont soumises à ladite loi ainsi qu'à celles de la législation et de la réglementation en vigueur... ${ }^{964}$ A cet effet, l'Administration chargée de la gestion des ressources en eau devra dresser un inventaire établissant le degré de pollution des eaux continentales, en fonction des critères physiques, chimiques, biologiques et bactériologiques. Cet inventaire est révisé périodiquement ou chaque fois qu'une pollution exceptionnelle affecte l'état de ces eaux ${ }^{965}$. L'on peut multiplier les exemples et l'on constatera que les formules adoptées par les différents Etats de l'Afrique subsaharienne sont presque similaires ${ }^{966}$. DROBENKO B. dira à cet égard que «le statut de domanialité publique » constitue le plus souvent une référence au regard des garanties qu'il est susceptible de conférer ${ }^{967}$. Il faut préciser que ce statut définit l'ensemble des règles qui garantissent la protection de la ressource en eau, organise les rapports de voisinage et précise les conditions d'utilisation de la ressource. Aussi, importe-t-il de délimiter le domaine public de l'eau.

\section{La délimitation du domaine public de l'eau}

L'opération de délimitation peut être organisée soit par voie réglementaire soit par voie législative. Mais elle implique dans tous les cas les autorités exécutives. En application de la Loi d'orientation relative à la gestion de l'eau au Burkina un décret d'application, a précisé les conditions de délimitation du domaine public de l'eau. En effet, la délimitation s'opère, notamment sur la base de données diverses, hydrométriques et hydrogéologiques ${ }^{968}$. La

961 Ordonnance $n^{\circ} 85-144$ du 4 juillet 1986 portant code de l'eau.

$962 C f$. article 5 de la loi n²010-004 du 14 juin 2010 portant Code de l'eau au Togo.

$963 C f$. article 2 de la loi n ${ }^{\circ} 02-006 /$ du 31 jan.2002 portant Code de l'eau en République du Mali.

$964 C f$. Loi ${ }^{\circ}$ 96/12 DU 5 aout 1996

$965 C f$. Article 26 de la loi précitée.

$966 C f$. article 11 de la LOI n98-755 du 23 décembre 1998 Portant Code de l'Eau en République de Côte d'Ivoire. Voir également Art. 2. De la Loi n 81-13 du 4 mars 1981 portant Code de l'Eau en République du Sénégal :« Les ressources hydrauliques font partie intégrante du domaine public. Ces ressources sont un bien collectif et leur mise en exploitation sur le territoire nationale est soumise à autorisation préalable et à contrôle ».

967 DROBENKO B., Le droit à l'eau : une urgence humanitaire, précité, p. 137.

$968 C f$. Article 4 décret n²005-193/PRES/PM/MAHRH/MFB du 4 avril 2005 portant procédure de délimitation des limites des dépendances du domaine public de l'eau. 
procédure retenue, assez lourde, compte tenu des atteintes susceptibles d'être portées à la propriété privée impose le déroulement d'une enquête publique et l'avis d'organes consultatifs tels que l'organe délibérant de la région ou le comité technique de l'eau. La délimitation des dépendances du domaine public est prononcée par arrêté conjoint du Ministre chargé de l'eau, des domaines et de la santé 969 .

Aux termes de l'article 11 du Code de l'eau de la Mauritanie, les limites des eaux du domaine public sont fixées par des arrêtés conjoints des ministres chargés de l'hydraulique et de l'Equipement après enquête; ces limites sont en principe fixées à partir du niveau atteint par les eaux avant tout débordement. Les articles 12 et 13 prévoient respectivement que les limites des eaux du domaine public peuvent être fixées d'après l'interprétation des données hydrométriques, hydrologiques, botaniques ou autres et que cette délimitation ne peut être modifiée que dans les mêmes formes.

D'autre part, les actions en reconnaissance de droits acquis sur les terrains compris dans une délimitation doivent être intentées sous peine de forclusion, dans un délai d'un an à compter de la date de publication de l'arrêté de délimitation et lorsqu'il y a changement des limites naturelles des cours d'eau délimités, les riverains intéressés peuvent adresser une demande de nouvelles délimitations au ministre chargé de l'hydraulique qui doit instruire la demande en liaison avec le ministre chargé de l'Equipement ${ }^{970}$. Dans l'opération de délimitation du domaine public de l'eau, il est constaté que certains codes de l'eau excluent du domaine les piscines, les citernes, les bassins d'agréments, les puits et forage, et un certain nombre d'ouvrages privés destinés à recueillir et à drainer les eaux ${ }^{971}$. Par ailleurs, il est défendu d'une part, de dépasser de quelque manière que ce soit, notamment par des constructions, sur les limites des francs-bords de cours d'eau temporaires ou permanents, de lacs, de périmètres de protection, de sources ainsi que sur les limites d'emprise des aqueducs, conduites d'eau, canaux de navigation, d'irrigation ou autres périmètres de protection faisant partie du domaine public de l'eau sauf avec autorisation expresse de l'autorité compétente, et d'autre part, de placer à l'intérieur des limites du domaine public de l'eau tout obstacle entravant la navigation, le libre écoulement des eaux et la libre circulation sur les francs-bords ${ }^{972}$.

\section{B. La portée de l'admission de l'eau comme élément du domaine public}

En admettant que l'eau est un élément du domaine public, on obtiendra une protection des ressources en eau ce qui facilitera l'instauration d'un régime d'utilisation et d'exploitation.

\footnotetext{
$969 C f$. Article 3 du décret précité.

970 Voir les articles 15 et 16 du code de la Mauritanie.

$971 C f$. article 9 de la loi n²010-004 du 14 juin 2010 portant Code de l'eau au Togo.

972 Voir article 10 du Code de l'eau du Togo précité.
} 


\section{La protection du domaine public de l'eau}

La protection du domaine est organisée autour de trois principes fondamentaux à savoir l'inaliénabilité, l'imprescriptibilité et l'insaisissabilité. Légitimement, l'on peut s'interroger sur le sort de l'eau dans un contexte de libéralisation du secteur de l'eau. Les règles de protection garantissent la conservation de la ressource en eau et le maintien de son affection à l'utilité publique $^{973}$. Considérée comme le caractère de tout ce qui n'est pas aliénable, l'inaliénabilité frappe toutes les ressources en eau qui font partie du domaine public.

Le principe de l'inaliénabilité du domaine public a des origines diverses. Du droit romain à la Révolution française, l'on constate que le principe a fait l'objet de différentes appréciations ${ }^{974 .}$ Alors que dans le droit romain, la question n'est pas du tout abordée, dans l'ancien droit, l'inaliénabilité est admise mais elle est limitée au domaine de la Couronne ${ }^{975}$. Ce n'est qu'avec la Révolution française que le principe de l'inaliénabilité a connu une évolution significative ${ }^{976}$. Toutefois, il ne faut pas perdre de vue que le principe suscite toujours des polémiques juridiques. De telle sorte que certains auteurs se sont demandés : «Faut-il supprimer le principe de l'inaliénabilité du domaine public ? »977.

Le principe de l'inaliénabilité des ressources en eau a nécessairement une portée absolue alors que les ouvrages ou installations pourraient faire l'objet de la procédure de déclassement, et, ainsi, sortir du domaine public pour être cédés comme un bien privé978. Sur le caractère absolu de l'inaliénabilité, l'illustration du code de l'eau en vigueur en République du Mali est très édifiante. En effet, selon les termes de l'article 3, «l'eau ne peut faire l'objet d'appropriation privative que dans les conditions fixées par les dispositions en vigueur et dans le respect des droits coutumiers reconnus aux populations rurales pourvu qu'ils ne soient pas contraires à l'intérêt public $\gg{ }^{979}$. Ce que confirme la jurisprudence administrative française à travers une décision du Conseil d'Etat rendue en 1990980. En effet, selon cette jurisprudence, un organisme privé ne peut pas disposer d'un domaine public.

Quant à l'imprescriptibilité, elle découle du principe de l'inaliénabilité et a pour intérêt de prémunir la puissance publique contre la prescription acquisitive ${ }^{981}$ et de se voir dépossédée des

973 LOPA DUFRENOT M., précité, p 73.

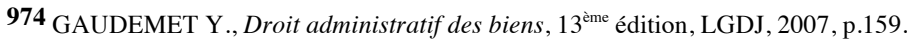

975 ROUSSELET A., La règle de l'inaliénabilité du domaine de la Couronne.Etude doctrinale de 1566 à la fin de l'Ancien Régime, Trav. Rech., Paris II, LGDJ, 1997.

976 GAUDEMET Y., précité.

977 DELALOY G., « Faut-il supprimer le principe de l'inaliénabilité du domaine public ? », RDP 2006.575. Voir aussi, SIMONIAN-GINESTE H., «L'avenir du principe de l'inaliénabilité du domaine public », Revue de droit immobilier, 1989, p. 169.

978 LOPA DUFRENOT M., précité, p 73.

$979 C f$. loi n ${ }^{\circ} 02-006 /$ du 31 jan.2002 portant Code de l'eau en République du Mali.

980 CE, section, 1990, Association Saint Pie V et Saint Pie X de l'orléanais.

981 Principe permettant après l'écoulement d'un certain délai de faire acquérir un droit réel à celui qui en fait l'exercice. 
dépendances du domaine à son insu. Enfin, l'insaisissabilité des dépendances du domaine public complète cette énumération. Ce principe interdit aux personnes de droit privé titulaires d'une créance à l'égard des personnes publiques de recourir aux voies d'exécution de droit privé en vue de son recouvrement. Enfin, on peut noter que le domaine public de l'eau est également protégé contre les entraves à la circulation et tout acte susceptible de contribuer à la diminution de leur valeur. En effet, il est interdit de jeter dans le lit des cours d'eau des objets susceptibles d'encombrer ce lit ou y provoquer des atterrissements et de traverser les conduites, aqueducs ou canalisations à ciel ouvert inclus dans le domaine public de l'eau, avec des véhicules ou animaux en dehors des passages spécialement réservés à cet effet et de laisser pénétrer les bestiaux dans les emprises des canaux d'irrigation ou d'assainissement ${ }^{982}$.

\section{L'instauration d'un régime d'utilisation et d'exploitation}

L'utilisation et l'exploitation du domaine public ont été organisées par le législateur à travers les différents codes sus cités. En effet l'utilisation est soumise dans la plupart des cas à un encadrement soigneusement gradué. Le constat est que l'on va du régime simple au régime d'utilisation intense. Au Togo, l'utilisation est soumise aux régimes de l'utilisation libre, de la déclaration, de l'autorisation et de la concession ${ }^{983}$.

Le régime de l'utilisation libre n'est soumis à aucune autorisation ni déclaration. En se référant au code de l'eau du Togo, "est libre l'utilisation des eaux à des fins domestiques, limitée à la satisfaction des besoins individuels et familiaux, à l'hygiène des personnes, des habitations et des animaux domestiques et à l'arrosage des jardins, à condition que la profondeur de captage, la capacité de puisage et le volume d'eau prélevé ne dépassent pas les seuils arrêtés par le ministre chargé de l'eau » de même que "l'accumulation artificielle des eaux pluviales tombant sur fonds privé à condition que ces eaux demeurent sur ce fonds et que leur utilisation soit conforme aux prescriptions édictées par les lois et règlements en vigueur $»^{984}$.

Lorsque l'utilisation du domaine public de l'eau nécessite la réalisation de travaux de captage des eaux souterraines équipés de moyens d'exhaure ou requiert la réalisation de puisards et puits traditionnels à usage domestique prélevant de l'eau de la nappe phréatique ne dépassant pas les seuils fixés par arrêté du ministre chargé de l'eau, l'utilisation est subordonnée à une déclaration si le dépassement des seuils établis par les autorités publiques est avéré985. Les deux autres régimes admis sont considérés comme une réponse à l'urgence de protéger le domaine public contre les dégradations et les risques de leur détournement.

Le régime de l'autorisation régit les cas d'activités dont les impacts sur les écosystèmes hydrauliques peuvent s'avérer irréversibles. Aussi, certaines législations citent « les utilisations

\footnotetext{
$982 C f$. Article 10 du Code de l'eau du Togo.

983 Cf. Article 11 du même code

$984 C f$. Article 12 du même code.

985 Cf. Article 13.
} 
des eaux de surface pratiquées au moyen d'ouvrages et installations permanents susceptibles de présenter des dangers pour la santé et la sécurité publique, de modifier substantiellement le niveau, le mode d'écoulement ou le régime des eaux, et de porter atteinte à la qualité de l'eau » et une série d'activités touchant directement les eaux souterraines ${ }^{986}$.

Le régime de concession nous intéresse particulièrement car il s'incruste parfaitement dans la problématique de nos recherches. En effet, sont soumis au régime de la concession, tous les aménagements et les exploitations des sources minérales et thermales ainsi que les prélèvements, l'accumulation et l'utilisation des eaux de surface et souterraines effectués au moyen d'ouvrages, installations et travaux permanents, et destinés à la production et distribution d'eau potable, ou à la production d'énergie électrique, dans le cadre d'un service public. D'autre part, la réalisation et l'exploitation d'ouvrages, installations et travaux destinés à l'alimentation de réseaux d'irrigation dans le cadre d'un service public, sont soumis au régime de la concession ${ }^{987}$. Les concessions de service public fondées sur l'utilisation des eaux sont accordées, pour une durée déterminée, aux personnes morales publiques ou privées ou aux personnes physiques exerçant une activité présentant un caractère d'intérêt général ${ }^{188}$.

Il faut préciser que les aménagements, ouvrages et travaux soumis au régime de la concession font l'objet d'une étude d'impact environnemental préalable dans les cas prévus par la législation sur l'environnement. Le contenu, la méthodologie et les procédures des études d'impact environnemental sont réglementés par le ministre chargé de l'environnement, en accord avec le ministre chargé de l'eau.

Alors que le législateur togolais distingue quatre différents types de régime, au Sénégal et au Mali, les textes en vigueur distinguent seulement les autorisations et les concessions ${ }^{989}$. De même, le législateur burkinabè est allé pratiquement dans le même sens en optant pour les autorisations et les déclarations ${ }^{990}$. L'accès à l'eau étant devenu de plus en plus compliqué suite à l'extension effrénée de l'urbanisation, des entreprises privées se sont lancées dans l'implantation d'ouvrages hydrauliques notamment les forages. Malheureusement, la majorité de ces ouvrages ne sont pas installés conformément aux dispositions du code de l'eau. Le phénomène n'est pas propre à l'Afrique subsaharienne, puisqu'en droit français, le Conseil d'État observe que l'administration ne connaît pas avec précision le taux de non-déclaration par type d'installations, ouvrages, travaux ou activités ${ }^{991}$. L'ampleur du phénomène de nondéclaration présente un inconvénient grave pour la connaissance des prélèvements et du cycle de

$986 C f$. Article 14 Code de l'eau du Togo: ( les activités de recherche des eaux souterraines ; le captage d'eau souterraine par forage, galerie drainante, canalisation ou par tout autre dispositif équipé d'un moyen d'exhaure; le curage, l'approfondissement, l'élargissement, le redressement et la régularisation des cours d'eau, temporaires ou permanents ; l'extraction de pierres, du sable et du gravier du lit et des berges des cours d'eau, des lacs et des canaux...)

987 Cf. Article 19 du même code.

$988 C f$. Article 27 de la loi n 81-13 du 4 mars 1981 portant Code de l’Eau au Sénégal.

$989 C f$. Article 7 et suivants de la loi n 81-13 du 4 mars 1981 portant Code de l'Eau au Sénégal. Voir également les articles 19, 20 et 21 du Code de l'eau du Mali.

$990 C f$. Article 24 de la loi nº02-2001 du 8 février 2001 portant loi d'orientation relative à la gestion de l'eau.

991 CE, L'eau et son droit, Rapport du CE 2010, La documentation, 2010, p. 217. 
l'eau ${ }^{992}$. Alors que le régime de déclaration et celui d'autorisation sont censés apporter, entre autres retombées, à la puissance publique chargée de la bonne utilisation de la ressource une information exacte sur l'ampleur des prélèvements, le non respect des formalités peut parfois démontrer la faiblesse de la machine administrative ${ }^{993}$.

\section{§ 2. L'eau en tant que patrimoine commun ou refus de sa marchandisation}

Le patrimoine est loin d'être une notion stabilisée et il demeure difficile à appréhender car il se situe dans un " entre-deux » dont les cadres théoriques standards ont du mal à rendre compte. Pensée au départ dans la tradition juridique héritée du Code civil comme une notion strictement individuelle, l'application du patrimoine aux objets naturels et culturels a conduit à porter un nouveau regard sur cette notion et à adopter, à partir des années 1960, l'expression de « patrimoine commun » pour les ressources naturelles, et notamment pour l'eau ${ }^{994}$.

Ce changement sémantique amène à réinterroger les couples individu-communauté, gestionnaire-usager, public-privé, court terme long terme, local-global, conduisant à mobiliser le territoire, l'histoire et l'identité comme des marqueurs du patrimoine commun. Notion dynamique (A), elle semble hostile à toute marchandisation (B).

\section{A. Une notion dynamique}

Envisager l'eau comme un patrimoine commun comme l'ont fait le législateur ivoirien ${ }^{995}$ et son homologue camerounais ${ }^{996}$, implique de revenir brièvement sur la notion telle qu'elle est définie en droit interne et en droit international. D'autre part, il faut préciser que l'analyse économique s'est, de fait, désintéressée de l'eau pendant une longue période, dans la mesure où

992 MIQUEL G., Rapport $n^{\circ} 2152$ sur la qualité de l'eau et de l'assainissement en France, Office parlementaire d'évaluation des choix scientifiques et technologiques, 18 mars 2003.

993 MONTGINOUL M. et RINAUDO J.-D., Quels instruments pour gérer les prélèvements en eau souterraine ? Le cas du Roussillon, CEMAGREF, UMR G-Eau, Montpellier.

994 La Charte européenne de l'eau, proclamée par le Conseil de l'Europe en 1968, reconnaît dans l'un de ses principes que «l'eau est un patrimoine commun dont la valeur doit être reconnue de tous. Chacun a le devoir de l'économiser et d'en user avec soin ». Cette notion a également été inscrite dans la loi française sur l'eau de 1992 et dans la Directive cadre européenne sur l'eau, adoptée en 2000.

995 Cf. Article 7 de la Loi n $98-755$ du 23 décembre 1998 portant Code de l'Eau : «L'eau fait partie du patrimoine commun national. Sa protection, sa mobilisation et sa mise en valeur, dans le respect des équilibres naturels, sont d'intérêt général. Elle ne peut faire l'objet d'appropriation que dans les conditions déterminées par les dispositions de la présente loi ».

$996 C f$. aussi article 2 de la loi n 98-005 du 14 avril 1998 portant régime de l'eau au Cameroun : « l'eau est un bien du patrimoine commun de la nation dont l'Etat assure la protection et la gestion et en facilite l'accès à tous ». 
cette ressource, présumée disponible en quantité illimitée, était considérée comme sans valeur ${ }^{997}$. Ce postulat d'abondance a été battu en brèche lorsque l'eau douce a commencé à se raréfier et à se dégrader, à mesure que la population mondiale et, par voie de conséquence, les besoins s'accroissaient.

\section{En droit interne}

Issu d'une construction doctrinale réalisée à partir de dispositions juridiques éparses ${ }^{998}$, le patrimoine est un objet familier du droit privé français. Relevant d'une logique individuelle, il constitue une extension juridique de la personne afin de permettre l'exécution de ses obligations sur ses biens. Il recouvre deux actions principales, dont l'une tend à l'appropriation et l'autre à la transmission. Dans le domaine de l'appropriation, le patrimoine est un outil juridique fondamental du libéralisme économique. En assurant la solvabilité actuelle ou future du sujet de droit, il joue comme une garantie pour les créanciers et comme une légitimation du droit des personnes quasiment illimité sur les choses. En matière de transmission, le patrimoine assure la continuation patrimoniale du de cujus (celui dont il s'agit de régler la succession) par ses successeurs. Alors qu'en économie le patrimoine et le capital peuvent se rejoindre, selon cette conception juridique, on ne gère pas un patrimoine exactement de la même manière que l'on gère un capital. On gère un patrimoine pour le transmettre et non pour l'accroître comme on le ferait pour un capital ${ }^{999}$. Dans cette perspective, on s'aperçoit que la notion devient facile à déplacer. On passe de l'individuel au familial, du familial au national, du national à l'international sans qu'elle ne devienne vague pour autant. Cette élasticité conduit à sortir du strict patrimoine individuel pour envisager l'existence de biens communs inscrits dans un patrimoine commun. C'est, d'ailleurs, par cette extension que la notion juridique de patrimoine est appliquée à la nature et à la culture, notamment dans la définition des patrimoines mondiaux au cours du XX $\mathrm{XX}^{\text {ème }}$ siècle ${ }^{1000}$.

Ceci signifie principalement que la nature et la culture doivent être transmises de génération en génération, indéfiniment, comme cela se fait de père en fils pour les biens privés. L'approche collective du patrimoine relève, en ce sens, d'une éthique qui consiste, pour un individu ou une organisation, à placer au premier rang de ses préoccupations le souci constant de préserver les libertés de choix de ceux qui viendront après lui. Pour cela, il convient de ne pas les engager malgré eux dans des voies qui pourraient les mener à des impasses ${ }^{1001}$. C'est pourquoi sa protection, sa mobilisation et sa mise en valeur, dans le respect des équilibres naturels, sont

997 Voir le paradoxe de l'eau et du diamant, chez Adam SMITH (1776).

998 AUBRY et RAU, 1873

999 BELAIDI N. et EUZEN A., « De la chose commune au patrimoine commun. Regards croisés sur les valeurs sociales de l'accès à l'eau », De Boeck Université, Monde en développement, 2009/01 - n 145, pages 55 à 72.

1000
1001
1 Idem.. 
considérées comme étant d'intérêt général. En effet le patrimoine commun ne peut faire l'objet d'appropriation que dans les conditions déterminées par les dispositions de la loi ${ }^{1002}$.

\section{En droit international}

En droit international public, la seule qualification existante qui puisse se rapprocher, dans son essence, des biens communs est celle de patrimoine commun de l'humanité. Le patrimoine substitue une logique complexe qui prend en compte les usages multiples qu'appellent les espaces et les ressources naturelles et met en place des réseaux de droit d'usage et de contrôle débordant les découpages issus de la propriété et de la souveraineté1003.

La qualification de patrimoine commun de l'humanité n'a pas cependant été utilisée jusqu'ici pour désigner des ressources hors de la juridiction des Etats, malgré le fait qu'historiquement ce soient d'abord les ressources de base de notre environnement qui aient été ainsi qualifiées ${ }^{1004}$. Paradoxalement, l'être humain qui n'est pas sujet de droit international, est le destinataire des droits de l'homme. Dès lors, l'humanité se retrouve destinataire de certaines règles du droit international. Le concept d'humanité ouvre ainsi l'espace de l'universalité à la base de la pensée kantienne qui exige de « briser le cercle de proximité qui obligeait seulement à l'égard du proche

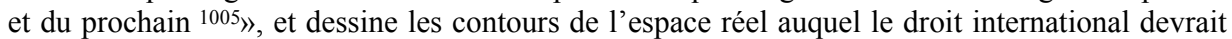
théoriquement s'appliquer, celui de la planète ${ }^{1006}$.

Dès lors, accorder à certaines ressources un statut de patrimoine commun de l'humanité oblige à tenir compte des ressources à travers les époques et les systèmes juridiques, du caractère universel, dans le temps et dans l'espace, de ces ressources ${ }^{1007}$.

Enfin, le droit international tout comme le droit interne, n'est rien de plus qu'un outil à la disposition des sociétés pour organiser les conditions du «vivre ensemble ». Le droit est ce par quoi une société présente le visage d'une puissance publique. Il doit donc évoluer avec la réalité pour que les normes qu'il contient demeurent utiles et pertinentes. Dans le domaine de l'eau, cette exigence d'évolution est particulièrement marquée si l'on considère que jusqu'au début du

1002 Cf. Article 7 de la Loi n $98-755$ du 23 décembre 1998 portant Code de l'Eau, précité.

1003 OST F., Le milieu un objet hybride qui déjoue la distinction public-privé, Paris, Presses Universitaires françaises, 1995, p.102.

1004 REMOND-GOUILlOUd M., Du droit de détruire, Essai sur le droit de l'environnement, Paris, Presses Universitaires, 1989, p. 122 et 145.

1005 REMOND-GOUILLOUD M., L'avenir du patrimoine, Esprit, n²16, 1995, p.70.

1006 PAQUEROT S., Eau douce, la nécessaire refondation du droit international, Presses de l'Université du Québec, 2005, p.11.

1007 OST F., La nature hors la loi : l'écologie à l'épreuve du droit, Paris, La découverte, 1995, p. 308. 
$\mathrm{XX}^{\text {ème }}$ siècle, les seules préoccupations reflétées par le champ du droit international concernaient les frontières et la navigation ${ }^{1008}$.

Complexe, juridiquement peu précis comme l'ont soutenu ses opposants lors des négociations de la Convention des Nations unies sur le droit de la mer ${ }^{1009}$, le concept de patrimoine commun de l'humanité n'en recèle pas moins, selon les auteurs ${ }^{1010}$, la reconnaissance d'un bien commun dépassant la simple addition des intérêts des Etats.

\section{B. Un concept s'opposant à la commercialisation de l'eau}

La communauté internationale s'est fixée comme priorité d'améliorer l'approvisionnement en eau et l'assainissement afin de veiller au respect des valeurs humaines fondamentales. L'accès à l'eau potable contribuerait largement à la satisfaction des besoins fondamentaux des populations. Chacun s'accorde à reconnaître que le droit à l'eau, récemment institué par les Nations unies 1011 , est "indispensable pour mener une vie digne» et qu'il est une "condition préalable à la réalisation des autres droits de l'homme ».

\section{Le constat de l'appropriation partielle de l'eau}

Avec l'émergence de ce droit de l'homme, l'eau apparaît comme un enjeu majeur tant au plan national, régional que mondial. L'observation $\mathrm{n}^{\circ} 15$ précise d'ailleurs que l'eau devrait être considérée comme un bien social et culturel et non essentiellement comme un bien économique. Elle ajoute que le droit à l'eau doit être exercé dans des conditions de durabilité afin que les générations actuelles et futures puissent en bénéficier ${ }^{1012}$.

Elle est susceptible d'avoir une importance simultanément internationale, nationale et locale et sa gestion soulève des problèmes régionaux. L'eau semble, par nature, n'appartenir à personne

1008 FENET A., «Droit de la mer, droit des cours d'eau internationaux : similitudes et convergences », ADMA, $1991, \mathrm{t} .11, \mathrm{p} .90$.

1009 Adoptée en 1982 à Montego Bay (Jamaïque), la Convention des Nations Unies sur le Droit de la Mer (CNUDM) est entrée en vigueur le 16 novembre 1994, après ratification de 60 États et de la Communauté européenne qui l'a également signée. Cette convention représente une étape importante dans l'application du droit international pour laquelle 12 ans de négociations ont été nécessaires (1982-1994). Elle définit les principes généraux de l'exploitation des ressources de la mer.

http://cms2.unige.ch/isdd/spip.php?article65

1010 BASLAR K., The concept of the common heritage of mankind in international law, 1998, The Hague/Boston.

1011 Conseil économique et social des Nations unies, 2002

1012 BELAIDI N. et EUZEN A., op. cit. 
et être offerte à l'usage commun. Pour autant, elle fait aussi l'objet d'une appropriation partielle, par exemple, de l'eau embouteillée.

Dans les deux hypothèses, l'eau n'a pas le même statut. Dans le premier cas, en étant liée à la protection d'intérêts supérieurs, l'eau apparait comme une chose commune. Dans cette optique, elle est inappropriable et n'intègre ni la catégorie de la propriété collective ni celle de la propriété individuelle. L'inappropriabilité des choses communes n'étant pas absolue, elle peut être affectée d'une certaine relativité. Il en est ainsi des choses communes qui se prêtent à une appropriation fragmentaire comme l'eau. Le fragment considéré est un bien, c'est le deuxième cas. La différence de nature entre les choses communes et les biens entraîne une différence de régime. Les biens sont, en principe, dans le commerce juridique.

Ce n'est que de manière exceptionnelle que leur commerce est interdit par la loi ou la jurisprudence. À l'inverse, les choses communes sont, en principe, hors du commerce juridique et, par là même, hors marché1013.

Toutefois, il ne faut pas en déduire que leur extra-commercialité découle de la nature des choses. Elle résulte plutôt d'une impossibilité juridique : le commerce des choses communes est indirectement interdit par le biais de leur inappropriabilité. Plus précisément, cette inappropriabilité est justifiée par les intérêts communs à tous les membres du corps social que la chose commune renferme ${ }^{1014}$. Le droit organise l'inappropriabilité afin de garantir l'usage commun. Partant, si le bien (l'eau embouteillée pour notre exemple) est dans le commerce juridique, l'appropriation partielle ne porte que sur une portion infime de la chose commune, et celle-ci demeure donc soustraite à la catégorie des biens. Mieux, cette réservation fragmentaire ne caractérise par les choses communes puisqu'elle ne concerne pas toutes les res communis. Cependant, le retrait d'une chose de la sphère d'appropriation et d'échange ne s'effectue pas en raison d'une nature propre. Cette soustraction est voulue et décidée par la société.

\section{Le statut de l'eau, une transaction sociale}

Selon DURKHEIM, «c'est l'opinion de chaque société qui fait que tels objets sont considérés comme susceptibles d'appropriation, tels autres non. Ce ne sont pas leurs caractères objectifs, tels que les sciences naturelles peuvent les déterminer, c'est la façon dont ils sont représentés dans l'esprit du public. Telle chose qui, hier, ne pouvait être appropriée l'est aujourd'hui et inversement ». En somme, c'est la société qui définit ce qui est appropriable ou non en fonction des valeurs défendues ${ }^{1015}$.

Finalement, toute la construction du concept de chose commune repose sur l'idée qu'il existe des intérêts communs à tous les membres du corps social qui s'incarnent, ou se matérialisent,

\footnotetext{
1013 Ibidem.

1014 Ibidem.

1015 BELAIDI N. et EUZEN A., supra.
} 
dans certaines choses du fait de la valeur sociale ou culturelle qu'elles représentent pour la collectivité.

Alors que du point de vue théorique, la notion de "patrimoine commun de l'humanité » amène à concevoir l'environnement comme le milieu de vie et, de ce fait, à lier l'homme, la société et la nature, la diversité des perceptions et des pratiques liées à l'usage de l'eau dans l'espace domestique, elle montre dans nos exemples qu'elle n'est pas, de manière absolue, considérée comme une chose commune, l'essentiel étant d'abord de satisfaire ses besoins et ses plaisirs $^{1016}$.

Le caractère inappropriable de la ressource n'est, en effet, pas évident vu les usages et les valeurs symboliques qu'elle véhicule. Cela pose avec acuité la question du partage de la ressource entre les individus appartenant à un même groupe, une même société, à l'échelle locale comme à l'échelle planétaire. Bien que la notion de patrimoine commun puisse amener à envisager toute dégradation de l'environnement comme une mise en danger des supports de vie, et par conséquent, comme une mise en péril du groupe humain dans lequel s'inscrit l'individu, la vision que la société a de son environnement tend à réduire ces potentialités. Celles-ci reposent sur ce que les membres de la société définissent comme inappropriable (et partant livré à l'usage commun) ou non, en fonction des valeurs qu'ils reconnaissent et défendent.

\section{Section II. L'apport du droit international de l'eau}

Il faut préciser d'emblée que l'essentiel de la contribution du droit international sur les ressources en eau, est fondé sur l'Observation générale $\mathrm{n}^{\circ} 15$ dont nous avons déjà fait mention tout au début de nos développements. Mais bien d'autres éléments sont intervenus dans l'affirmation du droit à l'eau. L'initiative de certaines organisations internationales qui ont tenté d'appréhender de manière générale ces enjeux, est restée marginale et n'a pas su redresser véritablement une situation qui continue à se détériorer à l'échelle planétaire, tant au regard de la dégradation des ressources en eau qu'à celui de leur distribution ${ }^{1017}$. Face aux enjeux des ressources en eau, la communauté internationale va réagir $(\S 1)$, et engager des actions en vue de leur protection $(\$ 2)$.

\section{§ 1. La communauté internationale face aux enjeux des ressources en eau}

Il est une pensée philosophique communément partagée selon laquelle toutes les sociétés ont la « terre en partage » et celle du patrimoine commun de « la terre mère » qui sont aujourd'hui traduites en droit. Un autre concept, celui du développement soutenable, tente d'en formaliser l'aspiration. Le développement soutenable, c'est « s'efforcer de répondre aux besoins du présent

\footnotetext{
1016 Ibidem.

1017 Ibidem.
} 
sans compromettre la capacité de satisfaire ceux des générations futures $»^{1018}$. Il repose sur l'idée que « chaque être humain, vivant et à venir, a droit à une vie digne de ce nom » comprenant bien sûr, l'accès aux ressources nécessaires pour vivre. Les Nations-Unies sont le grand architecte de ces nobles idées. En effet, se fondant sur sa mission de paix et de développement, les NationsUnies multiplient les grandes conférences au cours desquelles, sont prises des résolutions en matière de développement, de droits humains et de protection de l'environnement (A). Depuis la conférence de Stockholm de 1972, les Nations-Unies consacrent nombre de ces résolutions à la protection des ressources en eau (B).

\section{A. L'émergence de la question de l'eau dans les conférences onusiennes sur le social}

Depuis des décennies, l'eau douce est un sujet de préoccupation majeure, comme en témoigne l'intervention des ONG, qui se sont pourtant longtemps limitées aux enjeux de protection environnementale ou bien, concernant l'accès à l'eau potable, à des interventions caritatives. Mais, au début des années 1990, la fin de la Décennie internationale de l'eau, et surtout la considération de plus en plus générale des solutions du marché pour résoudre cette problématique, induira une intégration et une politisation progressive des interventions des ONG dans ce domaine ${ }^{1019}$. Ainsi, la question de l'eau va-t-elle s'inscrire à l'ordre du jour des conférences internationales sur le social.

\section{L'eau dans les préoccupations des conférences sur l'éducation, la population et l'habitat}

Ainsi, par exemple, parmi les sept objectifs prioritaires fixés pour l'an 2000 par le Sommet mondial des enfants, on retrouve nommément l'accès à l'eau potable, mais aussi trois autres objectifs qui y sont liés: une réduction du tiers des taux de mortalité pour les nourrissons et les moins de cinq ans; une diminution de moitié des taux de mortalité maternelle; l'accès à l'éducation de base ${ }^{1020}$. C'est dire que plus de la moitié des objectifs prioritaires de ce sommet concernait l'eau, directement ou indirectement.

Subséquemment, les Conférences sur la population (1994) et sur les établissements humains (1996) aborderont la question de l'eau, sous deux aspects principalement: d'une part, bien sûr, du point de vue de l'évolution démographique, préoccupante à cet égard, puisque les plus fortes augmentations de population prévisibles se situent dans des régions où la ressource en eau est déjà soit rare, soit en état de dégradation avancée, et où les infrastructures ne répondent déjà pas aux besoins immenses. Mais, par ailleurs, à l'occasion de ces conférences, ont aussi été développés et articulés les liens étroits entre droit au logement et droit d'accès à l'eau, le second

1018 Rapport BRUNDTLAND.

1019 Les interventions des ONG au Sommet de Johannesburg illustrent largement cette orientation.

1020 Sur l'éducation, qu'il suffise de mentionner que l'absence d'accès à l'eau lait en sorte que de nombreux enfants consacrent plusieurs heures par jour à aller chercher de l'eau, ce qui les empêche d'être à l'école, surtout les petites filles, H. GUISSE (1998), 
étant considéré comme un élément de définition d'un logement adéquat. De même, les enjeux reliés au droit à la santé qui dépend de l'assainissement de l'eau en zones fortement urbanisées ont été abordés ${ }^{1021}$. Ainsi, la Conférence sur la population et le développement du Caire affirmera-t-elle que: "l'approvisionnement en eau est l'un des problèmes les plus graves que connaissent les villes des pays en développement $»^{1022}$. Dans la Déclaration d'Habitat II, les chefs d'Etats ont réaffirmé leur «volonté d'assurer progressivement la pleine réalisation du droit à un logement convenable ${ }^{1023}$ », leur engagement à le mettre en œuvre ${ }^{1024}$ et leur définition de ce droit: "Chacun a droit de vivre convenablement avec sa famille, notamment de manger à sa faim, d'être correctement vêtu et logé et de bénéficier de systèmes d'approvisionnement en eau et d'assainissement $»^{1025}$.

\section{La préoccupation de l'eau dans les conférences sur le genre et l'alimentation}

En 1995, la IV ${ }^{\text {ème }}$ Conférence mondiale sur les femmes avait repris, elle aussi, cet enjeu en soulignant l'importance d'examiner le rôle spécifique des femmes dans l'utilisation et la préservation des ressources en eau et de garantir leur accès égal aux infrastructures, qu'il s'agisse de l'eau potable en elle-même ou d'un logement adéquat, comprenant cet accès à l'eau. On retrouve d'ailleurs cette considération du rôle spécifique des femmes dans de nombreuses déclarations liées à l'eau, à partir de la Déclaration de Dublin. La conférence de Beijing reprendra aussi à son compte la priorité qui traverse l'ensemble des interventions dans ce domaine depuis Mar del Plata: «que l'on assure qu'une eau propre soit disponible et accessible à tous d'ici l'an 2000 [...] et que l'on établisse des plans de conservation des eaux et des plans de nettoyage de l'eau polluée $e^{1026} »$.

En ce qui concerne le Sommet mondial sur l'alimentation de Rome, bien que l'eau ne soit pas explicitement mentionnée dans la Déclaration finale, il en est abondamment question dans le rapport.

La capacité de production agricole étant directement liée à la disponibilité en eau, il va de soi que la préservation des ressources hydriques est un élément incontournable de la sécurité alimentaire. Cependant, il faut souligner que cette déclaration, s'engageant à poursuivre l'objectif de sécurité alimentaire pour tous à travers un «système de commerce international orienté sur le marché », pose sous une nouvelle forme la question des conditions d'un développement durable ${ }^{1027}$ et de la répartition des ressources. En effet, comme plusieurs auteurs le soulèvent

\footnotetext{
1021 Nations-Unies, Déclaration d'Istanbul sur les établissements humains (1996).

1022 Nations-Unies, Conférence internationale sur la population et le développement (1 994), A/CONF. $171 / 1$ 3/Flev. 1 
régulièrement, la production agricole mondiale intégrée à la mondialisation des marchés a tendance à favoriser, même dans des zones à stress hydrique élevé, la production de denrées ou de produits demandant une forte irrigation. Les contradictions existantes entre compétitivité et durabilité ont été amplement démontrées, y compris en matière de production agricole et alimentaire.

\section{B. Une aspiration au droit largement exprimée au niveau international}

La mise en place d'un cadre normatif au plan international qui tienne compte des trois dimensions essentielles de la problématique de l'eau, de la paix, de la préservation des ressources et du respect des droits humains, apparaît encore aujourd'hui bien hypothétique. Au plan des enjeux environnementaux, bien qu'elle ne soit pas unanime, on reconnaît largement l'importance de prendre en compte la nature de la ressource, qu'il s'agisse du cycle hydrologique, du fonctionnement écosystémique de la circulation de l'eau. Nombreuses sont les personnes qui meurent chaque année du manque d'eau potable. En osant la comparaison comme Sylvie PAQUEROT l'avait essayée, on constate que le déficit en eau potable tue quotidiennement plus que la tragédie de World Trade Center. "S'il ne s'agit pas dans ce cas d'imposer volontairement la mort et ces atteintes massives à la dignité humaine, le défaut d'agir est flagrant », écrira -telle. Les Etats sont aujourd'hui très loin d'être représentatifs de la préoccupation globale de la communauté internationale à l'égard de l'eau, à la fois du point de vue de leurs actions et de leur engagements normatifs ${ }^{1028}$.

\section{L'apparition des tribunaux de l'eau}

La dimension du droit des populations aux ressources en eau a été intégrée en 1992, peu de temps avant le Sommet de la Terre, lors de la seconde session du Tribunal international de l'eau à Amsterdam ${ }^{1029}$. Cette session portera sur les problèmes de gestion de l'eau en Afrique, en Asie et en Amérique latine. Cette initiative illustre bien en quelque sorte l'aspiration au droit eu égard aux enjeux de l'eau.

Le Tribunal international de l'eau, lors de la session d'Amsterdam, s'appuiera sur une déclaration, élaborée par un groupe d'experts juridiques provenant de différentes régions du monde ${ }^{1030}$, sur la base des instruments existants tels que les résolutions de l'assemblée générale des Nations Unies, les déclarations des conférences internationales, ainsi que le travail de la

1028 PAQUEROT S., op. cit. 234.

1029 Dès 1981, des ONG hollandaises fondent le tribunal international de l'eau qui tiendra, en 1983 à Rotterdam, une première session portant sur la pollution des eaux en Europe de l'ouest.

1030 Réuni en mars 1991, le groupe de travail comprend 14 juristes provenant d'Asie, d'Afrique, de l'Amérique latine, des Etats Unis et des Pays-Bas. Voir Déclaration of Amsterdam, 1992. 
Commission du droit international ${ }^{1031}$. Le Tribunal appliquera les principes ainsi dégagés à l'ensemble des ressources en eau et non seulement aux ressources transfrontalières ${ }^{1032}$. La déclaration d'Amsterdam reprend et/ou énonce entre autres le principe de l'équité intergénérationnel, celui du droit à l'eau en quantité et qualité suffisante en tant que droit humain, celui du caractère inappropriable de l'eau, bien commun devant relever du stewardship ${ }^{1033}$ de l'humanité.

En février 1992 le Tribunal international de l'eau entendra douze cas sur les vingt-deux qui lui ont été soumis et constitue avant tout, au dire des responsables de cette initiative, un lieu de débat, un forum permettant de discuter publiquement de situations conflictuelles correspondant assez bien à la recommandation d'Action 21, appelant à la mise sur pied de mécanismes internationaux plus larges et efficaces pour entendre et résoudre les conflits dans le domaine du développement durable ${ }^{1034}$.

Malgré son nom le Tribunal n'en est évidement pas un et les décisions du jury sont des jugements éthiques sans portée juridique formelle. Ils sont pourtant fondés sur une procédure rigoureuse respectant les droits de la défense et les exigences de la preuve ${ }^{1035}$, ce qui lui confère une légitimité importante et explique les résultats non négligeables obtenus par cette action. On peut en effet constater qu'au delà de résultats généraux tels que la démonstration du besoin de mécanismes permettant aux populations de se faire entendre, malgré l'absence de portée juridique réelle, le tribunal d'Amsterdam aura eu une incidence concrète sur plusieurs dossiers entendus.

Dans certains cas, les accusés auront accepté leurs responsabilités et manifesté leur volonté de régler les situations conflictuelles. En Argentine, au Chili et au Pérou notamment, les audiences auront permis aux plaignants d'obtenir au moins en partie satisfaction et au Costa Rica, cela aurait permis d'ouvrir un débat public.

Ces résultats ne peuvent bien sûr être confondus avec la possibilité d'obtenir justice devant une véritable instance juridictionnelle, mais ils illustrent clairement la nécessité de mécanismes de justice indépendants. Plus encore, le fait que plusieurs des mis en cause aient accepté de se plier aux décisions et recommandations du jury montre bien la portée que pourraient avoir des mécanismes internationaux formels. A la suite de cette expérience d'autres tribunaux de l'eau ont d'ailleurs été mis sur pied ${ }^{1036}$, ce qui corrobore largement ces constats.

1031 Voir le processus et le contenu.

1032 Idem.

1033 Le concept de stewardship est aussi utilisé par Kemal BASLAR, the concept of the common heritage of mankind in international law, 1998.

1034 Paragraphe 39, 3-h.

1035 Le jury est d'ailleurs assisté d'un comité d'experts, étant donné la complexité de la matière à l'examen, Voir Background and Results, 1994.

1036 Le Tribunal centraméricain de l'eau ; www.tragua.com basé au Costa Rica ; C. G. CAUCHET, « Un tribunal éthique en Amérique centrale », Manière de voir ${ }^{\circ}$ 65, 2002. 


\section{Mais un arsenal juridique toujours en quête d'affirmation}

D'autre part, le GWP comme le Conseil mondial de l'eau où siègent les grandes entreprises du secteur de l'eau font désormais référence à Déclaration de 2002 faite par le comité des droits économiques, sociaux et culturels selon laquelle l'eau est un droit de l'homme ${ }^{1037}$. Pour le Conseil mondial de l'eau «tous les êtres humains devraient avoir accès à des quantités suffisantes d'eau douce et à des systèmes d'assainissements adéquats ».

De plus, malgré cet élargissement de la perspective et la reconnaissance de plus en plus répandue du fait que les principes de la communauté d'intérêts sont plus aptes à fournir des réponses juridiques utiles que les principes de la souveraineté limitée en matière de ressources hydriques, le débat entre développement et environnement, entre préservation et exploitation, pour sa part, reste largement ouvert au vu de l'évolution des déclarations portant sur le sujet.

En matière de préservation des ressources et des écosystèmes, les principes essentiels à l'intérêt public demeurent essentiellement dans le domaine de la lege ferenda. Aucun document légal, en effet, dans le cadre juridique contemporain, ne mentionne même la loi la plus fondamentale concernant 1 'eau; celle du cycle hydrologique ${ }^{1038}$. Seule la déclaration de La Haye de $1989^{1039}$ a " osé » exprimer clairement que les enjeux d'environnement et de ressources, à cause de leur portée globale, exigeaient de transcender la souveraineté pour imposer des normes universelles.

\section{§ 2. Le renforcement des actions de la communauté internationale}

La véritable tragédie de la crise de l'eau est son effet sur la vie quotidienne des populations pauvres, terrassées par le fardeau des maladies liées à l'eau, qui vivent dans des environnements dégradés et souvent dangereux, qui luttent pour que leurs enfants reçoivent une éducation, pour gagner leur vie et assurer leur subsistance. Le Programme des Nations-Unies pour l'Environnent (A) et l'Organisation des Nations-Unies pour l'Education, la Science et la Culture (B) œuvrent inlassablement pour la protection des ressources en eau, des actions qu'il faudrait nécessairement renforcer.

1037 SMETS H., «Incorporation du droit à l'eau potable dans l'ordre juridique national », in Environnement et renouveau de droits de l'homme, Actes du colloque de Boulogne -sur-Mer des 20 et 21 novembre 2003, La Documentation française, Paris, 2006, p. 29.

1038 SHIVA V., Water Wars, Cambridge, South Ends Press, 2001, p.76.

1039 Déclaration sur la protection de l'atmosphère 


\section{A. Le PNUE et les ressources en eau}

Le Rapport sur la mise en valeur des ressources en eau dans le monde publié en $2003^{1040 \text {, }}$ constate le doublement du nombre de pauvres n'ayant pas accès à des services adéquats d'approvisionnement en eau et d'assainissement et l'écart grandissant entre riches et pauvres et entre citadins et ruraux en ce qui concerne les services d'approvisionnement en eau et d'assainissement. Par ailleurs, le rapport dénonce le coût grandissant des catastrophes liées à l'eau, la baisse de la qualité des ressources en eau et des écosystèmes hydriques et le sous financement du secteur de l'eau. Il préconise la nécessité de renforcer la gouvernance dans le domaine de l'eau.

\section{L'implication du PNUE dans la protection des ressources en eau}

Depuis sa création, le Programme des Nations Unies pour l'environnement (PNUE) a œuvré dans le domaine de l'évaluation et de la gestion des ressources en eau en favorisant l'application d'approches collaboratives de la gestion de ces ressources. Après plus de 30 ans, l'eau reste une des grandes priorités du PNUE.

De nombreuses instances internationales ont consacré beaucoup de temps et d'efforts à la définition de mandats, de buts, d'objectifs et de cibles pour la gestion des ressources en eau. Parmi ces instances figurent le Conseil d'administration du PNUE; la Conférence des Nations Unies sur l'environnement et le développement, qui a abouti à Action 21 ; le Sommet du Millénaire; le Sommet mondial pour le développement durable ; et les douzième et treizième sessions de la Commission du développement durable des Nations Unies. Ces instances ont indiqué et, dans le cas du Conseil d'administration déterminé quels devraient être les mandats et les responsabilités du PNUE dans le domaine de l'eau.

La tâche consiste donc maintenant à mettre en œuvre ces mandats plutôt que d'en élaborer de nouveaux, c'est-à-dire à passer de la planification à l'action. Lors de l'établissement de la politique et stratégie de l'eau, le PNUE a pris en considération le fait que le Conseil d'administration/Forum ministériel mondial sur l'environnement du PNUE a également adopté, à sa vingt-troisième session, le Plan stratégique de Bali pour l'appui technologique et le renforcement des capacités ${ }^{1041}$. L'exécution des fonctions assignées au PNUE dans le domaine de l'eau, en particulier aux niveaux national et régional, s'inscrira dans le cadre d'une application cohérente du Plan stratégique de Bali à l'échelle du PNUE. Dans les activités qu'ils mèneront aux niveaux régional et national, le PNUE et ses partenaires s'efforceront de se compléter et contribueront à la consolidation des capacités nationales de gestion de l'environnement et à la prise en compte de l'environnement dans le développement économique

$\mathbf{1 0 4 0}$ http://unesdoc.unesco.org/images/0012/001295/129556f.pdf consulté le 5 juillet 2012.

1041 UNEP/IEG/3/4, annexe. 
et social (c'est-à-dire dans les stratégies nationales de réduction de la pauvreté et de développement durable).

La politique et stratégie de l'eau du PNUE a principalement pour but de faciliter une mise en œuvre coordonnée, efficace et rapide des fonctions qui lui ont été assignées dans le domaine de l'eau douce. Dans le contexte du Plan stratégique de Bali ${ }^{1042}$, l'impératif d'exécution est devenu encore plus pressant.

\section{Les mandats du PNUE dans le domaine de l'eau}

La politique du PNUE dans le domaine de l'eau est définie en partie par son mandat général, tel qu'il est énoncé dans les résolutions de l'Assemblée générale des Nations Unies, et par sa mission qui consiste à montrer la voie et à encourager la coopération pour protéger l'environnement tout en étant une source d'inspiration et d'information pour les Etats et les populations et un instrument de facilitation leur permettant d'améliorer la qualité de leur vie sans toutefois compromettre celle des générations à venir.

Pour amorcer le travail, le directeur exécutif du PNUE proposera lui-même une définition de ce concept qui d'entrée de jeu soulève les réticences des États. Il en circonscrit les contours $a$ contrario et considère que ce concept concerne un nombre limité d'États par opposition au patrimoine commun international. Il propose une liste, non exhaustive des ressources qui relèveraient de cette définition: " un système hydrologique international, y compris les eaux de surface et les eaux souterraines ... $\gg^{1043}$.

En 1997, le Conseil d'administration a adopté la Déclaration de Nairobi sur le rôle et le mandat du Programme des Nations Unies pour l'environnement ${ }^{1044}$ dans laquelle il a précisé ce mandat en y englobant en particulier les rôles convenus aux chapitres 17 (Océans) et 18 (Eau douce) d'Action 21.

Les décisions prises par le Conseil d'administration/Forum ministériel mondial sur l'environnement à ses dix-huitième, dix-neuvième, vingtième et vingt-deuxième sessions ont défini des programmes prévoyant les activités à mener par le PNUE dans les domaines des récifs coralliens, de la gestion intégrée des zones côtières et des bassins hydrographiques ainsi que de la lutte contre la pollution marine due aux activités terrestres. A sa dix-neuvième session et à sa cinquième session extraordinaire, le Conseil/ Forum a également demandé au PNUE d'accorder une priorité élevée à l'eau douce et d'aider les pays en développement à être mieux à même d'effectuer des évaluations concernant l'eau douce ainsi que d'élaborer et de mettre en œuvre des plans de gestion intégrée des ressources en eau dans le cadre d'une coopération régionale. A sa vingt-troisième session, le Conseil/Forum a outre défini les éléments d'une politique de l'eau,

\footnotetext{
1042 Plan stratégique de Bali pour l'appui technologique et le renforcement des capacités en matière de gestion des ressources en eau.

1043 Doc. UNEP/GC/44, p.41, paragr. 8, cité par J.T. CALASANS, p.238

1044 Décision 19/1 du Conseil d'administration.
} 
par exemple dans le domaine des eaux souterraines, qui faisaient défaut dans les politiques et stratégies précédentes. Le Conseil d'administration a en aussi assigné des mandats ${ }^{1045}$ dans les domaines du transfert de technologies pour la gestion des ressources en eau et la lutte contre la pollution, de l'assainissement urbain et rural, des eaux souterraines et de la promotion de la responsabilité sociale des entreprises, qui ont tous d'importantes contributions à apporter en matière de gestion intégrée des ressources en eau.

En 2000, la communauté internationale a défini les grandes priorités de développement en fixant les objectifs énoncés dans la Déclaration du Millénaire (Objectifs du Millénaire pour le développement). La réduction de la pauvreté et de la faim, l'amélioration de la santé humaine et la réalisation de la durabilité environnementale ont été considérées comme les principales priorités mondiales. En 2002, eu égard à la gravité de la situation dans le domaine de l'eau douce dans de nombreuses parties du monde et au fait qu'il est indispensable d'améliorer la gestion de l'eau pour atteindre les objectifs généraux de développement économique, les participants au Sommet mondial pour le développement durable ont adopté un objectif prévoyant l'élaboration de plans intégrés de gestion et d'utilisation rationnelle des ressources en eau d'ici 2005.

A sa huitième session extraordinaire, le Conseil d'administration/Forum ministériel mondial sur l'environnement a adopté l'Initiative de Jeju, dans laquelle il a souligné qu'une "gestion intégrée des ressources en eau comportant une approche écosystémique constitue un élément clé pour atteindre les cibles concernant l'eau, l'assainissement et les établissements humains ... et pour favoriser la croissance économique et la réalisation des cibles concernant la santé et la réduction de la pauvreté » ${ }^{1046}$.

\section{B. L'UNESCO et l'éthique de l'eau}

L'UNESCO, intervient dans le domaine de la gestion de l'eau pour deux raisons essentielles. D'une part, elle contribue au développement de la recherche scientifique, de l'éducation et de la coopération dans ce domaine. D'autre part, la défense du droit des hommes pour l'accès aux ressources en eau participe de la défense de leur dignité et des principes de base selon lesquels l'éducation et la culture ne peuvent être accessibles que dans la mesure où les gens ont un minimum de ressources pour vivre et non pas seulement pour survivre. Cette philosophie humanitaire marque l'institutionnalisation du problème de l'eau au niveau international depuis de nombreuses années, notamment dans le cadre de l'intervention des organisations spécialisées de l'ONU et des Communautés européennes.

Comme le fait remarquer Sylvie PAQUEROT, l'UNESCO a depuis longtemps pris en compte la problématique de l'eau puisqu'elle est responsable du programme hydrologique mondial, créé dans la foulée de la Conférence de Mar del Plata. Elle " héberge » aujourd'hui le World Water Assessment Program (WWAP), chargé de coordonner l'action de l'ensemble des composantes onusiennes dans le domaine de l'eau.

1045 Décisions 10/19, 11/7, 13/19, 21/1, 22/2, 22/6, 22/7 et 23/2 du Conseil d'administration.

1046 Rapport du Conseil d'administration/Forum ministériel mondial sur l'environnement sur les travaux de sa huitième session extraordinaire (UNEP/ GCSS.VIII/8), annexe II, par. 5. 


\section{L'exigence prioritaire de l'UNESCO en matière d'eau : l'éthique}

Il faut souligner que cette organisation met particulièrement l'accent sur la question de l'éthique, donc sur des valeurs et des règles qui devraient refléter ces valeurs, qu'elle aborde la question de l'eau, à travers plusieurs dimensions de la problématique: la gestion des différentes utilisations; la sécurité alimentaire; la santé et l'assainissement; les catastrophes naturelles liées à l'eau; la prise de décision et la gestion; l'eau et l'écologie; le rôle des femmes; l'histoire de l'eau et l'éthique sociale; les défis technologiques; les problèmes spécifiques relatifs à l'utilisation intensive des nappes phréatiques dans les pays arides; les conflits en rapport avec l'eau ${ }^{1047}$.

Conscient de la complexité de cette problématique aux multiples facettes, le groupe de travail mis en place pour en examiner les enjeux conviendra que "des trois grands facteurs qui contribuent à la situation de pénurie et au risque de conflit qui lui est lié, diminution ou dégradation des ressources, croissance démographique et inégalité de la répartition ou de l'accès, le dernier est souvent le plus décisif $\gg^{1048}$ et en ce sens accordera une attention particulière aux aspects liés à l'accès et à la redistribution.

Le but poursuivi est de contribuer à jeter les bases de la confiance, de la justice et de l'équité dans la disponibilité et l'accès des ressources en eau douce pour la communauté des nations ${ }^{1049}$.

Aussi, le groupe de travail fonde-t-il son approche sur des principes qu'il considère « universels » et directement applicables à l'eau: principe de la dignité humaine; principe de participation; principe de solidarité; principe d'égalité des êtres humains; principe du bien commun; principe d'économie, nous invitant au respect de la nature et à une sage utilisation de celle-ci ${ }^{1050}$. Sur cette base, les principes directeurs proposés ouvrent sur l'affirmation suivante: "Les principes de base à adopter commencent par la notion que tout être humain a droit à l'eau nécessaire pour satisfaire ses besoins de boisson, d'alimentation, de santé et de développement; encourager la perception de l'eau comme un produit de base revient à éloigner le public de sa dimension en tant que bien commun ${ }^{1051}$.

Il ne s'agit pas de revenir ici sur l'intégralité des principes développés. Mais, il importe de souligner les aspects qui nous permettent de situer la réflexion sur l'état du droit international en la matière, d'autant plus qu'il s'agit bien souvent de clarifier des controverses concrètes apparues ces dernières années: l'eau est-elle une commodité? Doit-on tolérer un certain degré de pollution ? Est-il acceptable que les développements urbains privent les paysans de leurs droits à l'eau en les rachetant même si cela entraîne un usage économiquement plus efficace? Quelles sont les limites acceptables de l'exploitation des eaux souterraines non renouvelables en zones arides ?...

1047 Au cours de quatre réunions ; Paris, 28 octobre 1998; Paris, 10 janvier 1999;Oslo (Norvège) 26 avril 1999 ; Almeria (Espagne) 31 juillet et 1er aout 1999.

1048 UNESCO, L'éthique de I 'utilisation de l'eau douce. vue d'ensemble (2000) sous commission de la COMEST Lord Selborne, prés. p. 36

1049 Idem.
1050 Idem.
1051 Idem. 
Ainsi, du point de vue de la répartition de cette ressource vitale, la dimension de droit humain est ici précisée comme excluant la considération de l'eau en tant que produit de base, celle-ci induisant la transformation des citoyens et citoyennes en consommateurs et consommatrices. Pour certains, encourager la conception de l'eau comme un produit de base revient à éloigner le public de sa réalité en tant que bien commun et du sens du devoir et de la responsabilité de tous à son égard. En d'autres termes, nous percevoir comme des citoyens de l'eau ou des consommateurs d'eau est lourd de conséquences sur le plan de l'éthique.

La sécurité alimentaire est un impératif moral; les demandes de l'industrie et les besoins en irrigation devraient être coordonnés afin de garantir le droit à l'eau des fermiers pratiquant une agriculture de subsistance, y compris le droit à l'utilisation et la collecte des eaux pluviales et l'accès â des sources suffisantes pour l'irrigation.

La perspective de droit humain adoptée entraîne non seulement une primauté des besoins humains de base mais également, du point de vue de la question du prix de l'eau, une dichotomie similaire à celle retenue à Rio, qui affirme la nécessité de fixer un juste prix à l'eau, au-delà des besoins essentiels, et en tant que remboursement à la société.

\section{Les autres recommandations de l'UNESCO en matière d'eau}

On pourrait ainsi considérer par exemple que le développement d'un réseau global d'informations sur la ressource où qu'elle se situe relève précisément des obligations communes et non de chaque Etat ou communauté, même si cette obligation est mise en œuvre de manière décentralisée, dans la mesure où l'usage commun de ces informations s'avère essentiel à la pérennité de la ressource prise globalement ${ }^{1052}$. La mise en place du programme hydrologique mondial, dans une certaine mesure, répondait à ce type de responsabilité, de même que la recension des eaux souterraines entreprise par l'UNESCO afin d'uniformiser les informations à cet égard pour faciliter la résolution des conflits ${ }^{1053}$.

De la même manière qu'ordonnateur et comptable sont dissociés en matière fiscale, il est indispensable que l'autorité qui fixe les règles soit séparée de l'opérateur du service, qu'il soit public ou privé. En effet, la part de réussite dépend fortement du fait que chaque intervenant au contrat doit se limiter à ses seules attributions et qu'on doit éviter au maximum l'empiètement sur le domaine d'autres participants. Ceux-ci doivent respecter ces règles constituant un contrat

\footnotetext{
1052 On se rappellera que dans la Convention de New York de 1997 portant sur les utilisations des cours d'eau internationaux autres que la navigation, si l'obligation d'échange d'informations est inscrite, rien n'est prévu en cas de non respect des obligations de coopération.

1053 L'UNESCO a lancé un programme international appelé International Shared Aquifer Resources (Isarm). Il a pour tâche de recenser au cours des six prochaines années les aquifères transfrontaliers et de les cartographier à l'échelle du globe. « Une étude qui sera très utile, car elle permettra de combler les lacunes dans les connaissances et d'homogénéiser les approches. Tous les pays en effet ne raisonnent pas avec les mêmes chiffres », estime Thierry POINTET, ingénieur hydrogéologue au Bureau de recherches géologiques et minières (BRGM) : C. GALUS, «Les nappes phréatiques abreuvent la moitié du monde » (2003) Le Monde, 20 mars.
} 
de service vers les usagers branchés sur le réseau, qu'ils soient domestiques ou institutionnels ou qu'ils concernent la desserte des services publics comme les écoles ou hôpitaux ${ }^{1054}$.

L'Etat doit fixer les règles et le cadre du service avant son éventuelle dévolution au secteur privé. Ces dispositions respectant les règles internationales doivent s'adapter à la spécificité culturelle de chaque Etat et de ses citoyens ce qui impose une certaine souplesse. Il en résulte que le contrat à respecter pour l'opérateur, qu'il soit public ou privé, comporte le respect des principes universels de bonne gestion, notamment l'équilibre recettes/dépenses, y compris celles concernant la sécurité du service et sa durabilité. Il doit s'adapter aux contraintes locales. En particulier le niveau du service doit tenir compte des possibilités financières des usagers et du temps nécessaire pour résorber les retards ${ }^{1055}$.

Dans le cadre de la gestion du service de l'eau, qu'il s'agisse d'une entité spéciale désignée comme telle par le législateur ou une collectivité existante, par exemple une commune, l'opérateur public peut déléguer à un opérateur privé tout ou partie du service, au motif la plupart du temps du trop grand coût à supporter : l'autorité en charge du réseau peut ne pas avoir fait les provisions nécessaires au fonctionnement du service et / ou elle n'est simplement pas compétente dans ce domaine. L'opérateur public garde par contre le contrôle du délégataire notamment pour le prix du service facturé à l'usager et l'application effective des règles formant contrat de service public édictées par le législateur. La mainmise de l'autorité publique assure l'équité du service pour tous et sa permanence auprès de l'usager ${ }^{1056}$.

Dans beaucoup de pays, l'opérateur public de base est la commune et plus particulièrement le Maire, ou un groupement de communes (syndicats intercommunaux par exemple). Le titulaire de l'autorité, élu par ses concitoyens, a un rôle essentiel pour apporter ou garantir aux usagers les moyens modernes indispensables à son confort et ses activités (voirie, énergie, police, soins, loisirs, évacuation des eaux de pluie) et doit avoir un droit de regard sur la desserte en eau, même s'il n'est pas un opérateur public. Ce rôle minimum doit lui être reconnu. Toutes les règles relatives à l'usager, à la protection de la ressource sont les mêmes pour l'opérateur quel qu'il soit. On interdit ainsi tout système de préférences pouvant résulter de la passation de marchés selon le mode du favoritisme. Cela implique que les contrats passés doivent à la fois être encadrés de la manière la plus précise possible lors de leur conclusion, mais aussi pendant leur suivi ${ }^{1057}$.

1054 Académie de l'eau, UNESCO - Colloque du 29 octobre 2002.

1055 Idem.

1056 Idem.

1057 Idem. 
Si, en 1995, 400 millions de personnes vivaient dans des Etats sous stress hydrique ${ }^{1058}$, ils seront 4 milliards en 2025 à connaître cette situation, avec toutes les conséquences sociétales, alimentaires, sanitaires, et économiques qui en résulteront. Majoritairement, pays de l'Afrique subsaharienne, leur indice de développement humain est alarmant ${ }^{1059}$. Prise isolément la question de l'eau se révèle très préoccupante.

Aussi, l'ampleur de la question de l'eau donne-t-elle aux diverses conférences et rencontres une importance croissante. Une importance confirmée par les enjeux juridiques et financiers de la reconnaissance du droit à l'eau.

Selon une déclaration du Conseil des droits de l'Homme des Nations Unies, "l'accès à l'eau potable fait partie de l'accès à la dignité humaine et s'intègre donc dans le cadre des droits de l'homme »1060. L'amélioration de l'accès à l'eau et de sa qualité sont des devoirs moraux. Ils doivent devenir des priorités stratégiques : la problématique de l'accès à l'eau fera indubitablement naitre des tensions dans les ensembles géographiques "à risque ", les plus soumis à la rareté de cette ressource. Il faudra donc saluer l'apport du droit international qui à travers la Convention de New York, pourtant critiquée, tente de contribuer à la résolution des différends liés à l'eau.

Il est donc urgent que des mesures soient prises pour préserver la ressource en eau. En ce sens il faut saluer le principe selon lequel « obligation est faite de ne pas causer de dommages appréciables aux autres États du fait de l'utilisation du cours d'eau par un État ${ }^{1061}$. Enfin, les États ont le devoir de se consulter et d'échanger des informations au sujet des effets éventuels de mesures projetées sur l'état d'un cours d'eau international. L'exercice de la souveraineté d'un État au droit des eaux coulant sur son territoire ne doit pas priver les autres États riverains du même droit car l'eau est une ressource naturelle partagée (Res communis) dans la mesure où son utilisation dans un État à des effets sur son utilisation dans un autre État.

\footnotetext{
1058 Moins de $500 \mathrm{~m}^{3}$ d'eau par habitant et par an

$\mathbf{1 0 5 9}$ Rapport sur le développement humain 2010.

1060 http://www .aidh.org/ONU_GE/conseilddh/08/7-resol-eau.htm

1061 Article 7.
} 
CONCLUSION DE LA IIEME PARTIE. 
L'effectivité du droit à l'eau exige une action d'envergure susceptible de conserver et de protéger les ressources en eau. Ceci explique pourquoi en ce début de millénaire, la protection de l'environnement en général et celle des écosystèmes fluviaux et lacustres en particulier sont devenues des préoccupations réelles tant sur le plan national qu'international.

Malgré la prise de conscience généralisée des problèmes de l'environnement vers les années 1970 et la prolifération des instruments juridiques de portée universelle et régionale qui l'ont suivis, l'état de la biosphère devient de plus en plus catastrophique : diminution considérable de la biodiversité, l'avancement effréné du désert, détérioration de la couche d'ozone et rareté de l'eau. Cette situation déplorable de l'état de la nature au niveau mondial résume la question de l'accès à l'eau et ces difficultés.

La rareté généralisée des ressources en eau douce, leur destruction progressive et leur pollution croissante que l'on constate dans de nombreuses régions du monde, ainsi que l'intrusion graduelle d'activités incompatibles, exigent une intégration de la planification et de la gestion des ressources en eau. Cette opération doit couvrir toutes les étendues d'eau douce interdépendantes, notamment les eaux de surface et les eaux souterraines, et tenir dûment compte des aspects quantitatifs et qualitatifs. Il est nécessaire de reconnaître la dimension multisectorielle de la mise en valeur des ressources en eau dans le contexte du développement socio-économique ainsi que les utilisations multiples de l'eau : approvisionnement et assainissement, agriculture, industrie, urbanisation, hydroélectricité, pisciculture en eau douce, transports, activités de loisirs, gestion des basses terres et autres. Des plans rationnels de mise en valeur des eaux de surface, des eaux souterraines et d'autres sources possibles doivent être appuyés en même temps par des mesures de protection des eaux et de limitation maximale du gaspillage. Il s'agira pour les multinationales, de prendre en compte la gestion intégrée des ressources en eau est fondée sur l'idée que l'eau fait partie intégrante de l'écosystème et constitue une ressource naturelle et un bien social et économique dont la quantité et la qualité déterminent l'affectation. Dans cette optique, les ressources en eau doivent faire l'objet de mesures de protection tenant compte du fonctionnement des écosystèmes aquatiques et de la pérennité de la ressource et visant à satisfaire ou à concilier les besoins en eau aux fins des activités humaines. La protection des ressources en eau peut « souffrir » également des problèmes de corruption. Ceci est souvent dû à la proximité relationnelle qui existe entre certains agents de contrôle ou certains procureurs avec les entreprises délégataires contrôlées. La pratique est remarquable dans les Etats où la corruption des agents de l'Etat est courante ${ }^{1062}$.

Dans la mise en valeur et l'utilisation des ressources en eau, il faut donner la priorité à la satisfaction des besoins fondamentaux et à la protection des écosystèmes. Il faut mettre un coup d'accélérateur sur les initiatives visant la refondation du droit international de l'eau. Aussi, est-il nécessaire de «trouver un équilibre entre l'indépendance des États riverains et leur souveraineté sur les ressources naturelles; un équilibre également entre les États d'amont et les États d'aval mais aussi entre les différentes utilisations de l'eau ». Comme Léon WALRAS ${ }^{1063}$, nous pouvons affirmer que l'État est mieux à même de gérer les services d'intérêt public : "Nous ne saurions, pour notre part, admettre ce parti pris de doter l'individu de toutes les vertus et l'État de tous les défauts. C'est là un abus des principes économiques. S'il est vrai que l'initiative individuelle, stimulée par la libre concurrence, effectue mieux que qui que ce soit les services d'intérêt privé,

1062 FAURE M., L'analyse économique du droit de l'environnement, op.cit, p. 303.

1063 Cité par LE MASNE P., op. cit. 
il l'est également que l'initiative collective, sous le contrôle de la publicité et de la discussion, effectue mieux que n'importe qui les services d'intérêt public »1064.

1064 LE MASNE P., op. cit., p. 7. 
Les chambardements que connaissent l'accès à l'eau et la distribution de l'eau suite à la libéralisation du secteur sont bien résumés dans "la parabole du phare maritime $»^{1065}$. C'est l'histoire d'un économiste anglais d'obédience libérale, qui constata, avec regret, qu'il existait des services qui ne pouvaient faire l'objet de privatisation, comme les phares maritimes. En effet, les phares peuvent être utilisés par tous les marins du monde, qu'ils aient participé ou pas au financement de leurs constructions ou de leur entretien.

Le petit-fils de notre économiste, néolibéral, réfuta cette analyse en se montrant plus radical et déclare que toute chose est « privatisable». Partant toujours de l'exemple du phare, celui-ci affirme qu'avec l'avancée des techniques, on pouvait imaginer un dispositif électronique qui rende inobservables les signaux émis par le phare pour des marins non-équipés d'un décodeur. L'idée fit recette et on ne fournit un décodeur qu'aux marins qui avaient participé au financement des phares. On assista alors, avec la disparition du service public, au naufrage de notre société et de nombreux navires, sous l'œil indifférent du marché. Il y a donc bien des services à rendre qui ne peuvent être satisfaits par la seule logique du marché.

Nous voudrions démontrer par cette anecdote que pour des Etats comme ceux de l'Afrique subsaharienne où la majorité des populations vivent sous le seuil de pauvreté, les choix politiques ne devraient être guidés que par la recherche de l'intérêt général. La non prise en compte de l'intérêt général aboutira inévitablement au drame de l'eau.

Ainsi, comme on le voit, les multinationales n'ont pas participé jusqu'à présent à la construction d'un accès démocratique à l'eau. Leur but, faut-il le rappeler, est de réaliser les profits et non rendre l'eau potable accessible aux pauvres alors que c'est la base même des arguments qu'elles utilisent face aux populations pauvres.

Les Etats ont le devoir de protéger leurs économies locales du marché mondial. Quand il s'agit de garantir la production alimentaire, du droit à la nourriture et du droit à l'eau il faut préférer les marchés locaux communs et régionaux au libre marché mondial.

Dans le passé, au lendemain de l'accession des États africains à la souveraineté internationale, l'État et les collectivités publiques choisissaient librement le mode d'exploitation de leurs services publics. Les «jeunes » États ne disposaient pas encore d'un cadre juridique adéquat à la gestion des services publics. Le choix entre la régie et la délégation de service public relevait de la pure opportunité. Mais de plus en plus, avec l'émergence et l'affirmation des processus démocratiques, ces États se dotent des outils juridiques nécessaires à une exploitation transparente de ces services publics. Au niveau national et au niveau sous-régional ${ }^{1066}$, le législateur se montre très dynamique. A titre illustratif, l'on peut citer la loi portant Code de marchés publics et délégations de service public en République Centrafricaine ${ }^{1067}$ ou le décret portant procédures de passation, d'exécution et de règlement des marchés publics et des

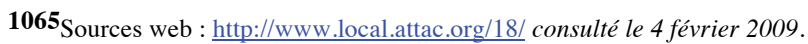

$1066 C f$. la directive $n^{\circ} 04 / 2005 / \mathrm{CM} /$ UEMOA du 09 décembre 2005 portant procédures de passation, d'exécution et de règlement des marchés publics et des délégations de service public dans l'UEMOA et la directive n05/2005/CM/UEMOA du 09 décembre 2005 portant contrôle et régulation des marchés publics et des délégations de service public dans l'UEMOA.
}

${ }^{1067}$ Loi nº8-017 du 6 juin 2008 
délégations de service public au Sénégal ${ }^{1068}$. De même, en France, le juge administratif vérifie par exemple que le refus de déléguer le service public ne constitue pas un abus de position dominante ${ }^{1069}$. Cette option libérale qui semble prévaloir en France fait école dans les États africains du sud du Sahara. La pression de la Banque mondiale et du Fonds monétaire sur les gouvernements de ces États, va les conduire à adopter une politique plutôt de tendance libérale. Celle-ci, de toute évidence, aura des conséquences contradictoires. Sur le plan social, les populations déjà en difficultés verront leur situation s'aggraver. Les infrastructures sociocollectives vont se dégrader. L'accès à l'eau sera compromis, car comme nous l'avons précisé précédemment la libéralisation du secteur de l'eau a des impacts négatifs importants sur la vie des populations africaines. Mais la traduction juridique de ces problèmes est l'effectivité du droit à l'eau.

D'abord le concept a mis du temps à s'imposer. Pendant longtemps, le droit à l'eau était absent du vocabulaire juridique. Il a progressivement émergé avant de s'affirmer le 28 juillet 2010 au niveau international. En effet, c'est au niveau des Nations Unies que vont être engagés des travaux au regard de la reconnaissance du droit à l'eau. Une première résolution, prise par l'Assemblée générale, précise dès 1999 en son article 12 «que le plein respect du droit au développement implique les principes suivants : le droit à la nourriture et à l'eau potable étant un droit fondamental, les gouvernements et la communauté internationale ont l'obligation morale de le promouvoir ». Ce premier acte majeur d'une reconnaissance spécifique va ensuite faire l'objet d'un double développement. Tout d'abord au sein du Haut commissariat aux droits de l'homme, avec notamment la sous-commission de la promotion et de la protection des droits de l'homme. Puis, une résolution de l'année 2000 va recommander à la Commission des droits de l'homme de l'ONU d'adopter une résolution relative à la reconnaissance du droit à l'eau ${ }^{1070}$. D'autre part, au sein du Conseil économique et social de l'ONU, la " gestation » du droit à l'eau va connaître d'avancées significatives. Au cours de la session de novembre 2002, le Comité des droits économiques, sociaux et culturels a adopté une observation générale relative au droit à $l^{\prime} e^{1071}$. Ainsi, pour la première fois, un texte intervenu au plus haut niveau du droit international public, détermine précisément les éléments constitutifs du droit à l'eau. Il aussi important de signaler que les sources du droit à l'eau ne sont pas seulement juridiques. On peut en trouver en dehors des textes juridiques ou toute autre norme. En effet, c'est une démarche qui serait plus ou moins liée à la conception jusnaturaliste du droit. C'est-à-dire l'idée que, comme le soutiennent les tenants de cette thèse, les droits de l'homme sont inhérents à la nature humaine et existent en dehors de l'intervention de la loi. A titre illustratif, l'on peut citer la Déclaration des droits de l'homme et du citoyen ${ }^{1072}$ qui évoque des "droits naturels, inaliénables et sacrés de l'homme». Ce que confirment FIALAIRE J. et MONDIELLI E. lorsqu'ils écrivent que "la Déclaration des droits de l'homme et du citoyen est largement inspirée par la théorie moderne $d u$ droit naturel »1073. Selon TAITHE Alexandre ${ }^{1074}$, le caractère vital de l'eau « en fait une

1068 Décret nº8 - 485 / P- RM du 11 août 2008

1069 CE, 18 mars 1988, n57893, Loupias, Recueil Lebon, T. 975.

1070 ONU, Haut Commissariat aux droits de l'Homme- Sous-commission de la promotion et de la protection des droits de l'homme, Résolution 2000/8, renvoyant au projet de décision E/CN, 4/2001/2 - E/CN, 4/Sub,2/2000/46.

1071 ONU, Conseil économique et social, comité des droits économiques, sociaux et culturels, 22ème session, 1129 novembre 2002.

1072 Déclaration des droits de l'homme et du citoyen du 26 août 1789.

1073 FIALAIRE J. et MONDIELLI E., Droits fondamentaux et libertés publiques, Paris, Ellipses, 2005, p. 54. 
ressource considérée comme sacrée, comme un don de la nature ou de Dieu». Ainsi, des premières vénérations animistes de sources, lacs et cours d'eau aux ablutions pratiquées par les musulmans, le rapport de l'eau à la vie est célébré dans toutes les religions à la fois comme symbole de purification et de renaissance ${ }^{1075}$.

Mais, pour THALES 1076 , ces germes et ces «animalcules» ont fondamentalement une nature aqueuse. Pour l'explication de tout, point n'est besoin de recourir au mystère, il suffit d'observer cette «materia prima» de toutes les idéologies, la substance la plus commune, la plus banale et la plus familière mais, dans le même temps, vitale qu'est l'eau ${ }^{1077}$. En 2003, l'Assemblée générale du Réseau européen chrétien pour l'environnement a exprimé sa conviction que "l'eau est une et nous unit à toute la création" et a déclaré que : "le droit d'accès à l'eau doit être reconnu et promu comme un droit humain fondamental qu'il faut mettre en cuvre par tous les moyens juridiques possibles ». Cette position a été confirmée en $2005^{1078}$. Le Saint Siège avait publié un message selon lequel : "le droit à l'eau est donc un droit inaliénable ». Au sein de la chrétienté, l'eau est utilisée pour plusieurs rites, notamment lors du baptême ou pour les lavements. Dans cette religion, l'eau est symbole de purification et d'assainissement ${ }^{1079}$.

La majorité des religions, croyances, philosophies et civilisations du monde ont valorisé l'eau. Elle est baptismale, bénite, vitale, purificatrice. En réalité, l'eau est l'élément autour duquel se développe l'écheveau de pratiquement toutes les croyances, de l'Egypte ancienne en passant par les animistes et l'Islam, même quand elles évitent les questions liées à la cosmogonie tel le bouddhisme ${ }^{1080}$.

Mais l'eau est aussi symbole de fécondité et le Coran ne manque pas de le rappeler : «Nous faisons descendre du ciel une eau bénie grâce à laquelle nous faisons crô̂tre les jardins ; le grain que l'on moissonne; les palmiers élancés porteurs de régimes bien ordonnés pour nourrir nos serviteurs $»^{1081}$.

En revisitant l'histoire, il apparait que l'eau est indissociable de la condition humaine au même titre que la nourriture et le logement. Les normes qui prévalent dans les sociétés sur les différents continents, y font référence. "Le droit à l'eau est inscrit dans les cultures humaines 》 affirmera B. DROBENKO ${ }^{1082}$. Pour les Chinois, le fonctionnement de l'univers en perpétuel

1074 TAITHE Alexandre, op. cit.

1075 GAITTI Jules, L'eau, mythes et symboliques, Paris, C.I.Eau, coll. Synthèse de l'eau, février 2001.

1076 Thalès de Milet appelé communément Thalès était un philosophe présocratique ionien né à Milet vers -625 et mort vers l'an 547 av. J.-C. Il fut l'un des Sept sages de la Grèce et le fondateur présumé de l'école milésienne.

1077 CONCHE M., «L'eau et les philosophes », Sciences au Sud, Spécial 2003.

1078 Selon le Saint-Siège (2003), le droit à l'eau est un « droit inaliénable ».

1079 Extrait de : www.unesco.org/water/fact figures/valeurs eau.shtml consulté le 25 mars 2007.

1080 LARBI BOUGUERRA M., «Symbolique et culture de l'eau», Les rapports de l'Institut Veolia Environnement $n^{\circ} 5$, p. 6 .

1081 Al-Qor'an , L , 9-11.

1082 DROBENKO B., précité, p.76. 
mouvement s'explique par l'interaction des fameux principes fondamentaux que sont le yin et le yang ainsi que les cinq agents que sont le bois, le feu, la terre, le métal et l'eau. En Afrique subsaharienne comme ailleurs, l'eau est omniprésente dans les croyances, en témoigne par exemple le «Dieu de l'Eau» des Dogon ${ }^{1083}$. Aussi, il y est affirmé que «la force vitale de la terre est l'eau. Dieu a pétri la terre avec de l'eau; de même, il fait du sang avec de l'eau. Même dans la pierre, il y a cette force $» . .$. Les croyances des peuples africains sont très souvent associées à l'eau, aux fleuves, aux lacs, aux puits. Au Ghana ancien ${ }^{1084}$, la légende de Ouagadougou (Wagadougou) parle d'un ancêtre-totem de la famille royale, le dieu Serpent, à qui l'on faisait des sacrifices et qui était le gardien d'un puits et le garant de la fécondité. Son extermination marquera, dit-on, le déclenchement de la sécheresse. Ces premiers épisodes de la vie des peuples sont marqués par le franchissement de fleuves dans des circonstances miraculeuses.... ${ }^{1085}$ Innombrables sont les significations symboliques attachées à l'eau dans les traditions et les cultures du Continent noir. De plus, elle a la capacité de guérir, de rajeunir voire de tuer puisqu'elle est en mesure de « faire sortir la vie de la mort et d'amener la mort sur la vie $»$.

Au bout du compte, c'est la dignité humaine qui est l'institution première et fondamentale des droits de $1^{\prime}$ homme ${ }^{1086}$. Or, dignité et religion sont indissociables, en d'autres termes, la pratique du culte est une composante incontournable de la sauvegarde de la dignité humaine. Aussi, faut-il préciser que l'essor de la promotion des droits fondamentaux a engendré une grande revendication par les sectes religieuses et les églises traditionnelles de la liberté de culte $^{1087}$. Si la liberté de culte est aujourd'hui reconnue dans la plupart des constitutions des Etats de l'Afrique subsaharienne, il est avéré que le droit à cette liberté ne peut être effectif sans l'accès à l'eau. Et comme nous l'avons démontré, l'eau est une référence de base aussi bien dans les pratiques cultuelles que dans les civilisations de par le monde entier.

Mais la proclamation de l'Assemblée générale des Nations Unies n'aura de portée réelle que si d'abord les Etats membres de l'ONU prennent les dispositions indispensables au niveau interne pour traduire ce succès en une réalité. D'autre part, il doit exister une solidarité entre ces Etats, une solidarité qui doit se traduire par le soutien des plus nantis aux moins nantis.

1083 Le peuple dogon est originaire des Monts mandingues, à la frontière guinéo-malienne. Animistes, ils ont refusé de se convertir à l'islam et ont dû s'exiler au XVIIIe siècle, remontant le fleuve Niger jusqu'aux reliefs protecteurs du plateau et de la falaise de Bandiagara.

1084 L'empire du Ghana (de ghana, « roi guerrier »), qui a existé de 750 environ à 1240, a constitué l'un des grands empires d'Afrique noire connus, s'étendant du moyen Sénégal à la région de l'actuelle Tombouctou. Désigné par ses habitants comme l'Empire Wagadou, il se fit connaître en Europe et en Arabie comme l'Empire du Ghana. Le royaume du Ghana s'est constitué au VIIIe siècle avec l'exportation d'or et de sel, important pour la conservation des aliments. Le terme Wagadou signifie "ville des troupeaux"; le mot dou est un terme de la langue Mandé qui signifie "ville" et que l'on retrouve dans nombre de lieux d'Afrique de l'Ouest (telle la capitale du Burkina Faso, Ouagadougou); le mot waga signifie approximativement «troupeau ».

1085 LARBi BOUgUERRA M., Symbolique et culture de l'eau, Les Rapports de l'Institut Veolia $\mathrm{n}^{\circ} 5$ :http://www.institut.veolia.org/fr/cahiers/symbolique-eau/mythes-eau/. Consulté le 12 septembre 2010.

1086 LÖWENTHAL P., «Ambiguïtés des droits de l'homme », Droits fondamentaux, $n^{\circ} 7$, janvier 2008 décembre 2009, p. 2 ; www.droits-fondamentaux.org consulté le 29 juin 2011.

1087 FIAILAIRE J. et MONDIELLI E. , précité, p . 469. 
Plus de vingt ans après le premier rapport sur le développement humain, la brillance conceptuelle et la pertinence de ce paradigme original du concept restent incontestables. Il est maintenant presque universellement admis que le succès d'un pays ou le bien-être d'un individu ne peut être mesuré strictement en termes monétaires. Le revenu est bien entendu crucial : sans ressources, tout progrès est difficile. Mais il est également essentiel de savoir si les gens ont la chance de vivre une vie longue et en bonne santé, s'ils ont ou non accès à une éducation et s'ils sont libres d'utiliser leurs connaissances et leurs talents pour façonner leurs propres destinées $^{1088}$.

L'insuffisance des ressources en eau, du moins en ce qui concerne sa qualité, et la nécessité d'y remédier sont évoquées tout le long de nos travaux. Tout comme le Comité préparatoire du Sommet mondial pour le développement durable l'avait constaté au moment où se préparait le Sommet de Johannesburg, « en matière de développement durable, les engagements existent, seuls font défaut les mécanismes de mise en œuvre $»^{1089}$.

L'affirmation du droit à l'eau et concomitamment le respect de la liberté d'entreprise relèvent d'un talent d'équilibriste. Nous avons vu l'importance de la question de l'accès à l'eau, une préoccupation légitime et vitale qui ne saurait attendre, même si certains auteurs estiment que c'est un droit de seconde génération. De même, la libéralisation du secteur de l'eau répondait à une conjoncture économique. La retarder ou la refuser, relèverait d'une gageure que ne pouvaient risquer les autorités des Etats de l'Afrique subsaharienne.

La complexité de la question du droit à l'eau est une réalité multiforme. Elle touche les lois du service public, la souveraineté permanente sur les ressources naturelles, la protection des ressources en eau et bien d'autres secteurs connexes.

Aussi, faut-il croire en la capacité du juge africain de pouvoir protéger non seulement les ressources en eau mais aussi le droit à l'eau, comme le fait déjà son homologue français considéré à juste titre par Laurent FONBAUSTIER comme "gardien de la ressource en eau ${ }^{1090}$. Il précise ainsi que si les litiges opposant les services publics industriels et commerciaux à leurs usagers sont classiquement de la compétence du juge judiciaire, certains contentieux relèvent néanmoins des juridictions administratives. En effet, selon une solution admise depuis les années $1930^{1091}$ la distribution d'eau est un service public industriel et commercial. Cette capacité du juge africain a été même illustrée au niveau régional. C'est le cas lorsque la République Démocratique du Congo a été condamnée sur plainte d'ONG en 1996 pour violation des droits nouveaux droits, tant économiques et sociaux que civils et politiques. La commission africaine des droits de l'Homme et peuples a estimé que la RDC avait violé l'article 16 de la Charte africaine.

\footnotetext{
1088 Rapport sur le développement humain 2010, Publié pour le Programme des Nations Unies pour le développement.

1089 Quatrième session - 7e séance plénière,

http://www.un.org/News/fr-press/docs/2002/ENVDEV648.doc.htm consulté le 17 mai 2011.

1090 FONBAUSTIER L., précité, p. 1068

1091 CE 6 mai 1931, Tondut, Lebon p. 477
} 
Relativement à la nature juridique de l'eau, la théorie des "res communes » avait prévalu. Elle reposait sur le fait que ces choses étaient si abondantes qu'il n'était pas utile de se les approprier. Mais la fin de l'abondance de la ressource hydraulique étant unanimement constatée, de nombreux Etats ont décidé d'étatiser ou de « domanialiser » l'eau. Il a en effet été constaté que l'effet pervers du statut de chose commune provenait du fait que tandis qu'un propriétaire se sent évidemment concerné par la conservation de son bien, le simple usager veut profiter du bien sans contraintes et limites ${ }^{1092}$.

Le problème se complique dès lors que cette eau, lorsqu'elle est disponible, est commune à plusieurs Etats. En effet, on estime qu'il existe plusieurs bassins fluviaux partagés entre deux ou plusieurs Etats. L'eau devient alors l'objet de convoitises et de débats quant aux règles de son partage et de sa distribution. C'est l'objet du droit international de l'eau que d'établir des processus d'entente et de règlements des différends à propos des eaux partagées entre plusieurs Etats. Les pouvoirs publics sont tenus d'exercer un contrôle sur le service de l'eau et de fixer les obligations découlant du service universel de l'eau. Quel que soit le mode de gestion adopté, les autorités doivent effectuer un contrôle sérieux et effectif sur le service de l'eau. Elles doivent examiner la qualité de l'eau, le degré d'assainissement, la continuité du service, la gestion en cas de pénurie, le contenu des cahiers des charges, la tarification, la participation des usagers et le financement des ouvrages, leur extension et leur réhabilitation. Ils peuvent aussi jouer un rôle utile pour informer les usagers et développer chez eux la volonté de ne pas gaspiller l'eau. En cas de privatisation du service de l'eau, les pouvoirs publics doivent pouvoir continuer de jouer un rôle important.

Il ne s'agit pas de dénoncer le modèle de la privatisation ou de préférer la gestion publique, mais de s'assurer que quelle que la gestion adoptée, que le droit à l'eau soit garanti et effectif. Car si son affirmation a été l'objet de nombreuses polémiques, son effectivité pourra également en pâtir.

S'il devient effectif, le droit à l'eau peut-il faire l'objet d'une appropriation ? La question mérite qu'on s'y attarde un peu en partant de l'exemple des eaux souterraines. En effet, les eaux souterraines sont en transit dans le sol. Au cours de tout laps de temps, une certaine quantité d'eau souterraine quitte un terrain tandis qu'une autre quantité y pénètre. Le droit de propriété ne s'exerce donc jamais sur les mêmes eaux souterraines. Le droit ne dispose pas d'instrument adéquat pour gérer de façon cohérente le passage d'un patrimoine à l'autre lorsque l'eau souterraine passe d'un terrain à l'autre. Cette migration soulève des problèmes regroupés sous deux aspects interdépendants. D'une part, l'entrée de l'eau souterraine dans le patrimoine d'un propriétaire lors de sa migration dans le sol qu'il possède et d'autre part, la sortie de l'eau souterraine du patrimoine d'un propriétaire lors de sa migration hors du sol qu'il possède. Sur le premier point, c'est-à-dire l'entrée de l'eau souterraine dans le patrimoine, la ressource peut $a$ priori être acquise par contrat, par succession, par occupation, par prescription, ou par accession ${ }^{1093}$. L'application de chacun de ces modes d'acquisition se butte à d'importants

1092 BRAMOULLE G., «L'appropriation des biens environnementaux », in Droit et environnement, précité note 58 , p. 58 citant ARISTOTE : « ce qui appartient à tout un chacun est le plus négligé, car tout individu prend le plus grand soin de ce qui lui appartient en propre, quitte à négliger ce qu'il possède en commun » La Politique, Livre II, chap. 3 .

1093 LAFOND P-C., Précis de droit des biens, Montréal, Éditions Thémis, 1999, p.987-988. 
obstacles $^{1094}$. Deuxièmement, l'occupation n'est possible que dans le cas des biens meubles sans propriétaire et elle implique la possession. Or, l'eau souterraine est généralement un immeuble approprié qui échappe à la possession du propriétaire du terrain tant qu'elle n'est pas captée ${ }^{1095}$. L'occupation ne permet donc pas l'acquisition de la ressource, à moins d'accepter une modification de la prémisse de départ selon laquelle la propriété du sol entraîne la propriété de l'eau souterraine.

Sur la question de la tarification de l'eau, le tabou portant sur la question de la gratuité a été brisé par l'Afrique du Sud. En effet, depuis février 2001, le Gouvernement sud-africain a changé de politique de l'eau et a décidé que l'eau « de base » serait fournie gratuitement en priorité à tous les pauvres et éventuellement à tout le monde. Depuis juillet 2001, chaque ménage devrait recevoir 200 litres d'eau gratuite par jour (6000 litres par mois correspondant à 25 litres pour huit personnes). L'effet de ce changement de politique a été d'augmenter le taux d'impayés, les ménages croyant que la nouvelle politique s'appliquait tout de suite à la totalité de l'eau distribuée ou à toutes les municipalités.

La régulation internationale des activités du secteur privé dans le domaine de la distribution d'eau potable est certainement l'un des enjeux de gestion de l'eau qui suscitent les débats les plus vifs. Elle est en effet très polarisée idéologiquement en même temps qu'épineuse du point de vue juridique. La demande de régulation globale est à l'évidence très forte et se trouve justifiée par la libéralisation des services d'eau dans le contexte de la mondialisation économique, par le caractère transnational des entreprises leaders dans ce domaine, et par une protection d'intérêts environnementaux et sociaux qui bénéficie à toutes les communautés humaines. A l'autre bout du spectre, le droit international, élaboré par et pour les États, paraît impuissant à répondre à ces attentes, à se saisir d'une question qui met en lumière le décalage existant entre la complexification des échanges transnationaux, la multiplication et l'interdépendance des activités et des acteurs, et son caractère intrinsèquement interétatique. L'évolution de l'implication du secteur privé transnational dans ce type de services pourrait laisser croire que le débat n'est qu'une tempête dans un verre d'eau ${ }^{1096}$.

L'on doit retenir que partout dans le monde, les ressources en eau, vitales, constituent un sujet d'inquiétude, justifiant le besoin urgent d'améliorer les politiques de gestion de l'eau. Il est impératif que les décideurs fassent en sorte que les ressources en eau soient utilisées pour assurer la survie et le développement économique des populations les plus démunies. Il est urgent de préciser que la libéralisation du comme et particulièrement celle du secteur de l'eau doit être gérée avec le plus grand soin dans le cadre de stratégies globales de développement qui englobent la santé, l'éducation, l'autonomisation des femmes, et le renforcement de l'Etat de droit $^{1097}$.

1094 Le contrat et la succession ne sont pas des modes d'acquisition pertinents pour appréhender la migration progressive de l'eau souterraine dans le sol.

1095 BANTON O., « Le capital eau : son potentiel et ses usages. Les eaux souterraines», 45, p.47, dans Jean-Pierre VILLENEUVE, Alain ROUSSEAU Sophie DUCHESNE, (dir)., Symposium sur la gestion de l'eau au Québec. Actes du Symposium. L'état de l'eau au Québec, Sainte-Foy, INRS-Eau, 1998 (Vol.2).

1096 RICHARD V., «Gestion privée de l'eau : où va le droit international ? » in Études et documents du Conseil d'État, p. 515.

1097 WEBER L., OMC, AGCS, Vers la privatisation de la société, Syllepse, 2003, p. 41. 
Au-delà de défis alimentaire et sanitaire et des solutions techniques envisagées, il s'agit de redéfinir des compromis sociaux qui permettront une gestion durable et équitable de la ressource. Les tensions suscitées par la commercialisation et la privatisation croissantes de l'eau révèlent un déficit d'intérêts commun, de valeurs communes aux utilisateurs. Donner un sens, créer un compromis, c'est ainsi conférer une nouvelle légitimité à des politiques qui vont établir des priorités dans les usages et des solidarités financières. Car la recherche d'un intérêt commun s'impose pour prévenir les tensions entre usagers urbains et agricoles, les conflits urbains et les contradictions issues de la reconnaissance progressive d'un droit de l'homme à l'eau ${ }^{1098}$.

En partant de l'hypothèse que le droit à l'eau peut s'inscrire sans discussion dans la catégorie des «droits-créances », on comprend que leur garantie implique nécessairement l'intervention d'aménagements destinés à concrétiser les exigences posées. C'est là la particularité principale de ces droits mais également, de manière consubstantielle, leur faiblesse originelle et inéluctable, qu'une comparaison générale avec la situation des « droits-libertés » illustre clairement. En effet, alors que les libertés, pour être satisfaites, ne sont censées réclamer des pouvoirs publics aucune intervention positive, l'individu pouvant en principe les exercer immédiatement du fait de sa seule autonomie, les " droits-créances » ne peuvent quant à eux offrir la perspective d'une jouissance immédiate. Leur définition même suppose en effet l'intervention de l'Etat. Leur satisfaction ne peut donc être aussi spontanée, à ce constat s'ajoutant celui selon lequel cette concrétisation doit être opérée par le débiteur même à l'encontre duquel la créance va s'exercer. Certains auteurs à l'instar de RIVERO J. et MOUTOUH H., estiment dès lors que les « droitscréances » sont marqués, sur le plan théorique, du sceau de la virtualité1099. Ces exigences dépendent par ailleurs toujours du niveau de développement économique et social. Mais quand on a les moyens pour s'acheter des armes destructrices, peut-on sincèrement se cacher derrière les difficultés économiques, pour s'extirper de l'obligation de fournir ou de mettre à portée, de l'eau à la population? Sans relancer le débat, on peut dire que l'inertie du Pouvoir, volontaire et/ou liée à des considérations matérielles, risquerait ainsi de laisser ces exigences à l'état de droits « mort-nés ».

Il est donc nécessaire de " mettre en place des mécanismes financiers et institutionnels qui permettent la maintenance et le remplacement des infrastructures et qui incitent à l'économie d'eau par une gestion fondée sur l'équité, la vérité des coûts et la transparence en matière de tarification, dans une démarche qui associe pouvoirs publics et usagers $»^{1100}$.

\footnotetext{
1098 TAITHE A., L'eau. Un bien? Un droit? Tensions et opportunités, Unicomm, 2008, p. 94

1099 RIVERO J. et MOUTOUH H., Libertés publiques, Tome I, PUF, $9^{\text {ème }}$ éd., 2003, p. 90.

1100 Cadre d'intervention sectoriel Eau et assainissement 2010-2012 de l'AFD, p8.
} 


\section{OUVRAGES GENERAUX}

1. A UCOC L., Conférences sur l'administration et le droit administratif, 3 vol., $3^{\text {ème }}$ édition, Dunod, 1885-1886, $866 \mathrm{p}$.

2. ABIKHZER F., La notion juridique d'humanité, Aix-en-Provence, Presses universitaires d'Aix-Marseille, 2005, tome I, p. 19 et suiv.

3. ADOUKI D.E., Droit international public, Tome 1, Paris, l'Harmattan, 2002, 336p.

4. ALLAND D., RIALS St. (dir.), Dictionnaire de la culture juridique, PUF, 2007, 1649 p.

5. ARDANT P., Institutions politiques et droit constitutionnel, $9^{\text {ème }}$ édition, Paris, LGDJ, $1997,608 \mathrm{p}$.

6. ARNAUD A.-J. (dir.), Dictionnaire encyclopédique de théorie et de sociologie du droit, LGDJ, $2^{\text {ème }}$ édition., 1993, $758 \mathrm{p}$.

7. ARTAUD A., Pour en finir avec le jugement de Dieu, Euvres Complètes, Gallimard, tome XIII, p. 104.

8. AUBERT J-L., Introduction au droit et thèmes fondamentaux du droit civil, Paris, Armand Colin, 2002, 342 p.

9. AUBIN E., Droit de l'aide et de l'action sociales, Gualino, $2^{\text {ème }}$ éd., 2008, 291p.

10. AUBY J.-F. et RAYMUNDIE O., Le service public, Le Moniteur, 2003, 563 p.

11. AUBY J-B. et DUTHEIL DE LA ROCHERE J. (dir.), Droit administratif européen, Bruylant 2007, $1017 \mathrm{p}$.

12. AUBY J-M. et DRAGO R., Traité $d u$ contentieux administratif, 2 vol. $3^{\text {ème }}$ édition LGDJ, 1984.

13. BATTIFOL H., La philosophie du droit, PUF, Que sais-je ?, 2000, 127 p.

14. BEAU M., L'art de la thèse, Paris, La découverte, 2003, 196p.

15. BENOÎT F-P., Le droit administratif français, Dalloz, 1968, 898 p.

16. BEQUET L. (dir.), Répertoire de droit administratif, 28 vol., Dupont, 1882-1911.

17. BERTHELEMY H., Traité élémentaire de droit administratif, $13^{\mathrm{eme}}$ édition, Rousseau, 1933 , $1016 \mathrm{p}$.

18. BERTHIAU D., Droit de la santé, Gualino éditeur, 2007, 273 p.

19. BETTINGER C., LE CHATELIER G., Les nouveaux enjeux de la concession, Paris, EFE, 1995, 496 p.

20. BON P., Dictionnaire constitutionnel, PUF, 1992, p.582.

21. BONNARD R., Précis de droit administratif, $4^{\text {ème }}$ édition, LGDJ, 1943, 786 p.

22. BORGETTO M. et LAFORE R., Droit de l'aide et de l'action sociale, Montchrestien, $6^{\text {ème }}$ édition, 2006, $687 \mathrm{p}$.

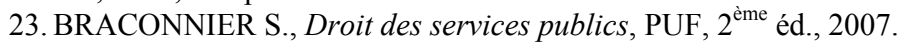

24. BRACONNIER S., Précis du droit des marchés publics, Le Moniteur, $3^{\text {ème }}$ édition, 2009.

25. BRAUD P., Sociologie politique, L.G.D.J., 7ème éd., 2004.

26. BREILLAT D., La hiérarchie des droits de l'homme, Mélanges ARDANT, LGDJ, 1999

27. BREILLAT D., Libertés publiques et droits de la personne humaine, Gualino, 2003.

28. BUENGERTHAL T. et KISS A., La protection internationale des droits de l'homme. Précis, Strasbourg/Arlington, N.P. Engel /Kehl, 1991, 261p.

29. BURDEAU G., Constitution, droits de l'homme et changement, dans l'ouvrage collectif Scritti in onore di Vzio Crisafulli, 2 vol., Padoue, CED, 1985.

30. BURDEAU G., Les libertés publiques, L.G.D.J, $3^{\text {ème }}$ éd., 1966.

31. CABRILLAC R., FRISON-ROCHE M.-A. et REVET T. (dir.), Libertés et droits fondamentaux, Dalloz, $14^{\text {ème }}$ éd., 2008, p. 490.

32. CAILLOSSE J., La constitution imaginaire de l'administration, PUF, 2008, 421p.

33. CAMBY J-P., Les libertés économiques, sous la direction de DRAGO G. et LOMBARD

M., Paris, Editions Panthéon-Assas, LGDJ, 2003, p. 25. 
34. CARBONNIER J., Flexible droit, pour une sociologie du droit sans rigueur, LGDJ, 2001, $10^{\text {ème }}$ édition. $493 \mathrm{p}$.

35. CATSIAPIS J., Guide du droit administratif, Ellipses, 2009.

36. CAUDAL S. (dir.), Les principes en droit, Economica, 2008, 384 p.

37. CHAGNOLLAUD D. et DRAGO G. (dir.), Dictionnaire des droits fondamentaux, Dalloz, 2006.

38. CHAMPEIL-DESPLATS V. et LOCHAK D., A la recherche de l'effectivité des droits de l'homme, Presses universitaires de Paris $\mathrm{X}, 2008,265$ p.

39. CHAPUS R., Droit administratif général, Tome 1 et 2, Montchrestien, $15^{\text {ème }}$ éd., 2001, 1427

p.

40. CHAPUS R., Droit du contentieux administratif, Montchrestien, $12^{\text {ème }}$ éd., 2006, $1560 \mathrm{p}$.

41. CHARDEAUX M.-A., Les choses communes, LGDJ, 2006, 487 p.

42. CHARVIN R., SUEUR J.-J., Droits de l'homme et liberté de la personne, $5^{\text {ème }}$ édition, Paris, Litec, 2007.

43. CHEROT J.-Y., Droit public économique, Economica, 2002, 712 p.

44. CHEVALIER J-M. et autres, L'idée de service public est-elle encore soutenable? PUF, 1999.

45. CHEVALLIER J. et LOCHAK D., Traité de science administrative, L.G.D.J., 1978.

46. CHEVALLIER J., Le service public, PUF, Collec. « Que sais-je ? », 2010, 127 p.

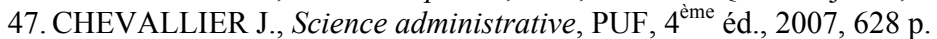

48. CLATRES P., La société contre l'Etat, Les éditions de Minuit, coll. Critique, 1974, 187 p.

49. CLUZEL-METAYER L., Le service public et l'exigence de qualité, Dalloz, Collection «La nouvelle Bibliothèque de Thèses", Paris, 2006, 634 p.

50. COLIN F., Droit public économique, Gualino, 2008.

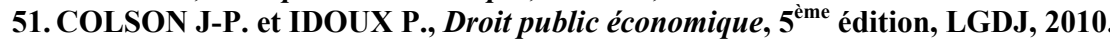

52. COMBACAU, J., Droit international public, $6^{\text {ème }}$ éd., Paris, Montchrestien, 2004, 809 p.

53. CORNU G. (dir.), Vocabulaire juridique, PUF, $8^{\text {ème }}$ édition, 2007, 986 p.

54. DAILLIER P. et PELLET A., Droit international public, $7^{\text {ème }}$ édition, 2002, 1510 p.

55. DAILLIER P., PELLET A., Droit international public, LGDJ, $7^{\text {ème }}$ édition., 2002, 1510 p.

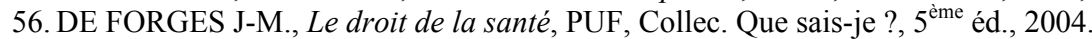

57. DE FOURVILLE O., Les procédures thématiques : une contribution efficace des Nations Unies à la protection des Droits de l'homme, Paris, Pédone, 1996, 139 p.

58. de GAUDUSSON J., L'usager du service public administratif, Paris, LGDJ, Collection «Bibliothèque de droit public », tome 115, 1974.

59. DE LAUBADERE A. et VENEZIA J.-C., Traité de droit administratif, Tome 3, $6^{\text {ème }}$ éd., L.G.D.J, 1997, 495 p.

60. De LAUBADERE A., Traité élémentaire de droit administratif, $1^{\text {ère }}$ édition, LGDJ, 1953.

61. DEBBASCH C. et COLIN F., Droit administratif, $9^{\text {ème }}$ édition, Economica, 2010, 706 p.

62. DEBBASCH C. et RICCI J.-C., Contentieux administratif, Dalloz, $7^{\text {ème }}$ éd., 2001.

63. DELAIRE Y., La délégation des services publics locaux, Berger-Levrault, 2008, 431 p.

64. DELVOLVE P., Droit Public de l'économie, Dalloz, 1998, 799 p.

65. DENQUEN J-M., Sur les conflits de libertés, Mélanges CHARLIER, 1981.

66. DIJON X., Droit naturel: Les questions du droit, Tome I, PUF, 1998, 618 p.

67. Du MARAIS B., Droit public de la régulation économique, Dalloz, coll. « Amphi», 2004, $602 \mathrm{p}$.

68. DUBOIS M.J.M., La rédaction scientifique, Estem, Belgique, 2005, 117p.

69. DUGUIT L., Les transformations du droit public, Armand Colin, 1913, 281p.

70. DUGUIT L., Traité de droit constitutionnel, Paris, Librairie de Boccard, $3^{\text {ème }}$ édition., tome $3,1930,856 \mathrm{p}$.

71. DUPRE de BOULOIS X., Droits et libertés fondamentaux, Paris, PUF, 2010, 302 p. 
72. DUPUIS G., GUEDON M.-J. et CHRETIEN P., Droit administratif, Sirey, 2009, 475 p.

73. DUPUY P-M., Droit international public, Dalloz, 2000, 731p.

74. ECKERT G., Droit public des affaires, Montchrestien, 2001

75. EISENMANN C., Centralisation et décentralisation, Paris, LGDJ, 1948, 330 p.

76. EISENMANN C., Cours de droit administratif, 2 vol., LGDJ, 1982-1983.

77. ERGEC R., Protection européenne et internationale des droits de l'homme, Bruxelles, Bruylant, 2004, 396 p.

78. ESMEIN A., Eléments de droit constitutionnel français et comparé, tome 1, Paris, Sirey, 1927 , p. 600.

79. ESPLUGAS P., Le service public, Connaissance du droit, Dalloz, $2^{\text {ème }}$ éd., 2002.

80. FAVOREU L., PHILIP L., Les grandes décisions du Conseil constitutionnel, Dalloz, 2005, 13e éd., $1065 \mathrm{p}$.

81. FEUER G. et CASSAN H., Droit international du développement, Précis Dalloz, 1991, p. 30.

82. FIAILAIRE J., Le droit des services publics locaux, LGDJ, Systèmes, 1998, 190 p.

83. FOILLARD Ph., Droit administratif, Paradigme, 2011, 434 p.

84. FOULETIER M., L'Equité et le droit public, LGDJ, Bibliothèque de droit public, t.229, 2002.

85. FRIER P.-L., Précis de droit administratif, Montchrestien, 2003, p. 227.

86. GAUDEMET Y., Droit administratif des biens, $13^{\text {ème }}$ édition, LGDJ, 2007, 619 p.

87. GODFRIN P. et M. DEGOFFE, Droit administratif des biens, 7e éd., Colin, 2005, 507 p.

88. GOHIN O., Institutions administratives, LGDJ, $5^{\text {ème }}$ édition, 2006, 854 p.

89. GONOD P. (dir.), Traité de droit administratif, tome 2, Dalloz, 2011, 711 p.

90. GOUNELLE M., Relations internationales, Dalloz, Collection 《Mementos $\gg, 6^{\text {ème }}$ édition, 2004, p.57.

91. GRAWITZ M., Méthodes des sciences sociales, Dalloz, $11^{\text {ème }}$ édition, France, 2001, 1019p.

92. GUETTIER C., Droits des contrats administratifs, Thémis Droit, PUF, 2011, 640 p.

93. GUETTIER C., Institutions administratives, Dalloz, $4^{\text {ème }}$ éd., 2008.

94. GUGLIELMI G., KOUBI G. et DUMONT G., Droit $d u$ service public, Domat, Montchrestien, $2^{\text {ème }}$ éd., 2007, $661 \mathrm{p}$.

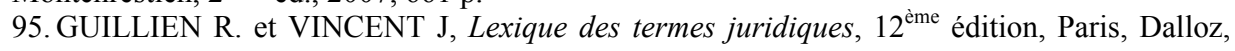
1999, 561p.

96. HAURIOU M., Notes d'arrêts sur les décisions du Conseil d'Etat et du Tribunal des conflits publiés au Recueil Sirey de 1792 à 1929, 3 vol., Sirey, 1929, réimprimé à La Mémoire du droit, 2000.

97. HAURIOU M., Précis de droit administratif et de droit public général, Paris, $4^{\text {ème }}$ édition., 1901, $896 \mathrm{p}$.

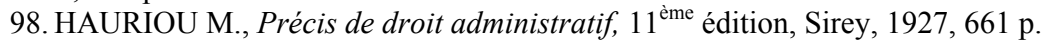

99. HUBRECHT H-G, Droit public économique, Dalloz, 1997, 320 p.

100. JEZE G., Les principes généraux du droit administratif, 6 vol. Giard, 1925-1936, réimprimé aux éditions Dalloz, vol. 2, 2004 ; vol. 1, 2005 et vol. 3, 2011.

101. KOUBI G. et autres, La gratuité une question de droit, l'Harmattan, 2003, 238 p.

102. LACHAUME J.-F., BOITEAU C. et PAULIAT H., Droit des services publics, $3^{\text {ème }}$ édition, Paris, Armand Colin, 2004.

103. LACHAUME J-F. (dir.), Droit administratif. Les grandes décisions de la jurisprudence, $15^{\text {ème }}$ édition, PUF, 2010, $1025 \mathrm{p}$.

104. LAMARCHE L., Perspectives occidentales du droit international des droits économiques de la personne, Bruxelles, Bruylant, 1995, pp. 443-444.

105. LEBRETON G., Liberté publiques et droits de l'homme, Paris, Sirey : Dalloz, 2009.

106. LESAGE M, Les interventions du législateur dans le fonctionnement de la justice : 
contribution à l'étude du principe de séparation des pouvoirs, Paris, L.G.D.J., 1960, 338 p. 107. LIGNIERES P., Partenariats Publics Privés, Litec, 2005, 440 p.

108. LINOTTE D., Droit public économique, Dalloz, $6^{\text {ème }}$ édition, 2006, 456 p.

109. LOMBARD M. et DUMONT G., Droit administratif, $9^{\text {ème }}$ édition, Dalloz, 2011, 646 p.

110. LONG M. (dir.), Les grands arrêts de la jurisprudence administrative, $18^{\text {ème }}$ édition, Dalloz, 2011, $1010 \mathrm{p}$.

111. MADIOT Y., Droits de l'Homme, Paris, Masson, 1990, $230 \mathrm{p}$.

112. MALLET-BRICOUT B., Droit des biens, Recueil Dalloz, 2007 p. 2490.

113. MAMONTOFF C., Domaine public et entreprises privées. La domanialité publique mise en péril par le marché, L'Harmattan, 2003, 504 p.

114. MARTENS P., La dignité humaine : bonne à tout faire des cours constitutionnelles? in Justice, éthique et dignité, textes réunis par GABORIAU S. et PAULIAT H., PULIM, pp. 143-158.

115. MATHIEU B. et VERPAUX M., Contentieux constitutionnel des droits fondamentaux, LGDJ, 2002, 808 p.

116. MATHIEU B., Le droit à la vie dans les jurisprudences constitutionnelles et conventionnelles européennes, Strasbourg, Ed. du Conseil de l'Europe, 2005, 120 p.

117. MATHIEU-IZORCHE M.-L., Droit civil. Les Biens, Sirey, 2006, $\mathbf{n}^{\circ} 246$ s.

118. MAURIZE M-O., Au delà de l'Etat. Le droit international et la défense des droits de l'homme, Paris, Amnesty International, 1992, 373 p.

119. MELIN-SOUCRAMANIEN F., Le principe d'égalité dans la jurisprudence du Conseil constitutionnel, Economica, 1997.

120. MENY Y., Dictionnaire constitutionnel, PUF, Paris, 1992, p. 337-340.

121. MESCHERIAKOFFA-S., Droit des services publics, PUF, 1997, p.175.

122. MICHAUD E., A la recherche de l'effectivité des droits de l'homme ", Paris, Presses Universitaires de Paris 10, 2008, p. 231.

123. MORAND-DEVILLER J., Cours de droit administratif, 12 ${ }^{\text {ème }}$, Montchrestien, 2011, 943 p.

124. MORANGE J., Manuel des droits de l'homme et libertés publiques, PUF, 2007, 278 p.

125. MOREAU J., Droit administratif, Droit fondamental, PUF, 1989, 569 p.

126. MORVAN P., Droit de la protection sociale, Litec, 3ème éd, 2007, 769 p.

127. MOULIN R. et BRUNET P., Droit public des interventions économiques, LGDJ, 2007, 327 p.

128. MOURGEON J., Les droits de l'homme, PUF, Collec. Que sais-je ?, $8^{\text {ème }}$ édition, 2003.

129. NGONDANKOY N.., Droits Congolais des droits de l'homme, éd. Academia Bruylant, coll. Bibliothèque de droit africain, Bruxelles, 2004, $489 \mathrm{p}$.

130. NGUYEN Q. D., DAILLER P., PELLET A., Droit international public, LGDJ, 2002, 1510 p.

131. NICINSKI S., Droit public des affaires, Montchrestien, $2^{\text {ème }}$ édition, 2010,720 p.

132. NICINSKI S., L'usager du SPIC, l'Harmattan, 2001.

133. OBERDORFF H., Droits de l'homme et libertés fondamentales, L.G.D.J., 2010, 540 p.

134. PECES-BARBA MARTINEZ G., Théorie générale des droits fondamentaux, traduction de I.A. PELE, Paris, LGDJ, 2004, 497 p.

135. PELLET A. et COT J-P., la Charte des Nations Unies Commentaire article par article, Paris/ Bruxelles, Economica/ Brusfort, 1985, pp 1009-1026.

136. PERROT R., Institutions judiciaires, Montchrestien, $13^{\text {ème }}$ éd., 2008, 506 p.

137. PLATON S., La coexistence des droits fondamentaux constitutionnels et européens dans l'ordre juridique français, LGDJ, 2008, 716 p.

138. PONTHOREAU Marie-Claire, "Constitutions, juges constitutionnels et droit à l'environnement " in L'environnement à quel prix ? sous la direction de MACKAAY Ejan et Hélène TRUDEAU, Les Editions Thémis, Université de Montréal, p.320. 
139. PONTIER J.-M., Droits fondamentaux et libertés publiques, Hachette, $2^{\text {ème }}$ éd., 2009, 158 p.

140. PONTIER J.-M., Les services publics, Hachette, 1996, 160 p.

141. RICHIER L., Droit des contrats administratifs, LGDJ, $7^{\text {ème }}$ édition, 2010, 713 p.

142. RIVERO J., Droit administratif, 1 ère édition, Dalloz, 1960, réimprimé 2011.

143. ROMAN D., Le droit public face à la pauvreté, L.G.D.J, 2002, 494 p.

144. ROSANVALLON P., 1992 (édition originale 1981), La crise de l'État-providence, Paris : Seuil, 184p.

145. ROSENBERG D., Le principe de souveraineté des États sur leurs ressources naturelles, Paris, L.G.D.J., 1983, 395 p.

146. ROUAULT M-C, BARBE V., et CANEL G., Droit administratif, Gualino Lextenso Editions, 2010,186 p.

147. ROUGET D., Le guide de la protection internationale des droits de l'Homme, éd. La Pensée Sauvage, Agir ensemble pour les Droits de l'Homme, Dijon, 2000, p.57.

148. ROUSSEAU D. et SUDRE F. (dir.), Conseil constitutionnel et Cour européenne des droits de l'homme, Actes du colloque de Montpellier des 20 et 21 janvier 1989, Ed. STH, 1990.

149. ROUSSEAU D., Droit constitutionnel, Montchrestien, 2004, 480 p.

150. ROUSSILlON H., Le Conseil Constitutionnel, Dalloz, Connaissances du droit, $6^{\text {ème }}$ éd., 2008, $145 \mathrm{p}$.

151. SAINT-JAMES V., La conciliation des droits de l'homme et des libertés en droit public français, PUF, 1995, 503 p.

152. SALMON J. (dir.), Dictionnaire de droit international public, Bruxelles, Bruylant, 2001, $1198 \mathrm{p}$.

153. SALMON J., Dictionnaire de droit international public, Bruxelles, Bruylant, 2001, pp 411-412.

154. SANTULLI C., Le statut international de l'ordre juridique étatique, Paris, 2001, p. 102.

155. SEILLER B., Droit administratif, 2 vol., $3^{\text {ème }}$ édition, Flammarion, 2010, 356 p.

156. SERIAUX A., Le droit naturel, PUF, Que sais-je, 1993, 128 p.

157. SUDRE F., Droit international et européen des droits de l'Homme, Paris, PUF, 2006, 786 p.

158. TCHIKAYA B., Mémento de la jurisprudence du droit international public, Les fondamentaux, $3^{\text {ème }}$ édition, Hachette, Paris, 2005,160 p.

159. TERRE D., Les questions morales du droit, Paris, PUF, Collection Ethique et philosophie morale, 2007, $354 \mathrm{p}$.

160. THAURY M., Leçons de droit public des activités économiques, Ellipses, 2011, 280 p.

161. TRAORE S., L'usager du service public, LGDJ, 2012, 212 p.

162. TRUCHET D, Droit administratif, $2^{\mathrm{ème}}$ édition, Thémis Droit PUF, 2011, $470 \mathrm{p}$.

163. TURPIN D., Droit constitutionnel, PUF, 2007

164. VALETTE J-P, Le service public à la française, Ellipses, 2000, p. 91-92.

165. VAN LANG A., Droit de l'environnement, $3^{\text {ème }}$ édition, Thémis Droit PUF, 2011, 520 p.

166. VASAK K, Les dimensions universelles des Droits de l'Homme, vol. 1, Bruxelles, Bruylant, 1990, 318 p.

167. VEDEL G. et DELVOLVE P., Droit administratif, 2 vol., $12^{\text {ème }}$ édition, PUF, 1992, 759 p.

168. VIALA A., Droits fondamentaux, in CHAGNOLLAUD et DRAGO G., Dictionnaire des droits fondamentaux, Paris, Dalloz, 2006, 751 p.

169. VLACHOS G., Principes généraux du droit administratif, Ellipses, 2000, 432 p.

170. WALINE J., Droit administratif, $22^{\text {ème }}$ édition, Dalloz, 2008, $702 \mathrm{p}$.

171. WALINE M., Droit administratif, Sirey, $8^{\text {ème }}$ édition, 1959, 932 p.

172. WALINE M., Manuel élémentaire de droit administratif, Sirey, $4^{\text {ème }}$ édition, 1946, 556 p.

173. WALINE M., Notes d'arrêts, tome 1, Dalloz, 2004, 852 p. 
174. WALINE M., Notes d'arrêts, tome 2, Dalloz, 2005, 742 p.

175. WALINE M., Notes d'arrêts, tome 3, Dalloz, 2006, 607 p.

\section{OUVRAGES SPECIALISES}

1. ALFANDARY E., Droit de l'eau : gestion et protection, Paris, MB Edition, 2003, 71 p.

2. BARLOW M. et CLARKE T., L'or bleu : l'eau, le grand enjeu du XXIe siècle, Fayard, 2002, $390 \mathrm{p}$.

3. BELAIDI N. et EUZEN A., De la chose commune au patrimoine commun. Regards croisés sur les valeurs sociales de l'accès à l'eau, De Boeck Université, Monde en développement, 2009/01 - n ${ }^{\circ} 145$, pages 55 à 72.

4. BERTHIAU D., Droit de la santé, Gualino éditeur, 2007, p. 18.

5. BOISSON de CHAZOURNES et SALMAN, Les ressources en eau et le droit international /Water resources and international law, Académie de droit international, 2005, 798 p.

6. BOISSON de CHAZOURNES L., DESGANE R., ROMANO C., Protection internationale de l'environnement, Recueil d'instruments juridiques, Paris, Pedone, 1998, 1117p.

7. BORDONNEAU M.-A., Regard juridique sur la double nature de l'eau, Ed. Johanet, 2009, 902 p.

8. BOSCO H., TANAWA E. et NGNIKAM E., L'eau potable au Cameroun. Approvisionnement en eau potable, Tome I, Yaounde, PUY, 2001, 359p.

9. BOURDIN J., Les finances des services publics de l'eau et de l'assainissement, Paris, Economica, 2007, 190 p.

10. BROWN-WEISS E., In Fairness to future Generations : International Law, Common Patrimony and Intergenerational Equity, New York, Transnational Publishers, 1998.

11. BRUNEL S., Les risques et les paradoxes de l'eau en Afrique, Conférence/Festival de géographie de Saint-Dié, 2003.

12. BURKE J., MARCUS M. et SAUVEPLANE C, «Groundwater and Society: Problems in Variability and Points of Engagement», 31, dans Salman Salman, dir., Groundwater : legal and policy perspectives, Washington, World Bank, 1999, p.38-41,

13. CANS R., La bataille de l'eau, Paris, Le Monde Editions, 1994, 219 p.

14. CANS R., La ruée vers l'eau, Paris, Editions Gallimard, 2001, 224 p.

15. CAPONERA D. A., Les principes du droit et de l'administration des eaux, droit interne et droit international, Paris, Editions Johanet, 2000, 348 p.

16. COHRE, AAAS, DDC et UN-HABITAT, Manuel de droit à l'eau et l'assainissement, un outil pour aider les décideurs et praticiens à développer des stratégies de mise en æuvre du droit de l'homme à l'eau et l'assainissement, Genève, 2008, 302p.

17. COMPAGNON Daniel, CONSTANTIN François, Administrer l'environnement en Afrique, Paris, Karthala-IFRA, 2000, 494 p.

18. CONAC F., CONAC G. (Dir.), La terre, l'eau et le droit en Afrique, à Madagascar et à l'Ile Maurice, Bruxelles, Bruylant, 1998, 759 p.

19. CONAC G., «Des politiques de l'eau aux droits de l'eau en Afrique, à Madagascar et à l'Ile Maurice : bilan et esprit d'une recherche ", in CONAC (F.), CONAC (G.) (Dir.), La terre, l'eau et le droit en Afrique, à Madagascar et à l'Ile Maurice, Bruxelles, Bruylant, 1998, 759 p.

20. DAOUDY M., Le partage des eaux entre la Syrie, l'Irak et la Turquie - Négociations, sécurité et asymétrie des pouvoirs, CNRS édition, 2005, $269 \mathrm{p}$.

21. DESIDERI J.-P. (dir.), Droit de l'environnement, Sup'Foucher, 2010, 287 p.

22. DONZIER J.F., La réforme des institutions clé de l'amélioration de la gestion de l'eau 
en Amérique Latine, in Vers une stratégie de gestion durable des fleuves, Les cahiers du CRIDEAU No8, PULIM, 2003, p. 45.

23. DOUMBE-BILLE S., "L'Agenda 21 et les eaux douces", dans M. PRIEUR et DOUMBE-BILLE S, Droit de l'environnement et développement durable, Limoges, PULIM, $719 \mathrm{p}$.

24. DROBENKO B. (sous la direction), Vers une stratégie de gestion durable des fleuves, Les cahiers du CRIDEAU Nº8, PULIM, 2003, p. 15.

25. DROBENKO B., Droit de l'eau, Gualino Editeur, 2007, 330 p.

26. DUPUY P.M., La responsabilité internationale des Etats pour dommages d'origine technologique et industrielle, Paris, Pedone, 1976, 309p.

27. DUROY S., La distribution d'eau potable en France. Contribution à l'étude d'un service public local, LGDJ, 1996, 436 p.

28. FAURE M., L'analyse économique du droit dans les pays de droit civil, dans DEFFAINS B., L'analyse économique du droit dans les pays de droit civil, Paris, CUJAS, 2002, 113-150.

29. FAURE M., L'analyse économique du droit de l'environnement, Bruxelles, Bruylant, 2007, $362 \mathrm{p}$.

30. FAURE M., SONG Y., China and international environmental liability, legals Remedies for Transboundary Pollution, New Horizons in environmental law, 2008, 360p.

31. FENET A., Droit de la mer, droit des cours d'eau internationaux: similitudes et convergences, ADMA, 1991, tome 11, p.90.

32. FEUER G., La théorie de la souveraineté sur les ressources naturelles dans les résolutions des Nations Unies, Paris, Université René Descartes, 1983, p.16.

33. GAITTI Jules, L'eau, mythes et symboliques, Paris, C.I.Eau, coll. Synthèse de l'eau, février 2001, $62 \mathrm{p}$.

34. GAONAC'H A., La nature juridique de l'eau, éd. Johanet, 1999

35. GARREC C., L'eau et la ville en droit communautaire: politique communautaire de l'eau douce et aménagement hydraulique de la ville, Apogée, Rennes, 1997, 141 p.

36. GAZZANIGA J-L., L'eau : usages et gestion, LITEC, 1998, 316 p.

37. GUILLOT Ph. Ch.-A., Droit de l'environnement, ${ }^{\text {ème }}$ édition, Ellipses, 2010, 320 p.

38. GUISELIN Emmanuel-Pie, "Droit au logement : quelle reconnaissance?", in. SEGAUD M., BRUN J. et DRIANT J.-C., Dictionnaire de l'habitat et du logement, Armand Colin, Paris, 2003.

39. HAUMONT F., «La subsidiarité et la protection de l'environnement», dans Francis Delpérée, dir., Le principe de subsidiarité, Bruxelles, Bruylant, 2002, p. 189.

40. HUGON Philippe, Géopolitique de l'Afrique, Paris, SEDES, 2007, 240 p.

41. INSERGUET-BRISSET V., Propriété publique et environnement, LGDJ, 1994, 320 p.

42. JACQUART A., L'équation du nénuphar, Calmann-Lévy, 1998, p.190.

43. KAMTO M., Droit de l'environnement en Afrique, EDICEF-AUPELF, France, 1996, 416p.

44. KISS A. et BEURIER, Droit international de l'environnement, $3^{\text {ème }}$ édition, 2004, 1001 p.

45. KISS A. et J.P. BEURIER, Droit international de l'environnement, Collections Etudes Internationales, Paris, Pedone, 2000, 424p.

46. LAMARCHE L., Perspectives occidentales du droit international des droits économiques de la personne, Bruxelles, Bruylant, 1995, pp. 443-444.

47. LAMARQUE J., Droit de la protection de la nature et de l'environnement, LGDJ, 1973, 974 p.

48. LAVIEILLE J.M., Droit international de l'environnement, France, Ellipses, 1998, 191p.

49. LAVIEILLE M., Droit international de l'environnement, Ellipses, $3^{\mathrm{eme}}$ édition, 2010, 368 p.

50. LECERF M., Droit des consommateurs et obligations des services publics, éd. d'organisation, Paris, 1999, p. 148. 
51. LESUEUR J-Y. et PLANE P., Les services publics africains à l'épreuve de l'assainissement : une évaluation économique et sociale, Paris, L'Harmattan, 1994.

52. LESUEUR J-Y. et PLANE P., Les services publics africains à l'épreuve de l'assainissement : une évaluation économique et sociale, Paris, L'Harmattan, 1994.

53. LOPA DUFRENOT M., L'accès à l'eau saine, un droit humain au Burkina Faso, L'Harmattan, 2009,183 p.

54. MAILlLARD DESGRÉES du LOÛ D., Droit des relations de l'administration avec ses usagers, PUF, 2000, p. 40.

55. MALINGREY Ph., Introduction au droit de l'environnement, $5^{\text {ème }}$ édition, Lavoisier, 2011.

56. MALJEAN-DUBOIS S., L'effectivité du droit européen de l'environnement, Contrôle de la mise en oeuvre et sanction du non-respect, La documentation française, 2000.

57. MARC P., Les cours d'eau et le droit, préf. R. ROMI, éd. Johanet, 2006.

58. MARC Ph., Les cours d'eau et le droit, Editions JOHANET, 2006.

59. MASSENET M. (dir.), Droits de propriété, économie et environnement. Les ressources en $e a u$, Dalloz Sirey, 1997, 372 p.

60. MODERNE, FRANK, «Le principe de subsidiarité fonctionnelle», dans Francis DELPEREE, dir., Le principe de subsidiarité, Bruxelles, Émile Bruylant, 2002, p. 395.

61. NADON O., «La responsabilité du pollueur et l'évolution de la notion de faute», dans Service de la formation permanente du Barreau du Québec, Développements récents en droit de l'environnement, Cowansville, Éditions Yvon Blais, 1996, p. 143,

62. NGNIKAM E. et TANAWA E., Les villes d'Afrique face à leurs déchets, Utbm, décembre 2006, 281p.

63. OST F., Entre droit et non-droit : l'intérêt - essais sur les fonctions qu'exerce la notion d'intérêt en droit privé, vol. 2, Droit et intérêt, éd. Fac. Univ. de Saint Louis, Bruxelles, 1990, p. 47.

64. OST F., Le milieu un objet hybride qui déjoue la distinction public-privé, Paris, Presses Universitaires françaises, 1995, p.102.

65. PAQUEROT S., "Ressources vitales et droits humains : peut-on sérieusement les dissocier? L'enjeu de l'eau douce " in DELAS (O.), DEBLOCK (Ch.), (Dir.), Le bien commun comme réponse politique à la mondialisation, Bruxelles, Bruylant, 2003, 444 p.

66. PAQUEROT S., Eau douce, la nécessaire refondation du droit international, Presses de l'Université du Québec, 2005, 246 p.

67.PÂQUES M., «Le principe de subsidiarité, la police administrative générale et la police de l'aménagement du territoire de l'environnement», dans Francis DELPEREE, dir., Le principe de subsidiarité, Bruxelles, Émile Bruylant, 2002, 240 p.

68. PEIGNOT B., «Protection de l'eau potable et agriculture», dans Max FALQUE et Michel MASSENET, dir., Droits de propriété, économie et environnement. Les ressources en eau, Paris, Dalloz, 2000, 507 p.

69. PELLETIER M-L., L'entreprise publique de service public: déclin et mutation, L'Harmattan, 2009, 452 p.

70. PETRELLA, Le manifeste de l'eau, Éditions Labor, Bruxelles, 1998 , p.2.

71. PEZON C., Le service d'eau potable en France de 1850 à 1995, Paris, Presses du CEREM, 2000, $450 \mathrm{p}$.

72. PNUD, Rapport mondial sur le développement humain 2006, Au-delà de la pénurie : Pouvoir, pauvreté et crise mondiale de l'eau, 552p.

73. PONTHOREAU M.-C., «Constitutions, juges constitutionnels et droit à l'environnement " in L'environnement à quel prix ? Sous la direction de Ejan MACKAAY et Hélène TRUDEAU, Les Editions Thémis, Université de Montréal, p.320.

74. POYDENOT A., Le droit international de l'eau, état des lieux, Les Notes d'analyse du CIHEAM, n²9 - février 2008, p. 4. 
75. PRIEUR M. (dir.), La mise en œuvre du droit international de l'environnement dans les pays francophones, PULIM, 2003, 579p.

76. PRIEUR M. et DOUMBE-BILLE S., Recueil francophone des traités et textes internationaux en Droit de l'environnement, Belgique, Bruylant, 1998, 719p.

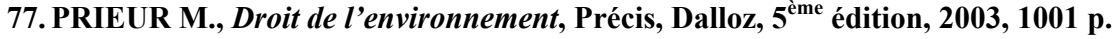

78. Programme des Nations Unies pour l'Environnement, Afrique. Atlas d'un environnement en mutation, Nairobi, Nations Unies, 2008.

79. PUTELAT E., La question de la justiciabilité des droits sociaux à l'épreuve du droit européen, Séminaire de droit européen, Université de Strasbourg, 2004-2005.

80. QUOC DINH N., P. DAILLIER, A. PELLET, Droit international public, L.G.D.J., 1999, Paris, 1709 p.

81. REMOND-GOUILLOUD M., Du droit de détruire, Essai sur le droit de l'environnement, Paris, Presses Universitaires, 1989, p. 122 et 145.

82. ROCHE P.A., L'eau au XXIe siècle : enjeux, conflits, marché, dans IFRI : Ramses 2001, Dunod, Paris 2001, 374 p.

83. ROMI R., BOSSIS G. et ROUSSEAUX S., Droit international et européen de l'environnement, Montchrestien, 2005, 368 p.

84. SADELEER N., Les principes du pollueur-payeur, de prévention et de précaution, Essai sur la genèse et la portée juridique de quelques principes du droit de l'environnement, Bruylant, Belgique, 1999, 347p.

85. Salif DIOP et Philippe REKACEWICZ, Atlas mondial de l'eau, Paris, Autrement, 2003, p.32.

86. SAOUT A., Théorie et pratique du droit de l'eau, Johanet, 2011, 474 p.

87. SHIVA V., La guerre de l'eau, Privatisation, pollution et profit, Paris, Paragon, 2003, 273

p.

88. SMETS H., De l'eau potable à un prix abordable, Académie de l'eau, 2008, p. 129-130.

89. SMETS H., Le droit à l'eau dans les législations nationales, AESN, Nanterre, octobre 2005.

90.SOHNLE J., Le droit international des ressources en eau douce : solidarité contre souveraineté, La documentation française, $2002,606 \mathrm{p}$.

91. SOHNLE J., Nouvelles tendances en matière de règlement pacifique des différends relatifs aux ressources en eau douce internationales, in BOISSON DE CHAZOURNES et SALMAN, Les ressources en eau et le droit international / Water resources and international law, Académie de droit international, 2005.

92. SORMAN G., La solution libérale, Fayard, 1984, p.270.

93. TAITHE A., L'eau. Un bien? Un droit? Tensions et opportunités, Unicomm, UE, 2008, 215 p.

94. TAITHE Alexandre, Partager l'eau. Les enjeux de demain, Paris, Éditions Technip, 2006, $168 \mathrm{p}$.

95. TEITGEN-COLLY C., La légalité de l'intérêt financier dans l'action administrative, Economica, 1981, p. 394.

96. TENIERE-BUCHOT P.F., L'eau, Rapport présenté à l'Académie des sciences morales et politiques à sa séance du 28 janvier 2002.

97. TIGNINO M., L'eau dans le processus de paix au Proche-Orient : éléments d'un régime juridique, Institut Universitaire de Hautes Études Internationales, Genève, Mai 2004, à la p. 14. Universitaires du Quebec, Sainte-Foy, 2005.

98. UHRY M., "Le droit au logement en Europe», in La ville à l'épreuve du libéralisme, Cahier Voltaire, Aitec, Paris, 2006.

99. VANDANA S., La guerre de l'eau, Paragon, 2003, $162 \mathrm{p}$.

100. WEBER L., OMC, AGCS, Vers la privatisation de la société, Syllepse, 2003, 302 p. 


\section{THESES ET MEMOIRES}

1. ABDOULAYE M. M., La gestion des ressources en eau dans le bassin conventionnel du Lac Tchad: état des lieux et perspectives, Master 2 en droit international de l'environnement, Université de Limoges, 2004.

2. ABIKHZER F., La notion juridique d'humanité. Thèse pour le Doctorat de l'Université Paul Cézanne (Aix-Marseille III), Droit, 2005, 620 p.

3. ABI-SAAB R., Droit humanitaire et conflits internes. Origine et évolution de la réglementation internationale, Thèse pour le Doctorat en Droit, Université de Genève, Pedone, $1986,280 \mathrm{p}$.

4. AHOULOUMA B. F., Contribution a l'étude du concept de développement durable : une application au secteur de l'eau douce au sein de l'union économique et monétaire ouest africaine (U.E.M.O.A.), Thèse, Université de Poitiers, 2008, 690 p.

5. AKUE S.A., Finances publiques et politiques de protection de l'environnement dans les pays d'Afrique de l'Ouest, Thèse, Université de Poitiers, 2003, 397 p.

6. ALACHI E. K., Orientations de la politique nigérienne d'exploitation et de gestion des ressources en eau, Mémoire DEA Droit et politique de l'environnement, Université de Lomé, Faculté de Droit, 2002-2003, 83 p.

7. AMADEI J.-P., Domaine public et décentralisation, Thèse, Montpellier I, 1996.

8. ASSEMBONI N.A. Epse OGUNJIMI, Le droit de l'environnement marin et côtier en Afrique occidentale : cas de cinq pays francophones, Thèse de doctorat, Université de Limoges, septembre 2006, 577p.

9. ATONTSA M. J. C., Droit international de l'environnement et mise en æuvre du droit a l'eau potable en Afrique centrale : le cas du Cameroun, Thèse, Université de Maastricht, 2011, 294 p.

10. ATONTSA M.J.C, L'apport du droit international de l'environnement pour l'accès à l'eau potable en Afrique centrale, Mémoire de Master, Université de Limoges, août 2007, 71p.

11. BALLANDRAS-ROZET Ch., Les techniques conventionnelles de lutte contre les pollutions et les nuisances et de prévention des risques technologiques, Thèse, Lyon, 2005, $877 \mathrm{p}$.

12. BEROUJON F., L'application du droit de la consommation aux gestionnaires de services publics, Eléments de réflexion sur l'évolution du droit des services publics, Thèse dactyl. Grenoble II, 2005, p. 340 s.

13. BIGOT C., Le droit à l'eau en droit international et en droit communautaire : contribution à l'étude d'un droit de l'Homme et des générations futures en émergence, Thèse pour le Doctorat de l'Université de Paris X-Nanterre, 2006, 504 p.

14. BIRRAUX D., Service public et entreprises privatisées, Université Pierre Mendès France, Mémoire Master II, 2006, p. 71.

15. BONNEL G., Le principe juridique écrit et le droit de l'environnement, Thèse de doctorat, Université de Limoges, $511 \mathrm{p}$.

16. BOUIN F., Tourisme et droit de l'environnement, Thèse, Limoges, 2000, 501 p.

17. BOURBEAU B., La notion d'affectation dans la théorie du domaine public, Thèse, Poitiers, 1980, 645 p.

18. BOYER-CAPELLE C., Le service public et la garantie des droits et libertés, Thèse dactylographiée, Université de Limoges, 2009, 732 p.

19. BURON C., La distinction entre les services publics administratifs et les services publics industriels et commerciaux, Thèse dactylographiée, Université d'Orléans, 1971, 2 tomes, 548 p.

20. CALASANS J. T., Le Concept de "ressource naturelle partagée». Application aux ressources en eaux : l'exemple de l'Amérique du Sud (1996), Thèse de doctorat en droit, Université Paris I - Panthéon- Sorbonne p. 314. 
21. CARAYON J., La cession de concession et permission, Thèse, Paris, 1934, 257 p.

22. CAUDAL-SIZARET S, La protection intégrée de l'environnement en droit public français, Thèse, Lyon, 1993, $729 \mathrm{p}$.

23. CLUZEL L., Le service public et l'exigence de qualité, thèse dactylographiée, Paris II, 2004, publiée aux éditions Dalloz 2006, 533 p.

24. COQUET E., Le domaine public colonial, Thèse, Poitiers, 1905, 181 p.

25. DABONNE E., Droits de l'Homme et lutte contre la pauvreté au Burkina Faso, Thèse pour le Doctorat de l'Université de Nantes, 2007, 270 p

26. DASSE F. O., Les instruments de protection des droits de l'Homme en Afrique subsaharienne, Thèse pour le Doctorat de l'Université de Nantes, 2001, $486 \mathrm{p}$.

27. DAVANTURE S., Les limites de l'application du droit sur les ressources naturelles: le cas des territoires palestiniens et du Sahara occidental, Université du Québec à MontréalLLM.

28. DAVID M., La décentralisation de l'environnement, essai sur l'administration de l'environnement par les collectivités locales, Thèse, Bordeaux, 2000, 476 p.

29. De DAVID-BEAUREGARD-BERTHIER O., La justification actuelle de la distinction entre le domaine public et le domaine privé, Thèse, Aix-Marseille III, 1994, 546 p.

30. De GAUDEMAR H., L'inaliénabilité du domaine public, Thèse, Paris II, 2006,321 p.

31. DRESSAYRE C., La mise en place du doit international de l'eau : mythe ou réalité ?, Thèse dactylographiée, Montpellier I, 2002, 437 p.

32. DRIS A., L'eau matière stratégique et enjeu de sécurité au 21ème siècle, DEA Sciences Politiques, Université Paris X, 82 p.

33. DUROY S., Contribution à l'étude d'un service public local : la distribution d'eau potable, Thèse, Paris II, 1992, 436 p.

34. DUVERGER M., L'affectation des immeubles domaniaux aux services publics, Thèse, Bordeaux, 1940.

35. ECKERT G., Le droit administratif et la commercialité, thèse, Strasbourg, 1994.

36. ERWANN L.C., La prise en compte de l'environnement par les règles locales d'urbanisme, Thèse de doctorat, Université de Paris I, janvier 1997, 714p.

37. ESPAGNO D., Essai sur la refondation de la notion du service public en droit administratif français, Thèse, Toulouse, 1998, 547 p.

38. ETOA S., Le passage des libertés publiques aux droits fondamentaux : analyse des discours juridiques français, Thèse pour le Doctorat de l'Université de Caen, 2010, 460 p.

39. FARINETTI A.,La protection juridique des cours d'eau, Thèse, Lyon III, 2010.

40. FERRIERA M., L'eau juridiquement durable, Thèse, Limoges, 2006

41. FEVRIER F., La spécificité des principes du service public, Thèse, Rennes I, 1999.

42. FOURMOND S., Occupations privatives du domaine public et droit des patrimoines, Thèse, Nantes, 2000.

43. FROUVILLE (de) O., L'intangibilité des droits de l'Homme en droit international public. Régime conventionnel des droits de l'Homme et droit des traités, Thèse pour le Doctorat de l'Université de Paris X-Nanterre, 2001, 682 p.

44. GARCERIES S., L'élaboration d'une notion juridique de service public industriel, Thèse dactylographiée, Université de Cergy-Pontoise, 2010, 850 p.

45. GARIDOU B., Recherche sur la théorie de la propriété publique en droit administratif français, Thèse, Toulouse I, 2003.

46. GAY L., Les " droits-créances " constitutionnels, Thèse pour le Doctorat de l'Université d'Aix-Marseille III, Droit, 2001, 562 p.

47. GAY L., Les droits-créances constitutionnels, thèse dactylographiée, Aix-en-Provence, 2001.

48. GERVAIS A., Le retrait des autorisations d'utilisation privative du domaine public, 
Thèse, Montpellier, 1942.

49. GNANGUI A., La Convention de Lomé IV et l'environnement, Université de Limoges, Faculté de Droit et des Sciences Economiques, Thèse de doctorat, décembre 1999, 442 p.

50. GOUGUIA E., L'ambiguïté de la distinction SPA/SPIC, Thèse dactylographiée, Université de Caen, 1990, $442 \mathrm{p}$.

51. HERTZOG R., Recherche sur la gratuité et la non gratuité des services publics, Thèse Strasbourg, 1972,p. 169.

52. HUBRECHT H.- G., Les contrats de service public, Thèse, Bordeaux, 1980.

53. KADOKALIH Y., L'application de la Convention des Nations unies sur la lutte contre la désertification au Togo, Mémoire de DEA, Université de Lomé (Togo), 2004, 72 p.

54. KASSERE A. F., La gestion intégrée des ressources en eau dans les Etats de l'Afrique de l'ouest, mémoire DEA, Université de Lomé, 2004, 87 p.

55. KONATE A., L'Organisation de l'Unité Africaine et protection juridique de l'environnement, Université de Limoges, Faculté de Droit et des Sciences Economiques, thèse, mars 1998, $631 \mathrm{p}$.

56. КОТОКО М., L'Intervention de l'Etat dans la gestion de l'eau potable au Togo, Mémoire DEA Droit et politique de l'environnement, Université de Lomé, Faculté de Droit, 1999-2000, 80 p.

57. KRISTIN B., L'antagonisme "commerce et environnement» ou le principe de développement durable comme fil conducteur pour la réconciliation des intérêts commerciaux et environnementaux sous l'article XX du GATT, Thèse de doctorat, Université de Laval, 2007.

58. LAFAGE P., Le pouvoir de gestion du domaine public, Thèse, Université de Savoie, 2000. 59. LAMBERT M.-L., La vie sauvage, enjeu du droit de l'environnement contemporain. Réflexions sur la C.I.T.E.S., Paris I, Panthéon - Sorbonne, 24 octobre 1997, 647 p.

60. LEBRETON J.-P., Les occupations du domaine public, Thèse, Paris, 1976.

61. LEDAIN D., Constitution, Convention européenne des droits de l'homme et droit des biens publics, Thèse, Pau, 2009.

62. LEMERCIER C., La part du droit administratif dans la gestion du domaine privé de l'Etat, Thèse, Paris II, 1990.

63. LY I., Problématique du droit de l'environnement dans le processus de développement économique et social d'un pays africain : l'exemple du Sénégal, Université CHIEKH Anta DIOP de Dakar, Thèse de doctorat en Droit, février 1994, 458 p.

64. MARBOUTY F., Le statut constitutionnel des services publics, thèse, Limoges, 1998.

65. MARQUES C., La stratégie communautaire de conservation du milieu marin depuis $1986-$ contribution à l'étude des principes d'intégration et de co-responsabilité., Thèse soutenue a l'Universite de Bretagne Occidentale, 2005, 442p.

66. MARQUES DE CARVALHO V., L'état et le secteur privé dans la gestion des services de l'eau au Brésil : une analyse juridique à partir de l'exemple français, Université de Paris I, Thèse, 2007

67. MERCURE F.P., L'évolution Du Concept de Patrimoine Commun de L'humanité Appliqué aux ressources naturelles, Thèse soutenue a l'Universite de Nice - Sophia Antipolis, 1998, 360p.

68. MOISAN M., Essai sur le droit et l'administration des cours d'eau en France, Thèse. Paris 1, 1996.

69. MOREL A., Contrat d'État et souveraineté étatique, Mémoire (Panthéon - Assas Paris II), 2001, $164 \mathrm{p}$.

70. MOUKOKO P. L'obligation de coopération en matière de conservation et de gestion des stocks chevauchants et des grands migrateurs, Thèse soutenue à Aix-Marseille, 2003, 747p.

71. OLAKA J-M., Le droit à l'eau, Thèse, Université Jean Moulin Lyon, 2008

72. OSSOMBO-YOMBO R., La gestion de l'eau potable dans les grandes agglomérations d'Afrique centrale. Contribution à l'étude d'un service public confronté à la mondialisation, 
Thèse, Université Jean Moulin - Lyon III, 2007, 646 p.

73. PAGNEY-VIARD, Rapports des droits de la santé et de l'environnement en matière de qualité de l'eau, Thèse, Paris II, 1998

74. PAQUEROT S., Le statut de l'eau douce en droit international : penser la res publica universelle, Thèse de doctorat, octobre 2003, 545p.

75. PEZ T., Le risque dans les contrats administratifs, Thèse, Paris II, 2006.

76. PLAGNET B., Evolution récente de la notion de SPIC, Thèse, Paris II, 1972, 421 p.

77. PLASKOWSKI S., La protection de l'eau dans les Etats en transition : l'action des Organisations internationales, Thèse, Paris, 2003. 1613 p.

78. QUIOT G., Aux origines du couple "gestion publique-gestion privée ». Recherche sur la formation de la théorie de la gestion privée des services publics, Thèse, Université de NiceSophia-Antipolis, 1992, 842 p.

79. RENARD MUGNIER A.-S., Le droit à l'eau, Thèse pour le Doctorat de l'Université du droit et de la santé (Lille 2), 2007, 449 p.

80. ROGER-MARBOUTY F., Le statut constitutionnel des services publics, Thèse Limoges, 1998.

81. SALOMON J., L'égalité des individus devant le service public, Thèse, Grenoble, 1954.

82. SEGUR L., La permissionde voirie, Thèse, Bordeaux, 1912.

83. SEHILI M.-H, La question des l'universalité des droits de l'homme dans les manuels relatifs aux droits et libertés, Master recherche Droit constitutionnel et théorie du droit, Université Montpellier 1,

84. SOHNLE J., Le droit international des ressources en eau douce; solidarité contre souveraineté, thèse dactylographiée, Université R. Schuman, Strasbourg, janvier 2000, 608 p.

85. SOSSOU BIADJA C. J., Décentralisation et coopération décentralisée au Benin: Vers la légitimation des espaces publics locaux pour le développement des collectivités locales, DEA, Université de Genève Institut Universitaire d'Etudes en Développement, 2004.

86. TAILlEFAIT A., L'évolution du droit et de la gestion des biens des collectivités territoriales, Thèse, Paris II, 1996.

87. TESTE P., Les services de distribution d'eau, gaz et électricité, Thèse, Paris, 1940.

88. TIEBLEY Y.D., La Côte d'Ivoire et la gestion durable des ressources naturelles marines, Thèse de doctorat, Université de Maastricht, mai 2010, 369p.

89. UNTERMAIER J., La conservation de la nature et le droit public, Thèse, Lyon, 1972, 814 p.

\section{ARTICLES ET CONTRIBUTIONS}

\section{1-EAU, RESSOURCES EN EAU ET THEMES CONNEXES}

1. ABI-SAAB G., «La souveraineté permanente sur les ressources naturelles» in Droit international, Bilan et perspectives, éd. Pedone, Paris, 1991, Tome II, pp 639-661.

2. ABOUBAKAR A., "L'initiative du Bassin du Niger (IBN) : développement durable et gestion intégrée d'un grand fleuve ", Afrique contemporaine, ${ }^{\circ} 209,2003$, p. 179-200.

3. ABSI H., " La protection de la ressource en eau au Maroc : un dispositif juridique déficient ", Environnement, ${ }^{\circ} 7,2005$, p. 15-24.

4. AgOSTINI R., "La politique de l'eau en France et ses évolutions", Les Cahiers du CRIDEAU, $n^{\circ}$ 6, 2002, p.87-92.

5. ALBUQUERQUE (de) C., Rapport de l'experte indépendante des Nations unies, ,A/HRC/15/31/Add. 1 
6. ANOUGOU J. P. Thiéry, «L'eau, bien public, bien privé : l'Etat, les communautés locales et les multinationales" in L'eau, patrimoine commun de l'humanité, Alternatives Sud, Vol. VIII, N4, 2001, p.163.

7. ARCARI M., « Le droit interétatique de l'eau : les principes matériels de la convention de New York (21 mai 1997)", in JEANCLOS Y. (Dir.), Les politiques de l'eau au XXIe siècle, Actes du colloque de Strasbourg, Tome II, CIRPES, 2005, p. 17-32.

8. AUTIN J.-L., « La gestion équilibrée de la ressource en eau », Environnement, ${ }^{\circ}$ 7, 2005, p. 95-97.

9. B'CHIR F., « La Commission consacre l'importance de l'eau en Afrique ", Marchés tropicaux, février 2004, p.317.

10. BAH A., DIALlO A. et MORIN R., "Approvisionnement en eau des ménages de Conakry », Afrique contemporaine 2007/ 1, N²21, p. 227-228.

11. BANGOURA D., "L'eau, enjeu de sécurité en Afrique ", in JEANCLOS Y. (Dir.), L'eau, arme stratégique pour le XXIe siècle ?, Actes du colloque de Strasbourg, Tome I, CIRPES, 2005, p. 101-112.

12. BARBERIS J. A., "Bilan de recherches de la section de langue française du centre d'étude et de recherche de l'Académie " dans Académie de droit international de La Haye, Centre d'étude et de recherche de droit international et de relations internationales, Droits et obligations des pays riverains des fleuves internationaux, La Haye, Martinus Nijhoff, 1990, 38.

13. BARLAW M., «A qui appartient l'eau ?», Projet Planète Bleue et Tony Clarke, Polaris Institute.

14. BARLOW M., "Privatisation de l'eau et des autres ressources environnementales", Source : wwww.urfig.org.

15. BASTIN J., « Pathologies hydriques ", Jeune Afrique l'Intelligent, n²248, du 8 au 14 février 2004, p. 53.

16. BELAIDI N. et EUZEN A., «De la chose commune au patrimoine commun. Regards croisés sur les valeurs sociales de l'accès à l'eau", De Boeck Université, Monde en développement, 2009/01 - $\mathrm{n}^{\circ} 145$, pages 55 à 72.

17. BENVENISTI E., "Collective action in the utilization of shared freshwater: the challenges of international water ressources law », A.J.I.L., 1996, p. 384-415.

18. BETHEMONT Jacques, «Le Nil, l'Égypte et les autres », Revue Vertigo, vol. 4, $\mathrm{n}^{\circ} 3$, décembre 2003.

19. BIED-CHARRETON M. et al., «La gouvernance des ressources en eau dans les pays en développement : enjeux nationaux et globaux », De Boeck Université, Mondes en développement, $2006 / 3 \mathrm{n}^{\circ} 135$, p. 49

20. BILLAUDOT F., « La planification des ressources en eau », RFDA, 1993, p. 1052.

21. BILLET $P$., « L'usage de l'eau mis en règle : entre droit des équilibres et équilibre des droits ", Environnement, ${ }^{\circ} 7,2005$, p. 35-39.

22. BILLET P., «La réorganisation de l'administration dans le domaine de l'eau et des missions du préfet coordonnateur de bassin ", Environnement, ${ }^{\circ} 7,2005$, p. 4.

23. BILLET $P$., «La régulation juridique des conflits liés à la gestion des eaux souterraines", $n^{\circ} 3$, R.J.E., 2001, p. 401.

24. BLANCHON David, « Les nouveaux enjeux géopolitiques de l'eau en Afrique australe ", Hérodote, $\mathrm{n}^{\circ}$ 102, 2001.

25. BLOMQUIST W., «Rôle de la propriété commune dans le gestion des ressources en eau», dans Max FALQUE et Michel MASSENET, dir., Droits de propriété, économie et environnement. Les ressources en eau, Paris, Dalloz, 2000, p. 227.

26. BOEGLIN-NAUMOVIC N., "De l'usage des cours d'eau internationaux comme frontières ", in BOISSON DE CHAZOURNES L. et SALMAN (S.M.A.) (Dir.), Les 
ressources en eau en droit international, Académie de droit international de La Haye, Martinus Nijhoff Publishers, Leiden/Boston, 2005, p. 133-165.

27. BOISSON de CHAZOURNES L., " Eaux internationales et droit international : vers l'idée de gestion commune ", in BOISSON de CHAZOURNES (L.), SALMAN (S.M.A.), Les ressources en eau et le droit international, Académie de droit international de La Haye, Martinus Nijhoff Publishers, Leiden/Boston, 2005, p. 3-43.

28. BOISSON DE CHAZOURNES L., CHARRIER B., CURTIN F., BOLLINGER J.-C., « Le cycle de l'eau dans la société ", Les Cahiers du CRIDEAU, $\mathrm{n}^{\circ}$ 6, 2002, p.79-86.

29. BONNARD H., "L'évolution du délit de pollution des eaux », in BOUTELET M. et FRITZ J.-C. (Dir.), L'ordre public écologique, Actes et débats de colloque, Dijon, les 6 et 7 février 2003, Bruxelles, Bruylant, 2005, pp. 175-188.

30. BOOKER A., "L'approvisionnement en eau des zones urbaines du Ghana : la réglementation d'un partenariat public-privé », Afrique contemporaine - Printemps 2003, pp. 171-192.

31. BORGETTO M., "Sur la nature juridique du service de distribution d'eau et le traitement jurisprudentiel du principe d'égalité ", RFDA 1993 p. 673.

32. BOTTON S., "Les multinationales de l'eau et les marchés du Sud", Débats et Controverses, juin 2007, $\mathrm{n}^{\circ} 1, \mathrm{p} .14$ et 15.

33. BOURNE C.B., "Freshwater as a scarce resource», Canadian Council of Int'l Law, p. 18 et $\mathrm{s}$.

34. BOURNE C.B., « The International Law Commission's Draft Articles on The Law of International Courses: Principles and Planned measures ", 3, Colardo Journal of Int. Env'l Law \& Politics, 1992, p.65 et s.

35. BRIAND A., LEMAITRE A., « La privatisation de la distribution d'eau potable en Afrique : une aubaine? », Actes de la journée d'étude "Les territoires d'eau », Université d'Artois, Arras, 26 mars 2004, p.98

36. BRUN A. et LASSERRE F., « La gestion par bassin versant : un outil de résolution des conflits ? ", Lex Electronica, vol.12 n² (Automne/Fall 2007), 19 p., http://www.lexelectronica.org/articles/v12-2/lasserre_brun.pdf.

37. BUIRETTE P., « Genèse d'un droit fluvial international général (Utilisations à des fins autres que la navigation) ", R.G.D.I.P. 1991, pp.5-70.

38. CAFLISCH L., " La convention du 21 mai 1997 sur l'utilisation des cours d'eau internationaux à des fins autres que la navigation ", A.F.D.I., 1997, pp.751-798.

39. CAFLISCH L., «Sic utere tuo ut alienum non laedas : règle prioritaire ou élément pour déterminer l'utilisation équitable et raisonnable d'un cours d'eau international ? ", in Internationales Recht auf See und Binnengewässern, Festschrift für Walter Müller, Zurich, Schulthess, 1998, pp. 27-47.

40. CAPONERA D. A., " Les eaux partagées et le droit international ", in Université SaintEsprit, Droit international et droit comparé des cours d'eau internationaux : éducation à une culture d'une eau partagée et protégée, Liban, CEDLUSEK, 2000, p. 85-92.

41. CAPONERA D. A., "Shared waters and international law» dans BLAKE G. H., HILDESLEY W. J., PRATT M. A., SCHOLFIEL C.H. (dir.), The peaceful management of transboundary Resources, Boston, GRANHAM et Trotman/Martinus Nijhoff, p. 121.

42. CATY G., « Histoire d'eau, Frotin « Curator aquarum », RA, 1966, p.339;

43. CAUDAL S., "Les redevances perçues par les agences de l'eau : évolutions et perspectives ", Environnement, ${ }^{\circ}$ 7, 2005, pp. 39-44.

44. CHAMPEIL-DESPLATS V, « Le service public dans les débats constituants de 1946 », in G. GUGLIELMI (dir.), Histoire et service public, PUF, 2004, pp. 244.

45. CHARBONNEAU S., «Un cadre juridique à venir pour une politique communautaire dans le domaine de l'eau : la directive 2000/60 du 23 octobre 2000 ", Droit de 
l'environnement, $\mathrm{n}^{\circ} 86,2001$, pp. 44-45.

46. CHAVRIER G., "La qualification juridique de l'eau des cours d'eau domaniaux », RFDA 2004 p. 928.

47. Chronique ONU, « Les échos de l'eau ", Chronique $O N U, n^{\circ} 1,2003$, pp. 29-30.

48. COLLARD D., « Le XXIe siècle sera-t-il celui des conflits et des guerres pour l' " or bleu " ? ", in JEANCLOS Y. (Dir.), L'eau, arme stratégique pour le XXIe siècle ?, Actes du colloque de Strasbourg, Tome I, CIRPES, 2005, pp. 9-23.

49. COLLIARD C.-A., " Evolution et aspects actuels du régime juridique des fleuves internationaux ", R.C.A.D.I., 1968, p. 345-439.

50. CONAC G., «Des politiques de l'eau aux droits de l'eau en Afrique, à Madagascar et à l'Ile Maurice : bilan et esprit d'une recherche ", in CONAC F., CONAC G. (Dir.), La terre, l'eau et le droit en Afrique, à Madagascar et à l'Ile Maurice, Bruxelles, Bruylant, 1998, pp. 23-32.

51. CONCHE M., «L'eau et les philosophes », Sciences au Sud, Spécial 2003.

52. CRINOT $L$., " L'accès à la terre et à l'eau en milieu rural : le contexte juridique en vigueur au Bénin ", in La terre, l'eau et le droit en Afrique, à Madagascar et à l'Ile Maurice, Bruxelles, Bruylant, 1998.

53. DAVID C., « Les batailles de l'or bleu », L'Expansion, juillet-août 2006, p.64.

54. DEGUIGNET P., «Le service public de la distribution d'eau potable », Administration, $n^{\circ} 96,1977$, p. 32 et $s$.

55. DIOUF M., "Privatisations des économies et des Etats africains » in L'Etat en voie de privatisation, Politique africaine $\mathrm{n}^{\circ} 73$, Karthala, 1999, p. 16.

56. DROBENKO B., "Aspects juridiques des services de l'eau ", Académie de l'eauUNESCO, octobre 2002 ;

57. DROBENKO B., « Directive eau : un cadre en trompe l'oeil », REDE, 2001, $\mathrm{n}^{\circ} 4, \mathrm{p} .2$;

58. DROBENKO B., "Les enjeux juridiques de la solidarité : l'exemple de l'eau ", Communication présentée au colloque international de Ouagadougou du 1er au 4 juin 2004 sur le thème « Développement durable : leçons et perspectives », disponible sur auf.org. ;

59. DROBENKO B., " Les nouveaux grands principes du droit moderne de l'eau ", Actes du colloque de Limoges, Presses universitaires de Limoges, $1^{\text {er }}$ trimestre 2002, publié également à la Revue brésilienne de droit de l'environnement, $\mathrm{n}^{\circ} 25$, janvier-mars 2002 ;

60. DROBENKO B., "Environnement : le défi solidaire" in Pour un droit commun de l'environnement, Mélanges en l'honneur de Michel PRIEUR, Dalloz, 2007, p. 103.

61. DROBENKO B., "L'eau et la gouvernance dans le bassin méditerranéen ", Communication au colloque d'Athènes des 19 et20 mars 2002.

62. DROUET D., "La recomposition du secteur de l'eau en Europe", Réseaux, Année 1989, Volume 7, Numéro 35, p. 37 - 51.

63. DUMONT H., "Systèmes internationaux de protection des droits de l'Homme", Syllabus de Cours, Université du Burundi, Chaire UNESCO en Education a la paix et Résolution Pacifique des conflits, 2008- 2009, inédit, p. 44.

64. DUPONT-RACHIELE J., PREVOST D. et RAYMOND S., «L'eau : un droit pour tous, un bien pour certains», (2004) Vol.17.1, R.Q.D.I., 39, p.42-57.

65. El Hadj GUISSE, « Rapport entre la jouissance des droits économiques, sociaux et culturels et la promotion de la réalisation du droit à l'eau potable et à l'assainissement », E/CN.4/Sub.2/2002/10 du 25 juin 2002, pp. 10-13.

66. ETIENNE J. et HENRY A, « Eau et assainissement en Afrique : croyances, modes et modèles ", in IDDRI, Eau : encadrer les partenariats public-privé, Paris, IDDRI, 2003, p. 2324.

67. FALQUE M., «Des droits de propriété sur l'eau, pourquoi pas?», dans Max Falque et FENET A., Droit de la mer, droit des cours d'eau internationaux: similitudes et 
convergences, $A D M A, 1991$, t.11, p.90.

68. FAURE M. et LEFEVERE J., « Protecting drinking water quality against contamination by pesticides: an alternative regulatory framework », Review of European Community and International Environmental Law, 1995, pp. 321-326.

69. FISETTE J. et SALMI M., « Le désengagement de l'État en Afrique et les initiatives locales : la naissance de nouvelles formes de partenariat. ", Cahiers de géographie du Québec, vol. $35, \mathrm{n}^{\circ}$ 95,1991 , p. 350.

70.FLAUSS J-F., «Le droit international des droits de l'homme face à la globalisation économique ", in Commerce mondial et protection des droits de l'homme, Bruylant, 2001, p. 225.

71. GAZZANIGA J.-L., « La génèse de la loi sur l'eau du 3 janvier 1992 », LPA, 19 oct. 1992, $\mathrm{n}^{\circ} 126$, p. 9.

72. GIBRIL N., ASSEMBONI A.N., «L'accès à l'information et à la participation du public à la prise de décisions publiques en droit africain de l'environnement ", in $L a$ protection de l'environnement au cour du système juridique international et du droit interne : Actes, valeurs et efficacité, Bruxelles, Bruylant, 2003, p. 257-292.

73. GLAVANY Jean, Rapport d'information sur " La géopolitique de l'eau ", http://www.assemblee-nationale.fr/13/rap-info/i4070.asp

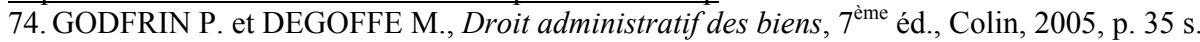

75. HALL D. et LOBINA E., Rapport réalisé à la demande de l'Internationale des Services Publics, novembre 2006, www.world-psi.org.

76. HENRY A., " Eau et assainissement en Afrique : croyances, modes et modèles », in IDDRI, Eau : encadrer les partenariats public-privé, Paris, IDDRI, 2003, p. 23-24.

77. HERTZOG R., «Les trois âges de la fiscalité de l'environnement », Les cahiers du CNFP, n³8, avril 1993, p. 136.

78. HUGON P., "Vers une nouvelle forme de gouvernance de l'eau en Afrique et en Amérique latine », Dalloz/I.R.I.S., Revue internationale et stratégique, 2007/2 - N 66, p. 65.

79. HUNT Paul, Reclaiming Social Rights: International and Comparative Perspectives (Aldershot, Dartmouth Publishing Company, 1996), p. 95 à 106.

80. JACKOWSKA Jonathan, "Des eaux et des idéaux: La question des villes du Sahel », Géopolitique de l'humanitaire, pp. 206-209.

81. JACQUEMOT P., "La désétatisation en Afrique subsaharienne. Enjeux et perspectives », Tiers-Monde, 1988, volume 29, $\mathrm{n}^{\circ} 114$, p. 271 et s.

82. JAGLIN S., « Services d'eau et construction métropolitaine au Cap (Afrique du Sud) : les difficultés de l'intégration urbaine », Revue française d'administration publique, $\mathrm{n}^{\circ}$ 107, 2003.

83. JAGLIN S., «La commercialisation du service d'eau potable à Windhoek, inégalités urbaines et logiques marchandes ", Flux $N^{\circ} 30$, octobre-décembre 1997, p. 16-29

84. JAGLIN S., «L'eau potable dans les villes en développement : les modèles marchands face à la pauvreté ", Tiers-Monde, Année 2001, Volume 42, Numéro 166, p. 278.

85.JAGLIN S., "Vingt ans de réformes dans les services d'eau urbains d'Afrique subsaharienne : une géographie de la diversité ", Séminaire de recherche du GDR Rés-EauVille (CNRS 2524) "L'eau à la rencontre des territoires", Montpellier, France, 27-28 et 29 mai 2004.

86. JAGLIN Sylvie, "Services d'eau et construction métropolitaine au Cap (Afrique du Sud) : les difficultés de l'intégration urbaine ", Revue française d'administration publique, $\mathrm{n}^{\circ} 107,2003$.

87. JAWORSKI V., « Répression de la pollution des eaux », Jurisclasseur Env. Fasc. 626.

88. KAMERI-MBOTE Patricia, « Eau, conflits et coopération : leçons tirées de l'expérience du bassin fluvial du Nil », Navigating Peace, $n^{\circ} 4$, avril 2008.

89. KAMTO M., "Le droit international des ressources en eau continentales africaines ", 
A.F.D.I., 1990.

90. KAUFFMANN C., «L'accès à l'eau potable et à l'assainissement en Afrique (d'après l'édition 2007 des Perspectives économiques en Afrique) ", Repères $n^{\circ}$ 41, Avril 2007, 2p. in www.oecd.org/dev/reperes.

91. KDHIR M., "Le principe de la liberté du commerce et de l'industrie : mythe ou réalité ?», Recueil Dalloz 1994 p. 30 et s.

92. KIPPING Martin, "Conflits et coopération liés à l'eau du fleuve Sénégal ", Revue Géocarrefour, vol. 80, $\mathrm{n}^{\circ} 4,2007$.

93. KOUANDA I. et MOUDASSIR M., «Social policies and private sector participation in water supply - the case of Burkina Faso », UNRISD, 2007.

94. KOUBI G, « La décomposition de la notion de gratuité en droit administratif français », JCP

95. KOUBI G., "Réflexions sur la gratuité dans le droit de la santé ", Revue de droit sanitaire et social 1999 p. 1.

96. LABERGE H., "Le temps de l'eau ». L'Agora, numéro spécial, « Le Sang de la terre », vol.6, no 2, 1999, p. 21.

97. LAIME M., « La marchandisation de l'eau s'accélère », Le Monde Diplomatique du 19 mars 2008.

98. LAMARQUE J., « La loi du 3 janvier 1992 sur l'eau », CJEG, 1993, p. 81.

99. LARBI BOURGUERRA M., "Eau et santé » in La mise en æuvre du droit à l'eau, Actes du XXIXème Congrès ordinaire de l'IDEF, Schultess, 2006, p. 125.

100. Le MOAL R. et BEURRIER J.-P., « Statut juridique de la qualité de l'eau », Rev. Dr. Rural $\mathrm{n}^{\circ} 244,1996$, p. 249.

101. LEBRIER S., " Les sanctions administratives de la police des eaux et des milieux aquatiques. Conditions de mise en œuvre de l'article L.216-1 du Code de l'environnement », RD.rur. 303, mai 2002, p.276.

102. LUSSON-LEROUSSEAU, « Les accords de produits de base, instruments de régulation des marchés », R.G.D.I.P. 1981, p.5-37.

103. MALLET-BRICOUT B., « Droit des biens », Recueil Dalloz 2007 p. 2490

104. M'BAYE K., «Les droits de l'homme en Afrique », Revue internationale de droit comparé, Année 1993, Volume 45, Numéro 3, p. 723 - 726.

105. MBEMBE A., "Du gouvernement privé indirect in l'Etat en voie de privatisation », Politique africaine $\mathrm{n}^{\circ} 73$, Karthala, 1999, pp. 103 à 104.

106. MCCAFFREY S.C., The Law of International Watercourses, Non-Navigational, Uses, Oxford University Press, 2001.

107. MENARD C., «Enjeux d'eau : la dimension institutionnelle», Tiers-Monde, Année 2001, Volume 42, Numéro 166, p. 259 - 274.

108. MERINO M., «L'eau : quels enjeux pour l'Afrique subsaharienne ? » Note de la FRS, ${ }^{\circ}$ 20/2008, p. 2.

109. MEUBLAT G., « De la gestion des fleuves internationaux en Afrique et du bassin du Sénégal en particulier " dans G. MEUBLAT (dir.) "Les nouvelles politiques de l'eau " (2001) Revue Tiers Monde, avril-juin, tome XLII, p. 439.

110. MEUBLAT G., " La politique de lutte contre la pollution des eaux, la mise en œuvre de la loi du 16 décembre 1964 », in Les politiques d'environnement, Editions Recherches, 1998, p. 67.

111. MEUBLAT G., "La rénovation des politiques de l'eau dans les pays du Sud ", TiersMonde, année 2001, Volume 42, Numéro 166, p. 249 - 257

112. MEUBLAT G., " De la gestion des fleuves internationaux en Afrique et du bassin du Sénégal en particulier », Revue Tiers Monde, $n^{\circ}$ 166, avril-juin 2001.

113. MILL J. S. cité par Galbraith Pierre LE MASNE (Chercheur au GEDES à l'Université de Poitiers), « Services publics et développement », p. 7.

114. MONTIALOUX Claire, "Service public et intérêt général », La Découverte, Regards 
croisés sur l'économie, 2007/2 - N², pages 25 à 26.

115. NDIAYE Tamsir, «L'Organisation pour la mise en valeur du fleuve Sénégal (OMVS) : une réussite à nuancer ? ", Lettre $p S$-Eau, $\mathrm{n}^{\circ} 47$, mars 2005.

116. NELSON N., " L'importance de l'eau pour les femmes africaines vivant dans un bidonville : le cas de Mathare Valley, faubourg de Nairobi (Kenya) », Tiers-Monde, Année 1980, Volume 21, Numéro 84, p. 866.

117. NILSSON D., «Public Service for a chosen few? Water and sanitation service provision in Kampala, Uganda, under colonial rule», RKTF Suède, Communication à la conférence de l'IWHA, décembre 2005, Paris.

118. NYASSOGBO G.K., "Comment une capitale devient macrocéphale en Afrique subsaharienne : Le cas de Lomé au Togo (Afrique de 1.Ouest) » in : TALOR J. LENGELLE J.G. and ANDREW C. : Capital Cities. Les capitales. Carleton University Press, Ottawa,1993, pp.325-342.

119. OUGUERGOUZ M. F., «La convention de Bamako sur l'interdiction d'importer en Afrique des déchets dangereux et sur le contrôle des mouvements transfrontaliers et la question des déchets dangereux produits en Afrique", In: Annuaire français de droit international, volume 38, 1992. pp. 871-884.

120. ÖZDEN Melik, «Le droit à la santé, Un droit humain fondamental stipulé par l'ONU et reconnu par des traités régionaux et de nombreuses constitutions nationales », Directeur $d u$ Programme Droits Humains du CETIM et Représentant permanent auprès de l'ONU.

121.PAPON P., « Mieux gérer l'océan mondial », Futuribles, février-mars 1999, n²39-240, p. 23-35.

122. PELLETIER M-L., L'entreprise publique de service public: déclin et mutation, L'Harmattan, 2009, p.51.

123. PIGOU A.C., The Economics of Welfare, London, Mac Millan, 1958

124. PLANE P. et LESUEUR J-Y., "Les services publics subsahariens: importance socioéconomique et évaluation des d'assainissement », Tiers-Monde, 1994, Volume 35, n²40, p. 780.

125. PLOYE François, « La planète est à sec ", Jeune Afrique, 5 février 2006.

126. RIBOT C. (dir.), «L'eau et droit. La gestion équilibrée de la ressource en eau », Actes du colloque de Montpellier, JCPE, juillet, 2005.

127. RICHARD V., Gestion privée de l'eau : où va le droit international ?Études et documents du Conseil d'État, p. 515.

128. RICHER L., « Les services de l'eau potable et de l'assainissement dans la loi sur l'eau. La loi à la traîne de la jurisprudence ", $A J D A 2007$ p. 1168.

129. ROYER P., "Le Massa et l'eau de Moussa. Cultes régionaux, " traditions » locales et sorcellerie en Afrique de l'Ouest ", Cahiers d'études africaines, Année 1999, Volume 39, Numéro 154, p. 337 - 366.

130. RUNYAN C., «Essai: Privatisation de l'eau, Etat de la Planète ", www.delaplanete.org / $\mathrm{N}^{\circ} 7$ janvier-février 2003.

131.SAKHO $P$. O., "La politique de privatisation du gouvernement: enjeux et perspectives ", Revue du Conseil économique et social $n^{\circ} 2$, févr.-avr. 1997, pp. 20-22

132. SALEM Gérard, "Crise urbaine et contrôle social à Pikine : bornes fontaines et clientélisme », Politique Africaine, $\mathrm{n}^{\circ}$ 45, mars 1992.

133. SANI ADAMOU M., "Problématique de la Privatisation de la Distribution de l'Eau en Afrique $»$, http://www.alternativeniger.org/spip.php?article145\&debut_articles_rubrique $=90$

134. SAYOUR A., " Aspects économiques et financiers du problème des distributions d'eau », RA, 1953, p.367.

135. SCHORN T.J, "Drinkable Water and Breathable Air: A Livable Environment as a Human Right ", Great Plains Natural Resources Journal, 2000, p. 123.

136. SCOTT C., "The interdependence and permeability of human rights norms: towards a 
partial fusion of the International Covenants on Human Rights», Osgoode Hall Law Journal, vol. 27, no 4, 1989; voir en particulier les pages 851 à 878.

137. SHIVA V., Water Wars, Cambridge, South Ends Press, 2001, p.76.

138. SHUVAL Hillel. I. « Le problème du partage de l'eau entre Israel et les Palestiniens A la recherche d'une solution équitable ", Revue Monde arabe-Maghreb-Machrek $\mathbf{n}^{\circ} 138$ oct. - déc. 1992, pp. 34 et s.

139. SIAT G., "La distribution de l'eau potable, un service public à repenser ? ", in JEANCLOS (Y.) (Dir.), Les politiques de l'eau au XXIème siècle, Actes du colloque de Strasbourg, Tome II, CIRPES

140. SIDIBE (D.F.), " La loi d'orientation relative à la gestion de l'eau au Burkina Faso ", in NIASSE (M.), IZA (A.), GARANE (A.), VARIS (O.), La gouvernance de l'eau, UICN, 2004.

141. SIGNOURET (M.), « L'eau, un droit universel ? » Jeune Afrique l'Intelligent, $\mathbf{n}^{\circ} 2248$, du 8 au 14 février 2004.

142. SIRONNEAU J., «Droit et gestion de l'eau, grandes tendances mondiales et applications récentes », RJE, 1998, n³.

143. SIRONNEAU J., Revue juridique de l'environnement, $n^{\circ} 3,1998$, p.317

144. SMETS (H.), « Incorporation du droit à l'eau potable dans l'ordre juridique national ", in Environnement et renouveau des droits de l'homme, Actes du colloque de Boulogne-sur$\operatorname{Mer}$ (20-21 novembre 2003).

145. SMETS H., «Examen critique du PPP », in Les hommes et l'environnement, Mélanges KISS A., Frison-Roche, 1998, p. 79.

146. THEYS J., "L'environnement au XXIe siècle. Entre continuité et ruptures », Futuribles, 1999, n²39-240, p. 12.

147. THIAM A., "Les problèmes de l'eau et d'accès a l'eau potable au Sénégal ", World Citizens Assembly on Water (WCAW), Kuala Lumpur (Malaysia), 27-30 October 2005.

148. THIAM A., "Les problèmes de l'eau et d'accès a l'eau potable au Sénégal », World Citizens Assembly on Water (WCAW), Kuala Lumpur (Malaysia), 27-30 October 2005.

149. THUNIS X., « Le principe du pollueur-payeur : de l'imputation des coûts à la détermination des responsabilités », in Stratégies de développement durable. Développement, environnement ou justice sociale?, 2009, pp. 169 et suiv.

150. TIGNINO M. et YARED D., « La commercialisation et la privatisation de l'eau dans le cadre de l'organisation mondiale du commerce", Revue québécoise de droit international, 2006, p. 178.

151. TREMOLET S., «Un point sur les privatisations de l'eau en Afrique », Annales des mines avril 2006, p. 61.

152. TREMOLET, S., «Multi-utilities and Access », Public Policy for the Private Sector, Note $\mathrm{n}^{\circ}$ 248, Groupe de la Banque mondiale, Washington, D.C., juin 2002.

153. TROUILLY P., "Le principe de la gestion équilibrée de la ressource en eau est-il devenu inutile? », Environnement, 7/2004, p. 7-9.

154. VENNETIER P., "Cadre de vie urbain et problèmes de l'eau en Afrique noire", Annales de Géographie, Année 1988, Volume 97, Numéro 540.

155. VERGNE $F$., " $L$ 'accès à l'eau potable : "privatisation " inéluctable ou pétrolisation coupable ? ", in Libération du 22 septembre 2008.

156. VINCENT Is., «Le prix de l'eau pour les pauvres : comment concilier droit d'accès et paiement d'un service ? », Afrique contemporaine - Printemps 2003, p. 122.

157. VINCENT Isabelle, «Le prix de l'eau pour les pauvres : comment concilier droit d'accès et paiement d'un service ? », Afrique contemporaine - Printemps 2003, p. 122.

158. VIRCOULON $T$., " L'eau gratuite pour tous ? L'exemple de la nouvelle politique de l'eau en Afrique du Sud, Afrique contemporaine " - Printemps 2003. 
159. WETHE J., Rapport: Systèmes énergétiques : Vulnérabilité - Adaptation - Résilience, HELIO International, 2009, p. 12.

160.ZIEGLER J., « Schizophrénie des Nations Unies », Le Monde diplomatique, novembre 2001, p.4.

\section{2-SERVICE PUBLIC ET AUTRES}

1. ARDANT P., «L'égalité des personnes en droit public ou la poursuite de l'insaisissable égalité concrète ", in La personne humaine sujet de droit, PUF, Publications de la faculté de Poitiers, 1994, p. 135.

2. AUBY J.-M., "Contribution à l'étude du domaine privé de l'Administration ", EDCE $1958, \mathrm{n}^{\circ} 12, \mathrm{p} .35$.

3. AUBY J.-M., «L'action domaniale », $A J D A$ 1983, 507.

4. AUBY J.-M., "Le problème de la domanialité publique des immeubles affectés à un service public ", in Mélanges Laborde-Lacoste, 1963, p. 11.

5. AMADEI J.-P., «Vers la constitutionnalisation du domaine public », LPA 9 août 1995, n'95, p. 19.

6. BELLOUBET-FRIER N., "L'égalité ", in Les droits fondamentaux, AJDA, n spécial, 1998, p. 152.

7. BELLOUBET-FRIER N., « Le principe d'égalité », AJDA 1998, p. 152.

8. BEROUJON F., «Le recul de l'établissement public comme procédé de gestion des services publics ", $R F D A 2008$ p. 26.

9. BIOY X., « La propriété éminente de l'Etat», RFDA 2006, 963.

10. BLANC F., "L'insaisissabilité des biens des établissements publics industriels et commerciaux, éléments pour une évolution », RDP, 2009,1553.

11. BRAIBANT G., « Nouvelles réflexions sur les rapports du droit et de l'équité ", Rev. fr. adm. publ. 1992, p. 687.

12. BROUANT J-P., "Domaine public et libertés publiques : instrument, garantie ou atteinte ? » $L P A, 15$ juillet 1994, p.21.

13. CAILLOSSE J., « Service public et concurrence - Le service public entre deux mythologies », Droit ouvrier, avril 2008, p. 199.

14. CHAMPEIL-DESPLATS V., "Services d'intérêt économique général, valeurs communes, cohésion sociale et territoriale », AJDA 1999 p. 959.

15. DE BELLESCIZE R., "Grandeur et servitude de la notion de service public constitutionnel ", $R F D A$ 2006, p. 1163 et s.

16. DELVOLVE P., "Constitution et contrats publics », Mélanges Franck Moderne, Dalloz, 2004, p. 469.

17. DELVOLVE P., «La question de l'application du droit de la consommation aux services publics », DA 1993, n 10, p. 3.

18. DELVOLVE P., "Les contradictions de la délégation de service public », AJDA 1996, p. 681.

19. DELVOLVE P., « Service public et libertés publiques », RFDA 1985, p. 2.

20. DONIER V., «Les lois du service public : entre tradition et modernité », RFDA 2006 p. 1219.

21. DOUENCE J-C., « La gestion du service public local », Rép. coll. loc. Dalloz, 6122-4.

22. DUGUIT L., « La situation des particuliers à l'égard des services publics », RDP, 1907, 411-439.

23. ESPLUGAS P., «A propos de la décision du Conseil constitutionnel de 23 juillet 1996 - Les télécommunications : un nouveau service public constitutionnel », $R A$ 1996, p. 510

24. ESPLUGAS P., «La constitutionnalisation du service public : un tigre de papier ? », RA 
1996, n² 290, p. 162.

25. ESPLUGAS P., « Le service universel », DA 2002, p. 6.

26. FAURE B., « Les droits fondamentaux des personnes morales », RDP 2008, p. 233.

27. FAURE B., « Les objectifs de valeur constitutionnelle : une nouvelle catégorie juridique ?», RFDC 1995, p. 47.

28. FAURE B., "Remarques sur les mutations du principe de la liberté de commerce et de l'industrie », RFDA 2004, p. 299.

29. FAVRET J.-M., « Le renforcement du rôle des autorités nationales dans la mise en œuvre du droit communautaire de la concurrence », AJDA 2004, p. 177.

30. HASTINGS M., "Les constellations imaginaires du service public », in S. DECRETON (dir.), Service public et lien social, L'Harmattan, 1999, p. 33.

31. HENNEBEL L., " Les droits intangibles », in E. BRIBOSIA et L. HENNEBEL (dir.), Classer les droits de l'homme, Bruylant, 2004, p. 195.

32. HENNION-MOREAU S., "Services sociaux et concurrence », RDSS 2006, n 5, p. 801.

33. HERTZOG R., Le prix du service public, AJDA 1997 p. 55

34. HERVOUET F., « Clarifier le régime du service public : la nécessité d'une directive cadre ? ", in H. PAULIAT (dir.), Service public, concurrence, régulation : le grand bouleversement en Europe ?, Colloque EUROPA, PULIM, 2008, p. 131.

35. IMBERT P.-H., « Droits des pauvres, pauvres droits, Réflexions sur les droits économiques, sociaux et culturels », RDP 1989, p. 739.

36. JACOBS N., « La portée juridique des droits économiques, sociaux et culturels », RBDI 1999, p. 19.

37. JACQUEMET A., " Monopoles des jeux de hasard et ordre public », AJDA 2007, p. 1282.

38. JEANNIN L., "Le principe de dignité en droit allemand ", in C. GIRARD et S. HENNETTE- VAUCHEZ, La dignité de la personne humaine, Recherche sur un processus de juridicisation, PUF, 2005, p. 169.

39. LEONETTI R., " La cession des immeubles publics », AJDA 2010, 2463.

40. MADIOT Y., " Service public et aménagement du territoire ", $A J D A$ 1997, p. 83.

41. MELLERAY F., «Ecole de Bordeaux, école du service public et école duguiste », RDP, 2001.1887

42. MESTRE J-L., « La notion de service public d'après les débats de l'Assemblée nationale constituante ", $E D C E, n^{\circ} 40,1989$, p. 187 et ss.

43. MODERNE F., "Les mutations des services publics en droit français ", in Service public et Communauté européenne : entre l'intérêt général et le marché, sous la direction de R. KOVAR et D. SIMON, La Documentation française, Tome 1, 1998, p. 3

44. MONPION A., «Le contrôle de l'interventionnisme économique public : l'affaiblissement du principe de liberté du commerce et de l'industrie ? " $A J D A 2008$ p. 232 et $\mathbf{s .}$

45. NIZARD L., «A propos de la notion de service public : mythes étatiques et représentations sociales », in Mélanges EISENMANN, CUJAS, 1975, p. 90.

46. PELISSIER G., « Le principe d'égalité en droit public », coll. Systèmes, LGDJ, 1996.

47. PIZZORUSSO A., "Le principe d'égalité dans la doctrine et dans la jurisprudence italiennes ". In : Sur le principe d'égalité", Extrait du Rapport public 1996, Paris, La documentation française, 1998, p. 209-219.

48. PLANE P. et LESUEUR J-Y., «Les services publics subsahariens : importance socioéconomique et évaluation des d'assainissement ", Tiers-Monde, 1994, Volume 35, ${ }^{\circ} 40$, p. 780.

49. RIVERO J., "Les deux finalités du service public industriel et commercial ", CJEG, 1994.

50. ROUBAN L., «Le service public face à l'opinion: la fin des clivages idéologiques ? ", 
Rev. $a d m .1998, \mathrm{n}^{\circ}$ 305, p. 645-652.

51.SANDEVOIR $P$., «Les vicissitudes de la notion de service public industriel et commercial ", Mélanges STASSINOPOULOS, p. 317

52. STIRN B., " La conception française du service public ", CJEG 1993, p. 299.

53. THIRY B., "Les conceptions de l'intérêt général dans l'Union Européenne ", EDCE, n'50, 1999, p. 403.

54. THOMAS I., « Le principe de participation des usagers au fonctionnement des services publics ", RFDA 2004 p. 330.

55. TRUCHET D., « Unité et diversité des grands principes du service public », $A J D A 1997$ p. 38

56. VEROT C., «L'utilité sociale sans le service public : à propos de la mission assurée par le gestionnaire privé d'un centre d'aide par le travail ", Revue de droit sanitaire et social 2007, p. 499.

57. VOISSET M., « Le service public autrement », RFDA 1995 p. 304.

\section{3-DROITS DE L'HOMME ET CONTRIBUTIONS APPARENTEES}

1. ABI-SAAB G., «La souveraineté permanente sur les ressources naturelles" in Droit international, Bilan et perspectives, éd. Pedone, Paris, 1991, Tome II, pp 639-661.

2. AHADZI K., "Droits humains et développements, théories et réalités", Revue Nigérienne de droit, $\mathrm{n}^{\circ} 3$, Décembre 2000, p. 51.

3. AUER A., "Les droits fondamentaux et leur protection ", in Pouvoirs $n^{\circ} 43$, La Suisse, pp. 87 ss

4. BADINTER R. et GENEVOIS B., "Normes de valeur constitutionnelle et degré de protection des droits fondamentaux", Rapport présenté par la délégation française à la VIIIème conférence des Cours constitutionnelles européennes (Ankara, 7-10 mai 1990), RFDA, 1990, pp. 317 s., spéc. pp. 331 s.

5. BADINTER R. et GENEVOIS B., «Normes de valeur constitutionnelle et degré de protection des droits fondamentaux », rapport présenté par la délégation française à la VIIIème conférence des Cours constitutionnelles européennes (Ankara, 7-10 mai 1990), RFDA, 1990, pp.

317 s., spéc. pp. $331 \mathrm{~s}$.

6. BASLAR K., The concept of the common heritage of mankind in international law, 1998, The Hague/Boston.

7. BERNARD M., « Existe-t-il un droit administratif ivoirien ?», Rev. Ivoirienne de droit 1970, $\mathrm{n}^{\circ} 4, \mathrm{p} 11$.

8. BIPOUN Woum, "Recherches sur les aspects actuels de la réception du droit administratif dans les Etats d'Afrique noire d'expression française : le cas du Cameroun », RJPIC 1972, p.359.

9. BOILLOT J-J., "Libéralisation sous contraintes : dette publique et dette extérieure ", Tiers-Monde, Année 1989, Volume 30, Numéro 119, p. 513 - 543

10. BORGETTO M., "L'irrésistible ascension des " "droits à" " ..., Informations sociales, n'81 pp. 4-16, 2000

11. BREILLAT D., « La hiérarchie des droits de l'homme » in Mélanges Philippe ARDANT, Droit et Politique à la croisée des cultures, Paris, LGDJ, 1999, pp. 353 s.

12. BURCHI S. et MELCHEM K., Groundwater in international law: compilation of treaties and other legal instruments, Rome, FAO \& UNESCO, 2005.

13. BURGORGUE-LARSEN L. (dir.), «Chronique de jurisprudence européenne comparée ", RDP, n॰3, 2001, p.709.

14. C.E.D.E.A.O., U.C.R.E., Observatoire Régional de l'Eau en Afrique de l'Ouest, Rapport final, Décembre 2006, 43 p. 
15. CANTERO A. et S. BOYTCHEV S., «Quelles avancées juridiques en matière " d'administration électronique »?,JCPA 7 nov. 2005 , p. 1639.

16. CARTON O., «De l'inutilité d'une constitutionnalisation du droit de l'environnement ? » in les Les Petites Affiches, $n^{\circ} 175,2005$, p. 3.

17. CHAMPEIL-DESPLATS V., «La liberté d'entreprendre au pays des droits fondamentaux ", Revue de droit du travail 2007, p. 19.

18. CHAVANE Bruno, «Bilan et perspectives des privatisations en Afrique francophone: Une étape de la démocratisation? »

19. CONAC G., " Le juge et la construction de l'Etat de droit en Afrique francophone », in Mélanges Guy Braibant, Paris, Dalloz, 2002, p.105-119.

20. DARBON D., " Le juge africain et son miroir : la glace déformante du transfert de jurisprudence administrative », in La justice en Afrique, Afrique contemporaine, 1990, $\mathrm{n}^{\circ} 156$ spécial, p.24.

21. DE BECHILLON D., «De quelques incidences du contrôle de la conventionalité internationale des lois par.

22. DECEZE D., Services publics, la grande braderie, Jean-Claude Gawsewitch Editeur, 2007.

23. DEL REY M.-J., «La notion controversée de patrimoine commun», ${ }^{\circ}$ 6, 2006, Recueil Dalloz, p. 388.

24. DELALOY G., « Faut-il supprimer le principe de l'inaliénabilité du domaine public ? », RDP 2006.575.

25. DELMAS-MARTY M., «Le crime contre l'humanité, les droits de l'homme et l'irréductible humain ", $R S C(3), 1994$, p.

26. ECOLE NATIONALE D'ADMINISTRATION, « La gestion de l'eau. Pour de nouvelles solidarités ", Actes du colloque Agro-ENA-X du 18 décembre 2003, Regard International, Paris, 2004

27. ENCOUA D., FLOCHEL L., «La tarification : du monopole à la concurrence régulée ", $A J D A$ 1997, p. 254.

28. FISETTE J. et SALMI M., « Le désengagement de l'État en Afrique et les initiatives locales : la naissance de nouvelles formes de partenariat. ", Cahiers de géographie du Québec, vol. 35, n 95, 1991, p. 350.

29. FLAUSS J-F., "Le droit international des droits de l'homme face à la globalisation économique ", in Commerce mondial et protection des droits de l'homme, Bruylant, 2001, p. 225.

30.FLAUSS J-F., Le droit international des droits de l'homme face à la globalisation économique, in Commerce mondial et protection des droits de l'homme, Bruylant, 2001, p. 225

31. FOYER J., «Les destinées du droit français en Afrique », Penant, 1962, p.6.

32. G. MARCOU, « Le bilan en demi-teinte de l'acte II. Décentraliser plus ou décentraliser mieux ? ", RFDA, 2008- 3. 295

33. GARAPON A., "Le sujet de droit ", Revue interdisciplinaire d'études juridiques, 1993, p. 69.

34. GAUTRON (J.C.), " Réflexions sur l'autonomie du droit public sénégalais », Annales Africaines , 1969, p.30;

35. GHEZALI M., Les nouveaux droits fondamentaux de l'homme, www.cidce.org/pdf/livre\%20rio/.../ghezali.pdf.

36. GREWE C., « Les droits sociaux constitutionnels », in : Constance GREWE et Florence BENOIT-ROHMER, Les droits sociaux ou la démolition de quelques poncifs, Strasbourg, Presses Universitaires de Strasbourg, 2003.

37. GUGLIELMI G. J., La notion d'administration publique dans la théorie juridique 
française. De la Révolution à l'arrêt Cadot (1789-1889), LGDJ, coll. "Bibliothèque de droit public ", tome 157, 1991, p. 265.

38. HAYEK F., Droit, législation et liberté, Tome 2 : Le mirage de la justice sociale, Quadrige-PUF, 1995, 221p.

39. HAYEK F., La route de la servitude, PUF, 1985, pp. 66-67.

40. HOUILLON G., et Jean RIVERO, «Démocratie et administration ", RFDA 2009 p. 1057.

http://www.ilo.org/public/french/employment/ent/papers/ippred1.htm

41. JACQUEMOT P., «La désétatisation en Afrique subsaharienne. Enjeux et perspectives ", Tiers-Monde, 1988, volume 29, $\mathrm{n}^{\circ} 114, \mathrm{p} .271$ et s.

42. KADDAR M., STIERLE F., SCHMIDT-EHRY B., et TCHICAYA A., " L'accès des indigents aux soins de santé en Afrique subsaharienne ", Tiers-Monde, Année 2000, Volume 41, Numéro 164, p. 903 - 925

43. KDHIR M., «Le principe de la liberté du commerce et de l'industrie : mythe ou réalité ? ", Recueil Dalloz 1994 p. 30 et s.

44. KOUBI G., «La pauvreté, comme violation des droits humains », Revue internationale des sciences sociales, 2004/2 - $\mathrm{N}^{\circ} 180$, p. 361.

45. KPODAR A., "Les droits catégoriels : philosophie et spécificité ", Revue béninoise des sciences juridiques et administratives, $\mathrm{n}^{\circ} 18,2007, \mathrm{p} .46$.

46. LARBI BOUGUERRA M., Les Rapports de l'Institut Veolia $n^{\circ} 5$ : Symbolique et culture de l'eau, p. 5.

47. LEBRETON G., Y-a-t-il un progrès du droit ? Dalloz 1991, c. 99

48. LETOURNEUR M., Les principes généraux du droit, EDCE, 1951.

49. M. MCFARLAND SANCHEZ-MORENO et T. HIGGINS, «No recourse : Transnational Corporations and the Protection of Economic, Social, and Cultural Rights in Bolivia", Fordham International Law Journal, vol 27 (2004), no 5, pp. 1663-1805., spéc. p. 1729.

50. MALAFOSSE de J., Le droit à la nature, Montchrestien, Paris, 1973

51. MALJEAN-DUBOIS S., «Le foisonnement des institutions conventionnelles" in IMPERIALLI Claude, L'effectivité du droit international de l'environnement, Economica, Paris, 1998, p. 41.

52. M'BAYE K., "Les droits de l'homme en Afrique", Revue internationale de droit comparé, Année 1993, Volume 45, Numéro 3, p. 723 - 726.

53. MESTRE J. -L., « Le Conseil constitutionnel, la liberté d'entreprendre et la propriété ", D. 1984. Chron.1.

54. MEYER-BISCH P., « Approche multidimensionnelle de l'effectivité des droits humains. Une fonction spécifique pour les droits culturels? ". Colloque sur l'effectivité des droits économiques, sociaux et culturels, fin et moyen de développement, Cotonou, du 18 au 20 novembre 2004, pp 2-3.

55. MICHAUD E., « Les études d'impact des politiques économiques internationales sur les droits de l'homme. » In A la recherche de l'effectivité des droits de l'homme, Paris, Presses Universitaires de Paris 10, 2008, p. 231.

56. MORALES V., "La protection juridictionnelle des droits fondamentaux : révélation d'une entente conceptuelle ", Congrès français du droit constitutionnel du 9, 10 et 11 juin 2005, Montpellier, 2005.

57. NGUEBOU TOUKAM J., «Les droits des femmes dans les pays de tradition juridique française ", Presses Universitaires de France, L'Année sociologique 2003/1 - Vol. 51, pages 89-108

58. NLEP R. G., «Le juge de l'administration et les normes internes, constitutionnelles ou infra constitutionnelles en matière de droits fondamentaux ", SOLON, Vol. I, n 1, 1999, pp. 135- 149. p.211. 
59. OLOWU D., «Evaluation des politiques de décentralisation en Afrique dans les années quatre-vingt-dix », R.F.A.P, ${ }^{\circ} 88$, octobre-novembre 1998, p.516.

60. OMC, Rapport mondial sur le commerce 2010, «le commerce des ressources naturelles ", p. 5, 46, 49, 67.

61. PACTEAU B., "La sécurité juridique, un principe qui nous manque? ", $A J D A, \mathrm{n}^{\circ}$ spécial juin 1995, p. 151.

62. PAVIA M. -L., "Eléments de réflexion sur la notion de droit fondamental ", Petites Affiches 6 mai 1994, pp. 6-13.

63. PELlOUX R., "Vrais et faux droits de l'homme. Problèmes de définition et de classification », RDP, 1981, pp. 53-68.

64. PICARD E., "Droits fondamentaux », in Dictionnaire de la culture juridique, Paris, PUF, 2003, p. 544.

65. PICARD E., L'émergence des droits fondamentaux en France, AJDA 1998, $\mathrm{n}^{\circ}$ spécial, pp. 6 ss.

66. PIERRE-CAPS S., "La constitution comme ordre de valeur », in Mélanges LAVROFF, Paris, Dalloz, 2005, p. 283.

67. POIRMEUR Y. et ROSENBERG D., La doctrine constitutionnelle et le constitutionnalisme français, in Les usages sociaux du droit, CURAPP, Paris, PUF, 1989, p. 242.

68. QUIRINY B., « Les droits de l'usager face au droit du marché », RFDA 2008 p. 20.

69. RACINE J-B, "L'ordre concurrentiel et les droits de l'Homme ", Mélanges en l'honneur d'A. PIROVANO, Edition Frison-Roche, 2003, p. 420

70. RACINE J-B, «L'ordre concurrentiel et les droits de l'Homme », Mélanges en l'honneur d'A. PIROVANO, Edition Frison-Roche, 2003, p. 420.

71. RIDEAU J., « La coexistence des systèmes de protection des droits fondamentaux dans la Communauté européenne et ses Etats membres ", AIJC, 1991, p.12.

72. RIVERO J., «Droit public et droit privé : conquête ou statu quo ? », D. 1947, Chroniques $X X V$, p. 69.

73. ROSENBAUM A., "Gouvernance et décentralisation : leçons de l'expérience ", R.F.A.P, n 88 , octobre-novembre 1988, p.507.

74. ROUSSEAU D., "Synthèse", in Le principe du respect de la dignité de la personne humaine: actes du Séminaire UniDem organisé à Montpellier du 2 au 6 juillet 1998 en coopération avec le Pôle universitaire européen de Montpellier et du Languedoc-Roussillon et la Faculté de droit - Commission européenne pour la démocratie par le droit, Strasbourg, Ed. du Conseil de l'Europe, 1999, pp. 109-111

75. ROUSSEAU, « La jurisprudence administrative à Madagascar », Penant 1971, p.281.

76. ROUSSELET A., La règle de l'inaliénabilité du domaine de la Couronne.Etude doctrinale de 1566 à la fin de l'Ancien Régime, Trav. Rech., Paris II, LGDJ, 1997.

77. SAKHO P. O., "La politique de privatisation du gouvernement : enjeux et perspectives ", Revue du Conseil économique et social $\mathrm{n}^{\circ}$ 2, févr.-avr. 1997, pp. 20-22.

78. SALES E., "Vers l'émergence d'un droit administratif des libertés fondamentales ?", $R D P, n^{\circ} 1,2004$,

79. SAVY F., La constitution des juges, D. 1983. Chron. 105.

80. SIMONIAN-GINESTE H., "L'avenir du principe de l'inaliénabilité du domaine public », Revue de droit immobilier, 1989, p. 169.

81. SINDJOUN (L.), « Esquisse de théorie de droit administratif camerounais », Penant, 1993, n ${ }^{\circ} 813$, p.330-390.

82. SIRINELLI M. et SORIN J., Questions de droit public, Ellipses, 2007, p. 164.

83. SMETS H., «Examen critique du PPP », in Les hommes et l'environnement, Mélanges KISS A., Frison-Roche, 1998, p. 79. 
84. SOLEIL S. et JAUME L., Centralisation/Décentralisation. Retour sur quelques certitudes historiques, $A J D A, 2005.760$.

85. TIZIO S., Entre Etat et marché. Une nouvelle régulation sanitaire pour les pays en développement? Tiers-Monde, Année 2004, Volume 45, Numéro 179, p. 643 - 663.

86. TURPIN D., République et décentralisation, AJDA 2003 p. 1577.

87. VIGNON Y. B., "La protection des droits fondamentaux dans les nouvelles constitutions africaines ", Revue nigérienne de droit, $\mathrm{n}^{\circ} 3$, déc. 2000, p. 80.

88. VIGNON Y., Réflexions sur la privatisation des entreprises publiques en Afrique, Annales de l'Université du Bénin, Série Droit Economie, Tome XVI, 1996, p. 214.

89. WACHMANN P., L'importation en France de la notion de droits fondamentaux, RUDH, 2004.

90. WETHE J., Rapport : Systèmes énergétiques : Vulnérabilité - Adaptation - Résilience, HELIO International, 2009, p. 12.

91. WILLYBIRO-SAKO J., "Des principes et convictions à une véritable protection juridique en Afrique», in MAUGENEST Denis et POUGOUE Paul-Gérard (dir.), Droits de l'Homme en Afrique Centrale, Colloque de Yaoundé (9-11 novembre 1994), Yaoundé, éd. Karthala, Presse de l'UCAC, 1994, pp.104-105.

92. YEM GOURI M., « Bilan de l'unité du droit administratif dans les pays d'Afrique noire francophone », Penant, 1988, nº797, p.293.

\section{SOURCES WEB}

- http://credof.u-paris10.fr/spip.php?article52

- http://wdsbeta.worldbank.org/external/default/WDSContentServer/IW3P/IB/2005/03/06/0 00090341_20050306101429/Rendered/PDF/wps3514.pdf

- http://www.academie-eau.org/IMG/pdf/DroitAlEau_01.pdf

- http://www.aidh.org/onu_ge/commission/62/images/bandeau_62.gif

- http://www.alternativeniger.org/spip.php?article145

- http://www.monde-diplomatique.fr/carnet/2008-03-19-La-marchandisation-de-l-eau

- http://www.sec.ecowas.int/sitecedeao/francais/adec12122000.htm

- http://www.un.org/french/waterforlifedecade/unwater.html.

- http://www.uneca.org/water/french.htm

- http://www.unesco.org/water/wwap/index_fr.shtml

- http://www.unesco.org/water/wwap/milestones/index_fr.shtml. (28/06/08).

- http://www.worldwaterday.org/thematic/hmnrights.html

- www.droits-fondamentaux.org

- www.france-libertés

- www.oecd.org/dev/reperes

- www.unfpa.org/swp

- www.world-psi.org. 


\section{Index thématique}

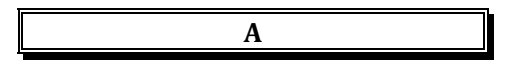

Accès à l'eau, un enjeu d'intérêt général

Action $21 \quad 45,260,261,287,289,290$

Admissible gratuité de la distribution de l'eau 155

Afrique du Sud $37,49,50,81,93,151,156,157,181$, $207,214,217,232,249,250,308,328,331$

Afrique subsaharienne $1,10,32,33,35,36,38,46,47$, $49,50,51,52,54,55,56,58,69,70,72,73$,

$76,77,78,80,82,83,86,87,88,90,91,92$, $96,102,114,115,119,125,126,130,140$ $142,145,146,148,149,155,167,170,182$, $183,185,186,187,188,196,203,206,218$, $219,222,225,236,242,244,248,250,257$ $258,259,270,271,273,277,296,302,305$, $306,322,328,329,330,336$

AGCS ou en anglais GATS pour General Agreement on Trade in Services.

Ajustement structurel

$74,82,86$

Alberti-Scott

$101,102,104,106,107$

Apport du droit international de l'eau 270, 283

arrêt Blanco

Art. 4 de la Déclaration de 1789

Art. 18 de la loi n $2010-006$ du 18 juin 2010 au Togo

Art. 44 alinéa 2 du code de l'eau de la République du Mali

Article 11 de la constitution de la IV ème $^{\text {è }}$ République du Togo

Article 27 de la loi $\mathrm{n}^{\circ} 81-13$ du 4 mars 1981 portant Code de l'Eau au Sénégal XE "Sénégal" .

Article 59 du Code de l'eau XE "Code de l'eau" en vigueur au Togo XE "Togo" .

Article 69 du code l'eau en vigueur au Mali

Article 76 de la loi ${ }^{\circ} 98-755$ du 23 décembre 1998 en Cote d'Ivoire.

Article 76 de la loi n $98-755$ du 23 décembre 1998 portant Code de l'Eau en Cote d'Ivoire.

Autogestion de l'eau

Autorités décentralisées

Autorités signataires

Autres cas de délégation

\section{B}

Banque Mondiale

Bénin $38,42,50,76$

Bretton Woods $38,42,50,76,93,176,207,259,327,338$
$10,35,56,71,208$

Burkina Faso $\quad 42,47,49,59,81,94,145,181,229,255$, $305,319,322,329,331$

\section{C}

CAA Marseille, 13 déc. 2001, Union fédérale des consommateurs
CAA Marseille, 4 juill. 2005

117

Cahier des charges

CE 12 nov. 2001, Commune de MontreuilBellay

CE 21 avr. 1961, Dame Agnesi,

CE 21 avr. 1961, Dame Agnési, Lebon,

CE 22 juin 1963, Synd. du personnel soignant de la Guadeloupe

CE 22 mai 2003, Commune de Théoule-sur$\mathrm{Mer}$

CE 6 mai 1931, Tondut

CE 6 mai 1931, Tondut, Lebon

CE 7 mai 1982, Verdier

CE ass. 10 avril 1996, Cayzeele,

CE Sect., 13 janv. 1961, Département du BasRhin, Lebon

CE, 10 janv. 1902, Cie nouvelle du gaz de Déville-Lès-Rouen.

CE, 13 mai 1977, Cie industrielle et financière des autoroutes

CE, 15 avril 1996, Préfet des Bouches-duRhône c/ Commune de Lambesc

CE, 19 juin 1964, Société des Pétroles Shell Berre,

CE, 19 octobre 1956, Société Le Béton

CE, ${ }^{\text {er }}$ février 1989, Socea-Balency

$\mathrm{CE}, 1^{\mathrm{er}}$ avril 1994, Commune de menton.

CE, 20 janvier 1964, Chambre syndicale des compagnies de change de Lyon et de Marseille

CE, 20 janvier 1964, Chambre syndicale des compagnies de change de Lyon et de Marseille, Rec. 20. ;

CE, 20 juillet 1990, Ville de Melun.

CE, 21 déc. 1906, Synd. des propriétaires et contribuables du quartier Croix- de Sergueytivoli.

CE, 22 novembre 1974, Fédération des industries françaises des articles de sport.

CE, 24 avril 1989, Sté Monégasque des entreprises $J$-B Pastor et fils

CE, 24 janv. 1990, Martinettti

CE, 28 octobre 1960, Martial de Laboulaye.

CE, 30 mars 1916, Cie d'éclairage de Bordeaux

CE, 7 avril 1916, Astruc.

CE, 9 février 1979, Société d'encouragement pour l'amélioration des races de chevaux.

CE, Ass., 10 juillet 1996, Sté Direct Mail Promotion,

CE, L'eau et son droit, Rapport du CE 2010, La documentation, 2010

CE, L'eau et son droit, Rapport du CE 2010, La documentation, 2010, p. 217.

CE, Sect., 13 oct. 1961, Sté des Ets CompanonRey,

Centre Européen de Droit et de

l'environnement.

Changements climatiques
128

61

108

101

63

61

101,306

101,306

103

123

101

119

123

124

76

78

121

116

64

64

116

123

79

121

123

64 
Charte africaine des droits de l'homme et des peuples

Charte des eaux du fleuve Sénégal

Chronique ONU

$17,37,110,200$

CNUCED

$22,228,229,273$ $274,276,277$

Code de l'eau du Mali

277

cohésion sociale et territoriale

$163,164,332$

Collectivités locales $\quad 16,40,73,88,150,257,259,260$, 322,324

Comité des droits économiques, sociaux et culturels $17,24,25,27,30,220,303$

concept de la qualité du service public 165

concession $\quad 33,73,76,83,90,112,114,117,118,119$, $120,121,124,125,126,127,128,129,133$, $134,135,140,141,147,192,202,211,221$, $276,277,312,322$

Conférence de Mar Del Plata

Conférence des Nations-Unies relatives aux établissements humains (Habitat II)

Conférence internationale de Nairobi

Consensus de Washington

Continuité territoriale

contours de la délégation

contrat d'affermage

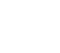

31

32

$170,180,258$

148,149

116 contrat de délégation de service public de l'eau 128

contrat intuitu personae

contrôle de la délégation de service public $\quad 130$

contrôle de légalité du contrat de délégation de service public

contrôle précédant la signature de la délégation

Convention africaine pour la conservation de la nature et des ressources naturelles

Convention d'affermage

Convention relative aux droits de l'enfant

Convention sur l'élimination de toutes les formes de discrimination à l'égard des femmes

Côte d'Ivoire $\quad 50,93,118,126,167,174,181,199,219$, $226,236,273,324$

\section{D}

Décentralisation $73,125,206,257,258,259,260,262$, $314,321,322,337,338$

Déchéance

Déclaration de Beijing.

$72,77,125,133,134,135$

Déclaration de Doha

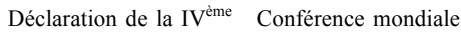
40,212 sur les femmes (1995). Beijing, article 2561.

Déclaration des droits de l'homme et du citoyen du 26 août 1789 .

Déclaration économique des Non alignés 227

Déclaration universelle des droits de l'Hom $102,112,113,114,115$ $117,120,124,125$

Développement durable $\quad 39,42,45,52,87,95,96,131$, $199,227,230,248,249,261,285,287,289$, $290,291,306,318,321,323,324,331$

Développement humain $\quad 23,37,145,200,207,215,216$, 270, 296, 306, 319

Dignité humaine $\quad 27,45,262,286,292,296,305,315$

Dimension économique de l'eau

Directive $\mathrm{N}^{\circ} 5 / 2005 / \mathrm{CM} / \mathrm{UEMOA}$ distribution d'eau potable $\quad 37,41,76,97,119,146,183$, $188,258,308,318,322,326$

distribution de l'eau $25,32,33,34,37,50,74,77,78$, $79,81,86,98,99,102,104,105,106,116$,

$125,140,151,155,166,170,172,180,183$, $184,185,186,206,208,210,243,245,247$, $250,255,257,260,262,270,302,331$

Domaine public de l'eau $127,272,273,274,275,276$

Droit à l'eau dans les instruments $1,10,22,23,24,25$,

$26,27,28,29,30,31,32,33,34,35,36,39$,

$40,41,42,43,44,45,46,47,48,49,50,51$,

$52,54,64,65,67,68,70,87,91,98,103$,

$121,128,130,150,163,164,172,174,185$,

$187,189,200,201,206,208,215,216,218$,

$228,236,239,240,242,243,244,245,249$,

$250,252,262,264,270,271,273,281,283$,

287, 288, 292, 296, 298, 302, 303, 304, 306,

$307,309,317,320,323,327,329,331$

droit à l'information

Droit au logement

droits des délégataires

droits fondamentaux $26,29,34,45,46,47,51,52,59$,

$60,64,68,69,70,163,164,233,249,305$,

$312,313,315,316,322,332,333,334,335$,

$336,337,338$

droits naturels, inaliénables et sacrés de

l'homme

303

droits-créances

$35,215,309,322$

\section{E}

Eau potable

$10,22,23,24,26,27,28,31,33,34,36$,

$37,38,39,41,42,45,48,49,50,54,58,74$,

$76,77,79,80,81,86,87,88,89,91,92,93$,

$94,97,103,106,119,121,127,128,130$,

$131,141,145,146,147,148,150,153,156$,

$157,163,166,174,180,181,183,185,186$,

$187,188,189,190,196,203,206,207,208$,

$210,211,214,216,222,231,233,240,242$,

$244,246,247,249,255,256,257,258,259$,

$260,262,271,281,284,285,286,288,296$,

$302,303,308,317,318,319,320,321,322$,

$323,326,327,328,329,330,331$

Ecole de Bordeaux

effectivité du droit à l'eau $1,46,47,49,50,51,52,65$, $130,236,239,240,242,243,244,252,270$, 298

enjeux des ressources en eau

283

étatisation pragmatique du secteur de l'eau

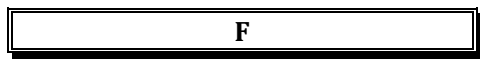

Forum mondial de l'eau

82,181

\section{G}

Galbraith Pierre LE MASNE

Gambie

Gestion privée du service public de l'eau

Gratuité de l'eau

Guinée

Guinée-Bissau

$209,222,229$

51,329

51,329
50,249

140

$63,181,207$ 


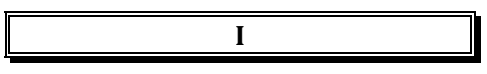

insaisissable principe de gratuité

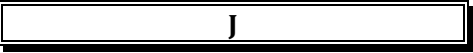

Jurisprudence Thomas

102,107

\section{$\mathbf{L}$}

la hiérarchie des droits fondamentaux

Libéralisation du secteur de l'eau 1, 32, 33, 40, 46, 51 , $52,53,111,112,113,172,173,179,180$ $188,190,205,236,242,275,306$

Liberté d'entreprendre $35,46,50,52,54,58,59,60,61$, $62,63,64,65,66,67,68,69,70,71,243$, 255

Libertés économiques $\quad 35,59,60,61,63,64,67,68,69$, 172,312

Loi N 97-028 du 15 janvier 1999 au Bénin XE "Bénin".

Loi n ${ }^{\circ} 002-2001 / \mathrm{AN}$ du 8 février 2001 portant loi d'orientation relative à la gestion de l'eau.

Loi n88-14 du 3 novembre 1988 instituant Code de l'environnement en vigueur au Togo XE "Togo" .

Lois de Rolland

140

lois du service public $140,141,142,155,157,158,159$ $160,161,162,165,166,306,332$

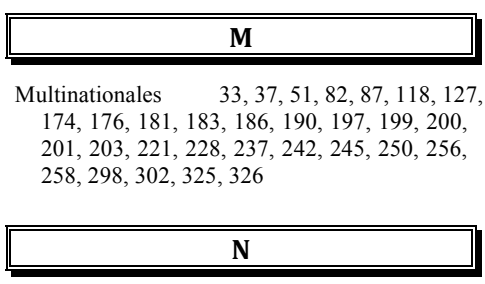

nationalisation $61,250,254,255,256,266$

Nationalisation

254

Nations unies,

248

Nations unies, Actions 21 du 13 juin 1992

Nations-Unies, Sommet mondial de

l'alimentation (1996). Déclaration et plan d'action, Rome.

Nature du lien de délégation

122

Niddam

108

Niger $\quad 16,19,35,50,76,80,81,82,91,103,168,181$, $207,230,249,305,324$

Nigéria

\section{$\mathbf{0}$}

Objectifs de développement du Millénaire.

Observation $n^{\circ} 15$

OCDE, Coopération pour le développement, Rapport 1986

OMC $33,39,146,177,208,215,221,225,226,227$, $228,308,320,337$
ONU, Conseil économique et social, comité des droits économiques, sociaux et culturels, 22ème session, 11-29 novembre 2002.

ONU, Haut Commissariat aux droits de l'Homme

Opérations de restructuration des secteurs publics et parapublics

organisations non gouvernementales $\quad 36,218,260,261$

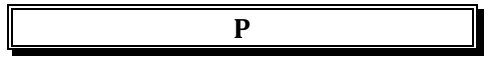

Parlement néerlandais

Partenariat public-prive

255,256

Plan National Eau Potable et Assainissement

PNUD 37, 38, 174, 196, 200, 214, 215, 216, 319

PNUE et les ressources en eau 289

principe d'accessibilité en pointillés $\quad 162$

principe d'égalité difficile à appliquer 144

Principe d'information 262

principe de continuité $\quad 112,143,148,149,159,161,236$

principe de la non-gratuité des services publics $\quad 152$

Principe de participation $161,262,264,265,266,292,334$

principe du pollueur-payeur $\quad 54,91,94,95,96,97,331$

Protocole à la convention d'Helsinki 39

Protocole de San Salvador

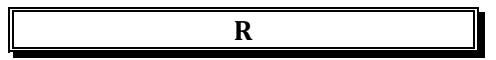

Rapport BRUNDTLAND.

Rapport d'information de M. Jean GLAVANY

Rapport d'Amnesty International,

RAPPORT DE LA CONFERENCE DES

NATIONS UNIES

L'ENVIRONNEMENT ET LE

DEVELOPPEMENT

RAPPORT MONDIAL SUR LE DÉ VELOPPEMENT HUMAIN 2006, Chapitre II

Rapport mondial sur le développement humain

XE "Développement humain" 2002 du PNUD.

Rapport sur le développement humain XE

"Développement humain" 2010.

réchauffement climatique

régie intéressée

régime d'utilisation et d'exploitation

Res communis

rupture de l'égalité devant et dans le service

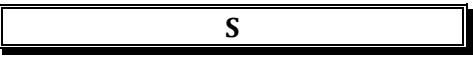

sanctions non pécuniaires

135,136

sanctions pécuniaires

134,135

Sénégal $\quad 18,42,46,50,59,75,81,119,120,126,135$

$146,167,174,181,192,199,207,228,255$,

$257,273,277,303,305,323,329,330,331$

service public constitutionnel $74,244,250,252,253,332$

$\begin{array}{lr}\text { service public d'intérêt local } & 243,257\end{array}$

\section{$\mathbf{T}$}

T. confl. 22 janv. 1921

TA Dijon, 9 mai 1995, Mme Bessis, 
TA Marseille, 12 avril 1994, Porelli,

123

Tarification $\quad 25,54,87,91,92,93,94,107,110,142$ $143,153,176,187,188,307,308,309,335$

TC, 2 mai 1977, Paraïso/Sté Lyonnaise des eaux

TC, 22 avril 1985, Laurent.

TC, 6 juin 1989, Préfet de Paris de la région Ilede-France.

22, 94, 96, 97, 119, 125 $127,128,129,207,229,236,258,259,273$,

$274,276,277,323,330$

\section{Index des auteurs}

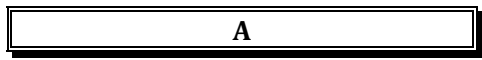

A UCOC L.

A. PELLET

ABDOULAYE M. M.

ABIKHZER F

ABI-SAAB G.

ABI-SAAB R.

ABOUBAKAR A.

312,321

324,334

ADOUKI D.E.

AHADZI K.

AHOULOUMA B. F.

AKUE S.A.

ALACHI E. K.

ALBUQUERQUE (de) C.

ALFANDARY E.

ALLAND D.

ANDREW C.

ARCARI M.

ARDANT P.

ARNAUD A.

ARTAUD A.

ATONTSA M. J. C

321

324

312

56,334

321

321

207, 321

324

317

312

86,330

325

312,332

312

191,312

240,321

AUBERT J-L.

AUBIN E.

AUBY J.-F.

AUBY J-B.

AUER A.

AUTIN J.-L.

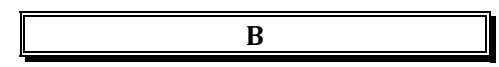

BADINTER R.

BANGOURA D.

BARBERIS J. A.

BARLOW M.

BASLAR K.

BASTIN J.

BATTIFOL $\mathrm{H}$.

BELAIDI N

BENVENISTI E.

BEROUJON F.

BERTHIAU D.

BETHEMONT Jacques

BETTINGER C.

BIED-CHARRETON M.
47,334

325

$176,317,325$

281,334

325

312

$279,281,282,317,325$

325

$191,192,194,321,332$

$30,312,317$

325

312

87,325

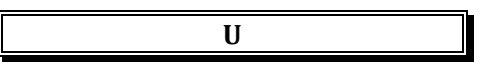

UNESCO et l'éthique de l'eau $95,96,208,291$

Union Européenne

263

Usager du service public

utilisateur-payeur

54,92

BIGOT C

BILLAUDOT F.

BILLET P.

BIRRAUX D.

BLANCHON David

BLOMQUIST W.

BOEGLIN-NAUMOVIC N.

BOISSON de CHAZOURNES L.

BOISSON de CHAZOURNES L.,

BOITEAU C.

BONNARD $\mathrm{H}$.

BONNARD R.

BOOKER A.

BORDONNEAU M.-A.

BORGETTO M.

BOSCO $\mathrm{H}$.

BOSSIS G.

BOTTON S.

BOURDIN J.

BOURNE C.B.

BOYER-CAPELLE C.

BRACONNIER S.

BREILLAT D.

Bretton Woods

BRIAND A

BROWN-WEISS E.

BRUNEL S.

BRUNET P.

BUENGERTHAL T.

BUIRETTE P

BURCHI S

BURDEAU G.

BURGORGUE-LARSEN L.

BURKE J.

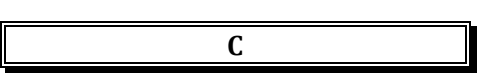

CAFLISCH L.

CAILLOSSE $\mathrm{J}$.

CALASANS J. T.

CAMBY J-P.

CANS R.

CAPONERA D. A.

CARAYON J.

CARBONNIER J.

CASSAN H.

CATY G.
32,321

325

325

143,321

325

325

325

317,326

317,326

$99,152,158,314$

326

191,312

326

317

$104,105,106,312,326,334$

317

320

146,326

317

$34,35,321$

$158,165,166,312$

$47,312,334$

$10,35,56,71,208$

188,326

317

317

315

312

326

334

312

334

317 
CAUDAL S.

313,326

CHAGNOLLAUD D.

CHAMPEIL-DESPLATS V 332, 335

CHAPUS R.

CHARBONNEAU S.

CHARDEAUX M.-A.

CHARVIN R.

CHAVANE Bruno

CHAVRIER G.

CHEROT J.-Y.

CHEVALIER J-M.

CHEVALLIER J.

CHRETIEN P.

Chronique ONU

CLARKE T.

CLATRES P.

CLUZEL-METAYER L.

CNUCED

COLIN F.

COLLARD D

COLSON J-P.

COMPAGNON Daniel, CONSTANTIN

François

CONAC F

CONAC G.

CONCHE M.

CORNU G.

COT J-P.

CRINOT L.

$17,37,110,200$

$34,78,141,148,159,313$

99,314

327

317

313

, 313

327

313

317

317,327

$37,38,317,327,335$

304, 327

313

315

327

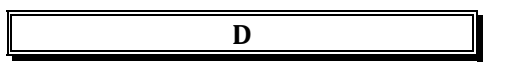

D. SIMON

162,333

DAILLER P.

DAILLIER P. et PELLET A.

315

DAOUDY M.

DARBON D.

DAVANTURE $S$.

DAVID C

DAVID M.

DE BECHILLON D.

De DAVID-BEAUREGARD-BERTHIER O.

DE FORGES J-M.

DE FOURVILLE O

De GAUDEMAR H.

de GAUDUSSON J.

De LAUBADERE A.

DE LAUBADERE A. et VENEZIA J.-C.

DEBBASCH C. et COLIN F.

$186,195,313$

DEBBASCH C. et RICCI J.-C. 313

DEFFAINS B.

DEGOFFE M.

DEGUIGNET P.

DELAIRE Y.

DELVOLVE P. $\quad 35,52,58,61,64,172,254,255,313$, 316,332

DENQUEN J-M.

DESGANE R.

DESIDERI J.-P.

DIJON X.

DONIER V.

DONZIER J.F.

DOUMBE-BILLE S.

DRAGO G.
318

222,328

327

313

$35,52,58,61,64,172,254,255,313$

313

317

317

313

$159,161,165,166,332$

270,317

318,320

$60,312,313,316$
DRAGO R.

193,312

DROBENKO B. 22, 24, 28, 33, 43, 48, 49, 98, 189, 228,

$236,245,270,271,273,304,318,327$

DROUET D.

Du MARAIS B.

DUBOIS M.J.M.

DUGUIT L.

DUMONT G.

DUMONT H.

DUPONT-RACHIELE J.

DUPRE de BOULOIS X.

DUPUIS G.

DUPUY P.M.

DUPUY P-M.

DUROY S.

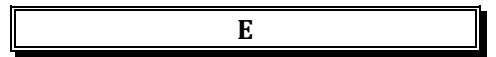

ECKERT G.

EISENMANN C.

Ejan MACKAAY

El Hadj GUISSE

ERGEC R.

ESMEIN A.

ESPLUGAS P.

ETIENNE J.

EUZEN A

EUZEN A.

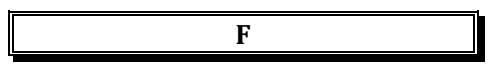

FALQUE M.

FAURE B.

FAURE M.

FAURE M. XE "FAURE M." ,

FAVOREU L.

FAVRET J.-M.

FENET A.

FEUER G.

FIAILAIRE J.

FISETTE J.

FLAUSS J-F.

FOILLARD Ph.

Fortuné B. AHOULOUMA

FOULETIER M.

Francis DELPEREE

FRIER P.-L.

FROUVILLE (de) O.

327

333

270, 298, 318, 328

$270,298,318$

47, 252, 253, 314

$281,318,327$

$198,199,202,314,318$

216,314

$73,74,328,335$

$40,328,335$

129, 314

248

314

319

158,314

322

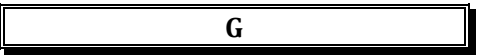

GAITTI Jules

GARCERIES S.

GARREC C.

GAUDEMET Y.

GAY L.

GAZZANIGA J.-L.

GAZZANIGA J-L.

GENEVOIS B.

GHEZALI M.

GIBRIL N.

GODFRIN P.

GOHIN O.

GONOD P
304, 318

$99,115,322$

318

275, 314

322

328

318

47, 334

335

328

222, 314, 328

314 
GOUNELLE M.

GRAWITZ M.

GREWE C.

GUEDON M.-J.

GUETTIER C.

GUGLIELMI G.

GUGLIELMI G. J.

GUILLIEN R.

GUILLOT Ph. Ch.-A.

GUISELIN Emmanuel-Pie

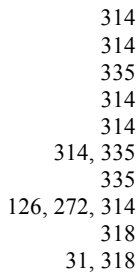

\begin{tabular}{lr}
\hline \multicolumn{1}{|c|}{ H } \\
\hline \hline HALL D. & 203,328 \\
HASTINGS M. & 333 \\
HAUMONT F. & 318 \\
HAURIOU M. & 35,336 \\
HAYEK F. & $24,315,319$ \\
Hélène TRUDEAU & 333 \\
HENNEBEL L. & 333 \\
HENNION-MOREAU S. & 110,328 \\
HENRY A. & \\
HERTZOG R. & \\
HERTZOG R., Recherche sur la gratuité et la & \\
$\quad$ non gratuité des services publics, & 151,323 \\
HUBRECHT H.- G. & 323 \\
HUBRECHT H-G. & 255 \\
HUGON P. & 221,328 \\
HUGON Philippe & 318 \\
HUNT Paul & 218,328
\end{tabular}

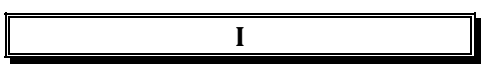

IDOUX P.

INSERGUET-BRISSET V.

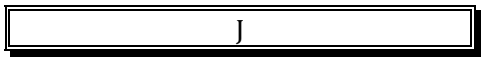

JACKOWSKA Jonathan

81,328

JACQUART A.

JACQUEMOT P.

217,318

JAGLIN S.

$73,328,336$

JAUME L.

JAWORSKI V.

JEANCLOS Y.

JEZE G.

74, 151, 210, 219, 236, 237, 328

338

325,327

78,314

\section{K}

KAMERI-MBOTE Patricia

KAMTO M.

KASSERE A. F.

KAUFFMANN C.

KDHIR M.

KIPPING Martin

KISS A. 97, 312, 318, 331, 337

KISS A.,

KOTOKO M.

KOUANDA I.

KOUBI G

KOUBI G.

KPODAR A.
328

318,328

323

329

$69,329,336$

$$
329
$$

$97,312,331,337$ 207,323 94,329

$99,151,153,155,156,209,314,329,336$ $99,153,155,156,209,314,329,336$ 240,336

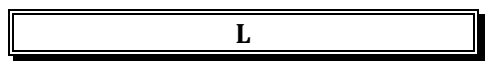

LABERGE H.

LACHAUME J.-F.

$99,158,314$

LACHAUME J-F.

152,314

182,329

LAIME M.

$44,314,318$

318,329

LAMARQUE J.

LARBI BOUGUERRA M.

LARBI BOURGUERRA M.

LAVIEILLE J.M.

LAVIEILLE M.

LE CHATELIER G.

Le MOAL R. et BEURRIER J.-P.

$304,305,336$

30,329

318

318

312

329

LEBRETON G. $\quad 314,336$

LEBRIER S. $\quad 232,329$

LECERF M. 265, 318

LEMAITRE A. $\quad 188,326$

LESUEUR J-Y. $\quad 56,76,319,330,333$

LIGNIERES P. $\quad 315$

LINOTTE D. $\quad 140,315$

LOBINA E. 203, 328

LOCHAK D. 313

LOMBARD M. $\quad 60,100,253,312,315$

LONG M. $\quad 72,315$

LOPA DUFRENOT M. 79, 167, 258, 261, 271, 275, 319

LUSSON-LEROUSSEAU 225, 329

M

M. DEGOFFE

314

M. MCFARLAND

336

MADIOT Y.

MAILLARD DESGRÉES du LOÛ D. $\quad 265,319$

MALAFOSSE de J.

MALINGREY Ph.

MALJEAN-DUBOIS S.

319

MALLET-BRICOUT B.

319,336

MAMONTOFF C.

MARBOUTY F.

MARC P.

MARC Ph.

MARCUS M.

MARQUES C.

MARTENS P.

MASSENET M.

MATHIEU B.

MATHIEU-IZORCHE M.-L.

$2,315,329$

315

323,324

223,319

319

317

323

315

319

MAURIZE M-O.

MBEMBE A.

MCCAFFREY S.C.

MELCHEM K.

MELIN-SOUCRAMANIEN F.

MENARD C.

MENY Y.

MERINO M.

MESCHERIAKOFFA-S

MESTRE J. -L.

MESTRE J-L.

MEUBLAT G.

MICHAUD E.

MILL J. S.

MODERNE F.

MONPION A.

MONTIALOUX Claire
51,315

315

329

329

334

315

$155,259,329$

315

58,329

315
336

$58,59,78,333$

$39,315,336$

51,329

162,333

61,333

77, 329 
MORAND-DEVILLER J.

MORANGE J.

MOREAU J.

MORVAN P.

MOULIN R

MOURGEON J.

$\mathbf{N}$

NADON O.

NDIAYE Tamsir

NELSON N.

NGNIKAM E.

NGONDANKOY N.

NGUYEN Q. D.

NICINSKI S.

NILSSON D.

NLEP R. G.

NYASSOGBO G.K.

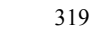

330
213,330

317,319

315

315

315

190, 330

336

330

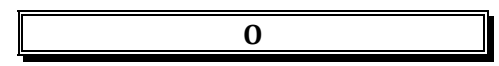

OBERDORFF $\mathrm{H}$.

315

OLAKA J.-M.

$43,44,323$

OLAKA J-M.

$43,44,323$

OLOWU D.

337

OSSOMBO-YOMBO R. 147, 183, 185, 188, 230, 323

OST F.

$153,280,319$

OUGUERGOUZ M. F.

ÖZDEN Melik

30,330

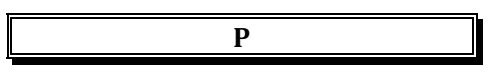

P. DAILLIER

PAPON P.

320

PAQUEROT S.

PÂQUES M.

PAULIAT H.

PECES-BARBA MARTINEZ G.

PEIGNOT B.

PELLET A.

PELLETIER M-L.

PELLOUX R.

PERROT R.

PEZON C.

PHILIP L.

Philippe REKACEWICZ

PICARD E.

PIGOU A.C

PLANE P.

PLASKOWSKI S.

PLATON S.

PLOYE François

PONTHOREAU M.-C.

PONTHOREAU Marie-Claire

PONTIER J.-M.

POYDENOT A.

PREVOST D.

PRIEUR M.

PRIEUR M. et DOUMBE-BILLE S

PUTELAT E.

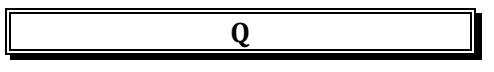

QUIOT G.

59,324

QUOC DINH N.

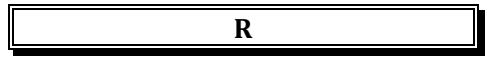

R. KOVAR

162,333

RAYMOND S.

162,333
327

REMOND-GOUILLOUD M. $\quad 280,320$

RIBOT C.

RICHARD V.

RICHER L.

330
308,330

RICHIER L.

RIVERO J.

ROCHE P.A.

ROMAN D.

ROMANO C.

ROMI R.

ROSANVALLON P.

ROSENBAUM A.

ROSENBERG D.

ROUBAN L.

$1,154,330$

316

$46,59,142,243,309,316,333,337$

320

316

317

140,320

316

260, 337

ROUGET D.

ROUSSEAU D.

ROUSSEAUX S.

ROUSSELET A.

ROUSSILLON H.

ROYER P.

RUNYAN C.

$202,316,337$

161,333

320

275,337

316

330

330

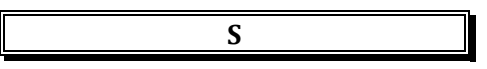

SADELEER N.

SAINT-JAMES V.

320

SAKHO P. O.

SALEM Gérard

SALES E.

Salif DIOP

SALMAN

SALMI M.

SALMON J.

SALOMON J.

SANDEVOIR P.

SANI ADAMOU M.

SANTULLI C.

SAOUT A.

SAVY F.

SAYOUR A.

SCOTT C.

SEILLER B.

SERIAUX A.

SHIVA V.

SHUVAL Hillel. I.

330,337

330

337

80,320

$317,320,325,326$

$73,74,328,335$

240, 316

154,324

142,334

206, 330

316

320

69,337

183,330

218,330

316

316

$236,288,320,331$

225,331

SIAT G.

SIGNOURET

SIMONIAN-GINESTE H.

331

75,337

79,337

50,331

SIRONNEAU J.

SMETS H. 24, 26, 27, 28, 33, 40, 49, 54, 87, 89, 91, 93, $94,97,163,185,288,320,331,337$

SOHNLE J.

SOLEIL S.

$202,320,324$

SORMAN G

142,320 
SOSSOU BIADJA C. J.

STIRN B.

SUDRE F.

SUEUR J.-J.

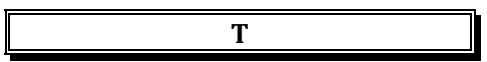

T. HIGGINS

TAITHE A.

TAITHE Alexandre

TALOR J. LENGELLE J.G.

TANAWA E.

TCHIKAYA B.

TEITGEN-COLLY C.

TENIERE-BUCHOT P.F

TERRE D.

THAURY M.

THEYS J.

THIAM A.

THUNIS X.

TIGNINO M.

TIGNINO M. et YARED D.

TIZIO S.

TREMOLET S.

TREMOLET, $S$.

TROUILLY P.

TRUCHET D.

243, 334

TURPIN D.

$78,100,117,120,145,149,154,172$,

$181,309,320$

$244,303,304,320$

86,330

317,319

316
154,320

56,320

316

316

$245,246,331$

146,331

$95,96,331$

320,331

331

338

119,331

219,331

246,331

316,338

\section{U}

UHRY M.
31,320

\begin{tabular}{|c|c|}
\hline \multicolumn{2}{|c|}{$\mathbf{V}$} \\
\hline VAN LANG A. & 97,316 \\
\hline VANDANA S. & 320 \\
\hline VEDEL G. & $45,46,47,59,243,316$ \\
\hline VENNETIER P. & 331 \\
\hline VERGNE F. & 331 \\
\hline VERPAUX M. & 315 \\
\hline VIALA A. & 316 \\
\hline VIGNON Y.B. & 338 \\
\hline VIGNON Y. & $65,176,338$ \\
\hline VINCENT Is. & 88,331 \\
\hline VINCENT J. & 126,272 \\
\hline VIRCOULON T. & $151,157,331$ \\
\hline VLACHOS G. & 316 \\
\hline
\end{tabular}

.

$83,166,168,177,334$

\begin{tabular}{|l|}
\hline $\mathbf{W}$ \\
\hline
\end{tabular}

WACHMANN P.

WALINE J.

WALRAS L.

WEBER L.

WETHE J.

WILLYBIRO-SAKO J

308,320

$145,332,338$

Y

YEM GOURI M.

338

\section{$\mathbf{Z}$}

ZIEGLER J.

ZIEGLER J.,

$86,180,198,199,332$

$86,180,199,332$ 
Résumé et mots-clés - Summary and keywords...................................................................9

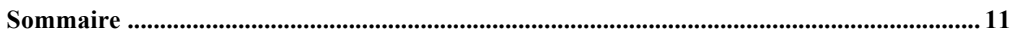

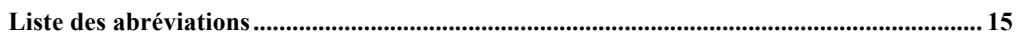

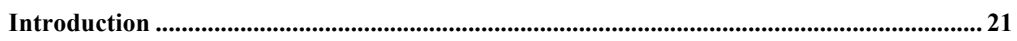

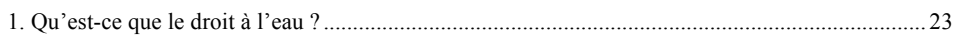

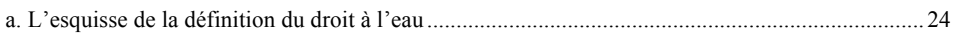

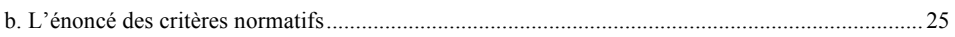

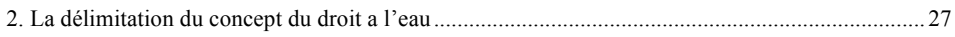

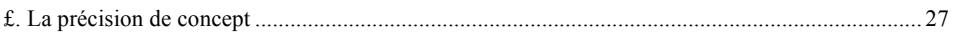

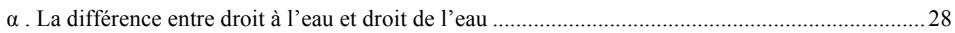

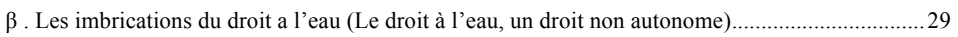

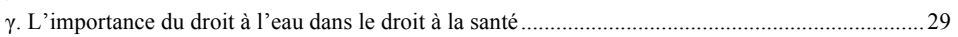

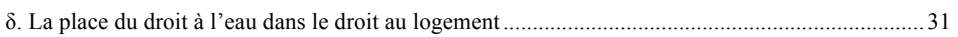

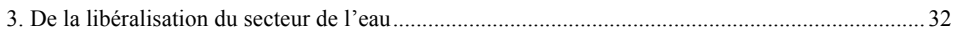

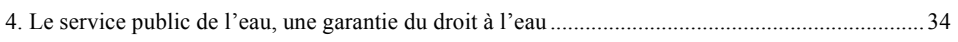

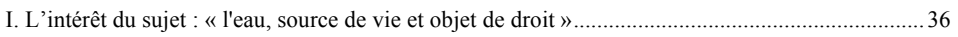

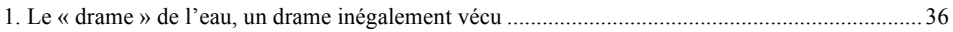

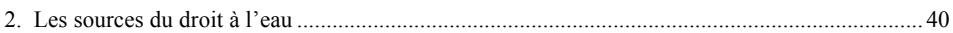

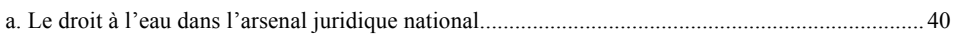

b. Le droit à l'eau dans les instruments internationaux ....................................................................... 41

$\alpha$. Le droit à l'eau dans les instruments sous-régionaux et régionaux .................................................... 42

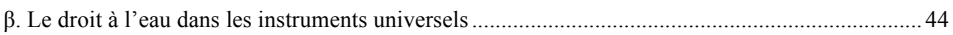

3. La question de l'existence du droit à l'eau et le débat sur la hiérarchie des droits fondamentaux. 45

4. La déclaration du 28 juillet 2010, une étape décisive dans le processus de ................................... 48

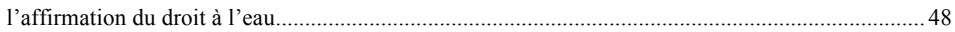

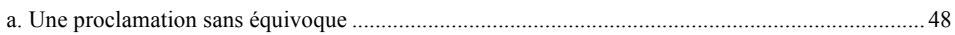

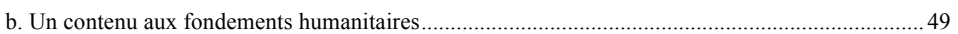

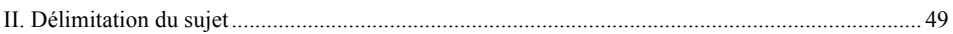

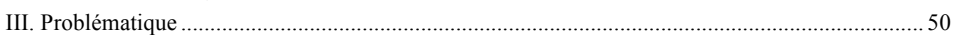

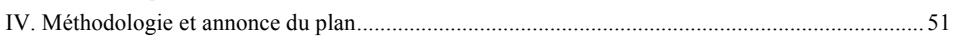

Partie I. La réalisation de la libéralisation du secteur de l'eau ..............................................53

Titre I. La pertinence de la désétatisation du secteur de l'eau en Afrique subsaharienne. 55

Chapitre I. La privatisation du secteur de l'eau juridiquement expliquée............................57

Section I. La privatisation du secteur de l'eau à l'aune du principe de liberté d'entreprendre ...........58

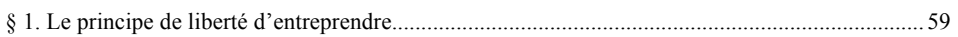

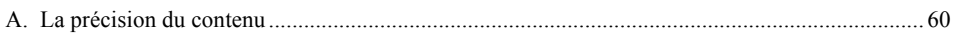

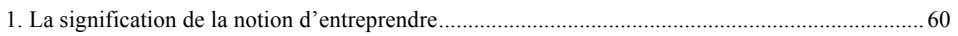

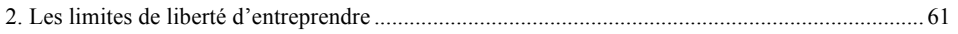

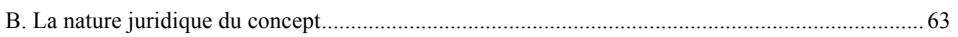

1. Une liberté économique et un droit fondamental........................................................................... 64

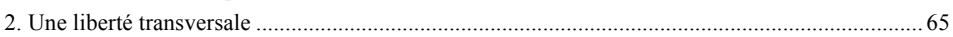

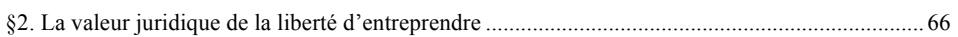

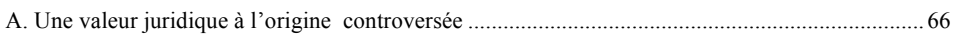

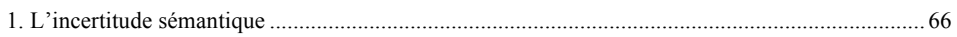

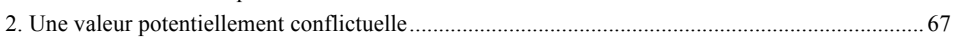

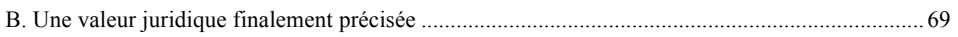

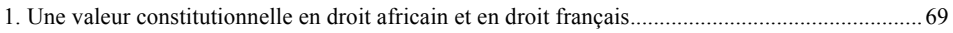

2. Une valeur constitutionnelle surestimée ....................................................................................... 71

Section II. La portée de la consécration du principe de la liberté d'entreprendre ................................ 71

$\S 1$. Le début de la désétatisation du secteur de l'eau ........................................................................ 72 


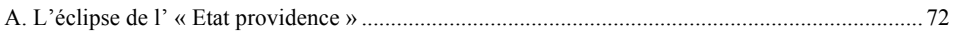

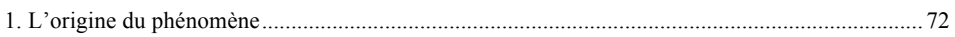

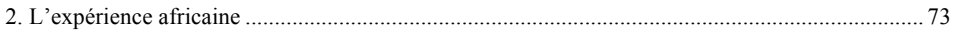

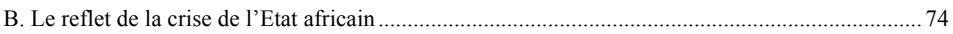

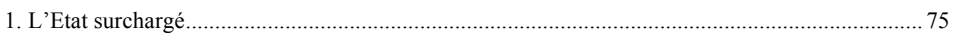

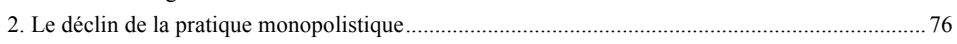

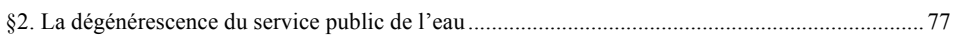

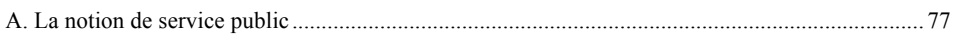

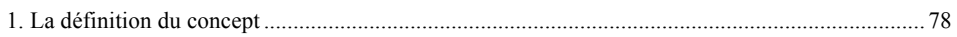

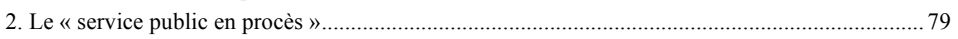

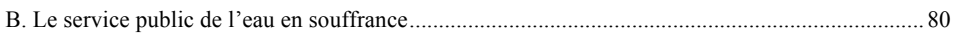

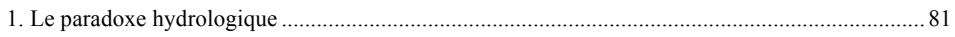

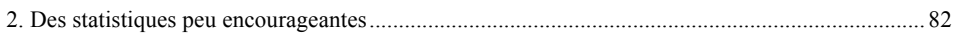

Chapitre II. La privatisation du secteur de l'eau économiquement expliquée.....................85

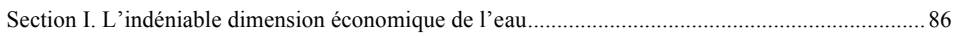

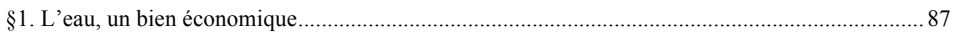

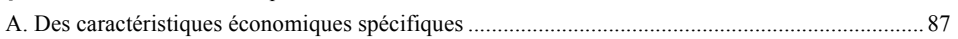

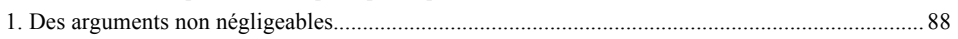

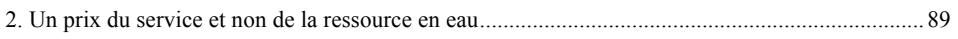

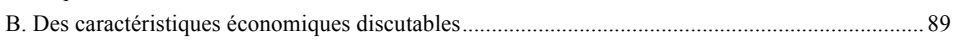

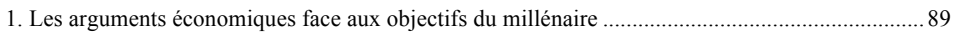

2. L'incertitude de l'Afrique subsaharienne face aux enjeux de l'eau ................................................ 90

§2. Les obligations financières des usagers de l'eau ...................................................................... 91

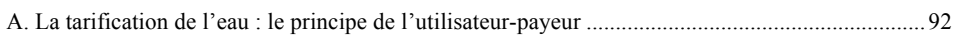

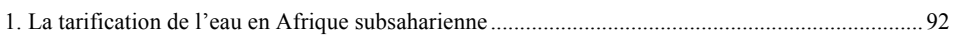

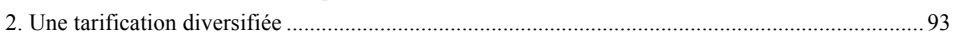

B. La prise en charge du coût de restauration et de préservation de la qualité de l'eau : le principe du

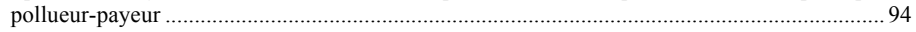

1. Les fondements de la théorie du principe du pollueur-payeur.........................................................95

2. L'eau et la mise en œuvre du principe du pollueur-payeur .............................................................. 97

Section II. Le service de distribution de l'eau, un service à dominante marchande............................98

§1. Un service revêtant en principe un caractère industriel et commercial ........................................ 98

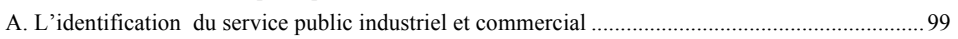

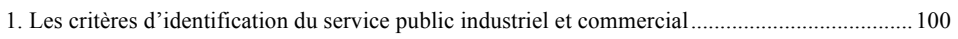

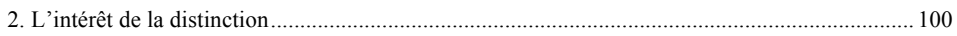

B. Le service de distribution d'eau, un service public à caractère industriel et commercial ............. 101

1. Le service de distribution d'eau, un service public industriel et commercial : la qualification du

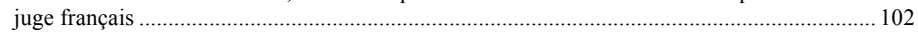

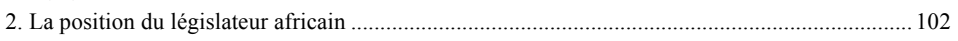

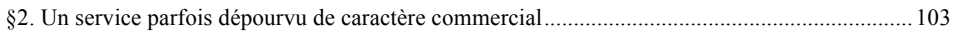

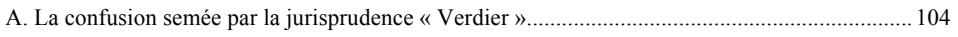

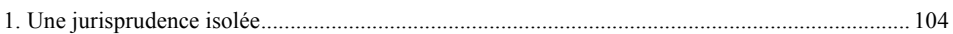

2. Une jurisprudence en marge de la législation en vigueur...................................................................... 105

B. La précarité de l'identification du caractère du service de distribution de l'eau. ............................. 106

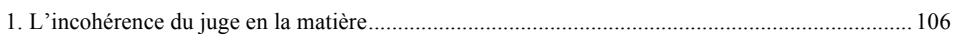

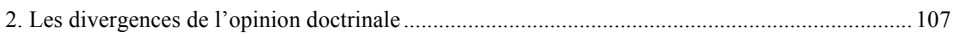

Conclusion du titre I de la Ière partie. ...................................................................................... 109

Titre II de la Ière partie : Les mécanismes juridiques de la................................................ 111

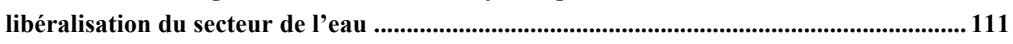

Chapitre I. le mode privilégié de la libéralisation du secteur de l'eau :..............................113

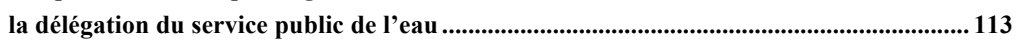

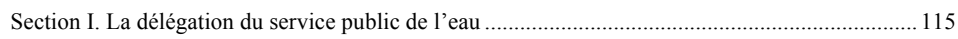


$\S 1$. Les contours de la délégation de service public

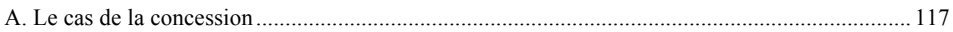

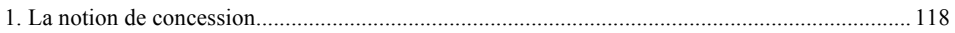

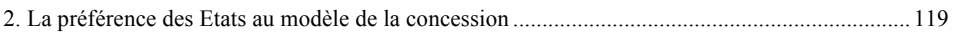

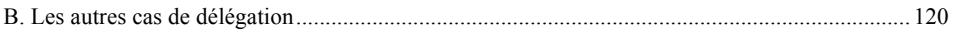

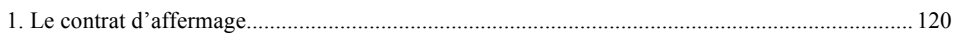

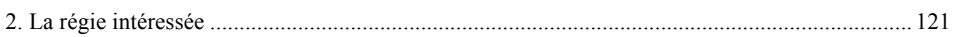

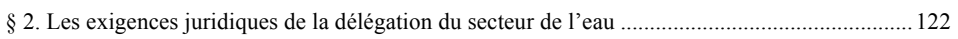

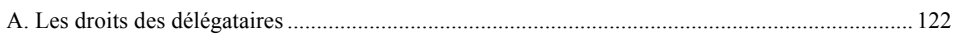

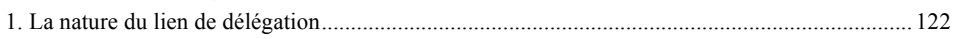

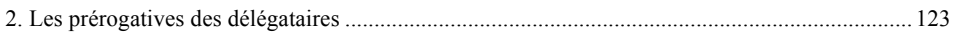

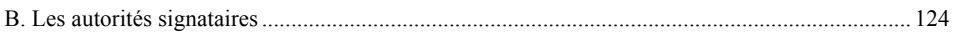

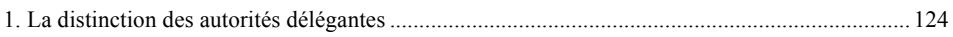

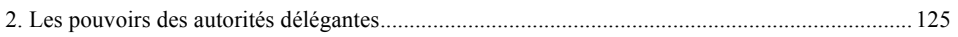

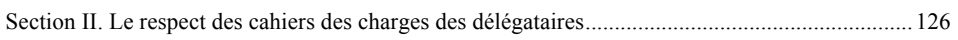

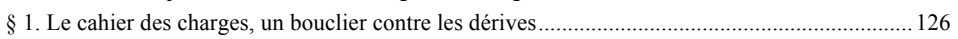

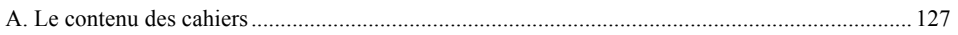

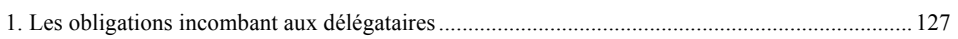

2. Le contrat de délégation de service public de l'eau, un contrat intuitu personae ........................... 128

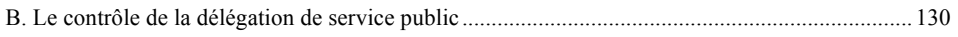

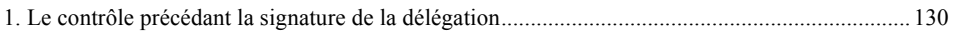

2. Le contrôle de légalité du contrat de délégation de service public.............................................. 131

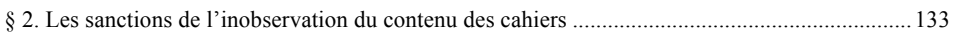

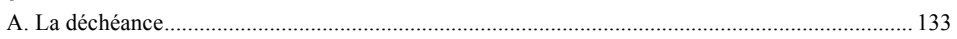

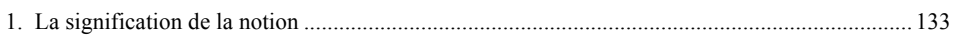

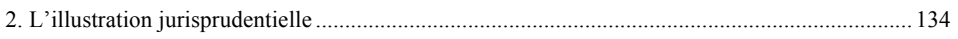

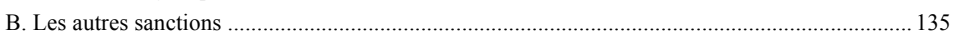

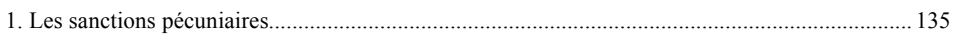

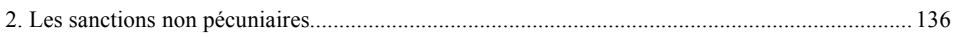

Chapitre II. La mise en berne inéluctable des principes du service public ......................... 139

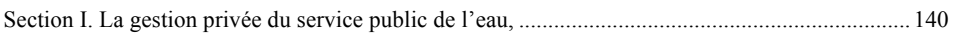

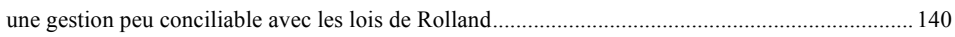

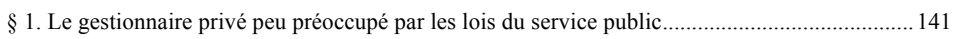

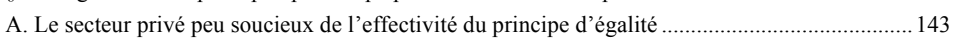

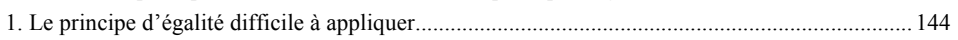

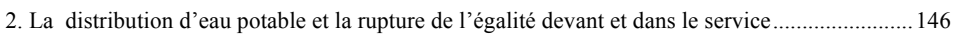

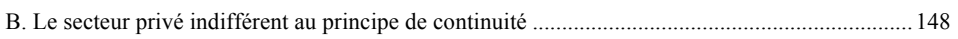

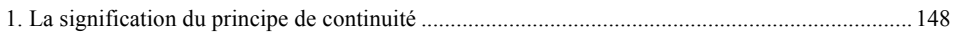

2. L'ineffectivité du principe de continuité dans les services publics ............................................ 149

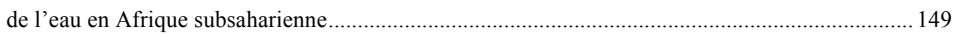

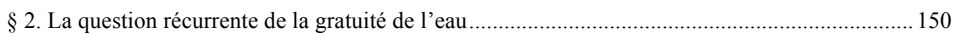

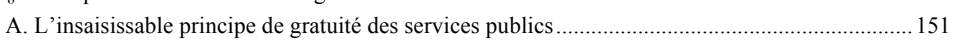

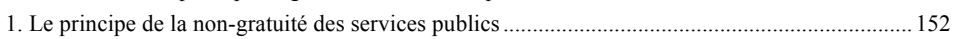

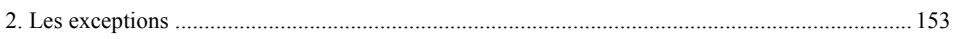

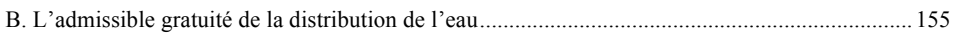

1. Les raisons d'instituer la gratuité de l'eau : la pauvreté des Etats de l'Afrique subsaharienne.... 155

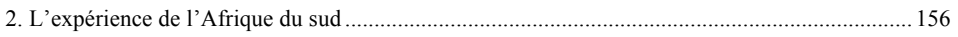

Section II. La gestion privée de l'eau et l'émergence des nouvelles lois du service public.............. 157

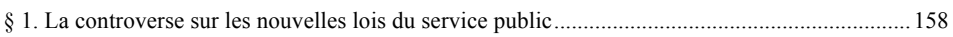

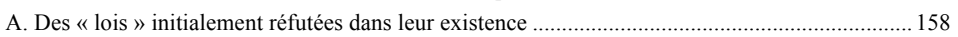

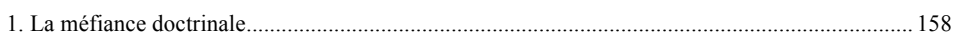

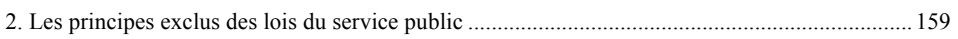




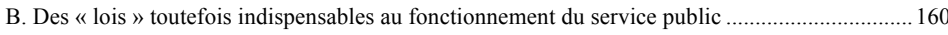

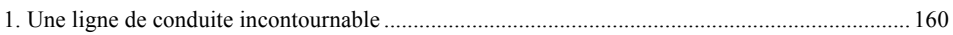

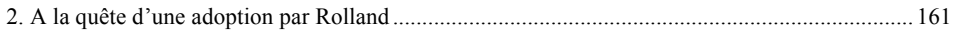

$\S 2$. Les désillusions de la privatisation par rapport aux lois émergeant du service public.............. 162

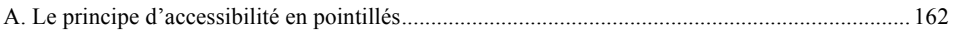

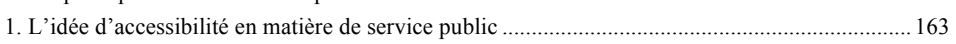

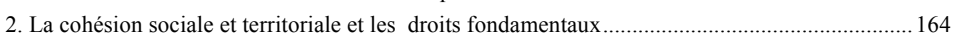

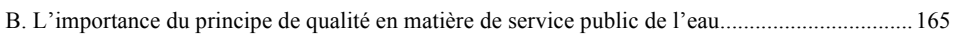

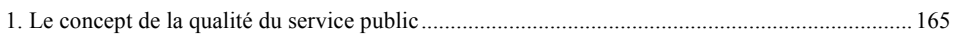

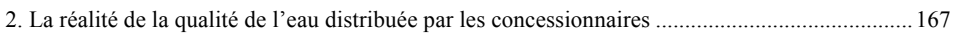

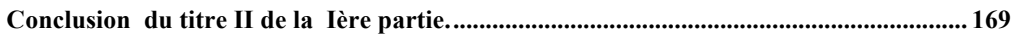

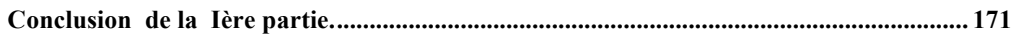

Partie II. Les aboutissements de la libéralisation du secteur de l'eau.................................. 173

Titre I. Les appréhensions suscitées par la privatisation du service public de l'eau ........ 175

Chapitre I. Les conséquences néfastes de la libéralisation du secteur de l'eau .................. 179

Section I. Les déconvenues engendrées par la libéralisation du secteur de l'eau................................ 180

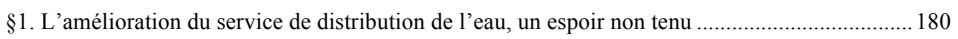

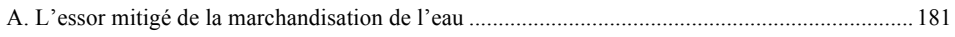

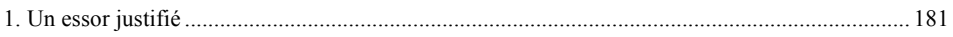

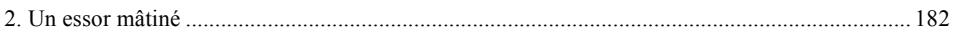

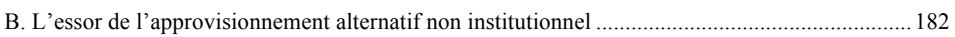

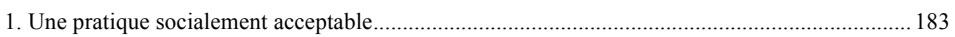

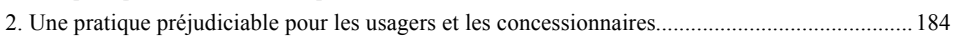

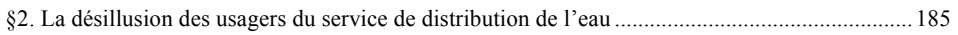

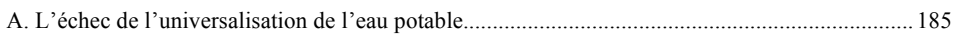

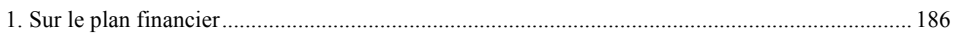

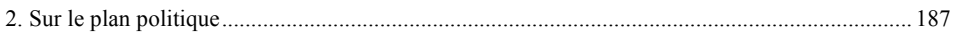

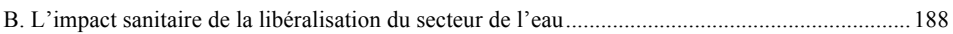

1. Une mise en péril de la sécurité alimentaire ................................................................................. 188

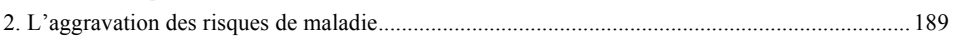

Section II. La libéralisation du secteur de l'eau, un déni de l'Etat .................................................... 190

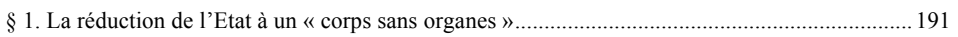

A. Le recul éphémère du procédé de l'établissement public industriel et commercial ...................... 191

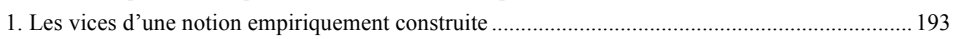

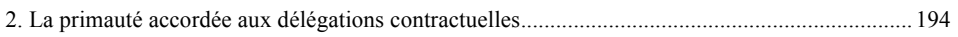

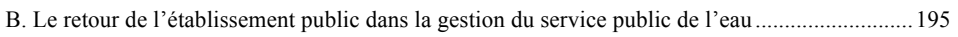

1. L'échec annoncé de la privatisation du secteur de l'eau en Afrique ............................................ 196

2. Les causes de l'échec de la privatisation du secteur de l'eau ......................................................... 197

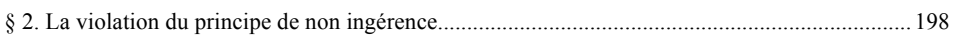

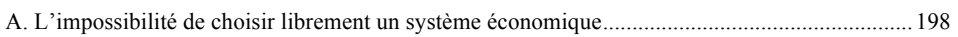

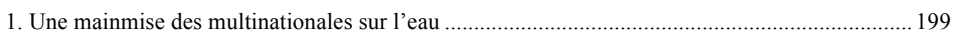

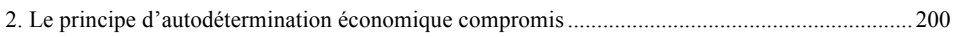

B. L'aliénation du droit de souveraineté permanente sur les ressources naturelles ............................200

1. La théorie du droit de souveraineté permanente sur les ressources naturelles .............................201

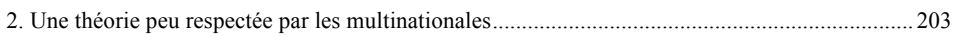

Chapitre II. La libéralisation du secteur de l'eau, .................................................................. 205

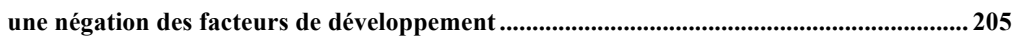

Section I. La privatisation du secteur de l'eau, un frein au développement social............................207

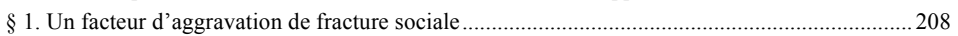

A. Les pauvres, principales victimes de la privatisation de l'eau .........................................................209

1. Le revenu est un facteur décisif pour l'accès à l'eau...................................................................... 209 
2. Les pauvres au centre des inégalités observées dans la distribution de l'eau...............................210

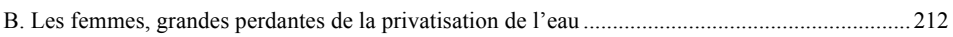

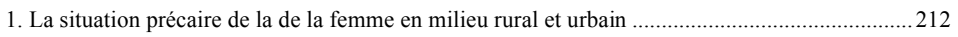

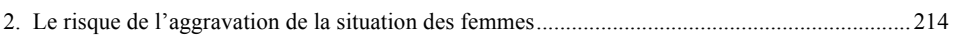

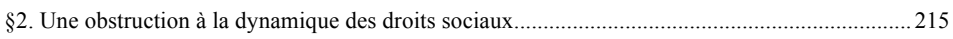

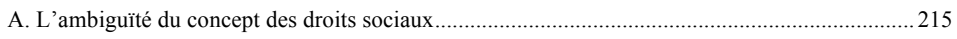

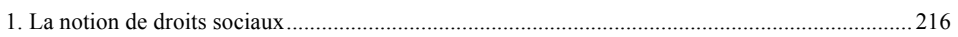

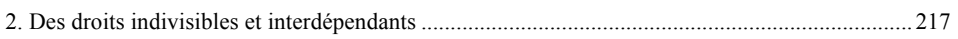

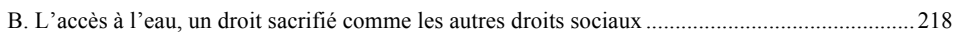

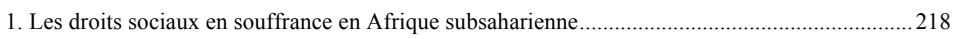

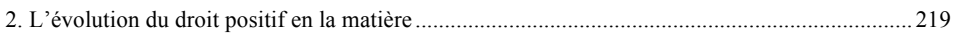

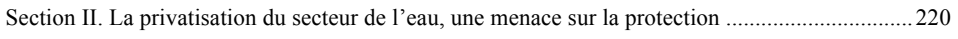

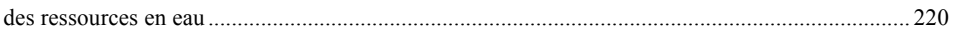

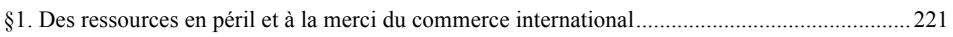

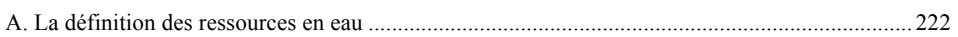

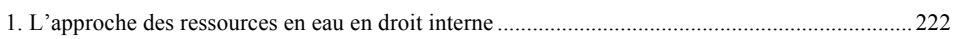

2. L'approche des ressources en eau en droit international .................................................................22 223

B. Le baroud d'honneur du Comité du commerce et de l'environnement de l'OMC ........................225

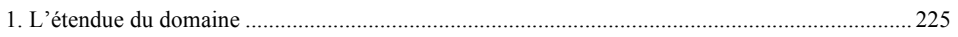

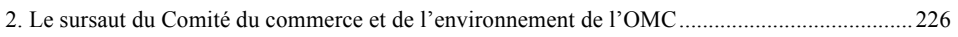

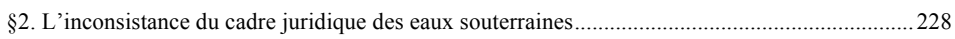

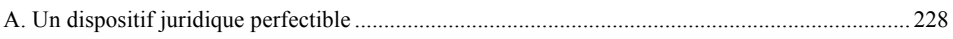

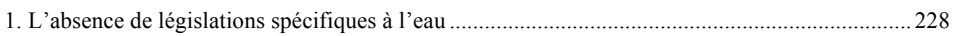

2. Les conséquences des insuffisances législatives ........................................................................22 230

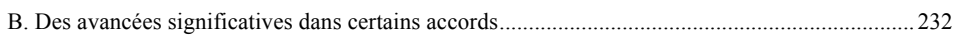

1. Les accords signés par les Etats africains en la matière …...........................................................22

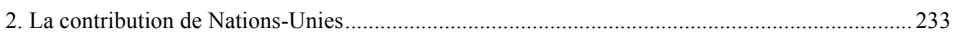

Conclusion du Titre I de la deuxième partie....................................................................2235

Titre II. La nécessaire amélioration des moyens de l'effectivité du droit à l'eau.............2239

Chapitre I. Le retour à une étatisation pragmatique du secteur de l'eau........................... 241

Section I. Le service public de l'eau, un service public objectivement d'intérêt général .................243

§1.L'évidence du caractère d'intérêt général du service public de l'eau en Afrique subsaharienne244

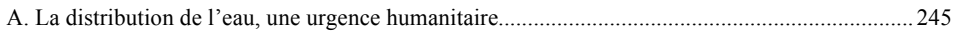

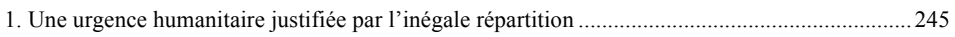

2. Une urgence humanitaire expliquée par des conséquences sociales alarmantes ...........................247

B. La promotion de l'accès à l'eau, un enjeu d'intérêt général. ........................................................2. 248

1. Le développement durable, une mission d'intérêt général ..............................................................248

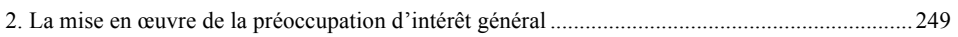

§2.La nécessité d'ériger la distribution de l'eau en service public constitutionnel .............................250

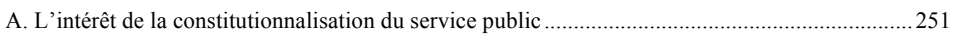

1. L'apparition de la constitutionnalisation du service public de l'eau ..............................................251

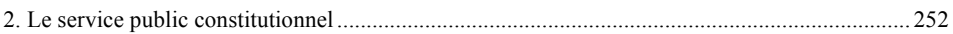

B. La conséquence de la constitutionnalisation : la nationalisation du secteur de l'eau ...................2.254

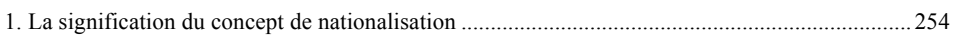

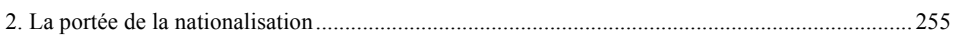

Section II. Le service public de l'eau, un service public d'intérêt local............................................225

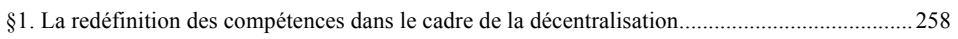

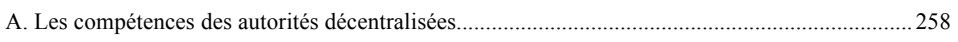

1. Une décentralisation consacrant le droit des collectivités à l'autogestion de l'eau.......................258

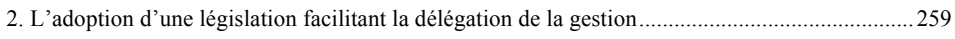

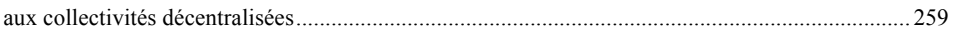

B. La compétence des acteurs autres que les communes...................................................................260 


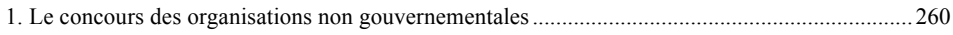

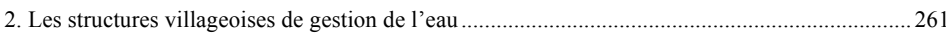

§2. Une gestion décentralisée de l'eau assortie des principes de gouvernance ..................................262

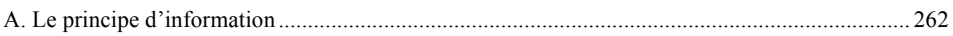

1. Le droit à l'information, un droit reconnu à l'usager du service public de l'eau ..........................2. 263

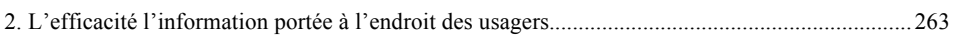

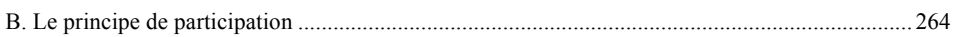

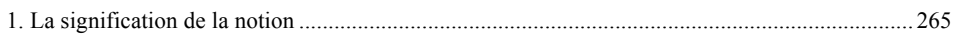

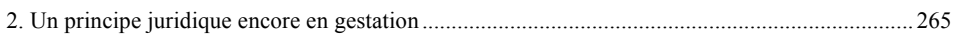

Chapitre II. La nécessité d'une meilleure protection des ressources en eau ..................... 269

Section I. Une protection de l'eau compromise par le statut juridique ambigü de l'eau ....................270

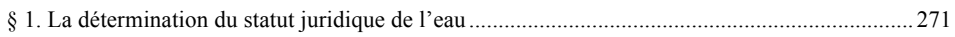

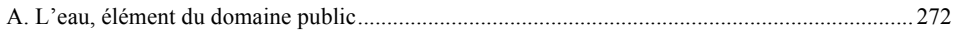

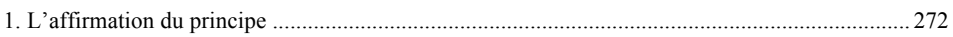

2. La délimitation du domaine public de l'eau ................................................................................ 273

B. La portée de l'admission de l'eau comme élément du domaine public .........................................274

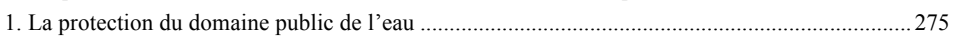

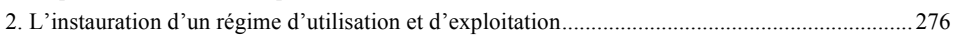

$\S 2$. L'eau en tant que patrimoine commun ou refus de sa marchandisation......................................2 278

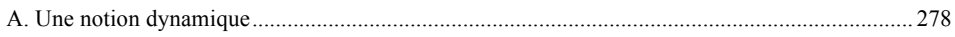

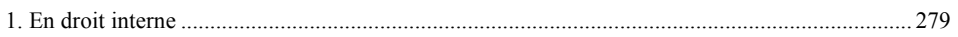

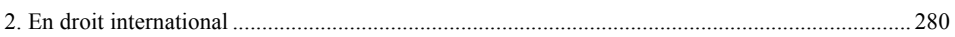

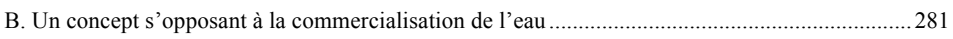

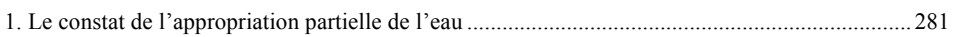

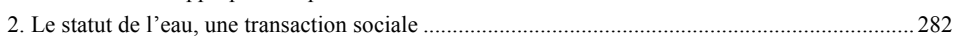

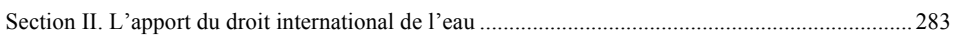

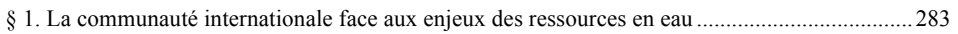

A. L'émergence de la question de l'eau dans les conférences onusiennes sur le social.....................284

1. L'eau dans les préoccupations des conférences sur l'éducation, la population et l'habitat...........284

2. La préoccupation de l'eau dans les conférences sur le genre et l'alimentation ..............................28

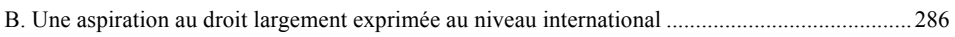

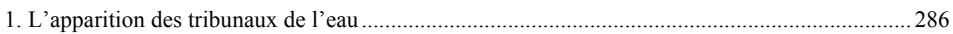

2. Mais un arsenal juridique toujours en quête d'affirmation...............................................................2. 288

§ 2. Le renforcement des actions de la communauté internationale ..................................................28

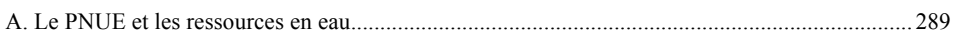

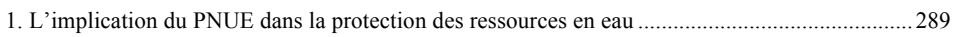

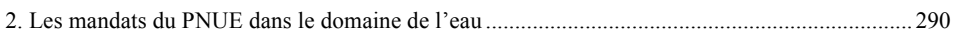

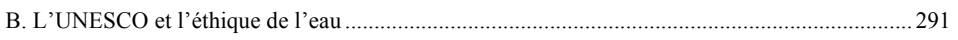

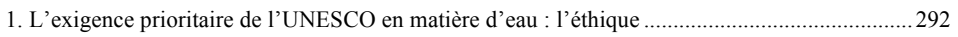

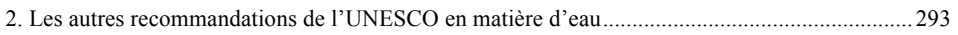

Conclusion du Titre II de la deuxième partie. .....................................................................295

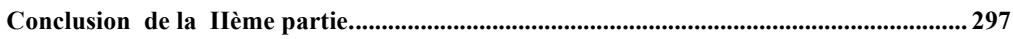

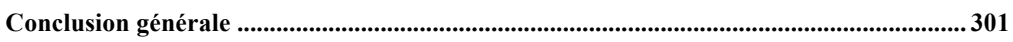

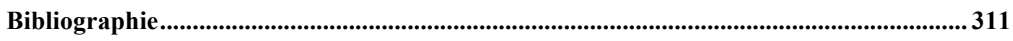

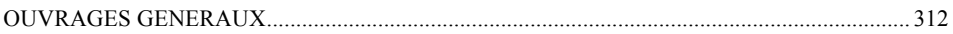

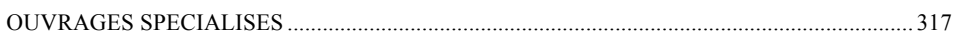

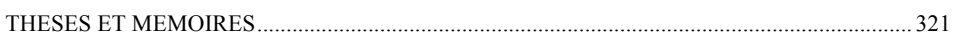

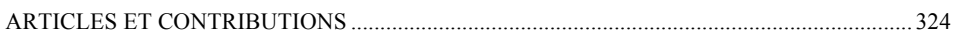

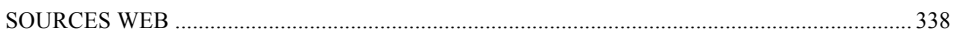

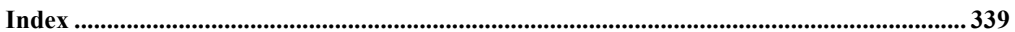

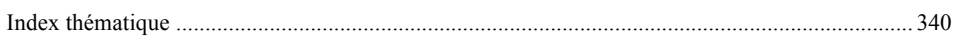

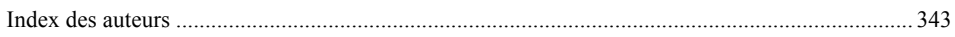




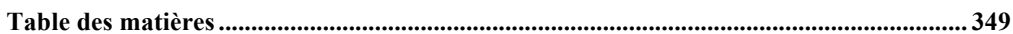

